\title{
Numerical Modelling of Seismic Wave Propagation through a Soft-sediment filled basin near Kinburn, ON
}

\author{
by
}

\author{
Stephen Crane
}

A thesis submitted to the Faculty of Graduate and Postdoctoral Affairs in partial fulfillment of the requirements for the degree of

Doctor of Philosophy

in

Earth Sciences

Carleton University

Ottawa, Ontario

(C) 2016, Stephen Crane 


\begin{abstract}
The unique geological conditions present around the Ottawa region provide the framework for strong amplifications of earthquake ground motions in areas with deep soil, and a basin structure of the bedrock surrounding the soil. Previous techniques used to identify the amplifications at a soil site compared to a rock site have been unable to account for the large amplifications recorded under these conditions. A soft-sediment filled basin was identified near Kinburn, Ontario and was instrumented with several seismometers to record the motions throughout the basin.
\end{abstract}

The basin was explored through several geophysical methods to determine the impedance boundary between the soft sediments and bedrock. A model was created from these measurements in order to complete numerical simulations of the basin. Several 2D profiles were derived from this model along the short and long axes of the basin. Numerical modelling was performed on the model of the basin using a spectral element method and varying physical properties for the material within the basin and for the rock surrounding the basin. These simulations delineate the amplifications within the basin which were caused by the transfer of energy from rock to soil, and those which were caused due to the shape and structure of the basin.

The numerical modelling was performed on both a 2D and 3D homogenous model of the region to recreate the ground motions recorded at a seismic recording station located close to the basin, but placed on a rock outcrop. The soft-sediment basin was added to the model and both the rock station and soil station at the centre of the basin were simulated for the 2013 
Ladysmith earthquake. These motions show similar properties for the first arrivals to the recorded motions at the same location as a result of this earthquake. The simulations were also used to identify different wave types and arrival times within the basin along with the expected motions at other locations within the basin. 


\section{Acknowledgements}

Firstly, I would like to thank my supervisors Dr. Dariush Motazedian and Dr. James Hunter. Their encouragement, guidance, and support were able to provide myself with the necessary knowledge and tools to complete this research project. I would also like to thank the research team, Amin, Sylvia, Sherry, Parisa, and Shutian who have worked on different aspects of parallel projects or similar projects. Their discussion about this topic and their work have helped me with my knowledge on the subject.

Thank you to my fellow grad students and the staff and faculty of the Department of Earth Sciences for the discussions on this topics and others, and to my family and friends for their encouragement, support, and interest in my studies.

Lastly, I would like to thank my loving wife Ashley. Her patience, support, and encouragement helped me greatly throughout my career.

This project was part of the Canadian Seismic Research Network (CSRN), which was funded by the Natural Sciences and Engineering Council of Canada. Financial assistance was provided in several forms by the Department of Earth Sciences, the Faculty of Graduate and Postdoctoral affairs, and the Government of Ontario.

The computing resources required for this project were provided by the High Performance Computing Virtual Laboratory (HPCVL) and Calcul Québec, both under Compute Canada. 


\section{Table of Contents}

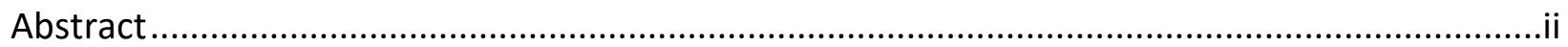

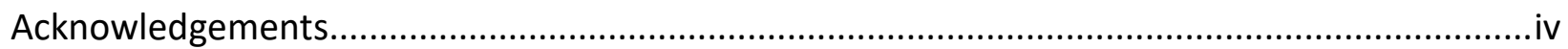

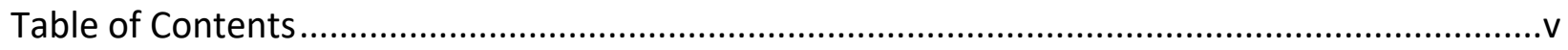

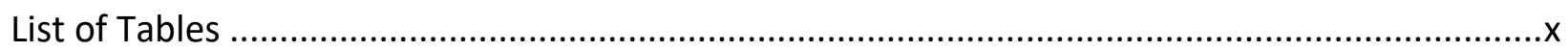

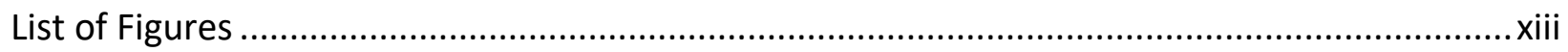

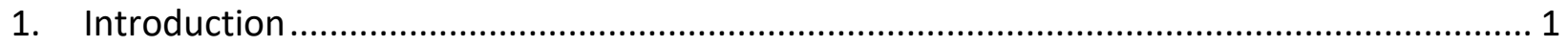

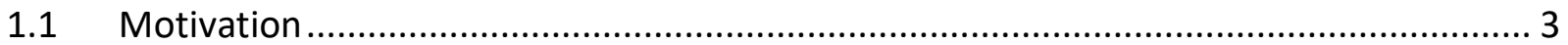

1.1.1 Seismic Hazard of Ottawa .......................................................................... 4

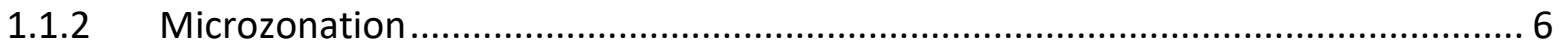

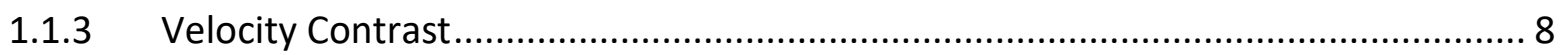

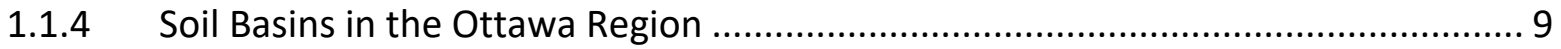

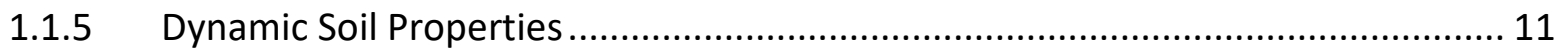

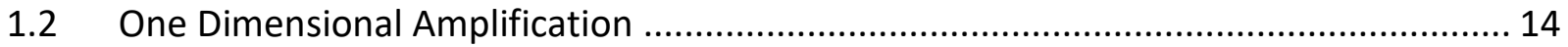

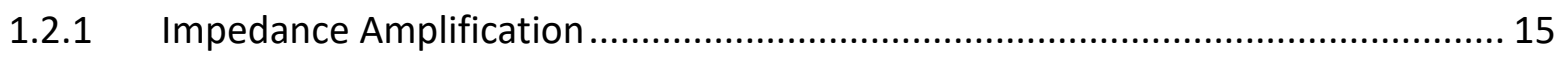

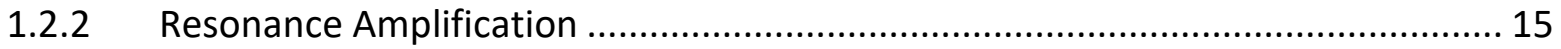

1.2.3 One Dimensional Modelling ......................................................................... 16

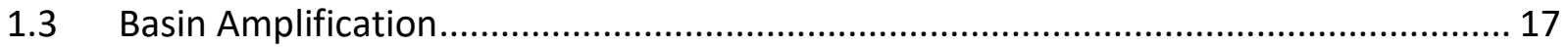

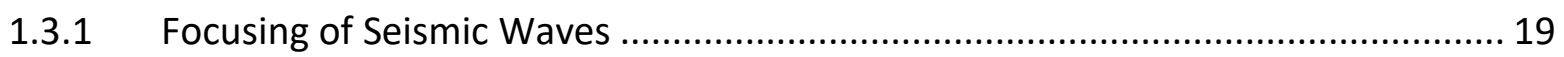

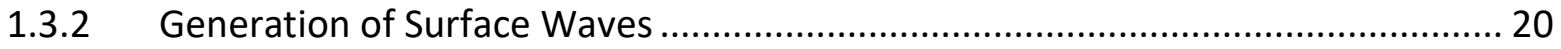

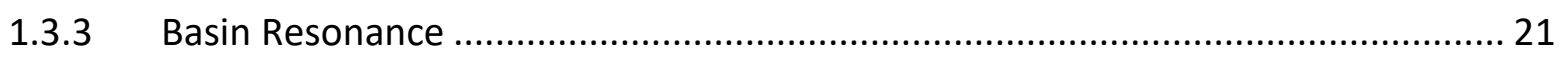

1.4 Comparison of Different Numerical Techniques .................................................. 22

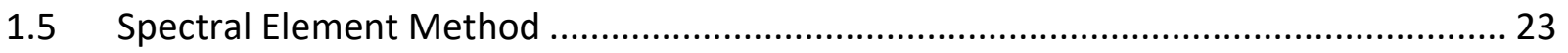

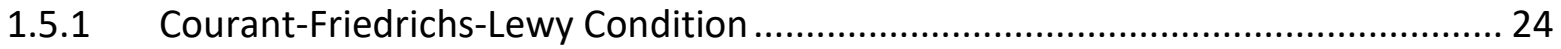

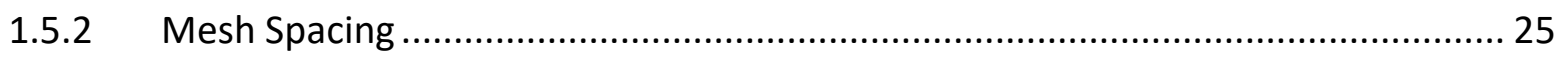

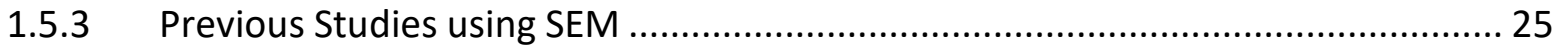

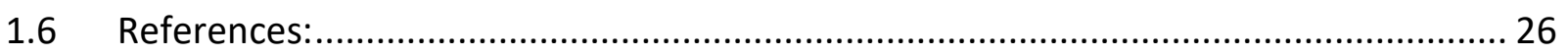

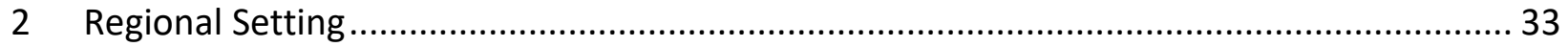

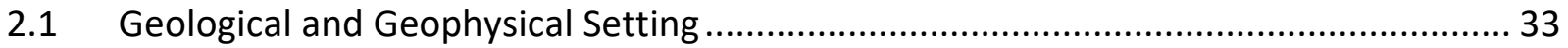

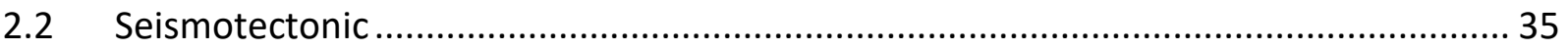




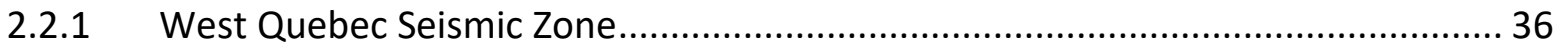

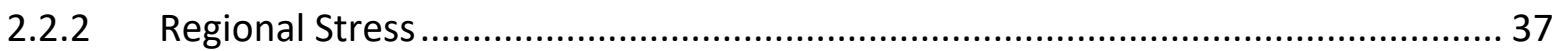

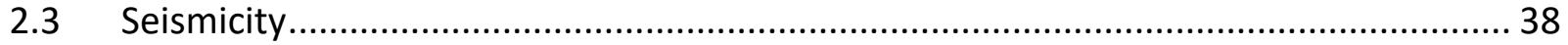

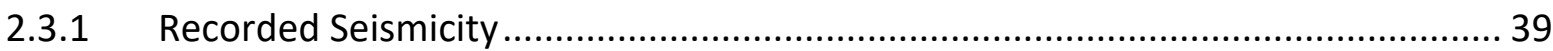

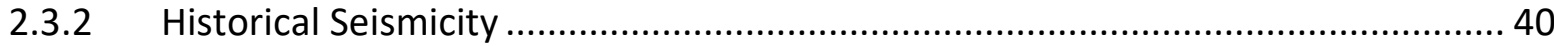

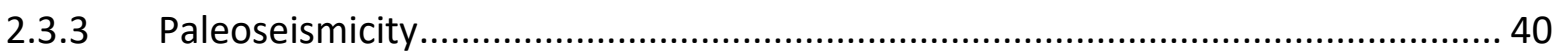

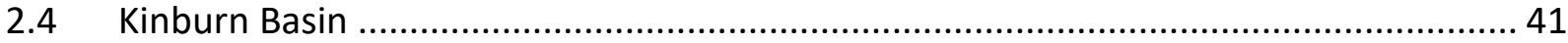

2.4.1 Kinburn Basin Model............................................................................................ 43

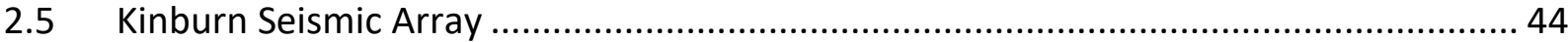

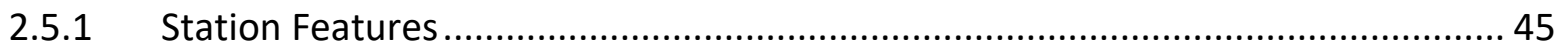

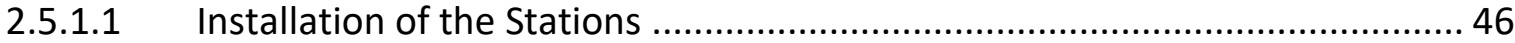

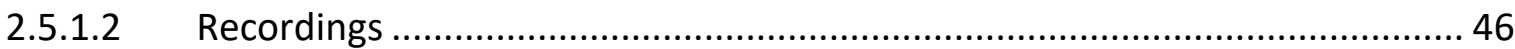

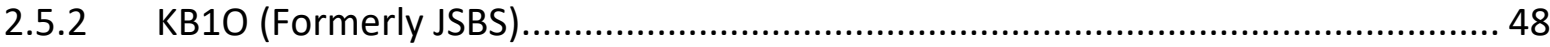

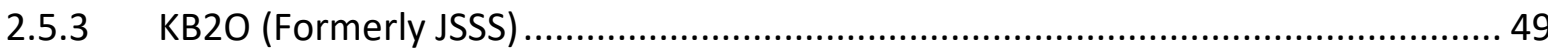

2.5.4 KB3О

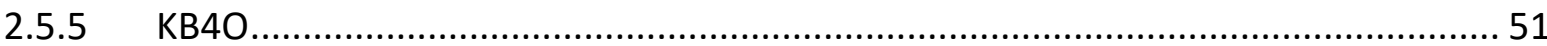

2.5.6 KB5O

2.5.7 KB6О

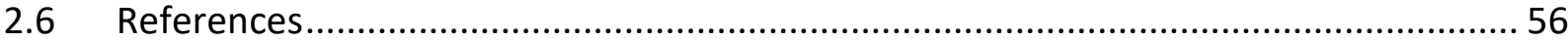

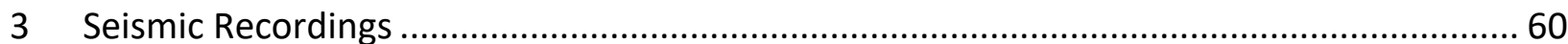

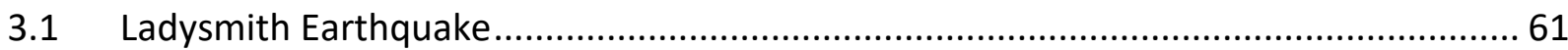

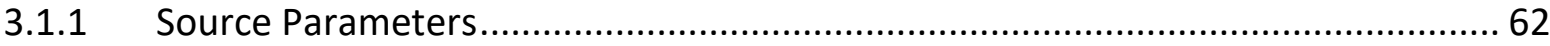

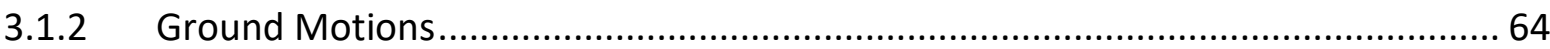

3.1.2.1 Basin Effects from Recorded Motions .......................................................... 68

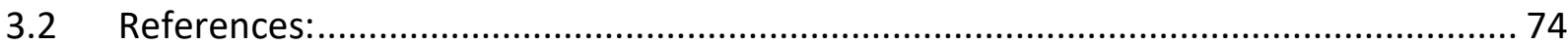

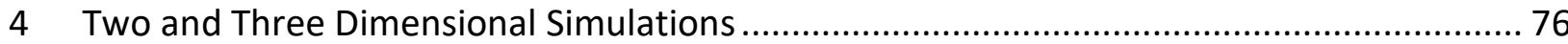

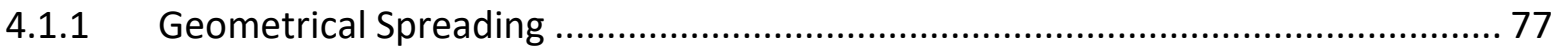

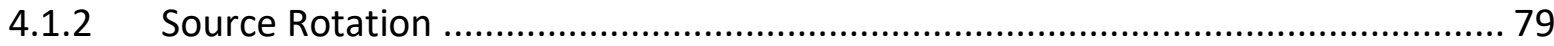

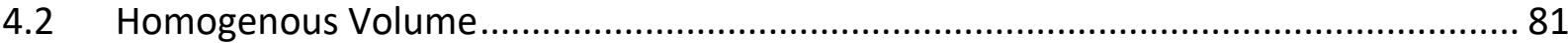

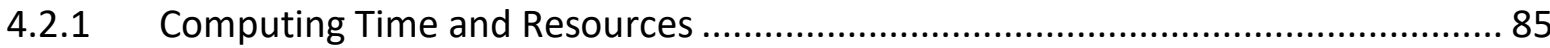




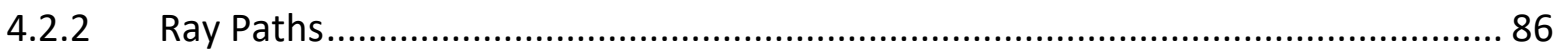

4.2.3 Unit X-direction Force Source ...................................................................... 87

4.2.4 Unit Z-direction Force Source .................................................................. 104

4.2.5 Applying Corrections to 2D Seismograms................................................. 111

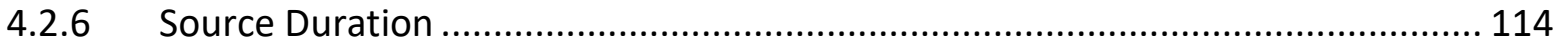

4.3 Ladysmith Earthquake: Rock Site ...................................................................... 118

4.3.1 Modelling using Moment Tensor Source from Bent et al. (2015) ...................... 120

4.3.2 Modelling using Global CMT Source ................................................................ 123

4.3.3 Modelling using Moment Tensor from Ma and Audet (2014) .......................... 125

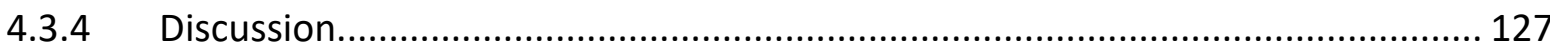

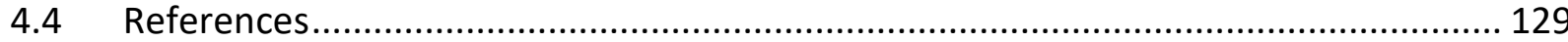

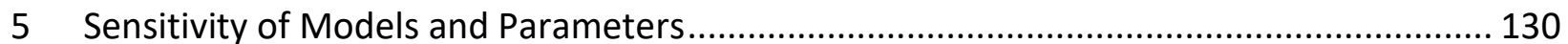

5.1 Factors affecting Computing Resources................................................................. 132

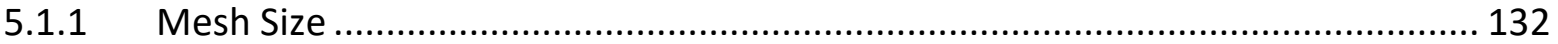

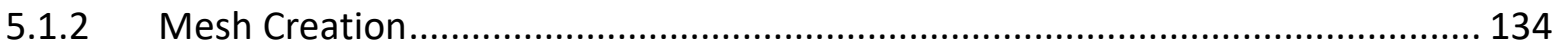

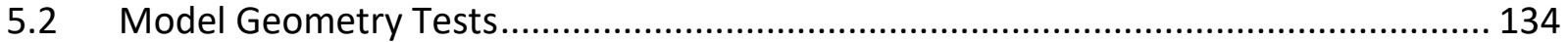

5.2.1 Model Sensitivity: Single Set of Rock Parameters .......................................... 139

5.2.2 Model Sensitivity: Two Sets of Rock Parameters .............................................. 143

5.2.3 Model Sensitivity: Three Sets of Rock Parameters .......................................... 146

5.2.4 Model Sensitivity: Layering Sets of Rock Parameters........................................ 149

5.3 Soil and Rock Parameter Tests ............................................................................... 154

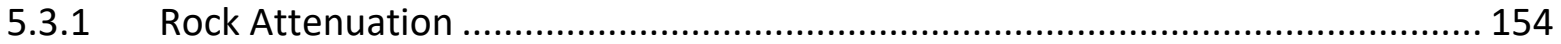

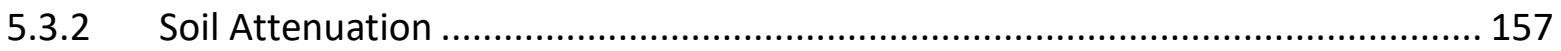

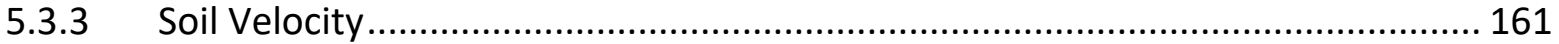

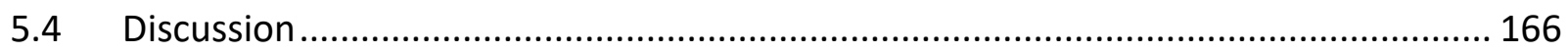

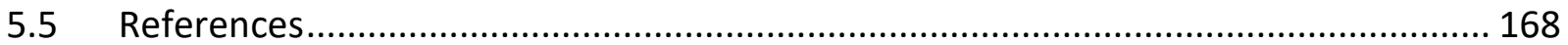

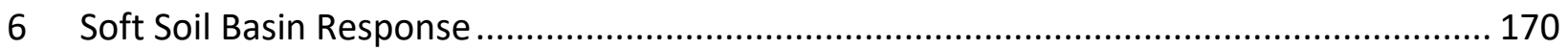

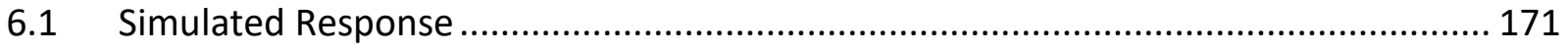

6.1.1 2D Rock and Soil Basins (Model I and Model II) ............................................ 176

6.1.2 2D Soil Layering and Basin Structure (Model III, Model IV and Model V) ........... 179 
6.1.3 2D and 3D Kinburn Basin Comparisons (Models VI, VII and VIII) ...................... 184

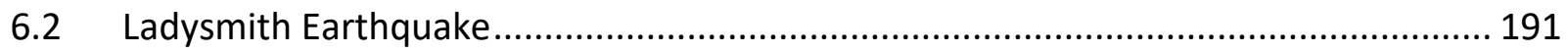

6.2.1 Time Series Matching using the Global Centroid Moment Tensor solution ........ 194

6.2.1.1 P-wave and S-wave Comparison ....................................................... 204

6.2.2 Spectral Acceleration Matching .............................................................. 208

6.2.2.1 P-wave and S-wave Comparison ....................................................... 216

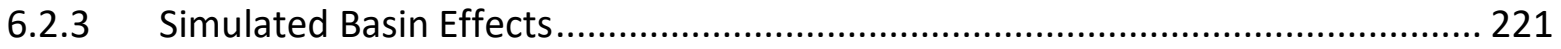

6.2.4 Simulated Motions at the Kinburn Seismic Array........................................... 225

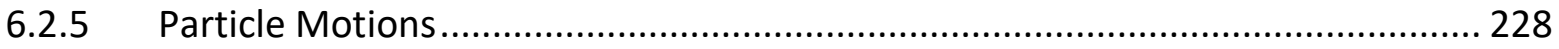

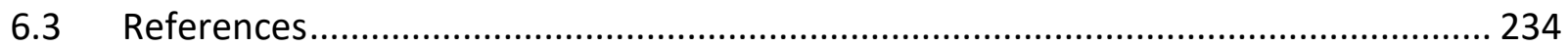

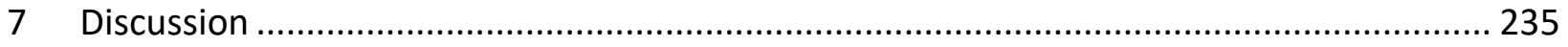

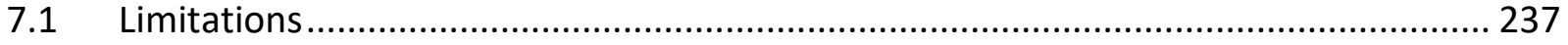

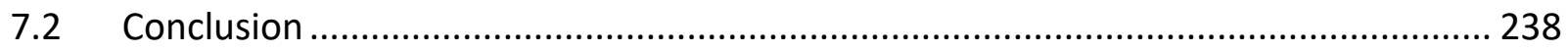

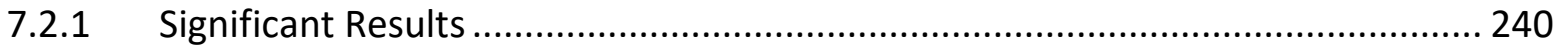

7.2.2 Implications on Seismic Hazard .............................................................. 240

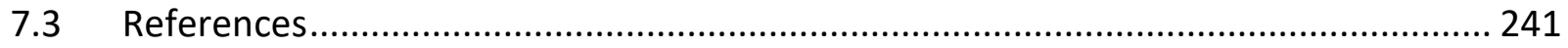

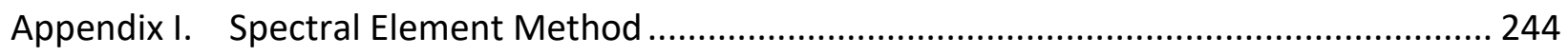

I.A. Constructing the Weak form of the momentum equation................................... 244

I.B. Mesh Construction and Elemental Representation ........................................... 245

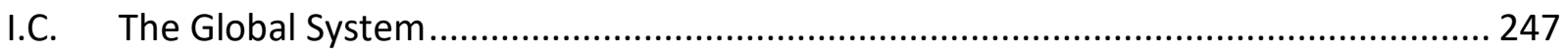

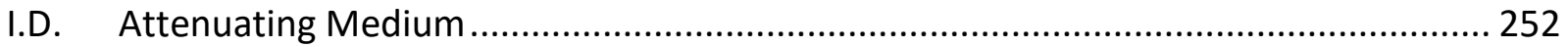

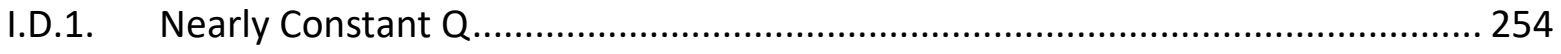

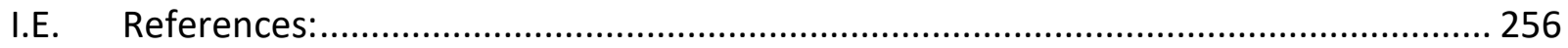

Appendix II. Simulation Properties by Chapter ............................................................ 258

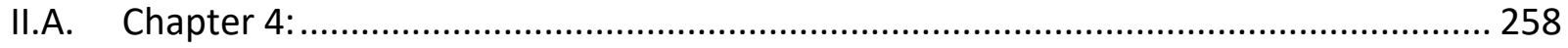

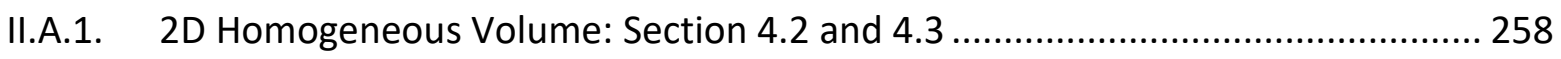

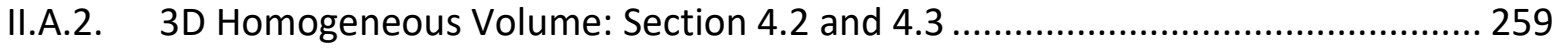

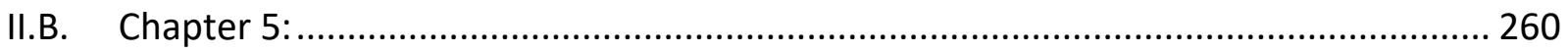

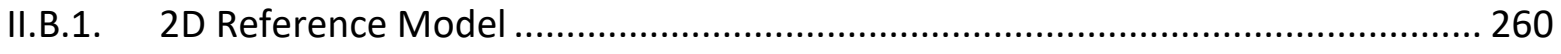

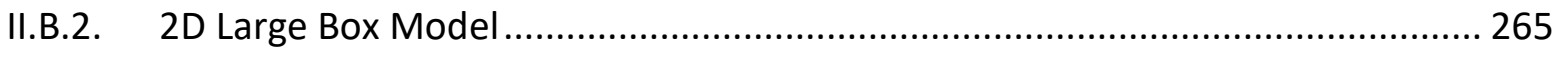




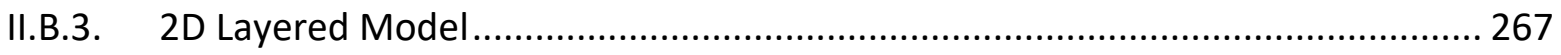

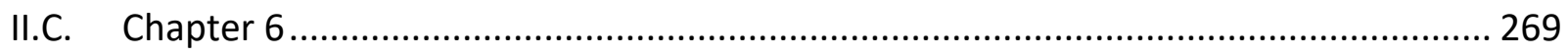

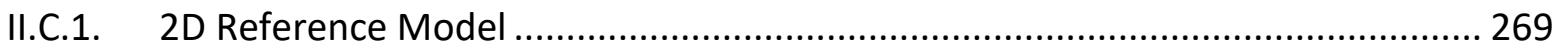

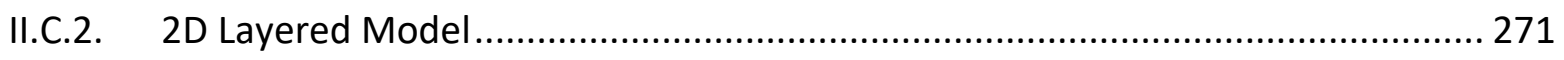

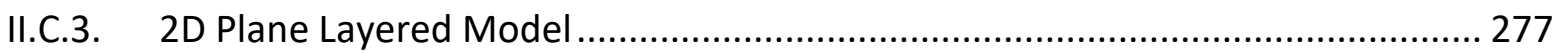

II.C.4. 2D Kinburn NESW Profile Model: Section 6.1.3............................................ 278

II.C.5. 2D Kinburn NWSE Profile Model: Section 6.1.3............................................. 279

II.C.6. $\quad 3 D$ Kinburn Basin Model: Section 6.1.3 ….................................................. 280

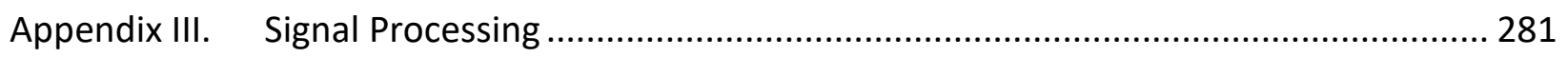

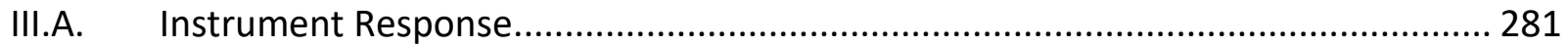

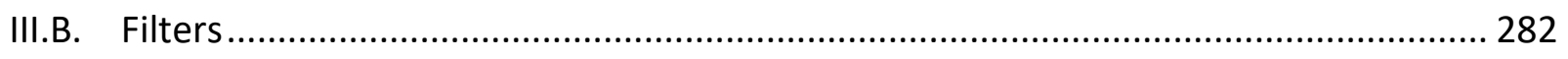

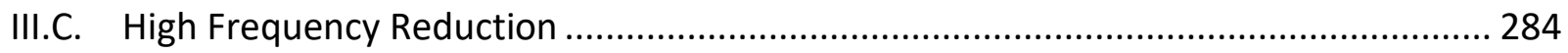

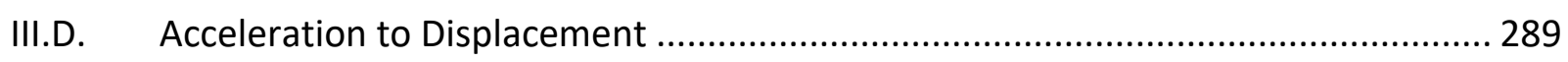




\section{List of Tables}

Table 2.1: The location and soil depth based on the HVSR measurements of each station in the Kinburn Seismic Array.

Table 2.2: A list of some of earthquakes recorded at the Kinburn Seismic array. ${ }^{*}$ The event name is usually a location near the epicentre and not unique to a specific event.

Table 3.1: The strike/dip/rake and Cartesian moment tensors for the May 17th, 2013 Ladysmith earthquake found by different methods. *The Cartesian moment tensor is multiplied by the seismic moment for the amplitude of the moment for each component. 63

Table 3.2: Peak motions recorded at KB1O and KB2O. 65

Table 4.1: Properties used for homogeneous simulations. These are consistent with the average eastern North America crust properties (Bent et al., 2015).

Table 4.2: Station in the 2D and 3D simulations. For the 3D simulations all stations have the $Y$ position of $10 \mathrm{~km}$. ${ }^{*}$ The distance column is the distance from the source.

Table 4.3: Theoretical travel time arrivals of different seismic phases based on ray paths for selected receiver $6,16,26,47$ and 57 . $P$ denotes a P-wave, $S$ denotes an S-wave, $n$ is a reflection from the near side $(X=0 \mathrm{~km}), \mathrm{p}$ denotes a $\mathrm{P}$-wave reflected from the free surface $(Z=0 \mathrm{~km})$, and an $\mathrm{s}$ denotes an $\mathrm{S}$-wave reflected off the free surface.

Table 4.4: A list of the 2D and 3D sources for the simulations of the Ladysmith earthquake. *The $2 \mathrm{D}$ moment tensors are rotated $164.4^{\circ}$ to correspond to $\mathrm{KB} 1 \mathrm{O}$ as the source-receiver azimuth.

TThe 3D moment tensors are in CMT format to correspond with the input for SPECFEM3D... 120 Table 5.1: A list of the tests performed to determine the sensitivity of different models or parameters.

Table 5.2: A list of the different parameters for rock and soil used in the simulations.

Table 5.3: A comparison of mesh size, time simulated and processing time between different simulations which used different models.

Table 5.4: List of parameters used for each surface in the different models for testing the effect of different models with one set of rock parameters.

Table 5.5: The peak values of the simulations using the different models with the reference parameters.

Table 5.6: List of parameters used for each surface in the different models for testing the effect of different models with two sets of rock parameters. 144

Table 5.7: Peak values of the simulations using two different rock types for each different model. 146

Table 5.8: List of parameters used for each surface in the Large Box Model for testing the effect of different sets of rock parameters.

Table 5.9: Peak values of the simulations using different rock parameters for the surfaces in the Large Box Model. 149 
Table 5.10: List of parameters used for each surface in the Layered Model for testing the effect

of different horizontal layering of the crust. 151

Table 5.11: Peak values of the simulations using different rock parameters for the surfaces in the Layered Model. 153

Table 5.12: List of parameters used for each surface in the reference model for testing the effect of different values of $Q$ for the rock.....

Table 5.13: Peak values from the simulations with a varying $Q$ value for the rock.

Table 5.14: List of parameters used for each surface in the reference model for testing the effect of different values of $Q$ for the soil.

Table 5.15: Peak values from the simulations with a varying $Q$ value for the soil.

Table 5.16: List of parameters used for each surface in the reference model for testing the effect of different velocities for the soil.

Table 5.17: Peak values from the simulations with a varying velocity for the soil.

Table 5.18: Error estimates on the simulations based on variability of input values.

Table 6.1: A list of the properties used for the different surfaces and volumes for the models in the test simulations.

Table 6.2: The peak values of the Model I and Model II.

Table 6.3: The properties of each surface used for the Ladysmith 2013 earthquake simulations. $\mathrm{CMT}$ is the global centroid moment tensor, and MTS is the moment tensor solution from Bent et al. (2015). 194

Table 6.4: Maximum acceleration values of the simulations for the global centroid moment tensor solution source at KB1O and KB2O. 1R indicates the simulation used one rock type, 2R is for a simulation which uses two rock types, Vs150 denotes the simulation used Soil Type I for the basin material, Vs250 denotes the simulation used Soil Type II for the basin material, and Vs275 denotes the simulation used Soil Type III properties for the basin material.

Table 6.5: Maximum acceleration values of the simulations for the global centroid moment tensor solution source at KB1O and KB2O for the P-wave and S-wave arrivals. 1R indicates the simulation used one rock type, $2 \mathrm{R}$ is for a simulation which uses two rock types, Vs150 denotes the simulation used Soil Type I for the basin material, Vs250 denotes the simulation used Soil Type II for the basin material, and Vs275 denotes the simulation used Soil Type III properties for the basin material. 207

Table 6.6: Maximum acceleration values of the simulations with the moment tensor source from Bent et al. (2015) at KB1O and KB2O. 1R indicates the simulation used one rock type, 2R is for a simulation which uses two rock types, Vs150 denotes the simulation used Soil Type I for the basin material, Vs250 denotes the simulation used Soil Type II for the basin material, and Vs275 denotes the simulation used Soil Type III properties for the basin material. 213 Table 6.7: Maximum acceleration values of the simulations with the moment tensor source from Bent et al. (2015) at KB1O and KB2O for only the P- and S-wave arrivals. 1R indicates the 
simulation used one rock type, $2 \mathrm{R}$ is for a simulation which uses two rock types, Vs150 denotes the simulation used Soil Type I for the basin material, Vs250 denotes the simulation used Soil Type II for the basin material, and Vs275 denotes the simulation used Soil Type III properties for

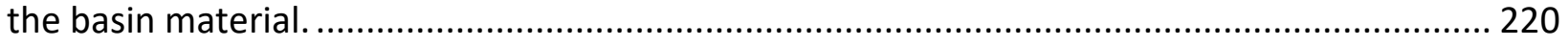

Table III.1: The zeros and poles of a CMG 3ESP by Guralp.............................................. 282 


\section{List of Figures}

Figure 1.1: a) An outline map of Canada with the epicentre of the May 17th, 2013 Ladysmith earthquake shown by the red star, and the black dashed outline of b) a map showing the West Quebec Seismic Zone (orange contours), the Ladysmith earthquake epicentre (red star), the outline of the Kinburn basin (blue square labelled KB), and the locations of Ottawa, Montreal,

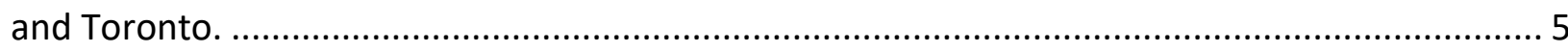

Figure 1.2: Vs30 map of the city of Ottawa from Motazedian et al. (2011)............................... 7

Figure 1.3: Map of Ottawa showing locations of data points collected for the seismic site classification map of Ottawa from Motazedian (2016)............................................................ 8

Figure 1.4: Spectral amplification within a soil basin for 9 earthquakes of M3.5 or greater and the Horizontal to Vertical spectral ratio (HVSR) at the recording station from Hayek (2016)...... 9 Figure 1.5: A To map of the City of Ottawa. The black squares outline the Kinburn Basin (left) and Orleans Basin (right). Adapted from Hunter et al. (2010).............................................. 11

Figure 1.6: A typical hysteresis loop. ........................................................................................ 12

Figure 1.7: Expected transfer function from rock to soil using one-dimensional modelling and the average measured spectral ratio from 9 earthquakes of M3.5 and above in the West Quebec Seismic Zone (from Hayek, 2016) ........................................................................ 14

Figure 1.8: Focusing and defocusing of seismic waves through an irregular shaped impedance boundary. 20

Figure 2.1: Recorded seismicity from SHEEF 2010 for Eastern Canada. The Western Quebec Seismic Zone is outlined by the orange dashed lines. The red circles indicate M0-M3 events, the green circles represent M3-M5 events, and the blue circles represent M5+ events. 39 Figure 2.2: The soil depth of the Kinburn Basin derived from the varying data sources: blue triangles are the HVSR measurements, black circles are the city of Ottawa boreholes and landstreamer data points, and the orange crosshair is the GSC borehole. Red diamonds are the locations of the Kinburn Seismic Array stations. 43

Figure 2.3: Looking Southward at station KB1O with the Kinburn basin in the background....... 49 Figure 2.4: A photograph of station KB2O being installed with a few members of the Kinburn Seismic array installation team. From left to right: Mingzhou Li, Isa Asudeh, Stephen Crane. ... 50 Figure 2.5: Station KB3O looking Northwest towards KB1O. The seismometer vault is to the left

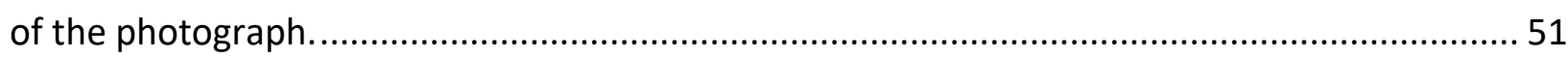

Figure 2.6: A photograph of station KB4O looking Eastward............................................. 52 Figure 2.7: A photograph of station KB5O looking West. The antenna is pointing in the direction

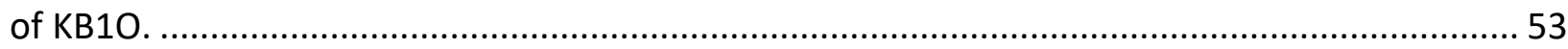

Figure 2.8: A photograph of station KB6O looking to the East.............................................. 54 Figure 2.9: Horizontal to vertical spectral ratios from tromino measurements near the final positions of the Kinburn Seismic Array stations. ................................................................. 55 
Figure 3.1: Strong ground motions recorded at stations KB1O and KB2O during the Ladysmith earthquake. 65

Figure 3.2: Fourier spectral accelerations recorded at station KB1O...................................6 66

Figure 3.3: Fourier spectral accelerations recorded at station KB2O....................................6 67

Figure 3.4: Transfer functions from KB1O to KB2O plotted on a log scale (left) and linear scale (right). The data is smoothed using a 5 point moving average method.

Figure 3.5: Horizontal to vertical ratios from the active recording at KB2O, and from the passive Tromino measurement at the same location for the N-S component (left) and E-W component (right).

Figure 3.6: The displacement time series for station KB1O and KB2O caused by the 2013 Ladysmith, M5.0 earthquake. The pairs of blue lines outline the time intervals for the particle motion plots. Note the vertical component (bottom) has a quarter of the displacement scale as the horizontal components (top and middle) in order to display these motions. 70 Figure 3.7: Particle motion plots for KB1O (top) and KB2O (bottom) for the P-wave recorded at each station. The grey dashed line is the receiver azimuth towards the earthquake epicentre. 71 Figure 3.8: Particle motion plots for KB1O (top) and KB2O (bottom) for the S-wave recorded at each station.

Figure 3.9: Particle motions for different time slices at KB2O. The time slices are 42-47 seconds (top), 55-60 seconds (middle), and 61-67 seconds (bottom). 73

Figure 4.1: The 2D model for the homogeneous simulation showing source and station locations.

Figure 4.2: The location of source and stations for the 3D model for the homogeneous simulation.

Figure 4.3: The first 5 seconds of the acceleration (left) and displacement (right) time series at the source for the 2D simulation (top) and 3D simulation (bottom) for the unit force in the $X$ direction source

Figure 4.4: The first 5 seconds of the acceleration (left) and displacement (right) time series at the source for the 2D simulation (top) and 3D simulation (bottom) for the unit force in the Zdirection source

Figure 4.5: An example of ray tracing through a two dimensional model. In this example a direct $\mathrm{P}$-wave $(\mathrm{P})$, both a $\mathrm{P}$ - and $\mathrm{S}$-wave reflected off the free surface resulting in a $\mathrm{P}$-wave ( $\mathrm{PP}$ and $\mathrm{SP}$ respectively), and lastly an S-wave reflected into a $\mathrm{P}$-wave off the near side boundary (SnP) are shown.

Figure 4.6: Acceleration time series at station numbers 6, 16, 26, 37, 47, and 57 for the 2-D simulation with a unit force in the X-direction. Distance is the distance from the source. The vertical lines are the theoretical wave arrivals for the different phases listed in Table 4.1. $P$ denotes a P-wave, $\mathrm{S}$ denotes an S-wave, $\mathrm{n}$ is a reflection from the near side $(X=0 \mathrm{~km}), \mathrm{p}$ denotes a $P$-wave reflected from the free surface $(Z=0 \mathrm{~km})$, and an $\mathrm{s}$ denotes an $\mathrm{S}$-wave reflected off the 
free surface. The labels are alternately placed between above and below the lines and they are listed in order of the next arrival.

Figure 4.7: Acceleration time series for station numbers 6, 16, 26, 37, 47, and 57 the 3-D simulation with a unit force in the X-direction. Distance is the distance from the source. The vertical lines are the theoretical wave arrivals for the different phases listed in Table 4.3. $P$ denotes a $\mathrm{P}$-wave, $\mathrm{S}$ denotes an $\mathrm{S}$-wave, $\mathrm{n}$ is a reflection from the near side $(\mathrm{X}=0 \mathrm{~km}), \mathrm{p}$ denotes a $P$-wave reflected from the free surface $(Z=0 \mathrm{~km})$, and an $\mathrm{s}$ denotes an $\mathrm{S}$-wave reflected off the free surface. The labels are alternately placed above and below the lines and they are listed in order of the next arrival.

Figure 4.8: X-component of motion for the receivers at the surface for the 2D simulation (top) and $3 \mathrm{D}$ simulation (bottom) for the simulations using the unit- $X$ direction force source. $P$ denotes a $\mathrm{P}$-wave, $\mathrm{S}$ denotes an $\mathrm{S}$-wave, $\mathrm{n}$ is a reflection from the near side $(\mathrm{X}=0 \mathrm{~km}), \mathrm{p}$ denotes a $P$-wave reflected from the free surface $(Z=0 \mathrm{~km})$, and an $\mathrm{s}$ denotes an $\mathrm{S}$-wave reflected off the free surface. For example: SnP is an S-wave that is reflected into a P-wave off the near side boundary

Figure 4.9: Z-component of motion for the receivers at the surface for the 2D simulation (top) and 3D simulation (bottom) for the simulations using the unit- $X$ direction force source. $P$ denotes a $\mathrm{P}$-wave, $\mathrm{S}$ denotes an $\mathrm{S}$-wave, $\mathrm{n}$ is a reflection from the near side $(X=0 \mathrm{~km}), \mathrm{p}$ denotes a $P$-wave reflected from the free surface $(Z=0 \mathrm{~km})$, and an $\mathrm{s}$ denotes an $\mathrm{S}$-wave reflected off the free surface. For example: $\mathrm{SnP}$ is an S-wave that is reflected into a P-wave off the near side boundary.

Figure 4.10: X-component of motion for the receivers at $20 \mathrm{~km}$ depth for the 2D simulation (top) and 3D simulation (bottom) for the simulations using the unit- $X$ direction force source. $P$ denotes a $\mathrm{P}$-wave, $\mathrm{S}$ denotes an $\mathrm{S}$-wave, $\mathrm{n}$ is a reflection from the near side $(\mathrm{X}=0 \mathrm{~km}), \mathrm{p}$ denotes a $P$-wave reflected from the free surface $(Z=0 \mathrm{~km})$, and an s denotes an $\mathrm{S}$-wave reflected off the free surface. For example: SnP is an S-wave that is reflected into a P-wave off the near side boundary.

Figure 4.11: Z-component of motion for the receivers at $20 \mathrm{~km}$ depth for the 2D simulation (top) and 3D simulation (bottom) for the simulations using the unit- $X$ direction force source. $P$ denotes a P-wave, $\mathrm{S}$ denotes an S-wave, $\mathrm{n}$ is a reflection from the near side $(X=0 \mathrm{~km}), \mathrm{p}$ denotes a $P$-wave reflected from the free surface $(Z=0 \mathrm{~km})$, and an $\mathrm{s}$ denotes an $\mathrm{S}$-wave reflected off the free surface. For example: $\mathrm{SnP}$ is an S-wave that is reflected into a P-wave off the near side boundary.

Figure 4.12: Particle motion plot of station number 16 for the 2D simulation. The displacement time series (bottom) outlines the different time slices used. P denotes a P-wave, $\mathrm{S}$ denotes an Swave, $n$ is a reflection from the near side $(X=0 \mathrm{~km}), y$ is a reflection from the $Y$-boundary (not present in $2 \mathrm{D}$ simulations), $\mathrm{p}$ denotes a $\mathrm{P}$-wave reflected from the free surface $(\mathrm{Z}=0 \mathrm{~km})$, and an $s$ denotes an S-wave reflected off the free surface. 
Figure 4.13: Particle motion plot of station number 16 for the 3D simulation. The time series (bottom) outlines the different time slices used. $\mathrm{P}$ denotes a $\mathrm{P}$-wave, $\mathrm{S}$ denotes an $\mathrm{S}$-wave, $\mathrm{n}$ is a reflection from the near side $(X=0 \mathrm{~km}), y$ denotes a reflection from the $Y$-boundary, $p$ denotes a $P$-wave reflected from the free surface $(Z=0 \mathrm{~km})$, and an $\mathrm{s}$ denotes an $\mathrm{S}$-wave reflected off the free surface.

Figure 4.14: Particle motion plot of station number 26 for the 2D simulation. The time series (bottom) outlines the different time slices used. $\mathrm{P}$ denotes a $\mathrm{P}$-wave, $\mathrm{S}$ denotes an $\mathrm{S}$-wave, $\mathrm{n}$ is a reflection from the near side $(X=0 \mathrm{~km}), \mathrm{p}$ denotes a $\mathrm{P}$-wave reflected from the free surface $(Z=0 \mathrm{~km})$, and an $\mathrm{s}$ denotes an $\mathrm{S}$-wave reflected off the free surface.

Figure 4.15: Particle motion plot of station number 26 for the 3D simulation. The time series (bottom) outlines the different time slices used. P denotes a $\mathrm{P}$-wave, $\mathrm{S}$ denotes an $\mathrm{S}$-wave, $\mathrm{n}$ is a reflection from the near side $(X=0 \mathrm{~km}), \mathrm{p}$ denotes a $\mathrm{P}$-wave reflected from the free surface $(Z=0 \mathrm{~km})$, and an $\mathrm{s}$ denotes an $\mathrm{S}$-wave reflected off the free surface. 101 Figure 4.16: Horizontal (left) and vertical (right) acceleration at time 1.05 seconds in the 2D simulation. The dots are the receivers, grey is the model volume and black is the acceleration. $\mathrm{P}$ denotes a P-wave, $\mathrm{S}$ denotes an S-wave. 102 Figure 4.17: Horizontal (left) and vertical (right) acceleration at time 3.55 seconds in the 2D simulation. The dots are the receivers, grey is the model volume and black is the acceleration. $P$ denotes a $\mathrm{P}$-wave, $\mathrm{S}$ denotes an $\mathrm{S}$-wave, $\mathrm{n}$ is a reflection from the near side $(\mathrm{X}=0 \mathrm{~km})$, and a $\mathrm{p}$ denotes a $P$-wave reflected from the free surface $(Z=0 \mathrm{~km})$. 103 Figure 4.18: Horizontal (left) and vertical (right) acceleration at time 6.55 seconds in the 2D simulation. The dots are the receivers, grey is the model volume and black is the acceleration. $P$ denotes a P-wave, $\mathrm{S}$ denotes an S-wave, $\mathrm{n}$ is a reflection from the near side $(\mathrm{X}=0 \mathrm{~km}), \mathrm{p}$ denotes a $P$-wave reflected from the free surface $(Z=0 \mathrm{~km})$, an s denotes an $\mathrm{S}$-wave reflected off the free surface, and $a b$ denotes a reflection from the bottom $(Z=-40 \mathrm{~km})$ 103 Figure 4.19: Horizontal (left) and vertical (right) acceleration at time 10.05 seconds in the 2D simulation. The dots are the receivers, grey is the model volume and black is the acceleration. $P$ denotes a $\mathrm{P}$-wave, $\mathrm{S}$ denotes an $\mathrm{S}$-wave, $\mathrm{n}$ is a reflection from the near side $(\mathrm{X}=0 \mathrm{~km}), \mathrm{p}$ denotes a $P$-wave reflected from the free surface $(Z=0 \mathrm{~km})$, an s denotes an S-wave reflected off the free surface, and $a b$ denotes a reflection from the bottom $(Z=-40 \mathrm{~km})$.... 104 Figure 4.20: Acceleration time series for station numbers 6, 16, 26, 37, 47, and 57 the 2D simulation with a unit force in the Z-direction. Distance is the distance from the source. The vertical lines are the theoretical wave arrivals for the different phases listed in Table 4.3. $P$ denotes a $\mathrm{P}$-wave, $\mathrm{S}$ denotes an $\mathrm{S}$-wave, $\mathrm{n}$ is a reflection from the near side $(\mathrm{X}=0 \mathrm{~km}), \mathrm{p}$ denotes a $P$-wave reflected from the free surface $(Z=0 \mathrm{~km})$, and an s denotes an $S$-wave reflected off the free surface. The labels are alternately labelled above and below the lines and they are listed in order of the next arrival. 105 
Figure 4.21: Acceleration time series for station numbers 6, 16, 26, 37, 47, and 57 the 3D simulation with a unit force in the Z-direction. Distance is the distance from the source. The vertical lines are the theoretical wave arrivals for the different phases listed in Table 4.3. $P$ denotes a P-wave, $\mathrm{S}$ denotes an S-wave, $\mathrm{n}$ is a reflection from the near side $(X=0 \mathrm{~km}), \mathrm{p}$ denotes a $P$-wave reflected from the free surface $(Z=0 \mathrm{~km})$, and an $\mathrm{s}$ denotes an $\mathrm{S}$-wave reflected off the free surface. The labels are alternately placed above and below the lines and they are listed in order of the next arrival. 106 Figure 4.22: X-component of motion for the receivers at the surface for the 2D simulation (top) and $3 D$ simulation (bottom) for the simulations using the unit- $Z$ direction force source. $P$ denotes a P-wave, $\mathrm{S}$ denotes an S-wave, $\mathrm{n}$ is a reflection from the near side $(X=0 \mathrm{~km}), \mathrm{p}$ denotes a $P$-wave reflected from the free surface $(Z=0 \mathrm{~km})$, and an $\mathrm{s}$ denotes an $\mathrm{S}$-wave reflected off the free surface. For example: SnP is an S-wave that is reflected into a P-wave off the near side boundary.

Figure 4.23: Z-component of motion for the receivers at the surface for the 2D simulation (top) and $3 D$ simulation (bottom) for the simulations using the unit- $Z$ direction force source. $P$ denotes a $\mathrm{P}$-wave, $\mathrm{S}$ denotes an $\mathrm{S}$-wave, $\mathrm{n}$ is a reflection from the near side $(X=0 \mathrm{~km}), \mathrm{p}$ denotes a P-wave reflected from the free surface $(Z=0 \mathrm{~km})$, and an $\mathrm{s}$ denotes an $S$-wave reflected off the free surface. For example: SnP is an S-wave that is reflected into a P-wave off the near side boundary

Figure 4.24: X-component of motion for the receivers at $20 \mathrm{~km}$ depth for the 2D simulation (top) and 3D simulation (bottom) for the simulations using the unit-Z direction force source. $P$ denotes a $\mathrm{P}$-wave, $\mathrm{S}$ denotes an $\mathrm{S}$-wave, $\mathrm{n}$ is a reflection from the near side $(\mathrm{X}=0 \mathrm{~km}), \mathrm{p}$ denotes a $P$-wave reflected from the free surface $(Z=0 \mathrm{~km})$, and an $\mathrm{s}$ denotes an $\mathrm{S}$-wave reflected off the free surface. For example: SnP is an S-wave that is reflected into a P-wave off the near side boundary..... 109

Figure 4.25: Z-component of motion for the receivers at $20 \mathrm{~km}$ depth for the $2 \mathrm{D}$ simulation (top) and 3D simulation (bottom) for the simulations using the unit-Z direction force source. $P$ denotes a $\mathrm{P}$-wave, $\mathrm{S}$ denotes an $\mathrm{S}$-wave, $\mathrm{n}$ is a reflection from the near side $(\mathrm{X}=0 \mathrm{~km}), \mathrm{p}$ denotes a $P$-wave reflected from the free surface $(Z=0 \mathrm{~km})$, and an $\mathrm{s}$ denotes an $\mathrm{S}$-wave reflected off the free surface. For example: SnP is an S-wave that is reflected into a P-wave off the near side boundary. 110

Figure 4.26: The first 10 seconds of the 3D time series and 2D corrected time series for station number 16 with different corrections applied. The time series is filtered using a 4-pole Butterworth lowpass filter with a cut-off frequency of $3.2 \mathrm{~Hz}$.

Figure 4.27: The first 15 seconds of the 3D time series and 2D corrected time series for station number 26 with different corrections applied for station number 26 . The time series is filtered using a 4-pole Butterworth lowpass filter with a cut-off frequency of $3.2 \mathrm{~Hz}$. 
Figure 4.28: The first 10 seconds of the 3D time series and 2D corrected time series with different corrections applied. The dominant frequency of the source for the 2D simulation was increased to match the half duration of the 3D simulation. The time series was filtered using a 4-pole Butterworth lowpass filter with a cutoff frequency of $3.2 \mathrm{~Hz}$.

Figure 4.29: The first 10 seconds of the 3D time series and 2D corrected time series with different corrections applied. The dominant frequency of the source for the 2D simulation was increased and the seismic moment was adjusted to match the half duration of the 3D simulation. The time series was filtered using a 4-pole Butterworth lowpass filter with a cutoff frequency of $3.2 \mathrm{~Hz}$. 116

Figure 4.30: The Fourier acceleration spectrum for the 2D simulation with corrected seismograms and the original Unit X-direction force source and for the 3D simulation.....

Figure 4.31: The Fourier acceleration spectrum for the 2D simulation with corrected seismograms and the adjusted source to match the 3D simulation.

Figure 4.32: A map showing the locations of the Ladysmith epicentre (star), and the Kinburn seismic array (black circles). The strike of the fault plane from Bent et al. (2015) is $350.4^{\circ}$ and the source to receiver azimuth for the epicenter to the station KB1O is $164.4^{\circ}$.

Figure 4.33: Radial and Vertical spectral accelerations using a moment tensor source from Bent et al. (2015). These have been filtered using an 8-pole Butterworth bandpass filter with corner frequencies 0.01 and $3.2 \mathrm{~Hz}$.

Figure 4.34: Radial and vertical time series for the Ladysmith recording at KB1O and the simulations with a moment tensor source from Bent et al. (2015). A Butterworth bandpass filter with corner frequencies 0.01 and $3.2 \mathrm{~Hz}$ was applied.

Figure 4.35: Radial and vertical time series for the Ladysmith recording at KB1O and the 2D simulation with $\mathrm{f}_{0}=2.2 \mathrm{~Hz}$. A Butterworth bandpass filter with corner frequencies 0.01 and 2.4 $\mathrm{Hz}$ was applied.

Figure 4.36: Radial and vertical time series for the Ladysmith recording at KB1O and the 3D simulation and $2 \mathrm{D}$ simulation with $\mathrm{f}_{0}=1.1 \mathrm{~Hz}$. A Butterworth bandpass filter with corner frequencies 0.01 and $1.2 \mathrm{~Hz}$ was applied. 123 Figure 4.37: Radial and Vertical spectral accelerations using the global centroid moment tensor. These have been filtered using an 8-pole Butterworth bandpass filter with corner frequencies 0.01 and $3.2 \mathrm{~Hz}$.

Figure 4.38: Radial and vertical time series for the Ladysmith recording at KB1O and the simulations with a global centroid moment tensor. A Butterworth bandpass filter with corner frequencies 0.01 and $3.2 \mathrm{~Hz}$ was applied. 124

Figure 4.39: Radial and vertical time series for the Ladysmith recording at KB1O and the 2D simulation with $\mathrm{fO}=1.66 \mathrm{~Hz}$. A Butterworth bandpass filter with corner frequencies 0.01 and $2.0 \mathrm{~Hz}$ was applied. 125 
Figure 4.40: Radial and vertical time series for the Ladysmith recording at KB1O and the 3D simulation and 2D simulation with $\mathrm{f}_{0}=0.83 \mathrm{~Hz}$. A Butterworth bandpass filter with corner frequencies 0.01 and $1.0 \mathrm{~Hz}$ was applied.

Figure 4.41: Radial and Vertical spectral accelerations using a moment tensor source from Ma and Audet (2014). These have been filtered using an 8-pole Butterworth bandpass filter with corner frequencies 0.01 and $3.2 \mathrm{~Hz}$.

Figure 4.42: Radial and vertical time series for the Ladysmith recording at KB1O and the simulations with a moment tensor source from Ma and Audet (2014). A Butterworth bandpass filter with corner frequencies 0.01 and $3.2 \mathrm{~Hz}$ was applied.

Figure 4.43: Radial and vertical time series for the Ladysmith recording at KB1O and the 2D simulation with $\mathrm{f}_{0}=1.83 \mathrm{~Hz}$. A Butterworth bandpass filter with corner frequencies 0.01 and 2.0Hz was applied.

Figure 4.44: Radial and vertical time series for the Ladysmith recording at KB1O and the 3D simulation and 2D simulation with $\mathrm{f}_{0}=0.94 \mathrm{~Hz}$. A Butterworth bandpass filter with corner frequencies 0.01 and $1.2 \mathrm{~Hz}$ was applied.

Figure 5.1: The Reference Model used in these simulations. The entire model with the mesh is shown in a), and b) displays a close up of a portion of the basin, surrounding surfaces and stations S1 and S2 as outlined in a). The white lines outline the mesh in the model. 136 Figure 5.2: The Large Box Model used in these simulations. The entire model with the mesh is shown in a), and b) displays a close up of a portion of the basin, surrounding surfaces and stations S1 and S2 as outlined in a). The white lines outline the mesh in the model.

Figure 5.3: The Layered Model used in these simulations. The entire model with the mesh is shown in a), and b) displays a close up of a portion of the basin, surrounding surfaces and stations S1 and S2 as outlined in a). The white lines outline the mesh in the model.

Figure 5.4: Acceleration time series resulted from the basin modeling for the Ladysmith earthquake for the horizontal component (left column) and vertical component (right column) recorded on the station S1 for the different models. The Reference model is the top row, the Large Box Model is the middle row and the Layered Model is the bottom row.

Figure 5.5: Acceleration time series resulted from the basin modeling for the Ladysmith earthquake for the horizontal component (left column) and vertical component (right column) recorded on the station S2 for the different models. The Reference model is the top row, the Large Box Model is the middle row and the Layered Model is the bottom row. Figure 5.6: Acceleration time series resulted from the basin modeling for the Ladysmith earthquake for the horizontal component (left column) and vertical component (right column) recorded on the station S1 for the different models with two rock types. The Reference model is the top row, the Large Box Model is the middle row and the Layered Model is the bottom row. 
Figure 5.7: Acceleration time series results from the basin modeling for the Ladysmith earthquake for the horizontal component (left column) and vertical component (right column) recorded on the station S2 for the different models with two rock types. The Reference model is the top row, the Large Box Model is the middle row and the Layered Model is the bottom row. 146

Figure 5.8: Acceleration time series resulted from the basin modeling for the Ladysmith earthquake for the horizontal component (left column) and vertical component (right column) recorded on the station S1 for the Large Box Model with different rock types. The reference simulation is the top row, the simulation with two rock types is the middle row and the simulation with three rock types is the bottom row. 148

Figure 5.9: Acceleration time series resulted from the basin modeling for the Ladysmith earthquake for the horizontal component (left column) and vertical component (right column) recorded on the soil station for the Large Box Model with different rock types. The reference simulation is the top row, the simulation with two rock types is the middle row and the simulation with three rock types is the bottom row.

Figure 5.10: Acceleration time series resulted from the basin modeling for the Ladysmith earthquake for the horizontal component (left column) and vertical component (right column) recorded on the station S1 for the Layered Model with different rock types. The reference simulation is the top row, the simulation with two rock types is the second row, the simulation with a progressive velocity profile is the third row, and the simulation with the velocity profile as found in Motazedian et al., (2013) is the bottom row. 152 Figure 5.11: Acceleration time series resulted from the basin modeling for the Ladysmith earthquake for the horizontal component (left column) and vertical component (right column) recorded on the station S2 for the Layered Model with different rock types. The reference simulation is the top row, the simulation with two rock types is the second row, the simulation with a progressive velocity profile is the third row, and the simulation with the velocity profile as found in Motazedian et al., (2013) is the bottom row. 153 Figure 5.12: Acceleration time series resulted from the basin modeling for the Ladysmith earthquake for the horizontal component (left column) and vertical component (right column) recorded on the station $S 1$ for the different $Q$ values for the rock. The reference simulation with $Q=1000$ is the top row, the simulation with $Q=750$ is the middle row, and the simulation with $Q=500$ is the bottom row. 156

Figure 5.13: Acceleration time series resulted from the basin modeling for the Ladysmith earthquake for the horizontal component (left column) and vertical component (right column) recorded on the station S2 for the different $Q$ values for the rock. The reference simulation with $Q=1000$ is the top row, the simulation with $Q=750$ is the middle row, and the simulation with $Q=500$ is the bottom row. 
Figure 5.14: Acceleration time series resulted from the basin modeling for the Ladysmith earthquake for the horizontal component (left column) and vertical component (right column) recorded on the station S1 for the different $Q$ values for the soil. The reference simulation with $Q=100$ is the top row, the simulation with $Q=50$ is the middle row, and the simulation with $Q$ $=20$ is the bottom row. 160

Figure 5.15: Acceleration time series resulted from the basin modeling for the Ladysmith earthquake the horizontal component (left column) and vertical component (right column) recorded on the station S2 for the different $Q$ values for the soil. The reference simulation with $Q=100$ is the top row, the simulation with $Q=50$ is the middle row, and the simulation with $Q$ $=20$ is the bottom row. 160

Figure 5.16: Acceleration time series resulted from the basin modeling for the Ladysmith earthquake for the horizontal component (left column) and vertical component (right column) recorded on the station S1 for the different velocity values for the soil. The reference simulation with $V s=150$ is the top row, the simulation with $V s=250$ is the middle row, and the simulation with $\mathrm{Vs}=350$ is the bottom row.....

Figure 5.17: Acceleration time series resulting from the basin modeling for the Ladysmith earthquake for the horizontal component (left column) and vertical component (right column) recorded on the station S2 for the different velocity values for the soil. The reference simulation with $V s=150$ is the top row, the simulation with $V s=250$ is the middle row, and the simulation with $\mathrm{Vs}=350$ is the bottom row... 163

Figure 5.18: Horizontal spectral accelerations for the simulations with different velocities for the soil. 164

Figure 6.1: Figures of the models for the first set of simulations: a) is the entire simulated model which shows the earthquake source and the S1 and S2 seismic stations, b) is the close up of Model I, with the basin filled with rock, and c) is the close up of Model II, with the basin filled with soil parameters.

Figure 6.2: Images of the models used for the second set of simulations: a) the entire model, b) the model with only two rock types, Model III, c) the model with two rock types and a horizontal soil layer, Model IV, and d) is the model with two rock types and the soil confined to a basin structure, Model V.....

Figure 6.3: The models for the NWSE transect of the Kinburn Basin. The top image is the 2D model and the bottom is a profile of the 3D model along the same axis.

Figure 6.4: The models for the NESW transect of the Kinburn Basin. The top image is the 2D model and the bottom is a profile of the 3D model along the same axis.

Figure 6.5: Acceleration time series at the receiver S1, for the model with only rock, Model I (top) and rock and soil model, Model II (bottom). The left column is the horizontal component and the right column is the vertical component of motion. 
Figure 6.6: Acceleration time series at the receiver S2, for the model with only rock, Model I (top) and rock and soil model, Model II (bottom). The left column is the horizontal component and the right column is the vertical component of motion.

Figure 6.7: Fourier acceleration spectrum at the receiver S2, for the horizontal motions (left) and vertical motions (right).

Figure 6.8: The spectral ratios for S2/S1 from Model I and Model II, and the spectral ratio at S2 for Model II divided by Model I.

Figure 6.9: Acceleration time series at the rock station, S1, for the rock only reference model, Model III (top), the model with a $100 \mathrm{~m}$ of soil, Model IV (middle) and the model with a basin structure, Model V (bottom). The left column is the horizontal component and the right column is the vertical component.

Figure 6.10: Acceleration time series at the soil station, S2, for the rock only reference model, Model III (top), the model with a $100 \mathrm{~m}$ of soil, Model IV (middle) and the model with a basin structure, Model V (bottom). The left column is the horizontal component and the right column is the vertical component. 182

Figure 6.11: Fourier acceleration spectrum for the different models for the horizontal component (left) and vertical component (right) at receiver S2.

Figure 6.12: Spectral ratios for Model IV and Model V divided by Model III at receiver S2. ..... 183 Figure 6.13: Acceleration time series for the vertical component of motion for the different models for receivers located at the surface (SM1, top), 150m depth (SM2, middle) and at the source (bottom).

Figure 6.14: Acceleration time series for the radial component of motion for the 3D model (Model VIII) and the 2D NESW model (Model VII) for receivers located at the surface (S1, top), $150 \mathrm{~m}$ depth (S2, middle) and at the source (bottom). 187

Figure 6.15: Acceleration time series for the radial component of motion for the 3D model (Model VIII) and the 2D NWSE model (Model VI) for receivers located at the surface (top), 150m depth (middle) and at the source (bottom).

Figure 6.16: Spectral accelerations for the horizontal (left) and vertical (right) components of motion in the 2D models, (Model VI and Model VII) and the 3D model (Model VIII). 188 Figure 6.17: Horizontal motions across the surface for the 2D NESW model, Model VII (left), and the radial motions along the NESW profile for the 3D model, Model VIII (right). An overlay of the basin surface is shown for each with a vertical exaggeration of about 10 . 189 Figure 6.18: Vertical motions across the surface for the 2D NESW model, Model VII (left), and the vertical motions along the NESW profile for the 3D model, Model VIII (right). An overlay of the basin surface is shown for each with a vertical exaggeration of about 10 . 190 Figure 6.19: Horizontal motions across the surface for the 2D NWSE model, Model VII (left), and the radial motions along the NWSE profile for the 3D model, Model VIII (right). An overlay of the basin surface is shown for each with a vertical exaggeration of about 10 . 190 
Figure 6.20: Vertical motions across the surface for the 2D NWSE model, Model VII (left), and the vertical motions along the NWSE profile for the 3D model, Model VIII (right). An overlay of the basin surface is shown for each with a vertical exaggeration of about 10 .

Figure 6.21: The modified 2D layered model for simulating the Ladysmith earthquake of 2013. Surface 1 is the basin, Surface 2 extends to a depth of $400 \mathrm{~m}$, and Surface 3 is the rest of the model.

Figure 6.22: Horizontal acceleration time series at station $\mathrm{KB} 1 \mathrm{O}$ for the simulations with the global centroid moment tensor solution source. 1R indicates the simulation used one rock type, $2 \mathrm{R}$ is for a simulation which uses two rock types, Vs150 denotes the simulation used Soil Type I for the basin material, and Vs250 denotes the simulation used Soil Type II for the basin material. 196

Figure 6.23: Vertical acceleration time series at station KB1O for the simulations with the global centroid moment tensor solution source. $1 R$ indicates the simulation used one rock type, $2 R$ is for a simulation which uses two rock types, Vs150 denotes the simulation used Soil Type I for the basin material, and Vs250 denotes the simulation used Soil Type II for the basin material.

Figure 6.24: Fourier acceleration spectrum for the horizontal (left) and vertical (right) components of motion at station KB1O. 1R indicates the simulation used one rock type, $2 \mathrm{R}$ is for a simulation which uses two rock types, Vs150 denotes the simulation used Soil Type I for the basin material, and Vs250 denotes the simulation used Soil Type II for the basin material.

Figure 6.25: Horizontal acceleration time series at station $\mathrm{KB} 2 \mathrm{O}$ for the simulations with the centroid moment tensor solution source. $1 R$ indicates the simulation used one rock type, $2 R$ is for a simulation which uses two rock types, Vs150 denotes the simulation used Soil Type I for the basin material, and Vs250 denotes the simulation used Soil Type II for the basin material.

Figure 6.26: Vertical acceleration time series at station KB2O for the simulations with the centroid moment tensor solution source. $1 R$ indicates the simulation used one rock type, $2 R$ is for a simulation which uses two rock types, Vs150 denotes the simulation used Soil Type I for the basin material, and Vs250 denotes the simulation used Soil Type II for the basin material.

Figure 6.27: The Fourier acceleration spectrum for the horizontal (left) and vertical (right) components of motion at station KB2O. $1 \mathrm{R}$ indicates the simulation used one rock type, $2 \mathrm{R}$ is for a simulation which uses two rock types, Vs150 denotes the simulation used Soil Type I for the basin material, and Vs250 denotes the simulation used Soil Type II for the basin material.

Figure 6.28: The spectral acceleration ratios for the horizontal (left) and vertical (right) components of motion for station KB2O/KB1O. 1R indicates the simulation used one rock type, 
$2 \mathrm{R}$ is for a simulation which uses two rock types, Vs150 denotes the simulation used Soil Type I for the basin material, and Vs250 denotes the simulation used Soil Type II for the basin material. 203

Figure 6.29: The windowed horizontal acceleration time series at station KB1O for the simulations with the global centroid moment tensor solution source. $1 \mathrm{R}$ indicates the simulation used one rock type, $2 \mathrm{R}$ is for a simulation which uses two rock types, Vs150 denotes the simulation used Soil Type I for the basin material, and Vs250 denotes the simulation used Soil Type II for the basin material. 204 Figure 6.30: The windowed vertical acceleration time series at station KB1O for the simulations with the global centroid moment tensor solution source. $1 \mathrm{R}$ indicates the simulation used one rock type, $2 \mathrm{R}$ is for a simulation which uses two rock types, Vs150 denotes the simulation used Soil Type I for the basin material, and Vs250 denotes the simulation used Soil Type II for the basin material. 205

Figure 6.31: The windowed horizontal acceleration time series at station KB2O for the simulations with the global centroid moment tensor solution source. $1 \mathrm{R}$ indicates the simulation used one rock type, $2 \mathrm{R}$ is for a simulation which uses two rock types, Vs150 denotes the simulation used Soil Type I for the basin material, and Vs250 denotes the simulation used Soil Type II for the basin material. ......................................................................................... 206 Figure 6.32: The windowed horizontal acceleration time series at station KB1O for the simulations with the global centroid moment tensor solution source. $1 R$ indicates the simulation used one rock type, $2 \mathrm{R}$ is for a simulation which uses two rock types, Vs150 denotes the simulation used Soil Type I for the basin material, and Vs250 denotes the simulation used Soil Type II for the basin material. 207

Figure 6.33: The spectral acceleration ratios for the horizontal (left) and vertical (right) components of motion for station KB2O/KB1O using the windowed time series. 1R indicates the simulation used one rock type, $2 \mathrm{R}$ is for a simulation which uses two rock types, Vs150 denotes the simulation used Soil Type I for the basin material, and Vs250 denotes the simulation used Soil Type II for the basin material. 208 Figure 6.34: Horizontal acceleration time series at station KB1O for the simulations with the moment tensor solution source from Bent et al. (2015). 1R indicates the simulation used one rock type, $2 \mathrm{R}$ is for a simulation which uses two rock types, Vs150 denotes the simulation used Soil Type I for the basin material, Vs250 denotes the simulation used Soil Type II for the basin material, and Vs275 denotes the simulation used Soil Type III properties for the basin material.

Figure 6.35: Vertical acceleration time series at station KB1O for the simulations with the moment tensor solution source from Bent et al. (2015). 1R indicates the simulation used one rock type, $2 \mathrm{R}$ is for a simulation which uses two rock types, Vs150 denotes the simulation used Soil Type I for the basin material, Vs250 denotes the simulation used Soil Type II for the basin 
material, and Vs275 denotes the simulation used Soil Type III properties for the basin material.

Figure 6.36: Horizontal acceleration time series at station $\mathrm{KB} 2 \mathrm{O}$ for the simulations with the moment tensor solution source from Bent et al. (2015). 1R indicates the simulation used one rock type, $2 \mathrm{R}$ is for a simulation which uses two rock types, Vs150 denotes the simulation used Soil Type I for the basin material, Vs250 denotes the simulation used Soil Type II for the basin material, and Vs275 denotes the simulation used Soil Type III properties for the basin material.

Figure 6.37: Vertical acceleration time series at station KB2O for the simulations with the moment tensor solution source from Bent et al. (2015). 1R indicates the simulation used one rock type, $2 \mathrm{R}$ is for a simulation which uses two rock types, Vs150 denotes the simulation used Soil Type I for the basin material, Vs250 denotes the simulation used Soil Type II for the basin material, and Vs275 denotes the simulation used Soil Type III properties for the basin material.

Figure 6.38: The Fourier acceleration spectrum at KB1O for the horizontal (left) and vertical (right) component for the simulations with the moment tensor solution from Bent et al. (2015). $1 R$ indicates the simulation used one rock type, $2 R$ is for a simulation which uses two rock types, Vs150 denotes the simulation used Soil Type I for the basin material, Vs250 denotes the simulation used Soil Type II for the basin material, and Vs275 denotes the simulation used Soil Type III properties for the basin material.

Figure 6.39: The Fourier acceleration spectrum at KB2O for the horizontal (left) and vertical (right) component for the simulations with the moment tensor solution from Bent et al. (2015). $1 R$ indicates the simulation used one rock type, $2 R$ is for a simulation which uses two rock types, Vs150 denotes the simulation used Soil Type I for the basin material, Vs250 denotes the simulation used Soil Type II for the basin material, and Vs275 denotes the simulation used Soil Type III properties for the basin material.

Figure 6.40: The spectral ratios for the horizontal (left) and vertical (right) components of motion at station KB2O divided by station KB1O. 1R indicates the simulation used one rock type, $2 \mathrm{R}$ is for a simulation which uses two rock types, Vs150 denotes the simulation used Soil Type I for the basin material, , Vs250 denotes the simulation used Soil Type II for the basin material, and Vs275 denotes the simulation used Soil Type III properties for the basin material.

Figure 6.41: The windowed horizontal acceleration time series at station KB1O for the simulations with the moment tensor solution source from Bent et al. (2015). 1R indicates the simulation used one rock type, $2 \mathrm{R}$ is for a simulation which uses two rock types, Vs150 denotes the simulation used Soil Type I for the basin material, Vs250 denotes the simulation used Soil Type II for the basin material, and Vs275 denotes the simulation used Soil Type III properties for the basin material. 
Figure 6.42: The windowed vertical acceleration time series at station KB1O for the simulations with the moment tensor solution source from Bent et al. (2015). 1R indicates the simulation used one rock type, $2 \mathrm{R}$ is for a simulation which uses two rock types, Vs150 denotes the simulation used Soil Type I for the basin material, Vs250 denotes the simulation used Soil Type II for the basin material, and Vs275 denotes the simulation used Soil Type III properties for the basin material.

Figure 6.43: The windowed horizontal acceleration time series at station KB2O for the simulations with the moment tensor solution source from Bent et al. (2015). 1R indicates the simulation used one rock type, $2 \mathrm{R}$ is for a simulation which uses two rock types, Vs150 denotes the simulation used Soil Type I for the basin material, Vs250 denotes the simulation used Soil Type II for the basin material, and Vs275 denotes the simulation used Soil Type III properties for the basin material.

Figure 6.44: The windowed horizontal acceleration time series at station KB1O for the simulations with the moment tensor solution source from Bent et al. (2015). 1R indicates the simulation used one rock type, $2 \mathrm{R}$ is for a simulation which uses two rock types, Vs150 denotes the simulation used Soil Type I for the basin material, Vs250 denotes the simulation used Soil Type II for the basin material, and Vs275 denotes the simulation used Soil Type III properties for the basin material. 220

Figure 6.45: The spectral acceleration ratios for the horizontal (left) and vertical (right) components of motion for station KB2O/KB1O using the windowed time series. $1 \mathrm{R}$ indicates the simulation used one rock type, $2 \mathrm{R}$ is for a simulation which uses two rock types, Vs150 denotes the simulation used Soil Type I for the basin material, Vs250 denotes the simulation used Soil Type II for the basin material, and Vs275 denotes the simulation used Soil Type III properties for the basin material. 221 Figure 6.46: Horizontal (left) and vertical (right) components of motion across the basin for the model with two rock types and Soil Type I properties, SIM VI. The dashed lines display the theoretical arrival times of the P-wave (P), S-wave (S) and an S-wave reflected off the bottom of the model (SbS).

Figure 6.47: Horizontal (left) and vertical (right) components of motion across the basin for the model with two rock types and Soil Type III properties, SIM IX. The dashed lines display the theoretical arrival times of the P-wave (P), S-wave $(\mathrm{S})$ and an S-wave reflected off the bottom of the model (SbS).

Figure 6.48: The smoothed horizontal (left) and vertical (right) components of the simulated Fourier acceleration spectrum at stations KB1O, KB2O, KB3O, and KB6O from SIM VIII. 226 Figure 6.49: The smoothed spectral ratios of the Fourier acceleration spectrum at stations KB2O, KB3O, and KB6O compared to KB1O from SIM VIII. 226 Figure 6.50: Simulated acceleration time series at stations KB1O, KB2O, KB3O and KB6O from 
Figure 6.51: Particle motion plots (top) at KB1O with the time slices outlined in the displacement time series (below).

Figure 6.52: Particle motion plots (top) at KB2O with the time slices outlined in the displacement time series (below).....

Figure 6.53: Particle motion plots (top) at KB3O with the time slices outlined in the displacement time series (below).....

Figure 6.54: Particle motion plots (top) at KB6O with the time slices outlined in the displacement time series (below). 233

Figure I.1: The mechanical model of a generalized Zener Body. 252

Figure III.2: Acceleration time series without a filter applied (top) and with a 4th order Butterworth bandpass filter with cutoff frequencies 0.1 and $5 \mathrm{~Hz}$ (bottom). 283

Figure III.3: Spectral acceleration without a filter applied and with a 4th order Butterworth bandpass filter with cutoff frequencies 0.1 and $5 \mathrm{~Hz}$.

Figure III.4: Recorded acceleration (top) and simulated acceleration (bottom) without any filters

applied.

Figure III.5: Recorded spectral acceleration and simulated spectral acceleration without any filters applied.

Figure III.6: Recorded spectral acceleration and simulated spectral acceleration with a Butterworth bandpass filter of $8^{\text {th }}$ order with cutoff frequencies of 0.3 and $2.5 \mathrm{~Hz}$ applied. .... 286 Figure III.7: Recorded acceleration (top) and simulated acceleration (bottom) Butterworth bandpass filter of 8 th order with cutoff frequencies of 0.3 and $2.5 \mathrm{~Hz}$ 287

Figure III.8: Recorded spectral acceleration and simulated spectral acceleration with a Butterworth bandpass filter of 8th order with cutoff frequencies of 0.3 and $2.5 \mathrm{~Hz}$ applied, and a 12th order low-pass Butterworth filter with cutoff frequency of $2.5 \mathrm{~Hz}$ applied to the recorded acceleration. 288

Figure III.9: Recorded acceleration (top) and simulated acceleration (bottom) Butterworth bandpass filter of 8 th order with cutoff frequencies of 0.3 and $2.5 \mathrm{~Hz}$, and a 12 th order low-pass Butterworth filter with cutoff frequency of $2.5 \mathrm{~Hz}$ applied to the recorded acceleration. 288 Figure III.10: The steps to calculating a displacement time series from acceleration data. The top plot is the original acceleration. The middle plot shows the calculated velocity, and the velocity time series with the instrument drift removed. The bottom plot is the resulating displacement time series. 290 


\section{Introduction}

Earthquakes have the potential to be a devastating natural disaster, causing significant property damage and loss of life. As the understanding of their processes and effects are being studied in more detail it is clear that many factors can be associated with the potential for strong motions and damage. It is evident that the local geology and sediment coverage can significantly affect the amplitudes and durations of strong motions produced by an earthquake. One historically significant and devastating example was the 1985 Ms 8.1 Michoacán earthquake. Mexico City, located 300 kilometers from the epicentre, had an estimated 6 billion dollars in damage and 10,000 casualties as a result of this earthquake. The city was built on a clay-filled basin which amplified the ground motions roughly 14 times greater than nearby bedrock and produced strong motions lasting nearly 3 times longer than on the bedrock. Most of the damage within the city occurred to buildings between 5-15 stories tall, displaying a significant amount of motion was constrained to a small spectral bandwidth (Reiter 1991; Singh et al., 1998; Roullé \& Chávez-García 2006). This earthquake showcased the importance of local site effects on predicting ground motions.

Local site effects can have a strong influence on ground motions at any particular location. Different locations have varying importance depending on whether the site is on outcropping bedrock or has a soil profile. The simplified site effect parameters have been included in the National Building Code of Canada (NBCC 2005, 2010 and 2015) and are based on the average shear wave velocity of the top 30 metres of material. The local site effects include: impedance amplification; resonance amplification; focusing or defocusing of waves; and basin effects 
(Hunter et al., 2010). These have been known to cause significant changes in an earthquake signal as evident from the 1985 Michoacán earthquake, the 1989 Loma Prieta earthquake, and the 1996 Northridge earthquake (Hanks and Krawinkler 1992; Teng and Aki 1996; Singh et al., 1998). The recordings from these earthquakes were the topics for early discussion of local site effects.

Commonly local site effects are split between one dimensional effects such as impedance amplification and resonance amplification, and three dimensional effects from the shape and structure of the subsurface. The one dimensional effects have been studied for different types of material including variations of thicknesses, types and combinations of gravel, sand, silt, and clay (Kwok and Stewart 2006; Boore and Atkinson 2008; Hunter et al., 2010). These studies have produced relatively reliable methods to calculate the amount of amplification when a seismic signal is propagated from the base layer, most often taken as the bedrock, through the different layers of material up to the surface. There are several different programs and methods used for these calculations; for example SHAKE, uses the equivalent linear method (Schnabel et al., 1972).

Although one dimensional effects are important, and for most areas they seem to be the dominant factor in deciding the amount of amplification in a seismic signal, it is becoming increasingly evident that in certain areas the subsurface structure is an additional important factor. This is the case in several areas in the Ottawa-Montreal region which are currently being studied, including the focus area of this research: the Kinburn Basin. These geologically young soft soil basins display increased seismic amplitudes and duration well beyond the predicted values obtained from one dimensional methods. This research is a contribution towards determining the effects of the three-dimensional shape of the subsurface, and using these effects 
to gain a better understanding of the three-dimensional subsurface effects on earthquake ground motions throughout the region.

\subsection{Motivation}

There have been significant strides in classifying the seismic risk across Canada throughout the last 50 years or so. This has taken several forms of research projects including: setting up a network of seismographs capable of detecting earthquakes in Canada; classifying the at risk areas based on the population or infrastructure and their proximity to seismic sources; and determining the expected ground motions based on potential earthquakes. These areas of research have allowed for a better understanding of the natural processes which can have a negative impact on infrastructure.

The seismic network across Canada has allowed for a better classification of seismic source zones. There are several different types of source zones around Canada, with the subduction setting causing the highest risk in Western Canada. There are several source zones in Eastern Canada, including the Western Quebec Seismic Zone (Basham et al., 1979), which is in close proximity to the cities of Montreal and Ottawa.

Adams and Halchuk (2003) produced the fourth generation of seismic hazard maps for Canada and determined the risk for 650 localities. They determined the city with the highest risk was Vancouver, based on its large population and the close proximity to several seismic source zones. The next two cities with the highest risk were Montreal and then Ottawa. Although these cities do not have the largest population of Canadian cities, they are both located either within or very near one of the seismic source zones, increasing their exposure to a potential earthquake. 
The National Building Code of Canada (NBCC 2005, 2010, 2015) recognizes the significant impact the top layers can have on ground motions from an earthquake at a specific site. The seismic provisions included in the NBCC use a combination of the expected ground motions for a region, determined from the seismic hazard maps (Adams and Halchuk, 2003), and the site specific conditions at a building location. These site conditions are based on the strength of the uppermost materials, whether it is rock or soil, and have amplification factors for the ground motions accordingly.

\subsubsection{Seismic Hazard of Ottawa}

Ottawa is the city determined to have the third highest seismic risk in Canada, according to Adams and Halchuk (2003). Located within the Western Quebec Seismic Zone (Basham et al., 1979) moderately sized earthquakes have occurred in the recent past and, according to the earthquake recurrence relations, there are expected earthquakes at medium and close distances. There are also reports of two historic great earthquakes in the region (Lamontagne et al., 2008), which could cause severe ground motions in this area. The National Capital Region, which includes the city of Ottawa and surrounding areas, has a large population and contains various types of critical and important infrastructure. Figure 1.1 shows the locations of the important locations used for this research project including the epicentre of the Ladysmith earthquake, the City of Ottawa, and the Kinburn Basin. 


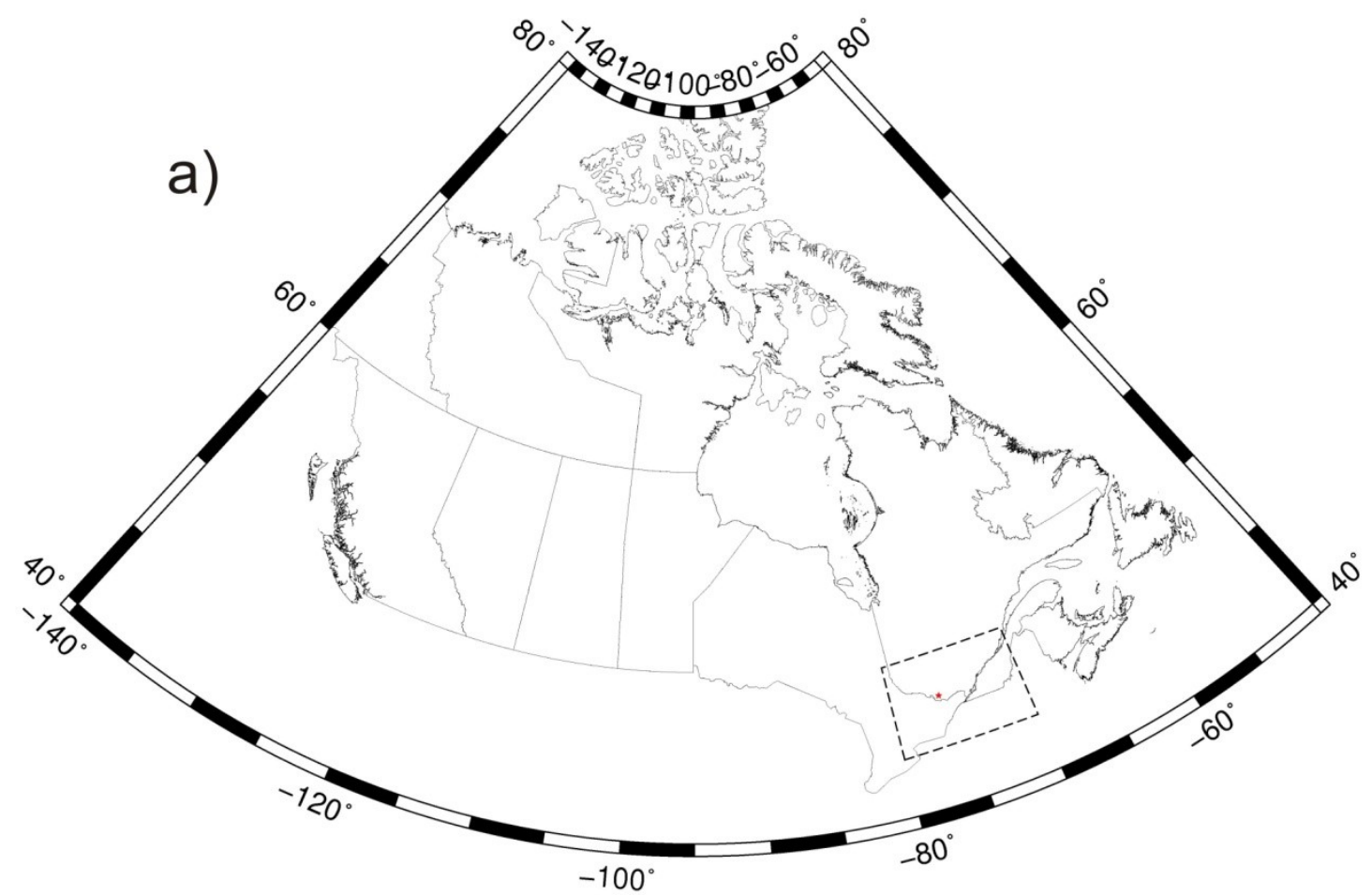

b) $-80^{\circ}$

$-75^{\circ}$

$-70^{\circ}$

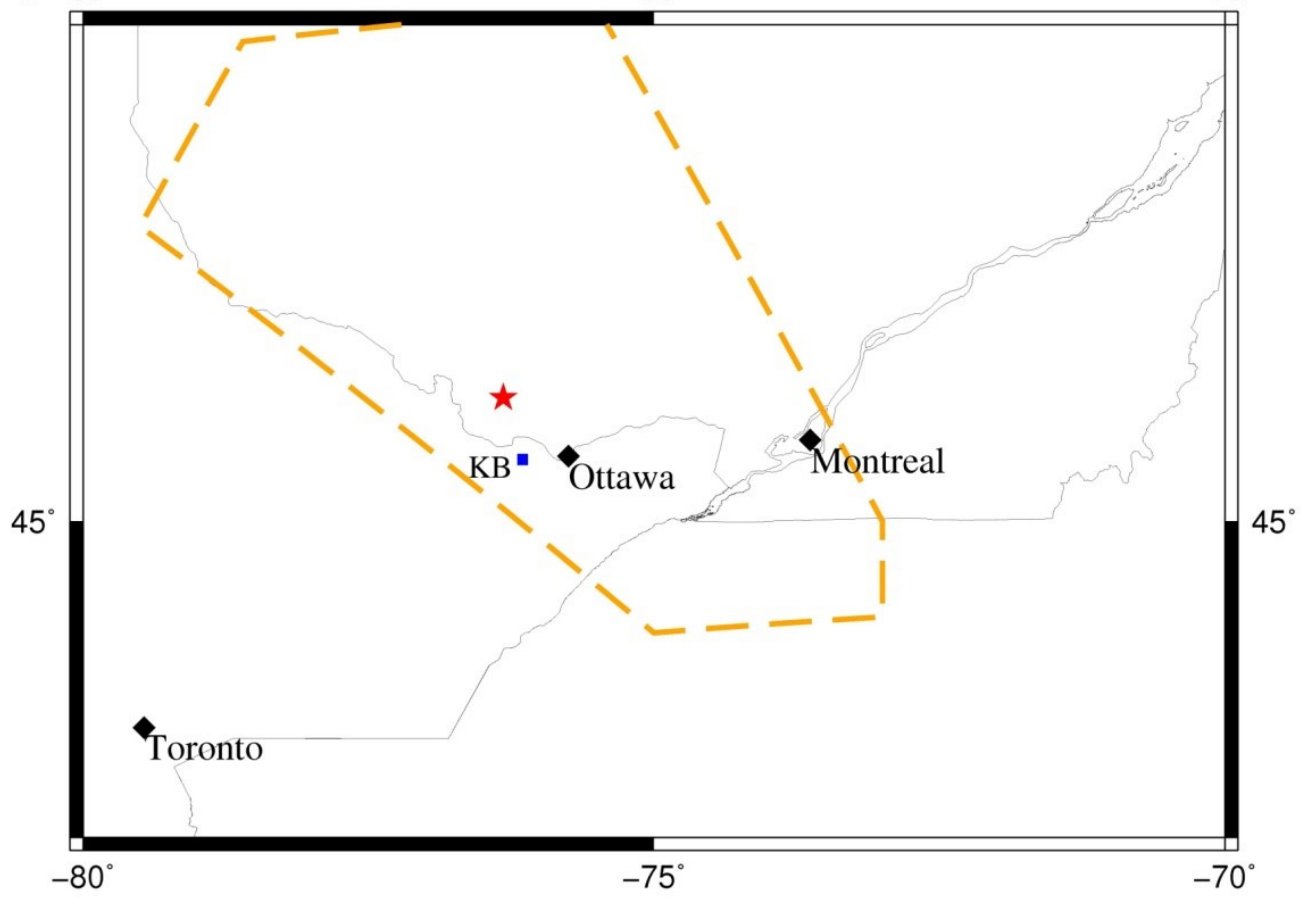

Figure 1.1: a) An outline map of Canada with the epicentre of the May 17th, 2013 Ladysmith earthquake shown by the red star, and the black dashed outline of b) a map showing the West Quebec Seismic Zone (orange contours), the Ladysmith earthquake epicentre (red star), the outline of the Kinburn basin (blue square labelled KB), and the locations of Ottawa, Montreal, and Toronto. 
The seismic risk assessment of Canadian localities has prompted several studies into reducing the exposure to seismic hazards in the cities with a greater risk. The Canadian Seismic Research Network (CSRN), a program funded by the Natural Sciences and Engineering Research Council of Canada (NSERC), was developed to determine and reduce the seismic risk in urban centres. This involved identifying the high risk infrastructure based on building materials, accurately quantifying seismic hazards, and then retrofitting the infrastructure to reduce the risk of failure under probable seismic stains. In Ottawa, one of the elements of this project was to assess the possible difference of ground motions across the city. As such a large geophysical project was undertaken to produce a microzonation map of the city.

\subsubsection{Microzonation}

The city of Ottawa has areas of varying soil thickness, glacial deposits and outcropping bedrock. These features along with a lot of other geological and geophysical data were collected and interpreted over several years to produce a microzonation map of Ottawa. The $\mathrm{V}_{\mathrm{s} 30}$ map, shown in Figure 1.2 (Hunter et al., 2010; Motazedian et al., 2011), displays the site class areas of Ottawa according to the Seismic Classification in the National Building Code of Canada (NBCC, 2010). The data points collected over the city were extensive as shown by Figure 1.3. This figure includes the different types of data collected for this project, including: seismic refraction surveys, various boreholes, and landstreamer lines. This project was a significant step in classifying the regions of higher hazard within the city of Ottawa. 


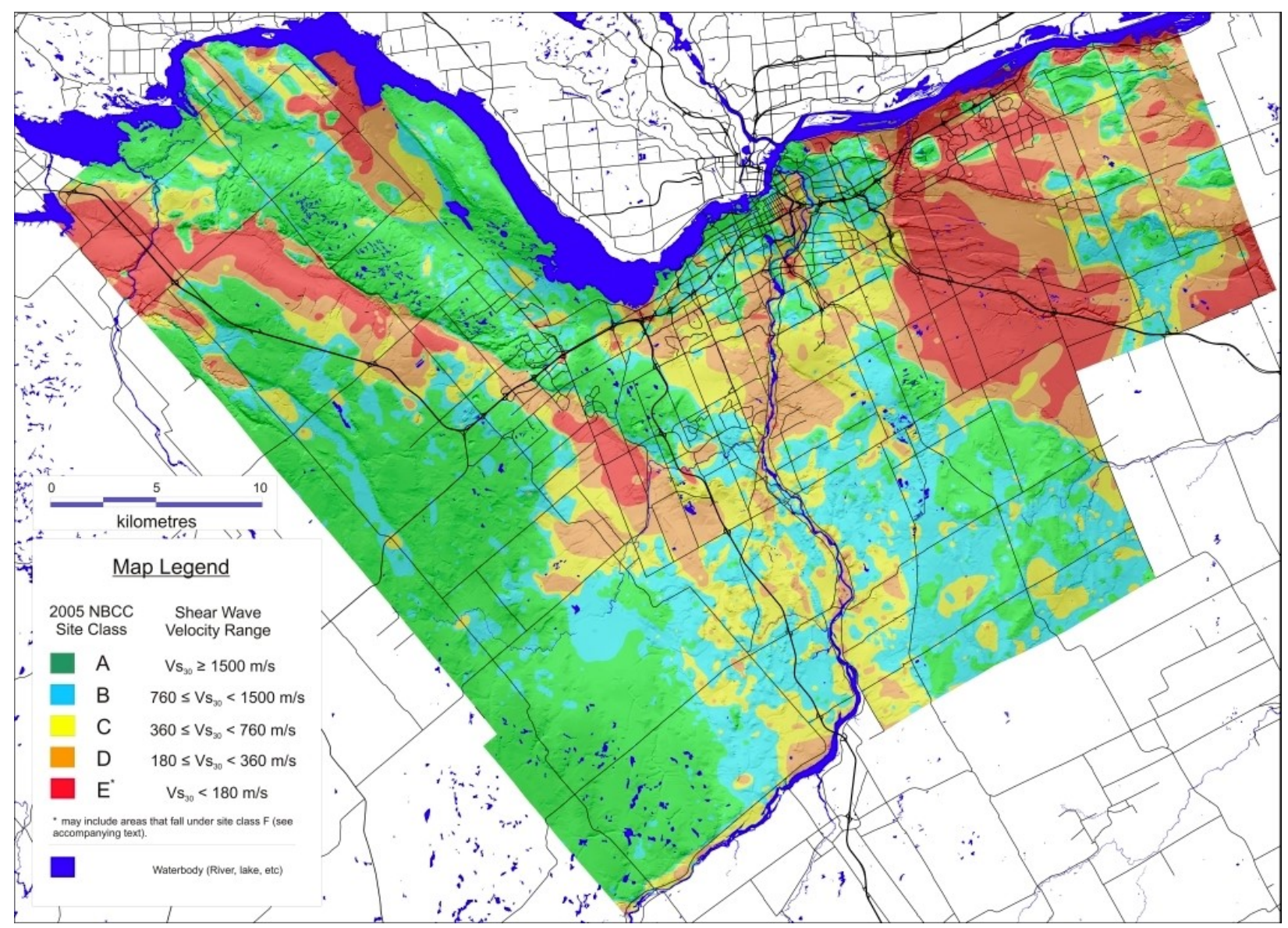

Figure 1.2: Vs30 map of the city of Ottawa from Motazedian et al. (2011). 


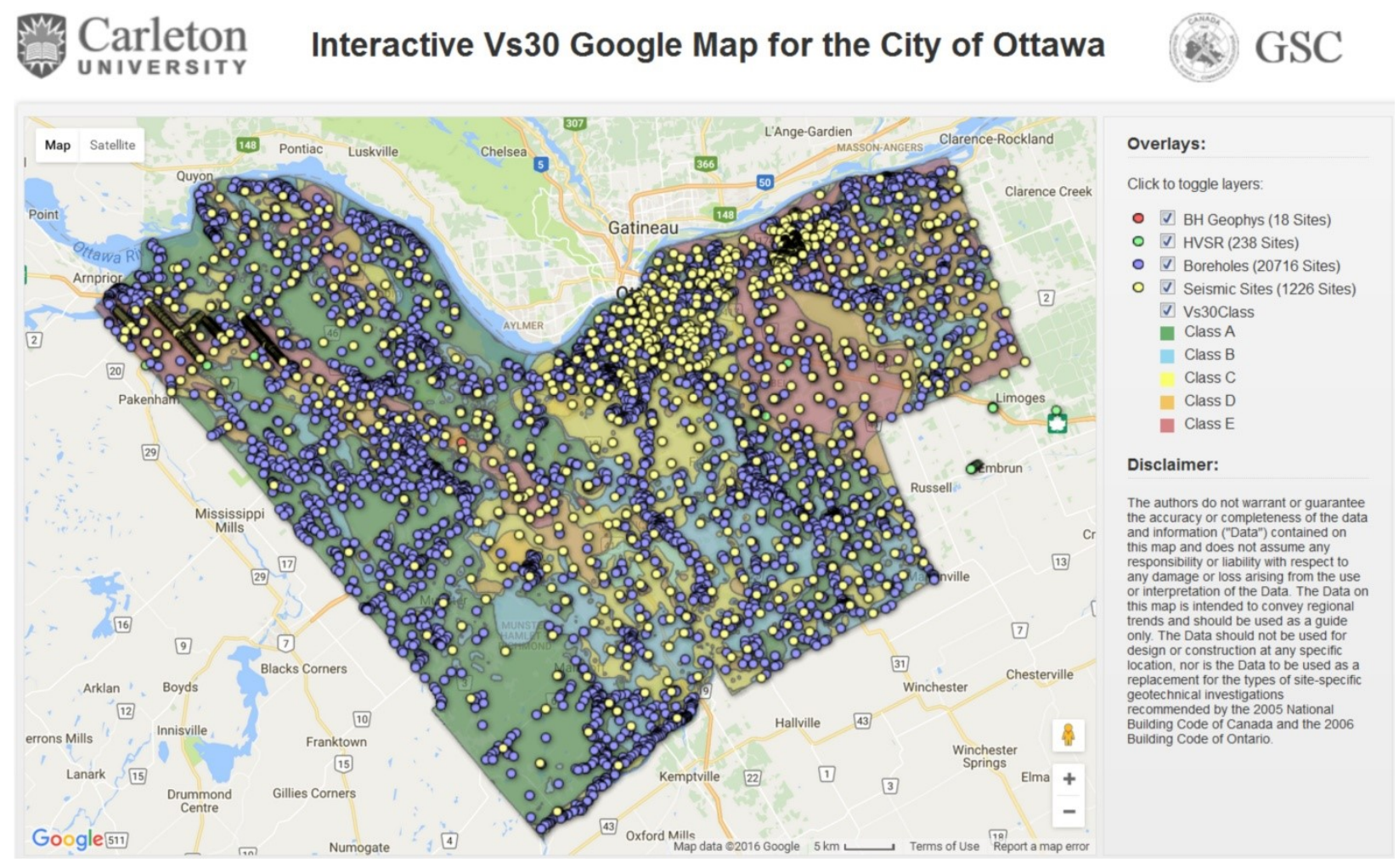

Figure 1.3: Map of Ottawa showing locations of data points collected for the seismic site classification map of Ottawa from Motazedian (2016).

\subsubsection{Velocity Contrast}

The velocity contrast is a large concern for the Ottawa region because the impedance between two layers can be extremely large. The combination of a very hard bedrock, with shear wave velocities around $2700 \mathrm{~m} / \mathrm{s}$, and geologically young soil deposits, with average shear wave velocities of $150 \mathrm{~m} / \mathrm{s}$, creates an impedance ratio of 18-20 from rock to soil (Crow, 2010; Hunter et al., 2010; Khaheshi Banab and Motazedian, 2010; Motazedian et al., 2011). This is a very large contrast compared to the impedance ratios used in the development of the soil site factors in the 2010 version of the NBCC. The soil amplification factors were developed from a typical soil with shear wave velocity between $300-500 \mathrm{~m} / \mathrm{s}$ over a typical Franciscan bedrock formation with shear wave velocities around $1100 \mathrm{~m} / \mathrm{s}$ (Borcherdt, 1994). These impedance ratios are often between 2-4 from the rock to soil. 
This discrepancy can have a large impact on what the soil factors should be, as the amplifications seen in the Ottawa area are much larger than those predicted using the soil factors. As seen in Figure 1.4, for weak ground motions the amplification from a rock station to a nearby soil station often exceeds 20 times at resonance frequencies, and can be as large as 80 times at the fundamental frequency. This is a large variation from the largest estimated amplification, which is around 5 times.

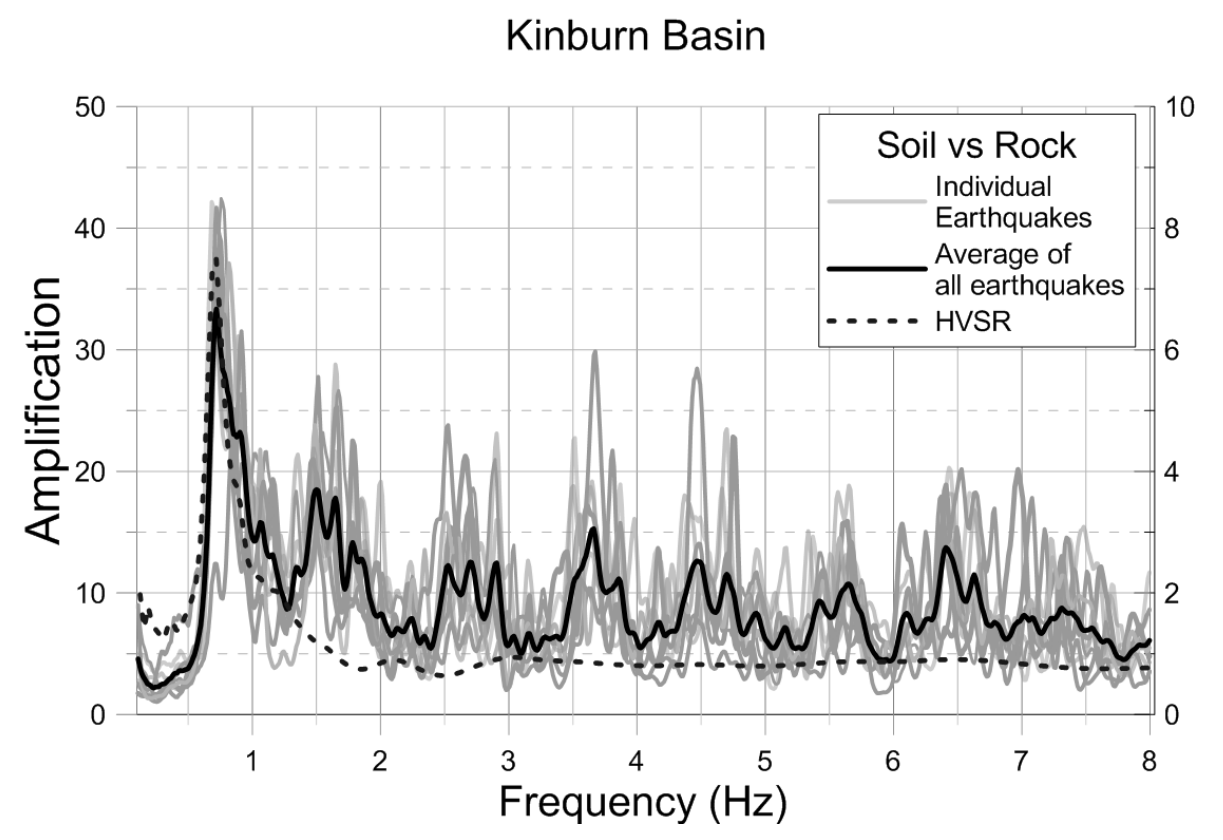

Figure 1.4: Spectral amplification within a soil basin for 9 earthquakes of M3.5 or greater and the Horizontal to Vertical spectral ratio (HVSR) at the recording station from Hayek (2016).

\subsubsection{Soil Basins in the Ottawa Region}

Another outcome of the microzonation project was the fundamental period map shown in Figure 1.5. This map correlates with soil depth better than the $\mathrm{V}_{\mathrm{s} 30}$ map, since the fundamental period is related to the depth and velocity of the layers below a site. This map indicates areas where the young soil is thick in concentrically oval (Kinburn) or circular (Orleans) shapes, both 
outlined in Figure 1.5. This is a strong indication that the bedrock fault structure is associated with soil filled basins.

These soil basins, along with one to the east of the city of Ottawa borders near Alfred, Ontario, were outfitted with a pair of broadband seismometers. One of the seismometers was placed as near the centre of the basins as possible, and the other was placed just outside the basin on a rock outcrop where possible. These seismometer pairs recorded several weak motion events which were studied by Hayek (2016). It was evident from this study that it was not possible to be explain the ground motions within the basin solely by a one-dimensional profile of soil and till layers below a station. 


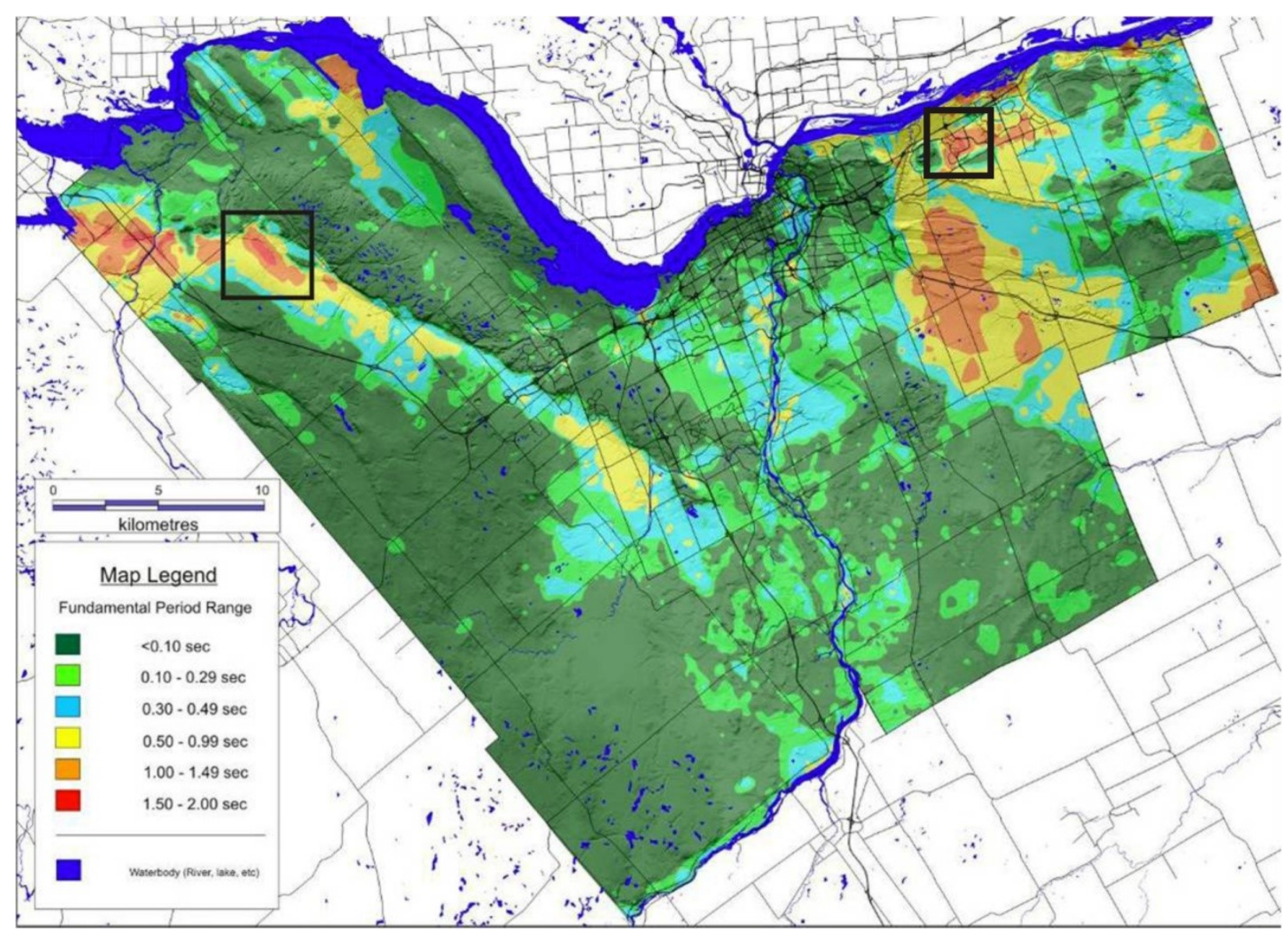

Figure 1.5: A To map of the City of Ottawa. The black squares outline the Kinburn Basin (left) and Orleans Basin (right). Adapted from Hunter et al. (2010).

\subsubsection{Dynamic Soil Properties}

The dynamic soil properties can have a large impact on ground motions at a given location, and exhibit very complex behaviour. There are many factors to consider for the response of soils to seismic loading, and one of the important factors is the strain amplitude (Finn, 1991; Hartzell et al., 2004). Soils are often assumed to behave linearly, however they display non-linear effects at larger strains, commonly with shear strains around $10^{-5}$ or $10^{-4}$. These non-linear effects are complicated to describe, yet essential for seismologists and geotechnical engineers. Therefore it has been the subject of many research projects including laboratory testing of soil strength, insitu soil testing, and measuring ground motions at soil stations from various earthquakes. 
A common explanation for this behaviour was proposed to be a hysteretic stress-strain relationship for unloading-reloading, based on numerous cyclic loading tests on various soil samples (Seed and Peacock, 1971; Hardin and Drnevich, 1972; Bonilla et al., 2005; Chatterjee and Basu, 2008). This hysteretic behaviour contains two parts: a backbone curve (or initial loading curve), and hysteresis loops, shown in Figure 1.6. Many backbone curves have been proposed, and detailed models can be found in Edrik (1987) and Finn (1988). A commonly used backbone curve is:

$$
\tau=G_{\max } \frac{\gamma}{\left(1+\left(G_{\max } \gamma\right) / \tau_{\max }\right)}
$$

where $\tau$ is the shear stress, $\gamma$ is the shear strain, $\tau_{\max }$ is the maximum shear stress, and $G_{\max }$ is the maximum shear modulus. The unloading-reloading fractions of the hysteresis loop is often explained using the modified Masing's rule (Masing, 1926). Using this rule, the unloading and reloading curves can be constructed from the backbone curve with the origin shifted to the reversal point and expanded by a factor of 2 .

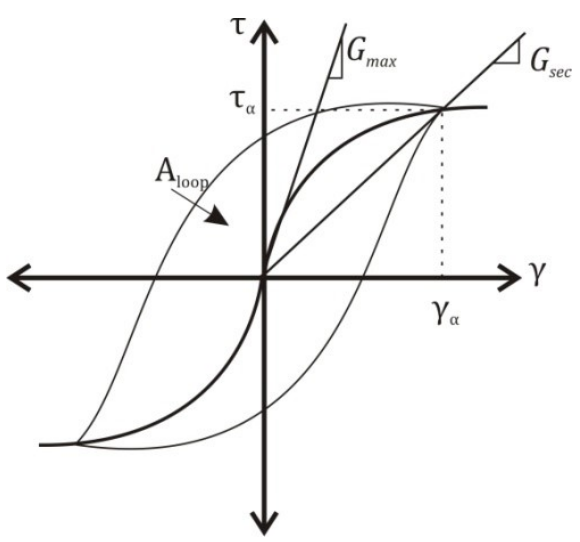

Figure 1.6: A typical hysteresis loop. 
These stress-strain loops display the variation of the shear modulus, $G$, with different strain levels. The shear modulus at low strains is related to the density, $\rho$, and shear wave velocity, $V_{s}$, of a soil by:

$$
G=\rho V_{s}
$$

As shown by the hysteresis loop, Figure 1.6 , the secant shear modulus, $G_{\text {sec }}$ is the average value over an entire loop and decreases with increasing strain.

In addition to the shear modulus variation, each hysteretic loop implies an energy loss or dampening, within a cycle. The dampening, $\xi$, from a hysteretic loop which assumes a viscous dampening, can be described by:

$$
\xi=\frac{W_{D}}{4 \pi W_{S}}
$$

where $W_{D}$ is the dissipated energy, and $W_{S}$ is the maximum energy stored in one cycle.

Hayek (2016) used an equivalent linear approach for a detailed one-dimensional model for the soil station at Kinburn to determine the expected response at that location. Using this method the dampening is described by the equivalent dampening ratio:

$$
\xi=\frac{W_{D}}{4 \pi W_{S}}=\frac{1}{2 \pi} \frac{A_{\text {loop }}}{G_{\text {sec }} \gamma_{\alpha}^{2}}
$$

where $A_{\text {loop }}$ is a measure of the energy dissipation. Using the appropriate curves for Leda Clay and the properties measured at the Kinburn soil station the expected transfer function from rock to soil is shown in Figure 1.7. 


\section{Transfer functions versus recorded spectral ratios}

Using west Quebec earthquakes with Kinburn basin profile

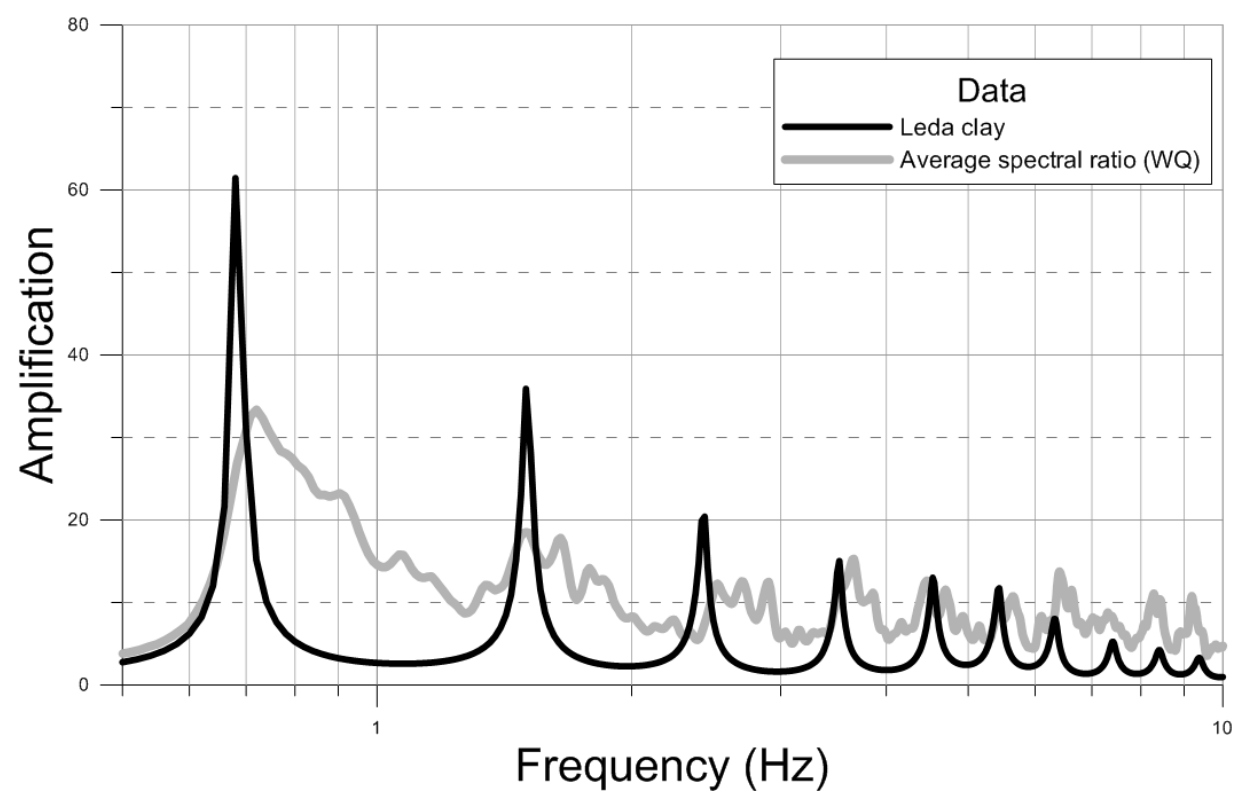

Figure 1.7: Expected transfer function from rock to soil using one-dimensional modelling and the average measured spectral ratio from 9 earthquakes of M3.5 and above in the West Quebec Seismic Zone (from Hayek, 2016).

Currently there are several research groups investigating the dynamic properties of Leda Clay, along with the impact of these effects on amplification of ground motions in different settings (LeBeouf et al., 2013; Motazedian et al., 2015).

\subsection{One Dimensional Amplification}

A change of amplitude of seismic waves occurs when a propagating wave travels through an impedance boundary. This is commonly related to a wave propagating from one material to another. The one-dimensional amplification effects in seismology are most commonly the effects of an upwards propagating seismic wave travelling from denser and faster material, into softer materials such as soil. The comparison is often determined from observing wave motions on an outcropping bedrock recording station, to a nearby station situated atop a soil profile. This 
comparison has found two different types of amplification: impedance amplification, and resonance amplification (Shearer and Orcutt, 1987; Kramer 1996; Hunter et al., 2010).

\subsubsection{Impedance Amplification}

A clear relationship between the amplitude of a seismic wave in bedrock and the amplitude of the corresponding wave in soil has been established over the past few years (Kramer, 1996). This relationship is dependent upon the different densities and shear wave velocities between the bedrock and soil. If the soil shear wave velocity is significantly less than the bedrock shear wave velocity then the wavelength is shortened and the amplitude is increased over a wide frequency band (Shearer and Orcutt, 1987). This relationship, when there is little attenuation present in the soil, is:

$$
A \sim\left(\frac{\rho_{r} v_{s r}}{\rho_{s} v_{S S}}\right)^{1 / 2}
$$

where $A$ is the amplification, $\rho_{r}$ is the density of the rock, $v_{s r}$ is the shear wave velocity of the rock, $\rho_{S}$ is the density of the soil, and $v_{S S}$ is the shear wave velocity of the soil at the ground surface (Hunter et al., 2010).

\subsubsection{Resonance Amplification}

If the impedance contrast between the soil and rock is large, then the other form of amplification which occurs is known as resonance amplification. This effect has gained increasing attention in the field of soil dynamics in the past several years. The theoretical implications have been introduced lately for teaching purposes by Dobry (2013) with the use of a rod with varying properties along its length, analogous to a soil and rock column. The important conclusions for this study are: the peak amplifications at the resonance frequencies, $f_{n}$ with $=0,1,2, \ldots$; and the 
contribution to ground surface motions from the successive wave arrivals and reflections, see Table 2 in Dobry (2013).

The resonant frequencies for any particular soil column without dampening correspond to the average shear wave velocity of the soil, $v_{\text {sav }}$, and the thickness of the soil, $H$, as:

$$
f_{n}=\left(\frac{v_{s a v}}{4 H}(2 n+1)\right) \text { for } n=0,1,2, \ldots
$$

The resonant amplification for the $f_{0}$ (fundamental frequency) is proportional to:

$$
A \sim\left(\frac{\rho_{r} v_{s r}}{\rho_{s} v_{s s}}\right)
$$

Comparing Eq. 1.7 to Eq. 1.5 it can be seen that the resonance amplification is greater than the impedance amplification at the fundamental frequency. Although both equations should contain an attenuation factor when dealing with a material such as soils, the attenuation factor would be dependent on the distance the wave travels. For the first arrival of each type of amplified wave, the comparison between amplified amplitudes can be completed using Eq. 1.5 and Eq. 1.7.

For interval shear wave velocities which follow a powerlaw curve of increasing velocity with soil depth above a resonant boundary, the effective resonant frequency can have a somewhat higher value than the one determined from Eq. 1.6 where only a single average shear wave velocity value is used (Dobry et al., 1976; Kausel 2013; Vrettos 2013).

\subsubsection{One Dimensional Modelling}

Extensive one dimensional modelling of ground motions corresponding to the centre of soil basins in the Ottawa region was recently completed by Hayek (2016). Several soil profiles of high detail were designed based on data located near a seismic recording station located within each 
basin. In the Kinburn Basin, the soil profile was obtained based on geotechnical borehole data located near the recording station. The profile was tested using various dampening ratios and modulus reduction curves and different types of input ground motions.

The conclusions from this work are summarized as follows. The soil layer requires the most detailed information for a successful modeling of the ground motions. Several dampening ratios and modulus reduction curves are acceptable at low strains, however these same curves may not hold at higher strains. The input motions have a significant effect on the resulting motions, therefore the input motions should have the same characteristics as expected earthquake motions at the location. Lastly there were several differences between recorded motions and those simulated using a one dimensional technique indicating that modelling the ground motions within the Kinburn Basin cannot be accurately simulated with a one dimensional approach.

\subsection{Basin Amplification}

As previously mentioned the shape and structure of the subsurface can have a significant effect on the ground motion at a particular site. This effect is highest if the subsurface structure is shaped like a basin. It has been theorized and shown in several studies that a basin shape to the bedrock or subsurface causes different types of amplification of ground motions. This includes creating surface waves at the basin edge boundaries (Bard and Bouchon 1980a; Bard and Bouchon 1980b; Kawase and Aki 1989; Kawase 1996; Hunter et al., 2010), focusing of waves when propagating through an impedance boundary (Graves et al., 1998; Semblat et al., 2002; 
Hunter et al., 2010), and the entire basin resonating at a frequency strongly dependent on the maximum depth of the basin (Bard and Bouchon, 1985).

There have been several studies which have shown some or all of these effects in a sedimentary basin (Frankel and Vidale, 1992; Frankel, 1993; Olsen et al., 1995; Olsen et al., 1996; Pritarka, 1999; Frankel and Stephenson, 2000; Joyner, 2000; Olsen, 2000a; Olsen, 2000b; Komatitsch et al., 2004; Frankel et al., 2007; Frankel et al., 2009; Molnar et al., 2014a; Molnar et al., 2014b), mostly across Western North America. These studies use three-dimensional numerical modelling techniques, usually finite difference, to compare the difference in strength and duration of seismic motions in an area when there is a contrast in the shear wave velocity of the sedimentary basin, and when the sedimentary basin has the same shear wave velocity as the surrounding bedrock types. These studies concluded that the durations and amplitudes of seismic ground motions are increased in an area with a basin structure, however identifying the cause of these amplifications proves to be difficult in most cases.

The methods above simulated ground motions in large sedimentary basins, and often did not include the top layer of material due to the implications for modelling. Modelling the top layer of soft sediments in numerical methods can present a difficult challenge, as the grid size must be reduced in order to maintain the accuracy. For the large sedimentary basins in those studies, the reduction in grid size would have drastically increased the computations, likely beyond what was available. However, this top layer of soil or soft sediments can have a significant impact on the ground motions as well. This has been determined from the one dimensional studies of local site effects (Hayek, 2016). In the Ottawa-Montreal region, the basins are smaller 
in size and can contain a combination of glacial and post-glacial sediments. The different types of basin effects are discussed below along with a description of the expected or observed impact on the ground motions.

\subsubsection{Focusing of Seismic Waves}

Waves travelling through an impedance boundary become reflected and refracted at certain angles depending on the properties of the different materials. If the subsurface structure resembles a bowl shape then the refracted wavefronts will change their angle towards a central area (Graves et al., 1998; Semblat et al., 2002; Hunter et al., 2010). This is known as focusing of seismic waves, shown in Figure 1.8. These wavefronts then interfere "coherently" and the resulting ground motions will be amplified.

This phenomenon can also behave the opposite way if the substructure shape resembles a hill. The wavefront will be refracted away from the centre, an effect which is known as defocusing, also shown in Figure 1.8. Less energy is directed towards the centre and the resulting surface ground motions will show a decrease in amplitude. During the course of this research it was shown that the subsurface impedance boundary is irregular and jagged in some areas, resulting in the possibility of both focusing and defocusing within the basin. 


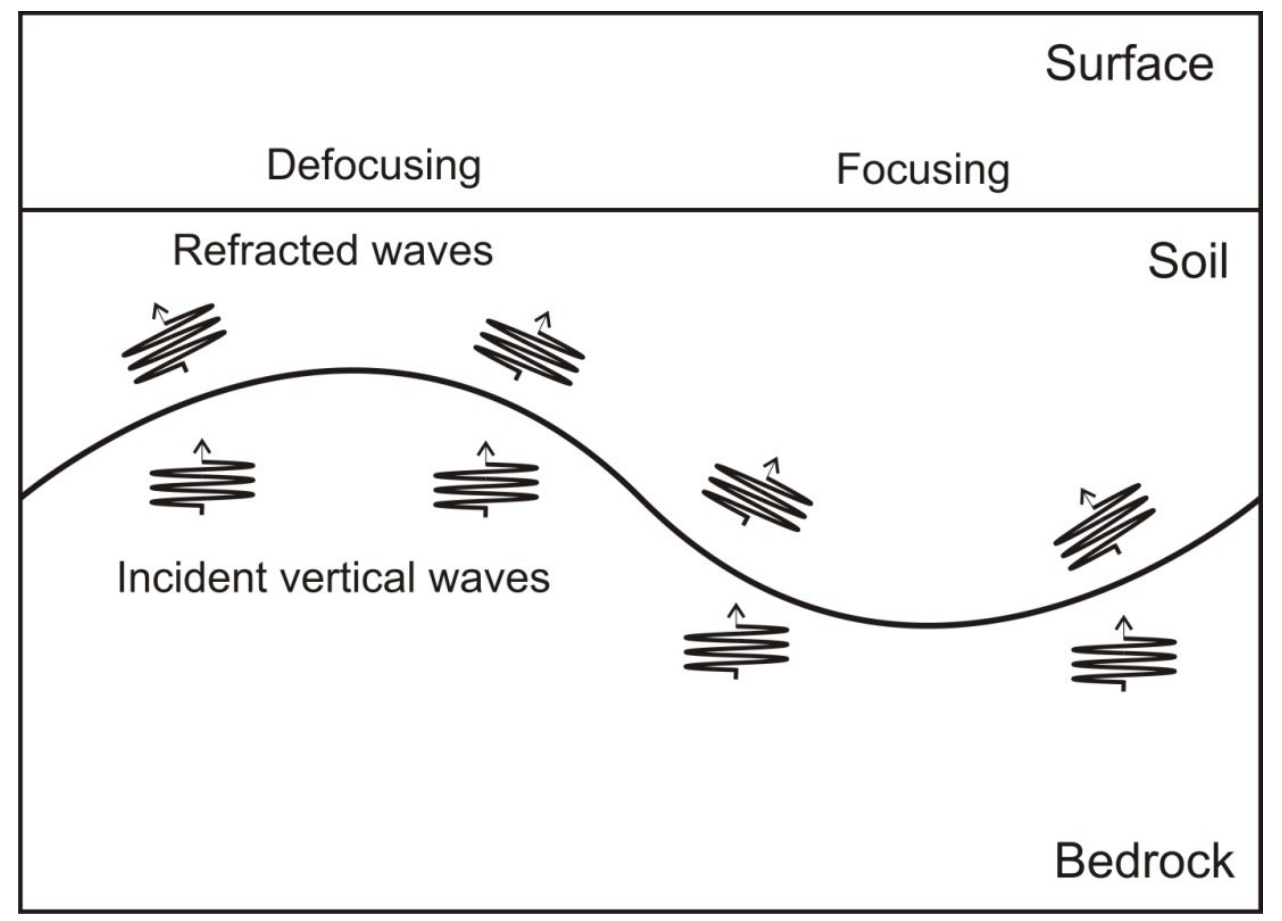

Figure 1.8: Focusing and defocusing of seismic waves through an irregular shaped impedance boundary.

\subsubsection{Generation of Surface Waves}

Apart from regionally generated surface waves usually a few hundred kilometres away from a large and shallow earthquake, surface waves can be locally generated by body waves interacting with a shallow soil/rock angled interface (Bard and Bouchon, 1980a; Bard and Bouchon, 1980b; Kawase and Aki, 1989; Kawase, 1996; Hunter et al., 2010). These locally generated surface waves are commonly referred to as "Basin Edge Effects" (Kawase, 1996) because they are commonly seen in sedimentary basins around the edges (Frankel and Vidale, 1992; Olsen, 2000a; Olsen, 2000b; Frankel et al., 2007; Frankel et al., 2009). In larger basins these surface waves are easier to track spatially, however in several cases such as this study, there are several reflected and refracted wave types travelling throughout the basin at any time. This will make it more difficult to track the generated surface waves even with an array of receivers throughout the basin. 
Several studies have attributed this basin edge effect to longer durations of motions within a basin (Kawase and Aki, 1989), along with increased damage to infrastructure around the surrounding area of the basin edges (Kawase, 1996). This effect should be important when considering ground motions within a basin setting, as it changes almost all aspects of the expected motion.

\subsubsection{Basin Resonance}

Basin resonance is a unique ground motion response which was discovered by Bard and Bouchon (1985) after performing a two-dimensional numerical simulation of deep sediment valleys. The response of the valley, at receivers placed at regular intervals across the surface of the valley, had a resonant frequency which was dependent upon the maximum depth and the shape ratio of the valley. The shape ratio is defined as the depth of the valley divided by half the length of the valley. The fundamental resonant frequency at the centre of the valley was shifted due to the shape ratio and did not match the expected one-dimensional fundamental frequency as found in Eq. 1.6. This was the case for each wave phase including $\mathrm{P}, \mathrm{SV}$, and $\mathrm{SH}$ waves. Other locations in the basin displayed a fundamental resonant frequency which was dependent on the maximum depth of the valley, and not the depth at each location that was recorded as expected. As this was derived using a numerical technique, it is unclear whether this is actual the behaviour of a valley or an artefact of the simulation method. It is expected to see this response when using other numerical methods. 


\subsection{Comparison of Different Numerical Techniques}

There are several numerical modelling techniques which could be used for this purpose, and similar research projects are underway. The most widely used method, in several variations, is the finite difference technique. This method uses a rectangular grid over the specified volume which usually has a consistent distance between grid points. However the distances between grid points in this method can be changed within a volume, if a staggered grid technique is used. Finite difference uses the distance between grid points as the differential operator to solve the equations of motion. Another method is a finite element technique, which uses shape functions over elements to solve a polynomial function over that element. The accuracy of a finite element method is dependent upon the order of polynomial function and the size of an element. One version of the finite element method is the spectral element method. This method uses a spectral solution over the different elements. In large simulations, the computational requirements for the finite element method are often far greater to achieve the same accuracy as the other methods provide. The spectral element method has a diagonal mass matrix by construction, reducing the computational requirements for this method.

These methods have proven to be accurate in simulating ground motions in basins, however the finite difference method is unable to fully capture the curvature of the impedance boundary between the basin and host rock due to the use of rectangular grids. The spectral element method, and higher order finite element methods can use a hexahedral mesh which conforms around the basin boundaries. The finite difference method also uses similar distances between grid points in the mesh, not dependent on the material in which the grid point is located. As discussed in more detail in sections 1.5.1 and 1.5.2, this can be problematic with the conditions 
present in this study. Either the mesh distance must be small, which causes an excessive increase in computation requirements due to unnecessary grid points in the rock, or the distance between grid points is larger, but all accuracy within the soil is lost except for the extremely long periods. The higher order finite element techniques would require a significant increase in the computational requirements for the simulations in this project.

\subsection{Spectral Element Method}

The spectral element method (SEM) is used to create synthetic seismograms for several receivers located within a region. It is a finite element method which uses a spectral solution to solve a large system of equations over a two or three-dimensional volume. A very brief description is provided, but for a more detailed explanation of the method and its implementation see for example Komatitsch and Tromp (1999). This implementation of the SEM is in the programs SPECFEM2D and SPECFEM3D (Komatitsch and Vilotte, 1998; Komatitsch and Tromp, 1999; Komatitsch et al., 2002; Komatitsch and Tromp, 2002a; Komatitsch and Tromp, 2002b). The method as discussed briefly below explains how SEM was implemented for the models in this study. A more technical description of this method is discussed in Appendix I.

A given volume is discretized into non-overlapping hexahedral mesh elements associated with the free surface and any internal boundaries, including the boundary of the basin. A "weak" formulation, or the integral form of the equations of motion, is solved over the discrete space. These equations of motion are solved throughout the volume, with the associated boundary conditions at the free surface and the absorbing boundary conditions at the edges of the volume. The global system is: 


$$
M \ddot{U}+C \dot{U}+K U=F
$$

where $U$ is the displacement vector of the global system, $M$ is the global mass matrix, $C$ is the global absorbing boundary matrix, $K$ is the global stiffness matrix, and $F$ is the source term. The global system, after being constructed, is solved in time using time steps which are marched forward using a classical explicit second order Finite Difference scheme.

\subsubsection{Courant-Friedrichs-Lewy Condition}

The Courant-Friedrichs-Lewy (CFL) condition is a necessary stability condition of numerical simulations which use an explicit time integration scheme. It was introduced by Courant, Friedrichs, and Lewy in 1928 (Courant et al., 1967). This condition places a restriction upon how large the time step can be for a given simulation. The Courant number, $\mathrm{C}$, is defined as:

$$
C=\Delta t \sum \frac{v_{x_{i}}}{x_{i}} \leq C_{\max }
$$

where $\Delta t$ is the time step, $x_{i}$ is the spatial direction, and $v_{x_{i}}$ is the velocity in the associated direction. In models with an isotropic velocity and varying mesh sizes the CFL condition on the size of the time step becomes:

$$
\Delta t \leq C \times \min _{\Omega}\left(\frac{d l}{v_{p}}\right)
$$

where $d l$ is the distance between two grid points, $v_{p}$ is the P-wave velocity, $\min _{\Omega}$ is the minimum value within the entire model volume, and $C \sim 0.3$ for the time stepping scheme and Lagrange polynomial degree used for these simulations. Appendix II shows that each simulation satisfies this condition along with the other important simulation parameters. 


\subsubsection{Mesh Spacing}

The spacing between grid points determines the frequency a simulation can be accurate up to, as the minimum resolution needed to resolve a frequency is at least 5 points per wavelength. The wavelength, $\lambda$, can be determined by the frequency, $f$, and the velocity of the material it is propagating through, $V$, and is given by:

$$
\lambda=V / f
$$

This restriction is critical for our project, as the spatial resolution needed in the soil is much smaller than the spatial resolution needed in the rock. However, the mesh along the impedance boundary must be consistent between the rock and soil layers. This causes the time step from needed for a stable computation to be small, based upon the CFL condition. The spectral element technique in this project uses 5 points per mesh element in each direction so the accuracy for any simulation is determined by the largest mesh size within the material with the lowest S-wave velocity, but the time step is constrained by the smallest mesh size within the material with the largest P-wave velocity.

\subsubsection{Previous Studies using SEM}

There have been many studies and applications of the spectral element method for research purposes. Specifically for the programs used in this study, SPECFEM2D and SPECFEM3D, the previous applications which are similar to this study include the modelling of several different basins around the world. These include the Los Angeles Basin, California (Komatitsch et al., 2004), the Taipei Basin, Northern Taiwan (Lee et al., 2008), the Grenoble valley, France (Chaljub et al., 2010), and the Mygdonian basin, Greece (Maufroy et al., 2015). 
Of these studies, the most similar in conditions to the Kinburn basin is the Mygdonian basin. This basin was modelled with a top layer of shear wave velocity of $200 \mathrm{~m} / \mathrm{s}$ and a bedrock with a shear wave velocity of $2600 \mathrm{~m} / \mathrm{s}$. There were several layers between the top layer and bedrock, and the maximum simulation frequency was $4 \mathrm{~Hz}$. The width of the basin is roughly 5-6 kilometers, however it is not confined to its length in the simulations. The spectral element method had a comparable performance to the other numerical methods of simulating the basin (Maufroy et al., 2015).

Although the Mygdonian basin shares some similarities with the Kinburn basin, there are a few significant differences. The Kinburn basin is confined in all directions, except for a small horseshoe valley near the town of Kinburn. Also, the Kinburn basin does not have the same layering in the sediments as the Mygdonian basin. The conditions of the Kinburn basin are unique to the Ottawa-Montreal regions, and attempts to complete numerical simulations for a very small basin with a very high impedance contrast to its surrounding has not been attempted. Therefore the numerical modelling of the Kinburn basin is a new challenge which provides the opportunity to better understand a very unique situation.

\subsection{References:}

Adams, J., and S. Halchuk, (2003). Fourth generation seismic hazard maps of Canada: Values for over 650 Canadian localities intended for the 2005 National Building Code of Canada. Geological Survey of Canada, Open File 4459, 1-155.

Bard, P.-Y. and M. Bouchon, (1980a). The seismic response of sediment-filled valleys. Part I. The case of incident SH waves, Bull Seismol Soc Am, 70, 1263-1286.

Bard, P.-Y. and M. Bouchon, (1980b). The seismic response of sediment-filled valleys. Part II. The case of incident $P$ and SV waves, Bull Seismol Soc Am, 70, 1921-1941. 
Bard, P.-Y. and M. Bouchon, (1985). The two-dimensional resonance of sediment-filled valleys, Bull Seismol Soc Am, 75, 519-541.

Basham, P. W., D. H. Weichert., and M. J. Berry (1979). Regional assessment of seismic risk in eastern Canada. Bull. Seism. Soc. Am. 69, 1567-1602.

Bonilla, L.F., R.J. Archuleta, and D. Lavallée (2005). Hysteretic and dilatant behavior of cohesionless soils and their effects on nonlinear site response; field data observations and modeling. Bull. Seism. Soc. Am., 95 (6): 2373-2395

Boore, D. M., and Atkinson, G. M. (2008). Ground-motion prediction equations for the average horizontal component of PGA, PGV, and 5\%-Damped PSA at Spectral Periods between $0.01 \mathrm{~s}$ and 10.0 s, Earthquake Spectra, 24, 99-138.

Borcherdt, R.D., 1994. Estimates of site-dependent response spectra for design (methodology and justification), Earthquake Spectra, 10(4): 617-653.

Chatterjee, P. and B. Basu (2008). Some analytical results on lateral dynamic stiffness for footings supported on hysteretic soil medium. Soil Dyn. Earthq. Eng., 28 (1), 36-43.

Chaljub, E., P. Moczo, S. Tsuno, P.-Y. Bard, J. Kristek, M. Käser, M. Stupazzini, and M. Kristeková (2010). Quantitative comparison of four numerical predictions of 3D ground motion in the Grenoble valley, France. Bull. Seismol. Soc. Am., 100, 1427-1455.

Crow, H. (2010). Low strain shear wave damping (Qs) measurements in Champlain Sea deposits using downhole geophysical and lab techniques. M.Sc. Thesis, Department of Earth Sciences, Carleton University.

Dobry, R. (2013). Radiation damping in the context of one-dimensional wave propagation: A teaching perspective, Soil Dyn. Earthq. Eng., 47, 51-61.

Dobry, R., I. Oweis, and A. Urzua (1976). Simplified procedures for estimating the fundamental period of a soil profile. Bull. Seis. Soc. Am., 66, 1293-321.

Edrik, M. (1987). Site response analysis, in Strong Ground Motion Seismology. D. Reidel Publishing Company, Dordrecht, Netherlands, 479-534.

Frankel, A. (1993). Three-dimensional simulations of ground motions in the San Bernardino valley, California, for hypothetical earthquakes on the San Andreas fault, Bull. Seis. Soc. Am., 83, 1020-1041.

Frankel, A., and J. Vidale (1992). A three-dimensional simulation of seismic waves in the Santa Clara valley, California, from a Loma Prieta aftershock, Bull. Seis. Soc. Am., 82, 2045-2074. 
Frankel, A., and W.J. Stephenson (2000). Three-dimensional simulations of ground motions in the Seattle region for earthquakes in the Seattle fault zone, Bull. Seis. Soc. Am., 90, 1251-1267.

Frankel, A., W.J. Stephenson, D.L. Carver, R.A. Williams, J.K. Odom, and S. Rhea, (2007). Seismic hazard maps for Seattle incorporating 3D sedimentary basin effects, nonlinear site response, and rupture directivity, USGS Open File Report 2007-1175.

Frankel, A., W.J. Stephenson, and D. Carver, (2009). Sedimentary basin effects in Seattle, Washington: Ground-motion observations and 3D simulations, Bull. Seis. Soc. Am., 99, 15791611.

Finn, W.D.L. (1988). Dynamic analysis in geotechnical engineering, Proceedings, Earthquake Engineering and Soil Dynamics II - Recent Advances in Ground Motion Evaluations. Geotechnical Special Publication, ASCE, 20, 523-591.

Finn, W.D.L. (1991). Geotechnical aspects of microzonation. Proceedings of the Fourth International Conference of seismic zonation, Stanford, California, 199-259.

Graves, R., (1996). Simulating seismic wave propagation in 3D media using staggered-grid finite differences, Bull. Seis. Soc. Am., 86, 1091-1106.

Graves, R., A. Pitarka, and P. Sommerville, (1998). Ground-motion amplification in the Santa Monica area: Effects of shallow basin-edge structure, Bull. Seis. Soc. Am., 88, 1224-1242.

Hanks, T.C., and H. Krawinkler (1991). The 1989 Loma Prieta, CA earthquake and its effects. Bull. Seis. Soc. Am., 81 (5).

Hardin, B.O. and V.P. Drnevich (1972). Shear modulus and damping in soils; design equations and curves Journal of the Soil Mechanics and Foundations Division, 98, 667-691

Hartzell, S., L.F. Bonilla, and R.A. Williams (2004). Prediction of nonlinear soil effects. Bull. Seis. Soc. Am., 94, 1609-1629.

Hayek, S. (2016). Seismic basin effects over soft-sediment filled basins in Ottawa, Canada. M.Sc. Thesis, Department of Earth Sciences, Carleton University.

Hunter, J.A., H.L. Crow, G.R. Brooks, M. Pyne, D. Motazedian, M. Lamontagne, A.J.-M. Pugin, S.E. Pullan, T. Cartwright, M. Douma, R.A. Burns, R. L. Good, K. Kaheshi-Banab, R. Caron, M. Kolaj, I. Folahan, L. Dixon, K. Dion, A. Duxbury, A. Landriault, V. Ter-Emmanuil, A. Jones, G. Plastow, D. Muir (2010). Seismic site classification and site period mapping in the Ottawa area using geophysical methods. Geological Survey of Canada. Open File 6273.

Joyner, W.B., (2000). Strong motion from surface waves in deep sedimentary basins, Bull. Seis. Soc. Am., 90, 6B, S95-S112.

Joyner, W.B., R.E. Warrick, and T.E. Fumal, (1981). The effect of Quaternary alluvium on strong ground motion in the Coyote Lake, California, earthquake of 1979, Bull. Seis. Soc. Am., 71, 1333-1349. 
Khaheshi Banab, K., and D. Motazedian (2010). Efficiency of the multichannel analysis of surface wave method for shallow and semi-deep loose soil layers overlaying a very high shear wave velocity bedrock, Int. J.Geophys. Article ID 403016, doi: 10.1155/2010/403016.

Kausel, E., (2013). On the frequencies of inhomogeneous soil strata: Dobry's paradox, Soil Dyn. Earthq. Eng., 47, 38-40.

Kawase, H., (1996). The cause of the damage belt in Kobe: 'the basin-edge effect', constructive interference of the direct $\mathrm{S}$ wave with the basin-induced diffracted/Rayleigh waves, Seis. Res. Lett., 67, 25-35.

Kawase, H., and K. Aki (1989). A study on the response of a soft basin for incident S, P, and Rayleigh waves with special reference to the long duration observed in Mexico City, Bull. Seis. Soc. Am., 79, 1361-1382.

Komatitsch, D. and J.P. Vilotte, (1998). The spectral element method: an efficient tool to simulate the seismic response of 2D and 3D geological structures, Bull. Seism. Soc. Am., 88, 368-392.

Komatitsch, D., and J. Tromp, (1999). Introduction to the spectral-element method for 3-D seismic wave propagation, Geophys. J. Int., 139, 806-822.

Komatitsch, D., and J. Tromp, (2002a). Spectral-element simulations of global seismic wave propagation - I. Validation, Geophys. J. Int., 149, 390-412.

Komatitsch, D., and J. Tromp, (2002b). Spectral-element simulations of global seismic wave propagation - II. Three-dimensional models, oceans, rotation, and self-gravitation, Geophys. J. Int., 150, 303-318.

Komatitsch, D., J.P. Vilotte, R. Vai, J.M. Castillo-Covarrubias, and F.J. Sanchez-Sesma, (1999). The spectral element method for elastic wave equations: application to 2D and 3D seismic problems, Int. J. Num. Meth. Eng., 45, 1139-1164.

Komatitsch, D., J. Ritsema, and J. Tromp (2002). The spectral-element method, Beowulf computing, and global seismology, Science, 298, 1737-1742.

Komatitsch, D., Q. Liu, J. Tromp, P. Süss, C. Stidham, and J. H. Shaw (2004). Simulations of ground motion in the Los Angeles basin based upon the spectral-element method, Bull. Seismol. Soc. Am., 94, 187-206.

Kramar, S. L. (1996). Geotechnical Earthquake Engineering, Prentice Hall, first edition. 
Kwok, A. O., and Stewart, J. P. (2006). Evaluation of the effectiveness of theoretical 1D amplification factors for earthquake ground-motion prediction, Bull. Seismol. Soc. Am. 96, 1422-1436.

Lamontagne, M., S. Halchuk, J. F. Cassidy, and G. C. Rogers (2008). Significant Canadian Earthquakes of the Period 1600-2006. Seism. Res. Lett. 79, 211-223.

LeBoeuf, D., S. Sivathayalan, D. Motazedian, G. Cascante, and H.L. Crow, (2013). Investigating the Dynamic Properties of Leda Clay, in proceedings, Eastern Section of the Seismological Society of America, LaMalbaie, QC October 2013.

Lee, S.J., H.W. Chen, Q. Liu, D. Komatitsch, B.S. Huang, and J. Tromp (2008). Three-dimensional simulations of seismic wave propagation in the Taipei basin with realistic topography based upon the spectral- element method, Bull. Seismol. Soc. Am., 98, 253-264, doi 10.1785/0120070033.

Masing, G., (1926). Eigenspannungen und vertfestigung beim messing. In Proceedings of the second international congress of applied mechanics, Zurich, Switzerland, 332-335 [in German].

Maufroy, E., E. Chaljub, F. Hollender, J. Kristek, P. Moczo, P. Klin, E. Priolo, A. Iwaki, T. Iwata, V. Etienne, F. De Martin, N.P. Theodoulidis, M. Manakou, C. Guyonnet-Benaize, K. Pitilakis, and P.-Y. Bard (2015). Earthquake ground motion in the Mygdonian basin, Greece: The E2VP verification and validation of 3D numerical simulation up to $4 \mathrm{~Hz}$. Bull. Seis. Soc. Am., 105, 1398-1418.

Molnar, S., J.F. Cassidy, K.B. Olsen, S.E. Dosso, and J. He (2014a). Earthquake ground motion and 3D Georgia Basin amplification in Southwestern British Colombia: Deep Juan De Fuca Plate scenario earthquakes, Bull. Seis. Soc. Am., 104, 301-320.

Molnar, S., J.F. Cassidy, K.B. Olsen, S.E. Dosso, and J. He (2014b). Earthquake ground motion and 3D Georgia Basin amplification in Southwestern British Colombia: Shallow Blind-thrust scenario earthquakes, Bull. Seis. Soc. Am., 104, 321-335.

Motazedian, D., J.A. Hunter, A. Pugin, and H. Crow, (2011). Development of a Vs30 (NEHRP) map for the city of Ottawa, Ontario, Canada. Can. Geotech. J., 48, 458-472.

Motazedian, D., J.A. Hunter, H. Torabi, S. Crane, S. Hayek, and H.L. Crow (2015). Investigation of soil amplification factors for the Ottawa area. Part I - Fa, in proceedings, 11 Canadian Conference on Earthquake Engineering, Victoria, BC, July 2015. 
Motazedian, D. (2016). Interactive Vs30 map for the city of Ottawa, available at: http://httpserver.carleton.ca/ dariush/Microzonation/vs30-map/google_map.html last accessed September 2016.

NBCC. (2005). Canadian commission on building and fire codes. National Building Code of Canada (NBCC), National Research Council Canada, Ottawa, Ont.

NBCC. (2010). Canadian commission on building and fire codes. National Building Code of Canada (NBCC), National Research Council Canada, Ottawa, Ont.

NBCC. (2015). Canadian commission on building and fire codes. National Building Code of Canada (NBCC), National Research Council Canada, Ottawa, Ont.

Olsen, K.B., (2000a). 3D Viscoelastic wave propagation in the Upper Borrego valley, California, constrained by borehole and surface data, Bull. Seis. Soc. Am., 90, 134-150.

Olsen, K.B., (2000b). Site amplification in the Los Angeles basin from three dimensional modelling of ground motion, Bull. Seis. Soc. Am., 90, S77-S94.

Olsen, K.B., J.C. Pechmann, and G.T. Schuster, (1995). Simulation of 3D elastic wave propagation in the Salt Lake basin, Bull. Seis. Soc. Am., 85, 1688-1710.

Olsen, K.B., and R.J. Archuleta, (1996). Three-dimensional simulation of earthquakes on the Los Angeles fault system, Bull. Seis. Soc. Am., 86, 575-596.

Pitarka, A., (1999). 3D elastic finite-difference modelling of seismic motion using staggered grids with nonuniform spacing, Bull. Seis. Soc. Am., 89, 54-68.

Reiter, L., (1991). Earthquake ground motion: Site effects, in Earthquake hazard analysis: Issues and insights, Columbia Univ. Press, NY, p.147-163.

Roullé, A., and F.J. Chávez-García, (2006). The strong ground motion in Mexico City: Analysis of data recorded by a 3D array, Soil. Dyn. Eq. Eng., 26, 71-89.

Schnabel, P. B., J. Lysmer, and H.B. Seed (1991). SHAKE: a computer program for earthquake response analysis of horizontally layered sites. Berkeley, University of California, Earthquake Engineering Research Centre Report ERRC 72-12, 88p.

Seed, H.B., and W.H. Peacock (1971). Test procedures for measuring soil liquefaction characteristics. Journal of the Soil Mechanics and Foundations Division, 97 (8), 1099-1119.

Seed, H.B., M.P. Romo, J.I. Sun, A. Jaime, and J. Lysmer, (1988). The Mexico earthquake of September 19, 1985 - Relationships between soil conditions and earthquake ground motions, Eq. Spec., 4, 687-729. 
Semblat J.F., P. Dangla, M. Kham, and A.M. Duval (2002). Seismic site effects for shallow and deep alluvial basins: in-depth motion and focusing effect. Soil Dyn Earthquake Eng, 22, 849-854.

Shearer, P.M. and J.A. Orcutt, (1987), Surface and near-surface effects on seismic waves - theory and borehole seismometer results, Bull. Seismol. Soc. Am., 77, 1168-1196.

Singh, S.K., E. Mena, and R. Castro, (1988). Some aspects of the source characteristics and ground motion amplifications in and near Mexico City from acceleration data of the September 1985, Michoacán, Mexico earthquakes, Bull. Seis. Soc. Am., 78, 451-477.

Teng, T., and K. Aki (1996). The Northridge, CA earthquake of January 1994. Bull. Seis. Soc. Am., 86, i. 1 B.

Vrettos, C., (2013). Dynamic response of soil deposits to vertical SH waves for different rigidity depth-gradients, Soil Dyn. Earthq. Eng., 47, 41-50. 


\section{Regional Setting}

The basin of focus in this study is near the old village of Kinburn located within the city limits of Ottawa. This basin was identified for further study from work completed creating a seismic microzonation map of the city (Hunter et al., 2010; Motazedian et al., 2011). It has a combination of soft soil overlying an irregularly shaped bedrock, and creates the possibility of a new study of seismic wave propagation within a small basin that has a high impedance contrast between the sediments and bedrock.

Several basins in the Ottawa-Montreal region share similar characteristics to Kinburn, and these basins are also potential sites for studying basin effects from earthquakes. Three of the identified basins were instrumented with seismometers for recording ground motions at a soil station and rock station. The soil station was placed as near the deepest part of the basin as identified at the time of installation, and the rock station was installed on the nearest outcrop of bedrock to the basin.

It was decided the Kinburn basin would be used for this study because there was a recent installation of an array of seismometer stations. The locations of the seismic stations in this array were chosen to provide the most information about seismic wave propagation within the basin as possible, based on what the expected effects would be at the time of installation. The Kinburn array replaced the soil-rock seismometer pair stations that were previously outfitted in this basin.

\subsection{Geological and Geophysical Setting}

The area surrounding Ottawa has a rich geological history as evident by the range of different ages and types of rock outcrops within the city. The Ottawa Valley is an extension of the St. 
Lawrence Platform consisting of Cambrian Sandstone and Ordivician limestone, shale, dolomite and sandstone (Harrison and Macdonald, 1980). The Paleozoic sequences are bound to the North and West by Precambrian bedrock of the Grenville Province (Johnson et. al., 1991). These are covered by late Quaternary deposits of a varying combination of unconsolidated till with an average thickness of $3.85 \mathrm{~m}$, a thin (often less than $2 \mathrm{~m}$ ) freshwater, fine-grained lacustrine sediment, overlain by a thick glaciomarine-marine sequence (Gadd, 1986).

For this study the main concerns are the seismic properties of the Precambrian bedrock as well as the thick glaciomarine-marine sequence. There is a thin cover of Paleozoic rock to the Southwest of the basin, but this cover in the area is less than $20 \mathrm{~m}$ thick. Furthermore, the seismic properties of interest for these Paleozoic rocks are similar to the Precambrian basement bedrock. For example, the interval shear wave velocities for the Paleozoic sequences range between 2800 to $2900 \mathrm{~m} / \mathrm{s}$, and for the Precambrian rocks an average interval shear wave velocity of $2783 \mathrm{~m} / \mathrm{s}$ (Hunter et al., 2010). These values were computed from refraction seismic data collected throughout the city.

The unconsolidated sediments within the basin are composed of both till and glaciomarine-marine sequence. However, the till is thin in comparison to the overlying thick glaciomarine and the effects on the ground motions from earthquakes are dominated by the younger and softer sediments. In Ottawa the average shear wave velocity for these sediments is about $150 \mathrm{~m} / \mathrm{s}$, although there is increase of velocity with thickness, $z$, given by (Hunter et al., 2010):

$$
V s_{a v}=123.86+0.88 z \pm 20.3 \mathrm{~m} / \mathrm{s} \text { for } 10 \mathrm{~m} \leq z \leq 100 \mathrm{~m}
$$


This linear relationship was derived from approximately 1000 measured data points throughout the city of Ottawa, and is a good approximation of the soft sediments within the Kinburn Basin.

\subsection{Seismotectonic}

Plate tectonics is able to explain the majority of earthquakes worldwide, as most earthquakes occur at plate boundaries. At these boundaries, the strength between tectonic plates is low compared to the stress of the tectonic plates moving relative one another. This also makes predicting the frequency and size of earthquakes possible, since several studies have attempted to relate these properties directly to the velocities of lithospheric plates (Mazotti, 2007; Stein, 2007).

Continental intraplate earthquakes account for a low percentage of the total number of earthquakes, and an even smaller amount of the total cumulative seismic moment released per year (Schulte and Mooney, 2005). These earthquakes occur in seemingly stable settings which makes it more difficult to determine the cause of these earthquakes. The lack of historical recordings of the moderate to large earthquakes and the short written history (about 300-400 years) in this region compared to the estimated large return periods (500-1000 years, or larger) makes estimating the frequency and size of these earthquakes more difficult (van Lanen and Mooney, 2007).

Eastern North America has several zones of active seismicity within an intraplate setting. These seismic zones are split into different regions based upon clusters of recorded seismicity and include the Western Quebec Seismic Zone, Charlevoix, New Madrid, Charleston, among 
others (Adams and Basham, 1991; Mazotti and Townend, 2010). These zones have been studied in more detail and several hypotheses have been proposed which could explain the source of the seismicity. Such explanations range from post-glacial isostatic rebound of a weakened crust, reactivation of an ancient rift system, and a hotspot trace (Forsyth, 1981; Adams and Basham, 1991; Wu and Mazzotti, 2007).

\subsubsection{West Quebec Seismic Zone}

The West Quebec Seismic Zone contributes most of the seismicity which affects our region of interest. This zone was originally contoured around a cluster of seismic activity in the Western portion of Quebec, north of Ottawa (Basham et al., 1979). At that time, it was difficult to constrain the boundaries of this zone based on geological features, because the error on earthquake epicentres was large. Forsyth (1981) tried to correlate the seismicity in this region with geological features. It was found that most of the seismicity is either correlated with the northeastern boundary of the Central Metasedimentary Belt, or near the junctions of rift systems which follow the Ottawa River, St. Lawerence River, and Lake Champlain, with most of the epicentres being located within the Grenville terrain. This zone appears to display seismicity in two separate bands (Adams and Basham, 1991) which, after refining the epicentre distribution, is separate enough to be distinct. After Adams and Basham (1991), one band of earthquakes follows the Ottawa River between Lake Temiskaming and Ottawa and is characterized by lower seismicity but larger events. The other band is slightly North of the first and strikes through Montreal. This band of seismicity has more frequent but lower magnitudes events. Both bands seem to merge into one zone Southeast of the St. Lawrence River. 
The source of seismicity in the WQSZ is constantly being challenged as it is located in an area that should be a stable continental interior. The general agreement towards the cause of earthquakes seems to be the reactivation of ancient normal rift faults due to horizontal compressive stress perpendicular to these faults (Forsyth, 1981; Adams and Basham, 1991). These fault systems may have been weakened by a thermal anomaly, a Mesozoic hot spot, which can be traced through the region by the monotonically increasing igneous crystallization ages along an inferred trace (Heaman and Kjaarsgaard, 2000; Ma and Eaton, 2007). Although the seismicity could also be influenced by stress induced by postglacial rebound (Wu and Mazotti, 2007), it is unlikely that this is significant for this seismic zone as it does not explain the linear trend of seismicity well (Ma and Eaton, 2007).

\subsubsection{Regional Stress}

The crustal stress in eastern North America has been studied extensively and through several methods (Adams and Bell, 1991; Zoback and Zoback, 1991; van Lanen and Mooney, 2007; Mazotti and Townend, 2010). The stress in this region is compressive and the principal axes of maximum stress is East-Northeast (Zoback and Zoback, 1991) for eastern North America, and Northeast for the West Quebec Seismic Zone (Mazotti and Townend, 2010). One of the axes is near vertical, but it is not the largest in magnitude (Adams and Bell, 1991). This is supported by several measurements including: focal mechanisms of moderate sized earthquakes; borehole breakouts; overcoring measurements; and several indirect measurements (Adams and Bell, 1991). The stress regime in this region indicates either strike-slip or reverse/thrust faulting for seismic events (Zoback and Zoback, 1991). 


\subsection{Seismicity}

The seismicity felt in the study region is dominated by the seismicity in the Western Quebec Seismic zone. A few larger earthquakes, which occur outside of the WQSZ and are mostly in the Charlevoix Seismic Zone (Basham et al., 1979; Adams and Bell 1991), are felt around the area of the Kinburn basin but are very infrequent. Teleseismic earthquakes are recorded on the weak motion instruments installed in the area. The WQSZ is characterized by a moderate level of seismic activity, with an earthquake occurring once every five days on average (Earthquakes Canada, 2016). Figure 2.1 shows the location of the epicenters of the earthquakes that have occurred in the region since 1985, with the symbol size and colour representing the preferred moment magnitude for each earthquake (Halchuk et al., 2015). 


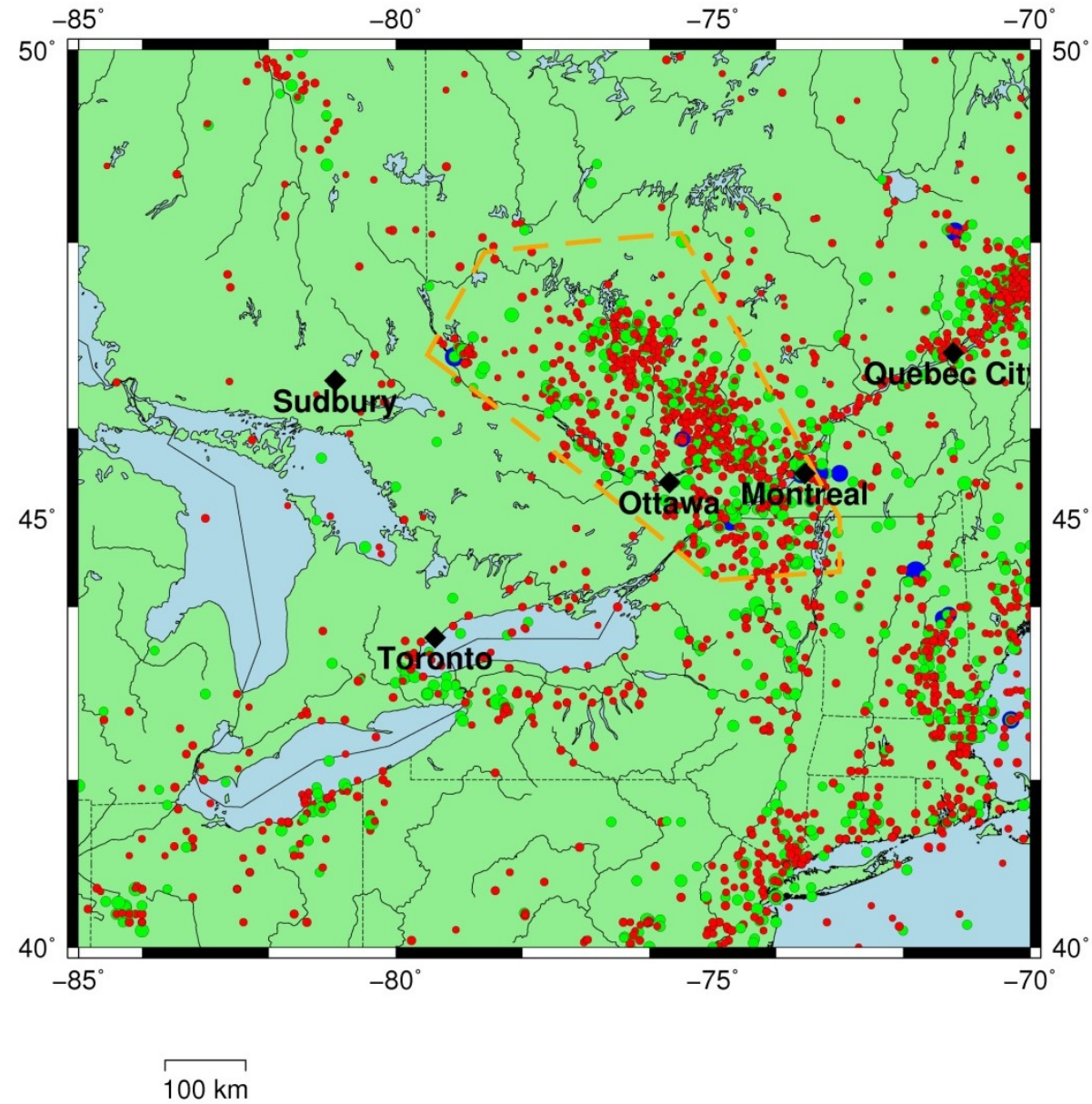

Figure 2.1: Recorded seismicity from SHEEF 2010 for Eastern Canada. The Western Quebec Seismic Zone is outlined by the orange dashed lines. The red circles indicate M0-M3 events, the green circles represent M3-M5 events, and the blue circles represent M5+ events.

\subsubsection{Recorded Seismicity}

The recorded seismicity in this region dating back to 1985 is available from Natural Resources Canada (NRCan) through their website (Earthquake Canada, 2016). This region has roughly 4000 earthquake epicentres located within its borders which have been used for the fifth generation of seismic hazard maps of Canada (Halchuk et al., 2015). The recording stations in the area are part of either the Southern Ontario Seismograph Network (SOSN) or the Canadian National Seismic Network (CNSN) and provide good coverage of Eastern Canada (Cassidy et al., 2007; 
Atkinson et al., 2014; Bent et al., 2015). The stations have been recording earthquakes for various amounts of time.

\subsubsection{Historical Seismicity}

The seismic region has seen several earthquakes of historical significance, and a few which have been felt by a large number of people. The significant earthquakes which caused varying degrees of damage include: in 1732 an earthquake near Montreal of Richter magnitude 5.8 (Leblanc, 1981); in 1935 Temiscaming area an earthquake of magnitude 6.2 occurred (Hodgson, 1936; Bent, 1996a); and in 1944 an earthquake of magnitude 5.6 occurred between Cornwall, Ontario and Massena, N.Y. (Hodgson, 1945; Bent, 1996b).

Other earthquakes which have been felt more recently are: in 1990 near Mont. Laurier a magnitude 5 occurred (Lamontagne et al., 1994); in 1996 and 1997 near Ste.-Agathe-des-Monts,

Quebec two earthquakes of magnitude 4.4 and 4.3 occurred (Bent et al., 1999); in 2010 a magnitude 5.0 earthquake occurred near Val-de-Bois, Quebec (Atkinson and Assatourians, 2010; Ma and Motazedian, 2012); and in 2013 a magnitude 4.6 earthquake with an aftershock of magnitude 4.1, 10 minutes later occurred near Ladysmith, Quebec (Atkinson et al., 2014; Bent et al., 2015).

\subsubsection{Paleoseismicity}

Several reports have suggested that significantly sized earthquakes have occurred within or very close to the city. Alysworth et al. (2000) dated landslides in the east of Ottawa which all occur within a specific date range suggesting a large local earthquake 4550 years before present. Another dating of a disturbed region of land near Alfred suggests an event that produced large 
ground disturbance in the area roughly 7060 years before present. Brooks (2013) found several large landslides in the Quyon Valley, in South-western Quebec, and ten other landslides in the Quyon-Ottawa region dated between 980 to 1060 calibrated years before present provide evidence of a paleoearthquake of $M_{w} \geq 6.1$ located within the Western Quebec Seismic Zone.

\subsection{Kinburn Basin}

Kinburn is a township located in the western portion of Ottawa. Beside this township is a roughly oval shaped soft soil basin shown in detail in Figure 2.2. The basin is roughly 7 kilometres along its long axis which follows John Shaw Rd. from Northwest to Southeast, and is about 5 kilometres along the short axis from Southwest to Northeast following Grants Side Rd. for a portion and then extending to Diamondview Rd. The northern and eastern portion of the basin is surrounded by Precambrian rocks which can be seen in outcrops beyond the extent of the basin, and by steep slopes of a strong reflector in the Landstreamer seismic section (Hunter et al., 2010). The southern and western portion of the basin appears to be underlain by Paleozoic rocks seen as a gradually sloping strong reflector in the seismic cross section. These are consistent with outcrops to the Southwest of the basin, however these outcrops are further away and not adjacent to the basin.

The basin is mostly filled with a high water content (low shear wave velocity) Holoceneage silt or silty clay deposit often referred to as Leda Clay. This material was deposited in the Champlain Sea during the last retreat of glaciers in the region. These sediments have been studied throughout the wide areal extent over which they appear (Fulton, 1987; Scott, 2003) and within the Kinburn basin by means of a Geological Survey of Canada (GSC) borehole (the orange 
crosshair in Figure 2.2) located roughly in the deepest extent of the basin (Medioli et al., 2012). Near surface (between 0-6 m), superimposed on the regional Vs-depth gradient, the velocity of the soil generally increases sharply with depth with a maximum velocity anomaly around $5 \mathrm{~m}$. This is interpreted to be caused by an overconsolidation of the clay from successive freeze-thaw cycles (Eden and Crawford, 1957).

There seems to be a thin layer of Pleistocene glacial deposits between the bedrock and Holocene sediments. This till or till-derived layer is thicker in the deeper portions of the basin and not evident close to the bedrock outcrops.

The depth of the basin, shown in Figure 2.2, is constrained by roughly 900 City of Ottawa waterwells, 286 Horizontal-to-Vertical Spectral Ratio (HVSR) measurements, 2 high resolution Landstreamer seismic lines (Pugin 2009), and a GSC logged borehole (Medioli et al., 2012). These measurements were interpolated using a first order linear Kriging method (Delfiner and Delhomme, 1975) to obtain a smoothed contour of the soil depth. The error on the soil depth at each location is smaller than the required error for the simulation, enabling the use of the interpolated grid for the purpose of a numerical model. 


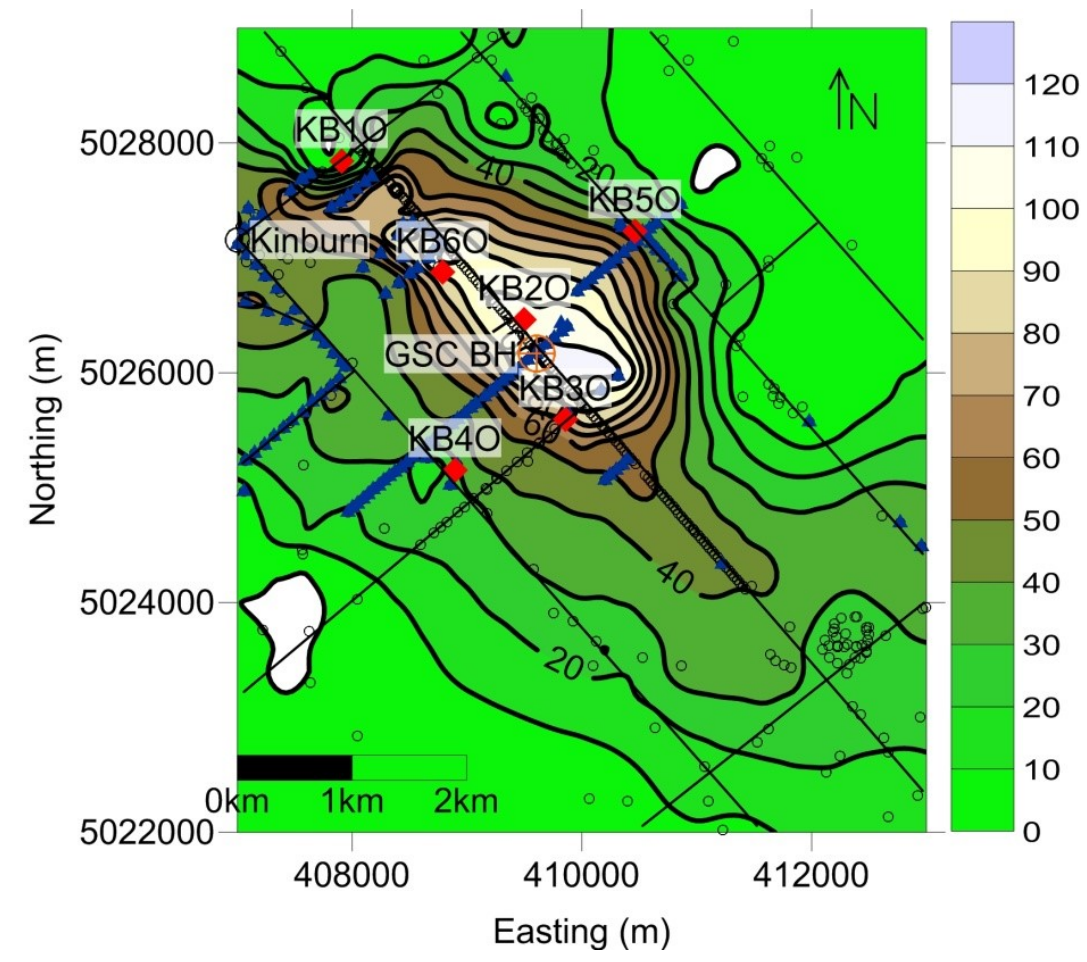

Figure 2.2: The soil depth of the Kinburn Basin derived from the varying data sources: blue triangles are the HVSR measurements, black circles are the city of Ottawa boreholes and landstreamer data points, and the orange crosshair is the GSC borehole. Red diamonds are the locations of the Kinburn Seismic Array stations.

\subsubsection{Kinburn Basin Model}

The Kinburn basin model was developed and refined over several years. A rough model was generated using the city of Ottawa waterwells in the region, along with the seismic landstreamer data, after being identified from the fundamental period map of Ottawa (see Figure 1.5). Using this model, some areas were identified as lower than desired resolution, due to the sparse coverage in certain places. Horizontal to Vertical Spectral Ratio (HVSR) measurements were completed along the landstreamer lines in the basin as a calibration for this method. Once calibrated, more of these measurements were completed in the areas which needed a better constraint on the depth to the impedance boundary. Another model was completed with the addition of these measurements and the target areas for seismic stations were identified. After consultation with the residents in the area, locations for the seismic array stations were 
suggested then confirmed. Before installing the stations, several HVSR measurements were completed in a higher density around these sites, to confine the shape of the subsurface of these locations to a higher resolution. The final model of the depth to the strong impedance boundary for the Kinburn basin was completed using all of this data collected.

\subsection{Kinburn Seismic Array}

Due to the availability of seismic stations from the POLARIS project, it was decided that this basin would be instrumented with an array of seismometers. This was set up in an attempt to determine the effects the size and shape of the Kinburn basin has on earthquake ground motions at different positions on the surface of the basin. After a detailed model of the subsurface topography was constructed to a reliable accuracy, the possible positions of each of the seismic stations were proposed and discussed in detail. The aim of the Kinburn Seismic Array is to provide the best possible configuration of an array within the basin to measure the different effects estimated to be affecting the surface ground motions.

The location of each of the six stations, KB1O to KB6O shown in Figure 2.2, were decided based on multiple factors including: availability of the location; subsurface structure based on soil depth model; and reference to other stations and possible sources of seismicity. One station, KB1O, is located on an outcrop of bedrock on the Northwest edge of the Kinburn basin. The soil depth at each station is constrained using HVSR measurements at each location and are shown in Table 2.1 along with the coordinates of each station. The stations were installed in a cross shape to try and capture as much information about seismic wave propagation through the basin as possible. This shape of the array should be able to show the waves travelling from one edge 
of the basin to the opposite in the directions which correspond to the basin axes. A brief description of each station is discussed below.

\begin{tabular}{|c|c|c|c|c|}
\hline \multirow{2}{*}{ Station Name } & \multicolumn{2}{|c|}{$\begin{array}{c}\text { Location in UTM coordinates (Zone } \\
\text { 18) }\end{array}$} & $\begin{array}{c}\text { Maximum H/V } \\
\text { Frequency (Hz) }\end{array}$ & $\begin{array}{c}\text { Depth to } \\
\text { Reflector }(\mathrm{m})\end{array}$ \\
\cline { 2 - 3 } & Northing $(\mathrm{m})$ & Easting $(\mathrm{m})$ & - & 0 (outcrop) \\
\hline KB1O & 5027845 & 407908 & $0.72 \pm 0.01$ & $101.2 \pm 0.9$ \\
\hline KB2O & 5026463 & 409499 & $0.84 \pm 0.01$ & $82.0 \pm 1.2$ \\
\hline KB3O & 5025592 & 409855 & $1.63 \pm 0.01$ & $32.4 \pm 0.3$ \\
\hline KB4O & 5025153 & 408894 & $1.31 \pm 0.01$ & $54.7 \pm 0.7$ \\
\hline KB5O & 5027234 & 410461 & $0.75 \pm 0.01$ & $96.0 \pm 1.1$ \\
\hline KB6O & 5026876 & 408786 & \\
\hline
\end{tabular}

Table 2.1: The location and soil depth based on the HVSR measurements of each station in the Kinburn Seismic Array.

\subsubsection{Station Features}

There are two different subgroups of the stations installed: the remote stations and powered stations. The powered stations have an AC power supply to each of them from surrounding infrastructure in order to run the electronics. The remote stations have a solar panel and several batteries within them to supply constant power. Each seismic station is equipped with a Guralp CMG 3ESP seismometer, a Nanometrics Taurus digitizer, a Freewave modem, a GPS antenna, a housing station with a power converter for the electronics, and a climate controller for the electronics box. The digitizers have been equipped with an $8 \mathrm{~GB}$ flash memory card to be used as a backup in case the base station, KB1O, is not able to connect over the network, or if any of the individual stations fails to communicate with the base station. The seismometer vaults are placed several feet from the electronics housing. The main station KB1O has an omnidirectional Laird technologies antenna and the other stations have a Larson, A Pulse Brand base station antenna directed at KB1O. Stations KB1O and KB2O also have strong motion accelerometers, Kinemetrics Etnas, installed beside the broad-band seismometers. These strong motion accelerometers are 
able to record stronger motions at these stations, but are not connected over the network. After an event triggers them, the data is recorded and then it must be collected by a field technician.

\subsubsection{Installation of the Stations}

Most of the stations were installed over the summer of 2013, and one was installed in October of the same year. The installation was completed by a group of technologists and contractors from the Geological Survey of Canada (GSC) and from the Polaris project. Everything was tested and calibrated at the GSC Anderson Road complex. The remote stations, KB3O and KB6O, had a deck built to elevate the housing box, and the powered stations have their housing box on a steel post affixed to a cement base. The seismic vaults at the soil stations were dug to a depth of at least 1 metre and a cement pad was poured to provide a firm stage which is well connected to the soil. These vaults are contained by a corrugated trench pipe to shield the seismometers. All stations were grounded using copper wire, and the remote stations have a grounding plate buried beside them.

\subsubsection{Recordings}

Records from the broad-band seismometers at these stations are available through the Automatic Data Request Manager (AutoDRM) on the Earthquakes Canada of the Natural Resources of Canada website (Earthquake Canada, 2016). The sampling rate for these stations is 100 samples per second, making the output signal resolvable up to $50 \mathrm{~Hz}$. The data retrieved is in raw form, so it has to be converted into ground motions and the instrument response removed after it has been obtained. This is completed based on the conversion:

$$
\operatorname{Ground} \text { Velocity }(\mathrm{m} / \mathrm{s})=\frac{\text { Amplitude }(\text { counts }) * A / D \text { conversion factor }(\mathrm{V} / \text { counts })}{\operatorname{Gain} * \text { Sensivity }(\mathrm{V} / \mathrm{m} / \mathrm{s})}
$$


The strong motion data from the Etnas are only available by collecting the data directly from the stations. These accelerometers record a predetermined time interval after a certain threshold of motion is exceeded. The data collected from these Etnas also need to have the instrument response removed, as discussed in Appendix I.

At the time of this research project, the Kinburn Seismic Array has only recorded several small earthquakes which were not suitable for numerical modelling. A list of some of the recordings available for the Kinburn Seismic Array is shown in Table 2.2 for both local and teleseismic events (K. Brewer, pers. comm.).

\begin{tabular}{|l|l|l|l|}
\hline Event Name* & Date of recording & $\begin{array}{l}\text { Magnitude } \\
(\mathrm{Mw})\end{array}$ & $\begin{array}{l}\text { Local (L) or } \\
\text { Teleseismic } \\
\text { (T) }\end{array}$ \\
\hline Barbados & & & T \\
\hline Saint-Remi & 20140218 & 6.5 & L \\
\hline Hawksberry & 20140207 & 3.1 & L \\
\hline Saint-Andre-Avellin & 20140309 & 2.0 & L \\
\hline Maniwake & 20140315 & 2.7 & L \\
\hline Chile & 20140330 & 3.0 & T \\
\hline Ferme-Neuve & 20140401 & 8.2 & L \\
\hline Mexico & 20140407 & 2.2 & T \\
\hline Port Alice & 20140418 & 7.2 & T \\
\hline Saint-Donat-De-Montcalm & 20140424 & 6.6 & L \\
\hline L'Original & 20140424 & 2.5 & L \\
\hline Mexico & 20140427 & 2.5 & T \\
\hline Panama & 20140531 & 6.2 & T \\
\hline Mid-Atlantic-ridge & 20140513 & 6.5 & T \\
\hline Mexico & 20140523 & 5.2 & T \\
\hline Aleutian & 20140531 & 6.2 & T \\
\hline Saint-Andre-Avellin & 20140623 & 7.9 & L \\
\hline Pembroke & 20140614 & 2.2 & L \\
\hline LaBelle & 20140628 & 2.0 & L \\
\hline Lordsberg New Mexico & 20140630 & 2.5 & T \\
\hline Mexico & 20140629 & 5.2 & T \\
\hline Saint-Andre-Avellin & 20140707 & 6.9 & L \\
\hline Pembroke & 20140614 & 3.0 & 2.9 \\
\hline
\end{tabular}




\begin{tabular}{|l|l|l|l|}
\hline L'Original & 20140819 & 2.5 & $\mathrm{~L}$ \\
\hline California & 20140824 & 6.0 & $\mathrm{~T}$ \\
\hline Gatineau & 20140922 & 2.0 & $\mathrm{~L}$ \\
\hline Nicaragua & 20141014 & 7.3 & $\mathrm{~T}$ \\
\hline L'Original & 20140819 & 2.5 & $\mathrm{~L}$ \\
\hline Saint-Andre-Avellin & 20141117 & 2.6 & $\mathrm{~L}$ \\
\hline Bourget & 20141212 & 2.0 & $\mathrm{~L}$ \\
\hline Gatineau & 20150101 & 2.2 & $\mathrm{~L}$ \\
\hline LaBelle & 20141224 & 2.6 & $\mathrm{~L}$ \\
\hline Salaberry & 20150119 & 2.6 & $\mathrm{~L}$ \\
\hline La Procatiere & 20150121 & 3.7 & $\mathrm{~L}$ \\
\hline Atlantic Ridge & 20150213 & 7.1 & $\mathrm{~T}$ \\
\hline Nepal & 20150425 & 7.9 & $\mathrm{~T}$ \\
\hline Bella Bella & 20150424 & 6.2 & $\mathrm{~T}$ \\
\hline La Procatierre & 20150628 & 3.3 & $\mathrm{~L}$ \\
\hline Vankleek & 20150715 & 3.9 & $\mathrm{~L}$ \\
\hline Ferme-Neuve & 20150726 & 3.0 & $\mathrm{~L}$ \\
\hline Mont Laurier & 20150727 & 3.1 & $\mathrm{~L}$ \\
\hline L'Annonciation & 20150707 & 3.2 & $\mathrm{~L}$ \\
\hline Val-de-bois & 20150805 & 3.4 & $\mathrm{~L}$ \\
\hline
\end{tabular}

Table 2.2: A list of some of earthquakes recorded at the Kinburn Seismic array. ${ }^{*}$ The event name is usually a location near the epicentre and not unique to a specific event.

\subsubsection{KB10 (Formerly JSBS)}

This station is located on a Precambrian rock outcrop on the Northwestern edge of the basin. Although slightly higher in elevation when compared to the other stations, it is a good estimate of the ground motions experienced by the bedrock underlying the basin. The location of this station was designed so that it would be either almost in line or adjacent with an earthquake source and the other Kinburn seismic array receivers. The HVSR measurement is shown in Figure 2.9. A photograph of the station looking Southward is shown in Figure 2.3. In the background the lower elevation of the basin can be seen. 


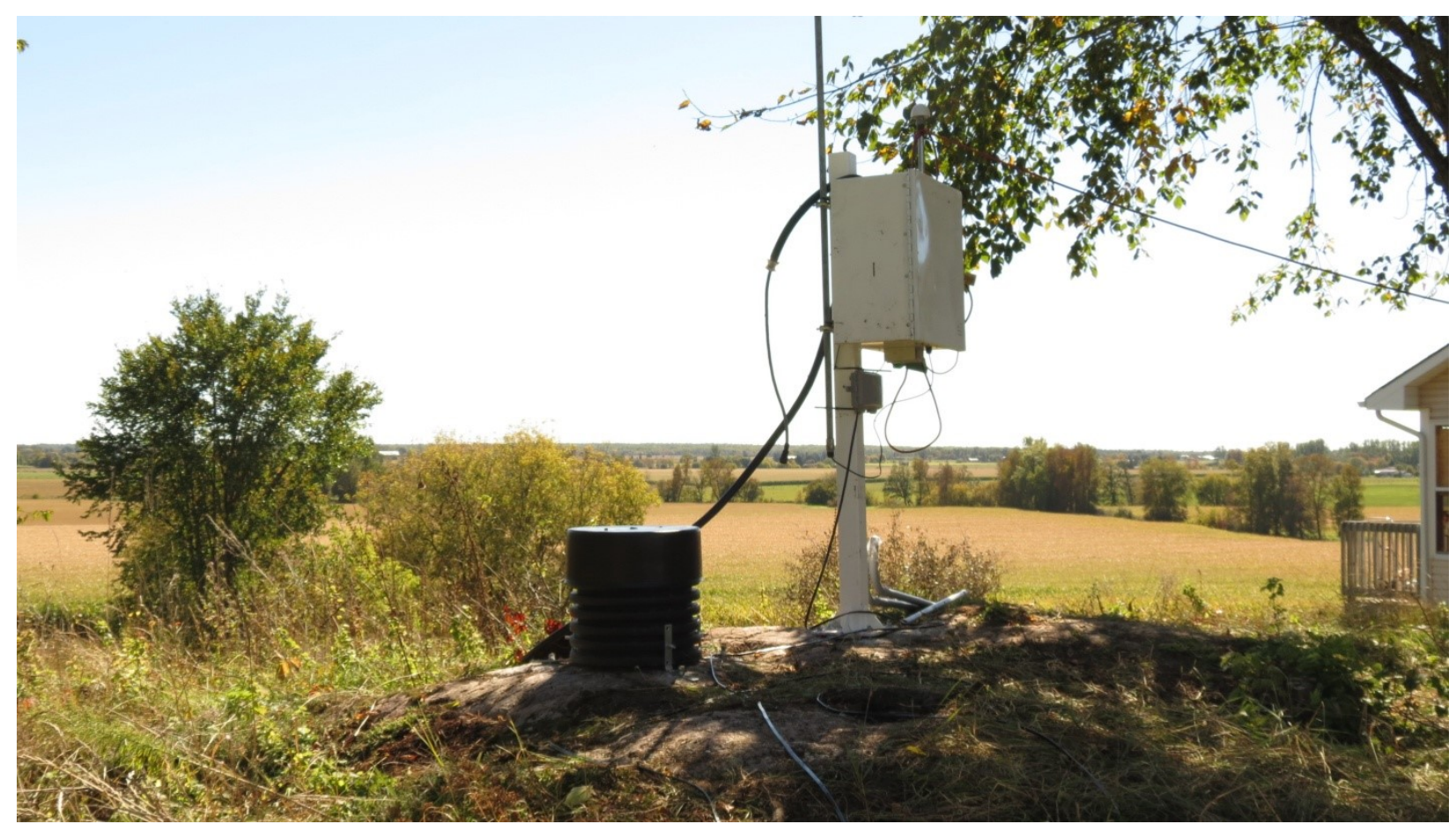

Figure 2.3: Looking Southward at station KB1O with the Kinburn basin in the background.

Due to its higher elevation and the proximity to power it was chosen as the base station for the array. It was equipped with and omnidirectional antenna to collect data from all the other stations. It was also connected to the internet to broadcast the collected information to a server at the Geological Survey of Canada (GSC).

\subsubsection{KB20 (Formerly JSSS)}

This station, located near what has been identified as the deepest portion of the Kinburn basin, is also near the geometric centre of the Kinburn basin. The depth to the reflector of this station is calculated to be $101.2 \pm 0.9$ metres from the HVSR measurement. The HVSR profile for this station is shown in Figure 2.9. Until the installment of the Kinburn Seismic Array, this location was the soil station to a rock-soil station pair as discussed earlier. A photograph of the seismic station along with a few members of the installation team is shown in Figure 2.4. 


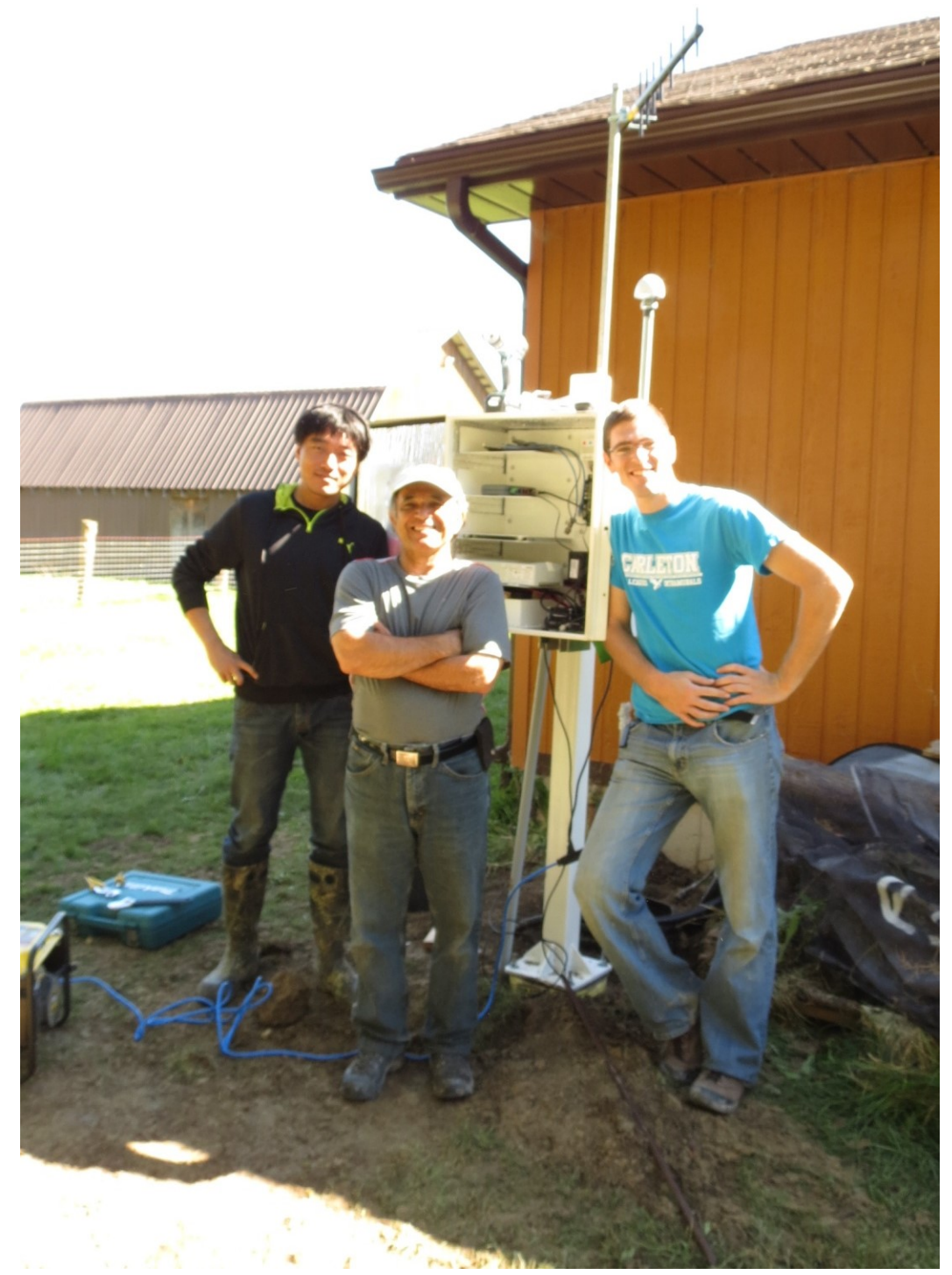

Figure 2.4: A photograph of station KB2O being installed with a few members of the Kinburn Seismic array installation team. From left to right: Mingzhou Li, Isa Asudeh, Stephen Crane.

\subsubsection{KB30}

The station KB3O is located near the Southeastern edge of the basin with a depth to the reflector at $82.0 \pm 1.2$ metres. The HVSR profile for this station is shown in Figure 2.9. This receiver was placed in line with $\mathrm{KB} 1 \mathrm{O}$ and $\mathrm{KB} 2 \mathrm{O}$ in an attempt to record waves travelling along the long axis of the Kinburn Basin. Figure 2.5 shows a photograph of this station looking Northwards towards station KB1O. 


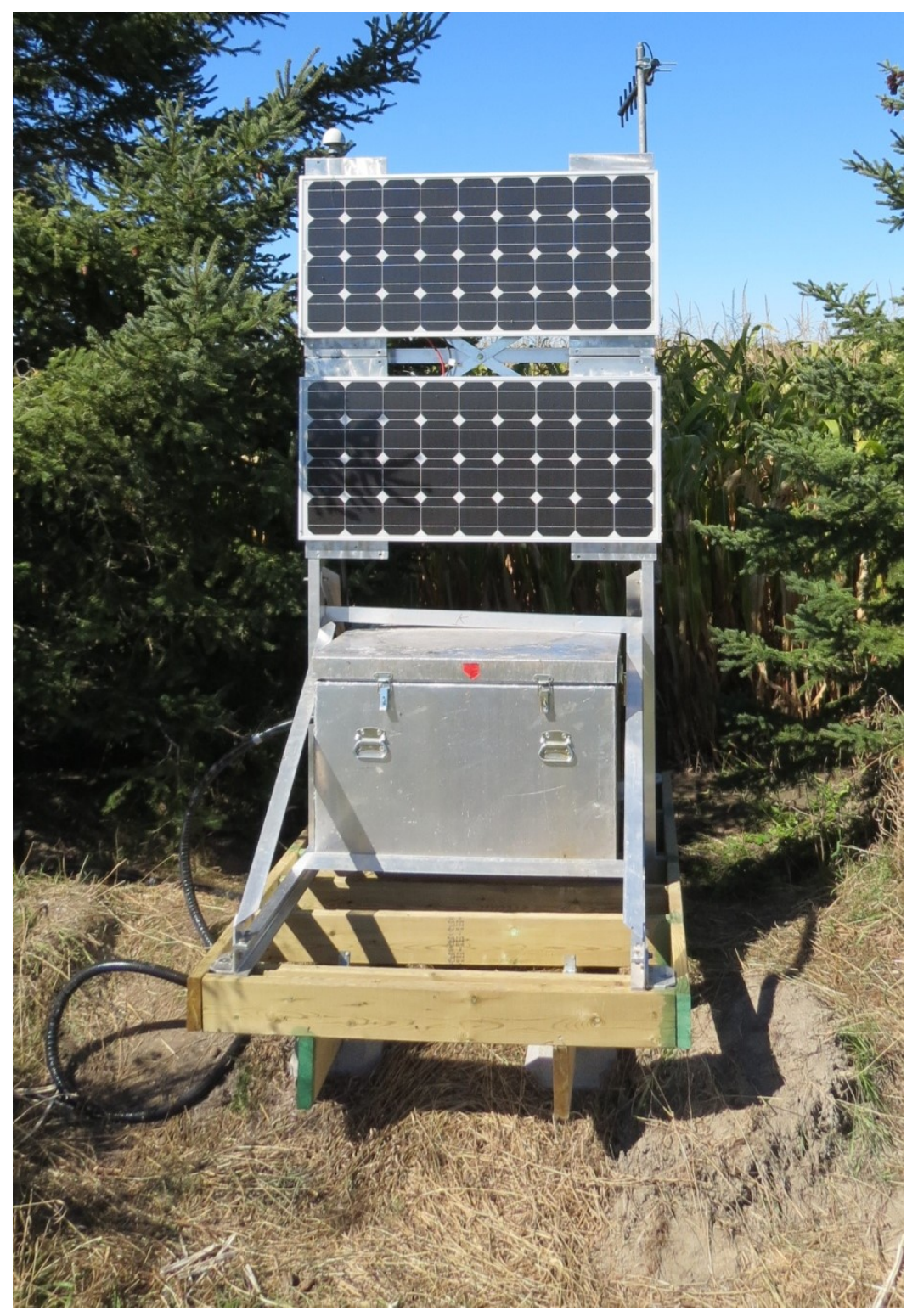

Figure 2.5: Station KB3O looking Northwest towards KB1O. The seismometer vault is to the left of the photograph.

\subsubsection{KB40}

The station KB4O is located near the Southwestern edge of the Kinburn basin. The HVSR profile for this station is shown in Figure 2.9 and shows a depth to the reflector of $32.4 \pm 0.3$ metres. This station has the least amount of material between the receiver and bedrock, compared to the other stations in the array. Figure 2.6 is a photograph of this station looking towards the East. 


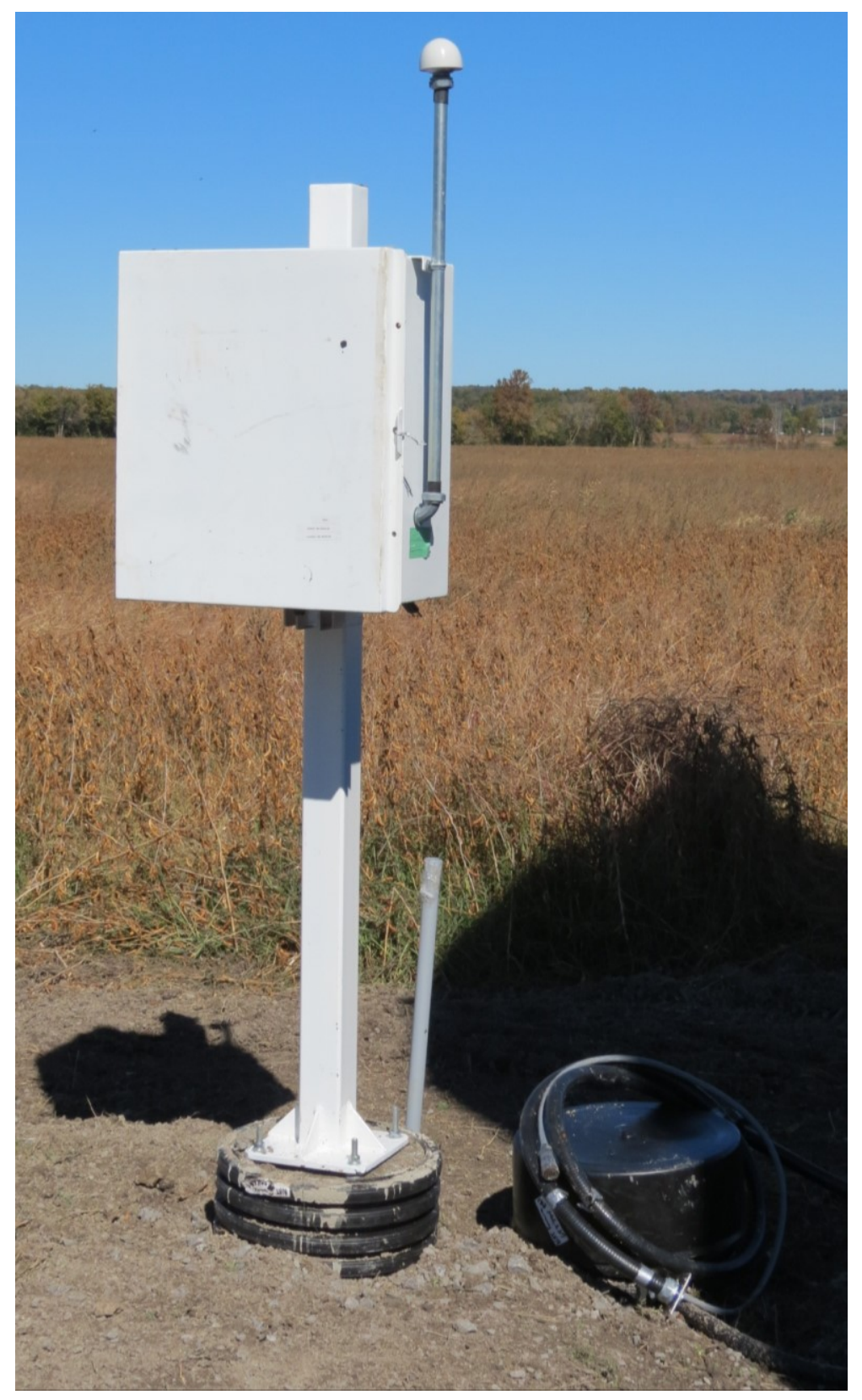

Figure 2.6: A photograph of station KB4O looking Eastward.

\subsubsection{KB50}

The station KB5O is located near the Northeastern edge of the Kinburn basin. This receiver has an estimated depth to reflector of $54.7 \pm 0.7$ metres as shown by the HVSR profile seen in Figure 2.9. It is roughly in line with stations $\mathrm{KB} 2 \mathrm{O}$ and $\mathrm{KB} 4 \mathrm{O}$ in an attempt to record waves travelling 
along the short axis of the Kinburn basin. Figure 2.7 shows a photograph of this station looking towards the West.

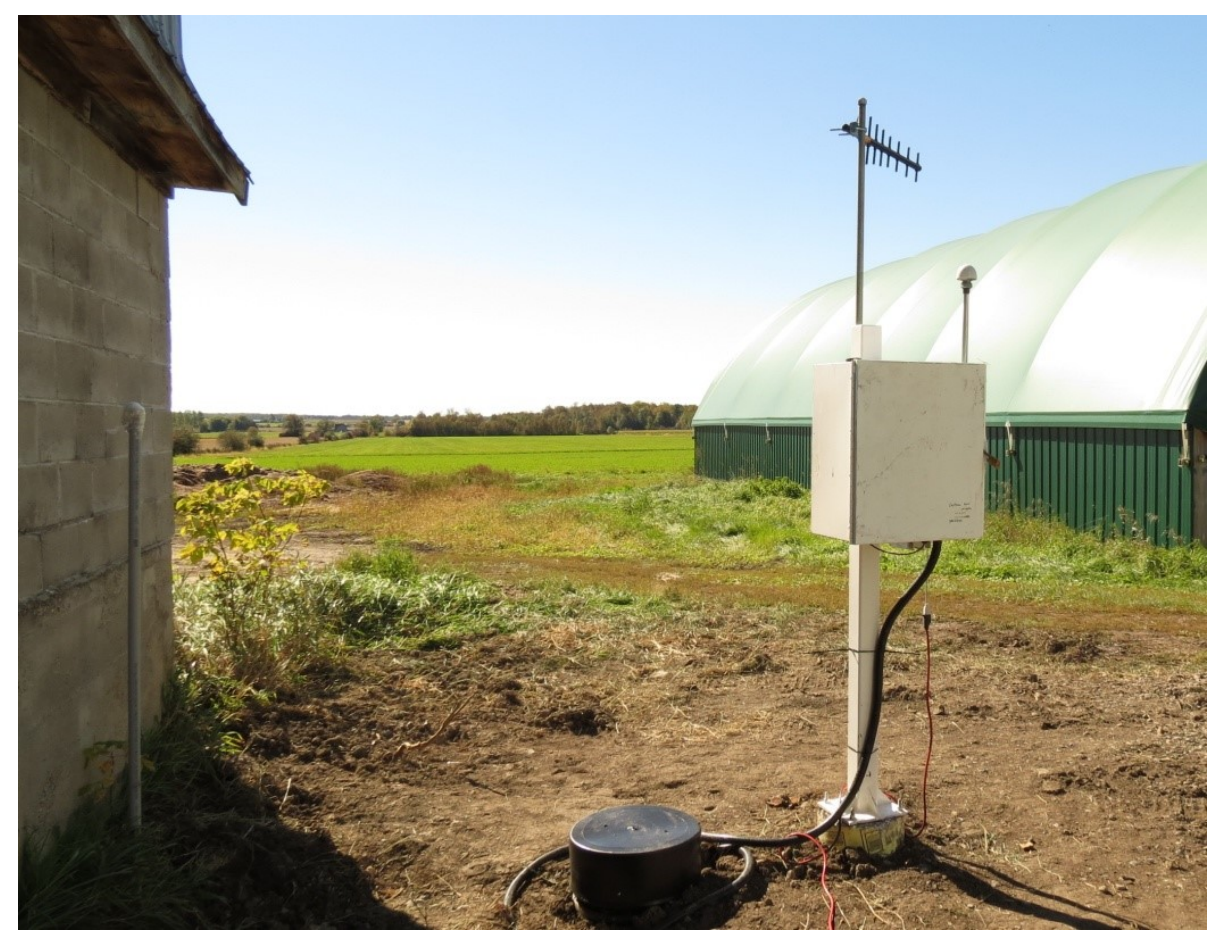

Figure 2.7: A photograph of station KB5O looking West. The antenna is pointing in the direction of KB1O.

\subsubsection{KB60}

The station KB6O is located near the Northwestern edge of the basin in an area that was mapped to be a have a larger depth to bedrock than the surrounding areas. This is identified through HVSR measurements along with a Landstreamer reflection profile along John Shaw road. The depth to reflector of this station is $96.0 \pm 1.1$ metres as shown by the HVSR profile in Figure 2.9. This station is in line with $\mathrm{KB} 2 \mathrm{O}$ and $\mathrm{KB} 3 \mathrm{O}$ in order to determine waves traversing the long axis of the basin. It is also in line between stations KB1O and KB2O giving a reference between the outcrop station and the station at the centre of the Kinburn basin. Figure 2.8 is a photograph of this station looking Eastward. 


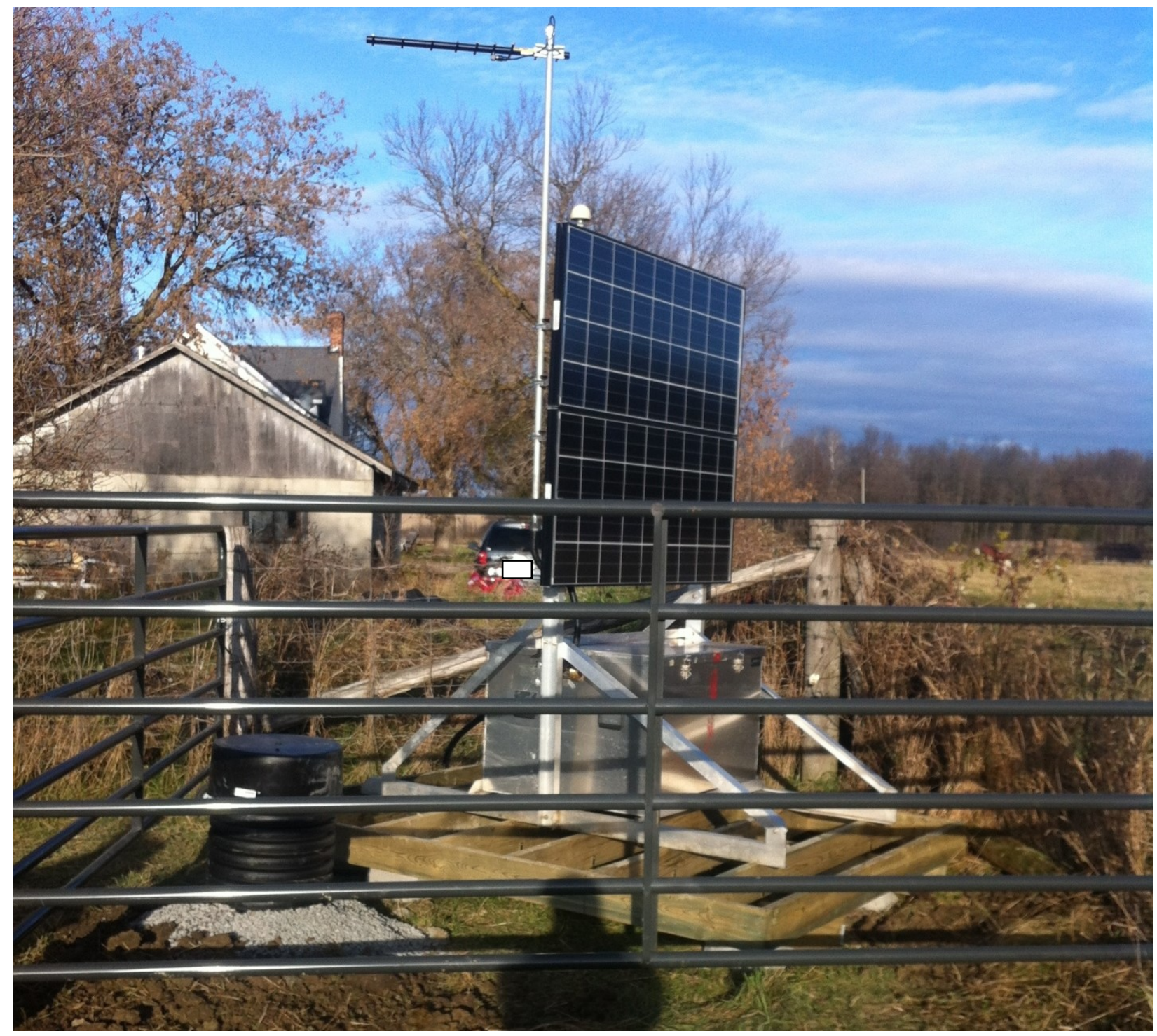

Figure 2.8: A photograph of station KB6O looking to the East. 


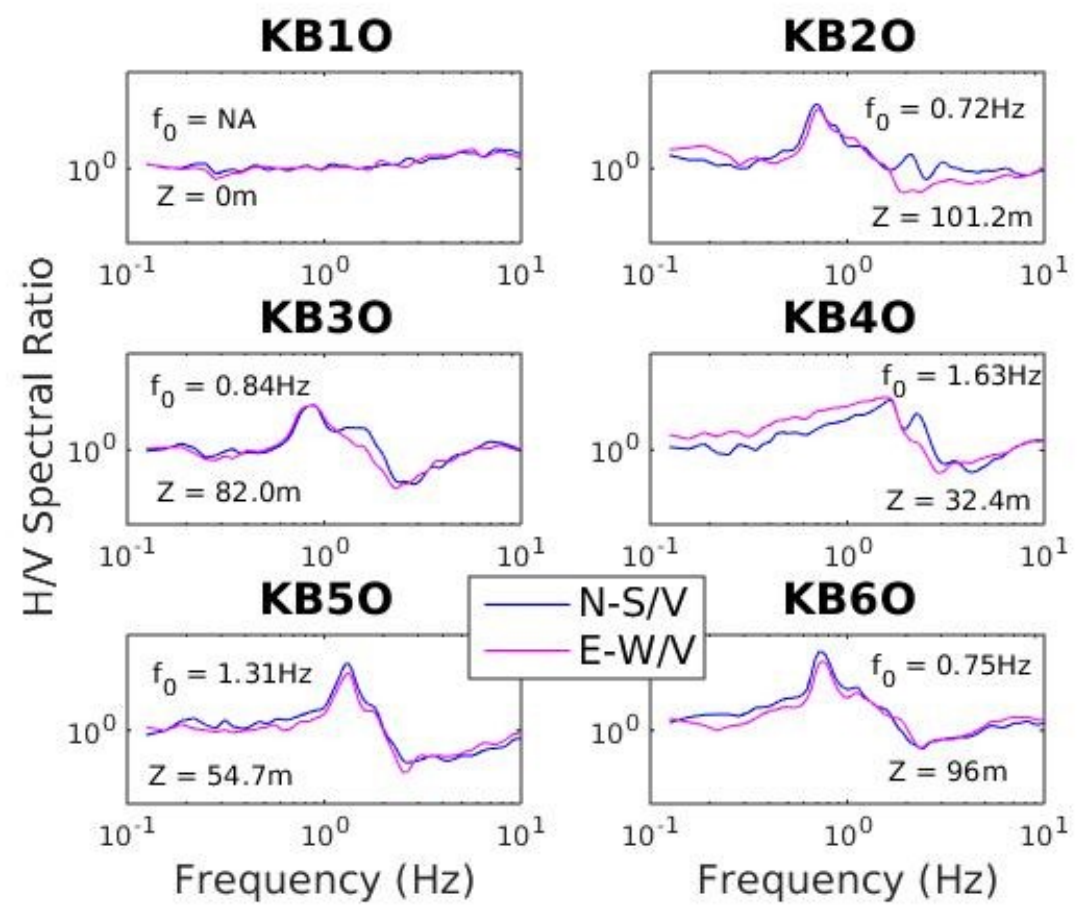

Figure 2.9: Horizontal to vertical spectral ratios from tromino measurements near the final positions of the Kinburn Seismic Array stations. 


\subsection{References}

Adams, J., and P. Basham (1991). The seismicity and seismotectonics of eastern Canada, in Neotectonics of North America, edited by D. B. Slemmons et al., pp. 261-276, Geol. Soc. of Am., Boulder, Colo.

Adams, J., and S. Bell (1991). Crustal stresses in Canada, in Neotectonics of North America, edited by D. B. Slemmons et al., pp. 261-276, Geol. Soc. of Am., Boulder, Colo.

Adams, J., and S. Halchuk, (2003). Fourth generation seismic hazard maps of Canada: Values for over 650 Canadian localities intended for the 2005 National Building Code of Canada. Geological Survey of Canada, Open File 4459, 1-155.

Atkinson, G., and K. Assatourians (2010). Attenuation and source characteristics of the 23 June 2010 M 5.0 Val-des-Bois, Quebec, earthquake. Seismol. Res. Lett., 81, 849-860.

Atkinson, G. M., K. Assatourians, and M. Lamontagne, (2014). Characteristics of the 17 May 2013 M 4.5 Ladysmith, Quebec earthquake, Seismol. Res. Lett., 85, 755-762, doi: $10.1785 / 0220130160$.

Aylsworth, J.M., D.E. Lawrence, and J. Guertin (2000). Did two massive earthquakes in the Holocene Induce widespread landsliding and near-surface deformation in part of the Ottawa Valley, Canada? Geology, 28, 903-906.

Basham, P. W., D. H. Weichert., and M. J. Berry (1979). Regional assessment of seismic risk in eastern Canada. Bull. Seism. Soc. Am. 69, 1567-1602

Bent, A. L. (1996a), An improved source mechanism for the 1935 Timiskaming, Quebec earthquake from regional waveforms, Pure Appl. Geophys., 146, 5-20

Bent, A. L. (1996b), Source parameters of the damaging Cornwall-Massena earthquake of 1944 from regional waveforms, Bull. Seismol. Soc. Am., 86, 489-497.

Bent, A.L., M. Lamontagne, and R.J. Wetmiller (1999). Two moderate earthquakes near SteAgathe-des Monts, Quebec, Seismol. Res. Lett., 70, 100-109

Bent, A.L., M. Lamontagne, V. Peci, S. Halchuk, G.R. Brooks, D. Motazedian, J.A. Hunter, J. Adams, C. Woodgold, J. Drysdale, S. Hayek, W. N. Edwards (2015). The 17 May 2013 M 4.6 Ladysmith, Quebec, Earthquake, Seismol. Res. Lett., 86, 460-476. doi: 10.1785/0220140138

Brooks, G. R. (2013). A massive sensitive clay landslide, Quyon Valley, southwestern Quebec, Canada, and evidence for a paleoearthquake triggering mechanism, Quarternary Research, 80, 425-434. 
Cassidy, J.F. A. Rosenberger, G.C. Rogers, T.E. Little, J. Toth, J. Adams, P. Munro, S. Huffmann, J.R. Pierre, H. Asmis and G. Pernica (2007). Strong motion seismograph networks in Canada, in Proceedings of the 9th Canadian Conference on Earthquake Engineering, Paper 1310.

Delfiner, P., and J.P. Delhomme, (1975). Optimum interpolation by kriging, in Display and analysis of spatial data, pp. 96-114, eds Davis, J.C. \& McCullagh, M.J., Wiley, London.

Earthquakes Canada Website at http://www.earthquakescanada.nrcan.gc.ca/index-en.php, Last accessed September, 2016.

Eden, W.J., and J.B. Crawford (1957), Geotechnical Properties of Leda Clay in the Ottawa Area; National Research Council of Canada, Research Paper No. 37 of the Division of Building Research, Ottawa, ON

Forsyth, D.A. (1981). Characteristics of the Western Québec Seismic Zone, Can. J. of Earth Sci. 18, 103-119.

Fulton, R.J. (1987). Quaternary geology of the Ottawa region, Ontario and Quebec. Geological Survey of Canada Paper 86-63.

Gadd, N.R., (1986). Lithofacies of Leda clay in the Ottawa Basin of the Champlain Sea. Geological Survey of Canada, Paper 85-21, $44 p$.

Halchuk, S., and J.A. Adams, (2004). Deaggregation of seismic hazard for selected Canadian cities, in Proceedings 13th World Conference on Earthquake Engineering, Vancouver, BC, Canada, August 1-6 2004, Paper 2470.

Halchuk, S., Allen, T.I., Rogers, G.C., and Adams, J., (2015). Seismic Hazard Earthquake Epicentre File (SHEEF2010) used in the Fifth Generation Seismic Hazard Maps of Canada; Geological Survey of Canada, Open File 7724.

Harrison, J E and MacDonald, G., (1980). Generalized bedrock geology Ottawa-Hull, Ontario and Québec. Geological Survey of Canada, A Series Map 1508A.

Heaman, L. M., and B. A. Kjarsgaard (2000). Timing of eastern North American kimberlite magmatism: Continental extension of the Great Meteor hotspot track? Earth Planet. Sci. Lett., 178, 253-268.

Hodgson, E. A. (1936), The Timiskaming earthquake of November 1st, 1935: The location of epicenter and determination of focal depth, J. R. Astron. Soc. Can., 30, 113-123.

Hodgson, E. A. (1945), The Cornwall-Massena earthquake, September 5, 1944, J. R. Astron. Soc. Can., 39, 5-13. 
Hunter, J.A., R.A. Burns, R.L. Good, J.M. Aylsworth, S.E. Pullan, D. Perret, and M. Douma, (2007). Borehole shear wave velocity measurements of Champlain Sea sediments in the OttawaMontreal region. Geological Survey of Canada, Ottawa, Ont. Open File 5345.

Hunter, J.A., H.L. Crow, G.R. Brooks, M. Pyne, D. Motazedian, M. Lamontagne, A.J.-M. Pugin, S.E. Pullan, T. Cartwright, M. Douma, R.A. Burns, R. L. Good, K. Kaheshi-Banab, R. Caron, M. Kolaj, I. Folahan, L. Dixon, K. Dion, A. Duxbury, A. Landriault, V. Ter-Emmanuil, A. Jones, G. Plastow, D. Muir (2010). Seismic site classification and site period mapping in the Ottawa area using geophysical methods. Geological Survey of Canada. Open File 6273.

Johnson, M.D., Armstrong, D.K., Sanford, B.V., Telford, P.G. and Rutka, M.A., (1991). Paleozoic and Mesozoic geology of Ontario. In Geology of Ontario, Ontario Geological Survey, Special Volume 4, Part 1, p. 907-1008.

Lamontagne, M., H. S. Hasegawa, D. A. Forsyth, G. G. R. Buchbinder, and M. Cajka (1994). The Mont-Laurier, Quebec, earthquake of 19 October 1990 and its seismotectonic environment. Bull. Seismol. Soc. Am., 84, 1505-1522

Lamontagne, M., S. Halchuk, J. F. Cassidy, and G. C. Rogers (2008). Significant Canadian Earthquakes of the Period 1600-2006. Seism. Res. Lett. 79, 211-223.

Leblanc, G. (1981), A closer look at the September 16, 1732 Montreal earthquake, Can. J. Earth Sci., 18, 539-550.

Ma, S., and D. W. Eaton (2007). Western Quebec seismic zone (Canada): Clustered, midcrustal seismicity along a Mesozoic hot spot track, J. Geophys. Res., 112, B06305, doi:10.1029/2006JB004827.

Ma, S., and D. Motazedian (2012). Studies on the June 23, 2010 North Ottawa Mw 5.2 earthquake and vicinity seismicity, J. Seismol., 16, 513-534. doi:10.1007/s10950-012-9294-7

Mazzotti, S., (2007). Geodynamic models for earthquake studies in intraplate North America, in Stein, S., and S. Mazzotti e.d., Continental Intraplate Earthquakes: Science, Hazard, and Policy Issues: Geological Society of America Special Paper 425, p 17-33, doi: 10.1130/2007.2425(02).

Mazzotti, S., and J. Townend, (2010). State of stress in central and eastern North American seismic zones, Lithosphere, 2, 76-83, doi: 10.1130/L65.1

Medioli, B.E., S. Alpay, H.L. Crow, D.I. Cummings, M.J. Hinton, R.D. Knight, C. Logan, A.J.-M. Pugin, H.A.J. Russell, and D.R. Sharpe (2012). Integrated data sets from a buried valley borehole, Champlain Sea basin, Kinburn, Ontario; Geological Survey of Canada, Current Research 20123, 16 p. doi: 10.4095/289597. 
Motazedian, D., J.A. Hunter, A. Pugin, and H. Crow, (2011). Development of a Vs30 (NEHRP) map for the city of Ottawa, Ontario, Canada. Can. Geotech. J., 48, 458-472.

NBCC. (2005). Canadian commission on building and fire codes. National Building Code of Canada (NBCC), National Research Council Canada, Ottawa, Ont.

NEHRP. (1994). Recommended provisions for seismic regulations of new buildings: Part 1, provisions. FEMA 222A. National Earthquake Hazards Reduction Program (NEHRP), Federal Emergency Management Agency, Washington, D.C.

Pugin, A.J.-M., S.E. Pullan, and J.A. Hunter (2009). Multi-component high resolution seismic reflection profiling; The Leading Edge, 28, 1248-1261 936-945.

Schulte, S., and W.D. Mooney (2005). An updated global earthquake catalogue for stable continental regions; reassessing the correlation with ancient rifts. Geophys. J. Int., 161, 707721.

Scott, J.S. (2003). A review of the geology and geotechnical characteristics of Champlain Sea clays of the Ottawa Valley with reference to slope failures, Geological Survey of Canada. Open File 4475 .

Stein, S., (2007). Approaches to continental intraplate earthquake issues, in Stein, S., and S. Mazzotti e.d., Continental Intraplate Earthquakes: Science, Hazard, and Policy Issues: Geological Society of America Special Paper 425, p 1-16, doi: 10.1130/2007.2425(01).

van Lanen, X., and W.D. Mooney (2007). Integrated geologic and geophysical studies of North American continental intraplate seismicity, in Stein, S., and S. Mazzotti e.d., Continental Intraplate Earthquakes: Science, Hazard, and Policy Issues: Geological Society of America Special Paper 425, p 101-112, doi: 10.1130/2007.2425(08).

Wu, P., and S. Mazzotti (2007). Effects of a lithospheric weak zone on postglacial seismotectonics in eastern Canada and the Northeastern United States, in Stein, S., and S. Mazzotti e.d., Continental Intraplate Earthquakes: Science, Hazard, and Policy Issues: Geological Society of America Special Paper 425, p 113-128, doi: 10.1130/2007.2425(09).

Zoback, M.D., and M.L. Zoback (1991). Tectonic stress field of North America and relative plate motions, in Neotectonics of North America, edited by D. B. Slemmons et al., pp. 261-276, Geol. Soc. of Am., Boulder, Colo. 


\section{Seismic Recordings}

There are many recordings of earthquakes which have occurred in Eastern Canada, even though earthquake seismicity is sparse in this region compared to tectonic plate boundary regions. Earthquakes are commonly recorded from the Western Quebec Seismic Zone (WQSZ) on the Canadian National Seismograph Network (CNSN) at a rate of about once every 3 to 5 days (Earthquakes Canada, 2016). The seismograph network in this area is well suited to detect earthquakes in the area and the waveforms are available for the public from these stations using the AutoDRM (Earthquakes Canada, 2016).

The seismograph stations are spread out over eastern Ontario and western Quebec, and are able to provide a good data set for studying small to moderate earthquakes which occur in the region (Atkinson et al., 2015; Bent et al., 2015). The moderately sized earthquakes, roughly magnitude 5, have a recurrence rate of about 10 years in the WOSZ (Basham et al., 1979). Previous studies on these earthquakes have provided a good determination of the characteristics and parameters for the propagation of seismic waves in the area (Atkinson and Assatourians, 2010; Atkinson et al., 2014).

Along with microzonation studies, Carleton University in Collaboration with Geological Survey of Canada (GSC), have installed several stations in eastern Ontario to study site effects in locations where there is an abundance of very soft soils overlying a firm bedrock, occasionally with a thin layer of glacial till between the two. These were installed as station pairs with identical sensors and have provided recordings that show a large difference in motions from rock to soil. Although the site effects can be easily shown using the station pair, the causes of such site effects 
are difficult to determine as there are a lot of possibilities. The station pairs were installed in soft soil basins, so the one dimensional site effects account for only a portion of the amplification between the soil recordings and the rock recordings. Hayek (2016) has studied the pairs of recordings in great detail.

\subsection{Ladysmith Earthquake}

The Ladysmith earthquake occurred on May 17th, 2013 at 09:43 EDT, about 18 kilometres northeast of Shawville, Quebec with a measured magnitude of M 4.6 (Bent et al., 2015). This earthquake was widely felt in the region and recorded (see Figure 3.1) by two identical strong motion stations (collocated with the two weak motion seismic stations of KB1O and KB2O) in the Kinburn array. It should be mentioned that recordings by two weak motion seismic stations of KB1O and KB2O were clipped. These stations at the time were named JSBS (now KB1O) and JSSS (now KB2O) and are the rock site and the soil site at the centre of the basin, respectively. The recordings show significant amplification within the basin even though the soil location is only slightly further from the source.

The earthquake itself was one of the best recorded earthquakes in the area for the coincidence of several stations of the US Transportable Array (USTA) having been deployed in the region during the time of the earthquake. One of these stations was close enough to use for a free depth determination of the hypocentre (Bent et al., 2015). This earthquake rupture was that of a reverse thrust motion on a northwest striking plane, and is a typical type of rupture of an earthquake in the WQSZ. The earthquake occurred within the Ottawa-Bonnechere graben, but the rupture does not match the orientation of the existing faults in that area (Atkinson et al., 
2014). The good coverage of recordings and the in-depth study of source and path characteristics of this earthquake make it a good choice to use for comparing simulations to an actual earthquake event (Atkinson et al., 2014; Ma and Audet, 2014; Bent et al., 2015).

\subsubsection{Source Parameters}

The deployment of the USTA in the region at the time of the earthquake has allowed a much more detailed study into the source parameters of this earthquake compared to others of this size occurring in the WQSZ. Bent and others (2015) were able to obtain a high quality solution from the regional moment tensor inversion method (Kao et al., 1998) using eight broadband stations with good azimuthal coverage relative to the epicentre. This inversion gives the strike/dip/rake as $132 / 49 / 63$ or $350 / 48 / 118$ and a depth of $12 \pm 2 \mathrm{~km}$ with the epicentre at $45.76^{\circ} \mathrm{N}$ and $-76.32^{\circ} \mathrm{E}$. Other methods and independent inversions give similar results for the fault plane including (Bent et al., 2015): an independent regional moment tensor inversion with a fault plane solution of $135 / 50 / 65$ or $351 / 46 / 117$ and depth of $13 \mathrm{~km}$ (Herrmann, 2013); a moment tensor solution based on vertical Rayleigh waves (Ma and Audet, 2014) gives a fault plane solution of 306/41/94 or 122/50/87; and clear first motions with the grid search algorithm (Snoke et al., 1984) gave a fault plane solution of $115 / 59 / 47$ or $356 / 52 / 139$. These are listed in Table 3.1, along with the Cartesian Moment Tensor solutions.

\begin{tabular}{|c|c|cc|}
\hline Source & Strike/Dip/Rake & Cartesian Moment Tensor* \\
\hline \multirow{3}{*}{ Bent et al., 2015 } & $132 / 49 / 63$ or & {$\left[\begin{array}{ccc}-0.147 & -0.475 & 0.291 \\
-0.475 & -0.736 & -0.138 \\
0.291 & -0.138 & 0.882\end{array}\right]$} \\
\hline \multirow{3}{*}{ Ma and Audet, 2014 } & $350 / 48 / 118$ & {$\left[\begin{array}{ccc}-0.700 & -0.459 & 0.029 \\
-0.459 & -0.298 & -0.040 \\
0.029 & -0.040 & 0.998\end{array}\right]$} \\
\hline
\end{tabular}




\begin{tabular}{|c|c|c|c|c|}
\hline Global CMT & $\begin{array}{c}143 / 45 / 79 \text { or } \\
338 / 46 / 101\end{array}$ & {$\left[\begin{array}{c}-0.226 \\
-0.435 \\
0.108\end{array}\right.$} & $\begin{array}{l}-0.435 \\
-0.756 \\
-0.081\end{array}$ & $\begin{array}{c}0.108 \\
-0.081 \\
0.982\end{array}$ \\
\hline Herrman, 2013 & $\begin{array}{c}135 / 50 / 65 \text { or } \\
351 / 46 / 117\end{array}$ & {$\left[\begin{array}{c}-0.123 \\
-0.446 \\
0.303\end{array}\right.$} & $\begin{array}{l}-0.446 \\
-0.770 \\
-0.081\end{array}$ & $\begin{array}{c}0.303 \\
-0.081 \\
0.893 \\
\end{array}$ \\
\hline $\begin{array}{l}\text { Bent et al., } 2015 \text { clear } \\
\text { first motion method } \\
\text { (from Snoke et al. 1984) }\end{array}$ & $\begin{array}{c}115 / 59 / 47 \text { or } \\
356 / 52 / 139\end{array}$ & {$\left[\begin{array}{c}-0.083 \\
-0.623 \\
0.460\end{array}\right.$} & $\begin{array}{l}-0.623 \\
-0.563 \\
-0.173\end{array}$ & $\begin{array}{c}0.460 \\
-0.173 \\
0.646\end{array}$ \\
\hline
\end{tabular}

Table 3.1: The strike/dip/rake and Cartesian moment tensors for the May 17th, 2013 Ladysmith earthquake found by different methods. *The Cartesian moment tensor is multiplied by the seismic moment for the amplitude of the moment for each component.

The preferred fault plane solution is converted to the moment tensor solution:

$$
\boldsymbol{M}=\left[\begin{array}{ccc}
-1.165 & -3.966 & 2.475 \\
-3.966 & -6.187 & -1.179 \\
2.475 & -1.179 & 7.352
\end{array}\right] \times 10^{15} \mathrm{Nm}
$$

where:

$$
\boldsymbol{M}=\left[\begin{array}{lll}
M_{x x} & M_{y x} & M_{z x} \\
M_{x y} & M_{y y} & M_{z y} \\
M_{x z} & M_{y z} & M_{z z}
\end{array}\right]
$$

and the individual components are calculated by (Aki and Richards, 1980):

$$
\begin{aligned}
& M_{x x}=-M_{0}\left(\sin \delta \cos \lambda \sin 2 \phi_{s}+\sin 2 \delta \sin \lambda \sin ^{2} \phi_{s}\right) \\
& M_{x y}=M_{y x}=M_{0}\left(\sin \delta \cos \lambda \cos 2 \phi_{s}+\frac{1}{2} \sin 2 \delta \sin \lambda \sin 2 \phi_{s}\right) \\
& M_{x z}=M_{z x}=-M_{0}\left(\cos \delta \cos \lambda \cos \phi_{s}+\cos 2 \delta \sin \lambda \sin \phi_{s}\right) \\
& M_{y y}=M_{0}\left(\sin \delta \cos \lambda \sin 2 \phi_{s}-\sin 2 \delta \sin \lambda \cos ^{2} \phi_{s}\right) \\
& M_{y z}=M_{z y}=-M_{0}\left(\cos \delta \cos \lambda \sin \phi_{s}+\cos 2 \delta \sin \lambda \cos \phi_{s}\right) \\
& M_{z z}=M_{0} \sin 2 \delta \sin \lambda
\end{aligned}
$$

where $\phi_{s}$ is strike, $\delta$ is dip, and $\lambda$ is the rake. This can be rotated into a source to receiver coordinate system by changing $\phi_{s} \rightarrow \Phi$, where $\Phi=\phi_{s}-\varphi$ and $\varphi$ is the receiver azimuth (Li et al., 2014).

The Global Centroid Moment Tensor found using the process described by Dziewonski et al. 1981, and Ekström et al. (2012) for this earthquake is given as: 


$$
\begin{aligned}
& M_{r r}=1.47 \times 10^{16} \mathrm{Nm} \\
& M_{t t}=-1.55 \times 10^{16} \mathrm{Nm} \\
& M_{p p}=-3.20 \times 10^{15} \mathrm{Nm} \\
& M_{r t}=1.34 \times 10^{15} \mathrm{Nm} \\
& M_{r p}=1.47 \times 10^{15} \mathrm{Nm} \\
& M_{t p}=1.47 \times 10^{15} \mathrm{Nm}
\end{aligned}
$$

where the depth is $22.15 \mathrm{~km}$, and the epicentre is at $45.8^{\circ} \mathrm{N}$ and $-76.41^{\circ} \mathrm{E}$. The centroid moment tensor is converted from the Cartesian moment tensor by:

$$
\begin{aligned}
& M_{r r}=M_{z z} \\
& M_{t t}=M_{x x} \\
& M_{p p}=M_{y y} \\
& M_{r t}=M_{x z} \\
& M_{r p}=-M_{y z} \\
& M_{t p}=-M_{x y}
\end{aligned}
$$

\subsubsection{Ground Motions}

The three component strong ground motions recorded at KB1O (rock) and KB2O (soil) are shown in Figure 3.1. The motions were recorded by identical three component Etnas adjacent to two broadband seismometers. The motions on the soil site of the broadband seismometers was clipped for all three components so the strong motion recordings are used. These recordings have the strong motion instrument responses removed and a baseline correction applied, as discussed in Appendix I. These motions show a significant amplification in the soil compared to the bedrock. Table 3.2 showing the peak amplitudes for each recording and the ratio between the soil and rock recordings. These stations are close together ( $2 \mathrm{~km}$ apart) and have similar epicentral distances from the Ladysmith earthquake, $41.7 \mathrm{~km}$ for KB1O and $43.5 \mathrm{~km}$ for KB2O, and similar source to receiver azimuths, $164.4^{\circ}$ for KB1O and $162.9^{\circ}$ for KB2O. 

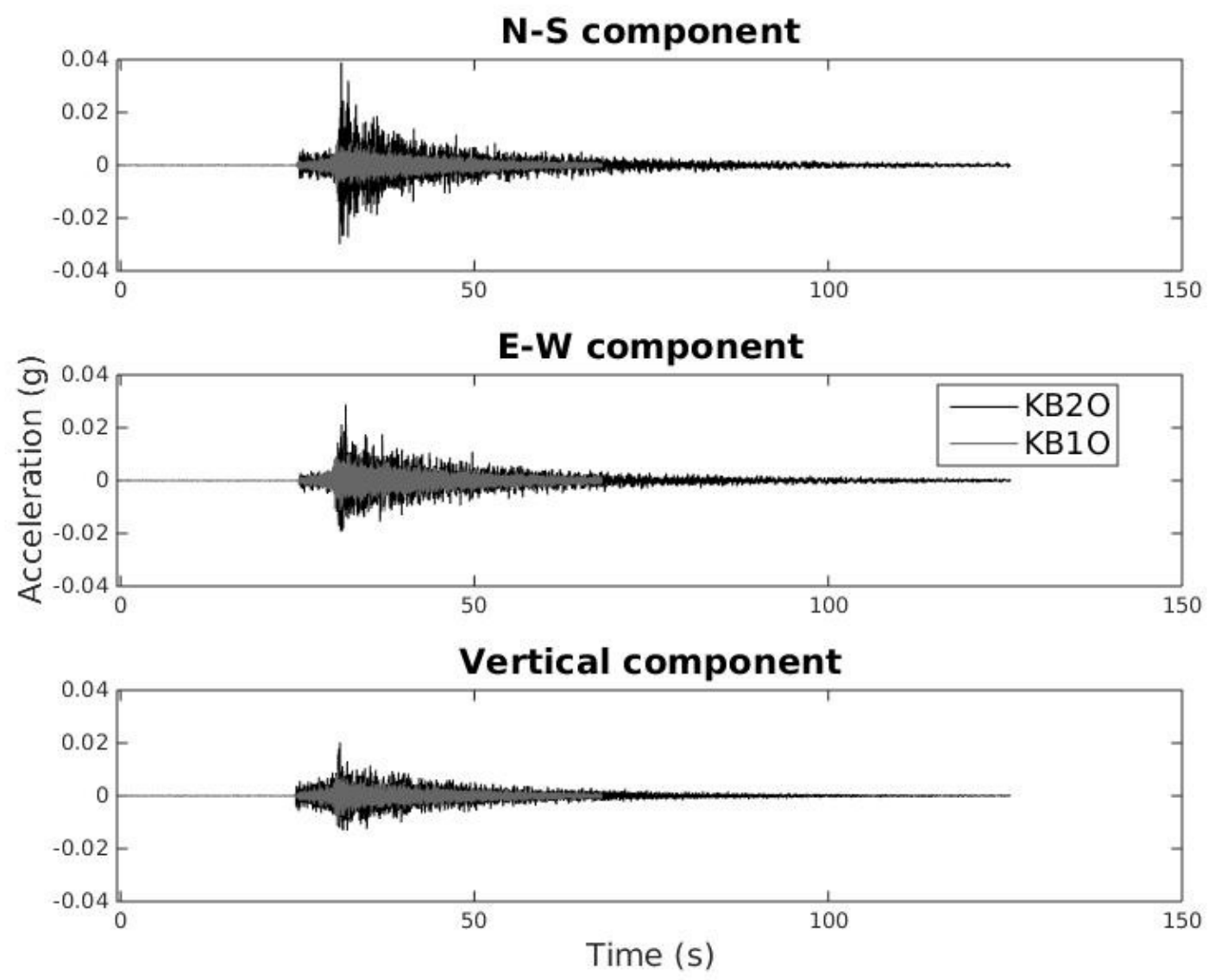

Figure 3.1: Strong ground motions recorded at stations $\mathrm{KB} 1 \mathrm{O}$ and $\mathrm{KB} 2 \mathrm{O}$ during the Ladysmith earthquake.

\begin{tabular}{|c|c|c|c|}
\hline Recording & $\begin{array}{c}\text { Peak motion in soil } \\
(\mathrm{g})\end{array}$ & $\begin{array}{c}\text { Peak motion in rock } \\
(\mathrm{g})\end{array}$ & Difference (Soil/Rock) \\
\hline N-S & 0.0389 & 0.0108 & 3.6019 \\
\hline E-W & 0.0275 & 0.0118 & 2.3305 \\
\hline Vertical & 0.0202 & 0.0114 & 1.7719 \\
\hline
\end{tabular}

Table 3.2: Peak motions recorded at KB1O and KB2O.

It is better, for these conditions, to look at the spectral amplification as these can significantly alter the ground motions between rock and soil sites. The Fourier spectral accelerations are shown in Figure 3.2 for KB1O and in Figure 3.3 for KB2O. Figure 3.4 shows the smoothed transfer function (soil spectral acceleration/rock spectral acceleration) between the 
two recordings. As shown there is a large amplification around the fundamental site period for KB2O.

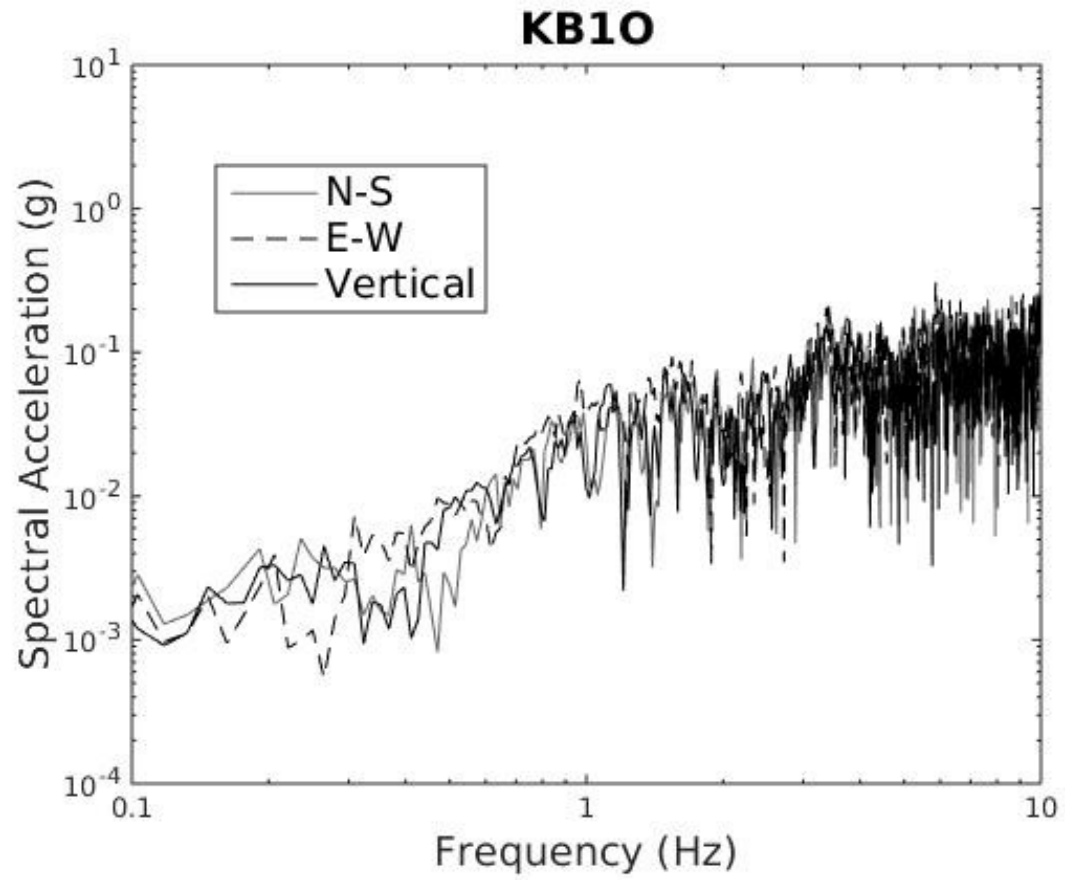

Figure 3.2: Fourier spectral accelerations recorded at station KB1O. 


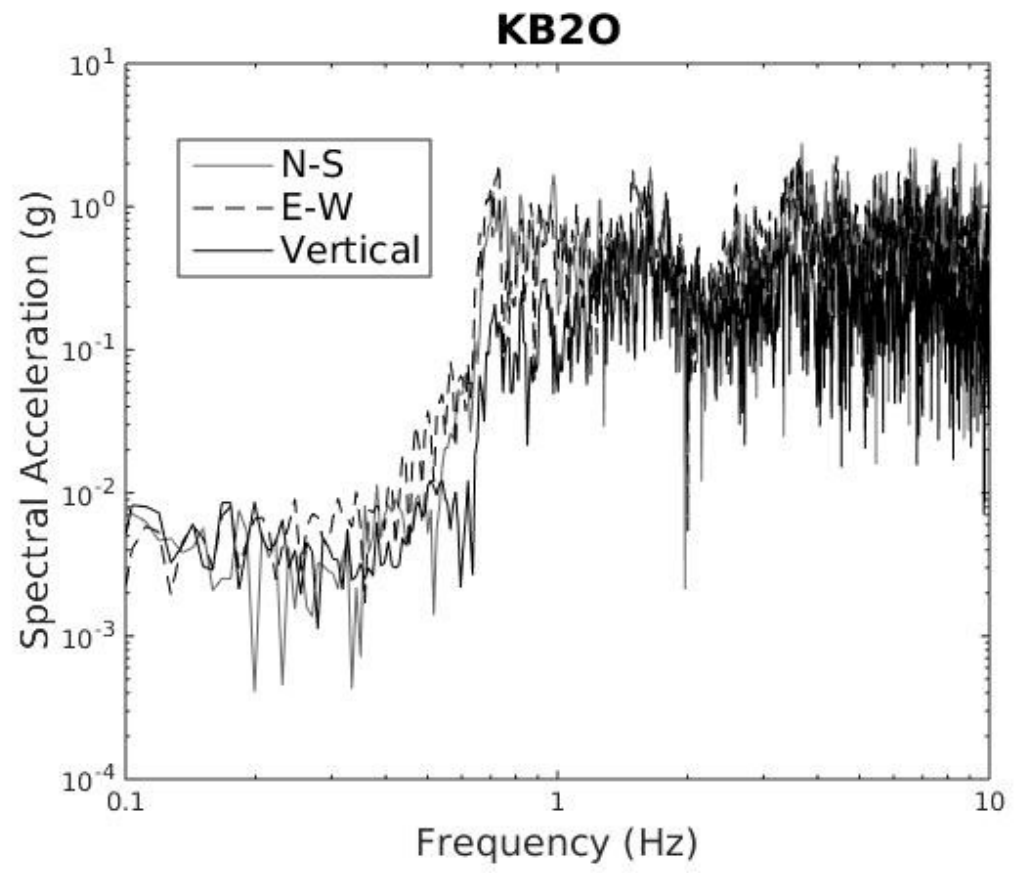

Figure 3.3: Fourier spectral accelerations recorded at station KB2O.
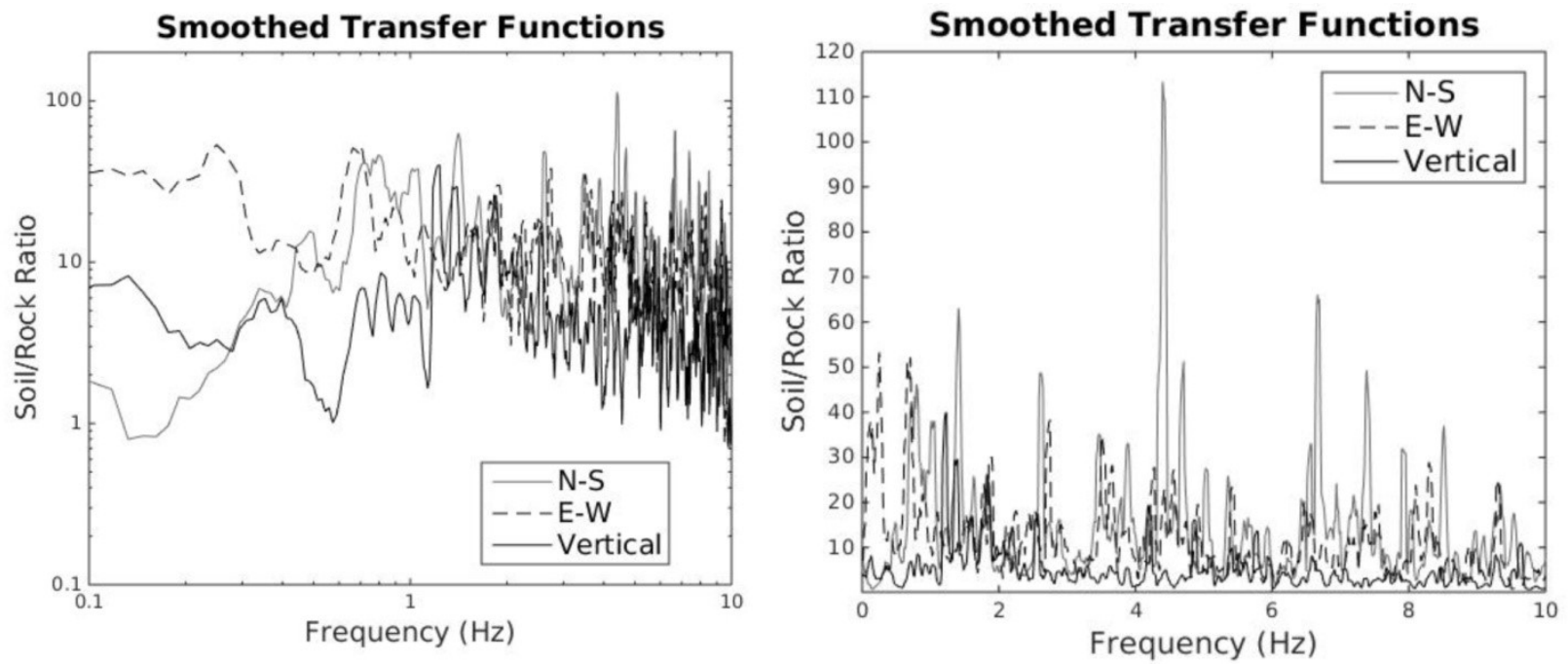

Figure 3.4: Transfer functions from KB1O to KB2O plotted on a log scale (left) and linear scale (right). The data is smoothed using a 5 point moving average method.

Figure 3.5 shows the horizontal to vertical spectral ratio (HVSR) for the strong motion recordings from the Ladysmith earthquake, and the HVSR from the passive method. From this it can be seen that the spectral ratio peaks near the fundamental period of the site, measured to 
be $0.72 \mathrm{~Hz}$ from the passive HVSR method. The spectral ratio peak of the active recording is slightly lower than that of the passive measurement, which could be due to several reasons. However, since the soil is extremely soft, one probable reason for a shift of the fundament period seen here could be due to nonlinear soil effects at different ground motion amplitudes (Yu et al., 1993).
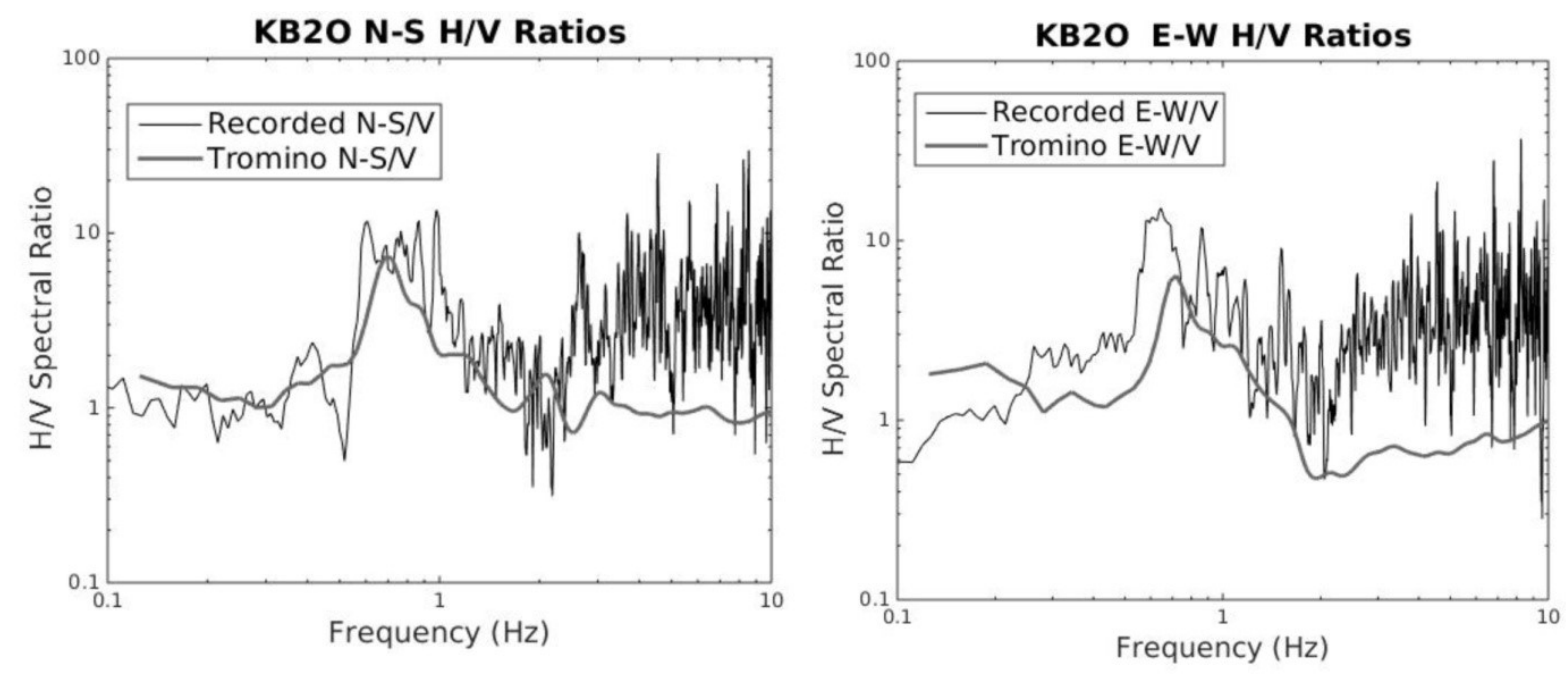

Figure 3.5: Horizontal to vertical ratios from the active recording at KB2O, and from the passive Tromino measurement at the same location for the N-S component (left) and E-W component (right).

\subsubsection{Basin Effects from Recorded Motions}

Although difficult, it may be possible to delineate some of the basin effects using the rock-soil seismic station pair. Hayek (2016) was able to indentify various components of the ground motion which were unexplained by one dimensional modelling at the soil station using the recordings at the rock station as input. These include a stronger amplification about a wider frequency band around the dominant frequency than expected, and particle motions within the basin being inconsistent with the particle motion recorded outside the basin. In her study, the particle motion was only completed on the Orleans rock-soil station pair, due to a misalignment problem with the other stations. The different components for KB1O and KB2O for the Ladysmith 
earthquake were aligned in the time domain based on the arrival of the first wave packets. Particle motion using these stations are likely not the correct particle motion, however a comparison between the particle motion from the soil to rock stations, processed in the same manner for both, can provide some insight into the differences of the particle motion between these stations.

The particle motion is found from the displacement for both KB1O and KB2O shown in Figure 3.1. The displacement is found by: integrating the acceleration over time, removing the instrument drift, then integrating over time again. Specific details about this method is explained in Appendix III. Particle motions are plotted for several time intervals including: the P-wave arrival; the S-wave arrival; and several other waveforms associated with stronger displacements recorded at the soil station. 

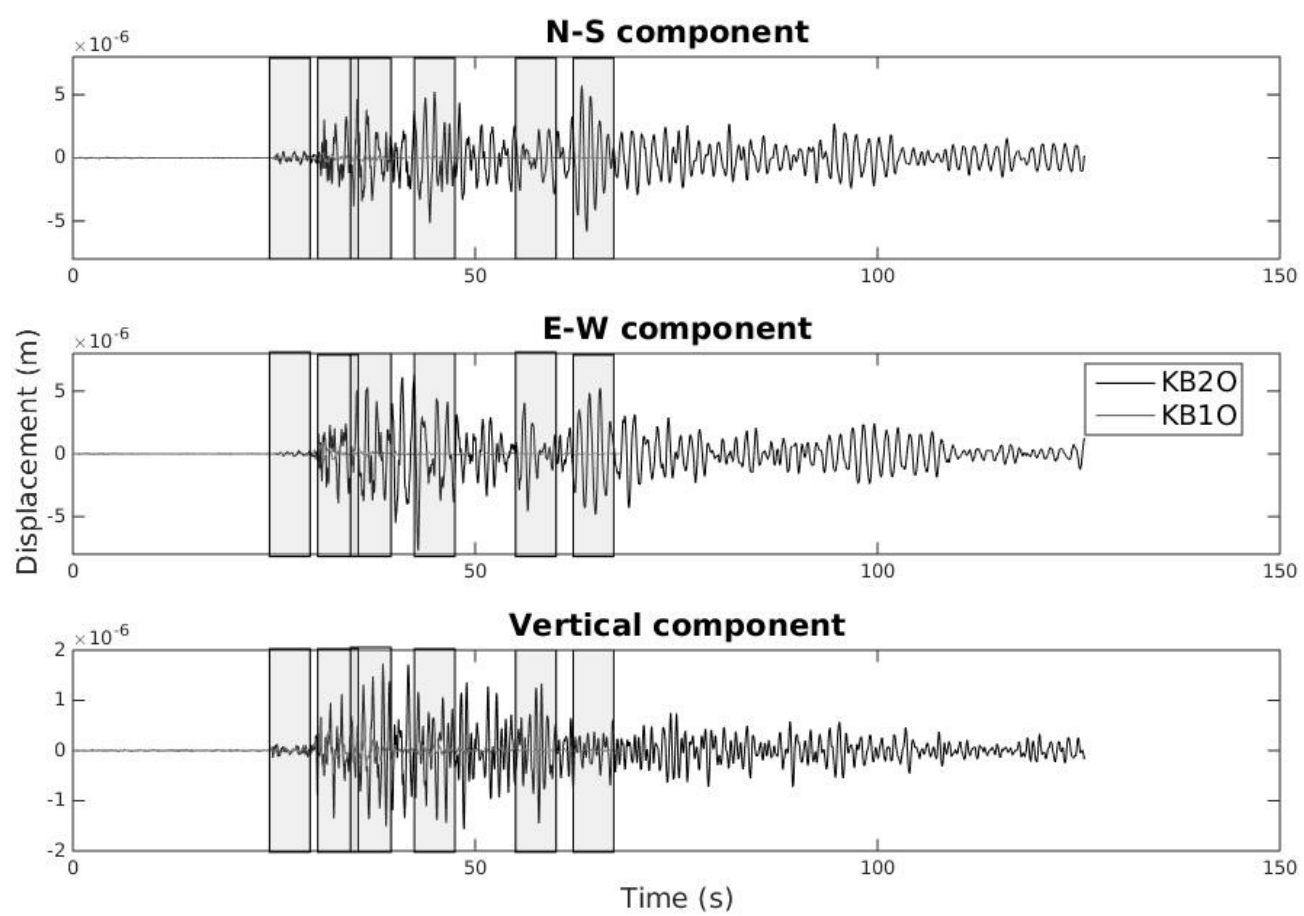

Figure 3.6: The displacement time series for station KB1O and KB2O caused by the 2013 Ladysmith, M5.0 earthquake. The pairs of blue lines outline the time intervals for the particle motion plots. Note the vertical component (bottom) has a quarter of the displacement scale as the horizontal components (top and middle) in order to display these motions.

The particle motion plot for the P-wave should display a strong directional dependence to the source of the earthquake, based on the particle motion caused by P-waves being the same as the direction of propagation of the wave. The particle motion for the P-wave for KB1O and KB2O is shown in Figure 3.7. The rock station, KB1O, shows the strong directional dependence seemingly rotated based on the location of the epicentre, displayed by the grey dashed line. The soil station within the basin seems to have a rotated directional dependence for the P-wave arriving at this location compared to the rock station. 


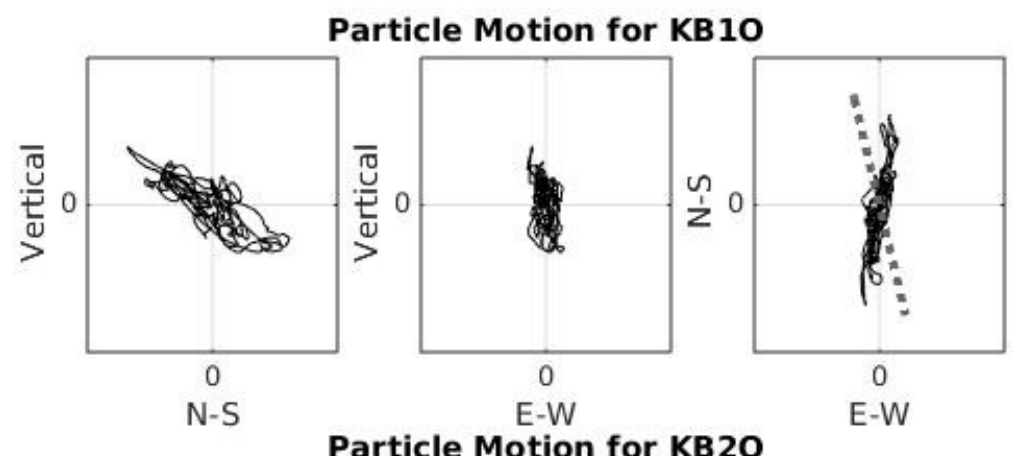

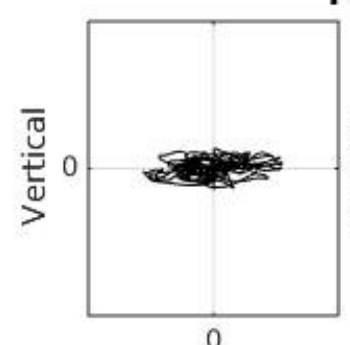

N-S

article Motion for KB

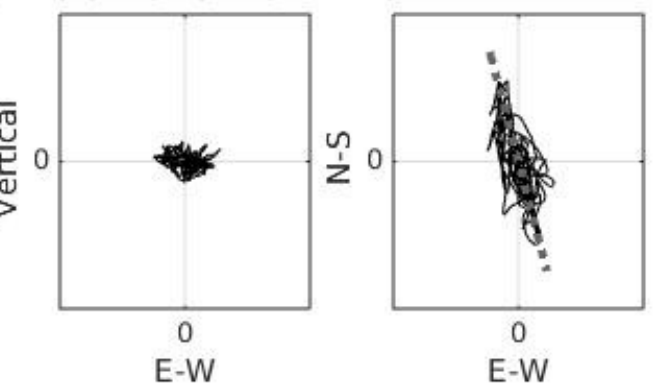

Figure 3.7: Particle motion plots for KB1O (top) and KB2O (bottom) for the P-wave recorded at each station. The grey dashed line is the receiver azimuth towards the earthquake epicentre.

The induced particle motion from S-waves being perpendicular to the direction wave propagation, should rotate the directional components from the P-wave. The particle motions for the time slice centred about the S-wave arrival at KB1O and KB2O is shown in Figure 3.8. There appears not to be any directional dependence that can be discerned from this data. Likely this is due to the high frequency nature of earthquakes in Eastern North America, and the short distance from the stations to the epicentre causing an overlap of the P-wave coda and the S-wave arrival. 


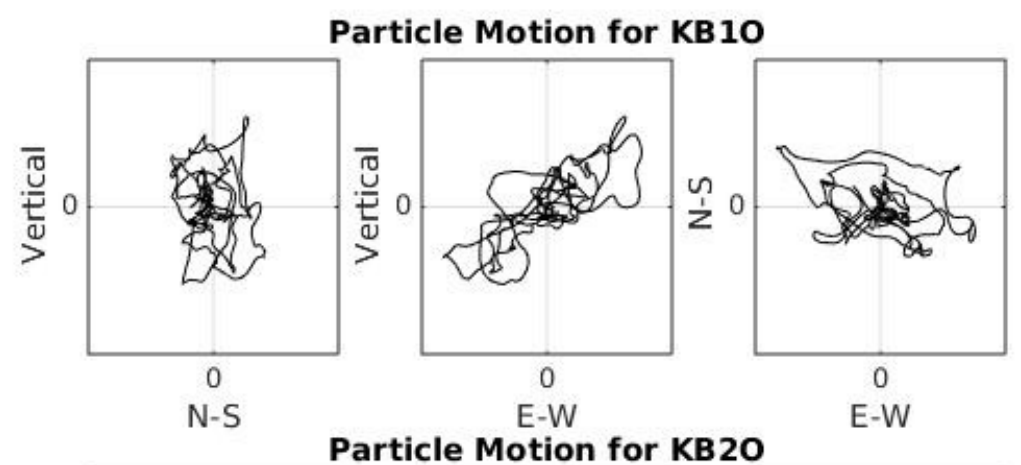

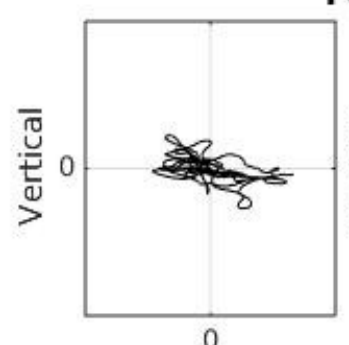

N-S

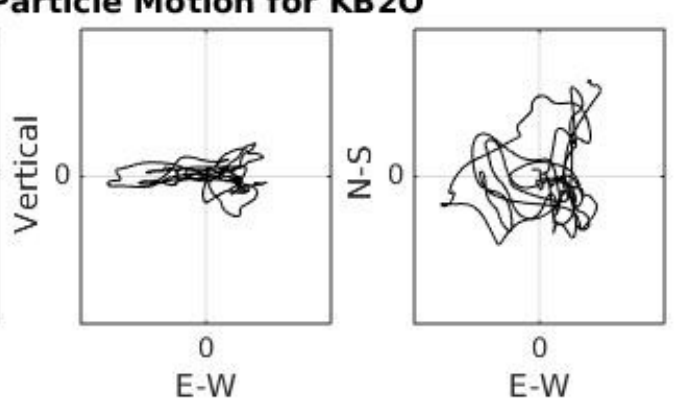

Figure 3.8: Particle motion plots for KB1O (top) and KB2O (bottom) for the S-wave recorded at each station.

The last particle motion plot is for three different time slices at KB2O where there appears to be a strong wave packet arriving due to increased displacement, shown in Figure 3.9. These time slices are $42-47$ seconds, 55-60 seconds, and 61-67 seconds as outlined by the displacement time series in Figure 3.6. These motions show a much stronger horizontal component in both the $\mathrm{N}-\mathrm{S}$ and E-W directions than the vertical component. This indicates that these are likely surface waves as both types of waves would have a stronger horizontal component than vertical component in this setting (Konno and Omachi, 1998). 

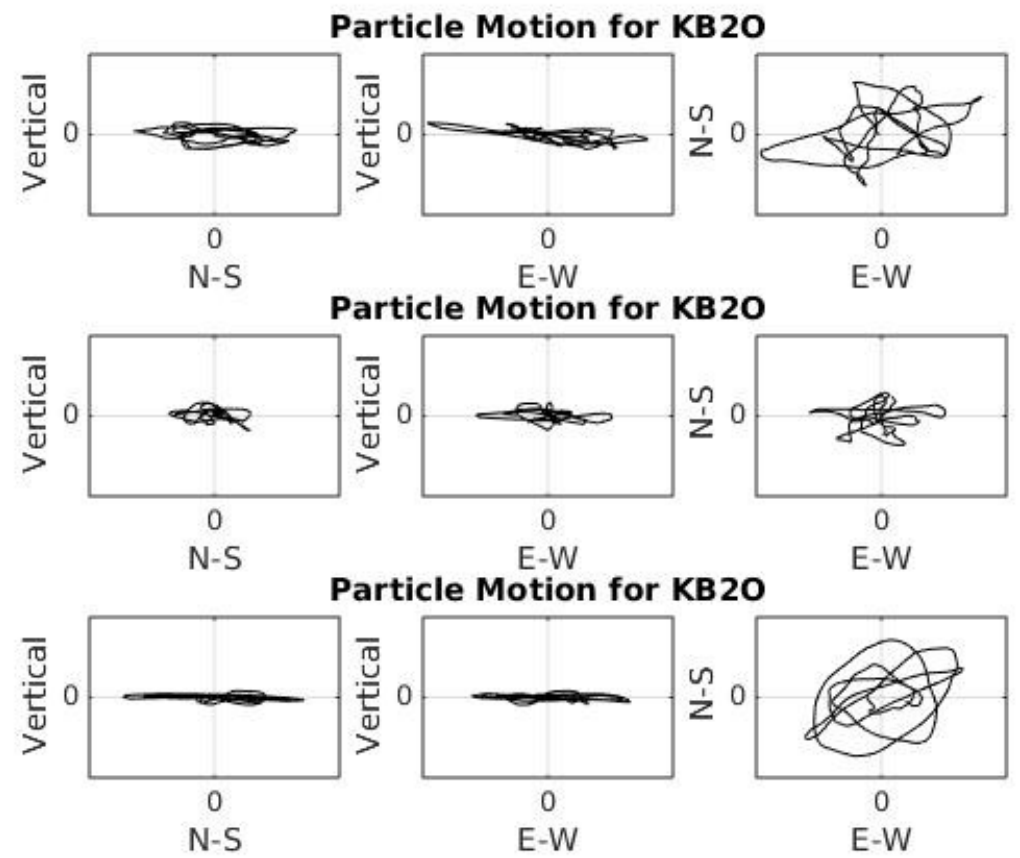

Figure 3.9: Particle motions for different time slices at KB2O. The time slices are $42-47$ seconds (top), 55-60 seconds (middle), and 61-67 seconds (bottom). 


\subsection{References:}

Aki, K. and P.G. Richards (1980). Quantitative Seismology: Theory and Methods, W.H. Freeman.

Atkinson, G., and K. Assatourians (2010). Attenuation and source characteristics of the June 23, 2010 Val-des-Bois, Quebec, earthquake, Seismol. Res. Lett., 81, 849-860.

Atkinson, G. M., K. Assatourians, and M. Lamontagne (2014). Characteristics of the 17 May 2013 M 4.5 Ladysmith, Quebec earthquake, Seismol. Res. Lett., 85, 755-762, doi: 10.1785/0220130160.

Basham, P. W., D. H. Weichert., and M. J. Berry (1979). Regional assessment of seismic risk in eastern Canada. Bull. Seism. Soc. Am. 69, 1567-1602

Basham, P. W., D. H. Weichert, F. M. Anglin, and M. J. Berry (1982). New probablilistic strong seismic ground motion map of Canada: A compilation of earthquake source zones, methods and results, Earth Physics Branch Open File 82-33, 205.

Bent, A.L., M. Lamontagne, V. Peci, S. Halchuk, G.R. Brooks, D. Motazedian, J.A. Hunter, J. Adams, C. Woodgold, J. Drysdale, S. Hayek, W. N. Edwards (2015). The 17 May 2013 M 4.6 Ladysmith, Quebec, Earthquake, Seismol. Res. Lett., 86, 460-476. doi: 10.1785/0220140138

Borcherdt, R. D. (1994). Estimates of site-dependent response spectra for design (methodology and justification), Earthq. Spectra, 10, no. 4, 617-653.

Dziewonski, A. M., T.-A. Chou and J. H. Woodhouse (1981). Determination of earthquake source parameters from waveform data for studies of global and regional seismicity, J. Geophys. Res., 86, 2825-2852. doi:10.1029/JB086iB04p02825

Earthquakes Canada Website at http://www.earthquakescanada.nrcan.gc.ca/index-en.php, Last accessed July 11th, 2016.

Ekström, G., M. Nettles, and A. M. Dziewonski (2012) The global CMT project 2004-2010:

Centroid-moment tensors for 13,017 earthquakes, Phys. Earth Planet. Inter., 200-201, 1-9. doi:10.1016/j.pepi.2012.04.002

Hayek, S. (2016). Seismic basin effects over soft-sediment filled basins in Ottawa, Canada. M.Sc. Thesis, Department of Earth Sciences, Carleton University.

Herrmann, R. (2013). North America moment tensor, Saint-Louis University, http://www.eas.slu.edu/eqc/eqc_mt/MECH.NA/MECHFIG/mech.html (last accessed June, 2016). 
Hunter, J.A., H.L. Crow, G.R. Brooks, M. Pyne, D. Motazedian, M. Lamontagne, A.J.-M. Pugin, S.E. Pullan, T. Cartwright, M. Douma, R.A. Burns, R. L. Good, K. Kaheshi-Banab, R. Caron, M. Kolaj, I. Folahan, L. Dixon, K. Dion, A. Duxbury, A. Landriault, V. Ter-Emmanuil, A. Jones, G. Plastow, D. Muir (2010). Seismic site classification and site period mapping in the Ottawa area using geophysical methods. Geological Survey of Canada. Open File 6273.

Kao, H., P. R. Jiang, K. F. Ma, B. S. Huang, and C. C. Liu (1998). Moment-tensor inversion for offshore earthquakes east of Taiwan and their implications for regional collision, Geophys. Res. Lett., 25, 3619-3622.

Khaheshi Banab, K., and D. Motazedian (2010). Efficiency of the multichannel analysis of surface wave method for shallow and semi-deep loose soil layers overlaying a very high shear wave velocity bedrock, Int. J.Geophys. Article ID 403016, doi: 10.1155/2010/403016.

Khaheshi Banab, K., M. Kolaj, D. Motazedian, S. Sivathyalan, J. A. Hunter, H. L. Crow, A. J.-M. Pugin, G. R. Brooks, and M. Pyne (2012). Seismic site response analysis for Ottawa, Canada: A comprehensive study using measurements and numerical simulations, Bull. Seismol. Soc. Am., 102, 19761993, doi: 10.1785/0120110248.

Konno, K., and T. Ohmachi (1998). Ground motion characteristics estimated from spectral ratio between horizontal and vertical components of microtremor. Bull. Seism. Soc. Am., 88, 228241.

Li, D., D. Helmberger, R.W. Clayton and D. Sun (2014). Global synthetic seismograms using a 2-D finite-difference method. Geophys. J. Int., 197 (2): 1166-1183.

Ma, S., and P. Audet (2014). The 5.2 magnitude earthquake near Ladysmith, Quebec, 17 May 2013: Implications for the seismotectonics of the Ottawa-Bonnechere graben, Can. J. Earth Sci., 51, 439-451, doi: 10.1139/cjes-2013-0215.

Motazedian, D., J.A. Hunter, A. Pugin, and H. Crow, (2011). Development of a Vs30 (NEHRP) map for the city of Ottawa, Ontario, Canada. Can. Geotech. J., 48, 458-472.

NBCC. (2005). Canadian commission on building and fire codes. National Building Code of Canada (NBCC), National Research Council Canada, Ottawa, Ont.

Snoke, J. A., J. W. Munsey, A. G. Teague, and G. A. Bollinger (1984). A program for focal mechanism determination by combined use of polarity and SV-P amplitude data, Earthq. Notes, 55, 15.

Yu, G., J. G. Anderson, and R. Siddharthan (1993). On the characteristics of nonlinear soil response, Bull. Seism. Soc. Am., 83, 218-244. 


\section{Two and Three Dimensional Simulations}

Numerical methods are a powerful tool for solving complex systems that are not solvable without the use of computing systems. With the advancement of computing power and the availability of larger computing resources they are becoming more widely used for a variety of different purposes. There are many different types of numerical simulations, each with their advantages and disadvantages when used for any specific purpose, (see Section 1.4 for examples).

In this study we employed the spectral element method (SEM) as described in Section 1.5, and Appendix I. Two and three dimensional (2D and 3D) implementations of this method were used to simulate recorded accelerograms and seismograms in the Kinburn Basin from the Ladysmith earthquake of 2013 (Bent et al., 2015). The differing simulations done in this study are important for determining the seismic hazard within this basin and similar basins. The possibility of accurate simulations of ground motions within the basin may lead to a more accurate estimation of ground motions to be expected from future earthquakes in the study area.

In order to directly compare the different types of simulations, a review of the mathematical approach of each simulation type is necessary. In this section the differences between a two dimensional and a three dimensional simulation using this method are explained. The largest difference in the theory between these two is the nonexistence of the third spatial direction in the two dimensional approach. The main cause for the amplitude difference arises from the manner by which the source is implemented: for a three dimensional simulation, the source is a point source at a given location; whereas, in a two dimensional approach the source is effectively a line source extending infinitely in a direction perpendicular to the model plane. 
In a two dimensional simulation using SPECFEM2D the plain strain of P-SV approximation is used (Pilant, 1979). The lack of a third dimension in 2D simulations does not affect the propagation of the $\mathrm{P}$ and SV phase of waves within the model plane since these phases do not have a component perpendicular to source-to-receiver plane. Therefore, the 3D simulations will include the propagation and effects of a $\mathrm{SH}$ wave in the simulations whereas the 2D simulations will not. This is most important for simulations which have components outside of a two dimensional plane. For our purpose this will be evident when comparing several receivers from the Kinburn array to a single source, as the receivers do not fall perfectly in line from the source.

\subsubsection{Geometrical Spreading}

The far field radiation patterns from a line source and a point source have been discussed in several textbooks and research papers (i.e. Chapman, 2004; Miksat et al., 2008; Li et al., 2014). The relationship between the two sources was studied to compare seismograms generated from $2 D$ to that of seismographs generated by $3 D$ simulations. This is completed by applying some additional steps to the 2D seismograms. Li et al. (2014) have studied the theoretical calculation for an explosive source in a fluid whole space. Based on these studies the 3D solution for the wavefield displacement $\left(V_{3 p s}\right)$ from a point source at the origin is:

$$
V_{3 p s}(x, y, z, t)=\frac{1}{R} \delta\left(t-\frac{R}{\alpha}\right)
$$

where $R=\sqrt{x^{2}+y^{2}+z^{2}}$ is the source to receiver distance, $\alpha$ is the P-wave velocity and $\delta$ is the Dirac delta function. 
After Aki and Richards (1980, p. 226), a $2 \mathrm{D}$ line displacement source solution, $V_{2 l s}$, along the $y$-direction was obtained by integrating the point source solutions along this line as given by

$$
\begin{gathered}
V_{2 l s}(x, z, t)=\int_{-\infty}^{\infty} V_{3 p s}(x, y, z, t) d y \\
=\frac{2 H(t-R / \alpha)}{\sqrt{t^{2}-R^{2} / \alpha^{2}}} \\
=\frac{2 H(t-R / \alpha)}{\sqrt{t-R / \alpha} \sqrt{t+R / \alpha}} \\
\approx \sqrt{\frac{2 \alpha}{R}} \frac{H(t-R / \alpha)}{\sqrt{t-R / \alpha}}
\end{gathered}
$$

where $R=\sqrt{x^{2}+z^{2}}$ is the distance in $2 \mathrm{D}$ and $H(t)$ is the Heaviside step function. The last approximation holds since the main contribution to $V_{2 l s}$ is the singularity at the P-wave arrival time, $t=R / \alpha$.

From a $2 \mathrm{D}$ displacement seismogram it is possible to obtain a $3 \mathrm{D}$ point source displacement seismogram at the same position by:

$$
V_{3 p s}(x, 0, z, t)=\frac{1}{\pi} \sqrt{\frac{1}{2 R \alpha}} \frac{d}{d t}\left[\frac{1}{\sqrt{t}} * V_{2 l s}(x, z, t)\right]
$$

A similar result can be found using ray theory for each individual arrival in general 2D media (Cerveny, 2001)

$$
V_{3 p s}(x, 0, z, t)=\frac{1}{\pi} \sqrt{\frac{1}{2 F}} \frac{d}{d t}\left[\frac{1}{\sqrt{t}} * V_{2 l s}(x, z, t)\right]
$$


where the factor $F=\int_{\text {ray }} v d s$ is an integration of a wave travelling with velocity $v$ along a ray path $d s$. This ray path differs for each seismic phase and thus each phase in the seismogram must be treated separately (Miksat et al., 2008).

Although this above correction has been derived for displacement seismograms, it can be also applied to velocity or acceleration seismograms. This is shown through the communicative property of convolution (Bracewell, 2000):

$$
(f * g)^{\prime}=f^{\prime} * g=f * g^{\prime}
$$

Taking a time derivative for each side of Eq. 4.4 the result is:

$$
\dot{V}_{3 p s}(x, 0, z, t)=\frac{d}{d t}\left(\frac{1}{\pi} \sqrt{\frac{1}{2 F}} \frac{d}{d t}\left[\frac{1}{\sqrt{t}} * V_{2 l s}(x, z, t)\right]\right)
$$

where $\dot{V}_{3 p s}$ is the velocity seismogram. If the ray path $F$ is not time dependent, then:

$$
\dot{V}_{3 p s}(x, 0, z, t)=\frac{1}{\pi} \sqrt{\frac{1}{2 F}} \frac{d}{d t}\left[\frac{1}{\sqrt{t}} * \dot{V}_{2 l s}(x, z, t)\right]
$$

This shows that the same correction from a displacement seismogram can be applied to the velocity seismogram without loss of information. The same process can be applied to prove that this correction is valid when applied to an acceleration seismogram.

\subsubsection{Source Rotation}

The difference in the evaluation methods in the simulations arise from the absence of a third dimension in the 2D simulations. In a 2D simulation the limitation is the use of only three of the moment tensor components for as motion associated with the y-direction is not addressed. Even 
though the y-direction is not included, it is still possible to simulate the P-SV seismic waves. The far-field radiation pattern for $\mathrm{P}, \mathrm{SV}$ and $\mathrm{SH}$ waves from a moment tensor source are (Chapman, 2004, p. 123):

$$
\begin{gathered}
P\left(M ; \phi_{1}, \phi_{2}\right)=\left(M_{x x} \cos ^{2} \phi_{1}+M_{y y} \sin ^{2} \phi_{1}+M_{x y} \sin 2 \phi_{1}\right) \sin ^{2} \phi_{2} \\
+M_{z z} \cos ^{2} \phi_{2}+\left(M_{z x} \cos \phi_{1}+M_{y z} \sin \phi_{1}\right) \sin 2 \phi_{2} \\
S V\left(M ; \phi_{1}, \phi_{2}\right)=1 / 2\left(M_{x x} \cos ^{2} \phi_{1}+M_{y y} \sin ^{2} \phi_{1}-M_{z z}\right. \\
\left.+M_{x y} \sin 2 \phi_{1}\right) \sin 2 \phi_{2} \\
+\left(M_{z x} \cos \phi_{1}+M_{y x} \sin \phi_{1}\right) \cos 2 \phi_{2},
\end{gathered}
$$

$\operatorname{SH}\left(M ; \phi_{1}, \phi_{2}\right)$

$$
\begin{aligned}
& =\left[1 / 2\left(M_{y y}-M_{x x}\right) \sin 2 \phi_{1}+M_{x y} \cos 2 \phi_{1}\right] \sin \phi_{2} \\
& +\left(M_{y z} \cos \phi_{1}-M_{z x} \sin \phi_{1}\right) \cos \phi_{2}
\end{aligned}
$$

Here $\phi_{1}$ and $\phi_{2}$ are the azimuth angle and inclination angle in spherical coordinates, respectively. So if the coordinate system is rotated such that the source and receiver both fall along the X-axis, (i.e. $\phi_{1}=0$ ) then the far-field radiation patterns become:

$$
\begin{gathered}
P\left(M ; 0, \phi_{2}\right)=M_{x x} \sin ^{2} \phi_{2}+M_{z z} \cos ^{2} \phi_{2}+M_{z x} \sin 2 \phi_{2}, \\
S V\left(M ; 0, \phi_{2}\right)=1 / 2\left(M_{x x}-M_{z z}\right) \sin 2 \phi_{2}+M_{z x} \cos 2 \phi_{2} \\
S H\left(M ; 0, \phi_{2}\right)=M_{x y} \sin \phi_{2}+M_{y z} \cos \phi_{2} .
\end{gathered}
$$

Using this coordinate system only $M_{x x}, M_{z z}$, and $M_{z x}$ contribute to the far-field P-SV waves and only $M_{x y}$ and $M_{y z}$ contribute to the far field SH wave. In this coordinate system the moment tensor strike is the effective source to receiver strike as discussed in Section 3.1.1. 


\subsection{Homogenous Volume}

The first comparison was completed with two homogenous volumes of the same material for both a 2D and a 3D simulation. Table 4.1 lists the properties used within the volumes. The 2D simulation is completed in the $(X, Z)$ plane with dimensions $60 \mathrm{~km} \times 40 \mathrm{~km}$, shown in Figure 4.1 . The source was placed at $(10 \mathrm{~km},-20 \mathrm{~km})$. The two-dimensional model had a mesh spacing of $200 \mathrm{~m}$. The 3D simulation had dimensions $60 \mathrm{~km} \times 20 \mathrm{~km} \times 40 \mathrm{~km}$, shown in Figure 4.2. The source for this model was placed at $(10 \mathrm{~km}, 10 \mathrm{~km},-20 \mathrm{~km})$, and it also had a mesh spacing of $200 \mathrm{~m}$. Table 4.2 lists the locations of each of the receivers, similar for both models, along with the reference distance from the source to each receiver. The time series data at each receiver was filtered using a 4-pole Butterworth Lowpass filter with a cut-off frequency of $3.2 \mathrm{~Hz}$ to remove high frequencies resulting from the simulation methods.

\begin{tabular}{|c|c|}
\hline Parameter & Value in Model \\
\hline Vp & $6500 \mathrm{~m} / \mathrm{s}$ \\
Vs & $3500 \mathrm{~m} / \mathrm{s}$ \\
Density & $2800 \mathrm{~kg} / \mathrm{m}^{3}$ \\
$Q$ & 1000 \\
\hline
\end{tabular}

Table 4.1: Properties used for homogeneous simulations. These are consistent with the average eastern North America crust properties (Bent et al., 2015). 


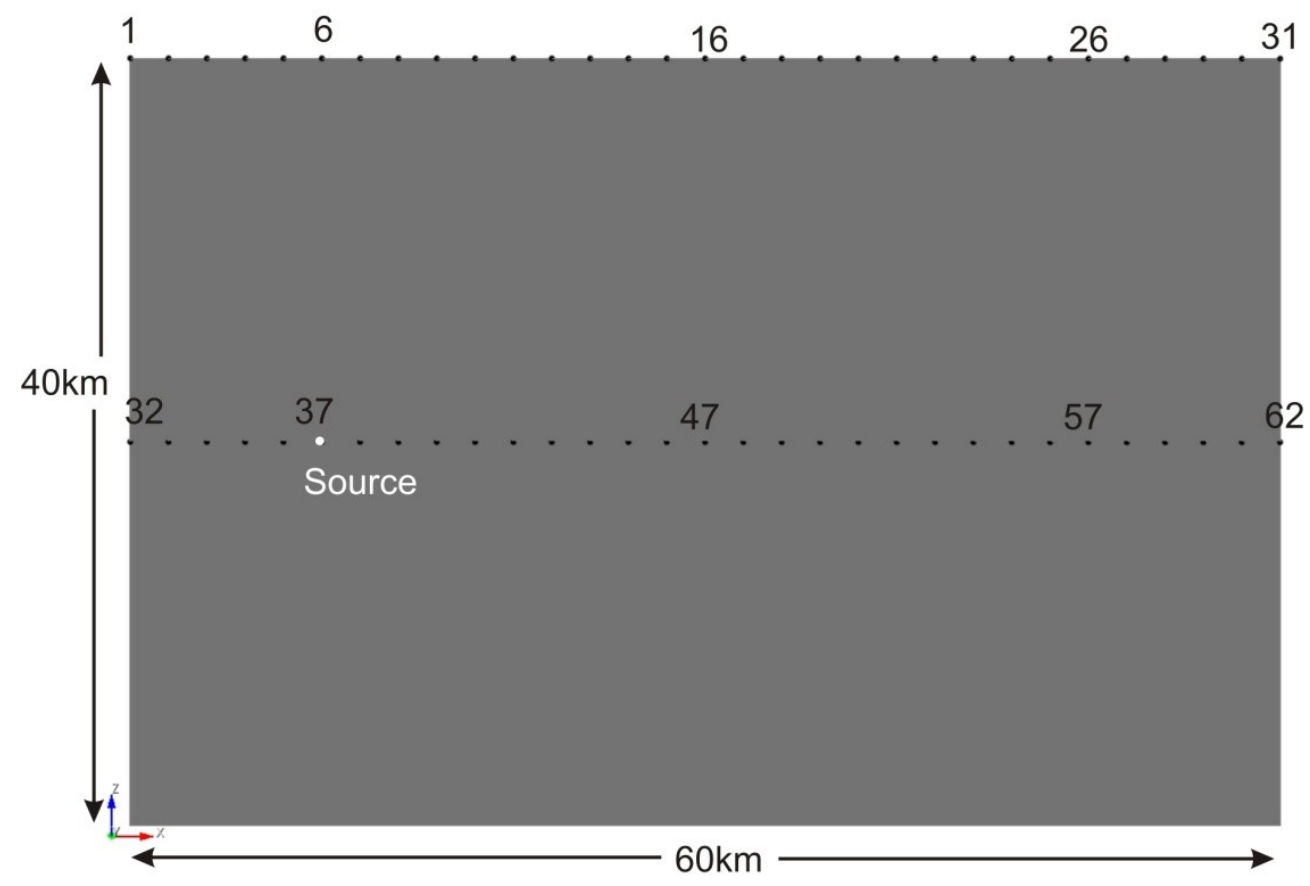

Figure 4.1: The 2D model for the homogeneous simulation showing source and station locations.

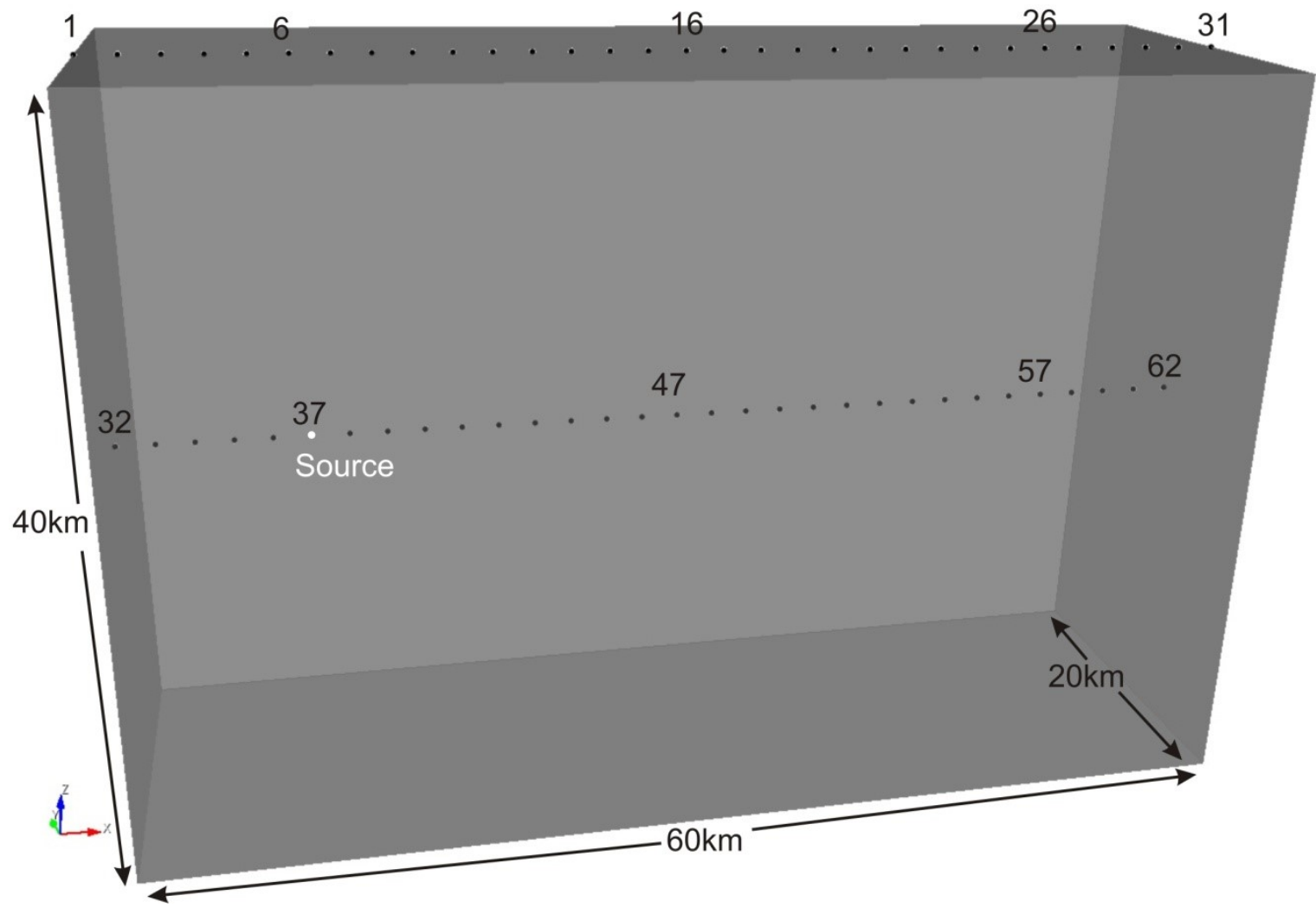

Figure 4.2: The location of source and stations for the 3D model for the homogeneous simulation. 


\begin{tabular}{|c|c|c|c|c|c|c|c|c|}
\hline $\begin{array}{c}\text { Station } \\
\#\end{array}$ & $\begin{array}{c}\mathrm{X}, \mathrm{Z} \\
\text { Position } \\
(\mathrm{km}, \\
\mathrm{km})\end{array}$ & $\begin{array}{c}\text { Distance } \\
(\mathrm{km})^{*}\end{array}$ & $\begin{array}{c}\text { Station } \\
\#\end{array}$ & $\begin{array}{c}\mathrm{X}, \mathrm{Z} \\
\text { Position } \\
(\mathrm{km}, \\
\mathrm{km})\end{array}$ & $\begin{array}{c}\text { Distance } \\
(\mathrm{km})^{*}\end{array}$ & $\begin{array}{c}\text { Station } \\
\#\end{array}$ & $\begin{array}{c}\mathrm{X}, \mathrm{Z} \\
\text { Position } \\
(\mathrm{km}, \\
\mathrm{km})\end{array}$ & $\begin{array}{c}\text { Distance } \\
(\mathrm{km})^{*}\end{array}$ \\
\hline 1 & $(0,0)$ & 22.4 & 22 & $(42,0)$ & 37.7 & 43 & $(22,-20)$ & 12.0 \\
\hline 2 & $(2,0)$ & 21.5 & 23 & $(44,0)$ & 39.4 & 44 & $(24,-20)$ & 14.0 \\
\hline 3 & $(4,0)$ & 20.9 & 24 & $(46,0)$ & 41.2 & 45 & $(26,-20)$ & 16.0 \\
\hline 4 & $(6,0)$ & 20.4 & 25 & $(48,0)$ & 42.9 & 46 & $(28,-20)$ & 18.0 \\
\hline 5 & $(8,0)$ & 20.1 & 26 & $(50,0)$ & 44.7 & 47 & $(30,-20)$ & 20.0 \\
\hline 6 & $(10,0)$ & 20.0 & 27 & $(52,0)$ & 46.5 & 48 & $(32,-20)$ & 22.0 \\
\hline 7 & $(12,0)$ & 20.1 & 28 & $(54,0)$ & 48.3 & 49 & $(34,-20)$ & 24.0 \\
\hline 8 & $(14,0)$ & 20.4 & 29 & $(56,0)$ & 50.2 & 50 & $(36,-20)$ & 26.0 \\
\hline 9 & $(16,0)$ & 20.9 & 30 & $(58,0)$ & 52.0 & 51 & $(38,-20)$ & 28.0 \\
\hline 10 & $(18,0)$ & 21.5 & 31 & $(60,0)$ & 53.9 & 52 & $(40,-20)$ & 30.0 \\
\hline 11 & $(20,0)$ & 22.4 & 32 & $(0,-20)$ & 10.0 & 53 & $(42,-20)$ & 32.0 \\
\hline 12 & $(22,0)$ & 23.3 & 33 & $(2,-20)$ & 8.0 & 54 & $(44,-20)$ & 34.0 \\
\hline 13 & $(24,0)$ & 24.4 & 34 & $(4,-20)$ & 6.0 & 55 & $(46,-20)$ & 36.0 \\
\hline 14 & $(26,0)$ & 25.6 & 35 & $(6,-20)$ & 4.0 & 56 & $(48,-20)$ & 38.0 \\
\hline 15 & $(28,0)$ & 26.9 & 36 & $(8,-20)$ & 2.0 & 57 & $(50,-20)$ & 40.0 \\
\hline 16 & $(30,0)$ & 28.3 & 37 & $(10,-20)$ & 0.0 & 58 & $(52,-20)$ & 42.0 \\
\hline 17 & $(32,0)$ & 29.7 & 38 & $(12,-20)$ & 2.0 & 59 & $(54,-20)$ & 44.0 \\
\hline 18 & $(34,0)$ & 31.2 & 39 & $(14,-20)$ & 4.0 & 60 & $(56,-20)$ & 46.0 \\
\hline 19 & $(36,0)$ & 32.8 & 40 & $(16,-20)$ & 6.0 & 61 & $(58,-20)$ & 48.0 \\
\hline 20 & $(38,0)$ & 34.4 & 41 & $(18,-20)$ & 8.0 & 62 & $(60,-20)$ & 50. \\
\hline 21 & $(40,0)$ & 36.1 & 42 & $(20,-20)$ & 10.0 & & & \\
\hline
\end{tabular}

Table 4.2: Station in the 2D and 3D simulations. For the 3D simulations all stations have the $\mathrm{Y}$ position of $10 \mathrm{~km}$. ${ }^{*}$ The distance column is the distance from the source.

Two Gaussian shaped time function sources were used for each model: a unit force applied in the positive $\mathrm{X}$ direction; and a unit force applied in the positive Z-direction. These sources were chosen to clearly demonstrate the propagation of P-wave and SV-wave phases throughout the models in each direction. Figure 4.3 and Figure 4.4 shows the acceleration and displacement recordings of the receiver located at the source in the model for each the unit Xdirection source and the unit Z-direction source, respectively. A strong effort was made to keep these source pulses to be similar in shape, but there are some differences; mainly the width of 
the wave, but also in the $2 \mathrm{D}$ model there was an extra peak, due to its implementation as an effective line source. These waveforms are easily traced through the models and reflections can be traced through the other receivers. The difference between the two sources are primarily the component of motion as shown by the differing displacements between Figure 4.3 and Figure 4.4. In all these cases all motion is in the horizontal component for the Unit-X source and in the vertical component for the Unit-Z source. As seen in Figure 4.3 the 2D simulation has a negative displacement at the source whereas the 3D simulation is positive. In Figure 4.4 the displacements for both the 2D and 3D models are positive which is due to the difference in the determination of the coordinate systems for the source between SPECFEM2D and SPECFEM3D. The source for the Z-component is not rotated from one program to the other, however with SPECFEM3D the horizontal components were rotated to align the positive X-direction with East and the positive Y-direction with North, resulting in a rotation of the source.
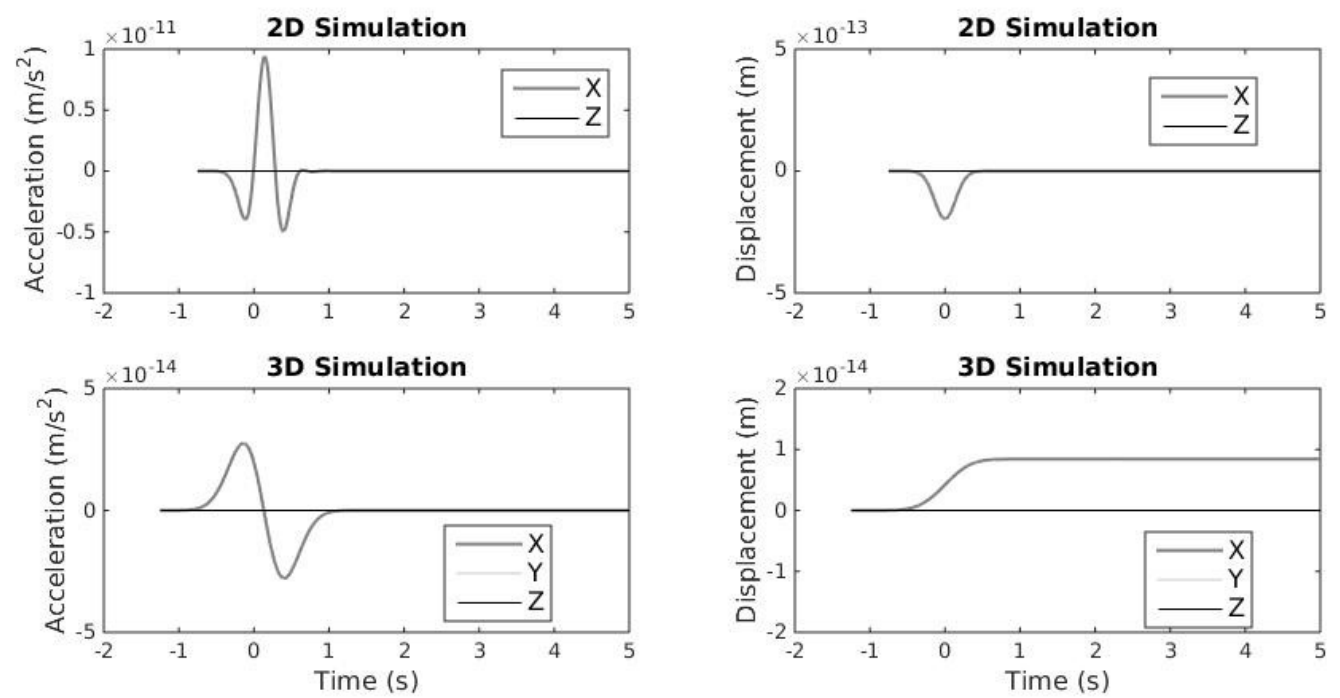

Figure 4.3: The first 5 seconds of the acceleration (left) and displacement (right) time series at the source for the 2D simulation (top) and 3D simulation (bottom) for the unit force in the X-direction source 

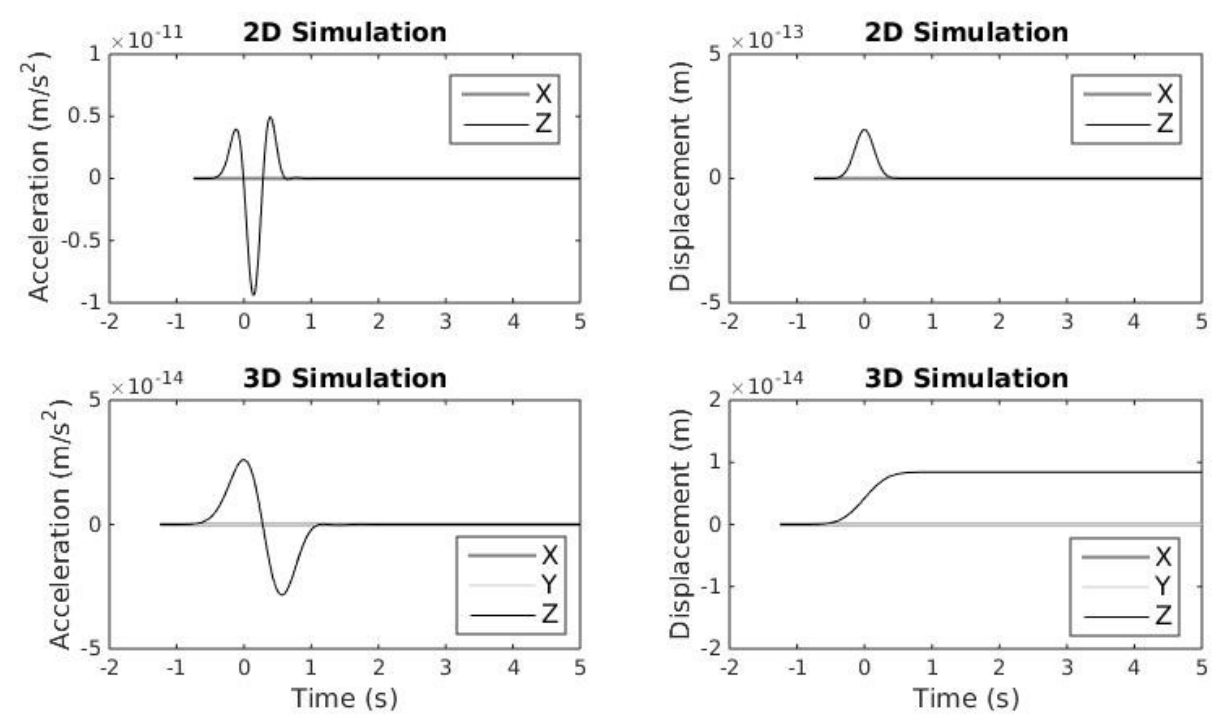

Figure 4.4: The first 5 seconds of the acceleration (left) and displacement (right) time series at the source for the 2D simulation (top) and 3D simulation (bottom) for the unit force in the Z-direction source

\subsubsection{Computing Time and Resources}

The computing resources needed for the 2D and 3D simulations varied greatly. Both models were run for 100,000 timesteps at 0.001 seconds per timestep. The 2D simulations were run on 8 cores of an Intel Core i7-4790k CPU for 3 hours and 4 minutes, which is equal to about 24.5 core*hours. The 3D models were run on 720 cores on AMD Opteron 6172 of the Mammouth parallèle II (Mp2) cluster at Université de Sherbooke for 29 hours and 33 minutes, equivalent to roughly 21,240 core*hours. The 3D simulation required $132 \mathrm{MB}$ per process of RAM memory, for a total of about 92GB, and the resulting simulated files were $1.8 \mathrm{~GB}$ in size. The $2 \mathrm{D}$ simulation used less than $16 \mathrm{~GB}$ total RAM memory and the resulting files were $476 \mathrm{MB}$ in size. This demonstrated that completing 2D simulations are much more preferential due to the significantly less amount of computing resources needed, even though 3D simulations included all spatial components.

Another consideration for 2D simulations was the model preparation for more complex systems. The processing time it takes to create a 2D meshed model compared to a 3D meshed 
model can be significant. For models such as the homogenous volumes as described above, the time difference was negligible. On the other hand, for a volume such as that containing the Kinburn basin, the creation of a 3D model with a suitable mesh could take a considerable amount of time, whereas creating a 2D profile with a suitable mesh could take a significantly less amount of time. In this study, after becoming familiar with the modelling and meshing programs, creating a 2D profile with a suitable mesh would take roughly a week, and creating a 3D model with a suitable mesh would take approximately a month of computing time.

\subsubsection{Ray Paths}

A principal component of the analysis in this study was the ability to trace wave phases throughout the model. This was essential for the corrections which are applied to the simulated two dimensional seismograms to convert them to the equivalent seismogram from a three dimensional simulation, as discussed in Chapter 4.2.5. The ray tracing method was used for determining the arrivals at any receiver. The ray paths were denoted using a slightly different labelling system than in common use for wave traces. P denotes a P-wave, S denotes an S-wave, $\mathrm{n}$ is a reflection from the near side $(X=0 \mathrm{~km}), \mathrm{p}$ denotes a $\mathrm{P}$-wave reflected from the free surface $(Z=0 \mathrm{~km})$, and an $\mathrm{s}$ denotes an $\mathrm{S}$-wave reflected off the free surface. An example of ray tracing is shown in Figure 4.5 for a direct P-wave (P), both a P- and S-wave reflected off the free surface resulting in a P-wave ( $\mathrm{pP}$ and $\mathrm{SP}$, respectively), and lastly an S-wave reflected into a $\mathrm{P}$-wave off the near side boundary ( $\mathrm{SnP})$. The wave arrival times based on the ray tracing method at selected receivers are listed in Table 4.3. 


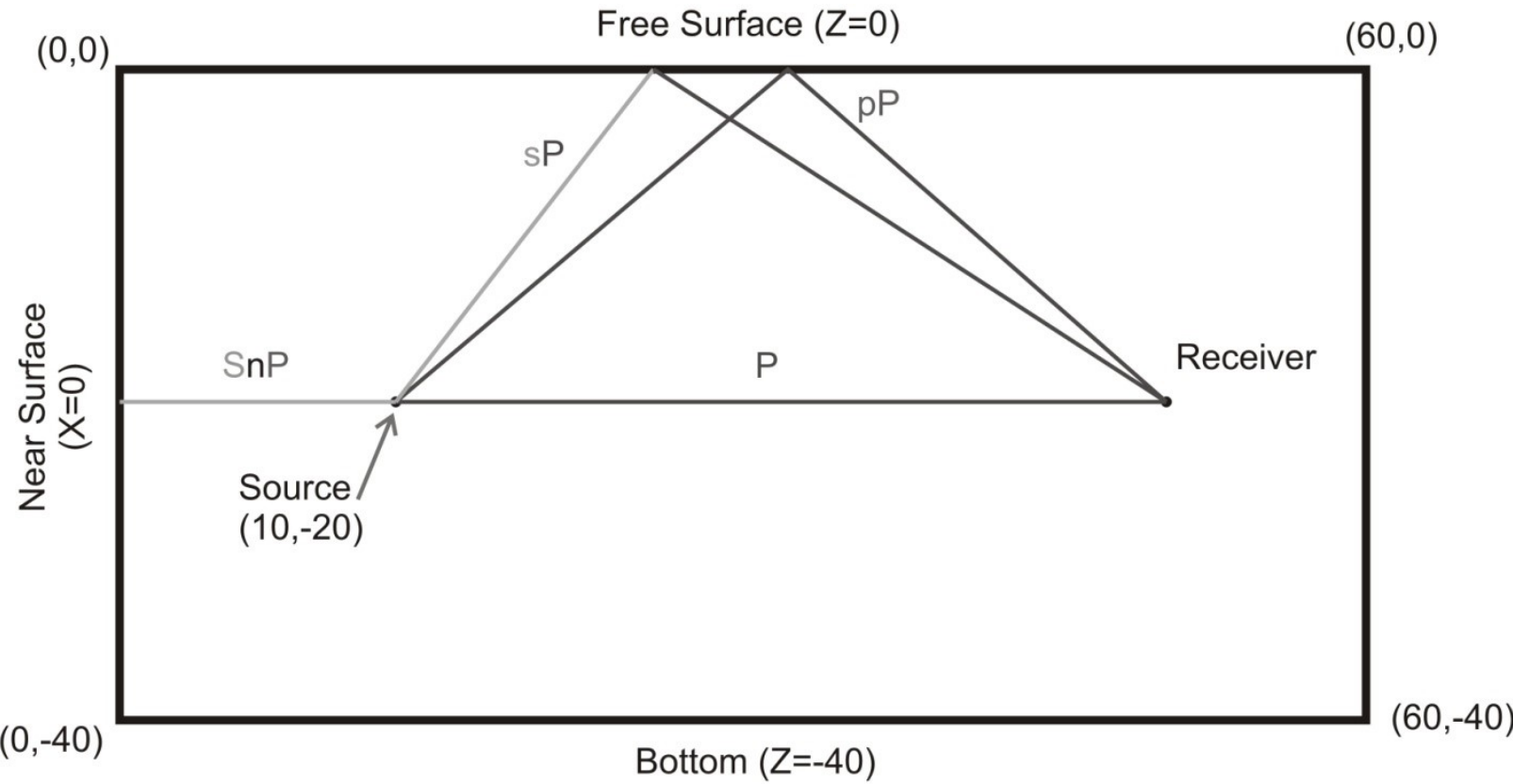

Figure 4.5: An example of ray tracing through a two dimensional model. In this example a direct P-wave (P), both a P-and S-wave reflected off the free surface resulting in a $\mathrm{P}$-wave ( $\mathrm{pP}$ and sP respectively), and lastly an S-wave reflected into a $\mathrm{P}$-wave off the near side boundary (SnP) are shown.

\begin{tabular}{|c|c|r|r|r|r|r|r|l|l|c|c|}
\hline \multirow{2}{*}{$\begin{array}{c}\text { Station } \\
\#\end{array}$} & \multirow{2}{*}{$\begin{array}{c}\text { Position } \\
(\mathrm{km}, \mathrm{km})\end{array}$} & $\begin{array}{c}\text { P- } \\
\text { wave }\end{array}$ & $\begin{array}{c}\text { S- } \\
\text { wave }\end{array}$ & PnP & SnP & PnS & SnS & pP & pS & sP & sS \\
\hline 6 & $(10,0)$ & 2.58 & 5.21 & 3.85 & 5.72 & 5.72 & 7.58 & - & - & - & - \\
\hline 16 & $(30,0)$ & 3.85 & 7.58 & 6.54 & 8.41 & 10.71 & 12.58 & - & - & - & - \\
\hline 26 & $(50,0)$ & 6.38 & 12.28 & 9.52 & 11.39 & 16.24 & 18.11 & - & - & - & - \\
\hline 47 & $(30,-20)$ & 2.58 & 5.21 & 5.65 & 6.97 & 9.61 & 10.93 & 6.93 & 8.29 & 9.57 & 13.30 \\
\hline 57 & $(50,-20)$ & 5.65 & 10.93 & 8.73 & 10.05 & 15.32 & 16.64 & 9.46 & 17.08 & 12.09 & 17.99 \\
\hline
\end{tabular}

Table 4.3: Theoretical travel time arrivals of different seismic phases based on ray paths for selected receiver 6, 16, 26, 47 and 57. $P$ denotes a $P$-wave, $S$ denotes an $S$-wave, $n$ is a reflection from the near side $(X=0 \mathrm{~km}), p$ denotes a $P$-wave reflected from the free surface $(Z=0 \mathrm{~km})$, and an $\mathrm{s}$ denotes an $\mathrm{S}$-wave reflected off the free surface.

\subsubsection{Unit X-direction Force Source}

Figure 4.6 shows the first 20 seconds of the acceleration time series for station numbers 6 (directly above the source), 16, 26, 37 (at the source), 47 and 57 for the $2 \mathrm{D}$ simulation, and Figure 4.7 shows the same ( $\mathrm{X}$ - and Z-components only) for the 3D simulation. The relative 
waveforms appear in both the $2 \mathrm{D}$ and $3 \mathrm{D}$ simulations with similar arrival times and characteristics based on their respective source waveforms. The theoretical arrival times of the waves, listed in Table 4.3, are displayed on Figure 4.6 and Figure 4.7 by the vertical lines. Although difficult to see due to the overlap of waves, the 3D simulation does show a few extra arrivals which are due to reflections off the $\mathrm{Y}$-boundaries. These are indicated through the absence of a waveform arrival in Figure 4.6 and the presence of a waveform arrival in Figure 4.7. One example of this is found directly after the $\mathrm{P}$ wave phase at station number 26 , appearing at time roughly equal to 7.5 seconds. The $2 \mathrm{D}$ simulation does not show a wave arriving at this time, however the $3 \mathrm{D}$ simulation does have an arrival not associated with any of the other rays. The stronger reflections from the free surface appear to be caused by reflections of the S-wave at the free surface. This effect is seen by the larger amplitude waves which occur at the same time of the sP and sS waves in station numbers 47 and 57. 

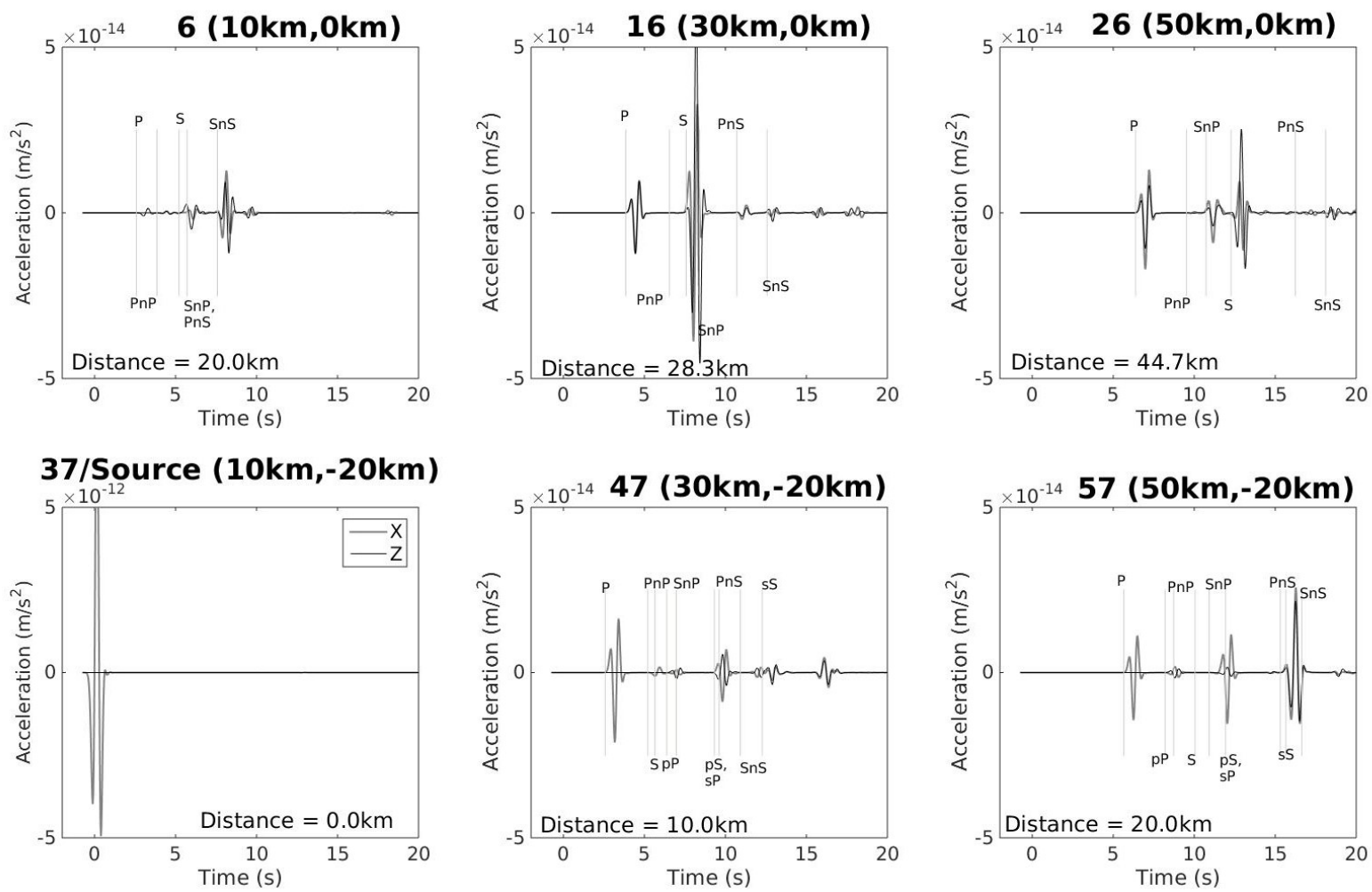

Figure 4.6: Acceleration time series at station numbers $6,16,26,37,47$, and 57 for the 2-D simulation with a unit force in the Xdirection. Distance is the distance from the source. The vertical lines are the theoretical wave arrivals for the different phases listed in Table 4.1. $\mathrm{P}$ denotes a $\mathrm{P}$-wave, $\mathrm{S}$ denotes an $\mathrm{S}$-wave, $\mathrm{n}$ is a reflection from the near side $(\mathrm{X}=0 \mathrm{~km}), \mathrm{p}$ denotes a $\mathrm{P}$-wave reflected from the free surface $(Z=0 \mathrm{~km})$, and an $\mathrm{s}$ denotes an $\mathrm{S}$-wave reflected off the free surface. The labels are alternately placed between above and below the lines and they are listed in order of the next arrival. 

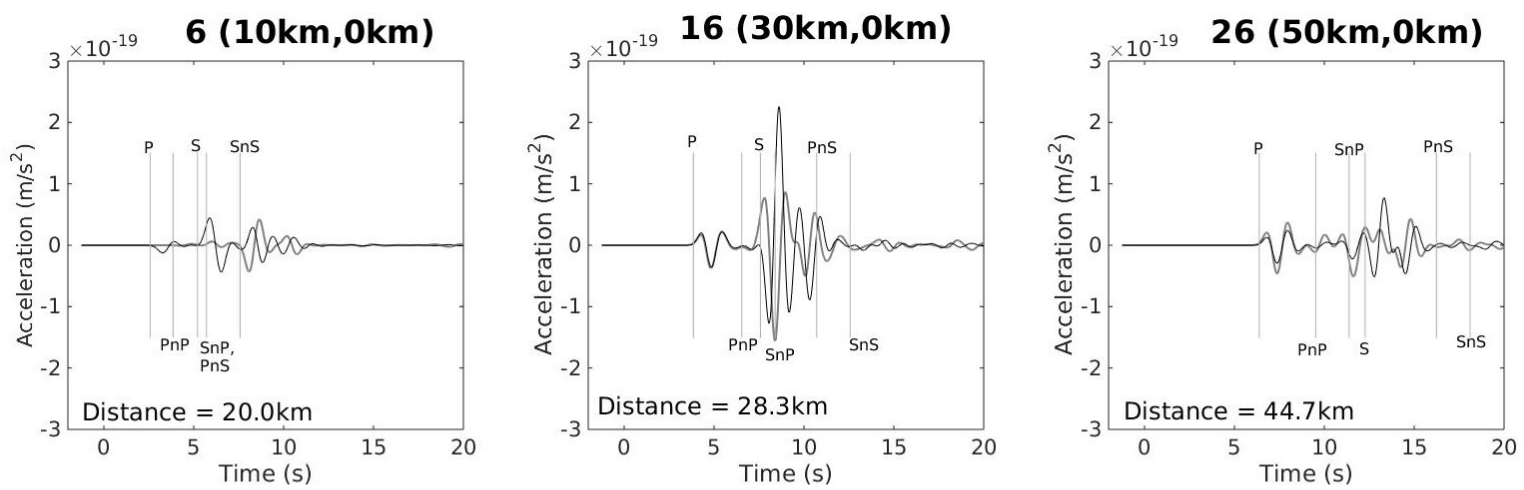

37/Source (10km,-20km)
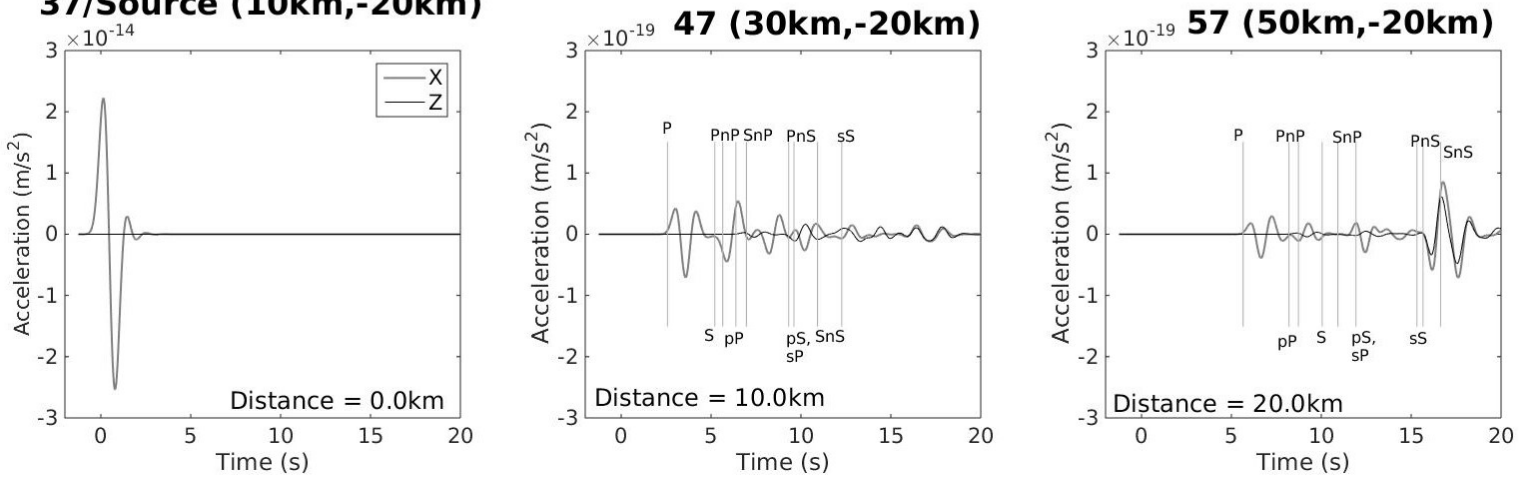

Figure 4.7: Acceleration time series for station numbers $6,16,26,37,47$, and 57 the 3-D simulation with a unit force in the $X-$ direction. Distance is the distance from the source. The vertical lines are the theoretical wave arrivals for the different phases listed in Table 4.3. $P$ denotes a $P$-wave, $S$ denotes an $S$-wave, $n$ is a reflection from the near side $(X=0 k m), p$ denotes a $P$-wave reflected from the free surface $(\mathrm{Z}=0 \mathrm{~km})$, and an $\mathrm{s}$ denotes an $\mathrm{S}$-wave reflected off the free surface. The labels are alternately placed above and below the lines and they are listed in order of the next arrival.

Figure 4.8 shows the X-component of motion and Figure 4.9 shows the Z-component of

motion for the first 25 seconds of the simulation for the receivers on the surface of the model, for station numbers 1 to 31, in both the 2D and 3D simulations. The red dashed lines are the theoretical arrival times for the P-wave and S-wave in the model. Both simulations show similar travel times and relative amplitudes for these waves. They also show indications of other travelling waves shown by the blue and green dashed lines. These waves are reflections from boundaries and are labelled based on the theoretical travel times of the waves from the associated ray paths. Figure 4.10 and Figure 4.11 show the X-component and Z-component of motion respectively, for the receivers in line at $20 \mathrm{~km}$ depth. The wave fronts traced through 
these figures in the same manner as before are based on the theoretical travel times and displayed using dashed lines. For the direct P-wave and S-wave only one direction of motion is shown for each; the motion is in the X-direction for the P-wave and Z-direction for the S-wave. 

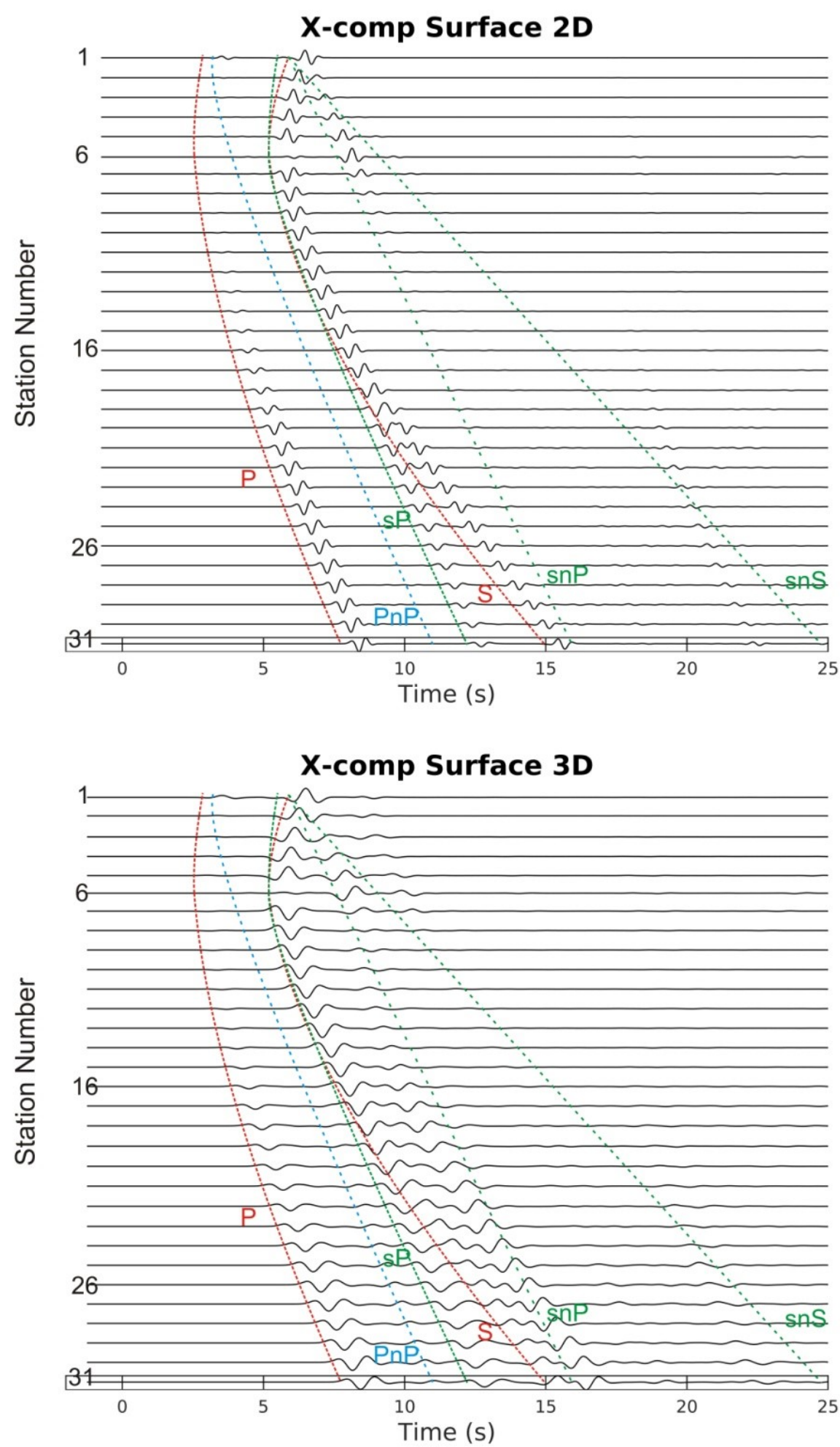

Figure 4.8: X-component of motion for the receivers at the surface for the 2D simulation (top) and 3D simulation (bottom) for the simulations using the unit- $X$ direction force source. $P$ denotes a $P$-wave, $S$ denotes an $S$-wave, $n$ is a reflection from the near side $(X=0 \mathrm{~km}), \mathrm{p}$ denotes a $\mathrm{P}$-wave reflected from the free surface $(\mathrm{Z}=0 \mathrm{~km})$, and an s denotes an $\mathrm{S}$-wave reflected off the free surface. For example: $\mathrm{SnP}$ is an S-wave that is reflected into a P-wave off the near side boundary. 

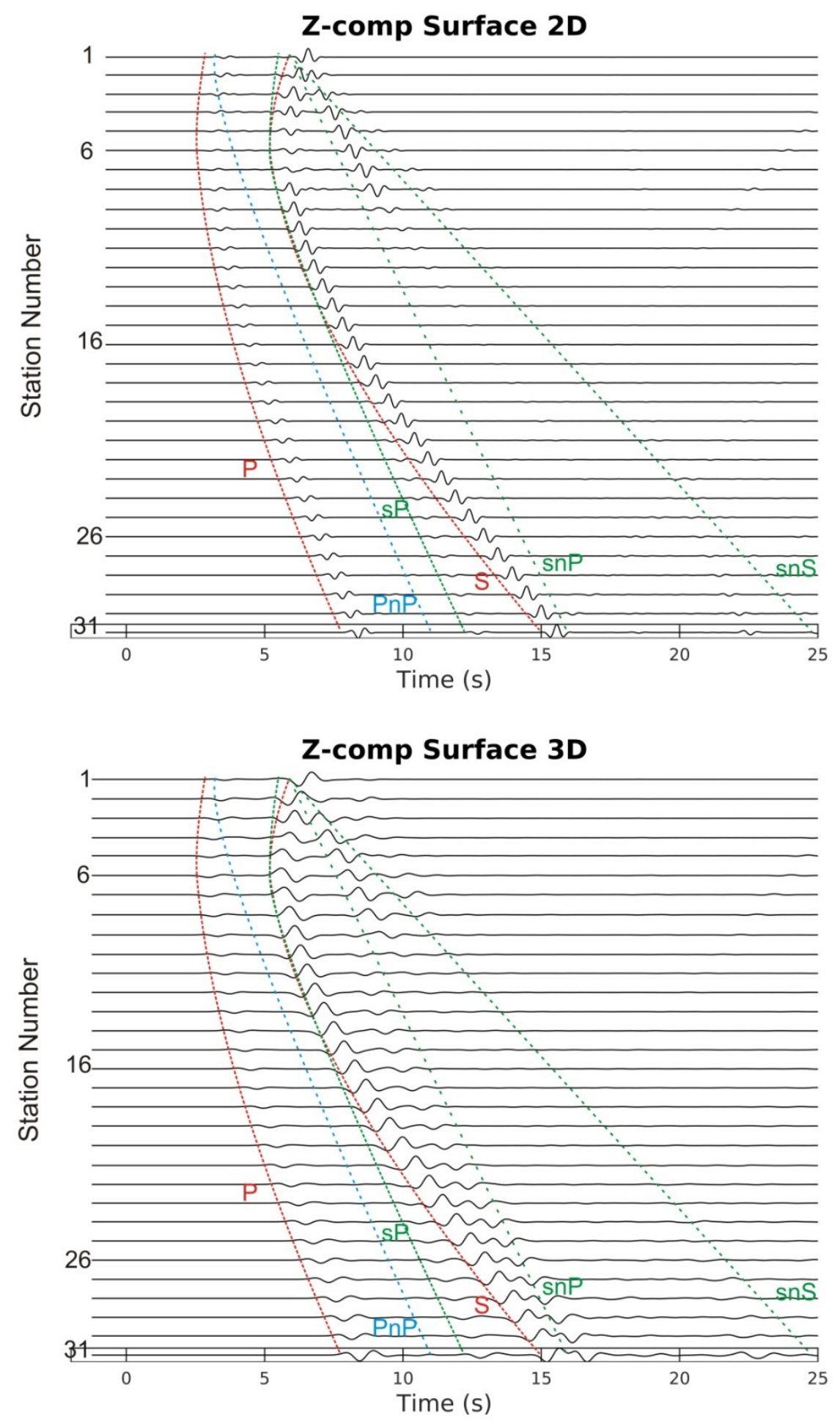

Figure 4.9: Z-component of motion for the receivers at the surface for the 2D simulation (top) and 3D simulation (bottom) for the simulations using the unit- $X$ direction force source. $P$ denotes a $P$-wave, $S$ denotes an $S$-wave, $n$ is a reflection from the near side $(X=0 \mathrm{~km}), \mathrm{p}$ denotes a $\mathrm{P}$-wave reflected from the free surface $(\mathrm{Z}=0 \mathrm{~km})$, and an s denotes an S-wave reflected off the free surface. For example: $\mathrm{SnP}$ is an S-wave that is reflected into a $\mathrm{P}$-wave off the near side boundary. 

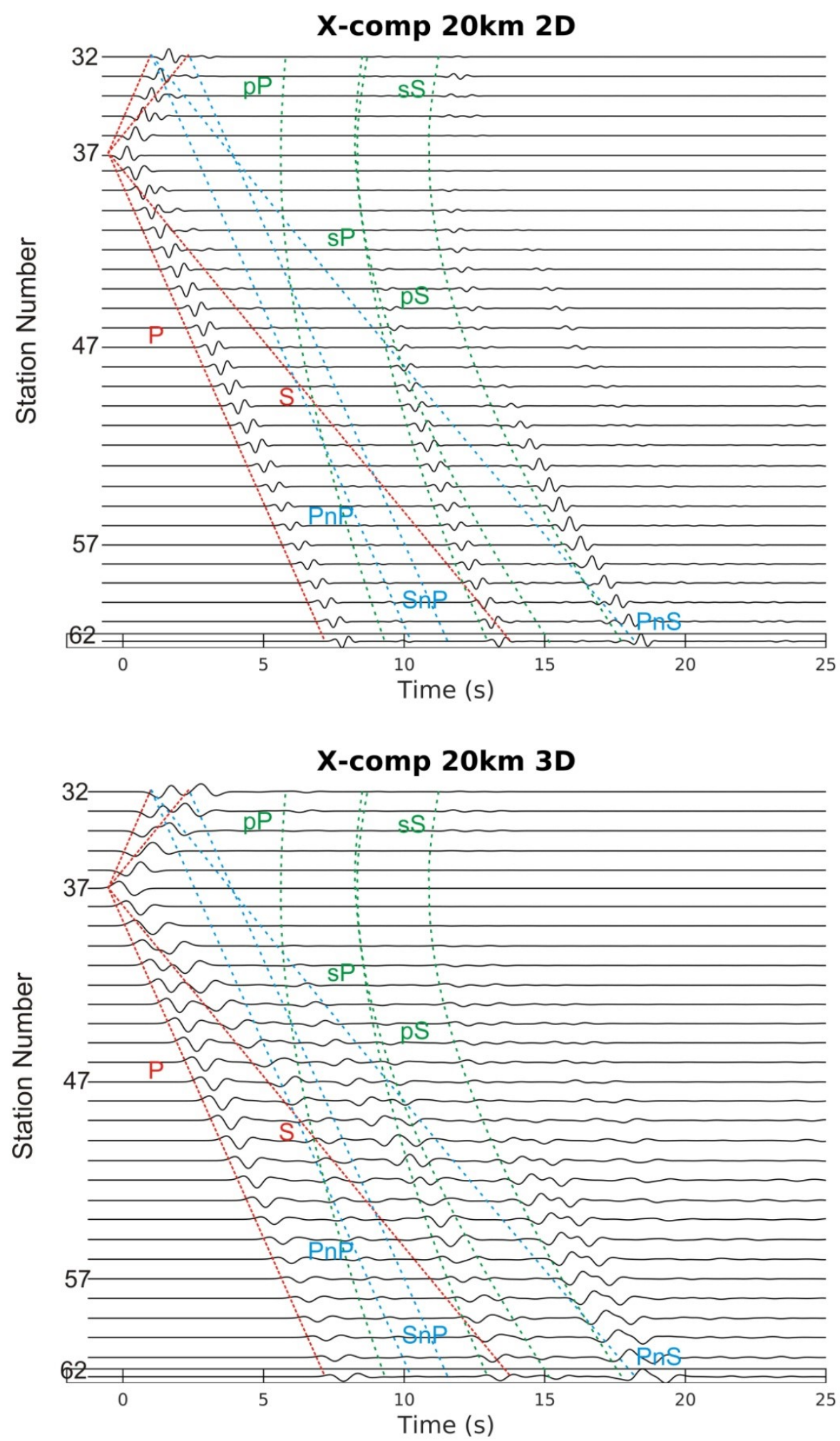

Figure 4.10: X-component of motion for the receivers at $20 \mathrm{~km}$ depth for the 2D simulation (top) and 3D simulation (bottom) for the simulations using the unit- $X$ direction force source. $P$ denotes a $P$-wave, $S$ denotes an $S$-wave, $n$ is a reflection from the near side $(X=0 \mathrm{~km}), \mathrm{p}$ denotes a $\mathrm{P}$-wave reflected from the free surface $(Z=0 \mathrm{~km})$, and an $\mathrm{s}$ denotes an $\mathrm{S}$-wave reflected off the free surface. For example: SnP is an S-wave that is reflected into a P-wave off the near side boundary. 

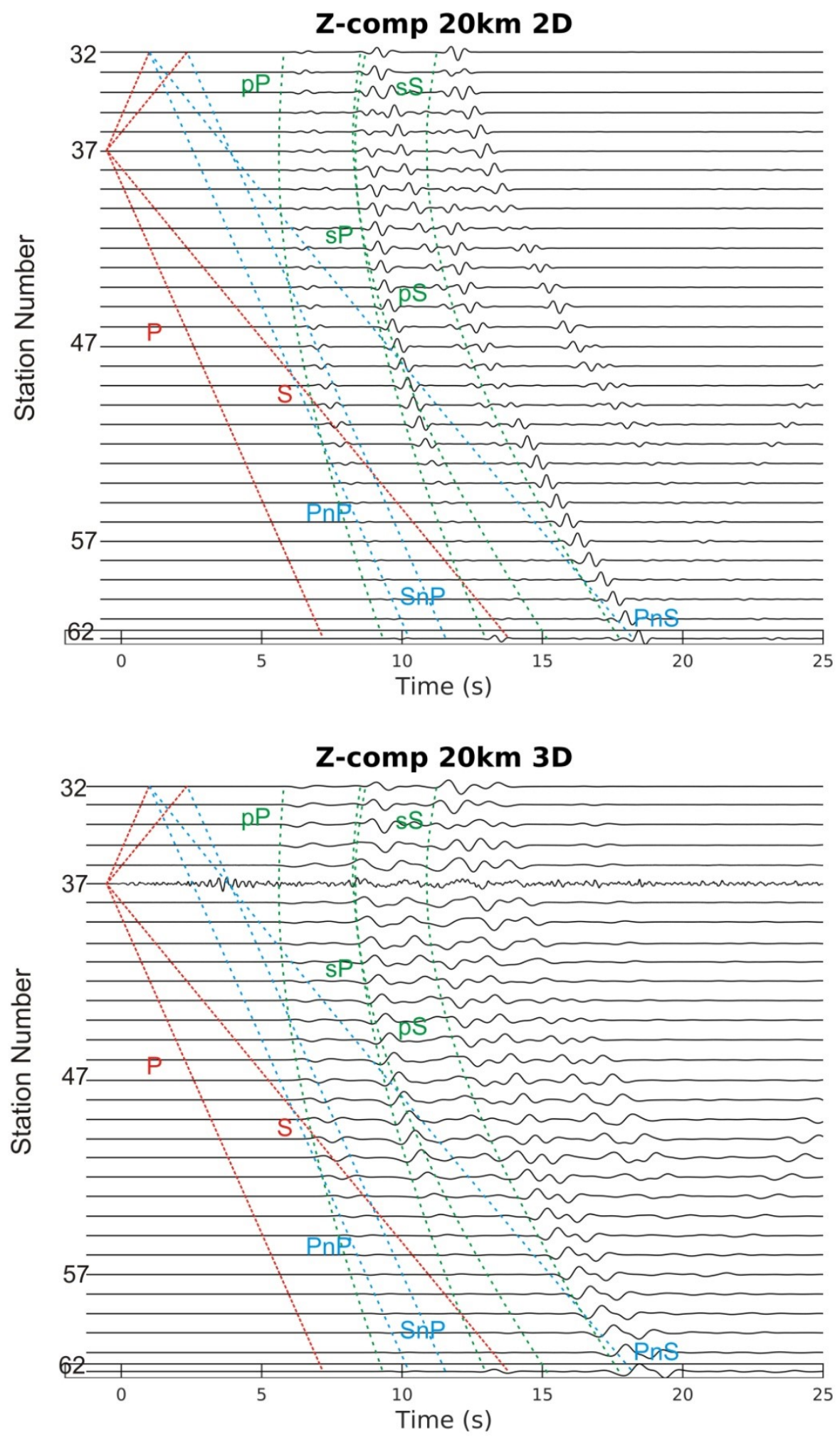

Figure 4.11: Z-component of motion for the receivers at $20 \mathrm{~km}$ depth for the 2D simulation (top) and 3D simulation (bottom) for the simulations using the unit- $X$ direction force source. $P$ denotes a $P$-wave, $S$ denotes an $S$-wave, $n$ is a reflection from the near side $(X=0 \mathrm{~km}), \mathrm{p}$ denotes a $\mathrm{P}$-wave reflected from the free surface $(Z=0 \mathrm{~km})$, and an $\mathrm{s}$ denotes an $\mathrm{S}$-wave reflected off the free surface. For example: SnP is an S-wave that is reflected into a P-wave off the near side boundary. 
Figure 4.12 shows the particle motion plots for the horizontal (X-component) and vertical (Z-component) motion at the different arrival times outlined in the time series for station number $16(X=30 \mathrm{~km}, \mathrm{Z}=0 \mathrm{~km})$ from the $2 \mathrm{D}$ simulation. The amplitudes of each plot are normalized to the corresponding time window. Figure 4.13 shows the particle motion for the 3D simulation with time windows outlined in the displacement time series. The P-wave arrival shows the particle motion primarily at $45^{\circ}$, as expected from its position relative to the source. From this plot it appears that the arrivals at time 7.5 s are surface waves, based on the retrograde elliptical motion occurring at this time. However, based on the theoretical arrival times, these could be a combination of a reflected $\mathrm{P}$-wave, $\mathrm{SnP}$, and the direct $\mathrm{S}$-wave, $\mathrm{S}$. The motions for these waves are better shown in Figure 4.14 and Figure 4.15 which are the particle motion plots for station number $26(X=50 \mathrm{~km}, Z=0 \mathrm{~km})$ for the $2 \mathrm{D}$ simulation and 3D simulation respectively. The arrivals of these waves are separated by a longer time. The same effect is seen in Figure 4.6 and Figure 4.7; the arrival times for these waves, $\mathrm{S}$ and $\mathrm{SnP}$, have been traced to occur at approximately the same time for station number 16 , but are slightly further apart at station number 26 . The other waves shown in Figure 4.12, Figure 4.13, Figure 4.14, and Figure 4.15 are reflected P- and S-waves from the boundaries, with the time windows are based on the theoretical arrival times, listed in Table 4.3 for these stations. The 3D simulation also shows an extra reflection seen best between roughly 9 to 11 seconds for station number 16 , which is an S-wave reflected from the Yboundaries, SyS. Although the boundary conditions at the model edges, not including the free surface, were designed to reduce the amplitude level of reflections as much as possible, there are still some reflections in the simulations due to the difficultly in implementing boundary conditions for numerical simulations as discussed in Appendix I. As seen by the particle motion 
and displacement time series plots, these reflections have a much lower amplitude than the direct arrivals, or any reflection from the free surface. This is important when correcting the 2D seismograms as discussed in section 4.2.5. 


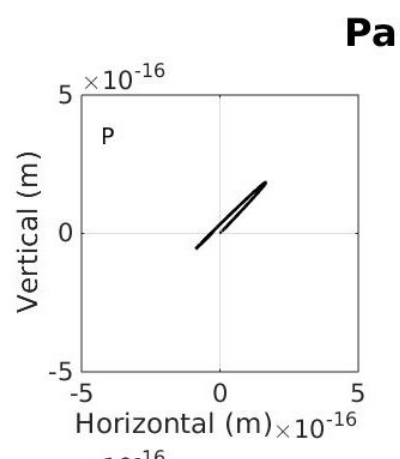

Particle Motion for Station Number $16(30 \mathrm{~km}, 0 \mathrm{~km})$
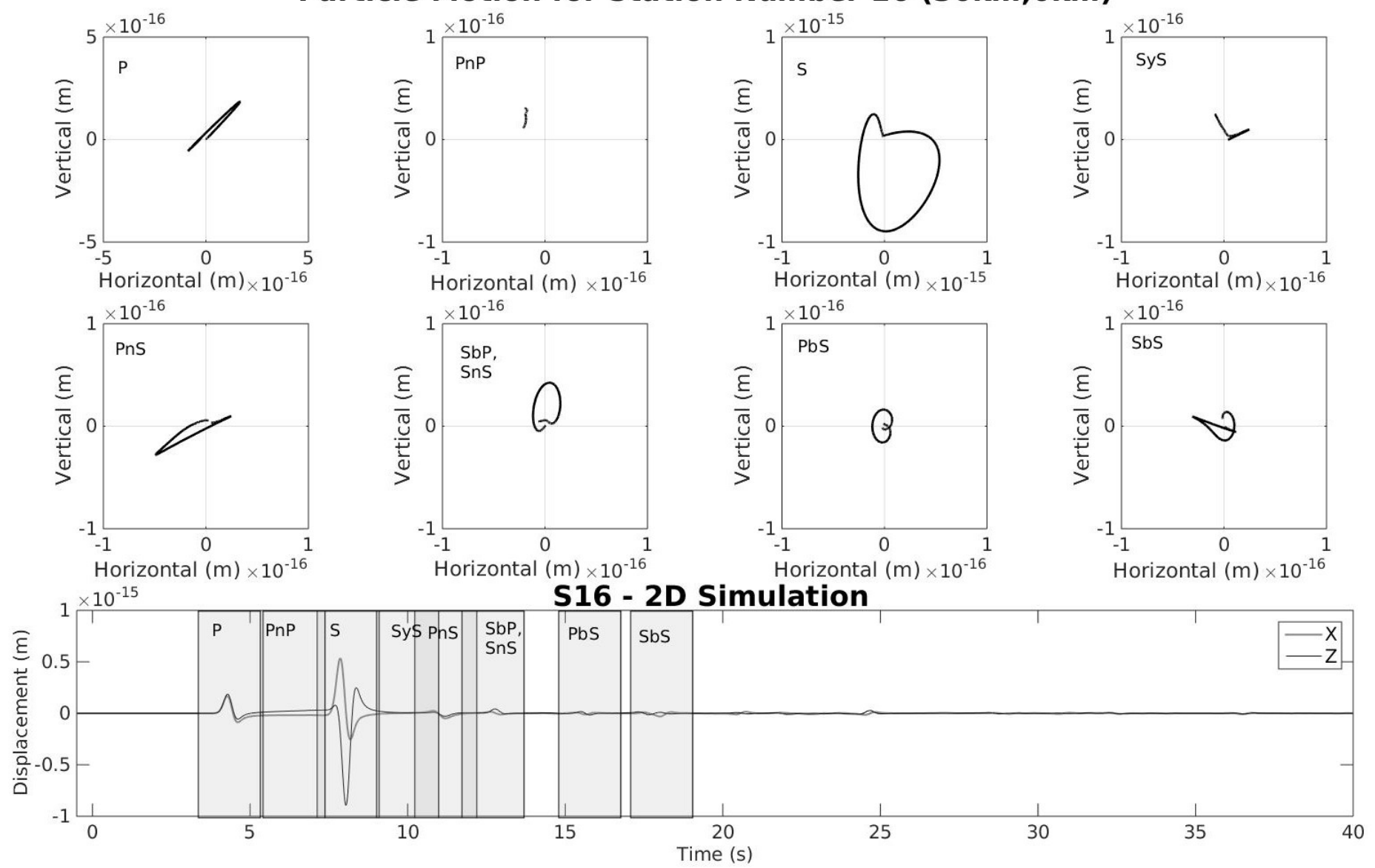

Figure 4.12: Particle motion plot of station number 16 for the 2D simulation. The displacement time series (bottom) outlines the different time slices used. $\mathrm{P}$ denotes a P-wave, $\mathrm{S}$ denotes an S-wave, $n$ is a reflection from the near side $(X=0 \mathrm{~km}), y$ is a reflection from the $Y$-boundary (not present in 2D simulations), $p$ denotes a $P$-wave reflected from the free surface $(Z=0 \mathrm{~km})$, and an $\mathrm{s}$ denotes an $\mathrm{S}$-wave reflected off the free surface. 
Particle Motion for Station Number 16 (30km,0km)
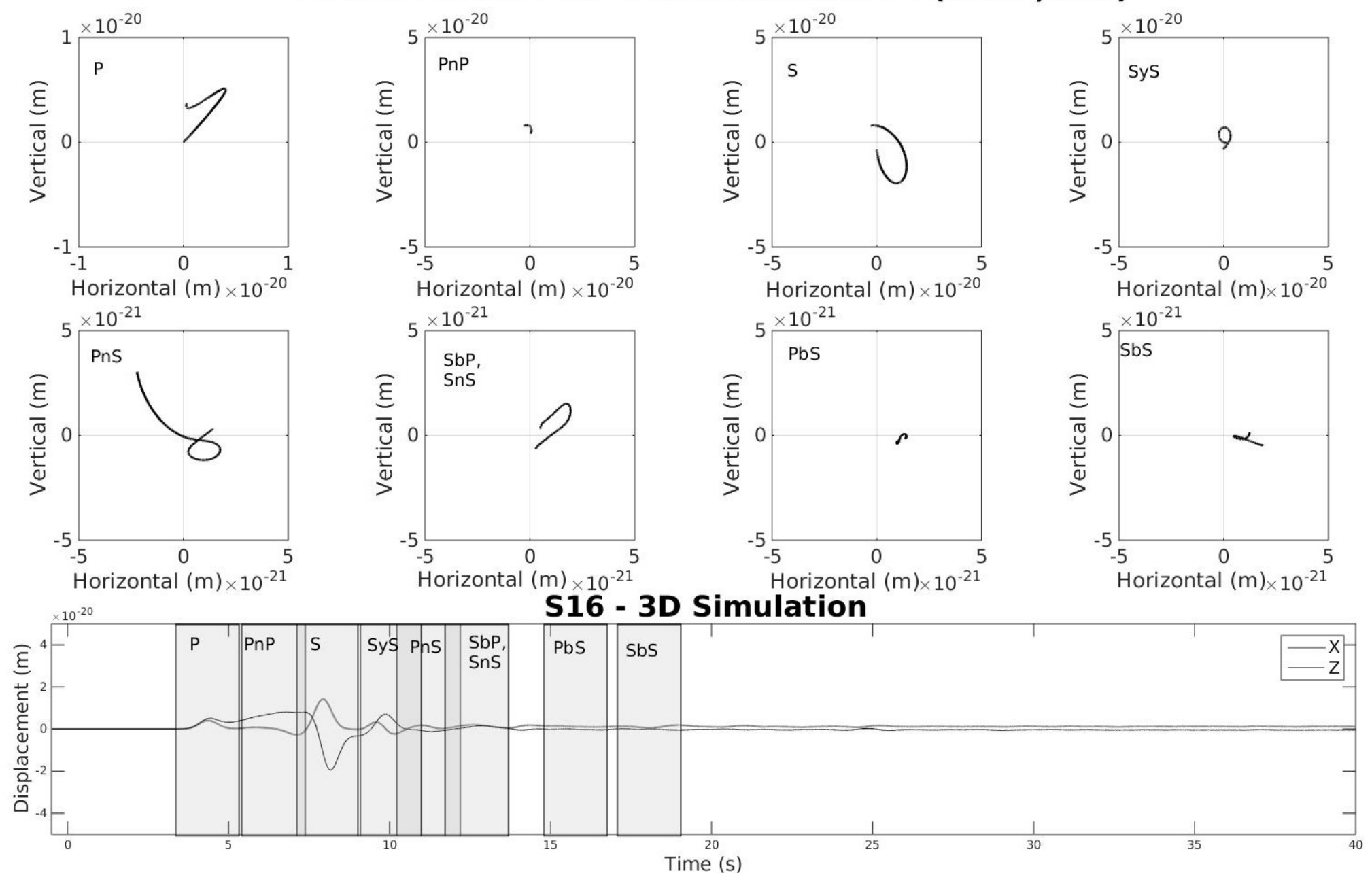

Figure 4.13: Particle motion plot of station number 16 for the 3D simulation. The time series (bottom) outlines the different time slices used. $\mathrm{P}$ denotes a P-wave, $\mathrm{S}$ denotes an Swave, $\mathrm{n}$ is a reflection from the near side $(\mathrm{X}=0 \mathrm{~km})$, $\mathrm{y}$ denotes a reflection from the $\mathrm{Y}$-boundary, $\mathrm{p}$ denotes a $\mathrm{P}$-wave reflected from the free surface $(\mathrm{Z}=0 \mathrm{~km})$, and an $\mathrm{s}$ denotes an S-wave reflected off the free surface. 


\section{Particle Motion for Station Number $26(50 \mathrm{~km}, 0 \mathrm{~km})$}
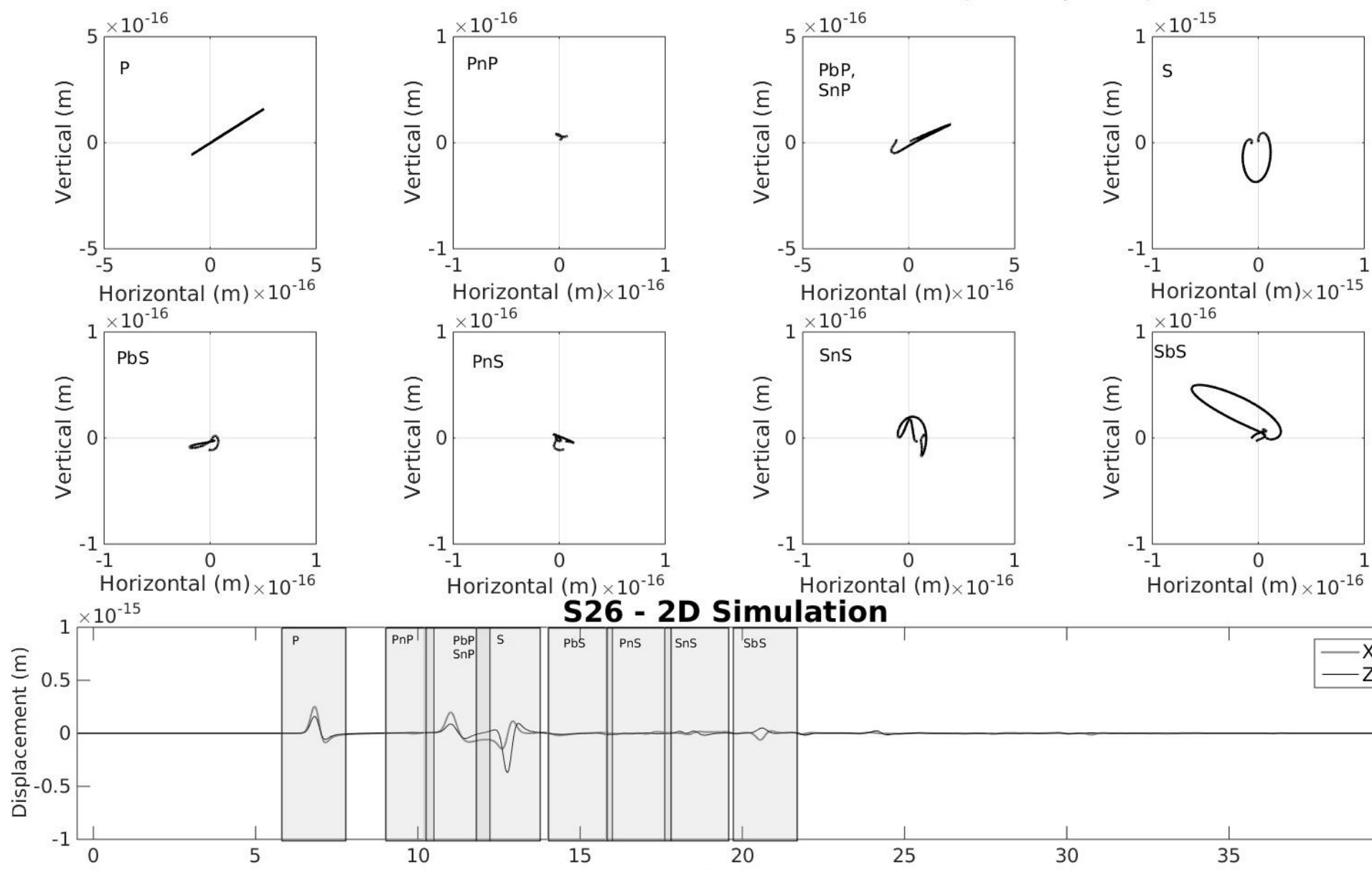

Horizontal $(\mathrm{m}) \times 10^{-16}$ Horizont
S26 - 2D Simulation

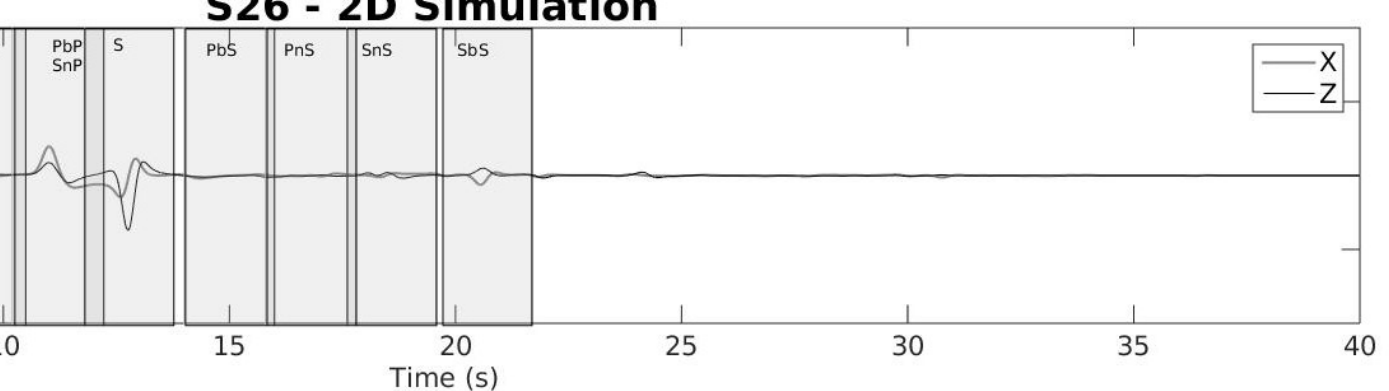

Figure 4.14: Particle motion plot of station number 26 for the 2D simulation. The time series (bottom) outlines the different time slices used. P denotes a P-wave, $\mathrm{S}$ denotes an Swave, $n$ is a reflection from the near side $(X=0 \mathrm{~km}), \mathrm{p}$ denotes a $\mathrm{P}$-wave reflected from the free surface $(\mathrm{Z}=0 \mathrm{~km})$, and an $\mathrm{s}$ denotes an $\mathrm{S}$-wave reflected off the free surface. 


\section{Particle Motion for Station Number 26 (50km,0km)}
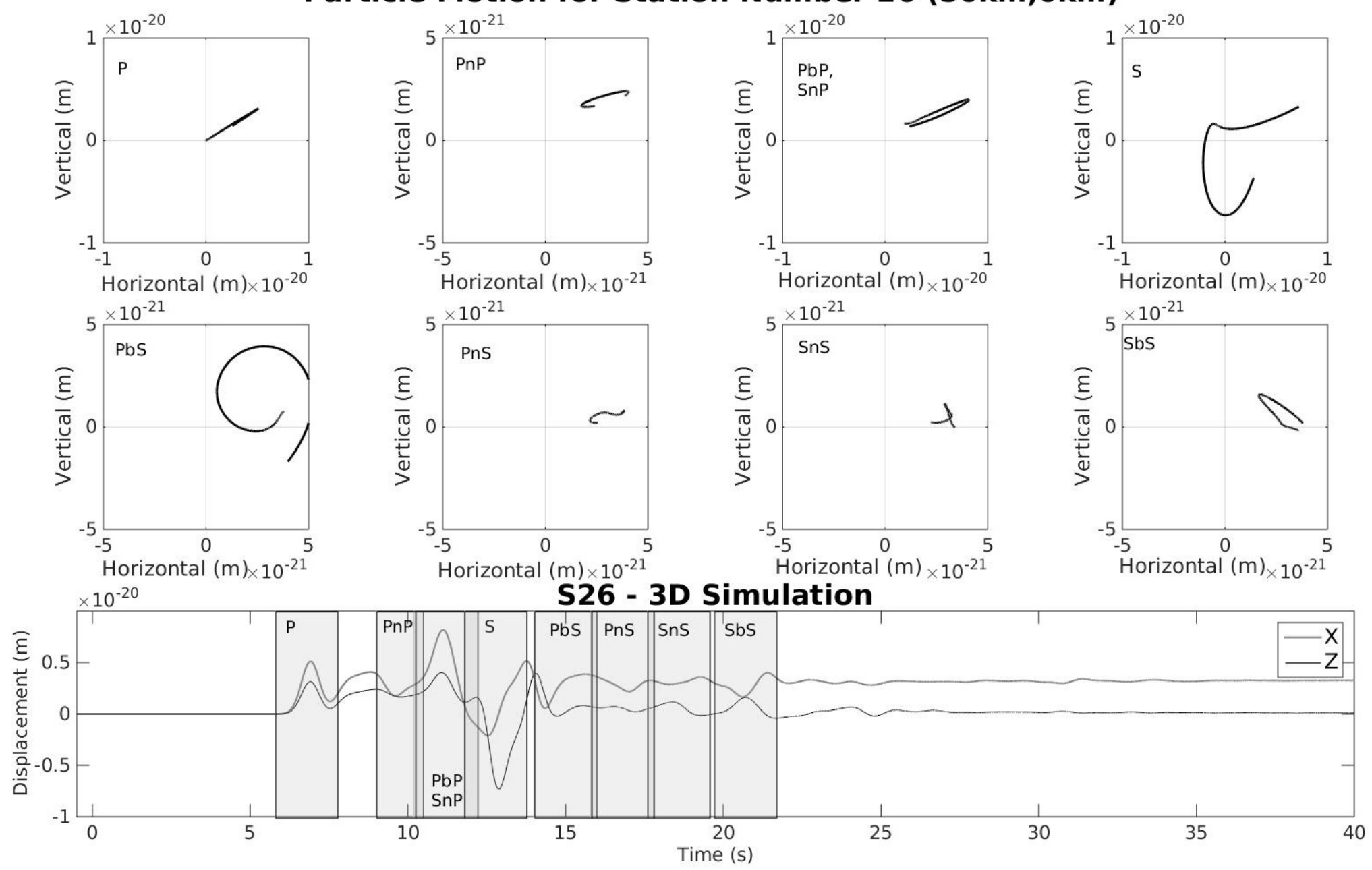

Figure 4.15: Particle motion plot of station number 26 for the 3D simulation. The time series (bottom) outlines the different time slices used. P denotes a P-wave, S denotes an Swave, $n$ is a reflection from the near side $(X=0 \mathrm{~km}), \mathrm{p}$ denotes a $\mathrm{P}$-wave reflected from the free surface $(\mathrm{Z}=0 \mathrm{~km})$, and an $\mathrm{s}$ denotes an $\mathrm{S}$-wave reflected off the free surface. 
A snapshot of the horizontal acceleration and vertical acceleration throughout the entire 2D model at different time slices are shown in Figure 4.16 for time 1.05s, Figure 4.17 for time $=$ 3.55s, Figure 4.18 for time $=6.55 \mathrm{~s}$, and Figure 4.19 for time $=10.05 \mathrm{~s}$. The different waves are labelled as it is easy to trace the different reflections with these snapshots. These snapshots of the acceleration within the model make it is possible to trace the different waves from the source, to the reflective boundary and then to the receiver. It is easy to see that as time progress the number of separate identifiable waves within the model increase, and that there are also incidents when several waves are arriving at a receiver at the same time.

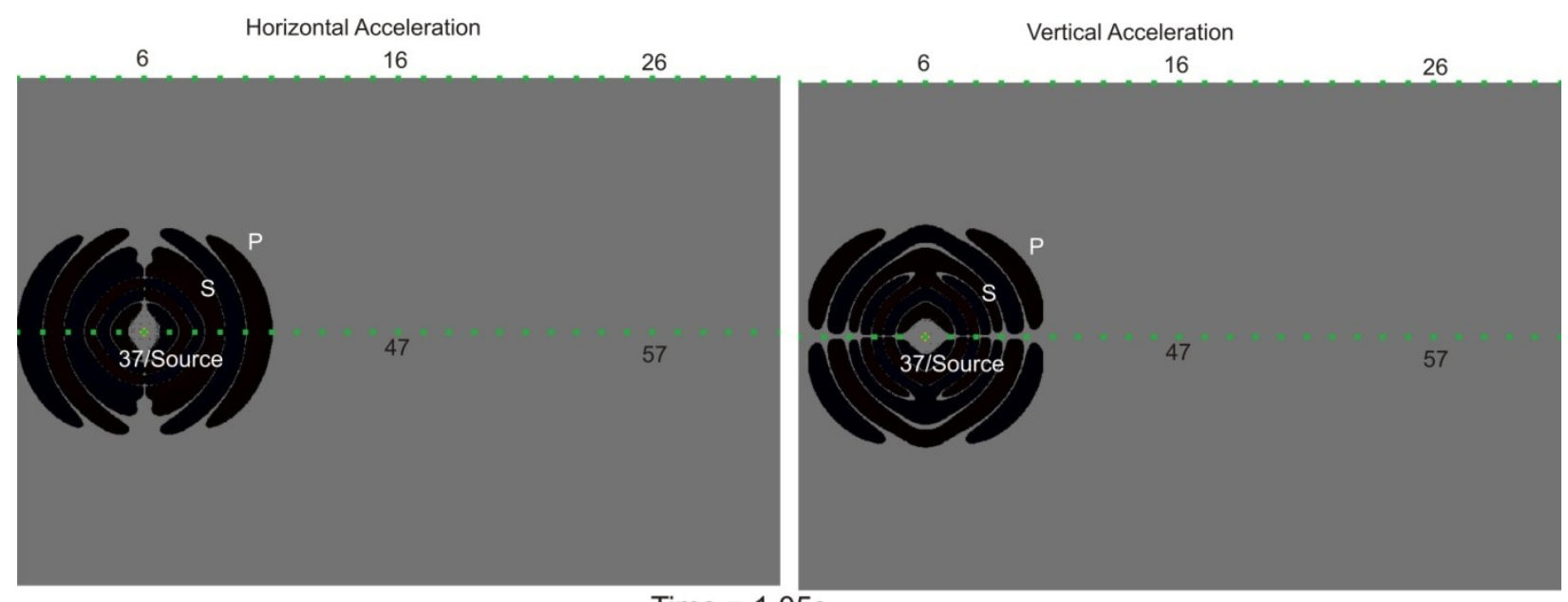

Time $=1.05 \mathrm{~s}$

Figure 4.16: Horizontal (left) and vertical (right) acceleration at time 1.05 seconds in the 2D simulation. The dots are the receivers, grey is the model volume and black is the acceleration. $\mathrm{P}$ denotes a P-wave, $\mathrm{S}$ denotes an S-wave. 


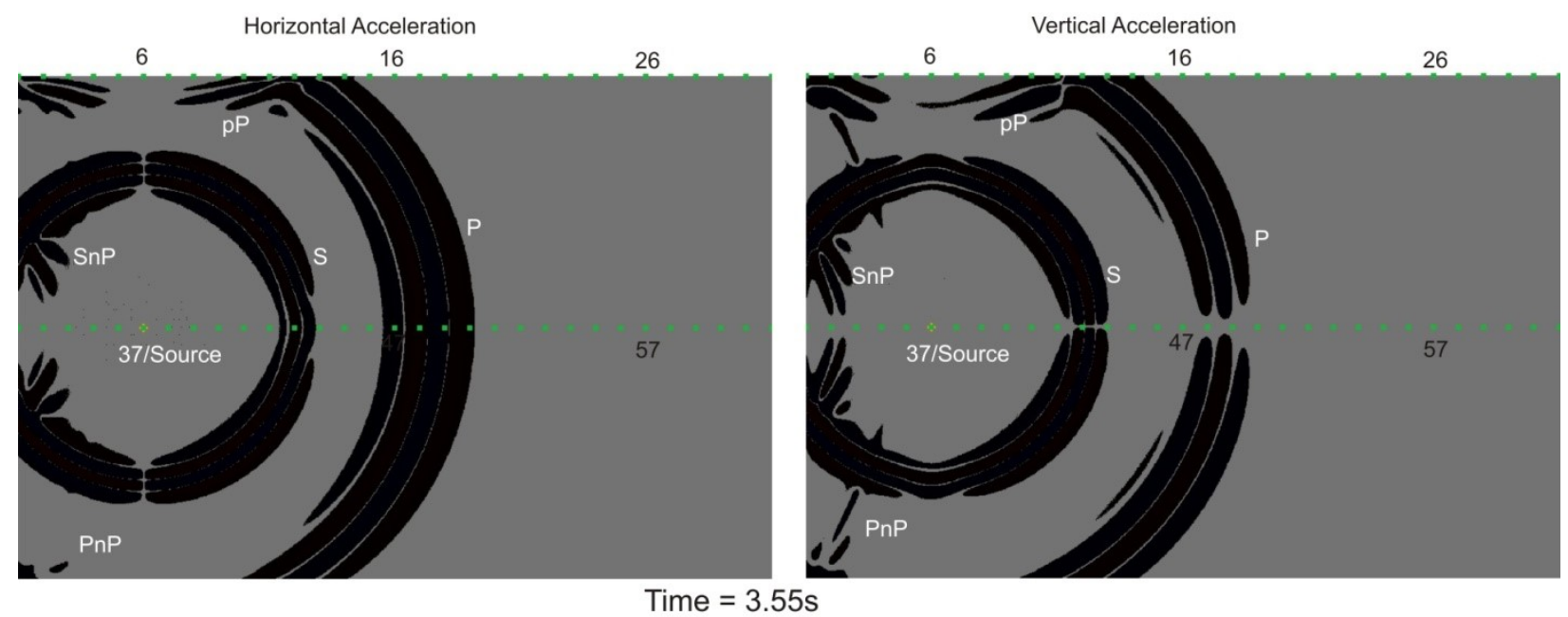

Figure 4.17: Horizontal (left) and vertical (right) acceleration at time 3.55 seconds in the 2D simulation. The dots are the receivers, grey is the model volume and black is the acceleration. $\mathrm{P}$ denotes a $\mathrm{P}$-wave, $\mathrm{S}$ denotes an $\mathrm{S}$-wave, $\mathrm{n}$ is a reflection from the near side $(X=0 \mathrm{~km})$, and a $\mathrm{p}$ denotes a $\mathrm{P}$-wave reflected from the free surface $(\mathrm{Z}=0 \mathrm{~km})$.

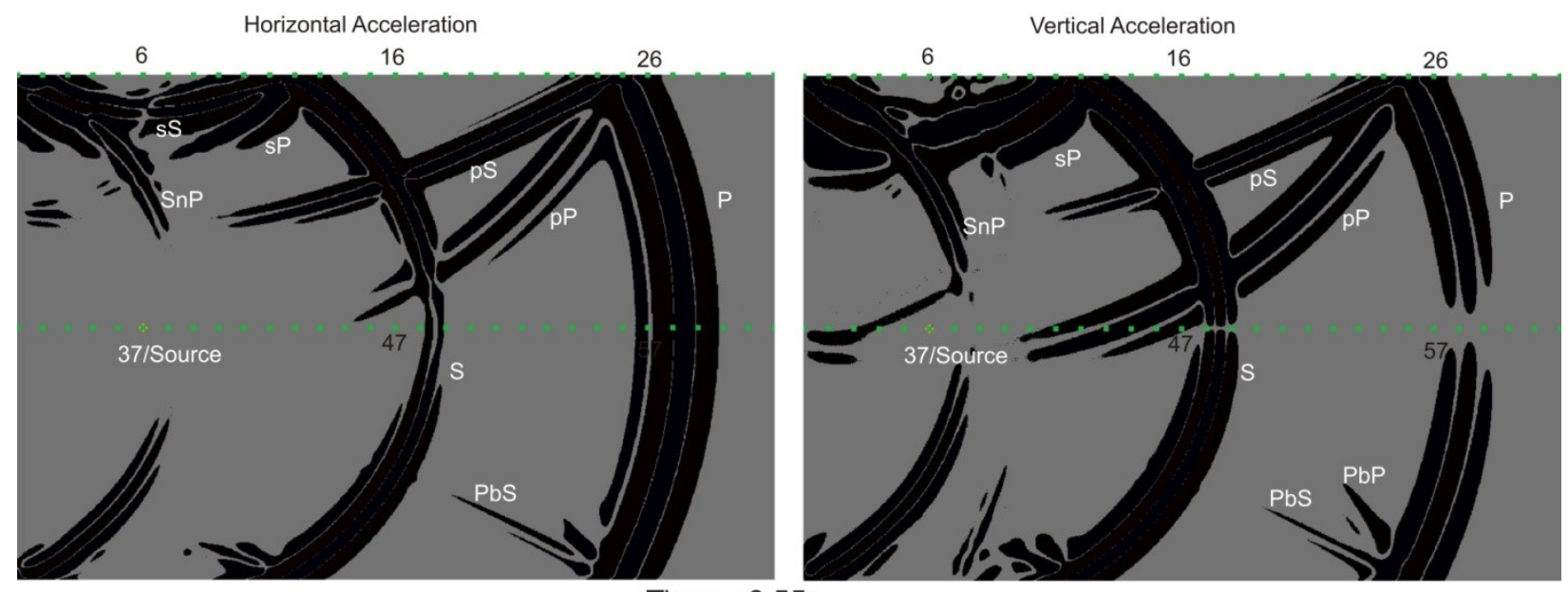

Time $=6.55 \mathrm{~s}$

Figure 4.18: Horizontal (left) and vertical (right) acceleration at time 6.55 seconds in the 2D simulation. The dots are the receivers, grey is the model volume and black is the acceleration. $\mathrm{P}$ denotes a $\mathrm{P}$-wave, $\mathrm{S}$ denotes an $\mathrm{S}$-wave, $\mathrm{n}$ is a reflection from the near side $(X=0 \mathrm{~km}), \mathrm{p}$ denotes a $\mathrm{P}$-wave reflected from the free surface $(Z=0 \mathrm{~km})$, an s denotes an $S$-wave reflected off the free surface, and $a b$ denotes a reflection from the bottom $(Z=-40 \mathrm{~km})$. 

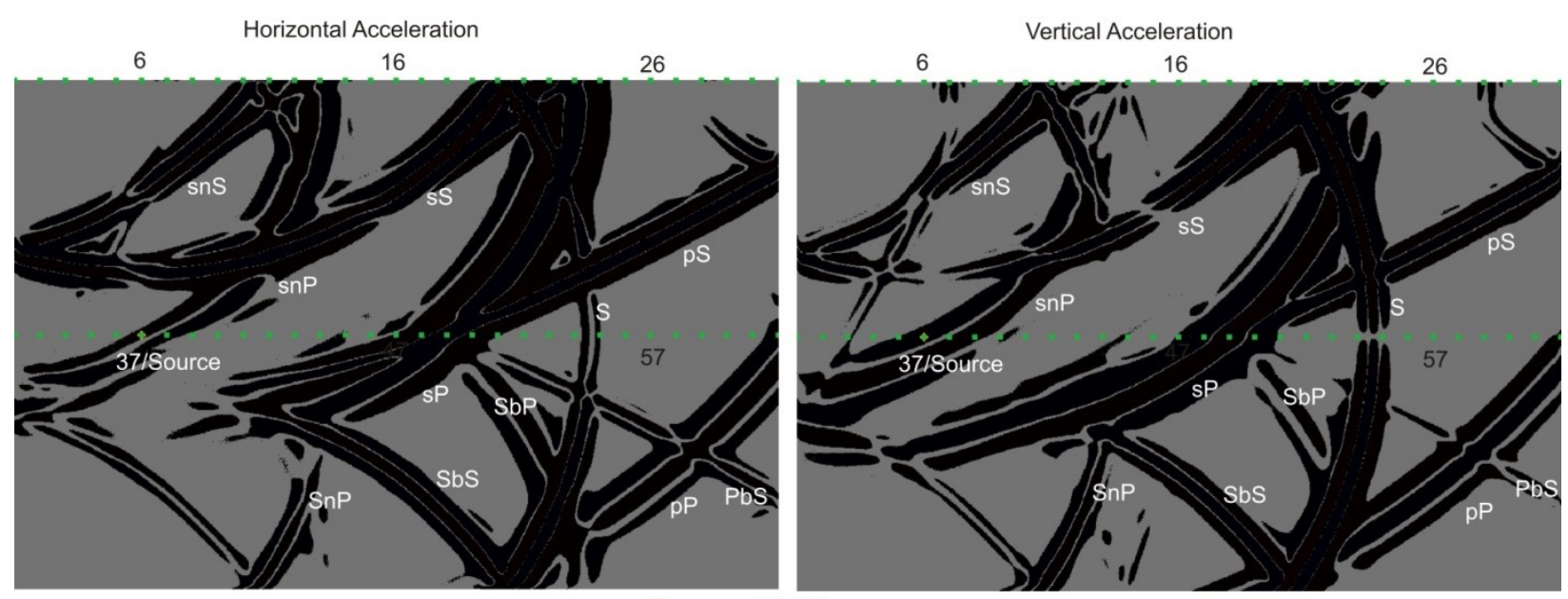

Time $=10.05 \mathrm{~s}$

Figure 4.19: Horizontal (left) and vertical (right) acceleration at time 10.05 seconds in the 2D simulation. The dots are the receivers, grey is the model volume and black is the acceleration. $\mathrm{P}$ denotes a $\mathrm{P}$-wave, $\mathrm{S}$ denotes an $\mathrm{S}$-wave, $\mathrm{n}$ is a reflection from the near side $(X=0 \mathrm{~km}), \mathrm{p}$ denotes a $\mathrm{P}$-wave reflected from the free surface $(Z=0 \mathrm{~km})$, an s denotes an $S$-wave reflected off the free surface, and $a b$ denotes a reflection from the bottom $(Z=-40 \mathrm{~km})$.

\subsubsection{Unit Z-direction Force Source}

The force source in the Z-direction has very similar characteristics to the force source in the X-

direction, except with a different polarization of the primary motion at the source and receivers.

The time series of the previously selected stations, 6, 16, 26, 37, 47, and 57 are shown in Figure

4.20 for the 2D simulation and Figure 4.21 for the 3D simulation. The wave fronts are labelled for each arrival, and the timing of each arrival is identical to the timing from the other source when the waves are present. The stronger reflections from the free surface in these simulations are Pwave reflections, as expected. These are also observed when comparing station numbers 47 and 57 between these two sources, as the strong reflections arrive earlier and are coincident with the theoretical arrival times of $\mathrm{pP}$ or $\mathrm{pS}$ waves. 

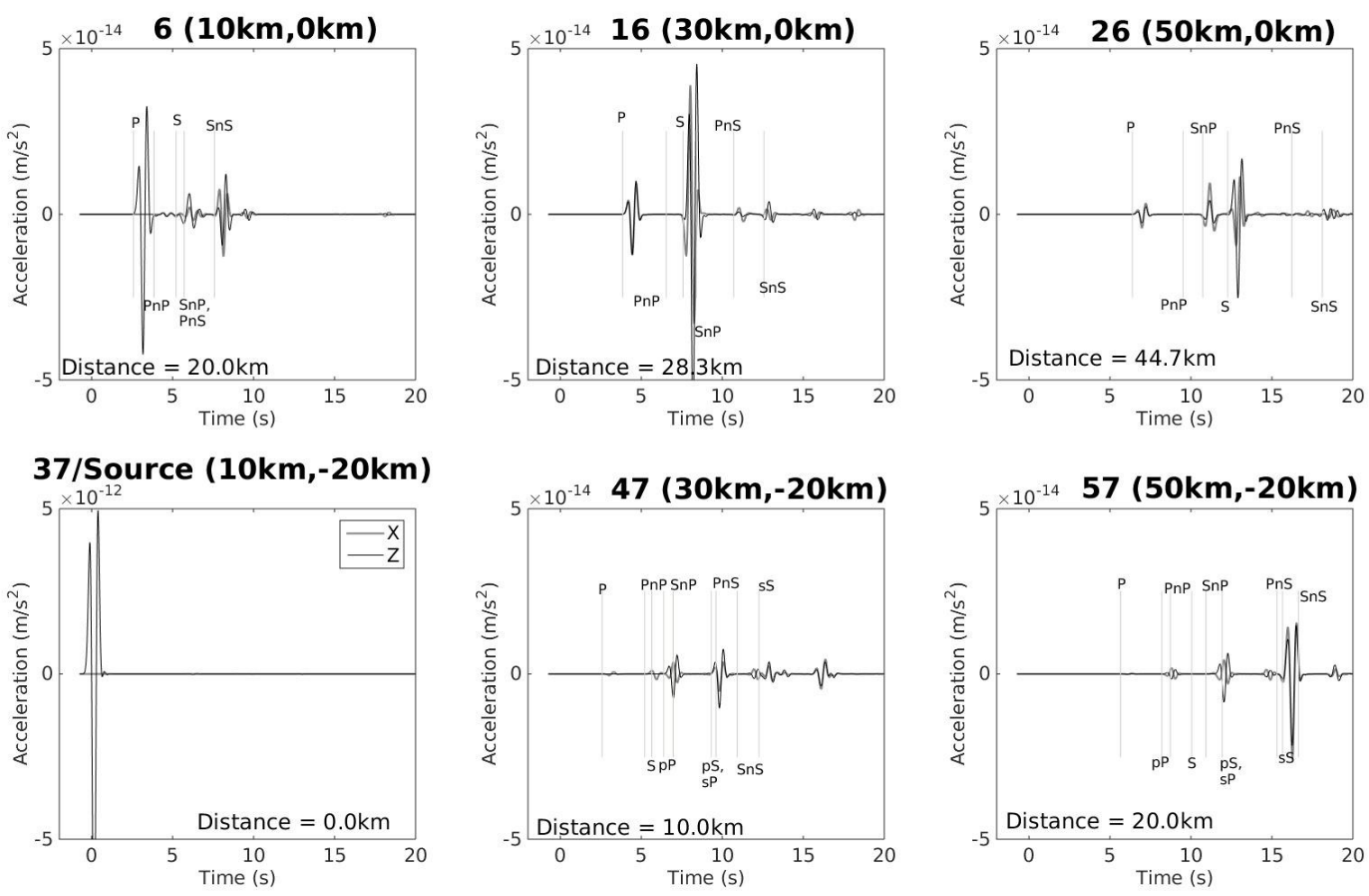

Figure 4.20: Acceleration time series for station numbers $6,16,26,37,47$, and 57 the 2D simulation with a unit force in the Zdirection. Distance is the distance from the source. The vertical lines are the theoretical wave arrivals for the different phases listed in Table 4.3. $\mathrm{P}$ denotes a $\mathrm{P}$-wave, $\mathrm{S}$ denotes an $\mathrm{S}$-wave, $\mathrm{n}$ is a reflection from the near side $(\mathrm{X}=0 \mathrm{~km}), \mathrm{p}$ denotes a $\mathrm{P}$-wave reflected from the free surface $(Z=0 \mathrm{~km})$, and an $\mathrm{s}$ denotes an $\mathrm{S}$-wave reflected off the free surface. The labels are alternately labelled above and below the lines and they are listed in order of the next arrival. 

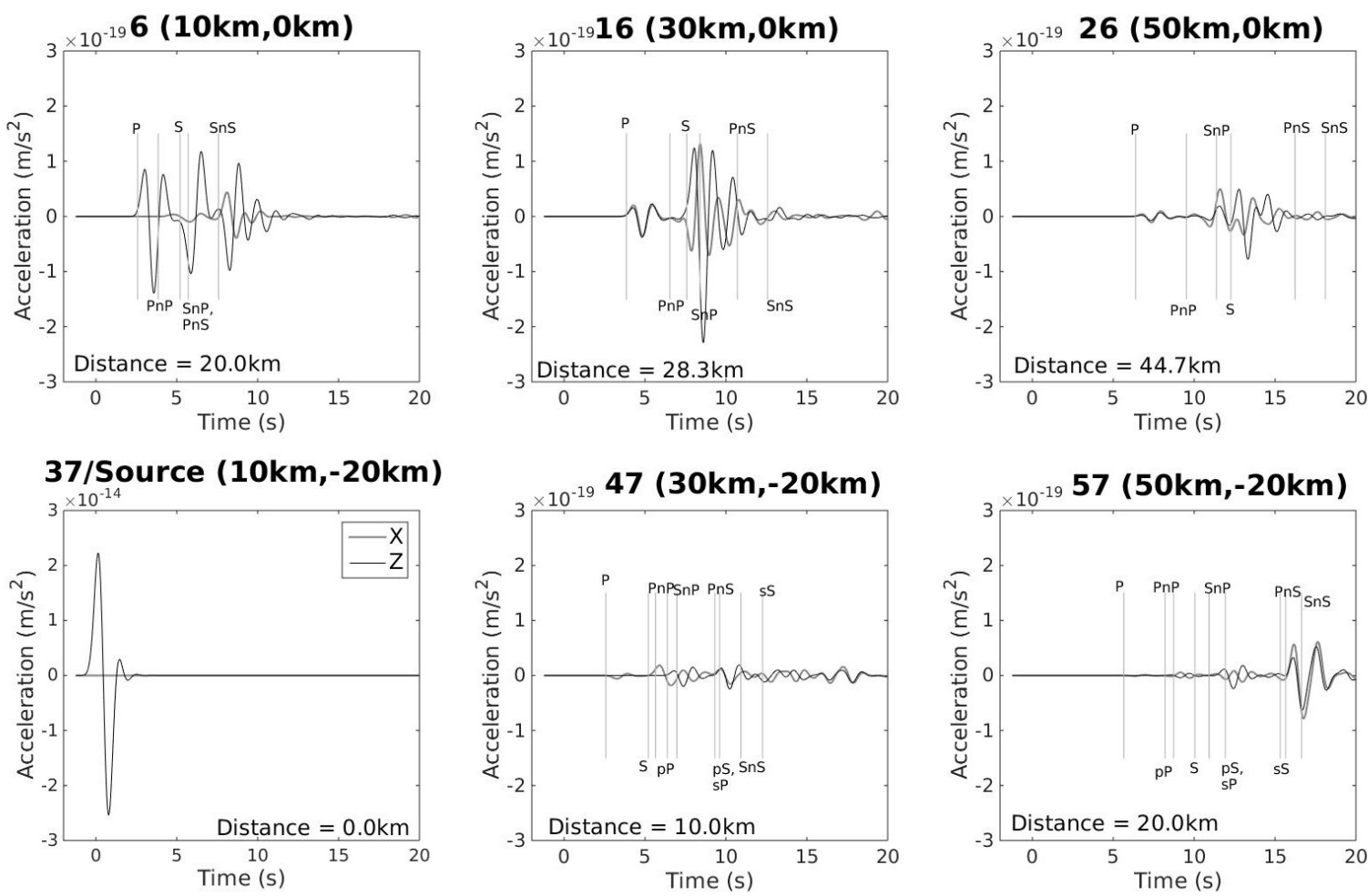

Figure 4.21: Acceleration time series for station numbers $6,16,26,37,47$, and 57 the 3D simulation with a unit force in the Zdirection. Distance is the distance from the source. The vertical lines are the theoretical wave arrivals for the different phases listed in Table 4.3. $P$ denotes a $P$-wave, $S$ denotes an $S$-wave, $n$ is a reflection from the near side $(X=0 \mathrm{~km}), p$ denotes a $P$-wave reflected from the free surface $(Z=0 \mathrm{~km})$, and an $\mathrm{s}$ denotes an $\mathrm{S}$-wave reflected off the free surface. The labels are alternately placed above and below the lines and they are listed in order of the next arrival.

Figure 4.22 and Figure 4.23 show the traces across the surface $(Z=0 \mathrm{~km})$ for the $X$ component and Z-component respectively. Figure 4.24 and Figure 4.25 show the motions for the receivers at $20 \mathrm{~km}$ depth for the $\mathrm{X}$-component and Z-component respectively. The theoretical arrival times of the wave fronts were traced through each set of receivers. The arrival times of each of these phase arrivals were identical to the arrivals from the unit force X-direction source. 

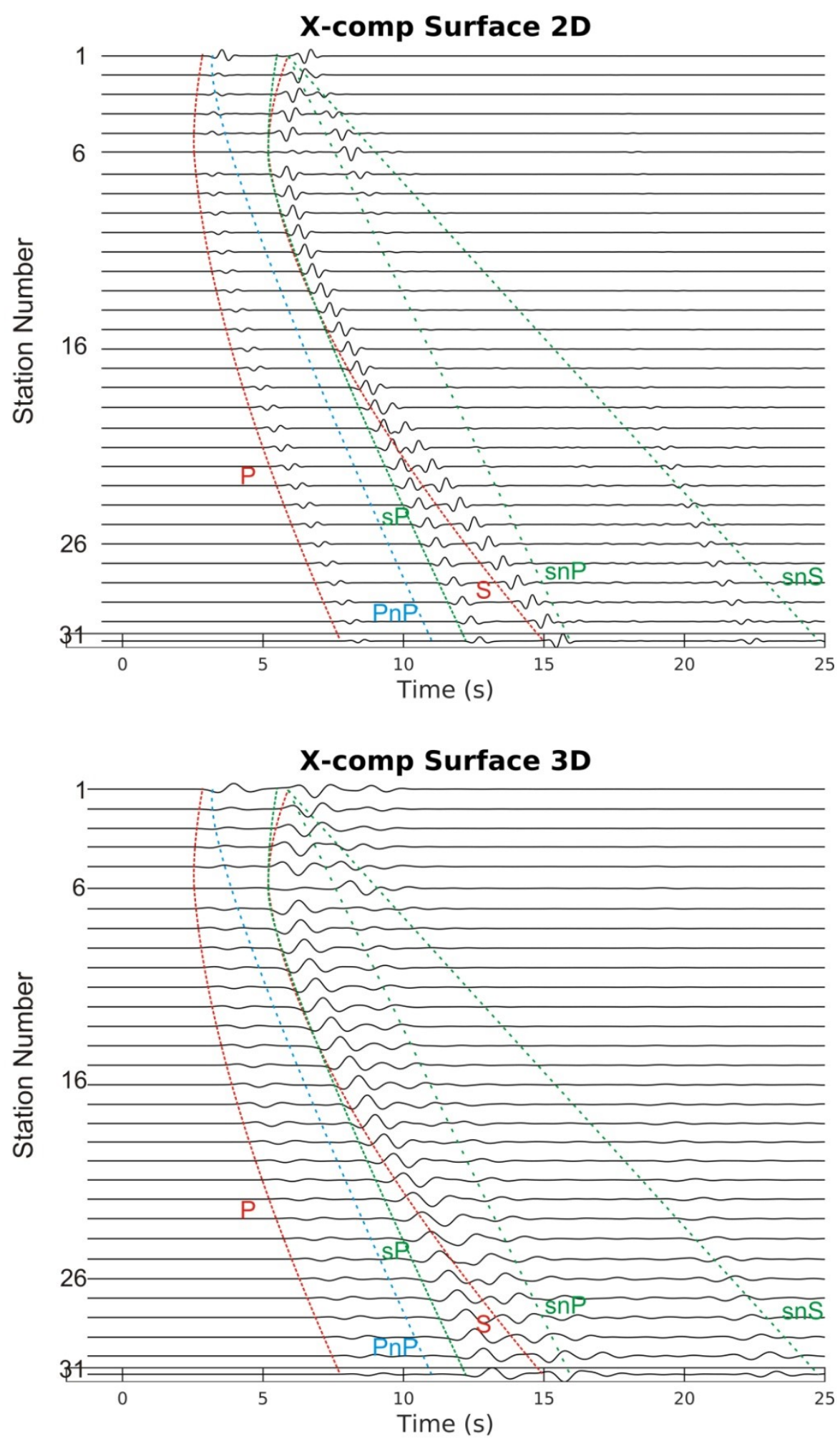

Figure 4.22: X-component of motion for the receivers at the surface for the 2D simulation (top) and 3D simulation (bottom) for the simulations using the unit- $Z$ direction force source. $P$ denotes a $P$-wave, $S$ denotes an $S$-wave, $n$ is a reflection from the near side $(X=0 \mathrm{~km}), p$ denotes a $P$-wave reflected from the free surface $(Z=0 \mathrm{~km})$, and an $\mathrm{s}$ denotes an $\mathrm{S}$-wave reflected off the free surface. For example: $\mathrm{SnP}$ is an S-wave that is reflected into a $\mathrm{P}$-wave off the near side boundary. 

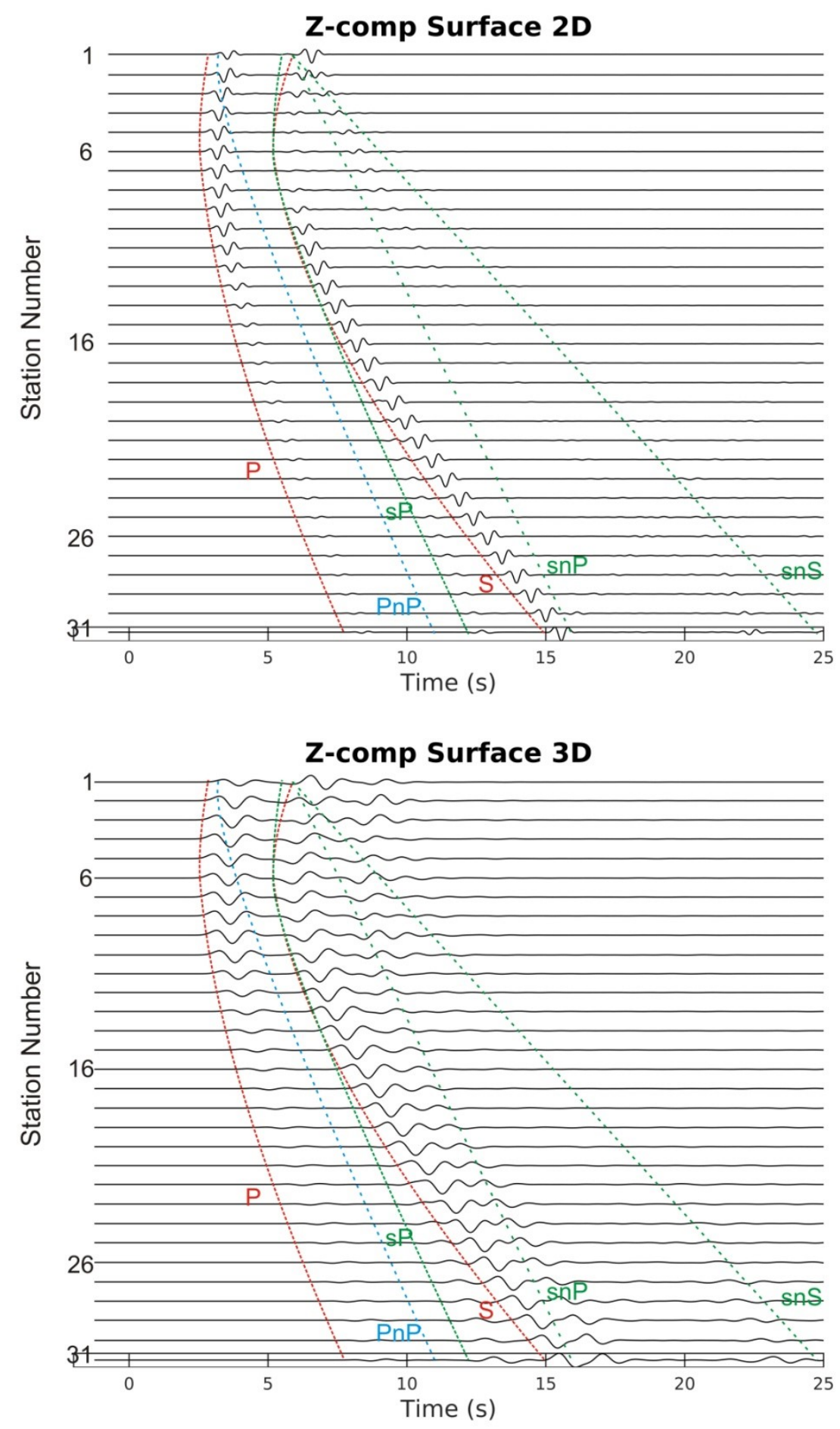

Figure 4.23: Z-component of motion for the receivers at the surface for the 2D simulation (top) and 3D simulation (bottom) for the simulations using the unit- $Z$ direction force source. $P$ denotes a $P$-wave, $S$ denotes an $S$-wave, $n$ is a reflection from the near side $(X=0 \mathrm{~km}), p$ denotes a $P$-wave reflected from the free surface $(Z=0 \mathrm{~km})$, and an $s$ denotes an $S$-wave reflected off the free surface. For example: SnP is an S-wave that is reflected into a P-wave off the near side boundary. 

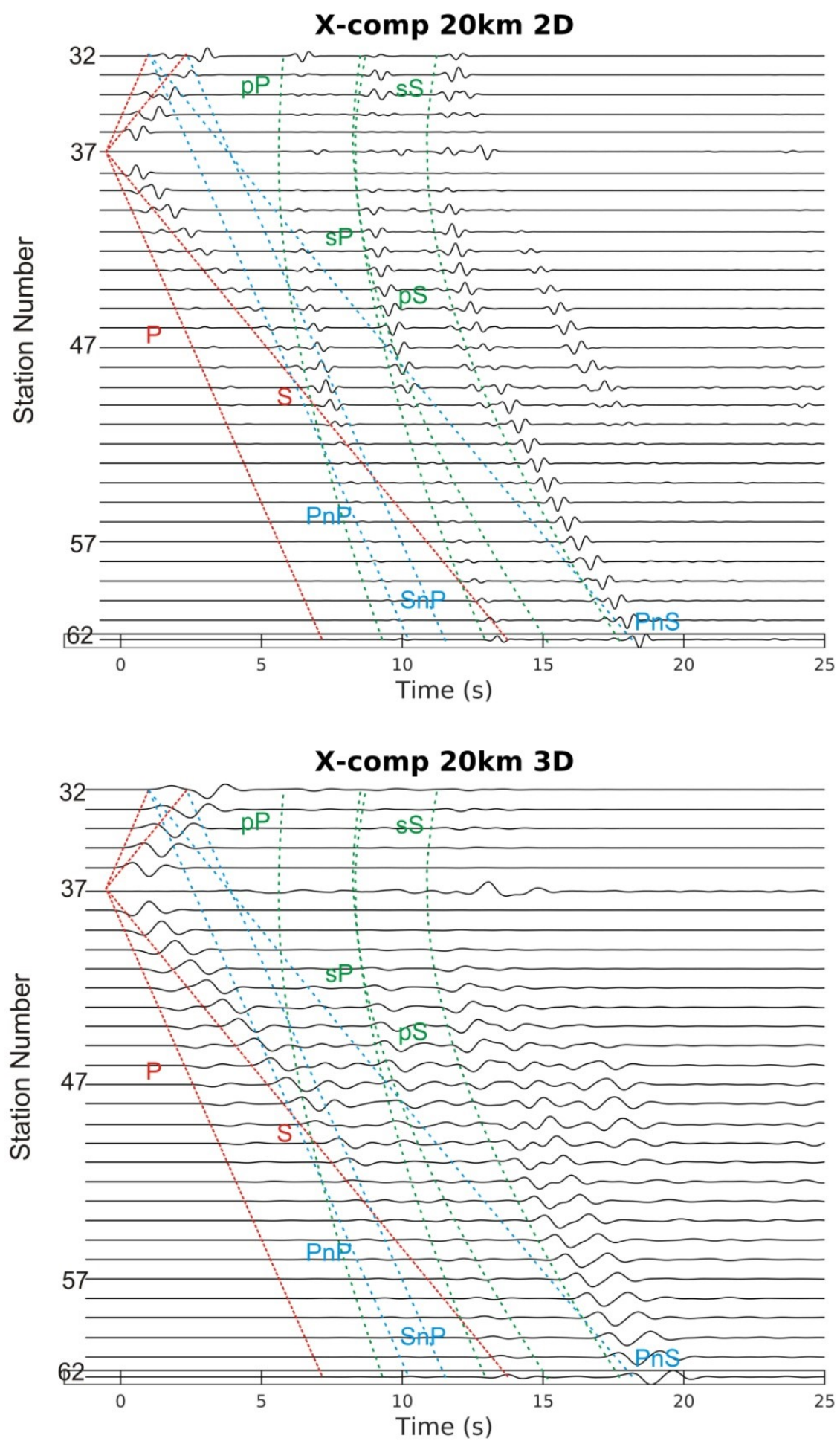

Figure 4.24: $\mathrm{X}$-component of motion for the receivers at $20 \mathrm{~km}$ depth for the 2D simulation (top) and 3D simulation (bottom) for the simulations using the unit- $Z$ direction force source. $\mathrm{P}$ denotes a $\mathrm{P}$-wave, $\mathrm{S}$ denotes an $\mathrm{S}$-wave, $\mathrm{n}$ is a reflection from the near side $(X=0 \mathrm{~km}), p$ denotes a $P$-wave reflected from the free surface $(Z=0 \mathrm{~km})$, and an $s$ denotes an $S$-wave reflected off the free surface. For example: SnP is an S-wave that is reflected into a P-wave off the near side boundary. 

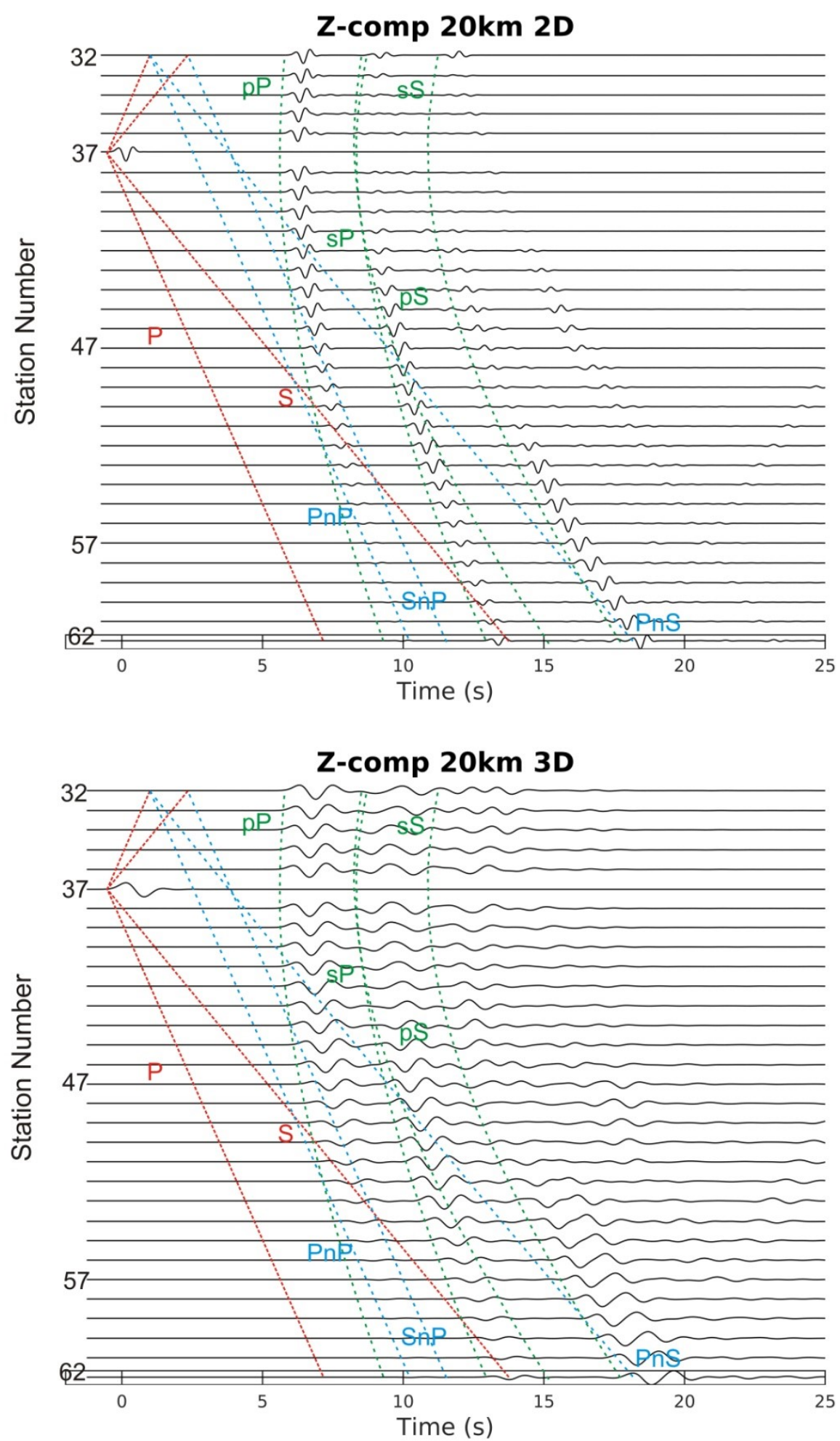

Figure 4.25: Z-component of motion for the receivers at $20 \mathrm{~km}$ depth for the 2D simulation (top) and 3D simulation (bottom) for the simulations using the unit- $Z$ direction force source. $P$ denotes a $P$-wave, $S$ denotes an $S$-wave, $n$ is a reflection from the near side $(X=0 \mathrm{~km}), p$ denotes a $P$-wave reflected from the free surface $(Z=0 \mathrm{~km})$, and an $s$ denotes an $S$-wave reflected off the free surface. For example: SnP is an S-wave that is reflected into a P-wave off the near side boundary. 


\subsubsection{Applying Corrections to 2D Seismograms}

As discussed above, it is possible to compare 2D and 3D simulations if corrections are applied to the $2 \mathrm{D}$ seismograms to change the effects of the source from a line source in $2 \mathrm{D}$ to a point source. These corrections are tedious as they must be completed on each arrival phase separately. An issue arises when the arrival of two separate phases occur over the same time. The question becomes: for which wave should the seismogram be corrected?

Figure 4.26 shows the first 10 seconds of a corrected acceleration time series at station number 16 in the 2D simulation, compared to the 3D simulation. There are several different corrections applied, as the PnP and S-wave phases overlap. The first correction was for the direct P-wave arrival between 4.35 and 8.35 seconds and direct S-wave arrival between 8.08 and 12.08 seconds. The second correction to the $2 \mathrm{D}$ seismogram was the direct $\mathrm{P}$-wave arrival as before and a reflected $\mathrm{P}$-wave from the near side ( $\mathrm{PnP})$ between 7.04 and 11.04 seconds. The third correction was for the direct P-wave arrival; reflected P-wave from the near side; and direct Swave arrival all with the same times as previous corrections. The direct P-wave arrival was the same for each correction as there was no overlap, and the corrected seismogram was very similar in amplitude as the 3D simulation pulse. Thus, it was difficult to determine which correction best corresponded to the 3D seismogram for the second peak. The correction with all 3 phases over predicted the amplitude, however the other two corrections were applied to the different components of the pulse differently. Examining the particle motion plots from before, Figure 4.12 and Figure 4.13, the S-wave and $\mathrm{PnP}$ arrival at this time appeared to be almost identical to one another as the amplitudes in the direction of the wave and perpendicular to the wave were 
comparable. It is difficult to decide for which phase the correction should be made when there is a strong overlap of two arrival phases at a particular time.
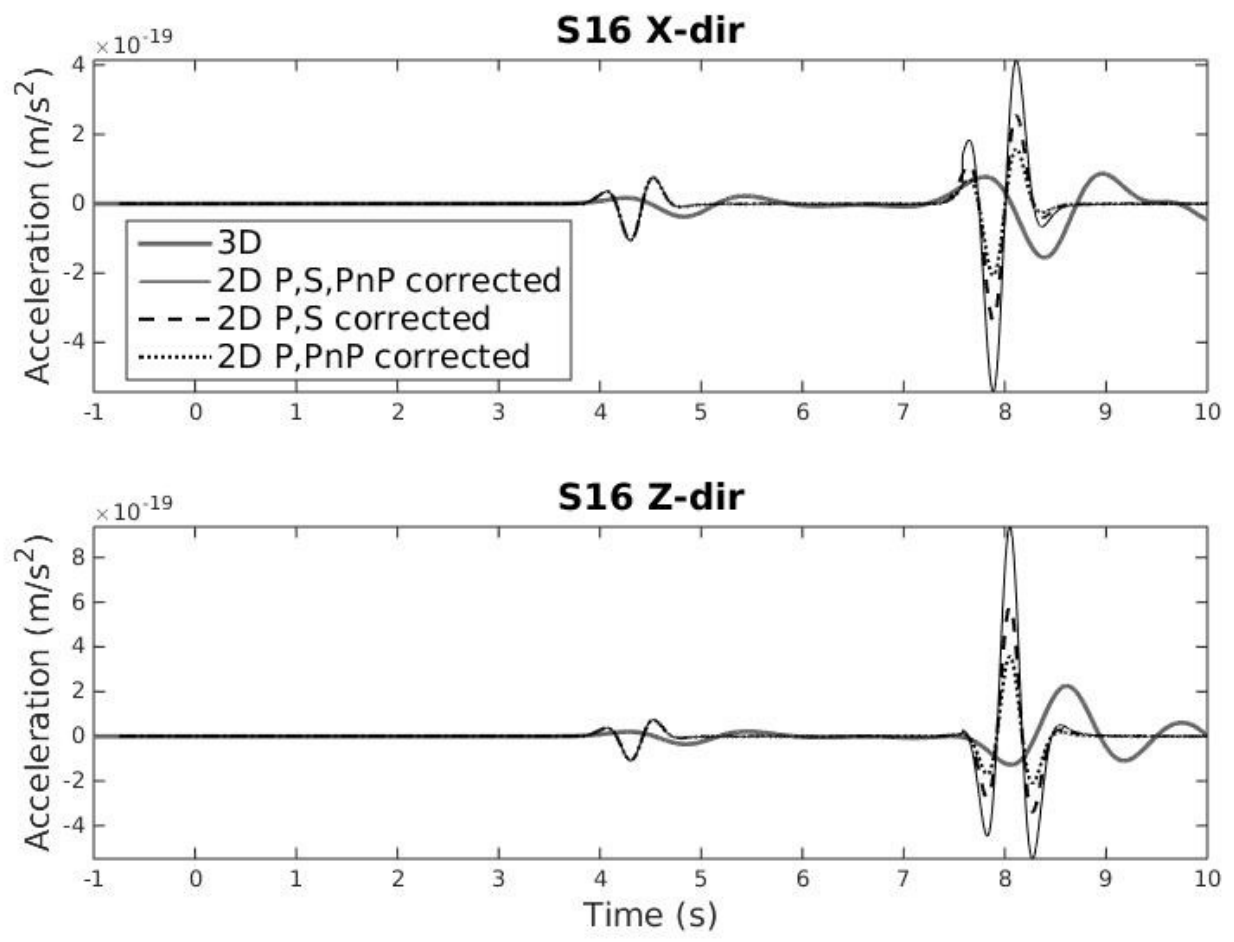

Figure 4.26: The first 10 seconds of the 3D time series and 2D corrected time series for station number 16 with different corrections applied. The time series is filtered using a 4-pole Butterworth lowpass filter with a cut-off frequency of $3.2 \mathrm{~Hz}$.

The final correction assumed that the superposition assumption holds true, that is when two waves interfere with one another their contribution can be added without one affecting the other. Although this may be appropriate, the correction did not separate the contribution from each wave. To correct for this completely, the seismogram would have had to be split into each arrival, corrected separately, and then summed together again. Although this may have been possible with the simplest of models, when heterogeneities (example a layered crust) were added, this would have become extremely difficult. 
Figure 4.27 shows the first 15 seconds of the 3D time series for station number 26 with the corrected 2D seismogram. The time windows for the corrections were adjusted for the arrivals of the different phases. Here the different arrivals are spread out more so their contributions do not overlap with one another. Applying the three different wave phase corrections as before, the 2D corrected seismogram was very comparable to the 3D seismogram. If we had only to correct 2 of these wave phases as before, the non-corrected waveform of the phase would have been significantly different from the 2D to 3D seismograms. Thus, the corrections which should be applied to a 2D seismogram must depend on the objectives and focus of calibration between 2D and 3D simulations.
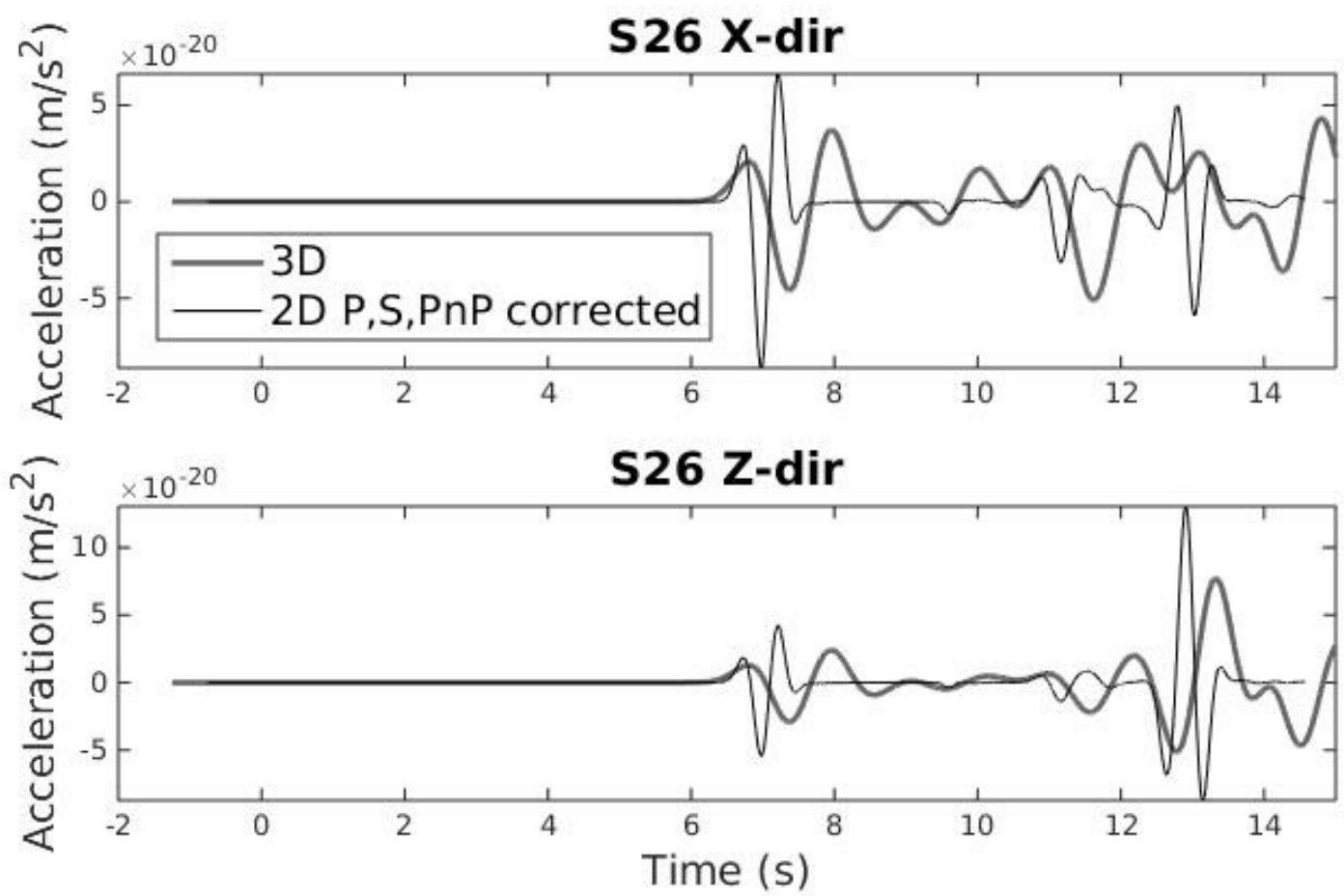

Figure 4.27: The first 15 seconds of the 3D time series and 2D corrected time series for station number 26 with different corrections applied for station number 26. The time series is filtered using a 4-pole Butterworth lowpass filter with a cut-off frequency of $3.2 \mathrm{~Hz}$. 


\subsubsection{Source Duration}

Previously the comparisons have been performed on the 2D and $3 D$ simulations where the sources were inputted so as to be identical to one another. It is clear the original pulse at the source is different from one another as shown by the time series in Figure 4.3 and Figure 4.4. The source duration can be adjusted in the $2 \mathrm{D}$ simulation to match the width of the $3 \mathrm{D}$ simulation, seen in Figure 4.28 where the dominant frequency of the 2D source was decreased by a factor of 2. Although the widths of the pulses match closely with one another, the comparative amplitudes are still different as discussed in Section 4.2.5.

As shown by Ekström (2012) the half duration, $t_{h d u r}$, is a function of seismic moment, $M_{0}$, given by:

$$
t_{\text {hdur }}=1.05 \times 10^{-8} M_{0}^{1 / 3}
$$

In order to match the amplitudes from a 2D simulation to a 3D simulation with a similar source, if the width of the source pulse is changed, then the seismic moment for the moment tensors has to be adjusted as well. This is shown by the corrected time series of station number 16 in Figure 4.29 , where both the width of the source and the seismic moment is adjusted to match the source of the $3 \mathrm{D}$ simulation. The waveforms from the $2 \mathrm{D}$ simulation are a better match to the 3D simulation waveforms for amplitude and duration, with a slight time difference due to the onset time of the source pulse difference from the 2D to 3D simulation. 

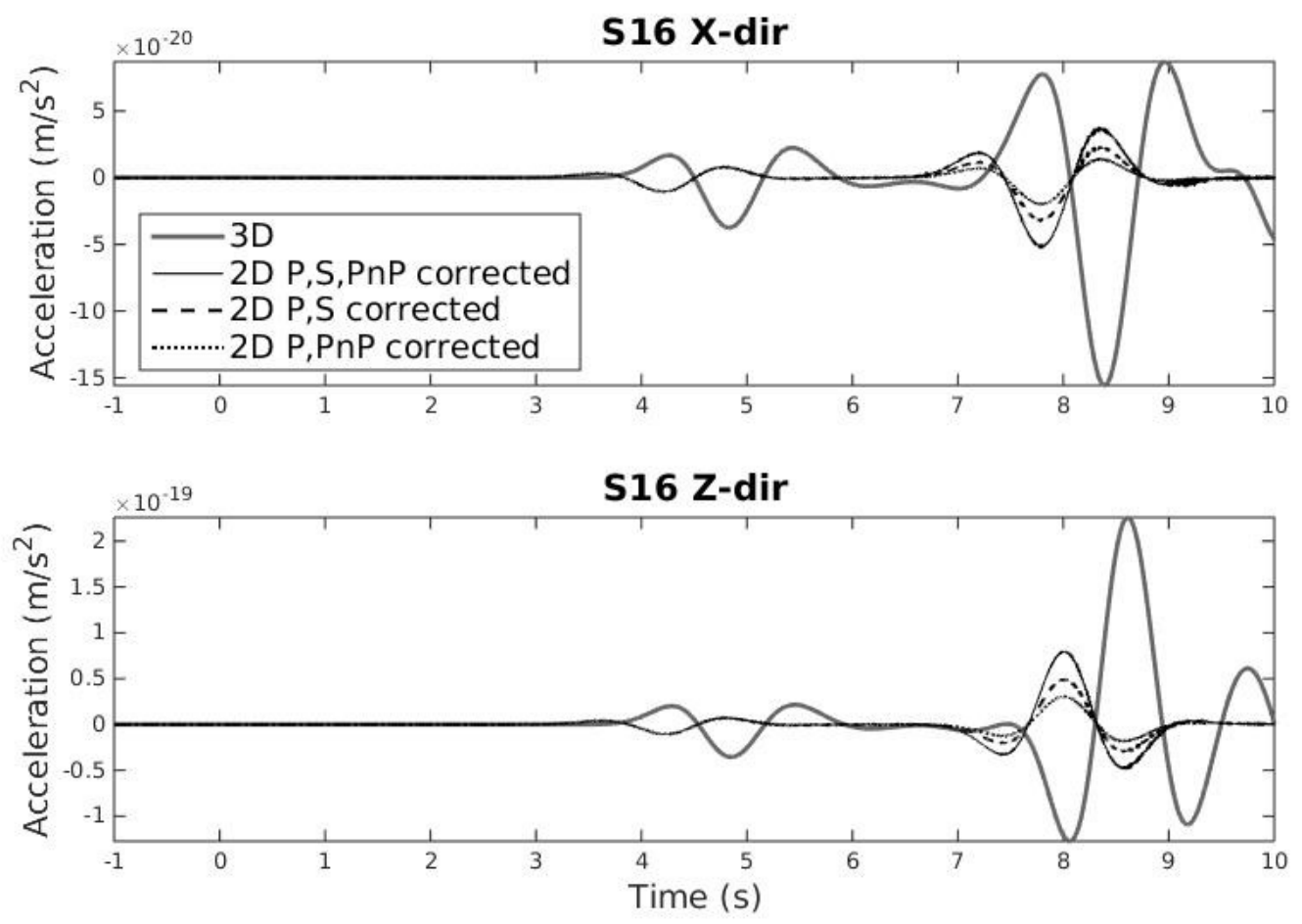

Figure 4.28: The first 10 seconds of the 3D time series and 2D corrected time series with different corrections applied. The dominant frequency of the source for the $2 \mathrm{D}$ simulation was increased to match the half duration of the 3D simulation. The time series was filtered using a 4-pole Butterworth lowpass filter with a cutoff frequency of $3.2 \mathrm{~Hz}$. 

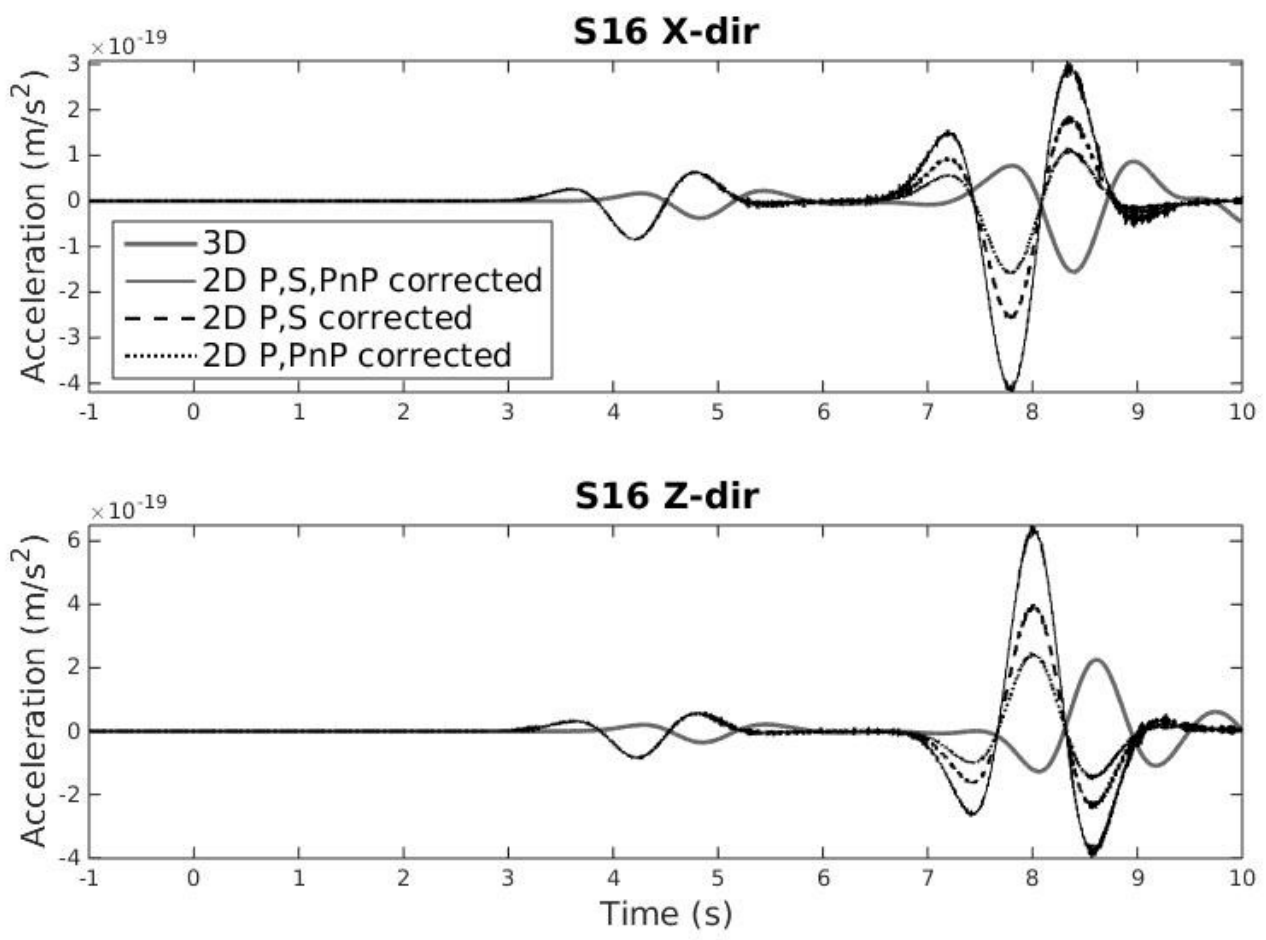

Figure 4.29: The first 10 seconds of the 3D time series and 2D corrected time series with different corrections applied. The dominant frequency of the source for the 2D simulation was increased and the seismic moment was adjusted to match the half duration of the 3D simulation. The time series was filtered using a 4-pole Butterworth lowpass filter with a cutoff frequency of $3.2 \mathrm{~Hz}$.

The frequency content in the simulations can be quite different as shown by the plots in Figure 4.30, of the Fourier acceleration spectrum of the corrected 2D simulation with the original source and the 3D simulation for station number 16 . This difference is associated with the source pulse as the dominant frequency was much higher for the 2D source than the 3D source. After correcting the source for both the half duration time and seismic moment, as discussed above, the frequency content of both the $2 \mathrm{D}$ and $3 \mathrm{D}$ simulation are very similar, as expected, shown in Figure 4.31 for station number 16. 

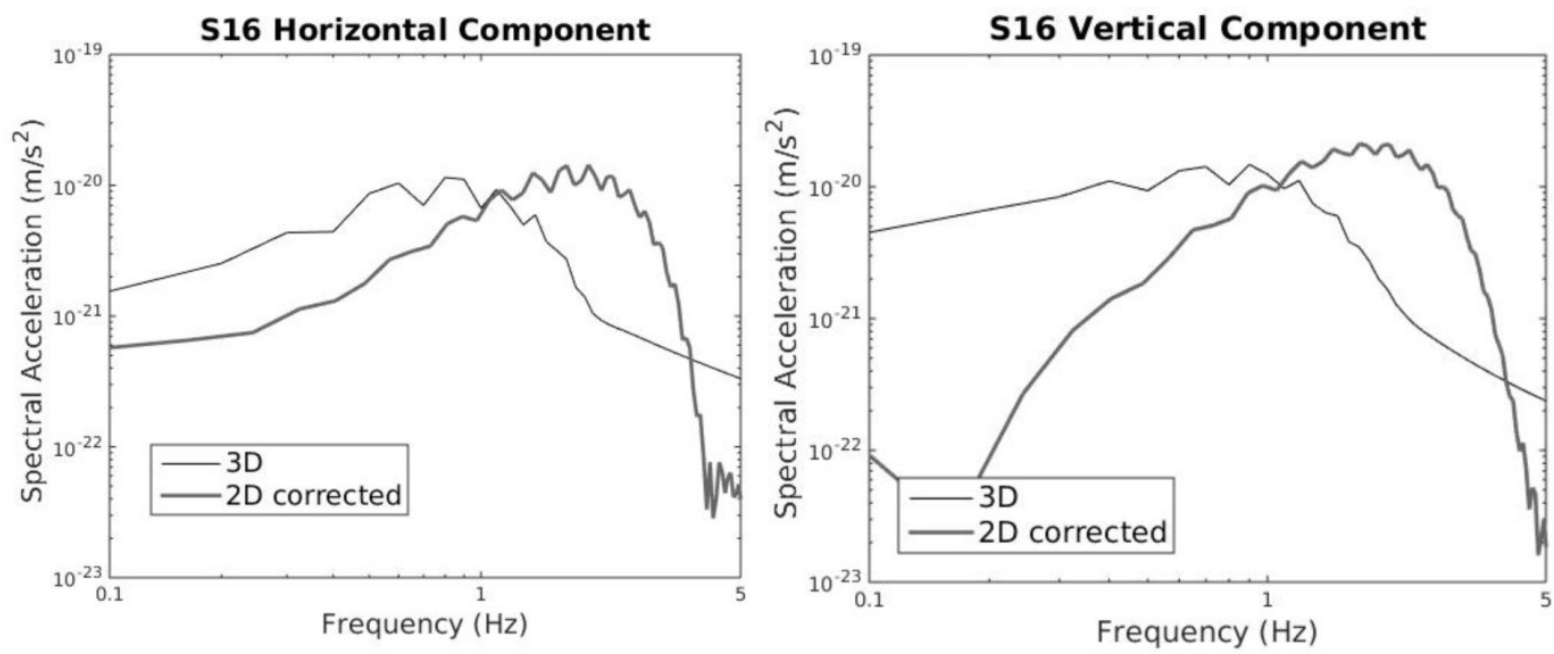

Figure 4.30: The Fourier acceleration spectrum for the 2D simulation with corrected seismograms and the original Unit X-direction force source and for the 3D simulation.
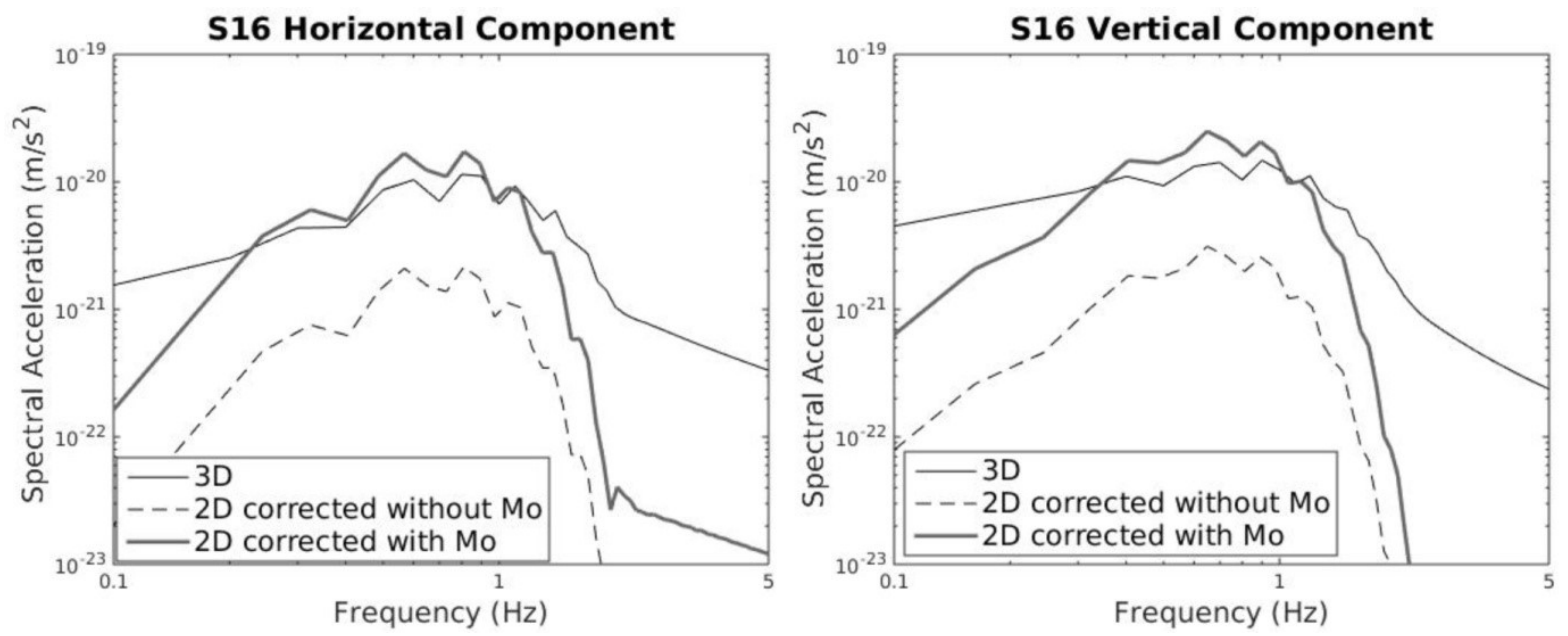

Figure 4.31: The Fourier acceleration spectrum for the 2D simulation with corrected seismograms and the adjusted source to match the 3D simulation. 


\subsection{Ladysmith Earthquake: Rock Site}

Similar to the homogeneous simulations described above, the station KB1O shown in Figure 4.32 was simulated using both a 3D and a 2D model. Three simulations were run on the 3D model: one with the moment tensor solution source as found in Bent et al. (2015); one with the global CMT source (Dziewonski et al., 1981; Ekström et al., 2012); and the third with a moment tensor derived from the fault plane solution and seismic moment from Ma and Audet (2014). The 2D models were run with a rotated moment tensor solution to correspond with KB1O being the source to receiver azimuth, and an adjusted moment tensor as described in Section 4.2.6 to match the 3D simulations. The models follow the properties described by the average rock conditions of eastern North America with a depth to Moho of 40 kilometres (EM40 from Bent et al., 2015). The simulation parameters are listed in Table 4.1 which are the same parameters used for the homogeneous simulations. 


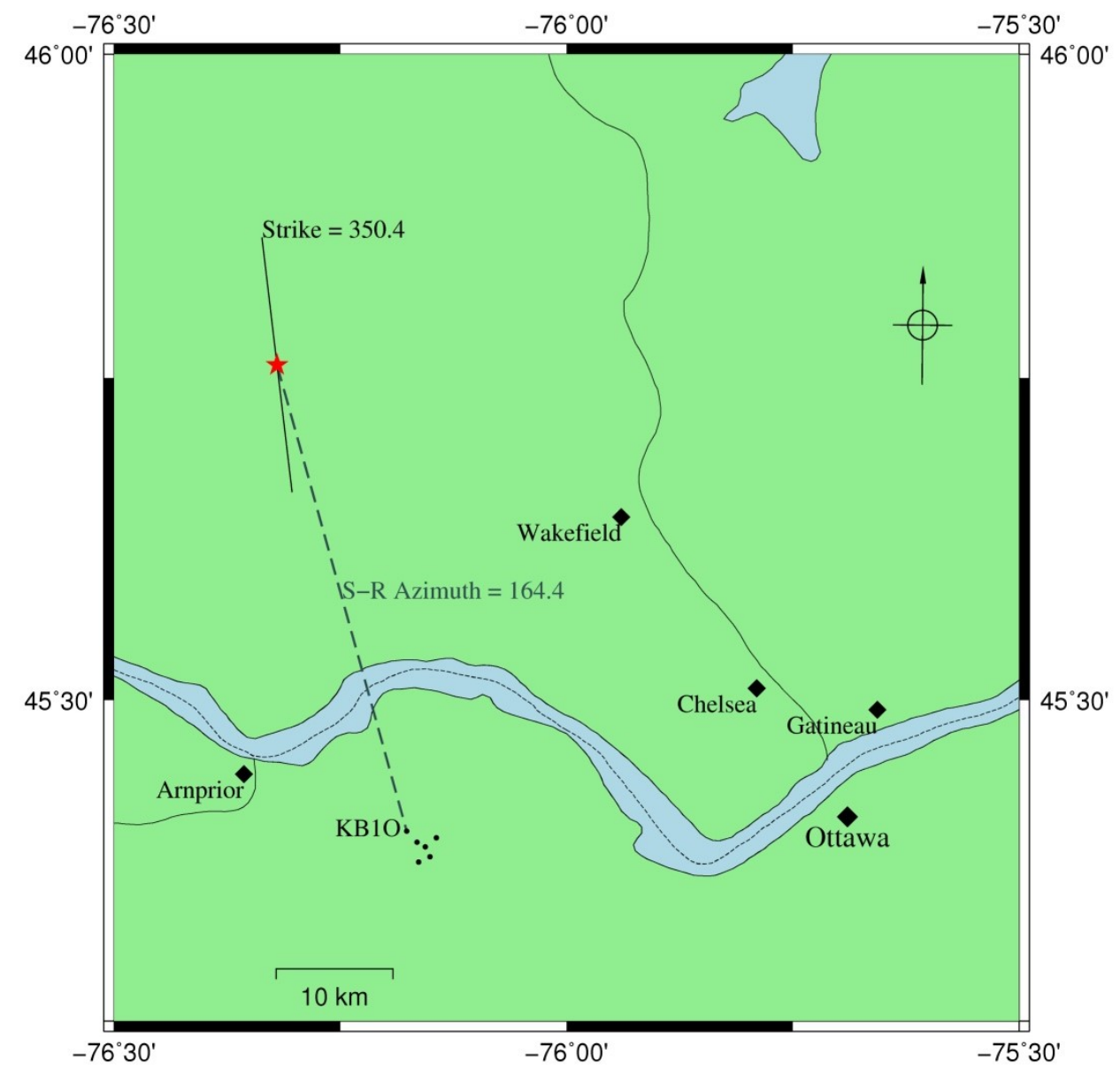

Figure 4.32: A map showing the locations of the Ladysmith epicentre (star), and the Kinburn seismic array (black circles). The strike of the fault plane from Bent et al. (2015) is $350.4^{\circ}$ and the source to receiver azimuth for the epicenter to the station KB1O is $164.4^{\circ}$.

As seen in Figure 3.1 and Figure 3.2, Section 3.1.2 the ground motions at KB1O have a very strong high frequency content. There are several reasons for this including: heterogeneities within the earth; a short source to receiver distance of about $42 \mathrm{~km}$; and a rupture which occurred along a fault plane. Although the mesh model is designed to be accurate up to $5 \mathrm{~Hz}$, the source is centered at frequencies between 0.4 and $2.2 \mathrm{~Hz}$. The models do not include the heterogeneities or a finite fault solution, so the simulations will be predominately lower frequencies than the recorded motions. A strong filter is applied to the recorded motions to reduce the impact of the higher frequencies as much as possible, however there will still be some high frequency content 
in the signal. This is discussed further in Appendix III. Although it is possible to simulate the higher frequency portion of a seismic signal, for example using a stochastic approach (Boore, 1998; Motazedian and Atkinson, 2005; Crane and Motazedian, 2014), the focus here is on the lower frequency content related to the moment tensor sources. These source are listed in Table 4.4.

\begin{tabular}{|c|c|c|c|}
\hline Source & 2D Moment Tensor $(\mathrm{Nm})^{*}$ & 3D Moment Tensor $(\mathrm{Nm})^{+}$ \\
\hline \multirow{2}{*}{ Bent et al., 2015 } & {$\left[\begin{array}{cc}0.524 & -2.700 \\
-2.700 & 7.352\end{array}\right] \times 10^{15}$} & {$\left[\begin{array}{ccc|}-1.165 & 3.966 & 2.475 \\
3.966 & -6.187 & 1.179 \\
2.475 & 1.179 & 7.352\end{array}\right] \times 10^{15}$} \\
\hline $\begin{array}{c}\text { Ma and Audet, } \\
2014\end{array}$ & {$\left[\begin{array}{cc}-5.628 & -1.684 \\
-1.684 & 13.04\end{array}\right] \times 10^{15}$} & {$\left[\begin{array}{ccc}-9.109 & 1.891 & 6.014 \\
1.891 & -3.931 & -0.149 \\
6.014 & -0.149 & 13.04\end{array}\right] \times 10^{15}$} \\
\hline Global CMT & {$\left[\begin{array}{cc}0.544 & -3.468 \\
-3.468 & 15.92\end{array}\right] \times 10^{15}$} & {$\left[\begin{array}{ccc}-3.20 & 1.43 & 7.03 \\
1.43 & -11.5 & 1.34 \\
7.03 & 1.34 & 14.7\end{array}\right] \times 10^{15}$} \\
\hline
\end{tabular}

Table 4.4: A list of the 2D and 3D sources for the simulations of the Ladysmith earthquake. *The 2D moment tensors are rotated $164.4^{\circ}$ to correspond to KB1O as the source-receiver azimuth. The 3D moment tensors are in CMT format to correspond with the input for SPECFEM3D.

\subsubsection{Modelling using Moment Tensor Source from Bent et al. (2015)}

The moment tensor used for these simulations is shown in Section 3.1.1, and in Table 4.4. This source has a shallow depth, at $12 \mathrm{~km}$, and a lower seismic moment, $8.372 \times 10^{15} \mathrm{Nm}$, in comparison to the other sources used. The filtered Fourier acceleration spectrum for each simulation is shown in Figure 4.33 for the radial and vertical component of motion. The 2D simulation with a dominant frequency, $f_{0}=2.2 \mathrm{~Hz}$ shows good agreement for the vertical component before the frequency cut-off, but consistently shows lower than expected values for the radial component of motion. The 3D simulation shows similar characteristics of motions up to about $1 \mathrm{~Hz}$, then it starts to fall off faster than the recording. This is due to the simulated frequencies being centred about $1 \mathrm{~Hz}$ then dropping before the filter corner frequency of $3.2 \mathrm{~Hz}$.

The $2 \mathrm{D}$ simulation with a similar source display values above the recorded motions for the frequencies below $1.5 \mathrm{~Hz}$, but a much quicker drop-off than the other simulations and recording. 

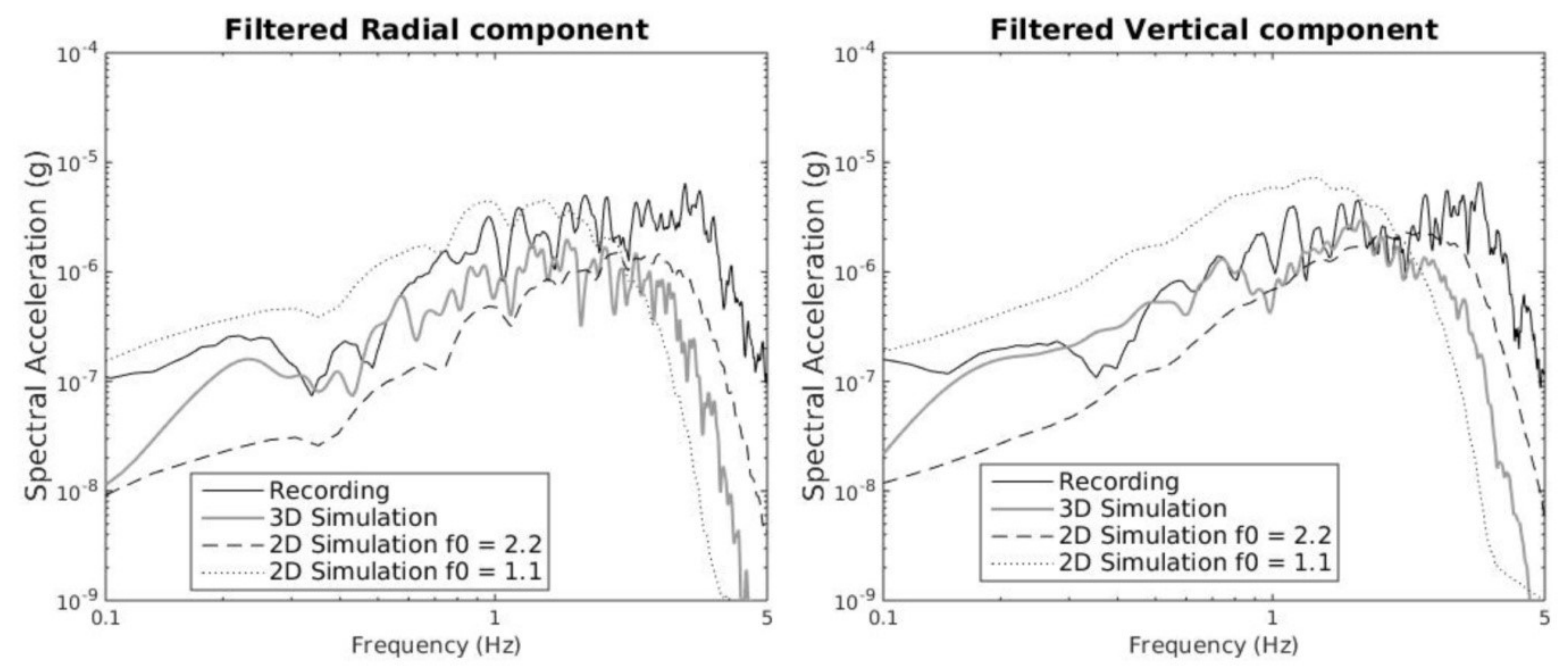

Figure 4.33: Radial and Vertical spectral accelerations using a moment tensor source from Bent et al. (2015). These have been filtered using an 8-pole Butterworth bandpass filter with corner frequencies 0.01 and $3.2 \mathrm{~Hz}$.

The filtered time series of the radial and vertical motions for all simulations and the recorded values is shown in Figure 4.34. Here the filter applied is an 8-pole Butterworth bandpass filter with cut-off frequencies of 0.01 and $3.2 \mathrm{~Hz}$ respectively. The recorded motions are larger the than the simulated values, since there is still a stronger contribution of higher frequencies even after a filter was applied. By applying filters around the simulation frequencies it was possible to get a better match between the peak values as seen in Figure 4.35, for a bandpass filter with corner frequencies 0.01 and $2.2 \mathrm{~Hz}$, as well as in Figure 4.36 which has a bandpass filter with corner frequencies 0.01 and $1.2 \mathrm{~Hz}$ applied. From these plots we see that the peak amplitudes matched closely between the 3D simulation, 2D simulation with $f_{0}=1.1 \mathrm{~Hz}$ and the filtered recording, however the $2 \mathrm{D}$ simulation with $\mathrm{f}_{0}=2.2 \mathrm{~Hz}$ still under-predicted the peak amplitude. 

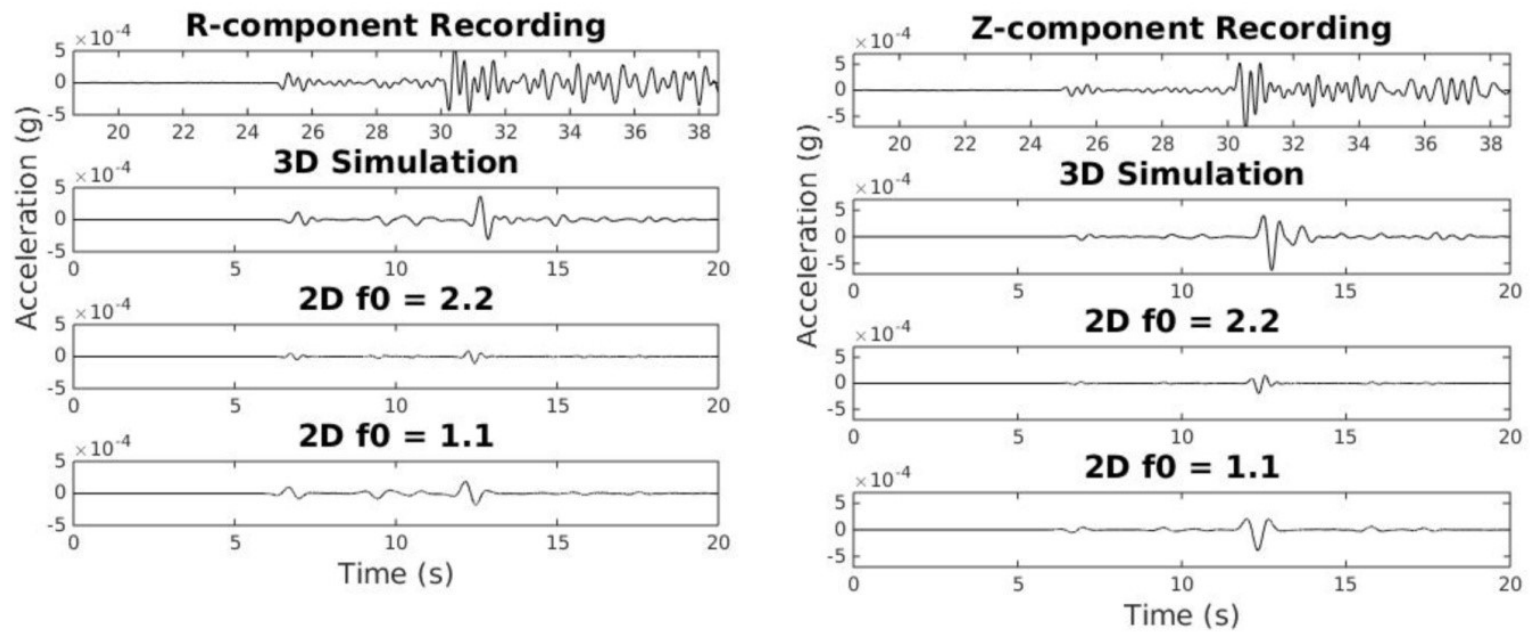

Figure 4.34: Radial and vertical time series for the Ladysmith recording at KB1O and the simulations with a moment tensor source from Bent et al. (2015). A Butterworth bandpass filter with corner frequencies 0.01 and 3.2 Hz was applied.
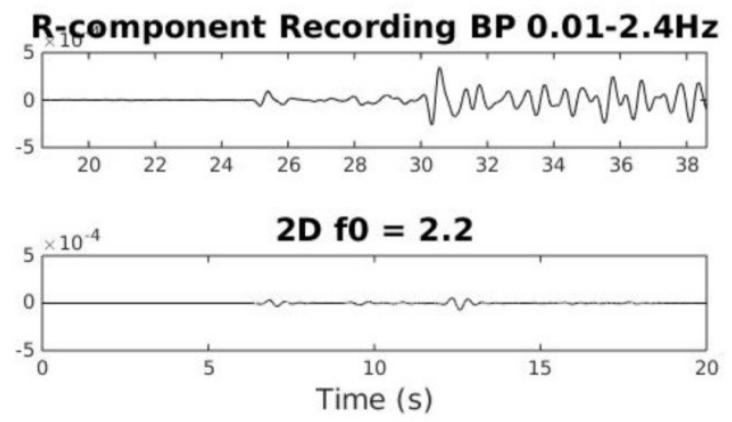
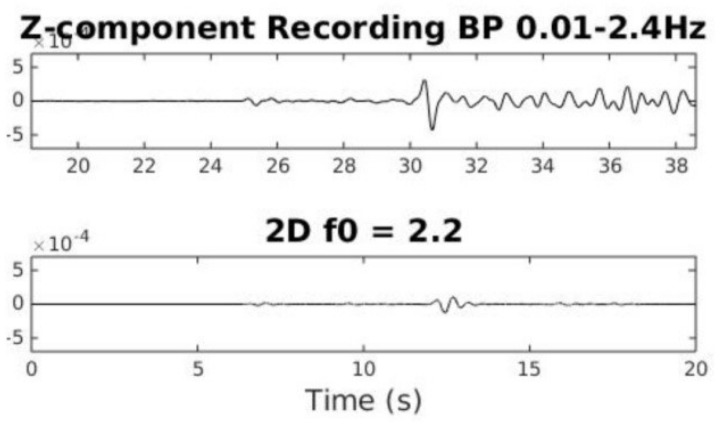

Figure 4.35: Radial and vertical time series for the Ladysmith recording at $K B 1 O$ and the $2 \mathrm{D}$ simulation with $\mathrm{f}_{0}=2.2 \mathrm{~Hz}$. A Butterworth bandpass filter with corner frequencies 0.01 and $2.4 \mathrm{~Hz}$ was applied. 

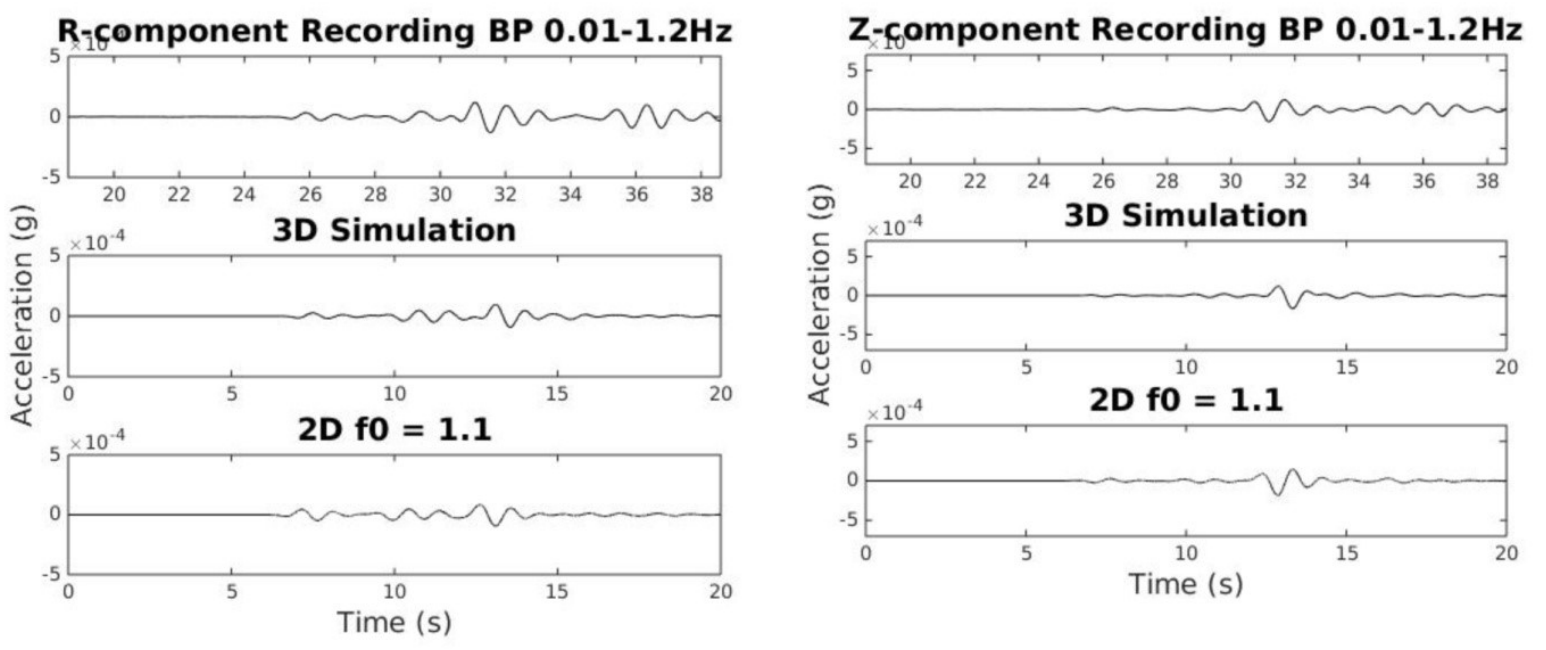

Figure 4.36: Radial and vertical time series for the Ladysmith recording at $\mathrm{KB} 10$ and the 3D simulation and 2D simulation with $\mathrm{f}_{0}=1.1 \mathrm{~Hz}$. A Butterworth bandpass filter with corner frequencies 0.01 and $1.2 \mathrm{~Hz}$ was applied.

\subsubsection{Modelling using Global CMT Source}

The global centroid moment tensor source was found from the global CMT project website (http://www.globalcmt.org). The process to determine this source is described by Dziewonski et al. (1981) and Ekström et al. (2012). This moment tensor was adjusted in the same manner as before for the $2 \mathrm{D}$ simulations. This source was the deepest with a depth of $22 \mathrm{~km}$ and also has the largest seismic moment at $1.52 \times 10^{16} \mathrm{Nm}$. The Fourier spectral acceleration for each simulation is shown in Figure 4.37. The 2D simulation with a $f_{0}=1.66 \mathrm{~Hz}$ is in good agreement for both the radial and vertical components of motion between approximately 0.2 to $2.5 \mathrm{~Hz}$, before the higher frequencies decay in the simulation. The $2 \mathrm{D}$ simulation with $\mathrm{fO}=0.83 \mathrm{~Hz}$ displays values which over predict the recorded spectral values for most of the spectra lower than $1 \mathrm{~Hz}$. The 3D simulation shows a similar frequency to the recorded motions up to about $0.8 \mathrm{~Hz}$, and then drops off very quickly. 

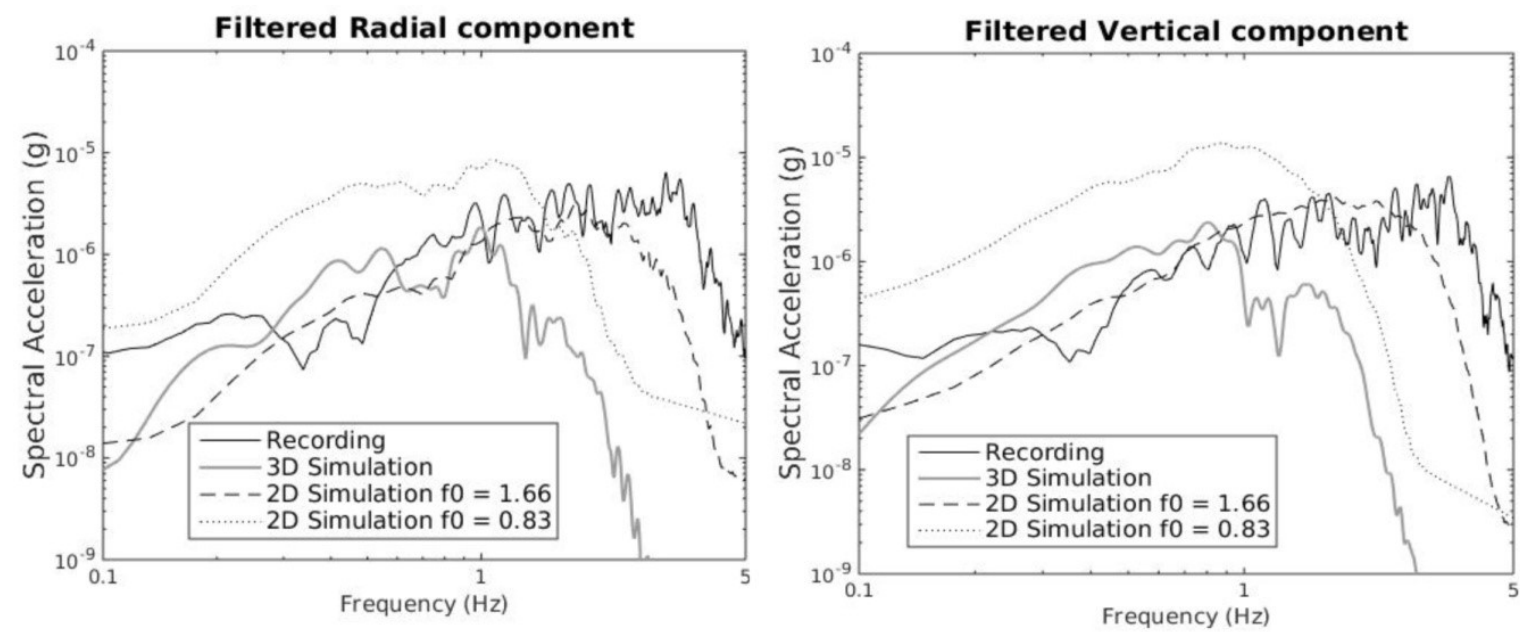

Figure 4.37: Radial and Vertical spectral accelerations using the global centroid moment tensor. These have been filtered using an 8-pole Butterworth bandpass filter with corner frequencies 0.01 and $3.2 \mathrm{~Hz}$.

The filtered time series, with a filter between 0.01 and $3.2 \mathrm{~Hz}$, is shown in Figure 4.38 for the recorded values and the different simulations. The peaks are larger for the recordings due to the dependence of their values on the higher frequencies. For better time series comparisons between recorded values and the simulations, narrower filters were applied as shown in Figure 4.39 and Figure 4.40 .
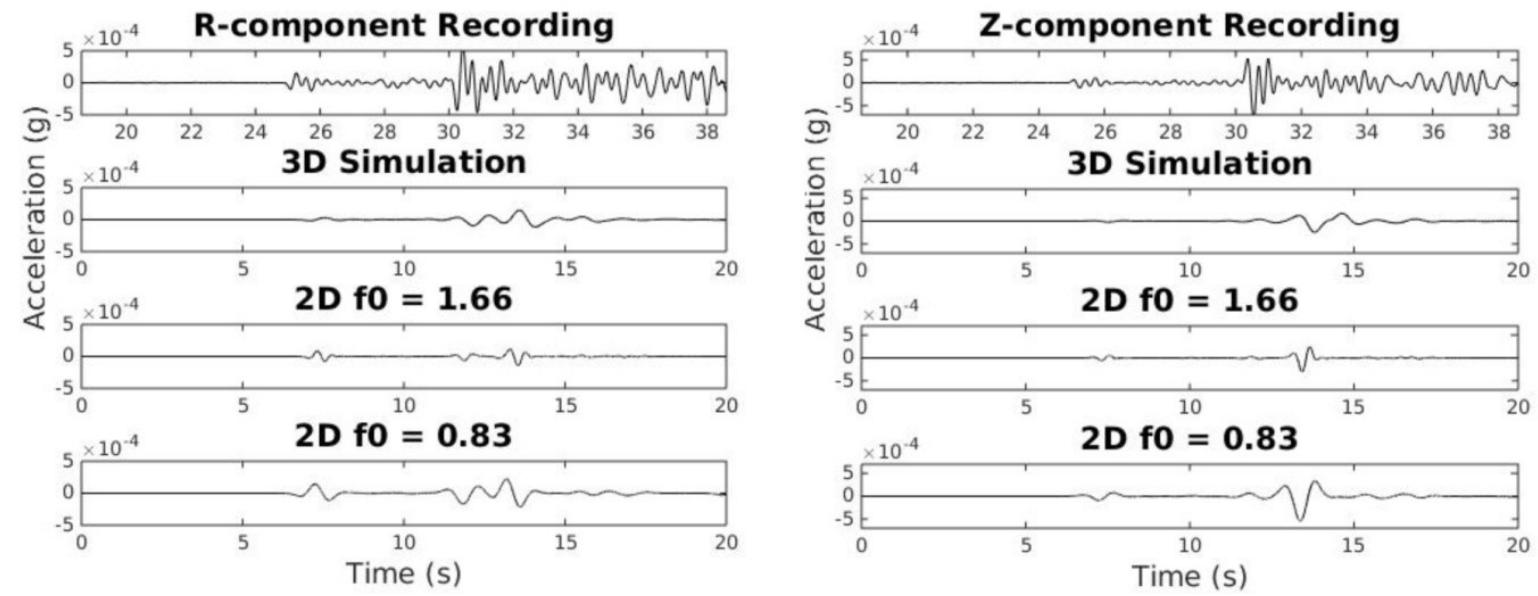

Figure 4.38: Radial and vertical time series for the Ladysmith recording at KB1O and the simulations with a global centroid moment tensor. A Butterworth bandpass filter with corner frequencies 0.01 and $3.2 \mathrm{~Hz}$ was applied. 
R-çomponent Recording BP 0.01-2.0Hz
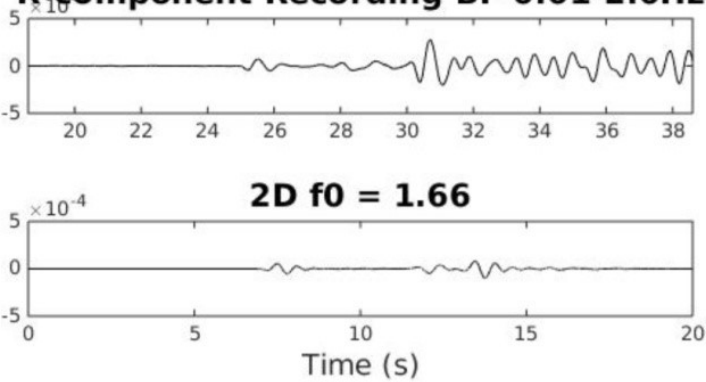

Z-component Recording BP 0.01-2.0Hz

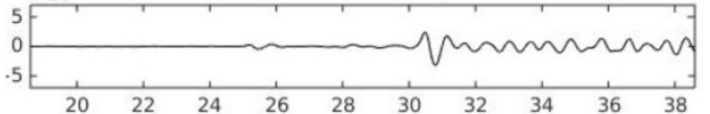

2D fO $=1.66$

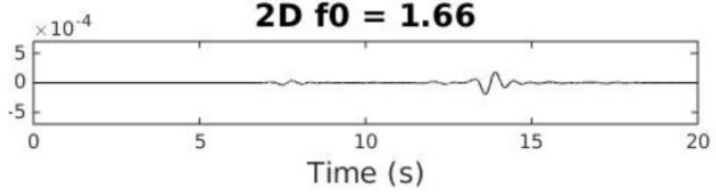

Figure 4.39: Radial and vertical time series for the Ladysmith recording at $K B 10$ and the $2 \mathrm{D}$ simulation with $\mathrm{fO}=1.66 \mathrm{~Hz}$. $\mathrm{A}$ Butterworth bandpass filter with corner frequencies 0.01 and $2.0 \mathrm{~Hz}$ was applied.
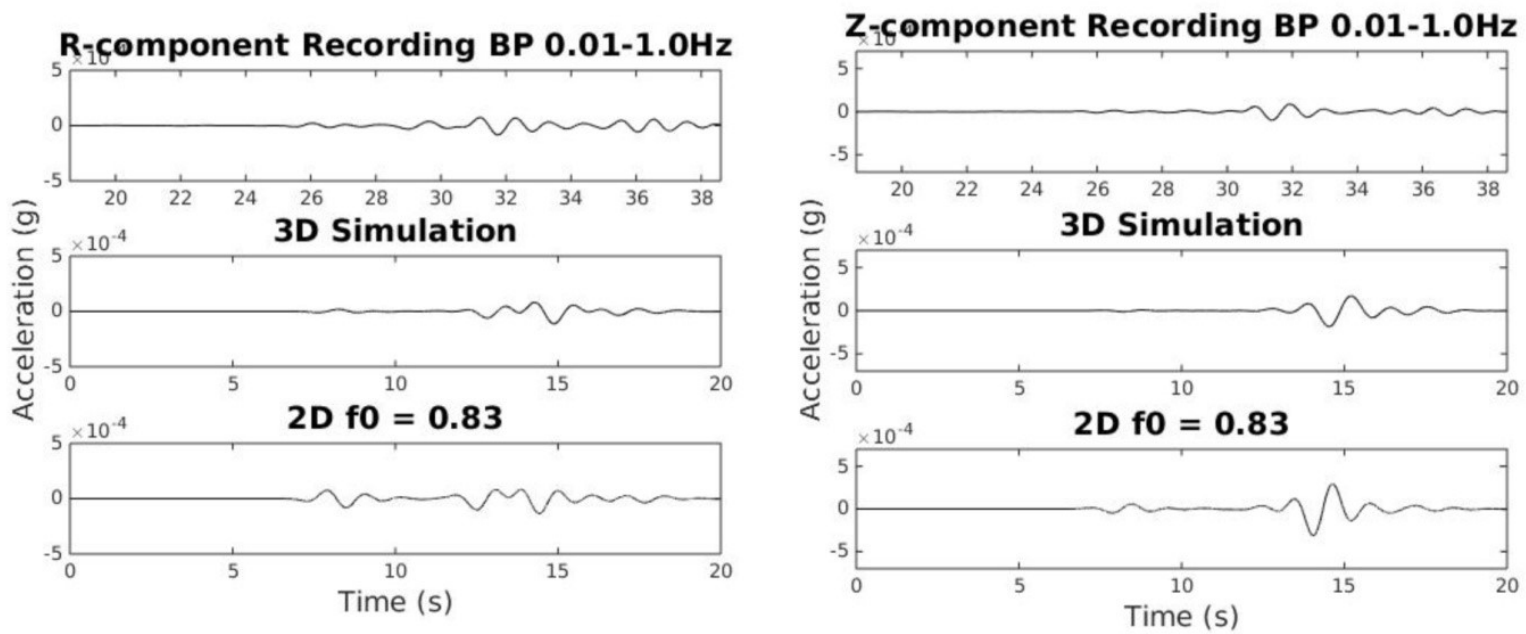

Figure 4.40: Radial and vertical time series for the Ladysmith recording at $\mathrm{KB} 10$ and the 3D simulation and 2D simulation with $\mathrm{f}_{0}$ $=0.83 \mathrm{~Hz}$. A Butterworth bandpass filter with corner frequencies 0.01 and $1.0 \mathrm{~Hz}$ was applied.

\subsubsection{Modelling using Moment Tensor from Ma and Audet (2014)}

This moment tensor source was derived from the fault plane solution found in Ma and Audet (2014), as described in Chapter 3.1.1. It has a depth of $14 \mathrm{~km}$ and the seismic moment is $1.32 \mathrm{x}$ $10^{16} \mathrm{Nm}$. The filtered Fourier spectral accelerations for each simulation compared to the recorded values are shown in Figure 4.41. The $2 \mathrm{D}$ simulation with a $\mathrm{f}_{0}=1.83 \mathrm{~Hz}$ shows a good agreement between the simulated spectra and the recorded spectra for frequencies below $2.5 \mathrm{~Hz}$. The other $2 \mathrm{D}$ simulation using this source, shows a larger than recorded spectral values for frequencies below $2 \mathrm{~Hz}$. The 3D simulation agrees well with the recorded values up to $0.9 \mathrm{~Hz}$. 

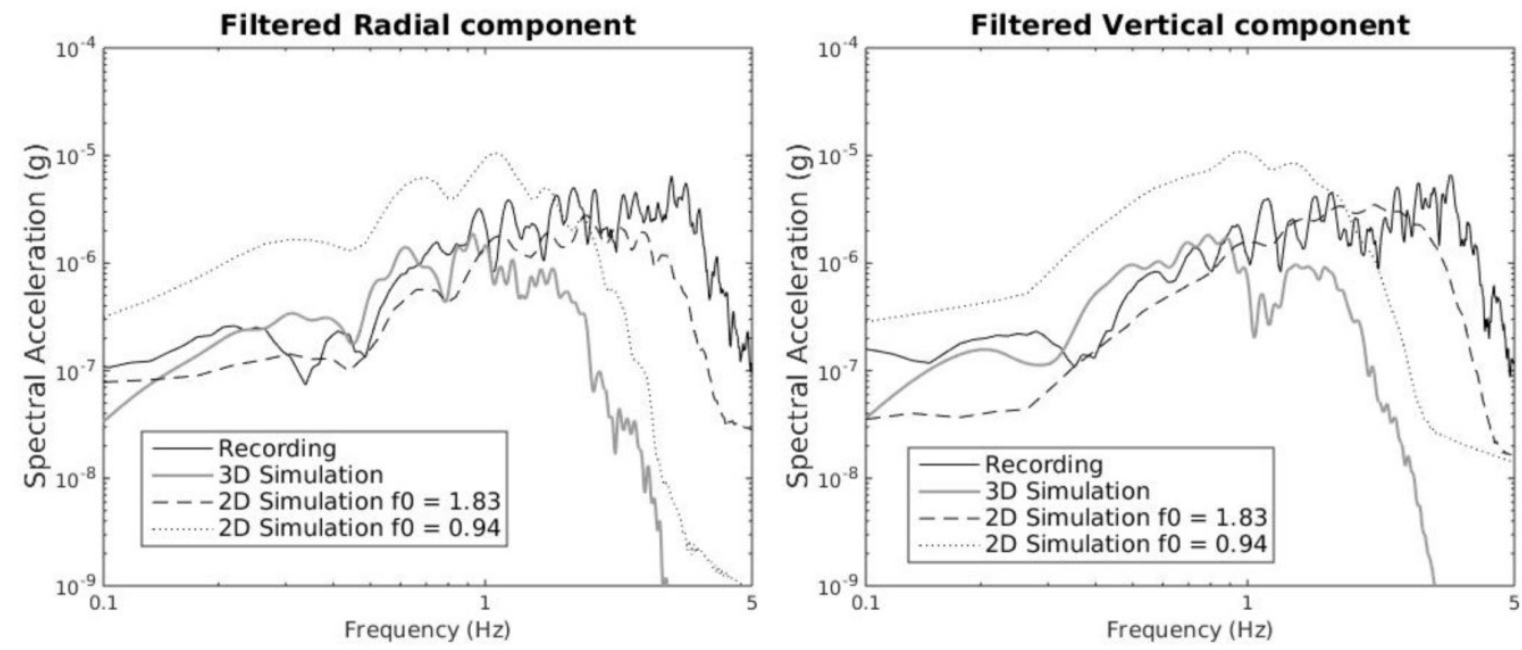

Figure 4.41: Radial and Vertical spectral accelerations using a moment tensor source from Ma and Audet (2014). These have been filtered using an 8-pole Butterworth bandpass filter with corner frequencies 0.01 and $3.2 \mathrm{~Hz}$.

The time series for each simulation and the recorded motions are shown in Figure 4.42, where a bandpass filter between 0.01 and $3.2 \mathrm{~Hz}$ was applied. Due to the abundance of higher frequencies within the recorded motions, the peak values are larger than the simulated values. The peak values between the simulated and recorded motions match better with a narrow filter centered around the dominant frequency of the simulations as shown in Figure 4.43 and Figure 4.44 .
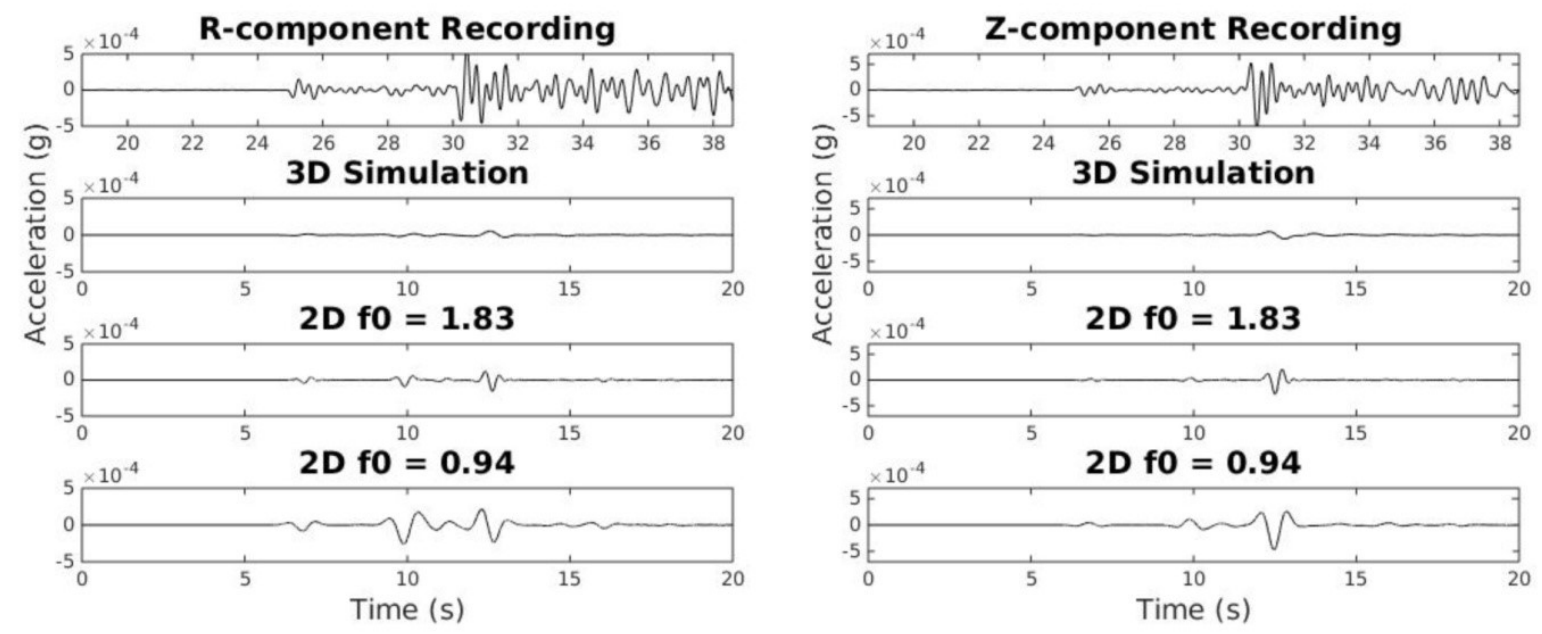

Figure 4.42: Radial and vertical time series for the Ladysmith recording at KB1O and the simulations with a moment tensor source from Ma and Audet (2014). A Butterworth bandpass filter with corner frequencies 0.01 and $3.2 \mathrm{~Hz}$ was applied. 
R-component Recording BP 0.01-2.0Hz
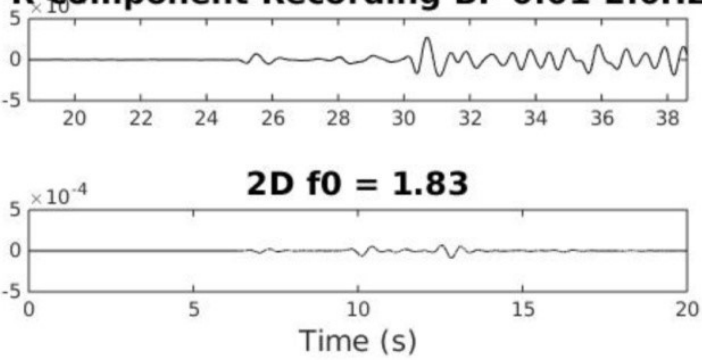
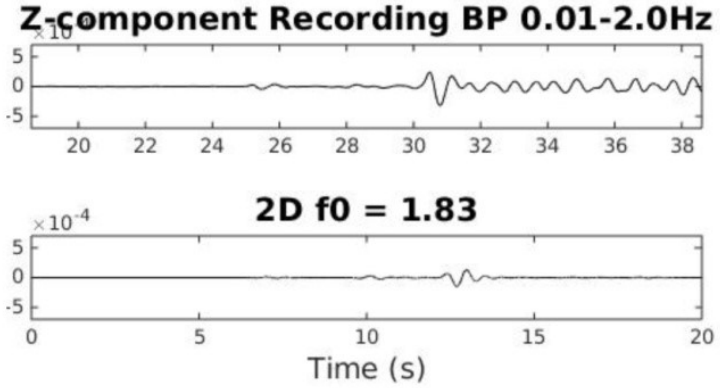

Figure 4.43: Radial and vertical time series for the Ladysmith recording at $K B 1 O$ and the $2 \mathrm{D}$ simulation with $\mathrm{f}_{0}=1.83 \mathrm{~Hz}$. $\mathrm{A}$ Butterworth bandpass filter with corner frequencies 0.01 and $2.0 \mathrm{~Hz}$ was applied.
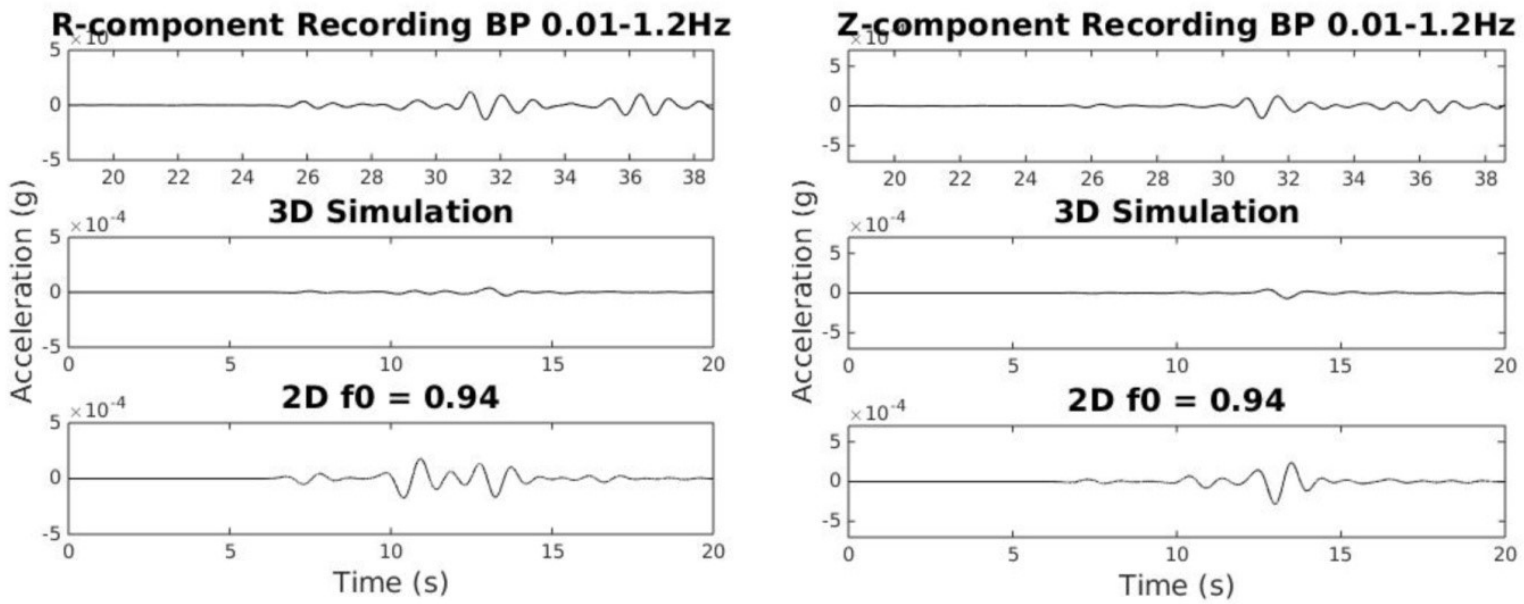

Figure 4.44: Radial and vertical time series for the Ladysmith recording at $K B 10$ and the 3D simulation and 2D simulation with $\mathrm{f}_{0}$ $=0.94 \mathrm{~Hz}$. A Butterworth bandpass filter with corner frequencies 0.01 and $1.2 \mathrm{~Hz}$ was applied.

\subsubsection{Discussion}

As seen in the previous sections, the 2D simulations with a higher dominant frequency better matched the spectral accelerations, whereas the 2D simulations with a lower dominant frequency better matched the peak values of the time series when a narrow filter was applied to the recorded motions. The 3D simulations were a combination of the two. These simulations matched the lower frequency portion of the recorded spectral accelerations, and sometimes matched the amplitude of the recorded acceleration time series if a strong filter was applied. There may be several reasons for the consistent performance between these simulations, however the main factor is likely the regional attributes of eastern North America. The moment 
tensor sources were derived from several filtered time series from stations which surround the earthquake, so it should be expected that these sources would match the recorded motions more closely. However due to the nature of seismicity in eastern North America, mainly the fact that earthquakes in this region are generally richer in higher frequencies than most other regions, a higher dominant frequency is a better representation of earthquake sources in this region.

The discrepancy between the different simulation methods becomes important when simulating motions within the basin. The ground motions in the Kinburn basin recording at station KB2O (the soil station which will be discussed in Section 6.2) showed a strong presence of frequencies around $0.72 \mathrm{~Hz}$ (Section 3.1.2, Figure 3.2 and Figure 3.3). Simulating the Ladysmith earthquake with a dominant frequency close to the KB2O fundamental site frequency may produce higher values around this frequency without necessarily simulating the proper site effects. However, if the earthquake is simulated with a higher dominant frequency away from the fundamental site period, then the presence of higher values at the fundamental site period could be explained as a contribution from either site or basin effects. 


\subsection{References}

Bent, A.L., M. Lamontagne, V. Peci, S. Halchuk, G.R. Brooks, D. Motazedian, J.A. Hunter, J. Adams, C. Woodgold, J. Drysdale, S. Hayek, W. N. Edwards (2015). The 17 May 2013 M 4.6 Ladysmith, Quebec, Earthquake, Seismol. Res. Lett., 86, 460-476. doi: 10.1785/0220140138

Bracewell, R.N. (2000). The Fourier Transform and its Applications, Third Edition: McGraw-Hill Book Co. New York.

Boore, D. M. (2003), Simulation of ground motion using the stochastic method, Pure and Applied Geophysics, 160, 635-676.

Chapman, C.H. (2004). Fundamentals of Seismic Wave Propagation, Cambridge Univ. Press.

Crane, S., and D. Motazedian (2014). Low-frequency scaling applied to stochastic finite fault modeling. J. of Seismol., 18 (1): 109-122

Dziewonski, A. M., T.-A. Chou and J. H. Woodhouse (1981). Determination of earthquake source parameters from waveform data for studies of global and regional seismicity, J. Geophys. Res., 86, 2825-2852. doi:10.1029/JB086iB04p02825

Ekström, G., M. Nettles, and A. M. Dziewonski (2012) The global CMT project 2004-2010: Centroid-moment tensors for 13,017 earthquakes, Phys. Earth Planet. Inter., 200-201, 1-9. doi:10.1016/j.pepi.2012.04.002

Global CMT project website: http://www.globalcmt.org last accessed August, 2016.

Li, D., D. Helmberger, R.W. Clayton, and D. Sun (2014). Global synthetic seismograms using a 2D finite-difference method. Geophys. J. Int., 197 (2), 1166-1183.

Ma, S., and P. Audet (2014). The 5.2 magnitude earthquake near Ladysmith, Quebec, 17 May 2013: Implications for the seismotectonics of the Ottawa-Bonnechere graben, Can. J. Earth Sci., 51, 439-451, doi: 10.1139/cjes-2013-0215.

Miksat J., T.M. Müller, and F. Wenzel (2008). Simulating three-dimensional seismograms in 2.5Dimensional structures by combining two-dimensional finite difference modelling and ray tracing. Geophys. J. Int., 174, 309-315.

Motazedian, D., and G. M. Atkinson (2005), Stochastic finite-fault modeling based on a dynamic corner frequency, Bull. Seism. Soc. Am., 95, 995-1010.

Pilant, W.L. (1979). Elastic Waves in the Earth. Elsevier Scientific Publishing Company. 


\section{Sensitivity of Models and Parameters}

There are several ways of constructing a suitable model for simulating ground motions. There are also several parameters within the simulations which can change the resulting ground motions to a varying degree. Some of the parameters used in this study are well constrained, whereas others are commonly estimated based on their performance within a simulation. Although the parameters were changed in the $2 \mathrm{D}$ models, the sensitivity of these parameters should apply to the $3 \mathrm{D}$ simulations as well, based on the fact that the $2 \mathrm{D}$ seismograms have been corrected to represent a 3D seismogram (Section 4.1.1). The sensitivity tests were completed on 2D simulations only in an effort to reduce the computing time where advanced computer systems are needed, since these tests using 3D simulations would have required significantly more computing time and resources.

Various tests were conducted by changing the properties of the simulations. These included changing the model on which a simulation was performed, varying the size and shape of different rock types in the simulations, and varying a specific parameter while holding the others unchanged. Table 5.1 lists the different sets of simulations and provides a brief description of the adjusted parameter, along with the variability observed in the peak acceleration. A list of the different soil and rock properties used in the simulation is displayed in Table 5.2. The percent difference in peak motions is defined by:

$$
\text { Percent Difference }=\frac{\left|A_{\text {peak }}^{\text {ref }}-A_{\text {peak }}^{\text {sim }}\right|}{\left|A_{\text {peak }}^{\text {ref }}\right|} \times 100 \%
$$


where $\left|A_{\text {peak }}^{\text {ref }}\right|$ is the absolute peak value in the reference simulation, and $\left|A_{\text {peak }}^{\text {sim }}\right|$ is the absolute peak value in the simulation. Although there are many possible error schemes, that of the percent difference of peak amplitudes provides a simple way of quantifying the amplitude changes (Miksat et al., 2008).

\begin{tabular}{|c|c|c|c|c|}
\hline Section & $\begin{array}{l}\text { Number of } \\
\text { Simulations }\end{array}$ & Summary & Values used & $\begin{array}{c}\text { Percent } \\
\text { Difference in } \\
\text { peak motion }\end{array}$ \\
\hline 5.2 .1 & 3 & $\begin{array}{l}\text { Different models using one } \\
\text { set of rock parameters }\end{array}$ & $\begin{array}{c}\text { Reference } \\
\text { Model, Large Box } \\
\text { Model, and } \\
\text { Layered Model }\end{array}$ & Less than $0.003 \%$ \\
\hline 5.2 .2 & 3 & $\begin{array}{l}\text { Different models using two } \\
\text { sets of rock parameters }\end{array}$ & $\begin{array}{c}\text { Reference } \\
\text { Model, Large Box } \\
\text { Model, and } \\
\text { Layered Model }\end{array}$ & $9-40 \%$ \\
\hline 5.2 .3 & 3 & $\begin{array}{l}\text { Different sets of rock } \\
\text { parameters using the } \\
\text { Large Box Model }\end{array}$ & Rock Types I-III & $2-51 \%$ \\
\hline 5.2 .4 & 4 & $\begin{array}{l}\text { Different sets of rock } \\
\text { parameters using the } \\
\text { Layered Model }\end{array}$ & Rock Types I-XIV & $0-46 \%$ \\
\hline 5.3 .1 & 3 & $\mathrm{Q}$ value of the rock & $1000,750,500$ & $1-4 \%$ \\
\hline 5.3 .2 & 3 & Q value of the soil & $100,50,20$ & $0-47 \%$ \\
\hline 5.3 .3 & 3 & Velocity of the soil (Vs) & $\begin{array}{c}150 \mathrm{~m} / \mathrm{s}, 250 \mathrm{~m} / \mathrm{s} \\
\text { and } 350 \mathrm{~m} / \mathrm{s}\end{array}$ & $0-18 \%$ \\
\hline
\end{tabular}

Table 5.1: A list of the tests performed to determine the sensitivity of different models or parameters.

\begin{tabular}{|c|c|c|c|c|}
\hline Name of Parameter set & $V p(\mathrm{~m} / \mathrm{s})$ & Vs $(\mathrm{m} / \mathrm{s})$ & Density $\left(\mathrm{kg} / \mathrm{m}^{3}\right)$ & Q value \\
\hline Rock Type I & 6500 & 3500 & 2800 & 1000 \\
\hline Rock Type II & 6000 & 2700 & 2600 & 1000 \\
\hline Rock Type III & 6250 & 3100 & 2700 & 1000 \\
\hline Rock Type IV & 6050 & 2730 & 2650 & 1000 \\
\hline Rock Type V & 6080 & 2790 & 2680 & 1000 \\
\hline Rock Type VI & 6130 & 2830 & 2700 & 1000 \\
\hline Rock Type VII & 6250 & 2990 & 2750 & 1000 \\
\hline
\end{tabular}




\begin{tabular}{|c|c|c|c|c|}
\hline Rock Type VIII & 6380 & 3250 & 2780 & 1000 \\
\hline Rock Type IX & 6110 & 3580 & 2740 & 1000 \\
\hline Rock Type X & 6090 & 3570 & 2740 & 1000 \\
\hline Rock Type XI & 6010 & 3530 & 2720 & 1000 \\
\hline Rock Type XII & 6180 & 3620 & 2760 & 1000 \\
\hline Rock Type XIII & 6280 & 3670 & 2780 & 1000 \\
\hline Rock Type XIV & 6680 & 3870 & 2880 & 1000 \\
\hline Soil Type I & 1600 & 150 & 1600 & 100 \\
\hline Soil Type II & 1700 & 250 & 1600 & 100 \\
\hline Soil Type III & 1800 & 350 & 1600 & 100 \\
\hline
\end{tabular}

Table 5.2: A list of the different parameters for rock and soil used in the simulations.

\subsection{Factors affecting Computing Resources}

The models in this study were designed based on two important factors: the stability for simulations with different parameters in the same model, and the amount of required computing resources needed to complete a simulation. Primarily, all models in this chapter were two dimensional ones, because they significantly reduced the processing time and memory needed for any simulation. This has been previously shown in Section 4.2.1, as the 2D simulation for a homogeneous volume was completed in 24.5 core*hours, and a 3D simulation was completed in 21,240 core*hours. It has also been shown in Appendix II, where 2D simulations had processing times between 20 and 220 core*hours, whereas the 3D simulations had processing times larger than 20,000 core*hours.

\subsubsection{Mesh Size}

One important factor affecting the computing time and memory of a simulation is the mesh size within a model. As discussed in Section 1.5.2, the size of the mesh determines the accuracy in a numerical simulation. However, the mesh size also has an impact on the size of the 
required time step for a stable solution, as also discussed in Section 1.5.1. The meshes within the models were designed to allow the required time step to be as large as possible, while maintaining the desired frequency accuracy of up to $5 \mathrm{~Hz}$.

The addition of soil to a model created a very high density mesh within and surrounding the surface. This can be seen in the reference model shown in Figure 5.1 for example, where the entire model had a mesh spacing of 400 metres, except the area surrounding the basin where the mesh was much denser. This greatly increased the processing time for these simulations, as each mesh point adds an additional 25 grid points for the solution (discussed in Appendix I). Comparing the run time for a simulation using the reference model, for example in Section 5.2.1, the processing time was around 60 core*hours for a total simulated time of 60 seconds. The processing time using the $2 \mathrm{D}$ homogeneous volume in Section 4.2 was 24.5 core*hours for 100 seconds of total simulated time. Adding the soil basin to a model more than doubled the processing time required for a simulation to a homogeneous model which is comparable in total size, even though the mesh within the homogeneous volume was half the size of the majority of the $2 \mathrm{D}$ reference model. A comparison between a simulation using the $2 \mathrm{D}$ homogeneous model, $2 \mathrm{D}$ reference model and $3 \mathrm{D}$ homogeneous model is shown in Table 5.3. Even though the 2D Reference model has significantly less total degree of freedom than the 2D Homogeneous model, the time step was much smaller resulting in more calculations to complete over a given time.

\begin{tabular}{|c|c|c|c|}
\hline Model & 2D Homogeneous & 2D Reference & 3D Homogeneous \\
\hline Mesh Spacing $(\mathrm{m})$ & 200 & $\begin{array}{c}\text { Varied between 5- } \\
400\end{array}$ & 200 \\
\hline $\begin{array}{c}\text { Number of time } \\
\text { steps }\end{array}$ & 100,000 & 600,000 & 100,000 \\
\hline Total time simulated & 100 seconds & 60 seconds & 100 seconds \\
\hline
\end{tabular}




\begin{tabular}{|c|c|c|c|}
\hline Number of Elements & 60,000 & 24,572 & $6,000,000$ \\
\hline $\begin{array}{c}\text { Total degrees of } \\
\text { freedom }\end{array}$ & $1,932,688$ & 795,324 & $1,200,941,412$ \\
\hline $\begin{array}{c}\text { Processing time } \\
\text { (core*hours) }\end{array}$ & 24.8 & 57.2 & $21,592.1$ \\
\hline
\end{tabular}

Table 5.3: A comparison of mesh size, time simulated and processing time between different simulations which used different models.

\subsubsection{Mesh Creation}

One other significant consideration for creation of the models, was the ability to create a suitable mesh for a numerical simulation. There are various conditions which had to be met in order for a simulation to remain stable. One of these properties is the shape of each element. Any individual element could not be skewed more than $75 \%$, and there could not be any negative Jacobians. These conditions were difficult to satisfy when there was a large change in the grid size and when there were curved boundaries, both of which were present in this study. A strict process to develop a mesh within one of these models was created in order to satisfy these conditions. Even using available advanced modelling and meshing software, this was a very time consuming process to ensure the model had a stable solution during simulations.

\subsection{Model Geometry Tests}

In this research three different model geometries were used for sensitivity tests:

(1) the Reference Model,

(2) a Large Box Model, and

(3) a Layered Model.

The Reference Model, shown in Figure 5.1 with the mesh, had 3 surfaces: the basin surface representing the in-fill material; a square surface surrounding the basin which extends roughly 400 metres in all directions from the basin; and the surface which represents the rest of the 
model. The basin surface was meshed using an unstructured grid of varying size between $20 \mathrm{~m}$ at the impedance boundary, represented as the boundary between surface 1 and surface 2 , to $10 \mathrm{~m}$ at the free surface. The second surface surrounding the basin was meshed with an increasing size from the basin edge to the outside edges of the surface. The last surface was meshed with a grid size of $400 \mathrm{~m}$ reducing its size twice as it reaches the edges of the second surface. The boundaries of these surfaces, along with all the others, were decided based on the ability to create a suitable mesh within the model, and to minimize the number of mesh elements. Portions of this mesh structure are shown in Figure 5.1. 


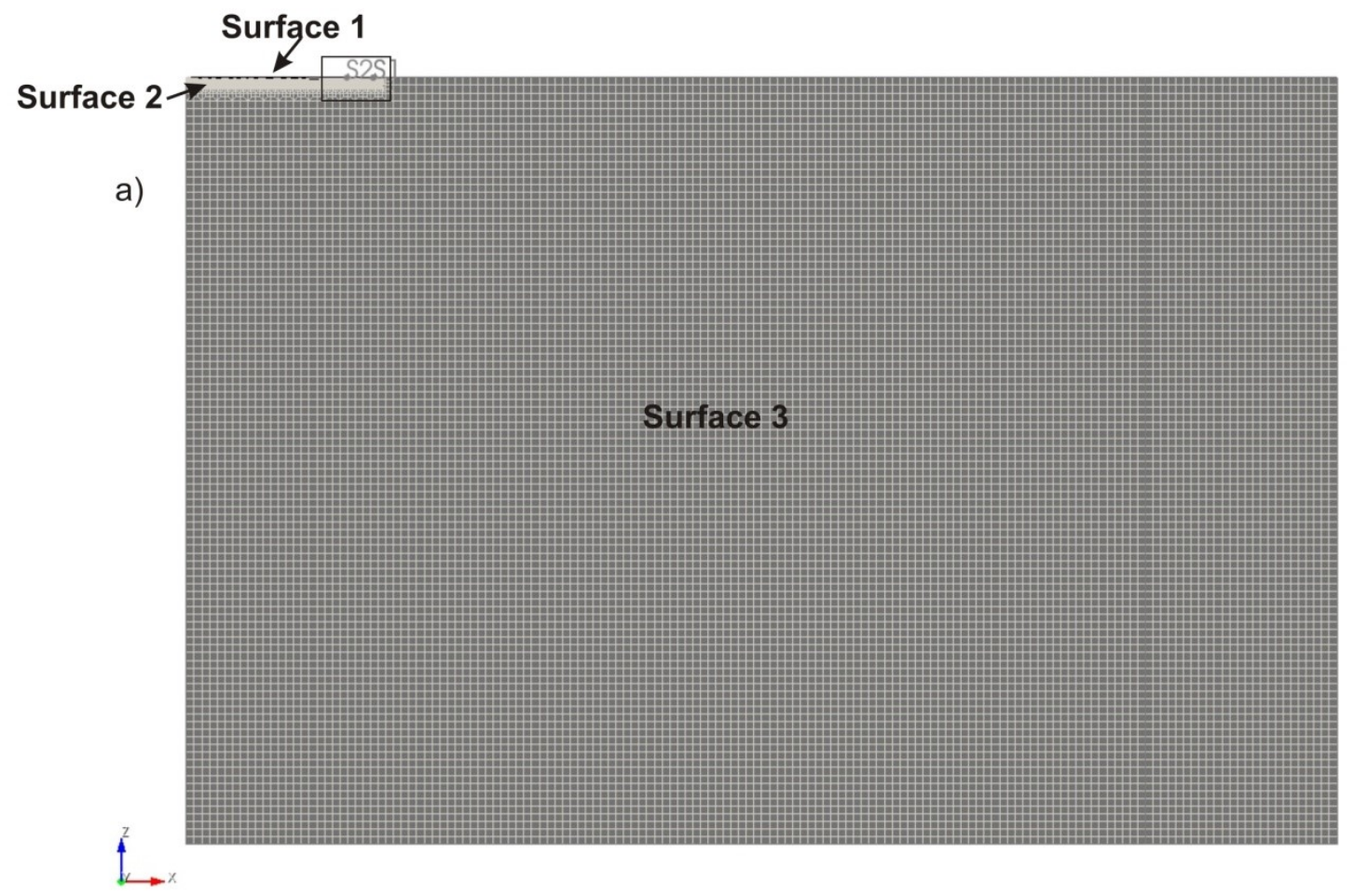

S2 Surface 1

S1

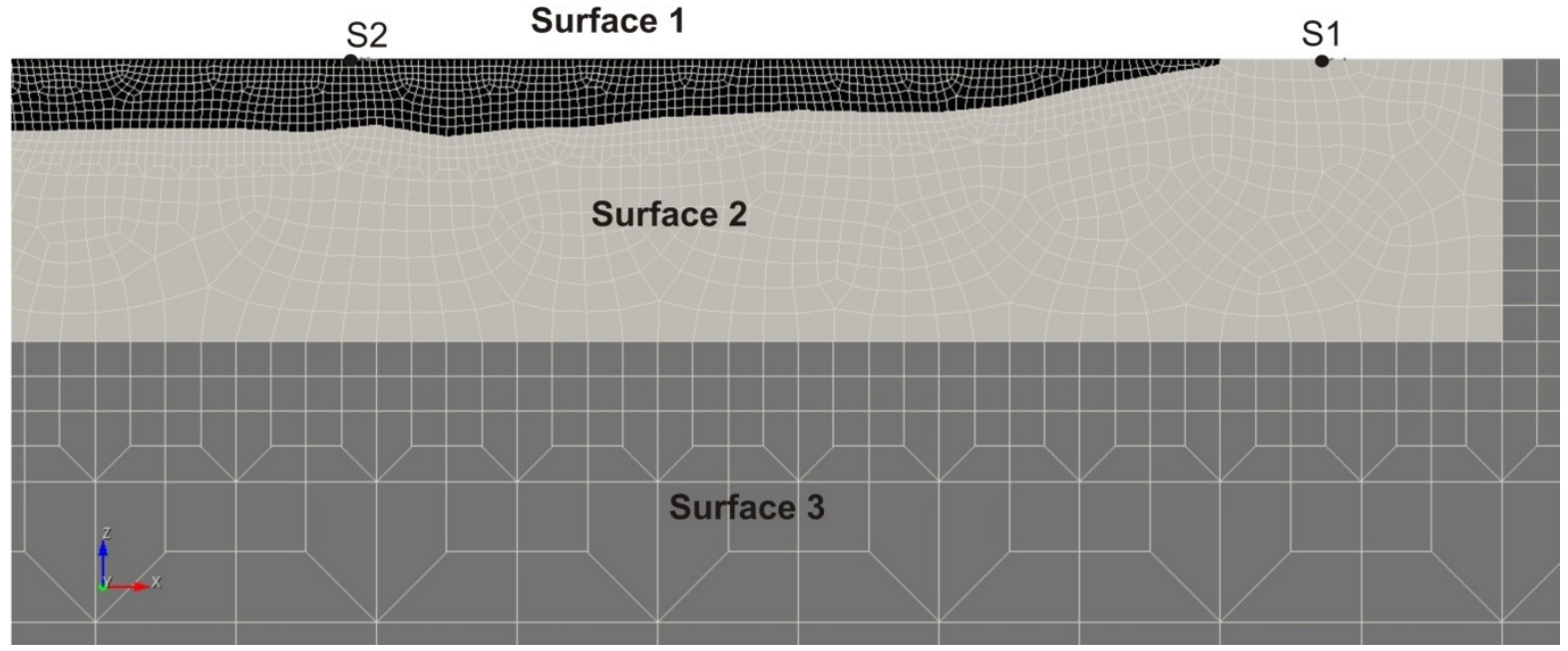

Figure 5.1: The Reference Model used in these simulations. The entire model with the mesh is shown in a), and b) displays a close up of a portion of the basin, surrounding surfaces and stations S1 and S2 as outlined in a). The white lines outline the mesh in the model.

The second model used for these tests was the Large Box Model, shown in Figure 5.2. This model was similar to the reference model, however it had an additional surface between the surface surrounding the basin and the rest of the model. This surface extended $4.0 \mathrm{~km}$ from the edge of the surface surrounding the basin. The mesh for this model was identical to the reference 
model where the large box contained the mesh which doubled in size from the surface surrounding the basin to the edges of the model. Portions of this mesh in this model are shown in Figure 5.2.
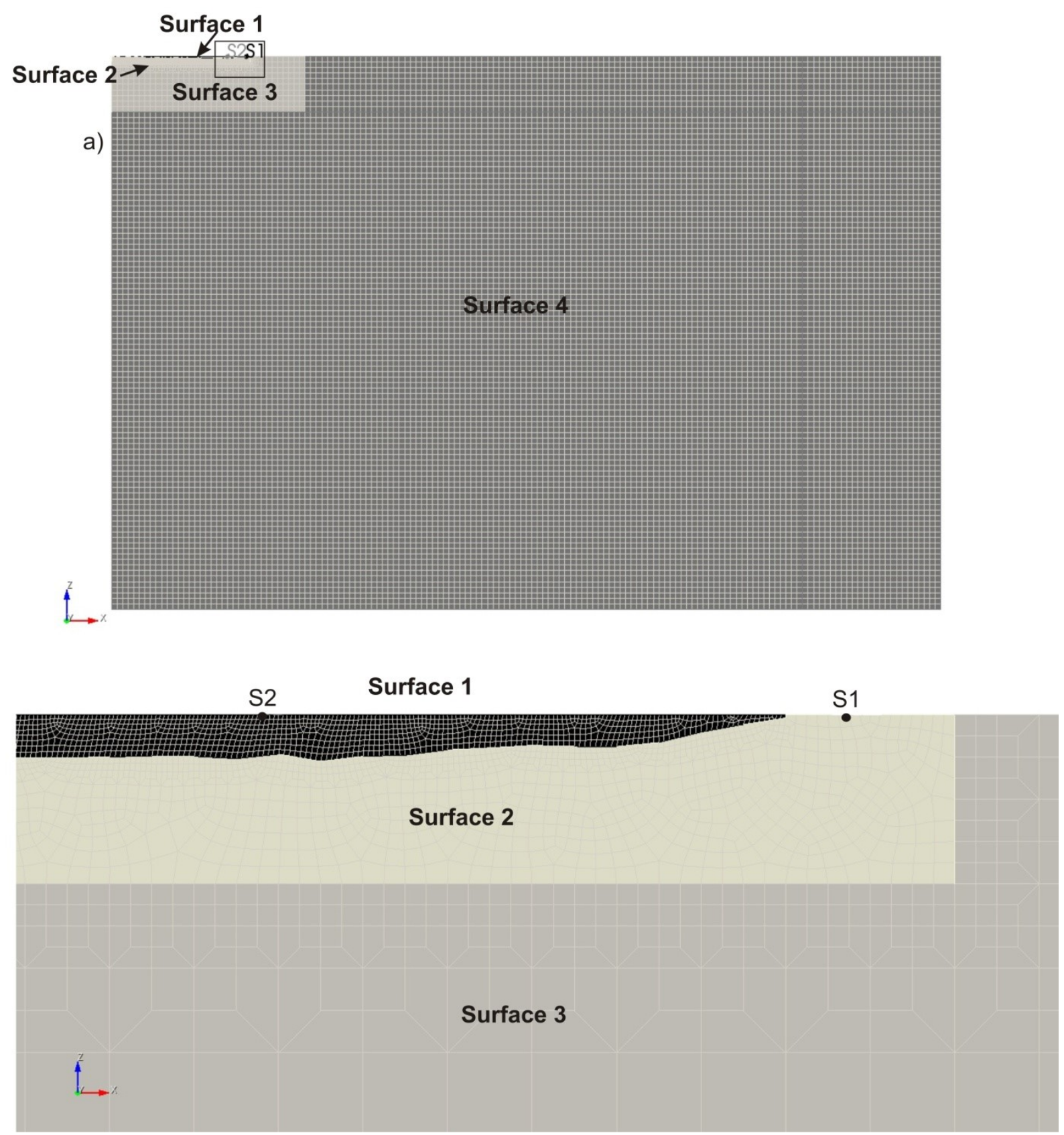

Figure 5.2: The Large Box Model used in these simulations. The entire model with the mesh is shown in a), and b) displays a close up of a portion of the basin, surrounding surfaces and stations S1 and S2 as outlined in a). The white lines outline the mesh in the model. 
The last model used was a Layered Model, shown in Figure 5.3. This model containd surfaces which represent different depth interfaces. The depths at which the boundaries between these surfaces occur were: $400 \mathrm{~m}, 1.2 \mathrm{~km}, 2.0 \mathrm{~km}, 4.0 \mathrm{~km}, 10 \mathrm{~km}, 20 \mathrm{~km}$, and $30 \mathrm{~km}$. These boundaries were chosen based on the ability to maintain the mesh size in each layer, and to enable the division of the model into layers which provided a good representation of the velocity profiles for the area (Motazedian et al., 2013). The mesh was similar to the other models, however the mesh surrounding the basin was slightly different from the others due to the construction of this surface. This mesh is shown in Figure 5.3. 


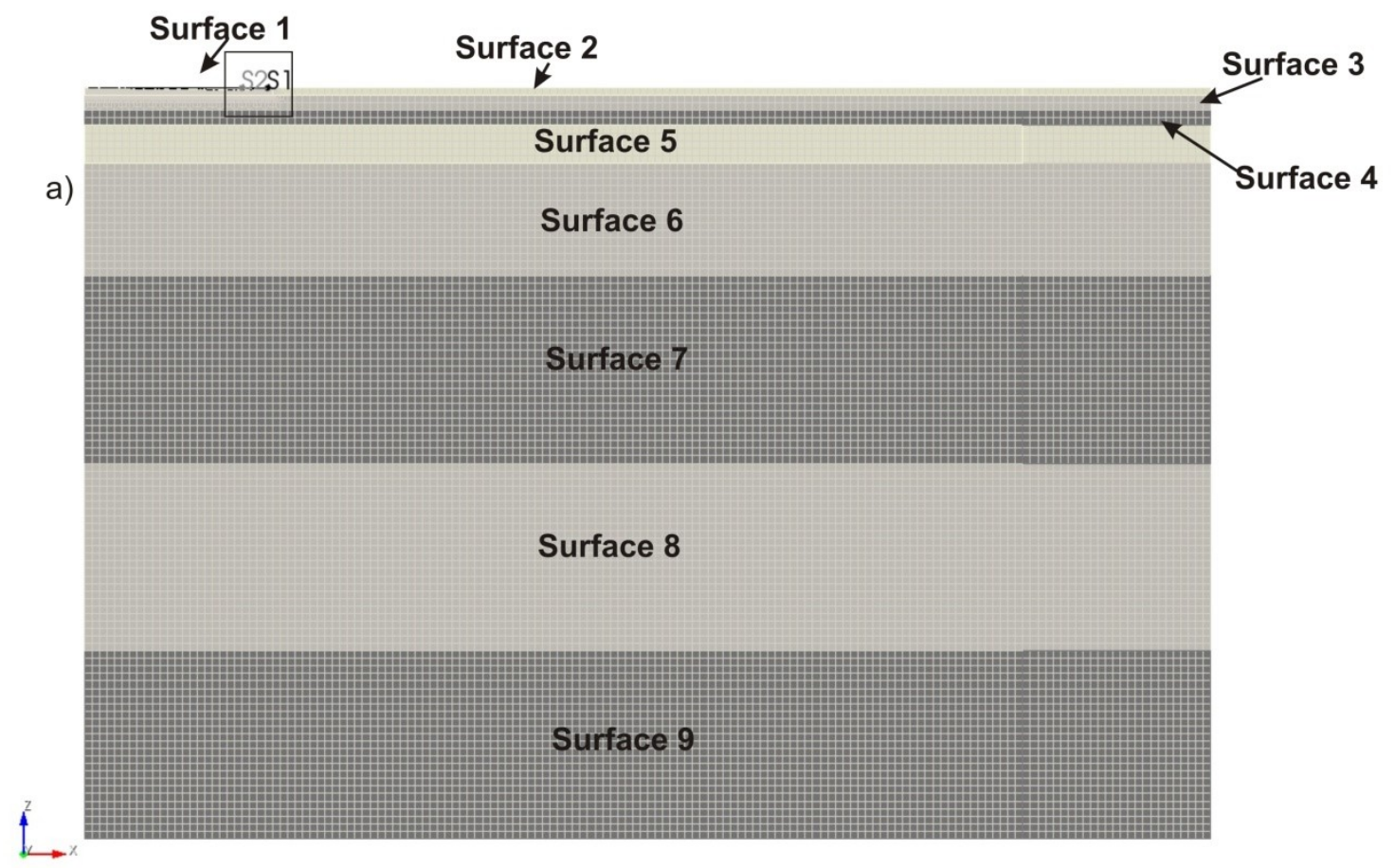

Surface 1

S2

S1

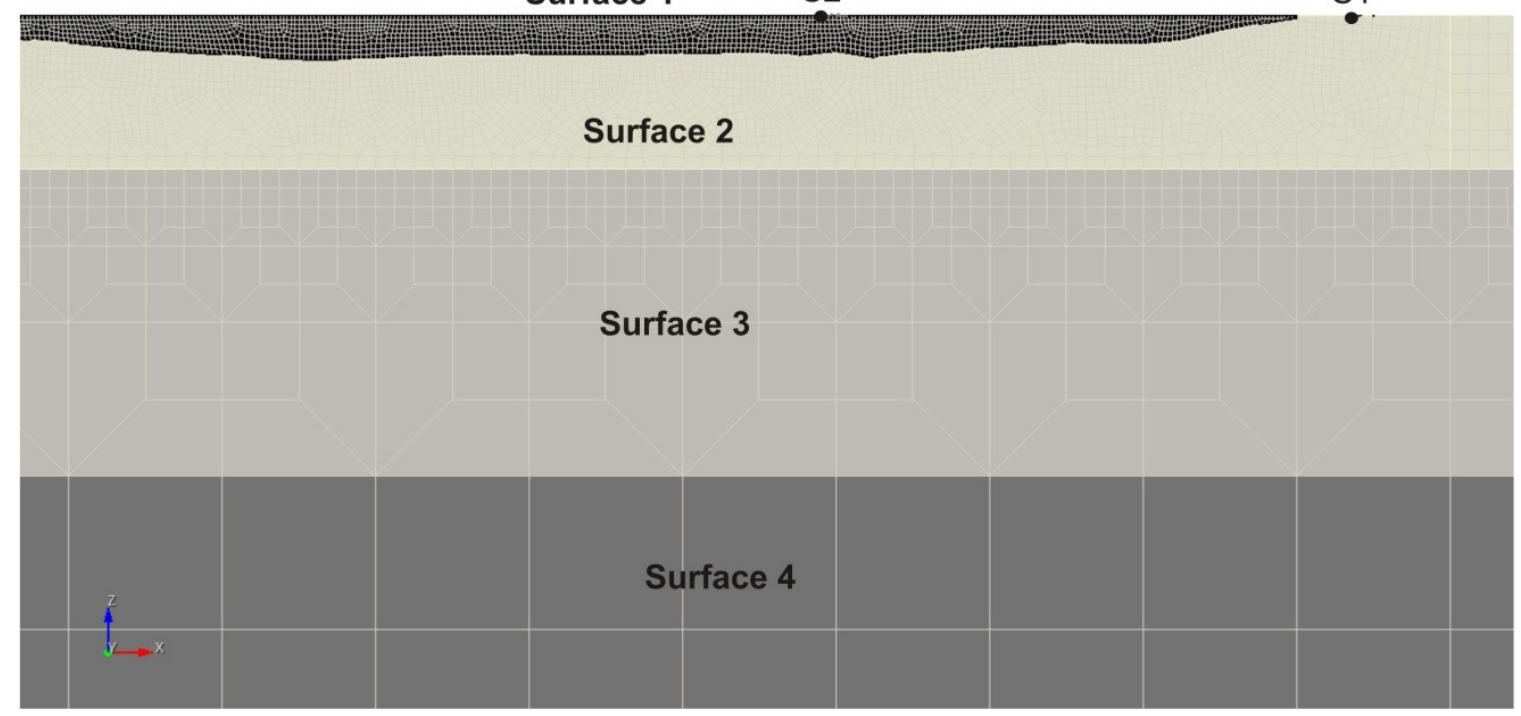

Figure 5.3: The Layered Model used in these simulations. The entire model with the mesh is shown in a), and b) displays a close up of a portion of the basin, surrounding surfaces and stations S1 and S2 as outlined in a). The white lines outline the mesh in the model.

\subsubsection{Model Sensitivity: Single Set of Rock Parameters}

The first test was performed on all three different models. The basin surface was represented as the average soil properties, Soil Type I (Hunter et al., 2010), and all other surfaces had the average rock properties of eastern North America, Rock Type I (Bent et al., 2015). Table 5.4 lists the 
properties for the surfaces in the different models for each simulation. The source for this simulation, and with all simulations in this chapter was the moment tensor source for the Ladysmith earthquake of 2013 (Bent et al., 2015).

The time series resulted from the basin modeling for the Ladysmith earthquake of each of the models for the horizontal and vertical components of motion are displayed in Figure 5.4 for the S1 receivers or the rock recording stations, and in Figure 5.5 for the S2 receivers or the soil recording stations. As can be seen in these Figures, all of the acceleration time series were nearly identical and the differences could be contributed to the internal error in the program from using different nodal points in the mesh. The differences in peak values are listed in Table 5.5, along with the percent difference from the reference model, as described by Eq. 5.1. The different models produce almost identical results from one another when the parameters were identical, so the differences produced in simulations were not dependent on the model used.

\begin{tabular}{|c|c|c|c|c|c|}
\hline $\begin{array}{c}\text { Surface } \\
\text { Number }\end{array}$ & $\begin{array}{c}\text { Name of } \\
\text { Parameter } \\
\text { set }\end{array}$ & Vp (m/s) & Vs (m/s) & $\begin{array}{c}\text { Density } \\
\left(\mathrm{kg} / \mathrm{m}^{3}\right)\end{array}$ & Q value \\
\hline \multicolumn{7}{|c|}{ Simulation 1: Reference Model (Figure 5.1) } \\
\hline Surface 1 & Soil Type I & 1600 & 150 & 1600 & 100 \\
\hline Surface 2 & Rock Type I & 6500 & 3500 & 2800 & 1000 \\
\hline Surface 3 & Rock Type I & 6500 & 3500 & 2800 & 1000 \\
\hline \multicolumn{7}{|c|}{ Simulation 2: Large Box Model (Figure 5.2) } \\
\hline Surface 1 & Soil Type I & 1600 & 150 & 1600 & 100 \\
\hline Surface 2 & Rock Type I & 6500 & 3500 & 2800 & 1000 \\
\hline Surface 3 & Rock Type I & 6500 & 3500 & 2800 & 1000 \\
\hline Surface 4 & Rock Type I & 6500 & 3500 & 2800 & 1000 \\
\hline \multicolumn{7}{|c|}{ Simulation 3: Layered Model (Figure 5.3) } \\
\hline Surface 1 & Soil Type I & 1600 & 150 & 1600 & 100 \\
\hline Surface 2 & Rock Type I & 6500 & 3500 & 2800 & 1000 \\
\hline Surface 3 & Rock Type I & 6500 & 3500 & 2800 & 1000 \\
\hline Surface 4 & Rock Type I & 6500 & 3500 & 2800 & 1000 \\
\hline Surface 5 & Rock Type I & 6500 & 3500 & 2800 & 1000 \\
\hline
\end{tabular}




\begin{tabular}{|c|c|c|c|c|c|}
\hline Surface 6 & Rock Type I & 6500 & 3500 & 2800 & 1000 \\
\hline Surface 7 & Rock Type I & 6500 & 3500 & 2800 & 1000 \\
\hline Surface 8 & Rock Type I & 6500 & 3500 & 2800 & 1000 \\
\hline Surface 9 & Rock Type I & 6500 & 3500 & 2800 & 1000 \\
\hline
\end{tabular}

Table 5.4: List of parameters used for each surface in the different models for testing the effect of different models with one set of rock parameters.

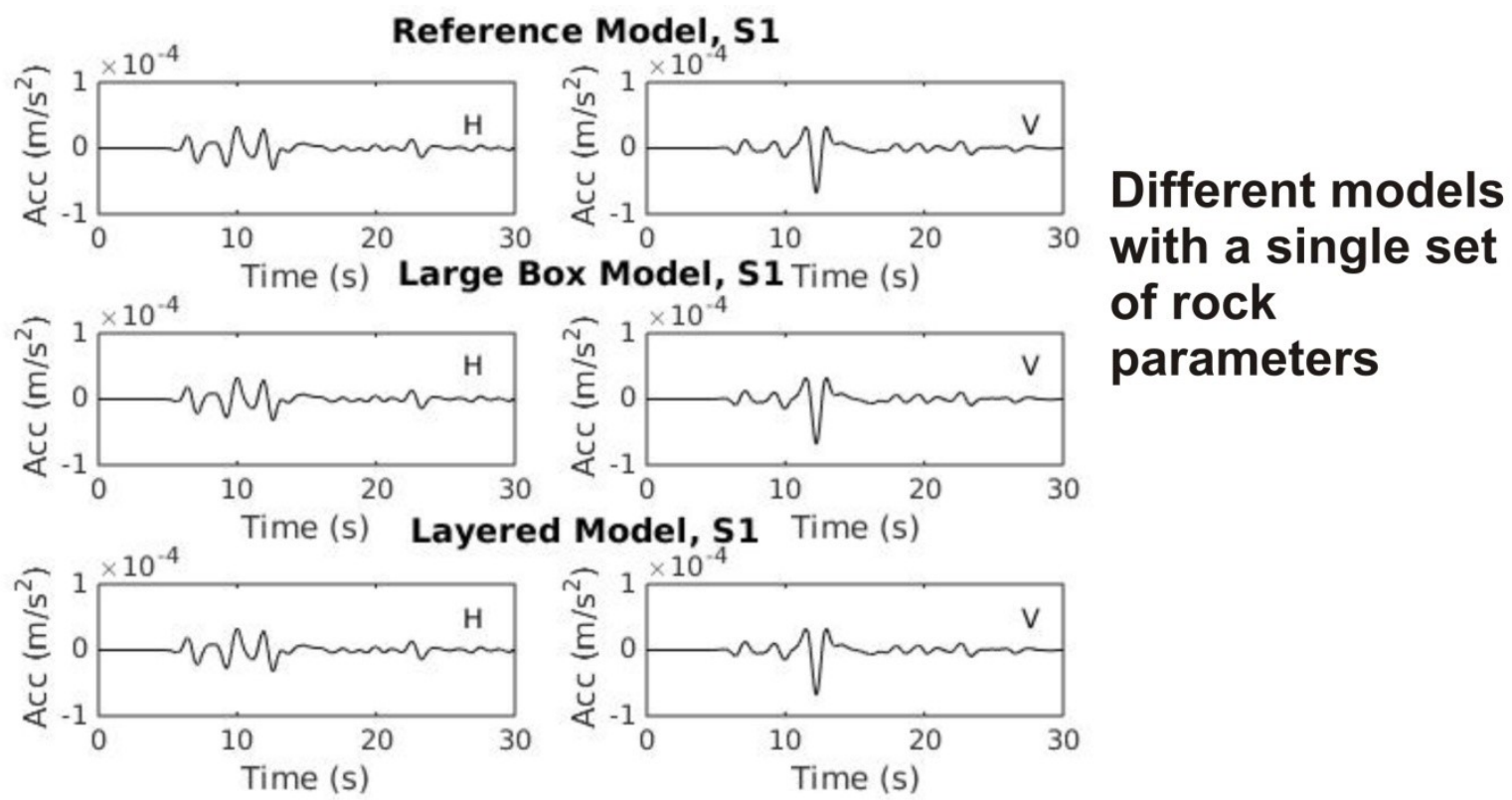

Figure 5.4: Acceleration time series resulted from the basin modeling for the Ladysmith earthquake for the horizontal component (left column) and vertical component (right column) recorded on the station S1 for the different models. The Reference model is the top row, the Large Box Model is the middle row and the Layered Model is the bottom row. 

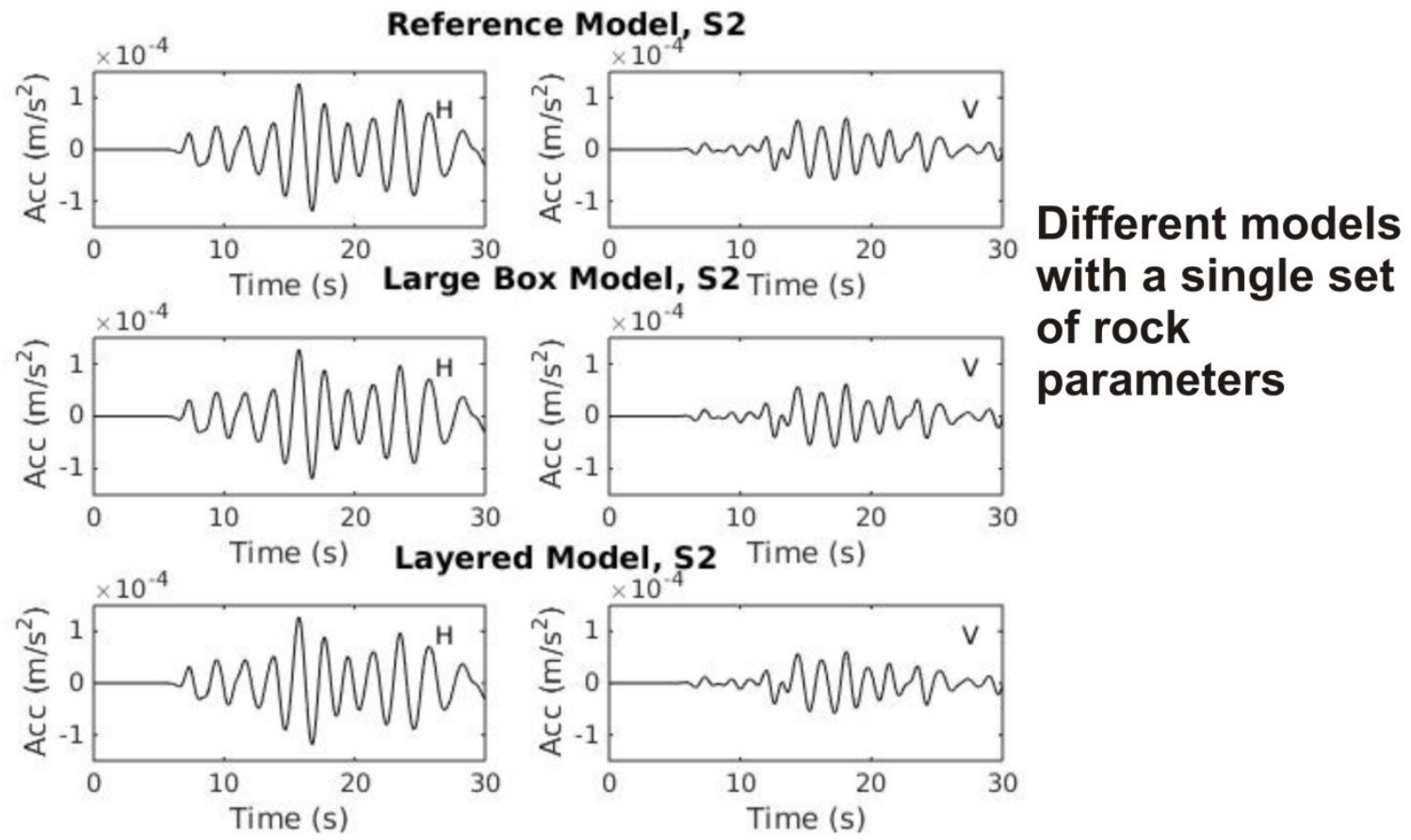

Figure 5.5: Acceleration time series resulted from the basin modeling for the Ladysmith earthquake for the horizontal component (left column) and vertical component (right column) recorded on the station S2 for the different models. The Reference model is the top row, the Large Box Model is the middle row and the Layered Model is the bottom row.

\begin{tabular}{|c|c|c|c|c|c|c|c|c|}
\hline \multirow{2}{*}{ Model } & \multicolumn{3}{|c|}{ Peak Value $\left(\mathrm{m} / \mathrm{s}^{2}\right)$} & \multicolumn{3}{c|}{$\begin{array}{c}\text { Peak Value Difference from } \\
\text { Reference (\%) }\end{array}$} \\
\cline { 2 - 9 } & \multicolumn{2}{|c|}{ Horizontal } & \multicolumn{2}{c|}{ Vertical } & \multicolumn{2}{c|}{ Horizontal } & \multicolumn{2}{c|}{ Vertical } \\
\cline { 2 - 9 } & $\mathrm{S} 1$ & $\mathrm{~S} 2$ & $\mathrm{~S} 1$ & $\mathrm{~S} 2$ & $\mathrm{~S} 1$ & $\mathrm{~S} 2$ & $\mathrm{~S} 1$ & $\mathrm{~S} 2$ \\
\hline $\begin{array}{c}\text { Small } \\
\text { Box } \\
\text { Model }\end{array}$ & $3.29 \mathrm{E}-05$ & $1.27 \mathrm{E}-04$ & $6.79 \mathrm{E}-05$ & $6.15 \mathrm{E}-05$ & & & & \\
\hline $\begin{array}{c}\text { Large } \\
\text { Box } \\
\text { Model }\end{array}$ & $3.29 \mathrm{E}-05$ & $1.27 \mathrm{E}-04$ & $6.79 \mathrm{E}-05$ & $6.15 \mathrm{E}-05$ & $\begin{array}{c}0.000 \\
0\end{array}$ & $\begin{array}{c}0.000 \\
0\end{array}$ & $\begin{array}{c}0.002 \\
9\end{array}$ & $\begin{array}{c}0.001 \\
6\end{array}$ \\
\hline $\begin{array}{c}\text { Layered } \\
\text { Model }\end{array}$ & $3.29 \mathrm{E}-05$ & $1.27 \mathrm{E}-04$ & $6.79 \mathrm{E}-05$ & $6.15 \mathrm{E}-05$ & 0.000 & 0.000 & 0.002 & 0.000 \\
9 & 0 & 9 & 0.000 \\
\hline
\end{tabular}

Table 5.5: The peak values of the simulations using the different models with the reference parameters. 


\subsubsection{Model Sensitivity: Two Sets of Rock Parameters}

The second test performed on the different models had two sets of rock parameters between the source and receivers. The first rock type is the same as given previously and had the average rock properties of the crust in eastern North America. The second rock type had the average rock properties as measured from surface measurements in the Ottawa region (Hunter et al., 2010). The basin surface had the same soil properties as before. Table 5.6 lists the different properties used for each surface in the different models for this set of simulations.

The reference model had the second rock type surrounding the basin for about $400 \mathrm{~m}$ in each direction. The Large Box Model had the second rock type surrounding the basin for roughly $4 \mathrm{~km}$ in each direction. The Layered Model had the second rock type for the top layer which was down to $400 \mathrm{~m}$ depth but extended across the entire model. This set of simulations tested the effect of a softening rock over different configurations within the models included: confinement very closely to the basin boundary (Reference Model simulation), confinemnet at a greater distance to the basin boundary (Large Box Model simulation), and within the same depth all across the model (Layered Model simulation).

\begin{tabular}{|c|c|c|c|c|c|}
\hline $\begin{array}{c}\text { Surface } \\
\text { Number }\end{array}$ & $\begin{array}{c}\text { Name of } \\
\text { Parameter } \\
\text { set }\end{array}$ & Vp (m/s) & Vs (m/s) & $\begin{array}{c}\text { Density } \\
\left(\mathrm{kg} / \mathrm{m}^{3}\right)\end{array}$ & Q value \\
\hline \multicolumn{7}{|c|}{ Simulation 1: Reference Model (Figure 5.1) } \\
\hline Surface 1 & Soil Type I & 1600 & 150 & 1600 & 100 \\
\hline Surface 2 & Rock Type II & 6000 & 2700 & 2600 & 1000 \\
\hline Surface 3 & Rock Type I & 6500 & 3500 & 2800 & 1000 \\
\hline \multicolumn{7}{|c|}{ Simulation 2: Large Box Model (Figure 5.2) } \\
\hline Surface 1 & Soil Type I & 1600 & 150 & 1600 & 100 \\
\hline
\end{tabular}




\begin{tabular}{|c|c|c|c|c|c|}
\hline Surface 2 & Rock Type II & 6000 & 2700 & 2600 & 1000 \\
\hline Surface 3 & Rock Type II & 6000 & 2700 & 2600 & 1000 \\
\hline Surface 4 & Rock Type I & 6500 & 3500 & 2800 & 1000 \\
\hline \multicolumn{7}{|c|}{ Simulation 3: Layered Model (Figure 5.3) } \\
\hline Surface 1 & Soil Type I & 1600 & 150 & 1600 & 100 \\
\hline Surface 2 & Rock Type II & 6000 & 2700 & 2600 & 1000 \\
\hline Surface 3 & Rock Type I & 6500 & 3500 & 2800 & 1000 \\
\hline Surface 4 & Rock Type I & 6500 & 3500 & 2800 & 1000 \\
\hline Surface 5 & Rock Type I & 6500 & 3500 & 2800 & 1000 \\
\hline Surface 6 & Rock Type I & 6500 & 3500 & 2800 & 1000 \\
\hline Surface 7 & Rock Type I & 6500 & 3500 & 2800 & 1000 \\
\hline Surface 8 & Rock Type I & 6500 & 3500 & 2800 & 1000 \\
\hline Surface 9 & Rock Type I & 6500 & 3500 & 2800 & 1000 \\
\hline
\end{tabular}

Table 5.6: List of parameters used for each surface in the different models for testing the effect of different models with two sets of rock parameters.

The time series for each model is shown in Figure 5.6 for the receivers S1, on rock, and in Figure 5.7 for the receivers S2, on soil, and the peak values are listed in Table 5.7. The different models have started to diverge from one another as the different waves phases arrive at different times. The Large Box Model showed the greatest difference from the other two models with peak value differences between $10-40 \%$, and with the vertical component showing a large variation of the percent difference of peak values between both the soil stations, S2, and the rock stations, S1. The difference between the horizontal peak values were relatively similar, when compared to the Reference Model for both the S1 and S2 stations. This model had the surface with the Rock Type II parameters which reached a depth of about ten times greater than the other two models which caused the large variation in the peak motions. The Layered Model showed a large discrepancy between the peak values when compared to the reference site with the horizontal motion differing from about $36 \%$ for S1 and $11 \%$ for S2, and the vertical motion differed roughly $17 \%$ for the S1 and only $2 \%$ for S2. This model had the same depth of the softening rock as the 
reference model, however it extended across the entire model instead of being confined just to a small area surrounding the basin.
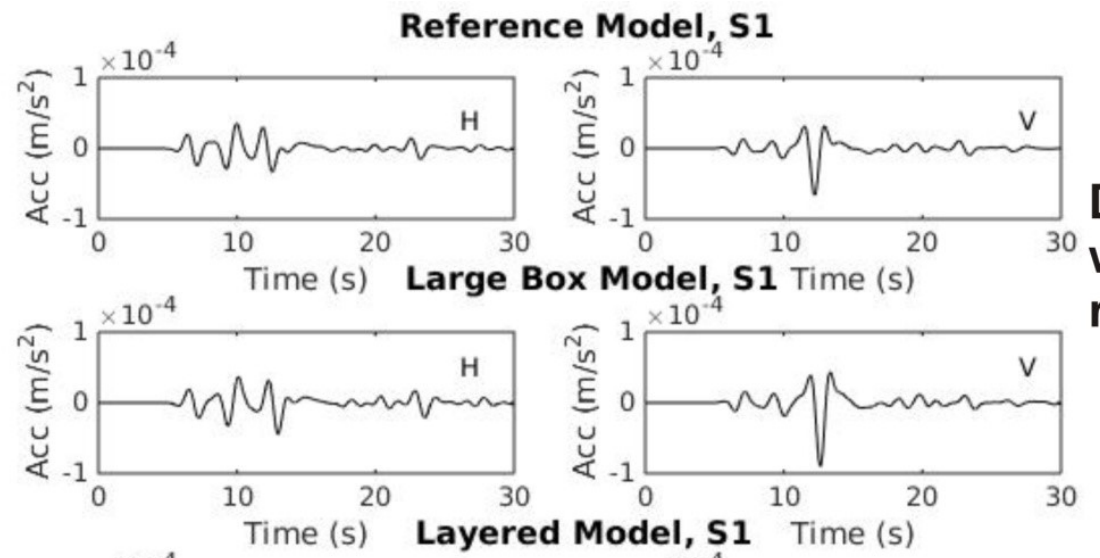

\section{Different models with two sets of rock parameters}
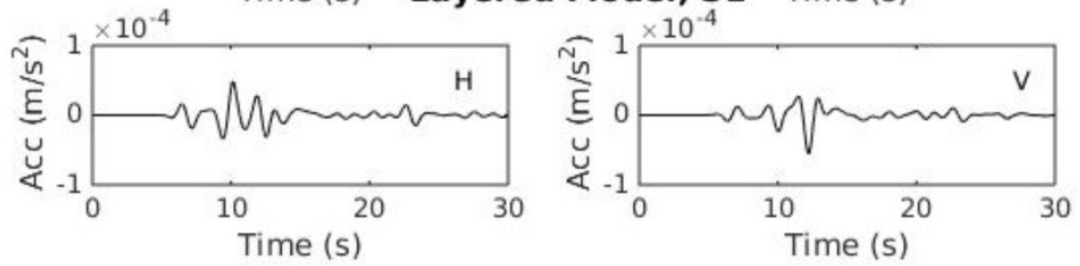

Figure 5.6: Acceleration time series resulted from the basin modeling for the Ladysmith earthquake for the horizontal component (left column) and vertical component (right column) recorded on the station S1 for the different models with two rock types. The Reference model is the top row, the Large Box Model is the middle row and the Layered Model is the bottom row. 

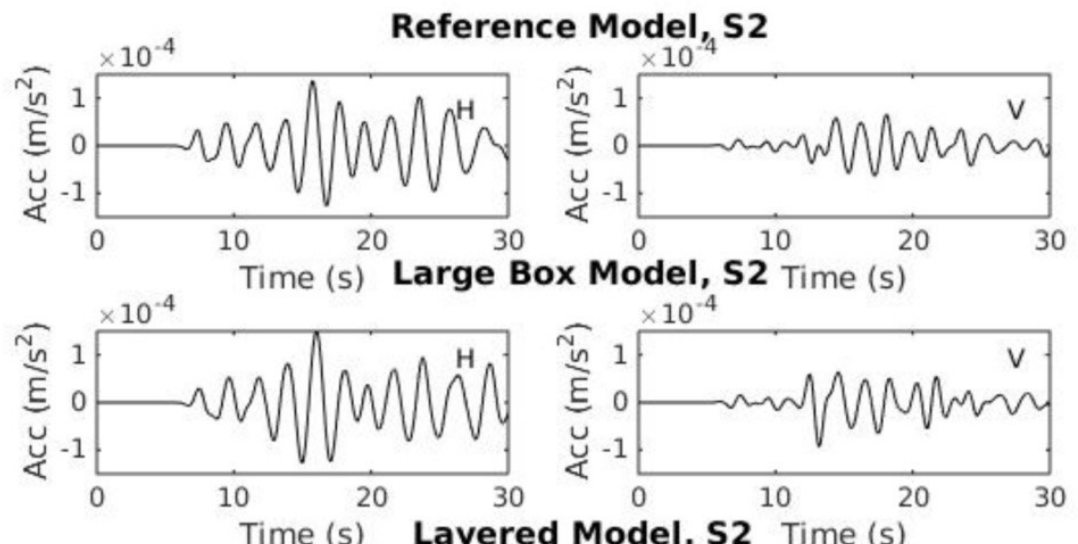

\section{Different models with two sets of rock parameters}
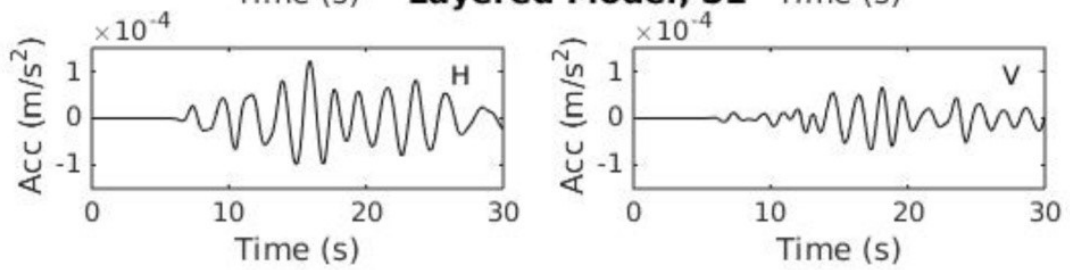

Figure 5.7: Acceleration time series results from the basin modeling for the Ladysmith earthquake for the horizontal component (left column) and vertical component (right column) recorded on the station S2 for the different models with two rock types. The Reference model is the top row, the Large Box Model is the middle row and the Layered Model is the bottom row.

\begin{tabular}{|c|c|c|c|c|c|c|c|c|}
\hline \multirow{2}{*}{ Model } & \multicolumn{4}{|c|}{ Peak Value $\left(\mathrm{m} / \mathrm{s}^{2}\right)$} & \multicolumn{4}{c|}{ Peak Value Difference from } \\
Reference (\%)
\end{tabular}

Table 5.7: Peak values of the simulations using two different rock types for each different model.

\subsubsection{Model Sensitivity: Three Sets of Rock Parameters}

The third test of model performance used the Large Box Model and changed the rock parameters from the reference simulation to a simulation with three rock types. The three rock parameters 
were: the average eastern North American crust properties, for the majority of the model; the average rock properties as measured at the surface (Hunter et al., 2010) surrounding the basin; and the third was a value between the two previous rock parameters, in order to act as a progressively weakening rock model from the average crust to the surface. Table 5.8 lists the different rock properties used for the models in these simulations.

\begin{tabular}{|c|c|c|c|c|c|}
\hline $\begin{array}{c}\text { Surface } \\
\text { Number }\end{array}$ & $\begin{array}{c}\text { Name of } \\
\text { Parameter } \\
\text { set }\end{array}$ & Vp (m/s) & Vs (m/s) & $\begin{array}{c}\text { Density } \\
\left(\mathrm{kg} / \mathrm{m}^{3}\right)\end{array}$ & Q value \\
\hline \multicolumn{7}{|c|}{ Simulation 1: Large Box Model (Figure 5.2) } \\
\hline Surface 1 & Soil Type I & 1600 & 150 & 1600 & 100 \\
\hline Surface 2 & Rock Type I & 6500 & 3500 & 2800 & 1000 \\
\hline Surface 3 & Rock Type I & 6500 & 3500 & 2800 & 1000 \\
\hline Surface 4 & Rock Type I & 6500 & 3500 & 2800 & 1000 \\
\hline \multicolumn{7}{|c|}{ Simulation 2: Large Box Model (Figure 5.2) } \\
\hline Surface 1 & Soil Type I & 1600 & 150 & 1600 & 100 \\
\hline Surface 2 & Rock Type II & 6000 & 2700 & 2600 & 1000 \\
\hline Surface 3 & Rock Type II & 6000 & 2700 & 2600 & 1000 \\
\hline Surface 4 & Rock Type I & 6500 & 3500 & 2800 & 1000 \\
\hline \multicolumn{7}{|c|}{ Simulation 3: Large Box Model (Figure 5.2) } \\
\hline Surface 1 & Soil Type I & 1600 & 150 & 1600 & 100 \\
\hline Surface 2 & Rock Type II & 6000 & 2700 & 2600 & 1000 \\
\hline Surface 3 & Rock Type III & 6250 & 3100 & 2700 & 1000 \\
\hline Surface 4 & Rock Type I & 6500 & 3500 & 2800 & 1000 \\
\hline
\end{tabular}

Table 5.8: List of parameters used for each surface in the Large Box Model for testing the effect of different sets of rock parameters.

Figure 5.8 shows the acceleration time series of the horizontal and vertical ground motions for the receivers at S1, the rock stations, and Figure 5.9 shows the same for the receivers at S2, the soil stations. It appeared as though there is no separation of wave phase arrivals, however the peak values differed significantly for some of the recordings. Table 5.9 lists the peak values for each of the recordings along with the percent difference from the reference simulation. The simulation with 3 rock types has the largest difference of peak value for the 
vertical component on the S2 with a $25 \%$ difference from the reference model. The horizontal peak values show similar differences of about $15 \%$ from this simulation to the reference simulation. The simulation with only two rock types, with the weaker rock having a larger area surrounding the basin, shows a significant difference from the reference model with differences between $18-51 \%$ in the vertical motion, but consistently differed by $33-36 \%$ in the horizontal motion.
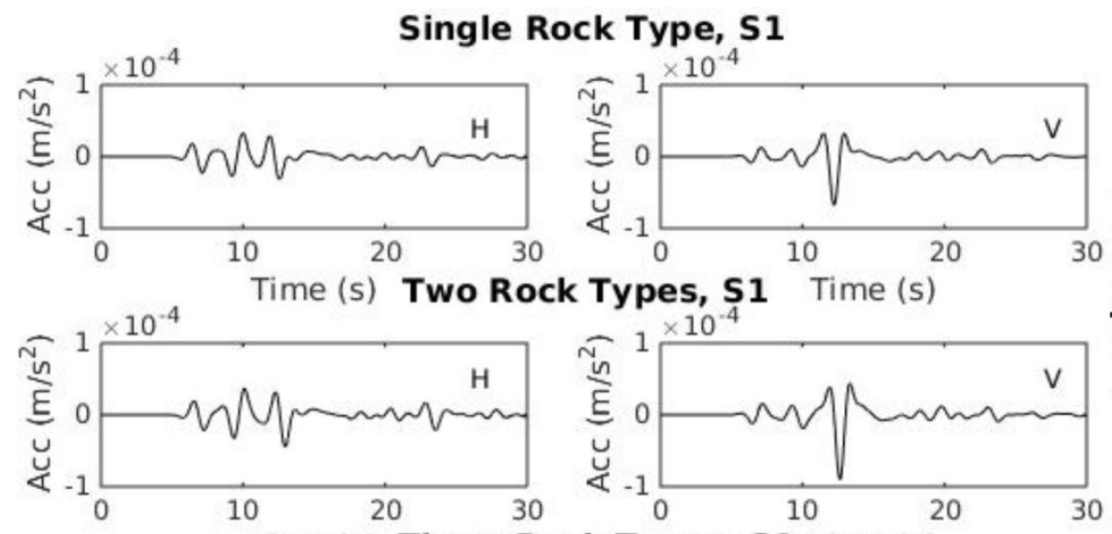

\section{Different sets of} rock parameters for the Large Box Model
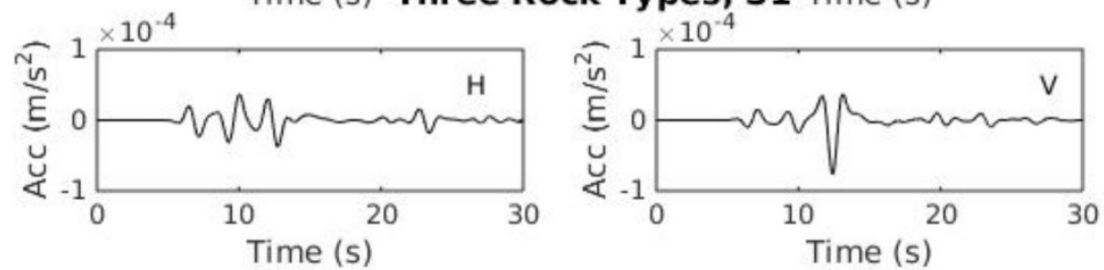

Figure 5.8: Acceleration time series resulted from the basin modeling for the Ladysmith earthquake for the horizontal component (left column) and vertical component (right column) recorded on the station S1 for the Large Box Model with different rock types. The reference simulation is the top row, the simulation with two rock types is the middle row and the simulation with three rock types is the bottom row. 

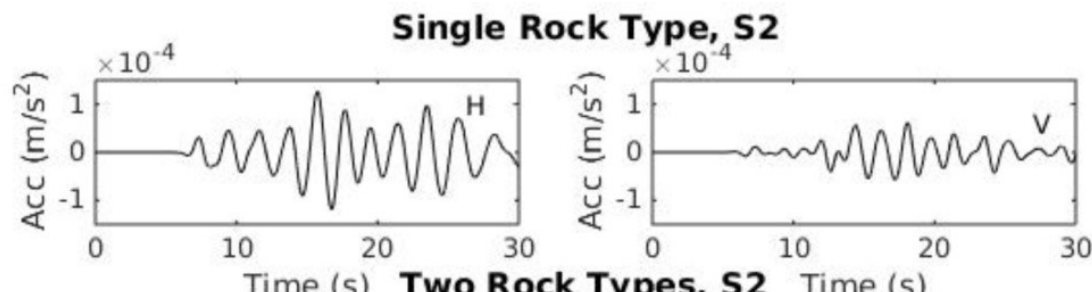

Different sets of

Time (s) Two Rock Types, $\mathbf{5 2}$ Time (s)
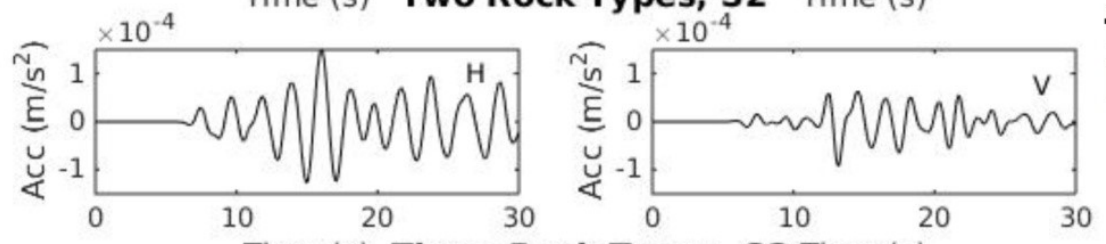

rock parameters for the Large Box Model
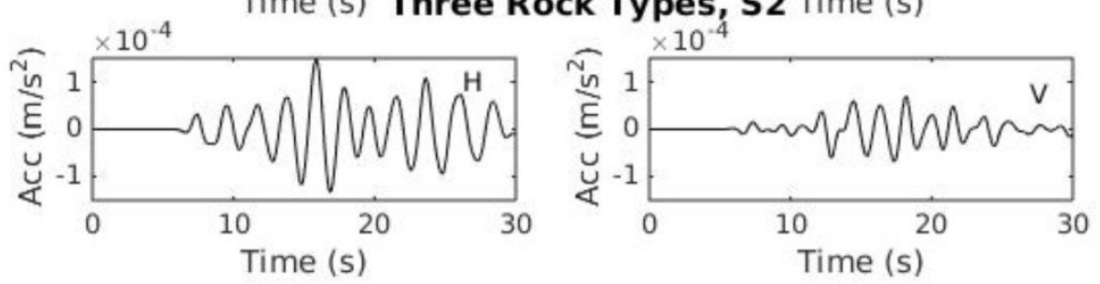

Figure 5.9: Acceleration time series resulted from the basin modeling for the Ladysmith earthquake for the horizontal component (left column) and vertical component (right column) recorded on the soil station for the Large Box Model with different rock types. The reference simulation is the top row, the simulation with two rock types is the middle row and the simulation with three rock types is the bottom row.

\begin{tabular}{|c|c|c|c|c|c|c|c|c|}
\hline \multirow{2}{*}{$\begin{array}{c}\text { Large Box } \\
\text { Model }\end{array}$} & \multicolumn{4}{|c|}{ Peak Value $\left(\mathrm{m} / \mathrm{s}^{2}\right)$} & \multicolumn{3}{c|}{$\begin{array}{c}\text { Peak Value Difference from } \\
\text { Reference (\%) }\end{array}$} \\
\cline { 2 - 9 } & \multicolumn{2}{|c|}{ Horizontal } & \multicolumn{2}{c|}{ Vertical } & \multicolumn{3}{c|}{ Horizontal } & \multicolumn{2}{c|}{ Vertical } \\
\cline { 2 - 9 } & $\mathrm{S} 1$ & $\mathrm{~S} 2$ & $\mathrm{~S} 1$ & $\mathrm{~S} 2$ & $\mathrm{~S} 1$ & $\mathrm{~S} 2$ & $\mathrm{~S} 1$ & $\mathrm{~S} 2$ \\
\hline $\begin{array}{c}\text { Referenc } \\
\text { e }\end{array}$ & $3.29 \mathrm{E}-05$ & $1.27 \mathrm{E}-04$ & $6.79 \mathrm{E}-05$ & $6.15 \mathrm{E}-05$ & & & & \\
\hline $\begin{array}{c}2 \text { Rock } \\
\text { Types }\end{array}$ & $4.46 \mathrm{E}-05$ & $1.50 \mathrm{E}-04$ & $9.05 \mathrm{E}-05$ & $9.29 \mathrm{E}-05$ & 35.80 & 18.01 & 33.36 & 51.04 \\
\hline $\begin{array}{c}3 \text { Rock } \\
\text { Types }\end{array}$ & $3.78 \mathrm{E}-05$ & $1.47 \mathrm{E}-04$ & $7.69 \mathrm{E}-05$ & $6.89 \mathrm{E}-05$ & 15.23 & 1.95 & 15.01 & 25.80 \\
\hline
\end{tabular}

Table 5.9: Peak values of the simulations using different rock parameters for the surfaces in the Large Box Model.

\subsubsection{Model Sensitivity: Layering Sets of Rock Parameters}

The last test of model geometry involved the Layered Model to test the performance of a horizontally layered crust. Table 5.10 lists the different properties for the layering that is used.

The first simulation was the reference simulation with only the average eastern North American 
crustal properties. The second simulation had a thin layer of average rock properties as measured at the surface. The third simulation had a progressive weakening of the crust from the average eastern North America rock properties at depth, to the average rock properties as measured at the surface. The last simulation used a velocity profile for the region as found by Motazedian et al., (2013) using Rayleigh wave dispersion from the 2010 M5.2 Val-de-Bois earthquake.

\begin{tabular}{|c|c|c|c|c|c|}
\hline $\begin{array}{l}\text { Surface } \\
\text { Number }\end{array}$ & $\begin{array}{c}\text { Name of } \\
\text { Parameter } \\
\text { set }\end{array}$ & $\mathrm{Vp}(\mathrm{m} / \mathrm{s})$ & Vs $(\mathrm{m} / \mathrm{s})$ & $\begin{array}{l}\text { Density } \\
\left(\mathrm{kg} / \mathrm{m}^{3}\right)\end{array}$ & Q value \\
\hline \multicolumn{6}{|c|}{ Simulation 1: Layered Model (Figure 5.3) } \\
\hline Surface 1 & Soil Type I & 1600 & 150 & 1600 & 100 \\
\hline Surface 2 & Rock Type I & 6500 & 3500 & 2800 & 1000 \\
\hline Surface 3 & Rock Type I & 6500 & 3500 & 2800 & 1000 \\
\hline Surface 4 & Rock Type I & 6500 & 3500 & 2800 & 1000 \\
\hline Surface 5 & Rock Type I & 6500 & 3500 & 2800 & 1000 \\
\hline Surface 6 & Rock Type I & 6500 & 3500 & 2800 & 1000 \\
\hline Surface 7 & Rock Type I & 6500 & 3500 & 2800 & 1000 \\
\hline Surface 8 & Rock Type I & 6500 & 3500 & 2800 & 1000 \\
\hline Surface 9 & Rock Type I & 6500 & 3500 & 2800 & 1000 \\
\hline \multicolumn{6}{|c|}{ Simulation 2: Layered Model (Figure 5.3) } \\
\hline Surface 1 & Soil Type I & 1600 & 150 & 1600 & 100 \\
\hline Surface 2 & Rock Type II & 6000 & 2700 & 2600 & 1000 \\
\hline Surface 3 & Rock Type I & 6500 & 3500 & 2800 & 1000 \\
\hline Surface 4 & Rock Type I & 6500 & 3500 & 2800 & 1000 \\
\hline Surface 5 & Rock Type I & 6500 & 3500 & 2800 & 1000 \\
\hline Surface 6 & Rock Type I & 6500 & 3500 & 2800 & 1000 \\
\hline Surface 7 & Rock Type I & 6500 & 3500 & 2800 & 1000 \\
\hline Surface 8 & Rock Type I & 6500 & 3500 & 2800 & 1000 \\
\hline \multicolumn{6}{|c|}{ Simulation 3: Layered Model (Figure 5.3) with weakening crust } \\
\hline Surface 1 & Soil Type I & 1600 & 150 & 1600 & 100 \\
\hline Surface 2 & Rock Type II & 6000 & 2700 & 2600 & 1000 \\
\hline Surface 3 & Rock Type IV & 6050 & 2730 & 2650 & 1000 \\
\hline Surface 4 & Rock Type V & 6080 & 2790 & 2680 & 1000 \\
\hline Surface 5 & Rock Type VI & 6130 & 2830 & 2700 & 1000 \\
\hline Surface 6 & Rock Type VII & 6250 & 2990 & 2750 & 1000 \\
\hline Surface 7 & $\begin{array}{c}\text { Rock Type } \\
\text { VIII }\end{array}$ & 6380 & 3250 & 2780 & 1000 \\
\hline
\end{tabular}




\begin{tabular}{|c|c|c|c|c|c|}
\hline Surface 8 & Rock Type I & 6500 & 3500 & 2800 & 1000 \\
\hline Surface 9 & Rock Type I & 6500 & 3500 & 2800 & 1000 \\
\hline \multicolumn{2}{|c|}{ Simulation 4: Layered Model (Figure 5.3) } & with Motazedian et al. (2013) profile \\
\hline Surface 1 & Soil Type I & 1600 & 150 & 1600 & 100 \\
\hline Surface 2 & Rock Type IX & 6110 & 3580 & 2740 & 1000 \\
\hline Surface 3 & Rock Type IX & 6110 & 3580 & 2740 & 1000 \\
\hline Surface 4 & Rock Type IX & 6110 & 3580 & 2740 & 1000 \\
\hline Surface 5 & Rock Type X & 6090 & 3570 & 2740 & 1000 \\
\hline Surface 6 & Rock Type XI & 6010 & 3530 & 2720 & 1000 \\
\hline Surface 7 & Rock Type XII & 6180 & 3620 & 2760 & 1000 \\
\hline Surface 8 & Rock Type XIII & 6280 & 3670 & 2780 & 1000 \\
\hline Surface 9 & $\begin{array}{c}\text { Rock Type } \\
\text { XIV }\end{array}$ & 6680 & 3870 & 2880 & 1000 \\
\hline
\end{tabular}

Table 5.10: List of parameters used for each surface in the Layered Model for testing the effect of different horizontal layering of the crust.

The horizontal and vertical components of the acceleration time series are shown in Figure 5.10 for the stations located at S1 and Figure 5.11 for the stations located at S2. Table 5.11 lists the peak values at each receiver and the percent difference from the reference simulation. The wave phase arrivals between the reference simulation and the simulation with only 2 rock types are very similar to one another, however the peak varied from $46 \%$ for the horizontal motion and $18 \%$ in the vertical motion at the rock station, S1. The station located on the soil, S2, had less difference in the peak values at $4 \%$ for the horizontal motion and $10 \%$ for the vertical component. There was a different timing of the wave phase arrivals for the progressive velocity profile. The large S-wave peak appeared to lag behind the arrival times from the other simulations. There is also a significant difference in the waveform for this simulation, with two almost identical strength peaks in the vertical motion compared to the other simulations which only had one strong peak. The maximum values in these peaks differ from the reference simulation by $40 \%$ and $36 \%$ in the horizontal and vertical components of motion recorded at the station placed on rock, $\mathrm{S} 1$, and by $8 \%$ and $33 \%$ for the horizontal and vertical components at the 
station located on soil, S2. The velocity profile from Motazedian et al., (2013) shows similar characteristics to the reference at the recording on rock, S1, but a significantly different response in the soil recording S2. Mostly it appears that the strong arrival of the S-wave peak did not appear in this recording, which caused the difference in the peak horizontal motion being $32 \%$.
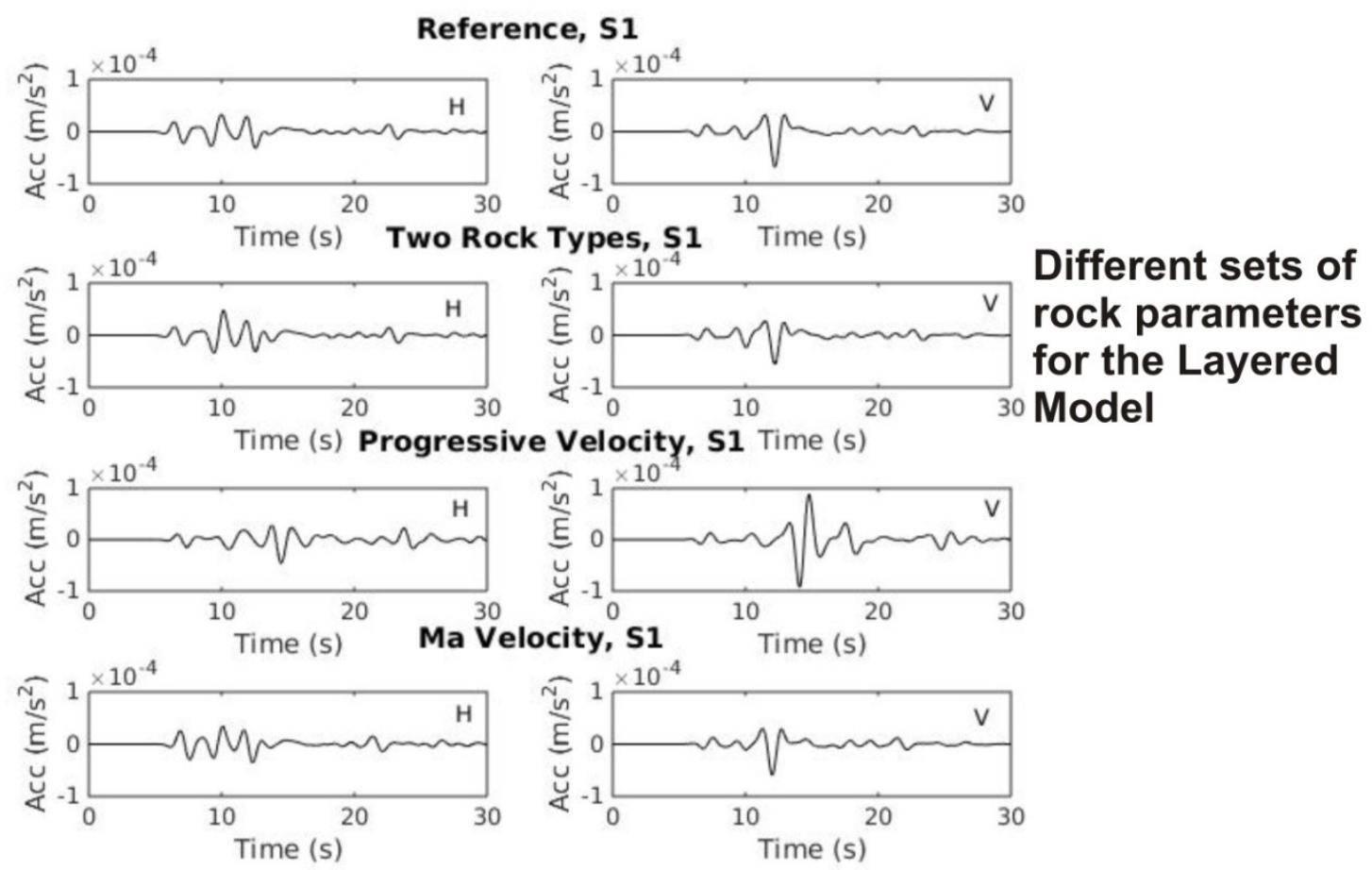

Figure 5.10: Acceleration time series resulted from the basin modeling for the Ladysmith earthquake for the horizontal component (left column) and vertical component (right column) recorded on the station S1 for the Layered Model with different rock types. The reference simulation is the top row, the simulation with two rock types is the second row, the simulation with a progressive velocity profile is the third row, and the simulation with the velocity profile as found in Motazedian et al., (2013) is the bottom row. 


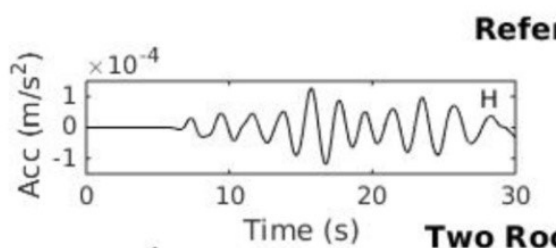

Reference, $\mathbf{5 2}$
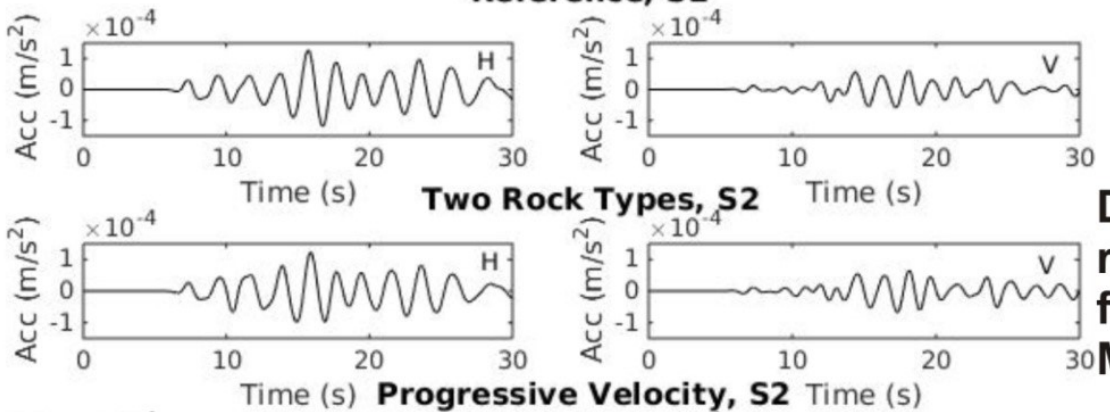

Different sets of rock parameters for the Layered Model
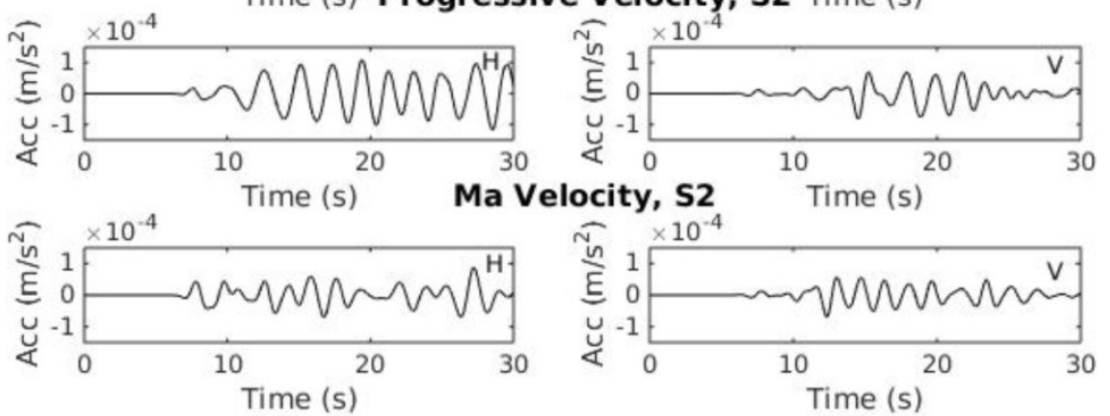

Figure 5.11: Acceleration time series resulted from the basin modeling for the Ladysmith earthquake for the horizontal component (left column) and vertical component (right column) recorded on the station S2 for the Layered Model with different rock types. The reference simulation is the top row, the simulation with two rock types is the second row, the simulation with a progressive velocity profile is the third row, and the simulation with the velocity profile as found in Motazedian et al., (2013) is the bottom row.

\begin{tabular}{|c|c|c|c|c|c|c|c|c|}
\hline \multirow{2}{*}{$\begin{array}{c}\text { Layered } \\
\text { Model }\end{array}$} & \multicolumn{4}{|c|}{ Peak Value $\left(\mathrm{m} / \mathrm{s}^{2}\right)$} & \multicolumn{4}{c|}{ Peak Value Difference from } \\
\cline { 2 - 9 } & \multicolumn{2}{|c|}{ Horizontal } & \multicolumn{2}{c|}{ Vertical } & \multicolumn{2}{c|}{ Horizontal } & \multicolumn{2}{c|}{ Vertical } \\
\cline { 2 - 9 } & $\mathrm{S} 1$ & $\mathrm{~S} 2$ & $\mathrm{~S} 1$ & $\mathrm{~S} 2$ & $\mathrm{~S} 1$ & $\mathrm{~S} 2$ & $\mathrm{~S} 1$ & $\mathrm{~S} 2$ \\
\hline Reference & $3.29 \mathrm{E}-05$ & $1.27 \mathrm{E}-04$ & $\begin{array}{c}6.79 \mathrm{E}- \\
05\end{array}$ & $6.15 \mathrm{E}-05$ & & & & \\
\hline $\begin{array}{c}2 \text { Rock } \\
\text { Types }\end{array}$ & $4.80 \mathrm{E}-05$ & $1.22 \mathrm{E}-04$ & $\begin{array}{c}5.54 \mathrm{E}- \\
05\end{array}$ & $6.74 \mathrm{E}-05$ & 46.07 & 3.70 & 18.31 & 9.61 \\
\hline $\begin{array}{c}\text { Progressive } \\
\text { velocity }\end{array}$ & $4.59 \mathrm{E}-05$ & $1.18 \mathrm{E}-04$ & $\begin{array}{c}9.25 \mathrm{E}- \\
05\end{array}$ & $8.16 \mathrm{E}-05$ & 39.56 & 7.58 & 36.30 & 32.75 \\
\hline $\begin{array}{c}\text { Motazedian } \\
\text { et al. (2013) } \\
\text { velocity } \\
\text { profile }\end{array}$ & $3.55 \mathrm{E}-05$ & $8.69 \mathrm{E}-05$ & $\begin{array}{c}5.89 \mathrm{E}- \\
05\end{array}$ & $6.90 \mathrm{E}-05$ & 8.0740 & 31.65 & 13.26 & 12.17 \\
\hline
\end{tabular}

Table 5.11: Peak values of the simulations using different rock parameters for the surfaces in the Layered Model. 


\subsection{Soil and Rock Parameter Tests}

There were three parameters tested on the reference model since the ground motions could have a strong dependence on these. These parameters included: the $Q$ value of the rock; the $Q$ value of the soil; and the velocity of the soil. The $Q$ values are directly responsible for the anelastic attenuation in each of the materials respectively, and there can be a large difference on how it is implemented. In the software SPECFEM2D and SPECFEM3D it is implemented as a constant value (see Appendix I for more detail), so they were tested to see which values are appropriate for the regional materials. In the models the soil velocity was implemented as a constant value, however as seen in Chapter 2.1 there was a dependence between soil velocity and depth. Using an average value for soil velocity simplified the simulation and could provide a good approximation of the soil response.

\subsubsection{Rock Attenuation}

The rock attenuation values used were $Q=1000,750$, and 500 . These are the typical range of seismic Q values for the region (Atkinson and Assatourians, 2010; Atkinson et al., 2014). There are several listed values for $Q$ values in eastern North America which were found based on different approaches. Woodgold (1990) estimated $Q=860 f^{0.33}$ for western Quebec and $Q=$

$750 f^{0.40}$ for the Ottawa area using coda waves. Atkinson et al. (2014) found $Q=416 f^{0.43}$ using the vertical component of the Fourier spectra from the Ladysmith earthquake. Mereu et al. (2013) estimated $Q=500 f^{0.5}$ as the average Q relationship for southern Ontario and western Quebec. 
Although these $Q$ values are frequency dependent, the programs used a nearly constant $Q$ to account for viscoelastic attenuation. The $Q$ value used is taken with the centre frequency equal to $2 \mathrm{~Hz}$, as this is roughly the dominant frequency in the simulations. Even though the difference between the $Q$ values tested was large, there should not be a significant difference in the output of the simulations. The anelastic attenuation along a regional scale was relatively low for lower frequencies. The attenuation, which is also described as the energy loss per cycle, depends on the wavelength, and the wavelengths of the low frequencies in the rock surfaces for these models were several kilometres in length.

A summary of the properties for the surfaces in the reference model for each simulation is listed in Table 5.12. The peak values resulted from previously discussed different scenarios for basin modeling and the percent difference from $Q=1000$ are listed in Table 5.13. The acceleration time series for the horizontal and vertical components are shown in Figure 5.12 for the station S1, and Figure 5.13 for the station S2. There is very little difference between the time series by using different $Q$ values for the rock, as the wave phases appear to be the same between all three simulations in both the station on the rock, S1, and the stations on the soil, $\mathrm{S} 2$. The differences between the peak values on the soil stations, S2, were minimal with the largest difference being $2 \%$ for the vertical component. The station S1 showed little difference as well with 1.6 and $3.2 \%$ for the horizontal component of $Q=750$ and $Q=500$, respectively, and 1.4 and $2.7 \%$ for the vertical component. The small difference in peak values for the large discrepancy of $Q$ values allowed for a large scope on the choice of the value of $Q$ for the rock without affecting the resulting ground motions to a large degree, as might be expected due to the close proximity of these stations to the earthquake epicenter. 


\begin{tabular}{|c|c|c|c|c|c|}
\hline $\begin{array}{c}\text { Surface } \\
\text { Number }\end{array}$ & $\begin{array}{c}\text { Name of } \\
\text { Parameter } \\
\text { set }\end{array}$ & Vp (m/s) & Vs (m/s) & $\begin{array}{c}\text { Density } \\
\left(\mathrm{kg} / \mathrm{m}^{3}\right)\end{array}$ & Q value \\
\hline \multicolumn{7}{|c|}{ Simulation 1: Reference Model (Figure 5.1) } \\
\hline Surface 1 & Soil Type I & 1600 & 150 & 1600 & 100 \\
\hline Surface 2 & Rock Type I & 6500 & 3500 & 2800 & 1000 \\
\hline Surface 3 & Rock Type I & 6500 & 3500 & 2800 & 1000 \\
\hline \multicolumn{7}{|c|}{ Simulation 2: Reference Model (Figure 5.1) } \\
\hline Surface 1 & Soil Type I & 1600 & 150 & 1600 & 100 \\
\hline Surface 2 & Rock Type I & 6500 & 3500 & 2800 & 750 \\
\hline Surface 3 & Rock Type I & 6500 & 3500 & 2800 & 750 \\
\hline \multicolumn{7}{|c|}{ Simulation 3: Reference Model (Figure 5.1) } \\
\hline Surface 1 & Soil Type I & 1600 & 150 & 1600 & 100 \\
\hline Surface 2 & Rock Type I & 6500 & 3500 & 2800 & 500 \\
\hline Surface 3 & Rock Type I & 6500 & 3500 & 2800 & 500 \\
\hline
\end{tabular}

Table 5.12: List of parameters used for each surface in the reference model for testing the effect of different values of $Q$ for the rock.
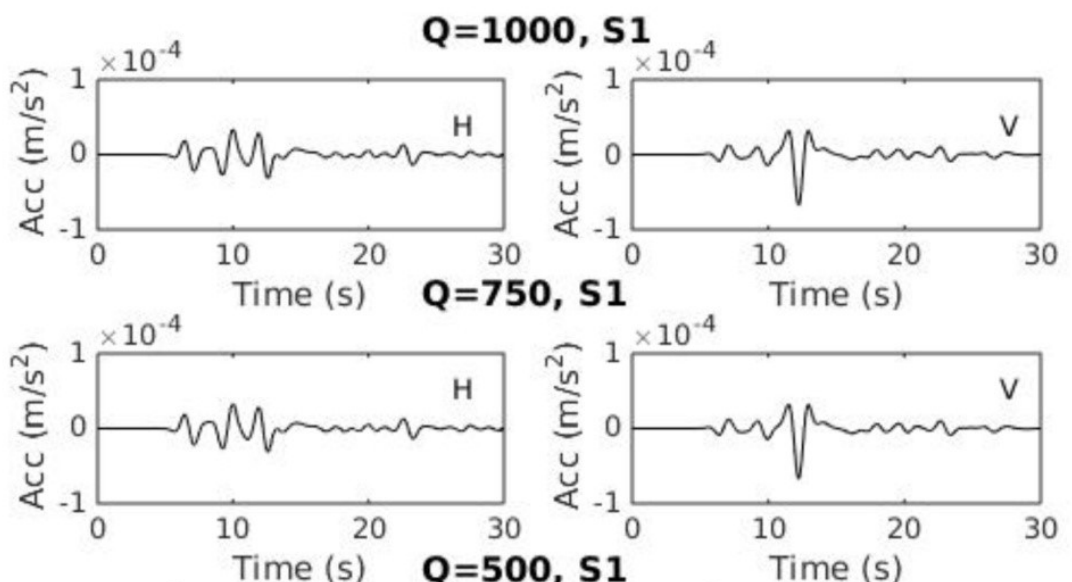

Changing the Rock $Q$ value for the Reference Model
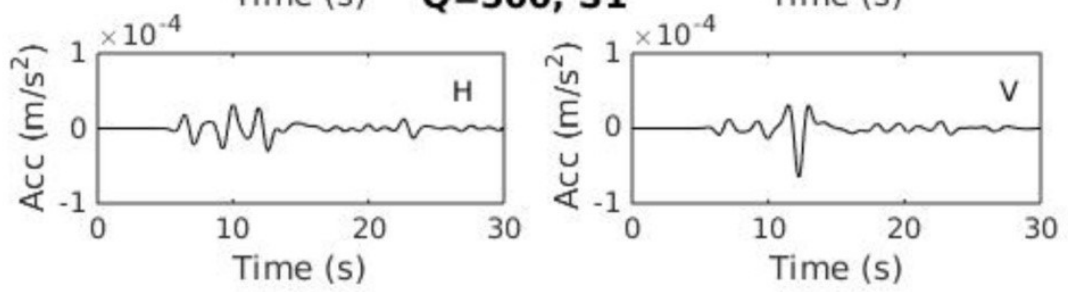

Figure 5.12: Acceleration time series resulted from the basin modeling for the Ladysmith earthquake for the horizontal component (left column) and vertical component (right column) recorded on the station S1 for the different $Q$ values for the rock. The reference simulation with $Q=1000$ is the top row, the simulation with $Q=750$ is the middle row, and the simulation with $Q$ $=500$ is the bottom row. 

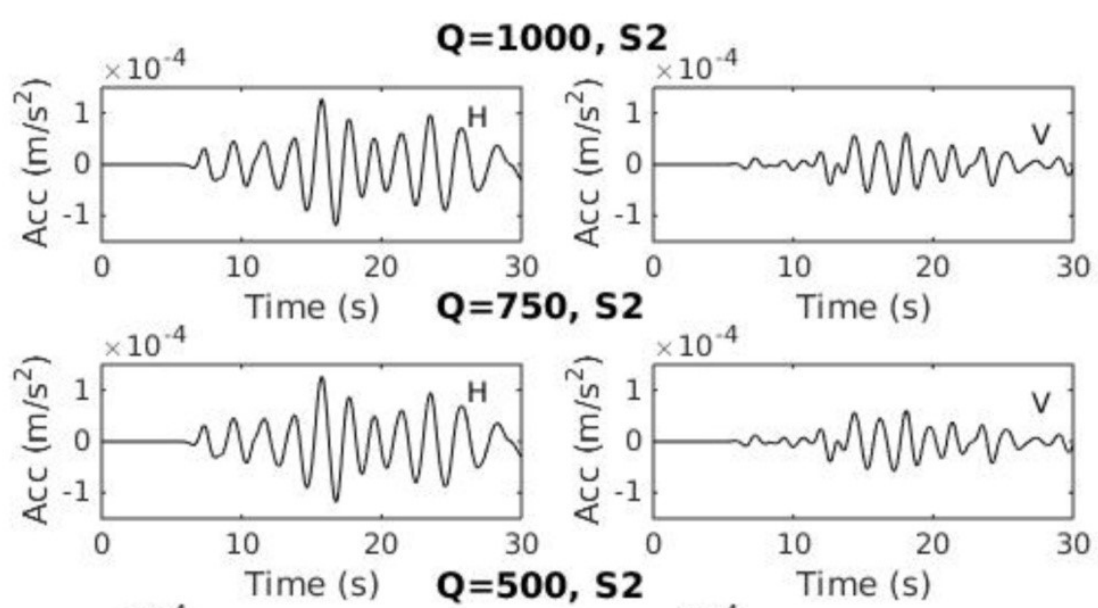

\section{Changing the Rock $Q$ value for the Reference Model}
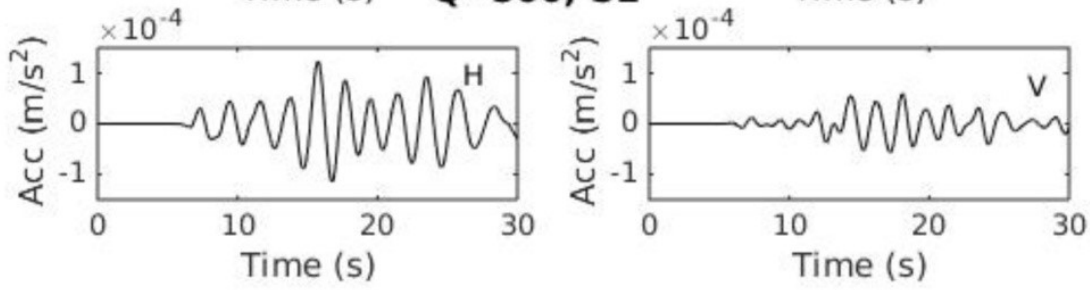

Figure 5.13: Acceleration time series resulted from the basin modeling for the Ladysmith earthquake for the horizontal component (left column) and vertical component (right column) recorded on the station S2 for the different $Q$ values for the rock. The reference simulation with $Q=1000$ is the top row, the simulation with $Q=750$ is the middle row, and the simulation with $Q$ $=500$ is the bottom row.

\begin{tabular}{|c|c|c|c|c|c|c|c|c|}
\hline \multirow{2}{*}{ Model } & \multicolumn{4}{|c|}{ Peak Value $\left(\mathrm{m} / \mathrm{s}^{2}\right)$} & \multicolumn{3}{c|}{$\begin{array}{c}\text { Peak Value Difference from } \\
\text { Reference (\%) }\end{array}$} \\
\cline { 2 - 9 } & \multicolumn{2}{|c|}{ Horizontal } & \multicolumn{2}{c|}{ Vertical } & \multicolumn{3}{c|}{ Horizontal } & \multicolumn{2}{c|}{ Vertical } \\
\cline { 2 - 9 } & $\mathrm{S} 1$ & $\mathrm{~S} 2$ & $\mathrm{~S} 1$ & $\mathrm{~S} 2$ & $\mathrm{~S} 1$ & $\mathrm{~S} 2$ & $\mathrm{~S} 1$ & $\mathrm{~S} 2$ \\
\hline $\mathrm{Q}=1000$ & $3.29 \mathrm{E}-05$ & $1.27 \mathrm{E}-04$ & $6.79 \mathrm{E}-05$ & $6.15 \mathrm{E}-05$ & & & & \\
\hline $\mathrm{Q}=750$ & $3.25 \mathrm{E}-05$ & $1.26 \mathrm{E}-04$ & $6.70 \mathrm{E}-05$ & $6.09 \mathrm{E}-05$ & 1.06 & 0.83 & 1.35 & 1.03 \\
\hline $\mathrm{Q}=500$ & $3.18 \mathrm{E}-05$ & $1.24 \mathrm{E}-04$ & $6.52 \mathrm{E}-05$ & $5.96 \mathrm{E}-05$ & 3.14 & 2.50 & 3.98 & 3.07 \\
\hline
\end{tabular}

Table 5.13: Peak values from the simulations with a varying $Q$ value for the rock.

\subsubsection{Soil Attenuation}

There is a wide range of $Q$ values listed in the literature for different types of soils, sands, clays, and gravels. These values have a wide range even within the same types of materials. There is little consensus on measurement techniques and specifically on in situ measurements(Crow et al., 2011). Although determination of anelastic attenuation over a regional scale in rock has been a subject of study, this effect has seen less attention for near surface soft soils. 
The soil attenuation values were set to $Q=100$ as the reference, and $Q=50,20$ for the other simulations. All the properties for each surface in the reference model for the simulations are listed in Table 5.14. These $Q$ values represent the range of values found using several different methods, and are common values found for clay and silty-clay materials (Crow, 2010; Crow et al., 2011). Based on the in situ measurements, it is likely that the $Q$ value for the material in this basin is 100 , however there are current projects under way which are trying to better determine this value along with other dynamic soil properties of this Leda Clay (LeBeouf et al., 2013; Motazedian et al., 2015).

The acceleration time series used for both components of motion for the S1 station is shown in Figure 5.14 and for the S2 station in Figure 5.15. The peak values and percent difference from the reference simulation are listed in Table 5.15. The ground motions at the S1 stations, on rock, varied only slightly with peak differences of less than $1 \%$ for the horizontal and vertical motions. This small variation might have been caused by a positive interference between the Swave arriving at this station and a reflection from the boundary of the basin. The ground motions recorded at the S2 stations, on soil, showed a large difference with a very significant decay of motions as the $Q$ value decreased. The peak values of these motions were also reduced as the $Q$ value decreased, with a $15 \%$ and $38 \%$ difference for the horizontal motions from the reference to a $Q$ value of 50 and 20 , respectively. The vertical peak motions were also decreased by $21 \%$ and $10 \%$ for the $Q$ values of 50 and 20 , respectively. This showed that there is a large possibility of error in the peak values due to the poorly constrained value of $Q$ in the models. With the lower attenuation values not only did the peak value decrease, but the resonance decreased as well. 
This was seen in the soil acceleration time series at recording station S2, where there are lower peaks after a strong arrival.

\begin{tabular}{|c|c|c|c|c|c|}
\hline $\begin{array}{c}\text { Surface } \\
\text { Number }\end{array}$ & $\begin{array}{c}\text { Name of } \\
\text { Parameter } \\
\text { set }\end{array}$ & Vp (m/s) & Vs (m/s) & $\begin{array}{c}\text { Density } \\
\left(\mathrm{kg} / \mathrm{m}^{3}\right)\end{array}$ & Q value \\
\hline \multicolumn{7}{|c|}{ Simulation 1: Reference Model (Figure 5.1) } \\
\hline Surface 1 & Soil Type I & 1600 & 150 & 1600 & 100 \\
\hline Surface 2 & Rock Type I & 6500 & 3500 & 2800 & 1000 \\
\hline Surface 3 & Rock Type I & 6500 & 3500 & 2800 & 1000 \\
\hline \multicolumn{7}{|c|}{ Simulation 2: Reference Model (Figure 5.1) } \\
\hline Surface 1 & Soil Type I & 1600 & 150 & 1600 & 50 \\
\hline Surface 2 & Rock Type I & 6500 & 3500 & 2800 & 1000 \\
\hline Surface 3 & Rock Type I & 6500 & 3500 & 2800 & 1000 \\
\hline \multicolumn{7}{|c|}{ Simulation 3: Reference Model (Figure 5.1) } \\
\hline Surface 1 & Soil Type I & 1600 & 150 & 1600 & 20 \\
\hline Surface 2 & Rock Type I & 6500 & 3500 & 2800 & 1000 \\
\hline Surface 3 & Rock Type I & 6500 & 3500 & 2800 & 1000 \\
\hline
\end{tabular}

Table 5.14: List of parameters used for each surface in the reference model for testing the effect of different values of $Q$ for the soil. 

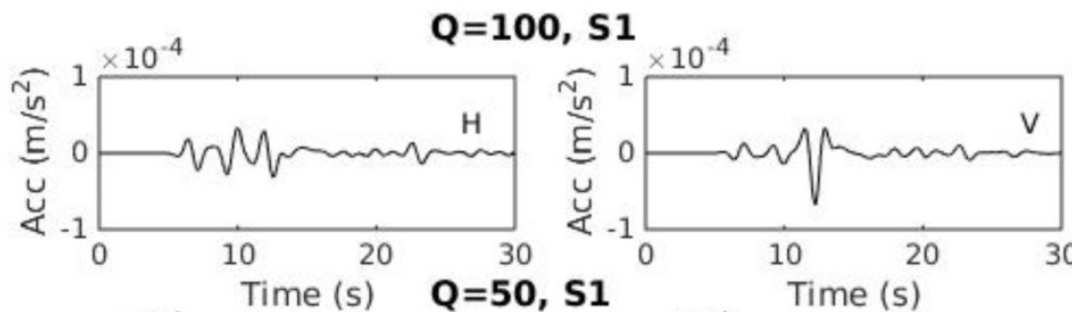

Changing the
Soil Q value for
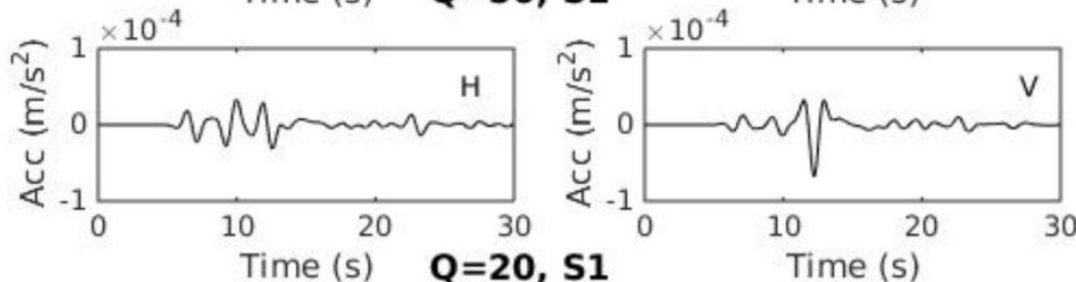
the Reference Model
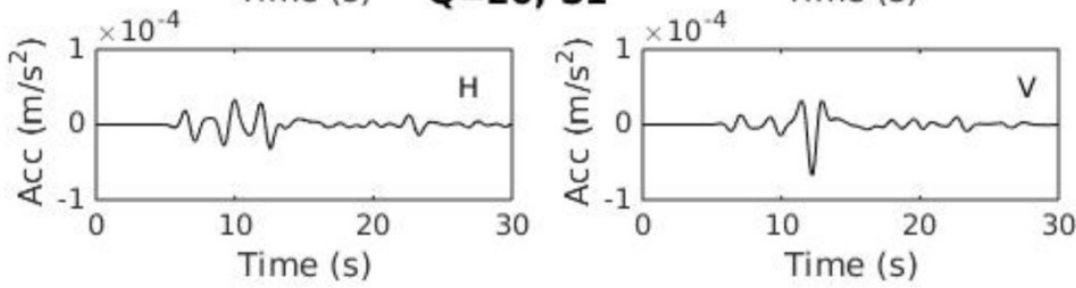

Figure 5.14: Acceleration time series resulted from the basin modeling for the Ladysmith earthquake for the horizontal component (left column) and vertical component (right column) recorded on the station S1 for the different $Q$ values for the soil. The reference simulation with $Q=100$ is the top row, the simulation with $Q=50$ is the middle row, and the simulation with $Q=$ 20 is the bottom row.
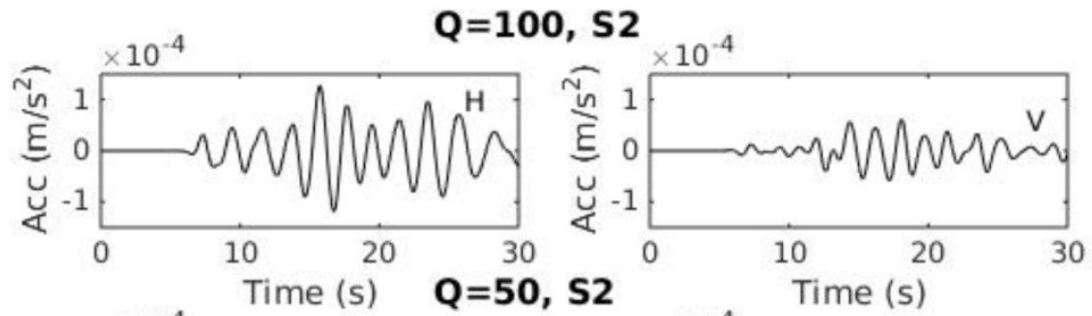

Changing the Soil $Q$ value for
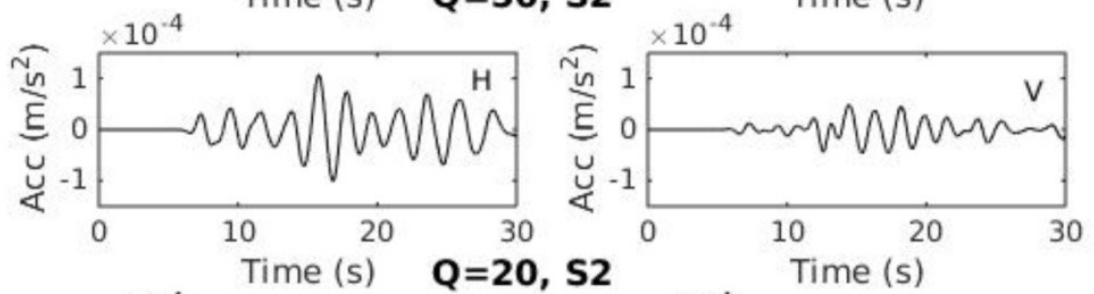
the Reference Model
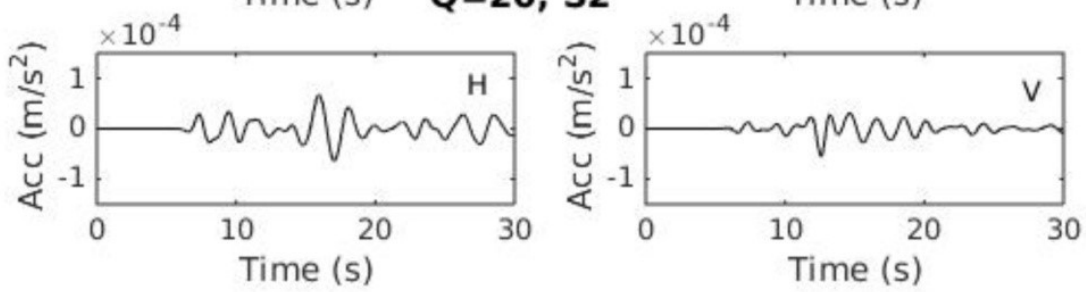

Figure 5.15: Acceleration time series resulted from the basin modeling for the Ladysmith earthquake the horizontal component (left column) and vertical component (right column) recorded on the station S2 for the different $Q$ values for the soil. The 
reference simulation with $Q=100$ is the top row, the simulation with $Q=50$ is the middle row, and the simulation with $Q=20$ is the bottom row.

\begin{tabular}{|c|c|c|c|c|c|c|c|c|}
\hline \multirow{2}{*}{ Model } & \multicolumn{4}{|c|}{ Peak Value $\left(\mathrm{m} / \mathrm{s}^{2}\right)$} & \multicolumn{3}{c|}{$\begin{array}{c}\text { Peak Value Difference from } \\
\text { Reference (\%) }\end{array}$} \\
\cline { 2 - 9 } & \multicolumn{2}{|c|}{ Horizontal } & \multicolumn{2}{c|}{ Vertical } & \multicolumn{3}{c|}{ Horizontal } & \multicolumn{2}{c|}{ Vertical } \\
\cline { 2 - 9 } & $\mathrm{S} 1$ & $\mathrm{~S} 2$ & $\mathrm{~S} 1$ & $\mathrm{~S} 2$ & $\mathrm{~S} 1$ & $\mathrm{~S} 2$ & $\mathrm{~S} 1$ & $\mathrm{~S} 2$ \\
\hline $\mathrm{Q}=100$ & $3.29 \mathrm{E}-05$ & $1.27 \mathrm{E}-04$ & $6.79 \mathrm{E}-05$ & $6.15 \mathrm{E}-05$ & & & & \\
\hline $\mathrm{Q}=50$ & $3.29 \mathrm{E}-05$ & $1.08 \mathrm{E}-04$ & $6.78 \mathrm{E}-05$ & $4.86 \mathrm{E}-05$ & 0.09 & 15.20 & 0.15 & 21.00 \\
\hline $\mathrm{Q}=20$ & $3.30 \mathrm{E}-05$ & $6.69 \mathrm{E}-05$ & $6.76 \mathrm{E}-05$ & $5.51 \mathrm{E}-05$ & 0.35 & 47.40 & 0.45 & 10.36 \\
\hline
\end{tabular}

Table 5.15: Peak values from the simulations with a varying $Q$ value for the soil.

\subsubsection{Soil Velocity}

The soil velocity values were set to $\mathrm{Vs}=150 \mathrm{~m} / \mathrm{s}$ for the reference simulation and $\mathrm{Vs}=250 \mathrm{~m} / \mathrm{s}$, and Vs $=350 \mathrm{~m} / \mathrm{s}$ for the other simulations. The properties for each surface in the reference model for the different simulations are listed in Table 5.16. The reference shear wave velocity value, Vs $=150 \mathrm{~m} / \mathrm{s}$, represented the approximate average value of the soil velocity in the basin $\left(V_{\mathrm{sav}}\right)$. The second value, Vs $=250 \mathrm{~m} / \mathrm{s}$ represented the maximum value of the average soil velocity at the depth of the basin ( $\mathrm{Vs}_{\mathrm{av}}$ at about $100 \mathrm{~m}$ depth). The last value, $\mathrm{Vs}=350 \mathrm{~m} / \mathrm{s}$, represented the velocity by combining the average soil velocity and the arithmetic mean velocity of a thin layer of glacial till.

Figure 5.16 shows the acceleration time series for the horizontal and vertical motions at the station S1, which were essentially identical to one another. Figure 5.17 shows the acceleration time series for the horizontal and vertical components at the station S2. Here the difference in time of the wave phase arrivals were shortened when the velocity of the soil increased as expected. Other than the differences in arrival times, the largest difference which could be seen was the dominant width of the peaks, which shorten as the velocity increased. This 
represented an increase in the fundamental site frequency response and could be seen better through the spectral accelerations as shown in Figure 5.18. The fundamental frequency was dependent upon the average shear wave velocity as shown by Eq. 1.6 and was discussed in Section 1.2.2. The fundamental site frequency changed through these simulations even though the dominant frequency of the source did not. Table 5.17 shows the peak values for each recording along with the percent difference from the reference simulation, the estimated fundamental site frequency from each simulation, and the predicted fundamental site frequency based on the depth and velocity at that location.

\begin{tabular}{|c|c|c|c|c|c|}
\hline $\begin{array}{c}\text { Surface } \\
\text { Number }\end{array}$ & $\begin{array}{c}\text { Name of } \\
\text { Parameter } \\
\text { set }\end{array}$ & Vp (m/s) & Vs (m/s) & $\begin{array}{c}\text { Density } \\
\left(\mathrm{kg} / \mathrm{m}^{3}\right)\end{array}$ & Q value \\
\hline \multicolumn{7}{|c|}{ Simulation 1: Reference Model (Figure 5.1) } \\
\hline Surface 1 & Soil Type I & 1600 & 150 & 1600 & 100 \\
\hline Surface 2 & Rock Type I & 6500 & 3500 & 2800 & 1000 \\
\hline Surface 3 & Rock Type I & 6500 & 3500 & 2800 & 1000 \\
\hline \multicolumn{7}{|c|}{ Simulation 2: Reference Model (Figure 5.1) } \\
\hline Surface 1 & Soil Type II & 1700 & 250 & 1600 & 100 \\
\hline Surface 2 & Rock Type I & 6500 & 3500 & 2800 & 1000 \\
\hline Surface 3 & Rock Type I & 6500 & 3500 & 2800 & 1000 \\
\hline \multicolumn{7}{|c|}{ Simulation 3: Reference Model (Figure 5.1) } \\
\hline Surface 1 & Soil Type III & 1800 & 350 & 1600 & 100 \\
\hline Surface 2 & Rock Type I & 6500 & 3500 & 2800 & 1000 \\
\hline Surface 3 & Rock Type I & 6500 & 3500 & 2800 & 1000 \\
\hline
\end{tabular}

Table 5.16: List of parameters used for each surface in the reference model for testing the effect of different velocities for the soil. 

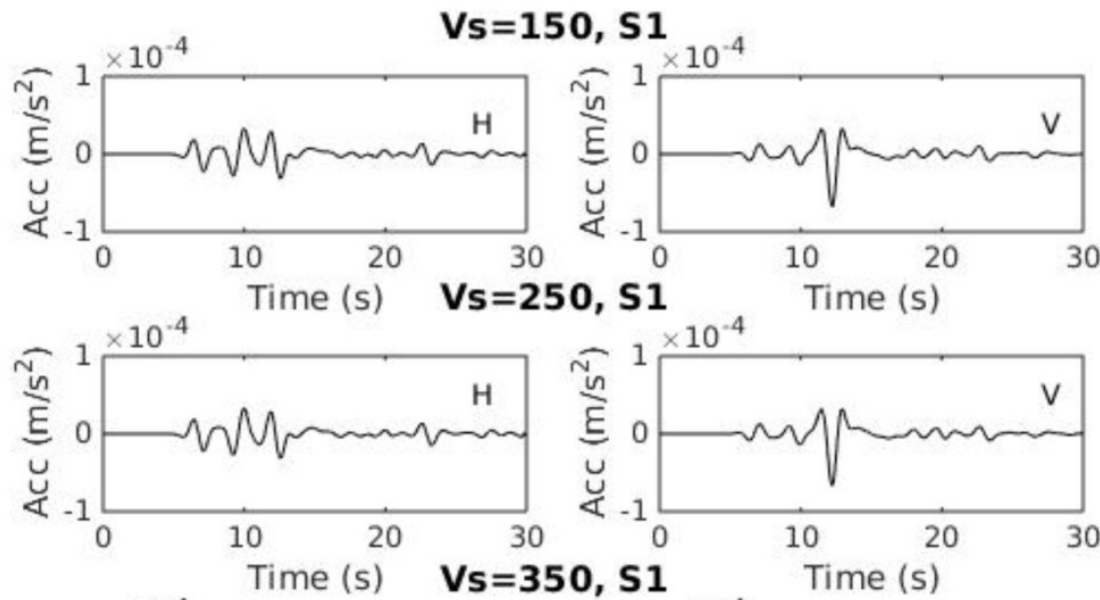

\section{Changing the Soil Velocity for the Reference Model}
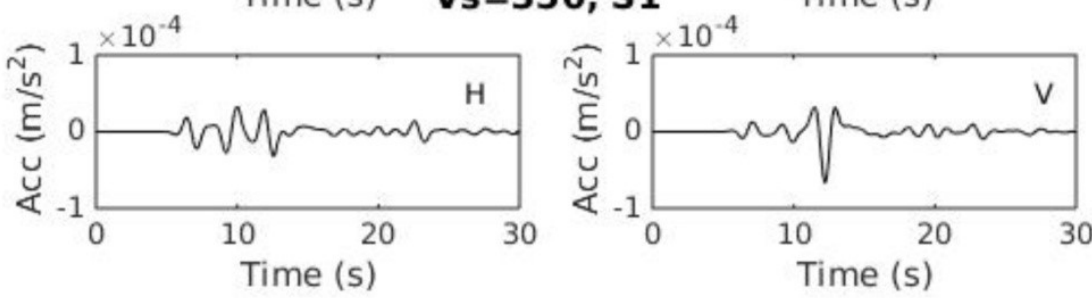

Figure 5.16: Acceleration time series resulted from the basin modeling for the Ladysmith earthquake for the horizontal component (left column) and vertical component (right column) recorded on the station S1 for the different velocity values for the soil. The reference simulation with $V s=150$ is the top row, the simulation with $V s=250$ is the middle row, and the simulation with $\mathrm{Vs}=350$ is the bottom row.
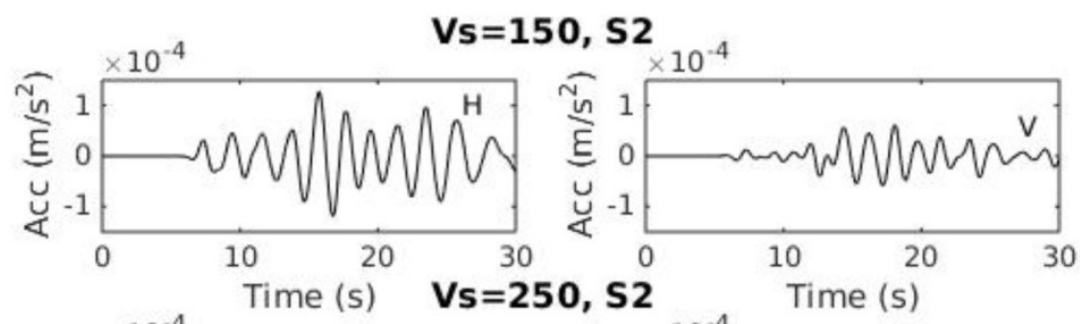

\section{Changing the Soil Velocity for}
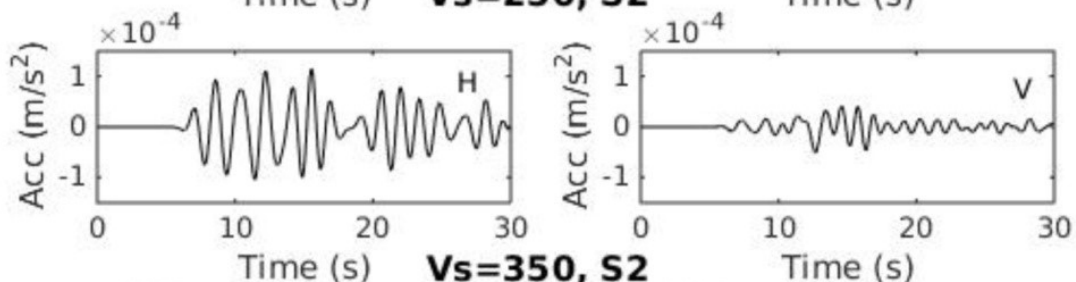
the Reference Model
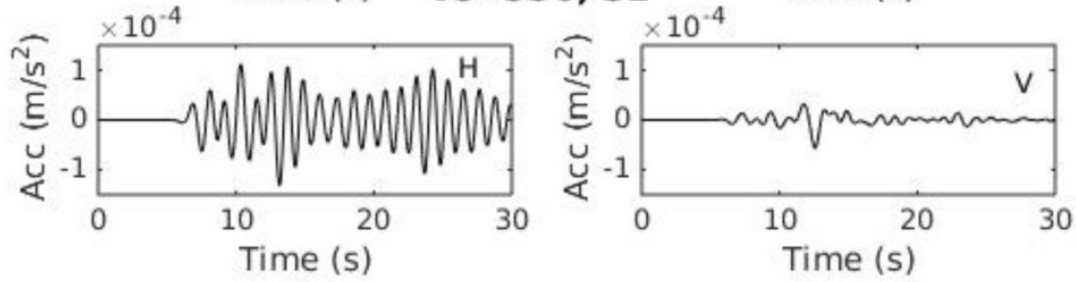

Figure 5.17: Acceleration time series resulting from the basin modeling for the Ladysmith earthquake for the horizontal component (left column) and vertical component (right column) recorded on the station S2 for the different velocity values for 
the soil. The reference simulation with $V s=150$ is the top row, the simulation with $V s=250$ is the middle row, and the simulation with Vs $=350$ is the bottom row.

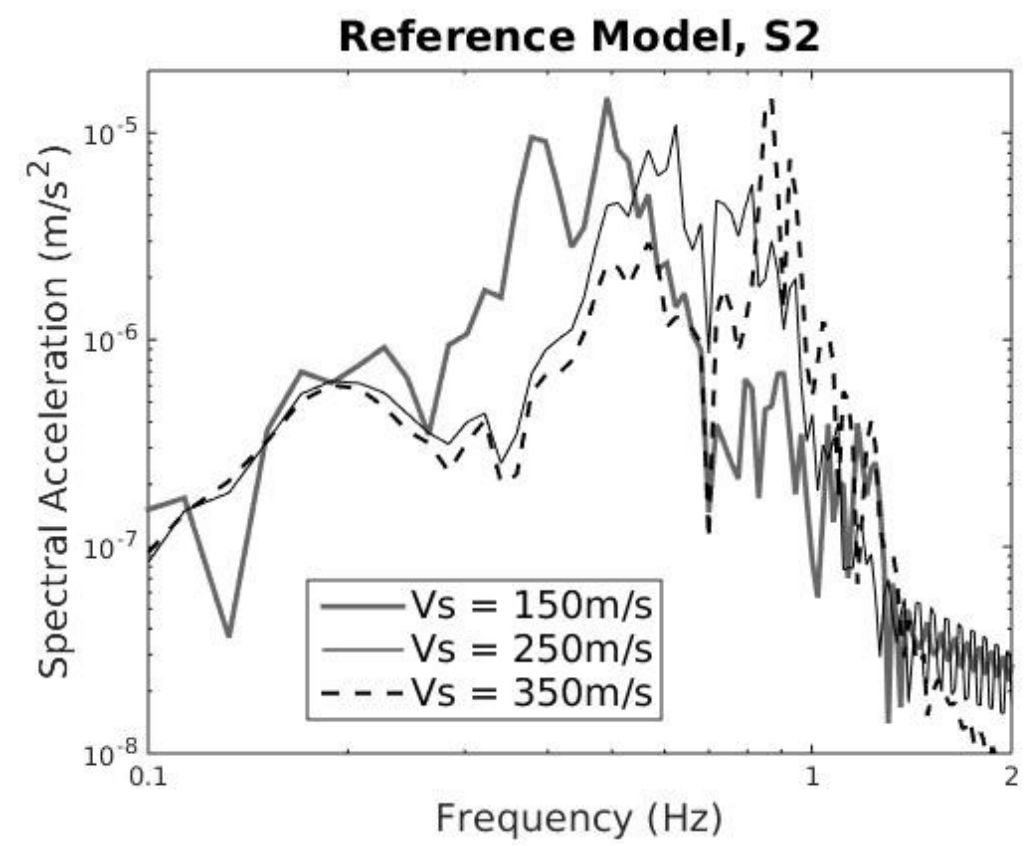

Figure 5.18: Horizontal spectral accelerations for the simulations with different velocities for the soil. 


\begin{tabular}{|c|c|c|c|c|c|c|c|c|c|c|}
\hline \multirow{3}{*}{ Model } & \multicolumn{4}{|c|}{ Peak Value $\left(\mathrm{m} / \mathrm{s}^{2}\right)$} & \multicolumn{4}{|c|}{$\begin{array}{l}\text { Peak Value Difference from Reference } \\
(\%)\end{array}$} & \multirow{3}{*}{$\begin{array}{l}\text { Simulated } \\
\text { Peak } \\
\text { Frequency } \\
(\mathrm{Hz})\end{array}$} & \multirow{3}{*}{$\begin{array}{l}\text { Estimated } \\
\text { Peak } \\
\text { Frequency } \\
\quad(\mathrm{Hz})\end{array}$} \\
\hline & \multicolumn{2}{|c|}{ Horizontal } & \multicolumn{2}{|c|}{ Vertical } & \multicolumn{2}{|c|}{ Horizontal } & \multicolumn{2}{|c|}{ Vertical } & & \\
\hline & S1 & $\mathrm{S} 2$ & S1 & S2 & S1 & $\mathrm{S} 2$ & S1 & S2 & & \\
\hline $\begin{array}{c}\mathrm{Vs}= \\
250 \mathrm{~m} / \mathrm{s}\end{array}$ & $3.25 \mathrm{E}-05$ & $1.15 E-04$ & $6.69 \mathrm{E}-05$ & $\begin{array}{c}5.06 \mathrm{E}- \\
05\end{array}$ & 1.13 & 9.35 & 1.44 & 17.70 & 0.7 & 0.62 \\
\hline $\begin{array}{c}\mathrm{Vs}= \\
350 \mathrm{~m} / \mathrm{s}\end{array}$ & $3.28 \mathrm{E}-05$ & $1.32 \mathrm{E}-04$ & $6.70 \mathrm{E}-05$ & $\begin{array}{c}5.63 \mathrm{E}- \\
05\end{array}$ & 0.28 & 4.10 & 1.35 & 8.53 & 0.9 & 0.86 \\
\hline
\end{tabular}

Table 5.17: Peak values from the simulations with a varying velocity for the soil. 


\subsection{Discussion}

The sensitivity tests were performed to quantify the amount of error produced by the simulations based on the possible variance of the input parameters. Although there are several references constraining each parameter to a varying degree, there was still some variability which exists when choosing the value for simulating ground motions from a particular earthquake. The choice of the value for each input parameter was selected based on the values found from previous studies, and the performance of the value in the test simulations. Table 5.18 lists the parameters used and the estimated error in the simulations, based on the parameters which were taken from the literature along with their associated error.

From the tests was is also possible to determine affect of the parameters the different aspects of the simulation. Most of these parameters effects were expected; however, some are not. For example: a change in soil attenuation should not affect the ground motions on station S1. However, as seen in Table 5.15, there was a difference between the peak values at the station S1 for the reference simulation and the other simulations of a maximum of $0.45 \%$. This was possibly caused by a positive interference pattern from a reflected wave from the basin boundary and the arrival of the S-wave. The intrinsic error in completing a numerical simulation, as was seen by the test of a single set of rock parameters using different models, was very small $(<0.003 \%)$, so it is unlikely that this intrinsic error is the cause of the difference of motions outside the basin.

When a layered model is used, having differing velocities in the rock layers, the shape of the entire waveform was changed, as seen in Figure 5.10 and Figure 5.11. The peak values of the 
motions also varied by a large percent (up to $36 \%$ ) when the rock parameters within the model were altered. Although there were surface measurements within the proximity of the basin, the crustal structure below the basin was not well confined, and could be a possible cause of the change in the shape of the arriving waveforms as well as a difference in the peak values.

\begin{tabular}{|c|c|c|c|}
\hline Parameter & Section & Value in literature & $\begin{array}{c}\text { Estimated Error on } \\
\text { Simulation }\end{array}$ \\
\hline Model & 5.2 .1 & N/A & Negligible \\
\hline $\begin{array}{c}\text { Size/Shape for rock } \\
\text { parameters }\end{array}$ & $\begin{array}{c}5.2 .2,5.2 .3, \text { and } \\
5.2 .4\end{array}$ & Not well constrained & $0-46 \%$ \\
\hline Rock Attenuation & 5.3 .1 & $\begin{array}{c}\text { Frequency } \\
\text { dependent, } \\
500 \leq \mathrm{Q} \leq 1500\end{array}$ & $<4 \%$ \\
\hline Soil Attenuation & 5.3 .2 & $\mathrm{Q}=100$ or $\mathrm{Q} \approx 20$ & $\begin{array}{c}\text { Up to } 50 \%, \text { but } \\
\text { probably }<21 \%\end{array}$ \\
\hline Soil Velocity & 5.3 .3 & $\begin{array}{c}\text { Variable, } \\
100 \mathrm{~m} / \mathrm{s} \leq \mathrm{V} \mathrm{s}_{\mathrm{av}} \leq 250 \mathrm{~m} / \mathrm{s}\end{array}$ & $<20 \%$ \\
\hline
\end{tabular}

Table 5.18: Error estimates on the simulations based on variability of input values. 


\subsection{References}

Atkinson, G., and K. Assatourians (2010). Attenuation and source characteristics of the June 23, 2010 Val-des-Bois, Quebec, earthquake, Seismol. Res. Lett., 81, 849-860.

Atkinson, G. M., K. Assatourians, and M. Lamontagne (2014). Characteristics of the 17 May 2013 M 4.5 Ladysmith, Quebec earthquake, Seismol. Res. Lett., 85, 755-762, doi: 10.1785/0220130160.

Bent, A.L., M. Lamontagne, V. Peci, S. Halchuk, G.R. Brooks, D. Motazedian, J.A. Hunter, J. Adams, C. Woodgold, J. Drysdale, S. Hayek, W. N. Edwards (2015). The 17 May 2013 M 4.6 Ladysmith, Quebec, Earthquake, Seismol. Res. Lett., 86, 460-476. doi: 10.1785/0220140138

Crow, H.L. (2010). Low strain damping measurements in Leda Clay using downhole geophysical and lab techniques. MSc thesis. Department of Earth Sciences, Carleton University;

Crow, H.L., J.A. Hunter, D. Motazedian (2011). Monofrequency in situ damping measurements in Ottawa area soft soils, Soil Dyn. Earthq. Eng., 31, 1669-1677

Hunter, J.A., H.L. Crow, G.R. Brooks, M. Pyne, D. Motazedian, M. Lamontagne, A.J.-M. Pugin, S.E. Pullan, T. Cartwright, M. Douma, R.A. Burns, R. L. Good, K. Kaheshi-Banab, R. Caron, M. Kolaj, I. Folahan, L. Dixon, K. Dion, A. Duxbury, A. Landriault, V. Ter-Emmanuil, A. Jones, G. Plastow, D. Muir (2010). Seismic site classification and site period mapping in the Ottawa area using geophysical methods. Geological Survey of Canada. Open File 6273.

LeBoeuf, D., S. Sivathayalan, D. Motazedian, G. Cascante, and H.L. Crow, (2013). Investigating the Dynamic Properties of Leda Clay, in proceedings, Eastern Section of the Seismological Society of America, LaMalbaie, QC October 2013.

Miksat J., T.M. Müller, and F. Wenzel (2008). Simulating three-dimensional seismograms in 2.5Dimensional structures by combining two-dimensional finite difference modelling and ray tracing. Geophys. J. Int., 174, 309-315.

Mereu, R., S. Dineva, and G. Atkinson (2013). The application of velocity spectral stacking to extract information on source and path effects for small-to-moderate earthquakes in southern Ontario, Seismol. Res. Lett., 84, 899-916.

Motazedian, D., Ma, S., and Crane, S. (2013). Crustal shear-wave velocity models retrieved from Rayleigh-wave dispersion data in northeastern North America. Bull. Seism. Soc. Am., 103, 2266-2276. doi:10.1785/0120120187. 
Motazedian, D., J.A. Hunter, H. Torabi, S. Crane, S. Hayek, and H.L. Crow (2015). Investigation of soil amplification factors for the Ottawa area. Part I - Fa, in proceedings, 11 Canadian Conference on Earthquake Engineering, Victoria, BC, July 2015.

Woodgold, C.R.D. (1990). Estimation of Q in eastern Canada using Coda Waves. Bull. Seism. Soc. Am., 80, 411-429. 


\section{Soft Soil Basin Response}

The earthquake shaking response due to a soft soil basin can be difficult to quantify when the impedance contrast of the soft sediment to the subsurface (firm soil or rock) is large and the basin is small. The Kinburn basin has those features, where the basin is only about $7 \mathrm{~km} \times 5 \mathrm{~km}$, and the impedance contrast is as large as 20 . There are several factors which need to be taken into account and many of their effects overlap with one another that occur in these basins. In the case of the Ottawa-Montreal region, basin effects have been proven to be difficult to detect when using only a single rock and soil seismometer pair (Hayek, 2016). The large impedance contrast between the soil and basement rock provides a lot of strong (1-D) amplification effects within the soil that can be seen from the large amplitudes and a long duration of strong motions recorded by seismometers placed on soil.

The recordings made between the rock and soil stations at the several basins in the Ottawa area provide a unique insight into the differences of ground motions although the two sites might be only a small distance apart from one another. Hayek, (2016) and Motazedian et al. (2015) have shown that the amplification seen within these basins is dependent on more than just a one-dimensional soil profile. Using several methods to determine the amplification of the soil station relative to the rock station, these authors have shown that there is a large gap between the expected 1D model values and the recorded motions.

This study uses several methods to attempt to separate the basin effects from the 1D soil effects through different simulations which could separate out these effects. The first set has been done to determine the difference in motion between the presence of a soil basin compared 
to a model with only rock properties. These are Model I, and Model II both of which are shown in Figure 6.1. The second set of simulations was designed to test the effects of a basin structure compared to a single semi-infinite layer of soil of the same thickness as beneath the recording station. These were shown using Models III, IV and V, all three of which are shown in Figure 6.2. The last set of simulations was designed to compare the effects of simulating a 3D model versus the two 2D models, which are cross sections of the short and long axes of the basin. This set uses Models VI, VII and VII, shown in Figure 6.3.

After testing the different possibilities to account for basin effects, an attempt to recreate the ground motions recorded from the Ladysmith Earthquake of 2013, on both the rock and soil recording stations, $\mathrm{KB} 10$ and $\mathrm{KB} 2 \mathrm{O}$ respectively, was made using several different simulations. These simulations use average parameter values of soil and rock as obtained in the literature as well as values based on their performance of recreating the motions. The models used for the simulations are also governed by those that require the least amount of computing resources, yet were still able to simulate the desired effects.

\subsection{Simulated Response}

The first set of simulations examines the effects of a model (Model I in Figure $6.1 \mathrm{~b}$ ) with a basin filled with rock, with the same parameters as the surrounding rock, compared to the same basin when it is filled with soft soil parameters (Model II in Figure $6.1 \mathrm{c}$ ). Both of these models are shown in Figure 6.1, and the properties of each surface is listed in Table 6.1. The results give the expected motions at the $\$ 2$ recording station when there is a large impedance contrast present compared to when there is not any impedance contrast in the model. 
The second set of simulations used a layered crust model. Three different models are simulated; Model III with a single horizontal soft rock layer at the top, Model IV with a horizontal soft rock layer overlaid by a horizontal soil layer of the same thickness as beneath the S2 station, Model V where the actual 2D geometry of soil-filled basin was considered. These models are shown in Figure 6.2 and the properties of each surface are listed in Table 6.1.

The last set of simulations compared: a Northwest to Southeast (NWSE) 2D Model; a Northeast to Southwest (NESW) 2D Model; and a 3D model of the basin, all having the same source. This source was place roughly 800 metres below the bottom of the basin, at the centre of each model. The NWSE 2D Model and a profile of 3D model along the same axis is shown in Figure 6.3. The NESW 2D Model and the profile of the 3D model along this axis is shown in Figure 6.4. Table 6.1 lists the different properties for the surfaces and volumes within these models. 
a)

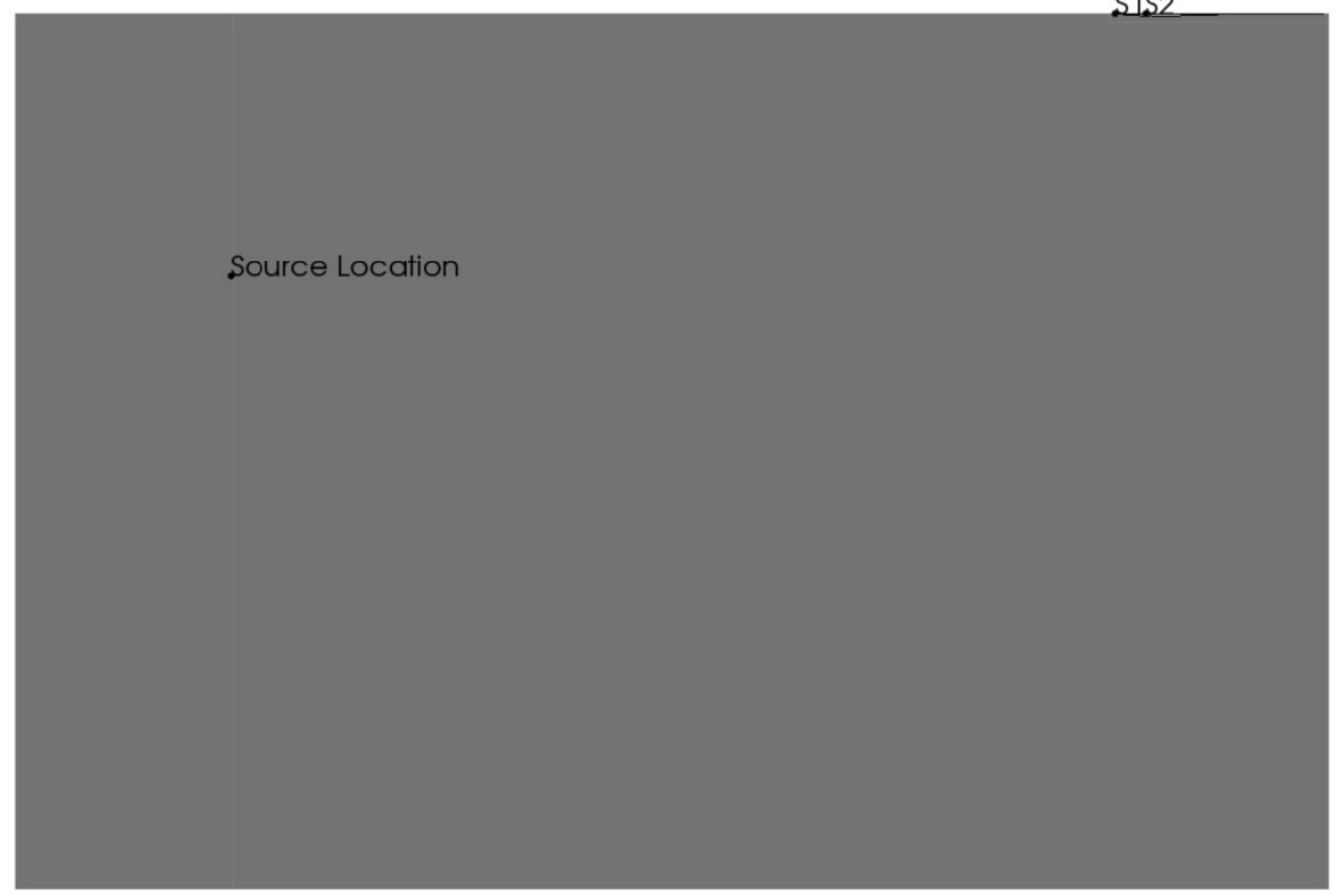

Model I

b) S1

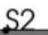

\section{Surface 2}

\section{Surface 1}

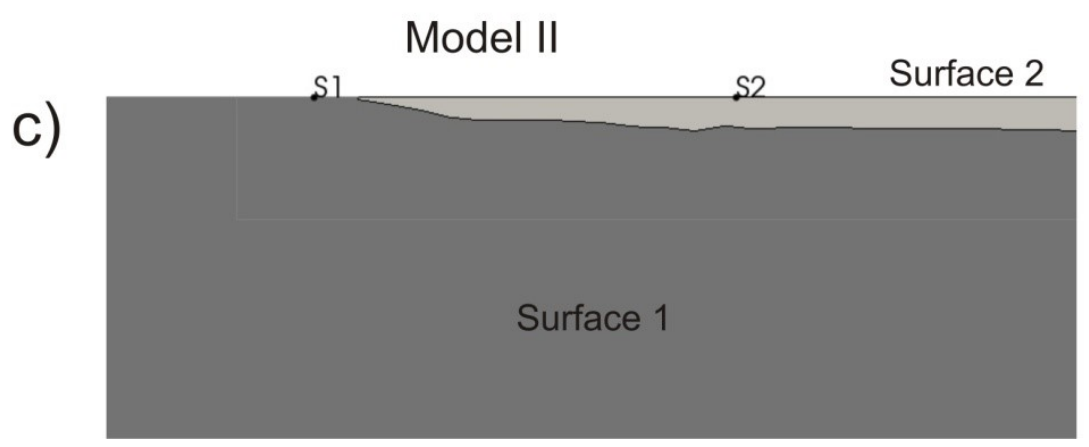

Figure 6.1: Figures of the models for the first set of simulations: a) is the entire simulated model which shows the earthquake source and the S1 and S2 seismic stations, b) is the close up of Model I, with the basin filled with rock, and c) is the close up of Model II, with the basin filled with soil parameters. 
a)

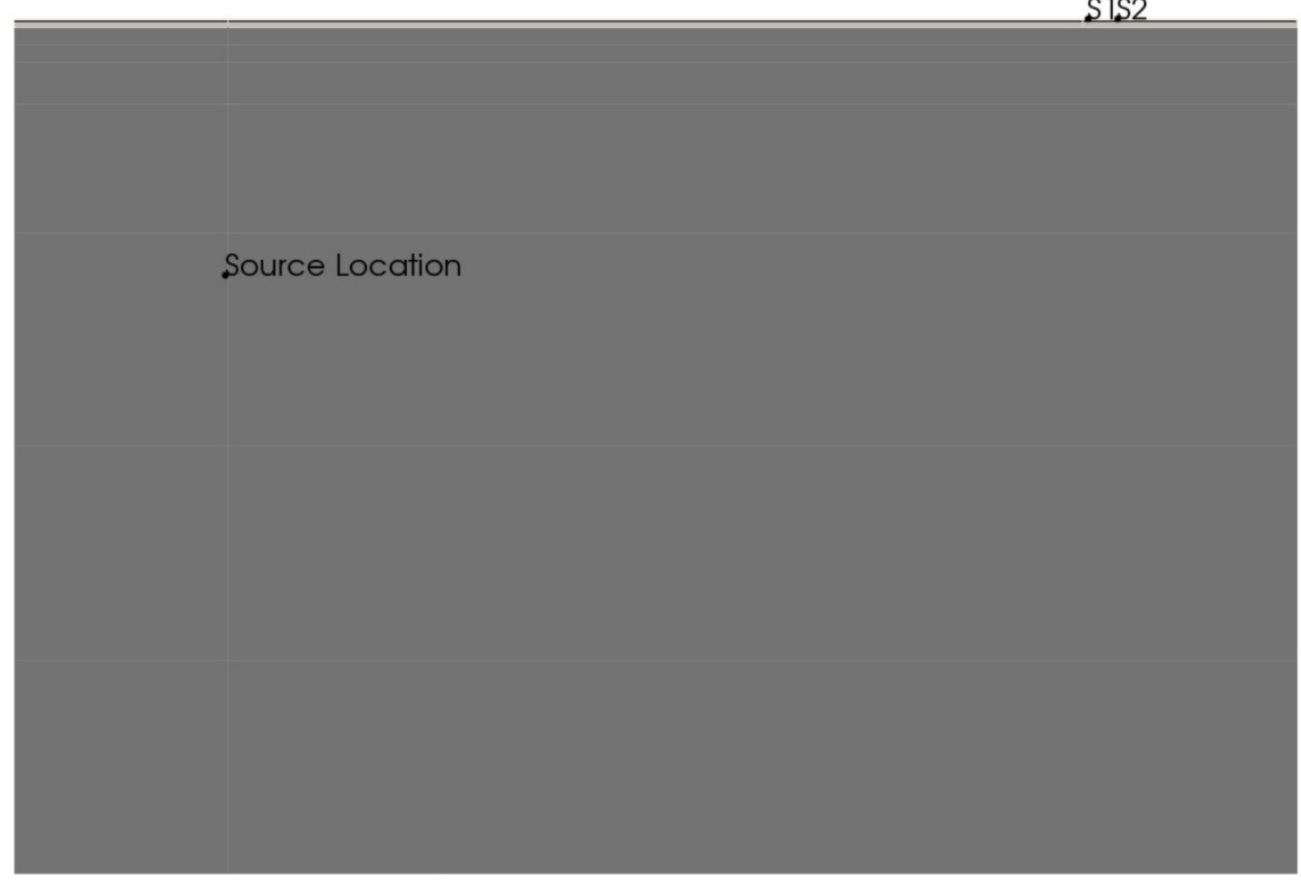

S1- Model III

b)

S1 $\quad$ S2

Surface 2

Surface 1
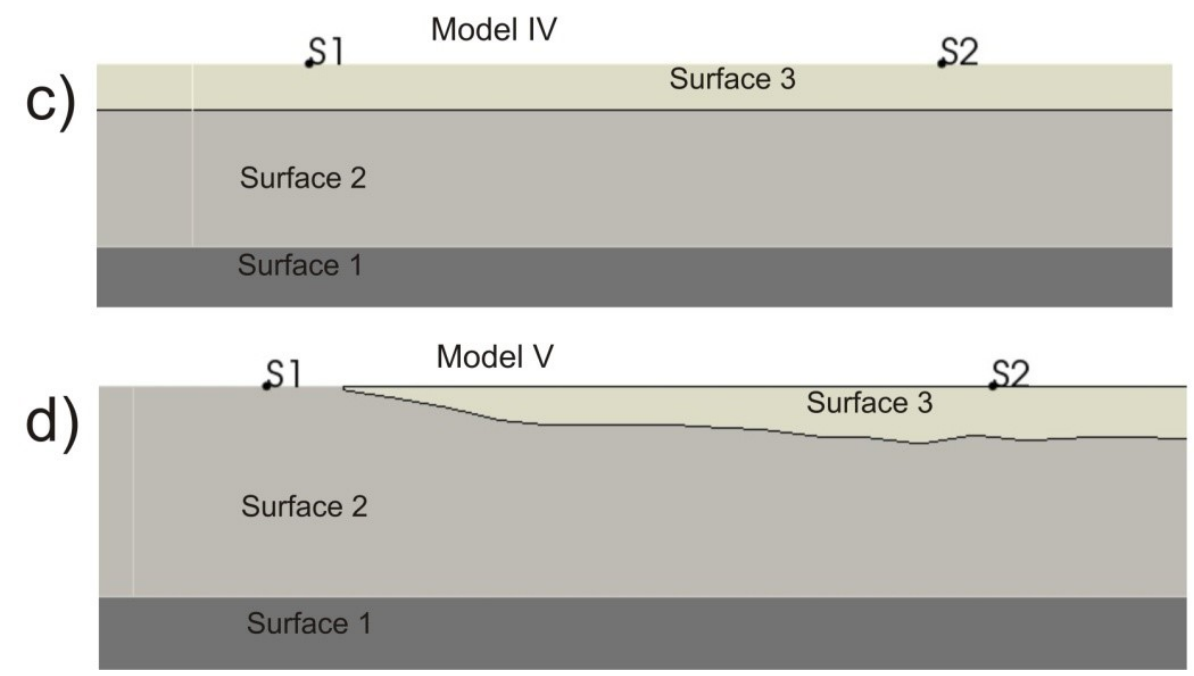

Figure 6.2: Images of the models used for the second set of simulations: a) the entire model, b) the model with only two rock types, Model III, c) the model with two rock types and a horizontal soil layer, Model IV, and d) is the model with two rock types and the soil confined to a basin structure, Model V. 
2D NWSE Model: Model VI

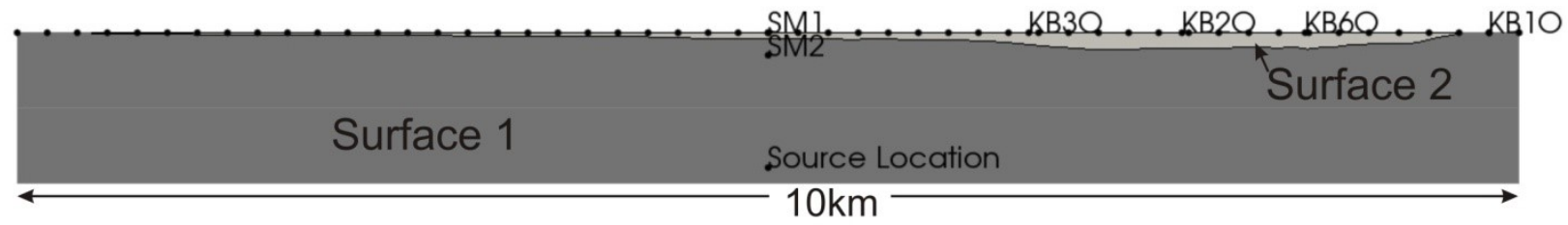

3D NWSE Profile: Model VIII

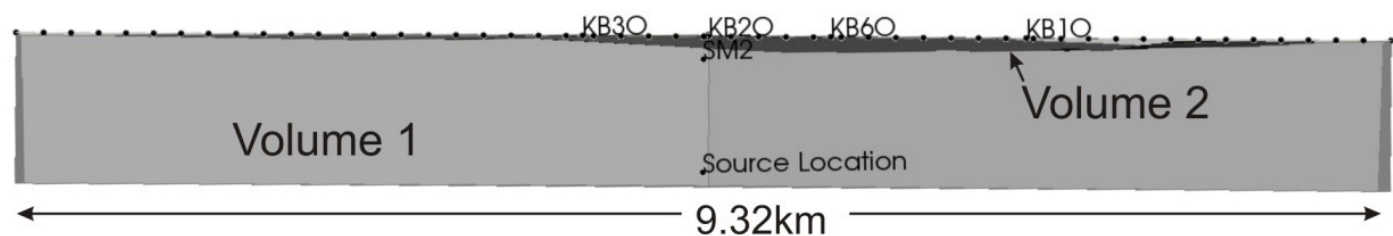

Figure 6.3: The models for the NWSE transect of the Kinburn Basin. The top image is the 2D model and the bottom is a profile of the 3D model along the same axis.

\section{D NESW Model: Model VII}

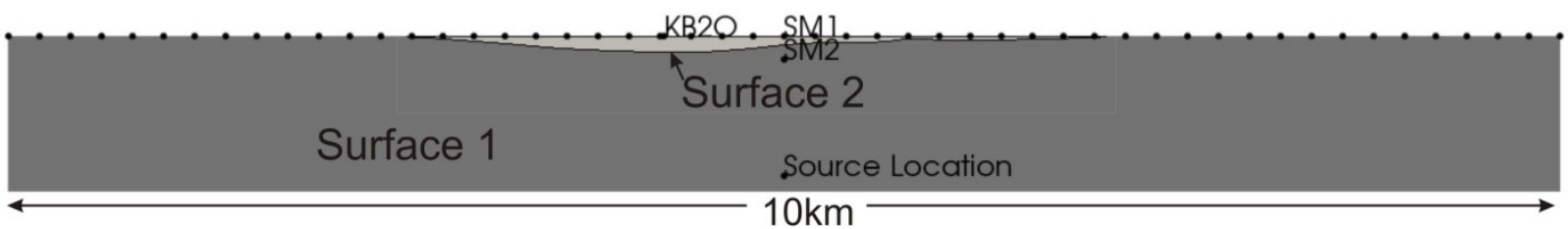

3D NESW Profile: Model VIII

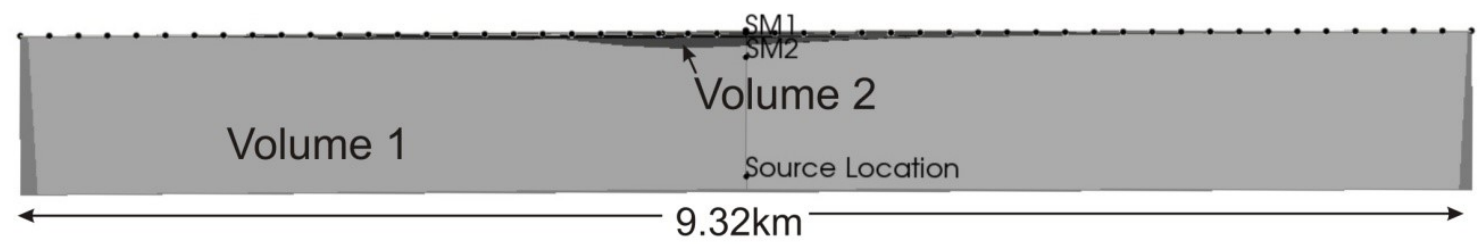

Figure 6.4: The models for the NESW transect of the Kinburn Basin. The top image is the 2D model and the bottom is a profile of the 3D model along the same axis.

\begin{tabular}{|c|c|c|c|c|c|}
\hline $\begin{array}{c}\text { Surface/Volume } \\
\text { Number }\end{array}$ & $\begin{array}{c}\text { Name of } \\
\text { Parameter } \\
\text { set }\end{array}$ & Vp (m/s) & Vs (m/s) & $\begin{array}{c}\text { Density } \\
\left(\mathrm{kg} / \mathrm{m}^{3}\right)\end{array}$ & Q value \\
\hline \multicolumn{7}{|c|}{ Model I: Rock Filled Basin (Figure 6.1 b) } \\
\hline Surface 1 & Rock Type I & 6500 & 3500 & 2800 & 1000 \\
\hline Surface 2 & Rock Type I & 6500 & 3500 & 2800 & 1000 \\
\hline \multicolumn{7}{|c|}{ Model 2: Soil Filled Basin (Figure 6.1 c) } \\
\hline Surface 1 & Rock Type I & 6500 & 3500 & 2800 & 1000 \\
\hline Surface 2 & Soil Type I & 1600 & 150 & 1600 & 100 \\
\hline
\end{tabular}




\begin{tabular}{|c|c|c|c|c|c|}
\hline \multicolumn{7}{|c|}{ Model III: Rock Layers (Figure 6.2 b) } \\
\hline Surface 1 & Rock Type I & 6500 & 3500 & 2800 & 1000 \\
\hline Surface 2 & Rock Type II & 6000 & 2700 & 2600 & 1000 \\
\hline \multicolumn{7}{|c|}{ Model IV: Rock and Soil Layers (Figure 6.2 c) } \\
\hline Surface 1 & Rock Type I & 6500 & 3500 & 2800 & 1000 \\
\hline Surface 2 & Rock Type II & 6000 & 2700 & 2600 & 1000 \\
\hline Surface 3 & Soil Type I & 1600 & 150 & 1600 & 100 \\
\hline \multicolumn{7}{|c|}{ Model V: Rock Layers and Soil Basin (Figure 6.2 b) } \\
\hline Surface 1 & Rock Type I & 6500 & 3500 & 2800 & 1000 \\
\hline Surface 2 & Rock Type II & 6000 & 2700 & 2600 & 1000 \\
\hline Surface 3 & Soil Type I & 1600 & 150 & 1600 & 100 \\
\hline \multicolumn{7}{|c|}{ Model VI: 2D NWSE Model (Figure 6.3 top) } \\
\hline Surface 1 & Rock Type I & 6500 & 3500 & 2800 & 1000 \\
\hline Surface 2 & Soil Type I & 1600 & 150 & 1600 & 100 \\
\hline \multicolumn{7}{|c|}{ Model VII: 2D NESW Model (Figure 6.4 top) } \\
\hline Surface 1 & Rock Type I & 6500 & 3500 & 2800 & 1000 \\
\hline Surface 2 & Soil Type I & 1600 & 150 & 1600 & 100 \\
\hline \multicolumn{7}{|c|}{ Model VIII: 3D Kinburn Basin Model (Figure 6.3 and Figure 6.4) } \\
\hline Volume 1 & Rock Type I & 6500 & 3500 & 2800 & 1000 \\
\hline Volume 2 & Soil Type I & 1600 & 150 & 1600 & 100 \\
\hline
\end{tabular}

Table 6.1: A list of the properties used for the different surfaces and volumes for the models in the test simulations.

\subsubsection{D Rock and Soil Basins (Model I and Model II)}

The comparison between a rock and a soil filled basin structure was completed, as shown by the models in Figure 6.1, with soil and rock parameters listed in Table 6.1. The source for these simulations was the moment tensor solution for the 2013 Ladysmith earthquake from Bent et al. (2015). Figure 6.5 shows the horizontal and vertical components of the simulated acceleration time series for receiver S1, the rock station for both Model I and Model II. It is clear that the simulated time series are very similar and the presence of the soil basin in Model II does not make a considerable difference at this receiver, as expected due to the similarity of the source to receiver paths. 
Figure 6.6 shows the acceleration time series at receiver S2, the rock station for Model I, and the soil station for Model II. There is a large amplification at the receiver S2 from Model II for both the horizontal and vertical motions. The horizontal component shows a stronger amplification and also a stronger resonance than the vertical component. The peak values of the simulations at this receiver is listed in Table 5.5, along with the percent difference, from Eq. 5.1 in Section 5. The resonance in Model II is confirmed by the strong presence of frequencies around $0.5 \mathrm{~Hz}$, the fundamental frequency of this location based on the soil depth, as seen in the smoothed Fourier acceleration spectrum shown in Figure 6.7. The horizontal amplification is larger, and the amplified frequencies have a larger bandwidth compared to the vertical component from Model I to Model II. Figure 6.8 shows the spectral ratio of receiver S2 for Model II compared to receiver S2 for Model I, along with the spectral ratios of S2/S1 for both Model I and Model II. The spectral ratio shows a significant increase, up to 10 times, for the fundamental frequency at this station. This spectral ratio between the different models represents the difference seen at this location between a soft soil basin as compared to a uniform rock in the area. 

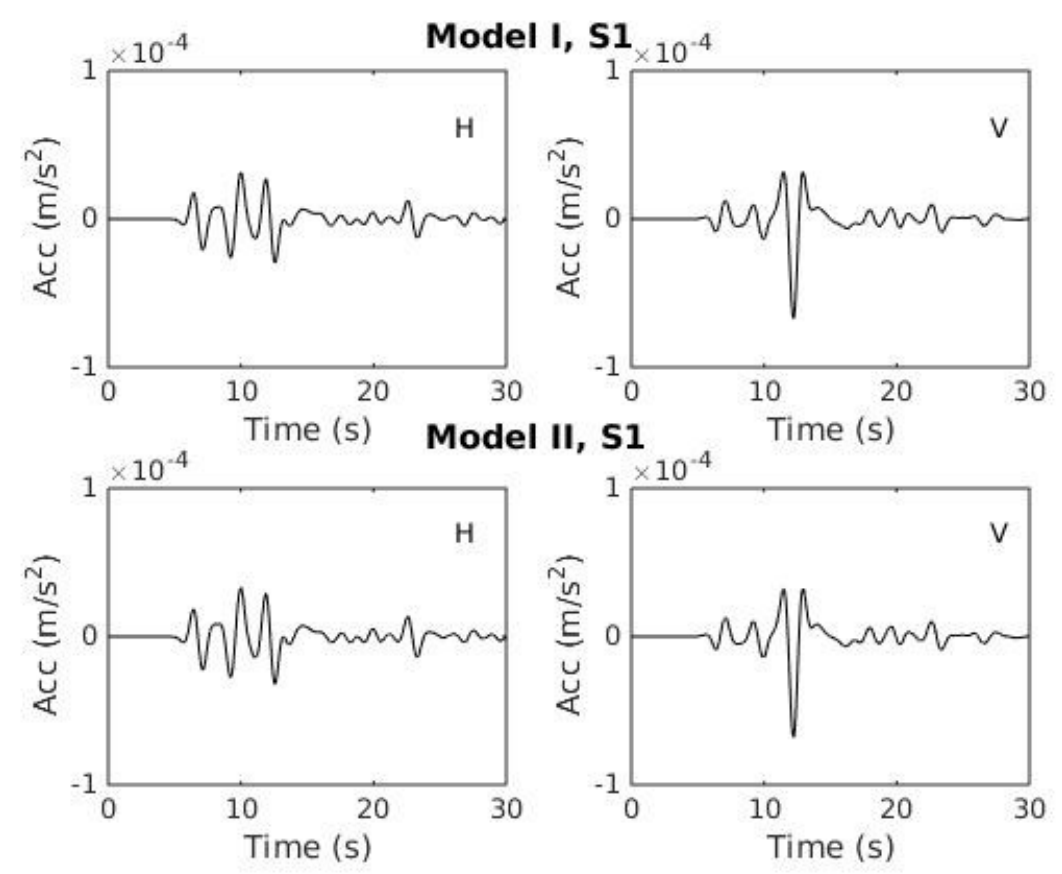

Figure 6.5: Acceleration time series at the receiver S1, for the model with only rock, Model I (top) and rock and soil model, Model II (bottom). The left column is the horizontal component and the right column is the vertical component of motion.
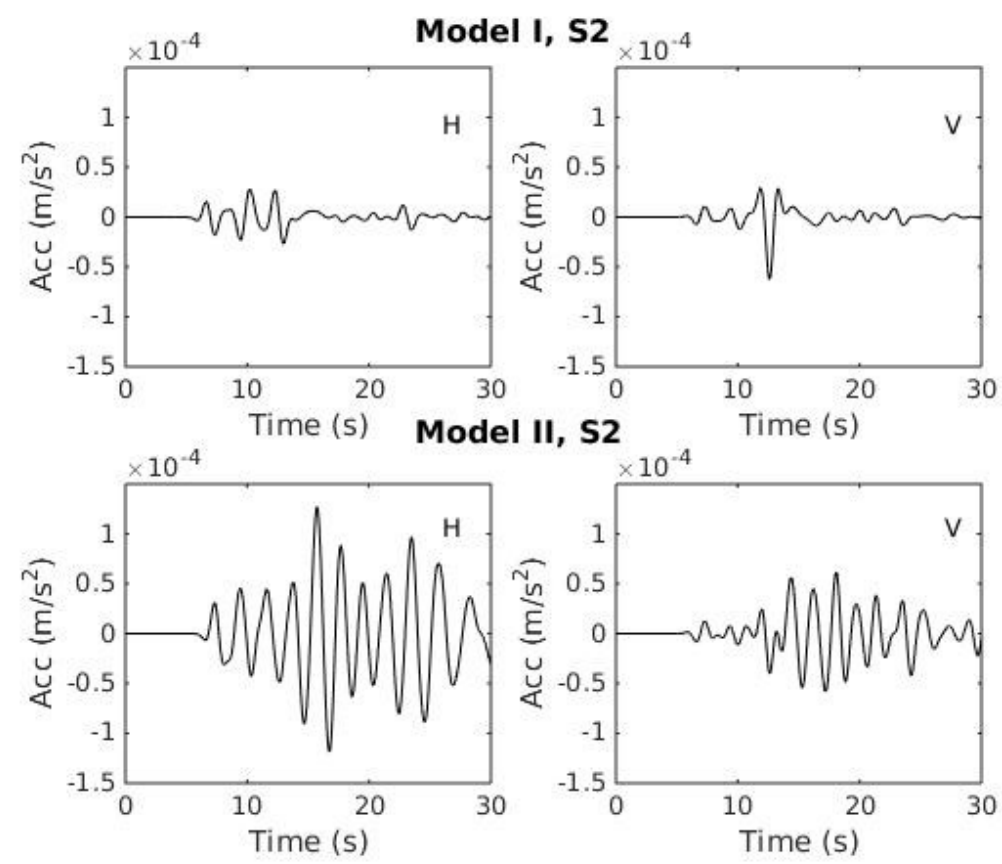

Figure 6.6: Acceleration time series at the receiver S2, for the model with only rock, Model I (top) and rock and soil model, Model II (bottom). The left column is the horizontal component and the right column is the vertical component of motion.

\begin{tabular}{|c|c|c|c|c|}
\hline \multirow{2}{*}{ Model } & \multicolumn{2}{|c|}{ Peak Value $\left(\mathrm{m} / \mathrm{s}^{2}\right)$} & \multicolumn{2}{c|}{$\begin{array}{c}\text { Percent Difference from Model I } \\
(\%)\end{array}$} \\
\cline { 2 - 5 } & Horizontal & Vertical & Horizontal & Vertical \\
\hline
\end{tabular}




\begin{tabular}{|c|c|c|c|c|c|c|c|c|}
\hline & S1 & S2 & S1 & S2 & S1 & S2 & S1 & S2 \\
\hline Model I & $3.29 \mathrm{E}-05$ & $2.79 \mathrm{E}-05$ & $6.79 \mathrm{E}-05$ & $6.26 \mathrm{E}-05$ & & & & \\
\hline Model II & $3.12 \mathrm{E}-05$ & $1.27 \mathrm{E}-04$ & $6.79 \mathrm{E}-05$ & $6.14 \mathrm{E}-05$ & 5.2 & 355.2 & 0.0 & 1.9 \\
\hline
\end{tabular}

Table 6.2: The peak values of the Model I and Model II.
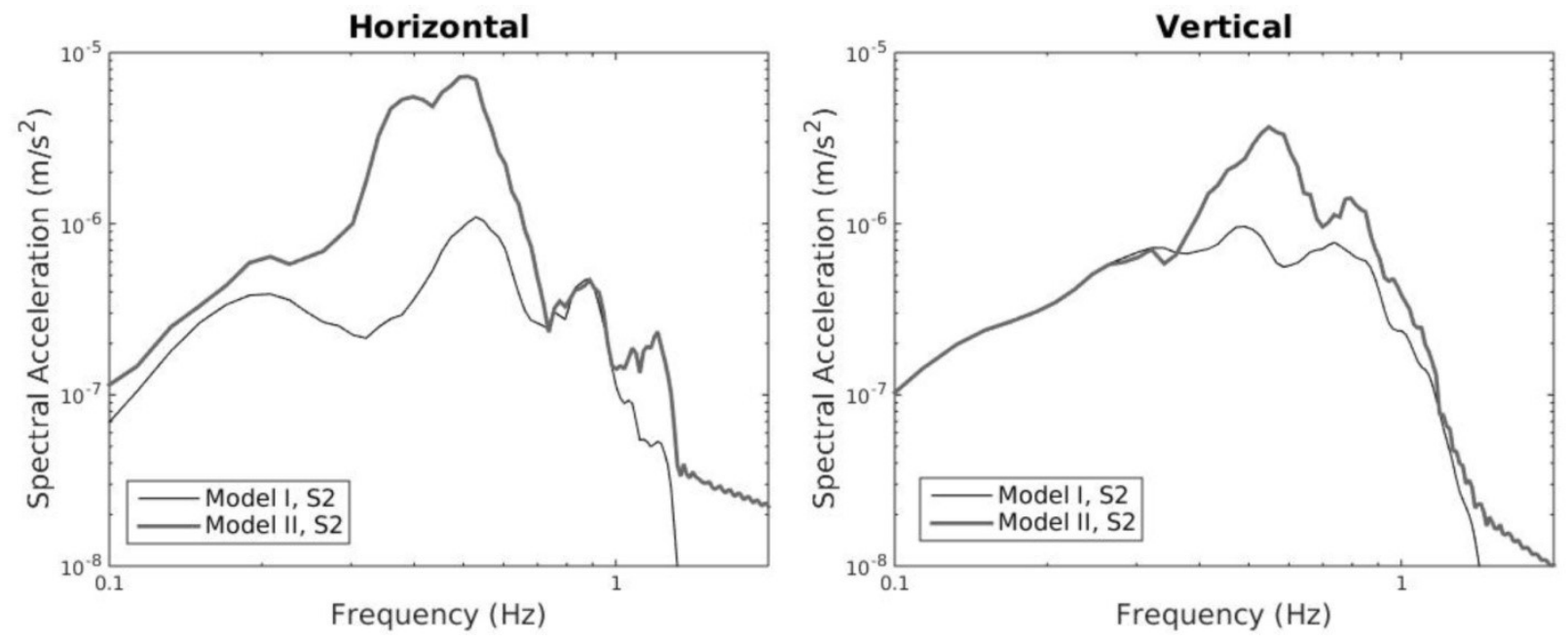

Figure 6.7: Fourier acceleration spectrum at the receiver S2, for the horizontal motions (left) and vertical motions (right).
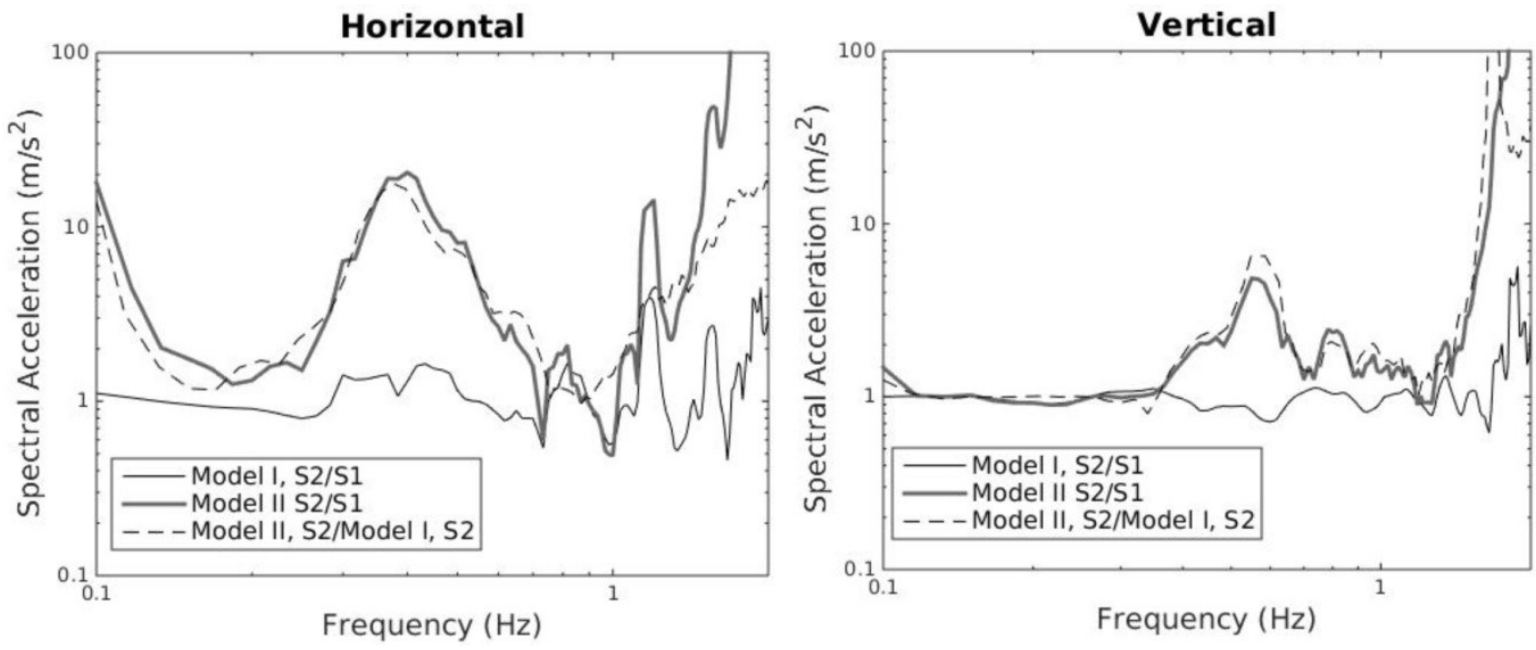

Figure 6.8: The spectral ratios for S2/S1 from Model I and Model II, and the spectral ratio at S2 for Model II divided by Model I.

\subsubsection{D Soil Layering and Basin Structure (Model III, Model IV and Model V)}

The simulations which compare a layering of weakening rock, a layering of weakening rock and soil, and a layering of weakening rock with a soil basin structure, were completed using the 
models shown in Figure 6.2. The reference simulation which uses Model III (Figure 6.2 b), had the top $400 \mathrm{~m}$ of the model with the rock parameters consistent with those which were measured at the surface (Hunter et al., 2010). The second simulation which used Model IV (Figure $6.2 \mathrm{c}$ ) had a layer with the soil properties which is $100 \mathrm{~m}$ in thickness with a layer of softer rock beneath which extends to a further $300 \mathrm{~m}$ in depth. For the last simulation, the soil was confined to a basin structure and used Model V, (Figure $6.2 \mathrm{~d}$ ). The soil recording station in the basin was positioned at a location where the soil depth was approximately $100 \mathrm{~m}$, as seen in Figure $6.2 \mathrm{~d}$. A list of all the properties used for each surface for these models is displayed in Table 6.1.

The horizontal and vertical components of the acceleration time series for the receivers at S1 are shown in Figure 6.9 and in Figure 6.10 for the receivers at S2. In Model III there is not much difference between the two recordings at S1 (Figure 6.9, top) and S2 (Figure 6.10, top), as expected due to the similarity between these two recording stations. There is only a change of about $2 \mathrm{~km}$ horizontal distance compared to the source which is roughly $42 \mathrm{~km}$ away, and were both located on the same material.

There is a large amplification at S1 from Model III compared to Model IV (Figure 6.9 top and middle). There is also a strong resonance seen in the soil layer which is strong and consistent in the horizontal component of motion. The model with a basin structure, Model V, displays some interesting differences from a horizontal layer of soil with the same thickness, Model IV, seen in Figure 6.9 (middle and bottom) and Figure 6.10 (middle and bottom). The amplification in the vertical component is larger within the basin model and there are separate packets showing energy predominantly at the resonance frequency. Although it seems there is a dominant 
frequency at which the site in the basin is resonating, the strength of the signal seems to be revived at later arrivals compared to one large arrival in the soil model, which decreases in amplitude over time.
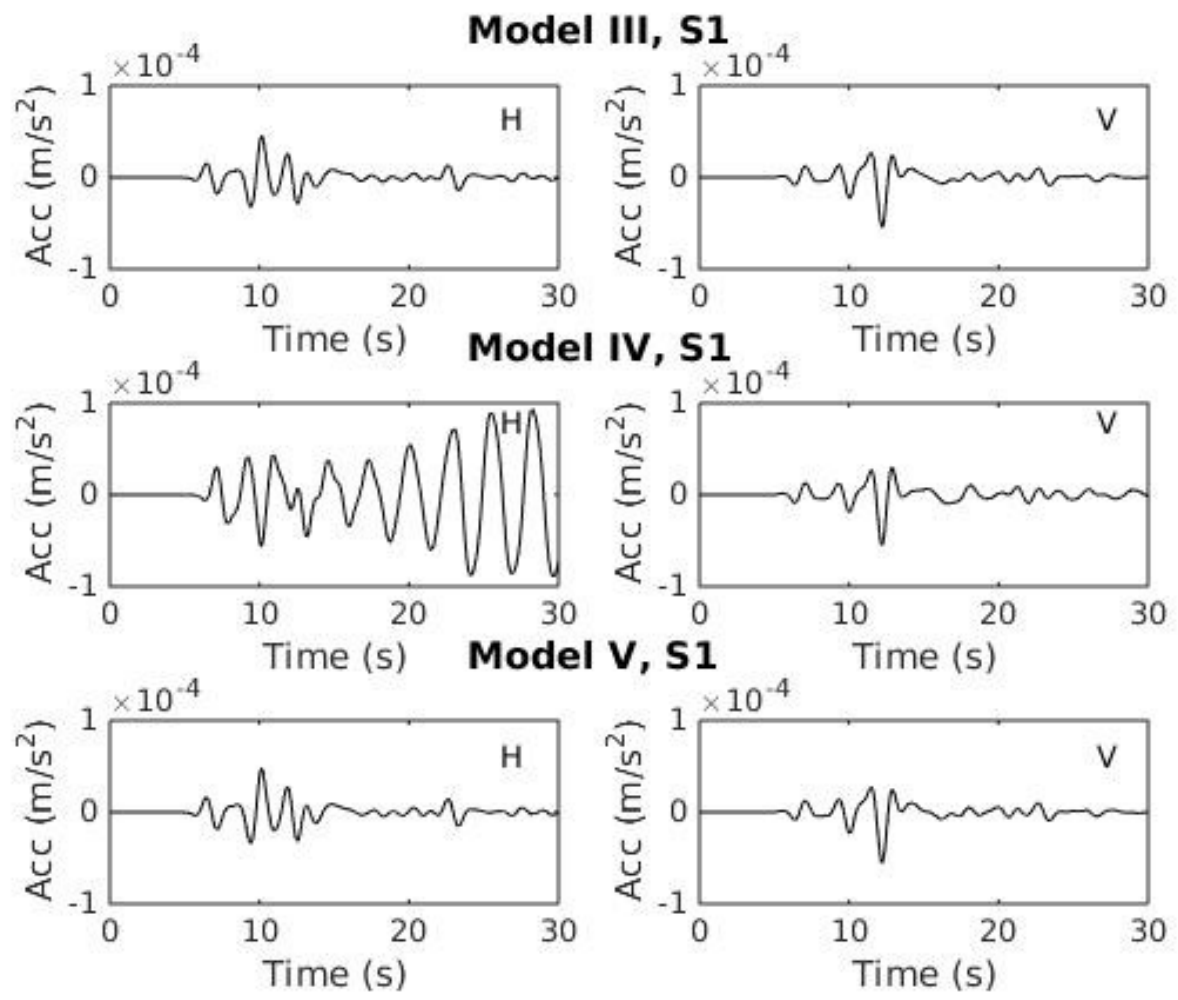

Figure 6.9: Acceleration time series at the rock station, S1, for the rock only reference model, Model III (top), the model with a $100 \mathrm{~m}$ of soil, Model IV (middle) and the model with a basin structure, Model V (bottom). The left column is the horizontal component and the right column is the vertical component. 

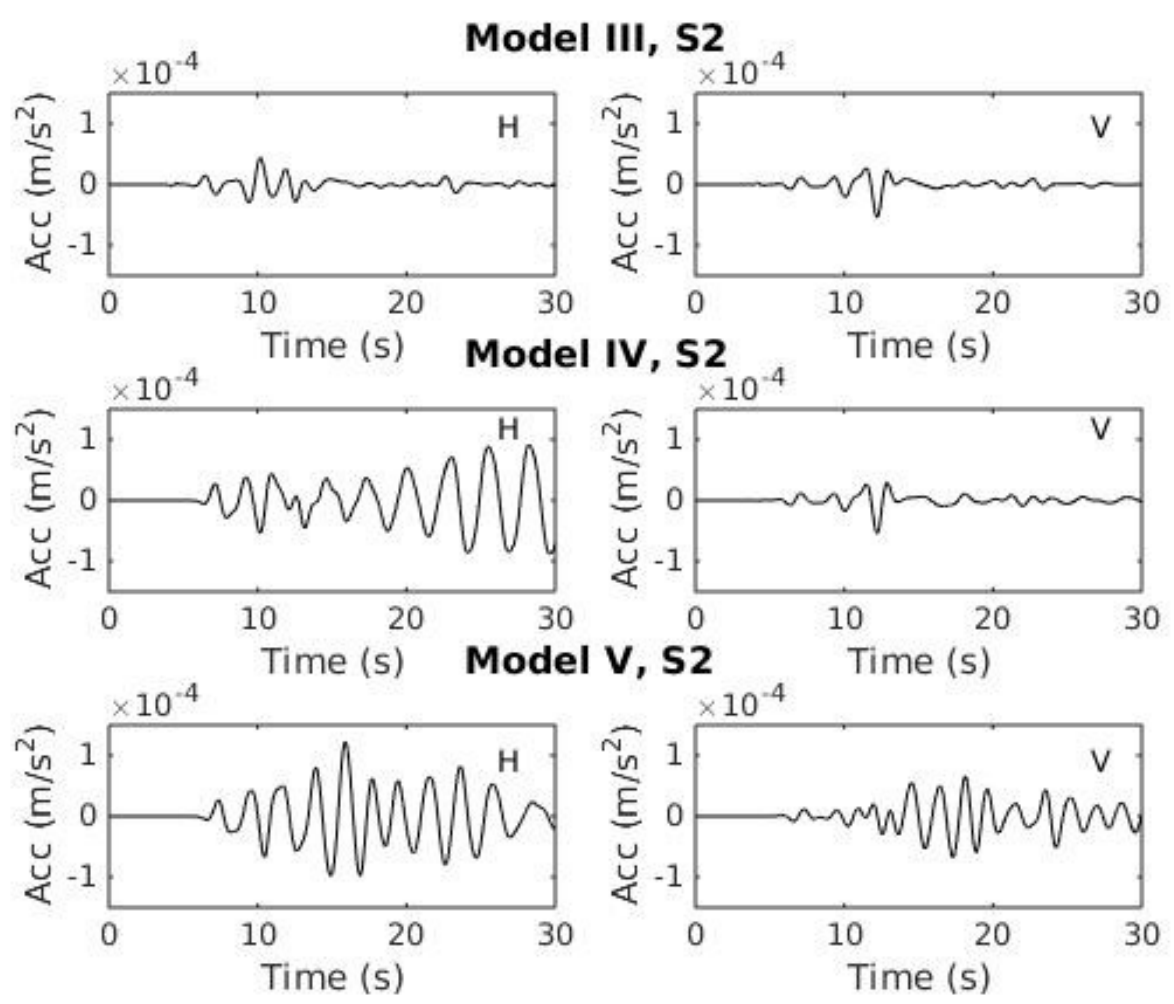

Figure 6.10: Acceleration time series at the soil station, S2, for the rock only reference model, Model III (top), the model with a $100 \mathrm{~m}$ of soil, Model IV (middle) and the model with a basin structure, Model V (bottom). The left column is the horizontal component and the right column is the vertical component.

The horizontal and vertical components of the simulated acceleration spectrum at the S2 receivers are shown in Figure 6.11. The model with a layer of soil (Model IV) shows a strong amplification at about $0.3 \mathrm{~Hz}$ in the horizontal component, and a slight increase at the same frequency for the vertical component. The basin model (Model V) displays a shift in this frequency, with the dominant frequency of this model around $0.5-0.6 \mathrm{~Hz}$ in the soil. There is also a larger increase in amplitude for the vertical spectra compared to the reference and soil layer models. The spectral ratios of Model IV and Model V, both divided by Model III, are shown in Figure 6.12. The horizontal component shows a stronger but narrow amplification for the horizontal layer of soil compared to just a continuous layer of weak rock. The horizontal component for the soil basin structure shows a wider band of frequencies over which the 
amplification is stronger. This effect has been seen by the actual recordings of earthquakes in this basin (Hayek, 2016). The vertical component does not display a large amplification for the layer of soil, Model IV, but does show stronger amplifications in the soil basin, Model V.
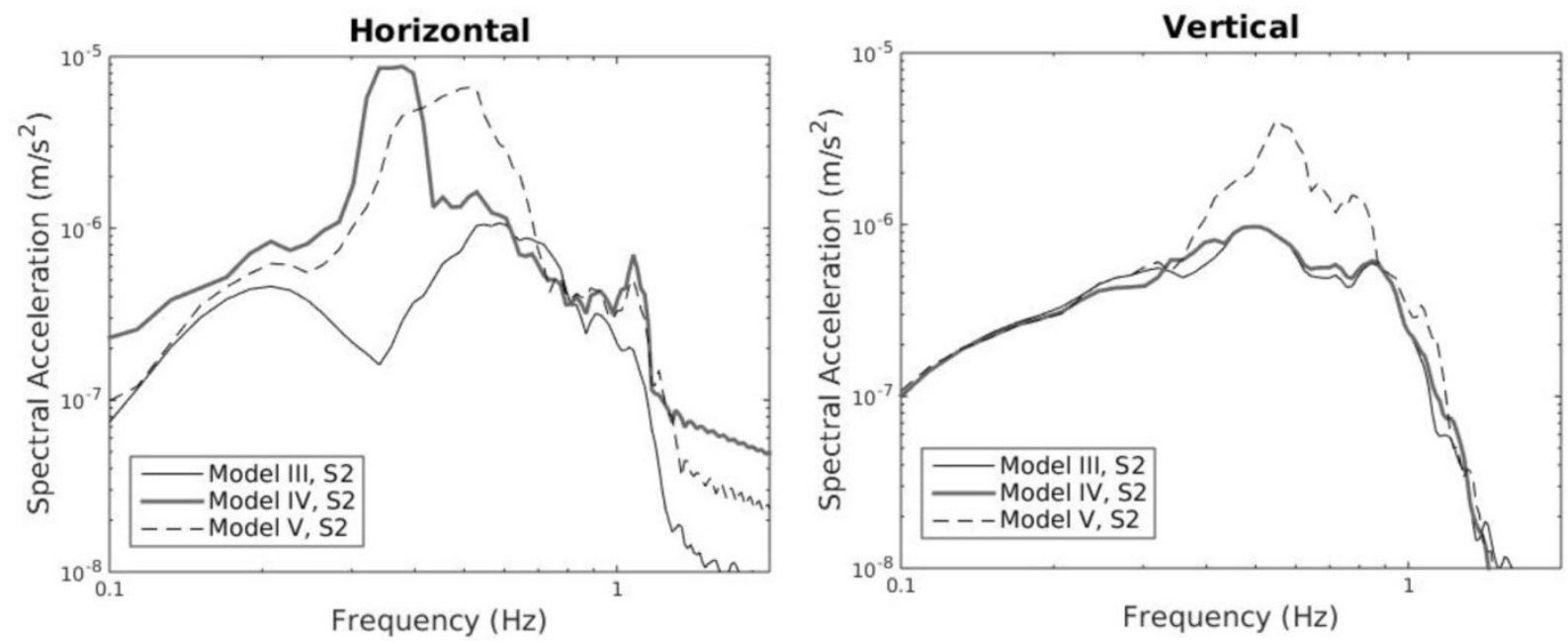

Figure 6.11: Fourier acceleration spectrum for the different models for the horizontal component (left) and vertical component (right) at receiver S2.
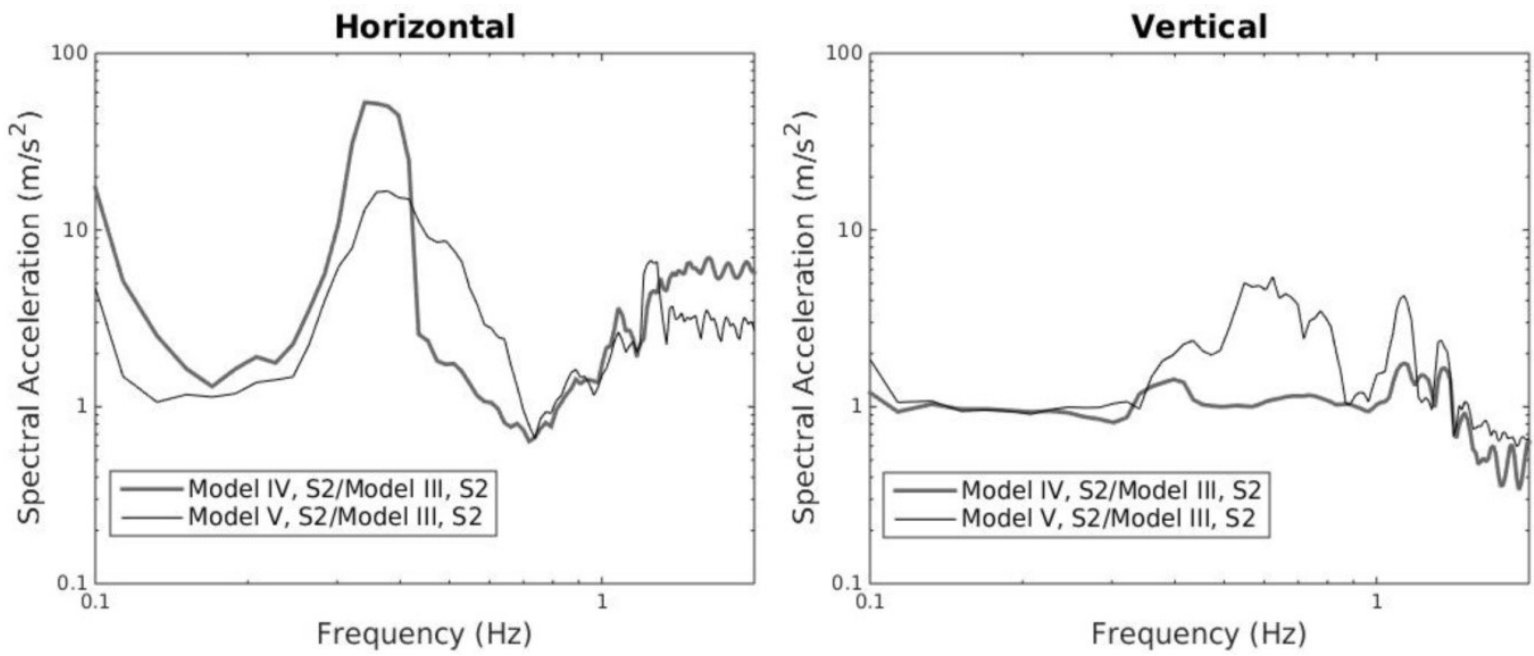

Figure 6.12: Spectral ratios for Model IV and Model V divided by Model III at receiver S2.

These simulations show there is a dependence on subsurface geometrical structure for the ground motions. The increased duration and resonance has different properties when there is just a horizontal layer of soil extending the entire model compared to when the soil is contained 
in a basin structure. These main effects can include: 1 ) increased amplitude at first arrivals for a basin structure; 2) later resonance effect seeming to be arriving in different packets within the basin; and 3) a wider band of amplified frequencies.

\subsubsection{D and 3D Kinburn Basin Comparisons (Models VI, VII and VIII)}

There were two 2D and one 3D simulations completed (Models VI, VII and VIII), using a unit upwards force source, to compare the effects of the shape of the basin on the ground motions at the surface. The 2D models were orthogonal profiles of the long (NWSE, Model VI) and short (NESW, Model VII) axis of the basin, and both extend to $10 \mathrm{~km}$ in length. The 3D model (Model VIII) is $6 \mathrm{~km} \times 7 \mathrm{~km}$ and contains the measured soil depth of the basin as outlined in Chapter 2.5, with a minimum soil depth of 10 metres. A NWSE profile of the 3D model is shown in Figure 6.3, and a NESW profile is shown in Figure 6.4. The minimum soil depth within this model was needed in order to create a stable mesh for numerical simulations.

The source was placed at the centre of the models at a depth of $900 \mathrm{~m}$ from the surface, for both 2D simulations (Model VI, and Model VII) and the 3D simulation (Model VIII). There were three receivers in line with the centre of the model for each: one receiver was located at the source; one located at 150m depth (SM2); and one at the surface (SM1), as shown in Figure 6.3 and Figure 6.4. The vertical motion for all three receivers in each of the models is shown in Figure 6.13. The radial motion for the 3D model (Model VIII) is aligned with the 2D NESW model (Model VII) and shown in Figure 6.14. The horizontal motion for the 2D NWSE model (Model VI) and the aligned horizontal motion from the 3D model (Model VIII) for the same receivers are shown in Figure 6.15. The vertical component is similar in all models with the dominant pulse travelling 
upwards from the source producing a strong resonating effect at the surface. However, each model displayed a different resonance frequency, as shown by the spectral acceleration in Figure 6.16. The horizontal motions for the NESW profile in the 3D model (Model VIII) as well as the horizontal motions from the NESW 2D model (Model VII) were similar at the surface (receiver SM1). The surface shows a strong pulse and resonances, however the motions at the source and at $150 \mathrm{~m}$ depth (receiver SM2) show a much stronger pulse in the $2 \mathrm{D}$ model, as seen in Figure 6.14. The horizontal motions from the 2D NWSE model (Model VI) and the motions in the NWSE direction for the 3D model (Model VIII) are different from one another, as seen in Figure 6.15. The surface receiver (SM1) in the 2D model has the strongest amplitudes arriving later than the 3D model. This provides some evidence to support the hypothesis that the impact of the shape of the basin will have an effect on the different motions as seen within the basin. 

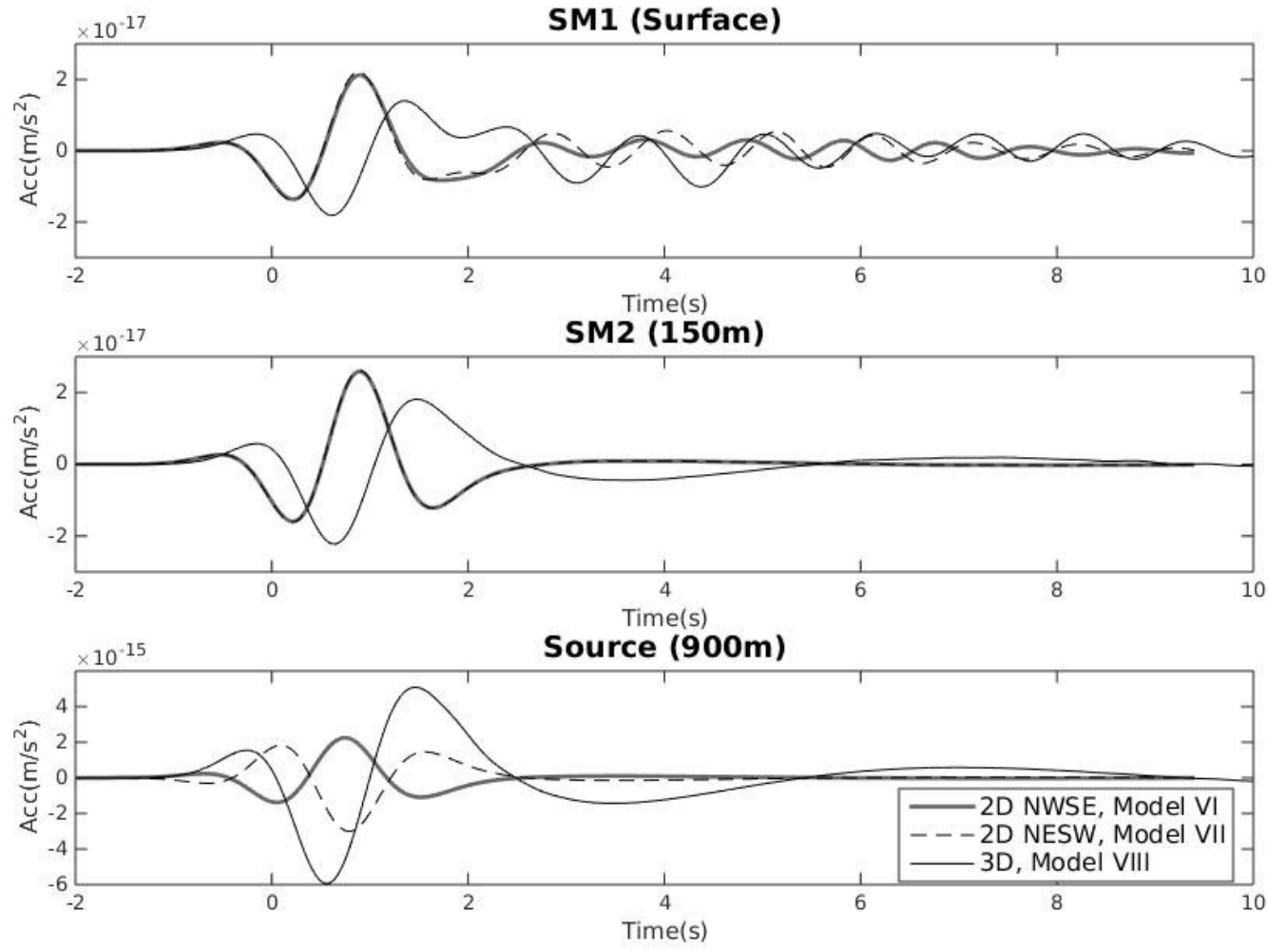

Figure 6.13: Acceleration time series for the vertical component of motion for the different models for receivers located at the surface (SM1, top), 150m depth (SM2, middle) and at the source (bottom). 

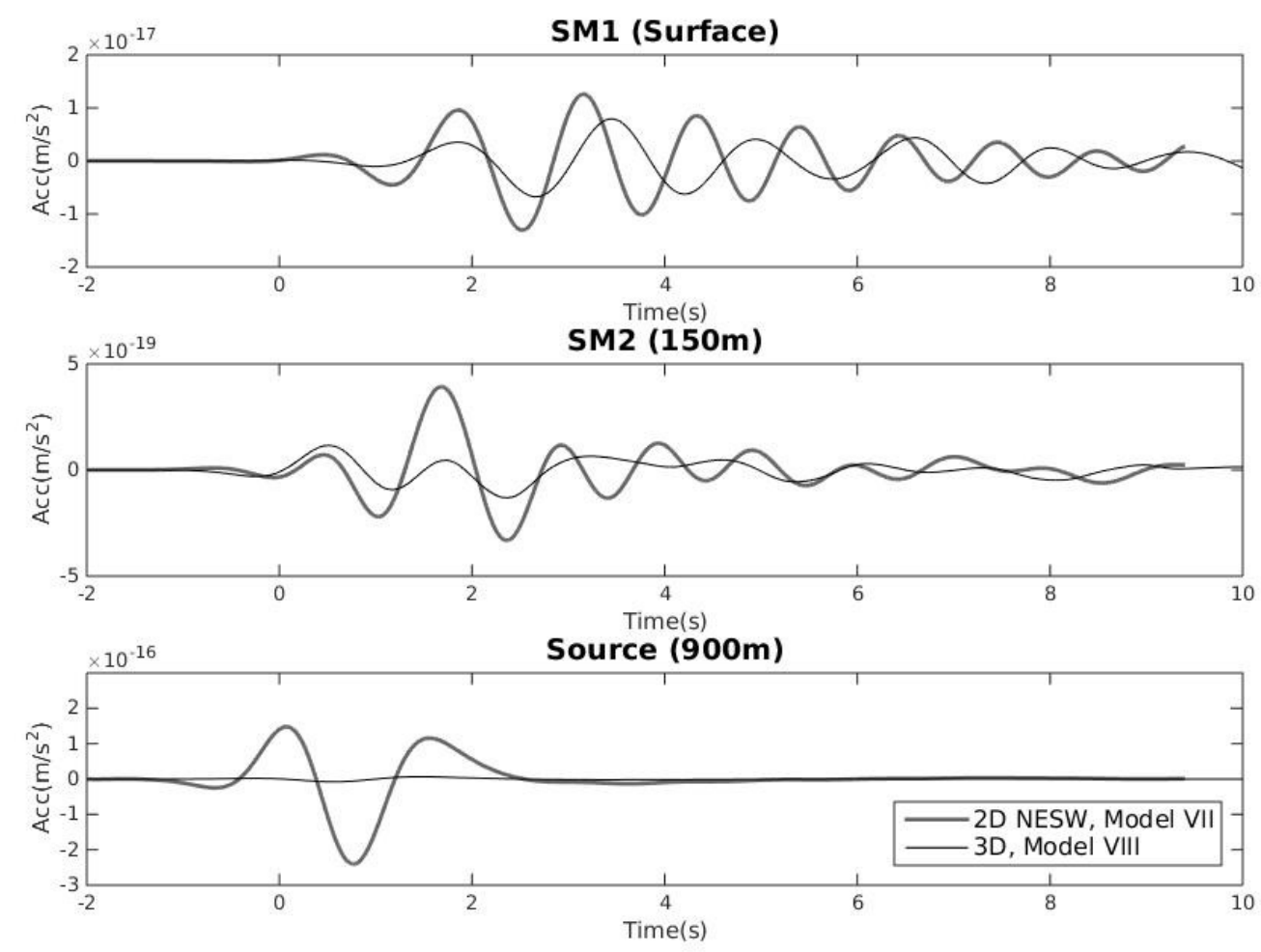

Figure 6.14: Acceleration time series for the radial component of motion for the 3D model (Model VIII) and the 2D NESW model (Model VII) for receivers located at the surface (S1, top), 150m depth (S2, middle) and at the source (bottom). 

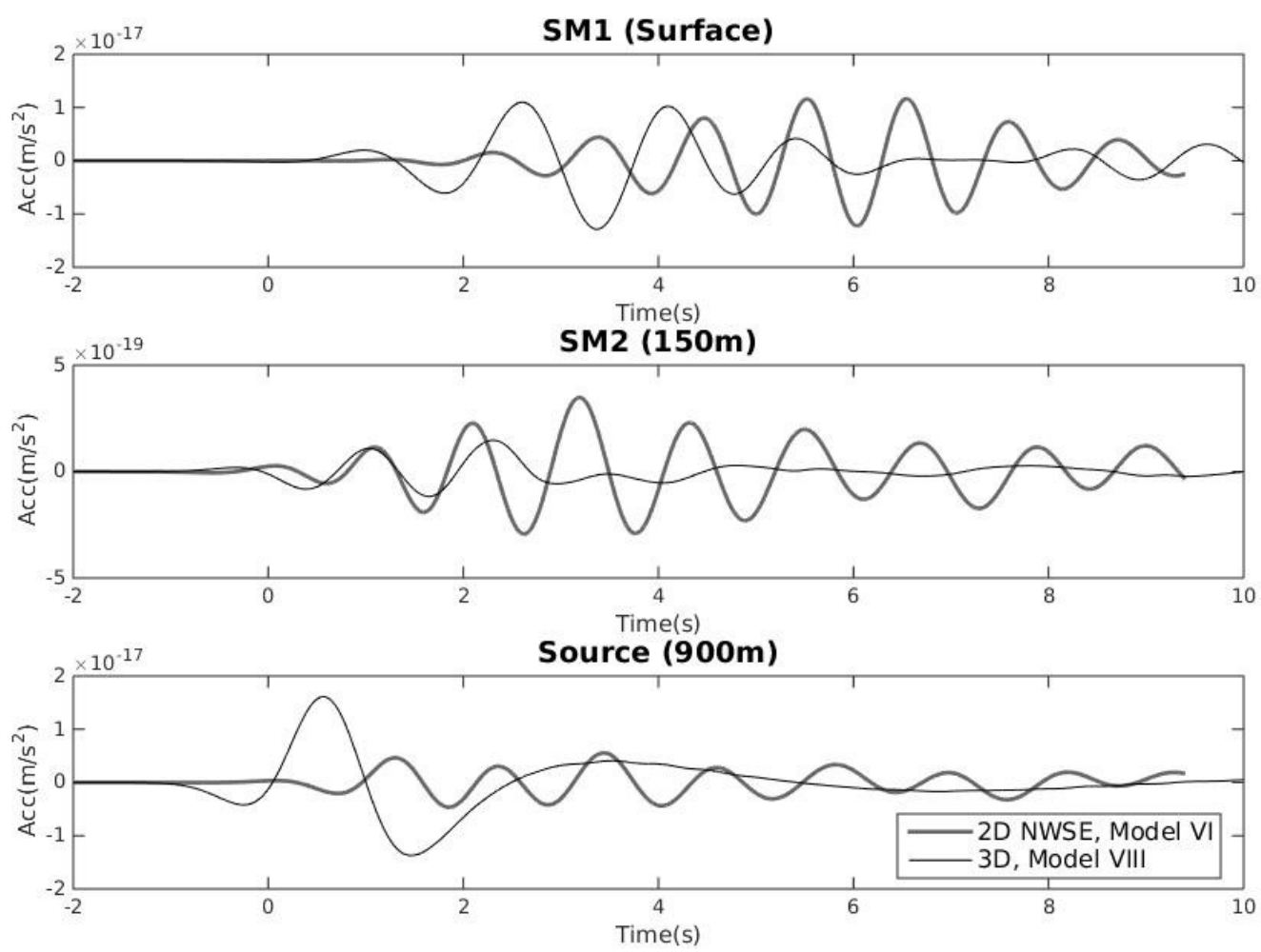

Figure 6.15: Acceleration time series for the radial component of motion for the 3D model (Model VIII) and the 2D NWSE model (Model VI) for receivers located at the surface (top), 150m depth (middle) and at the source (bottom).
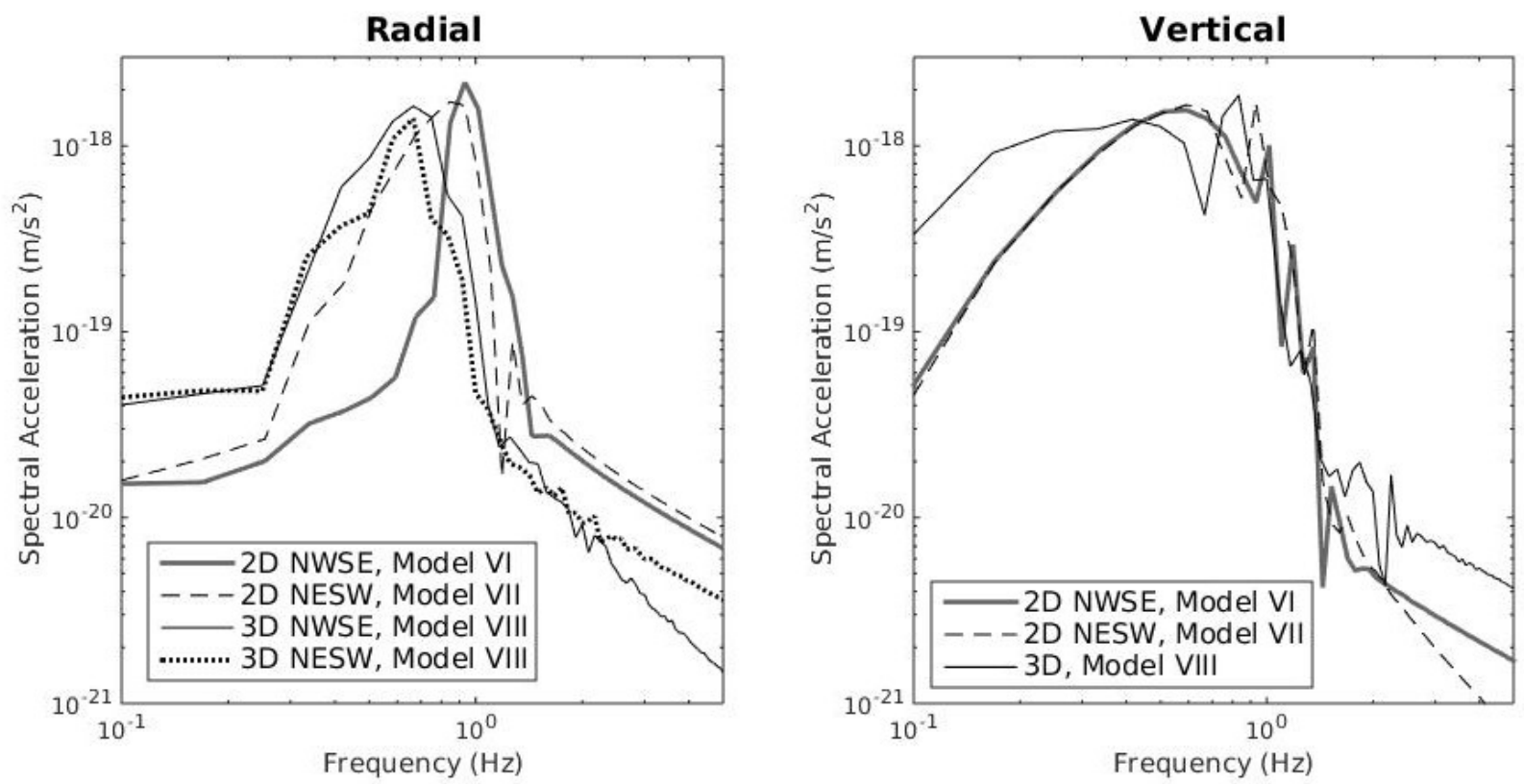

Figure 6.16: Spectral accelerations for the horizontal (left) and vertical (right) components of motion in the 2D models, (Model VI and Model VII) and the 3D model (Model VIII). 
Figure 6.17 shows the horizontal motions across the surface of the NESW profile in the 3D model (Model VIII) and 2D model (Model VII), and Figure 6.18 shows the vertical motions across the same models. The horizontal motions across the NWSE profile in the 3D model (Model VIII) and 2D model (Model VII) are shown in Figure 6.19 and the vertical motions are shown in Figure 6.20. These motions show the strongest resonances within the deepest portions of the basin for both the horizontal and vertical components. The NWSE profiles, Figure 6.19 and Figure 6.20, show a slightly longer time for the first arrival in the deepest portion of the basin in both components of motion, whereas this delay is less evident in the NESW profiles, Figure 6.17 and Figure 6.18. This delay is seen easier in the NWSE profiles because the deepest portion of the basin is off centre of the model and away from the source. In the NESW profiles the deepest part of the basin is almost centred within the model, so even though there is a slight time delay, it more difficult to discern in these figures.
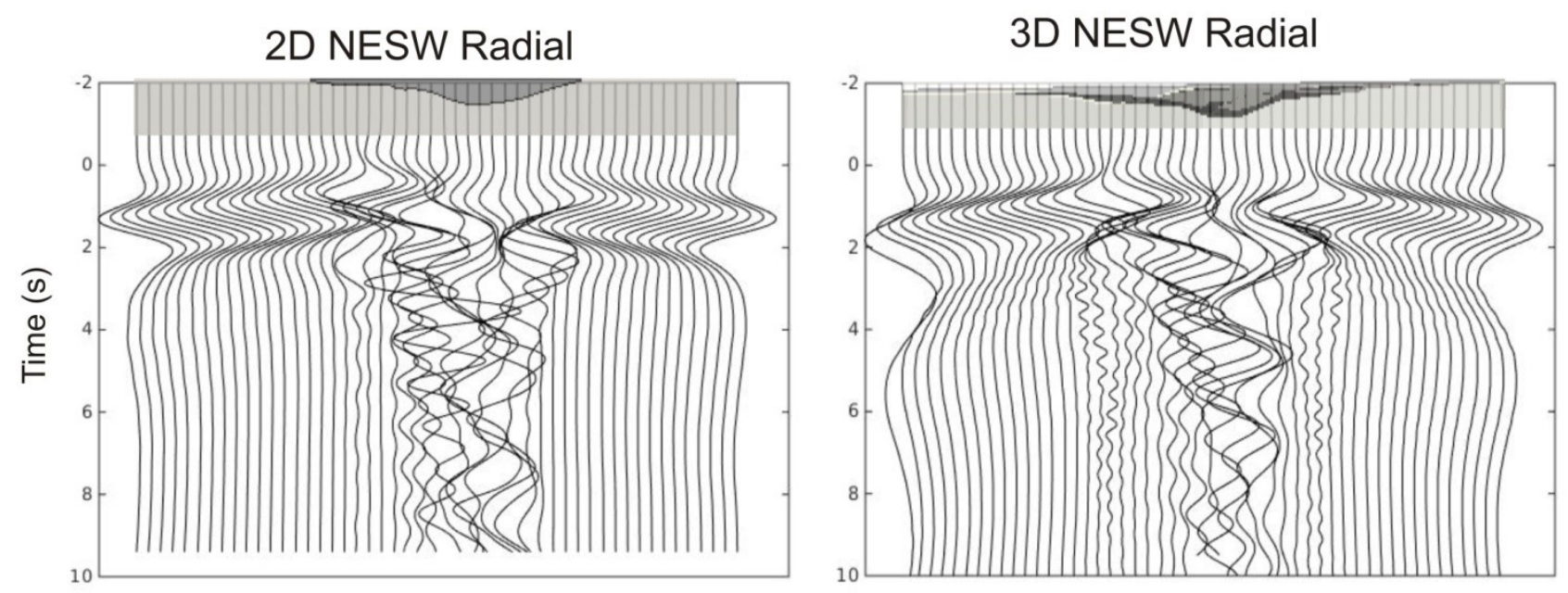

Figure 6.17: Horizontal motions across the surface for the 2D NESW model, Model VII (left), and the radial motions along the NESW profile for the 3D model, Model VIII (right). An overlay of the basin surface is shown for each with a vertical exaggeration of about 10 . 

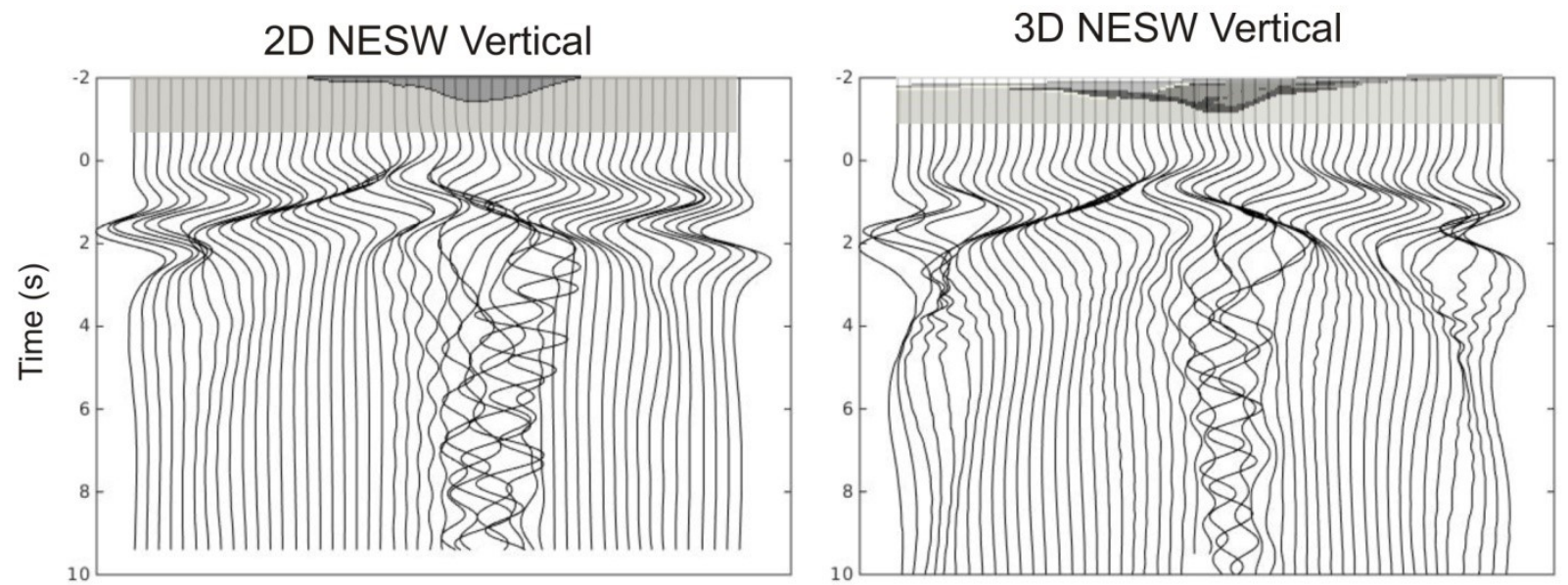

Figure 6.18: Vertical motions across the surface for the 2D NESW model, Model VII (left), and the vertical motions along the NESW profile for the 3D model, Model VIII (right). An overlay of the basin surface is shown for each with a vertical exaggeration of about 10.
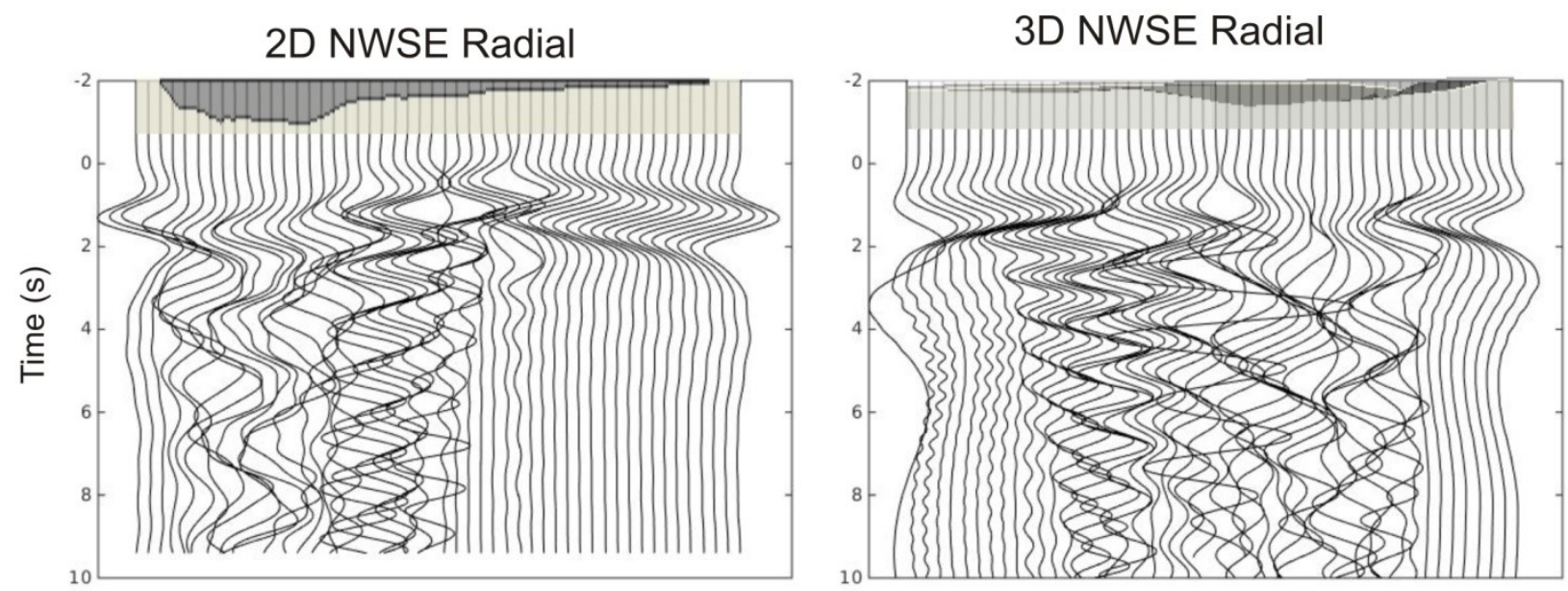

Figure 6.19: Horizontal motions across the surface for the 2D NWSE model, Model VII (left), and the radial motions along the NWSE profile for the 3D model, Model VIII (right). An overlay of the basin surface is shown for each with a vertical exaggeration of about 10. 

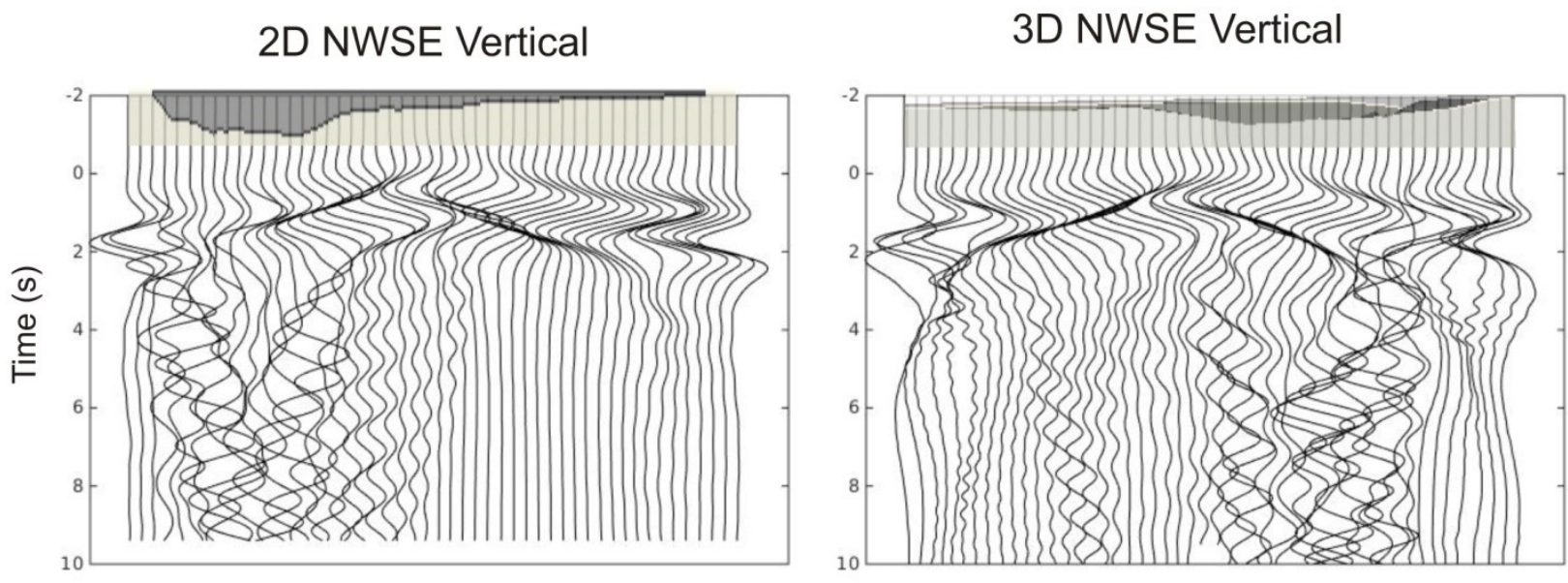

Figure 6.20: Vertical motions across the surface for the 2D NWSE model, Model VII (left), and the vertical motions along the NWSE profile for the 3D model, Model VIII (right). An overlay of the basin surface is shown for each with a vertical exaggeration of about 10.

\subsection{Ladysmith Earthquake}

The Ladysmith earthquake of 2013 (Bent et al., 2015) was simulated with a modified model of the 2D Layered Model from Chapter 5.2, and several different sources. The modified layered model is shown in Figure 6.21. The sources used for these simulations are identical to the ones used to determine the ground motions at KB1O in Chapter 4.3. The parameters within and surrounding the basin were adjusted in an attempt to create a simple, yet accurate, model of the Kinburn region. 


\section{Surface 3}

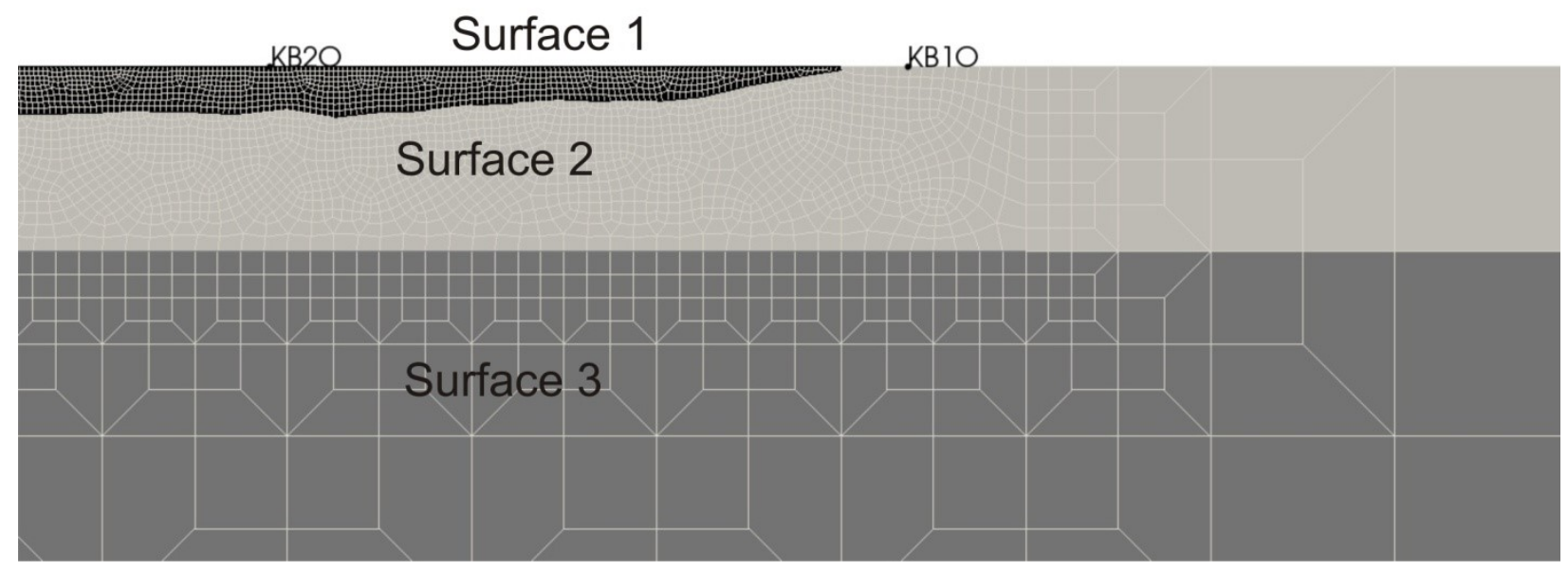

Figure 6.21: The modified 2D layered model for simulating the Ladysmith earthquake of 2013. Surface 1 is the basin, Surface 2 extends to a depth of $400 \mathrm{~m}$, and Surface 3 is the rest of the model.

The firm bedrock used for most of the model was the average value for the eastern North America crustal thickness (Bent et al., 2015). For the simulations with two rock types in the model, the top 400 metres of rock use the average values of rock as measured at the surface (Hunter et al., 2010). The soil within the basin was given several different model-dependent values used in different simulations. The properties for each surface in the model for each 
simulation are listed in Table 6.3. The simulations are compared to the recorded ground motions using a filtered acceleration time series and the spectral acceleration. As shown in Chapter 4.3, by applying this method it was possible to accurately match only one of these two for each simulation and the source had to be adjusted accordingly for each one.

\begin{tabular}{|c|c|c|c|c|c|}
\hline $\begin{array}{l}\text { Surface } \\
\text { Number }\end{array}$ & $\begin{array}{l}\text { Name of } \\
\text { Parameter } \\
\text { set }\end{array}$ & $\mathrm{Vp}(\mathrm{m} / \mathrm{s})$ & Vs (m/s) & $\begin{array}{l}\text { Density } \\
\left(\mathrm{kg} / \mathrm{m}^{3}\right)\end{array}$ & Q value \\
\hline \multicolumn{6}{|c|}{ SIM I: CMT Source, Single Rock type, Soil Vs=150m $/ \mathrm{s}$} \\
\hline Surface 1 & Soil Type I & 1600 & 150 & 1600 & 100 \\
\hline Surface 2 & Rock Type I & 6500 & 3500 & 2800 & 1000 \\
\hline Surface 3 & Rock Type I & 6500 & 3500 & 2800 & 1000 \\
\hline \multicolumn{6}{|c|}{ SIM II: CMT Source, Two Rock types, Soil Vs=150m/s } \\
\hline Surface 1 & Soil Type I & 1600 & 150 & 1600 & 100 \\
\hline Surface 2 & Rock Type II & 6000 & 2700 & 2600 & 1000 \\
\hline Surface 3 & Rock Type I & 6500 & 3500 & 2800 & 1000 \\
\hline \multicolumn{6}{|c|}{ SIM III: CMT Source, Single Rock type, Soil Vs=250m $/ \mathrm{s}$} \\
\hline Surface 1 & Soil Type I & 1600 & 150 & 1600 & 100 \\
\hline Surface 2 & Rock Type I & 6500 & 3500 & 2800 & 1000 \\
\hline Surface 3 & Rock Type I & 6500 & 3500 & 2800 & 1000 \\
\hline \multicolumn{6}{|c|}{ SIM IV: CMT Source, Two Rock types, Soil Vs=250m/s } \\
\hline Surface 1 & Soil Type I & 1600 & 150 & 1600 & 100 \\
\hline Surface 2 & Rock Type II & 6000 & 2700 & 2600 & 1000 \\
\hline Surface 3 & Rock Type I & 6500 & 3500 & 2800 & 1000 \\
\hline \multicolumn{6}{|c|}{ SIM V: MTS Source, Single Rock type, Soil Vs=150m/s } \\
\hline Surface 1 & Soil Type I & 1600 & 150 & 1600 & 100 \\
\hline Surface 2 & Rock Type I & 6500 & 3500 & 2800 & 1000 \\
\hline Surface 3 & Rock Type I & 6500 & 3500 & 2800 & 1000 \\
\hline \multicolumn{6}{|c|}{ SIM VI: MTS Source, Two Rock types, Soil Vs $=150 \mathrm{~m} / \mathrm{s}$} \\
\hline Surface 1 & Soil Type I & 1600 & 150 & 1600 & 100 \\
\hline Surface 2 & Rock Type II & 6000 & 2700 & 2600 & 1000 \\
\hline Surface 3 & Rock Type I & 6500 & 3500 & 2800 & 1000 \\
\hline \multicolumn{6}{|c|}{ SIM VII: MTS Source, Single Rock type, Soil Vs=250m/s } \\
\hline Surface 1 & Soil Type I & 1600 & 150 & 1600 & 100 \\
\hline Surface 2 & Rock Type I & 6500 & 3500 & 2800 & 1000 \\
\hline Surface 3 & Rock Type I & 6500 & 3500 & 2800 & 1000 \\
\hline \multicolumn{6}{|c|}{ SIM VIII: MTS Source, Two Rock types, Soil Vs=250m $/ \mathrm{s}$} \\
\hline Surface 1 & Soil Type I & 1600 & 150 & 1600 & 100 \\
\hline Surface 2 & Rock Type II & 6000 & 2700 & 2600 & 1000 \\
\hline
\end{tabular}




\begin{tabular}{|c|c|c|c|c|c|}
\hline Surface 3 & Rock Type I & 6500 & 3500 & 2800 & 1000 \\
\hline \multicolumn{7}{|c|}{ SIM IX: MTS Source, Two Rock types, Soil Vs=275m/s } \\
\hline Surface 1 & Soil Type I & 1600 & 150 & 1600 & 100 \\
\hline Surface 2 & Rock Type II & 6000 & 2700 & 2600 & 1000 \\
\hline Surface 3 & Rock Type I & 6500 & 3500 & 2800 & 1000 \\
\hline
\end{tabular}

Table 6.3: The properties of each surface used for the Ladysmith 2013 earthquake simulations. CMT is the global centroid moment tensor, and MTS is the moment tensor solution from Bent et al. (2015).

\subsubsection{Time Series Matching using the Global Centroid Moment Tensor solution}

The time series matching uses the global Centroid Moment Tensor solution (Section 4.3.2) as found by the method described in Dziewonski et al. (1981), and Ekström et al. (2012). This source has the strongest seismic moment, so it should produce the largest amplitudes for the ground motion which was found in Section 4.3. Four simulations were completed for this comparison. The first simulation, SIM I, used one rock type in the model and the $\mathrm{Vs}=250$ soil properties for the basin material. The second simulation, SIM II, used two rock types for the model and the Vs=250 soil properties. The third simulation, SIM III, contained one rock type in the model and the Vs=150 soil properties. The last simulation, SIM IV, used two rock types and the Vs=150 soil properties. The properties for the different materials are listed in Table 6.3 for each simulation.

The acceleration time series for the simulations and the recorded values were filtered with an 8-pole Butterworth bandpass filter between 0.1 and $2 \mathrm{~Hz}$. The recorded motions were then filtered with a 12-pole Butterworth low-pass filter with a cutoff frequency of $2 \mathrm{~Hz}$ to remove the higher frequencies as discussed in Appendix III. These acceleration time series are shown in Figure 6.22 for the horizontal component of motion and Figure 6.23 for the vertical component of motion. 
There is a consistent timing of the arrivals and amplitudes between the P-and S-waves for both the single-rock type, SIM I and SIM III, and the two-rock type models, SIM II and SIM IV. The peak values for the simulations and recorded motions are listed in Table 6.4. From the acceleration time series in Figure 6.22 and Figure 6.23, the arrivals of both the P-wave and Swave amplitudes are larger in the simulations for the horizontal and vertical motions compared to the recorded values. The duration of the motion is very different in both the horizontal and vertical components between the simulations and recorded motions, even after filtering out the higher frequencies. This indicates some higher frequency effects which are not being simulated in the model. This might include the failure to add finite fault effects for a moderately sized earthquake, along with other possibilities. Figure 6.24 is the Fourier acceleration spectrum for the horizontal and vertical motions at KB1O. The spectral accelerations are very similar; however, the simulated horizontal motions seem to have a lower peak frequency than the recorded motions. The vertical motions are almost identical, except for around $0.2 \mathrm{~Hz}$, where the recorded accelerations are weaker than the simulated accelerations. 


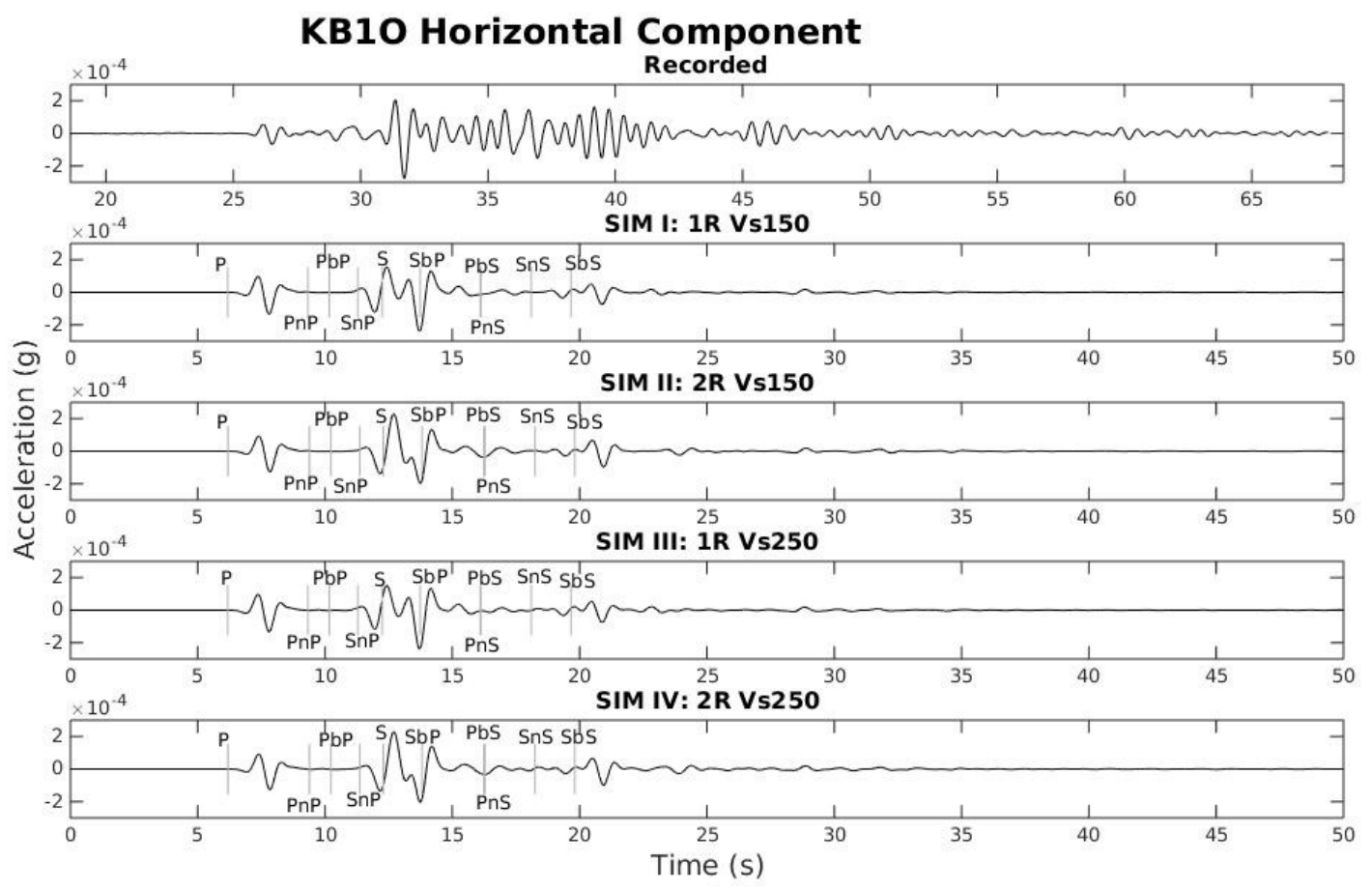

Figure 6.22: Horizontal acceleration time series at station KB1O for the simulations with the global centroid moment tensor solution source. $1 \mathrm{R}$ indicates the simulation used one rock type, $2 \mathrm{R}$ is for a simulation which uses two rock types, Vs 150 denotes the simulation used Soil Type I for the basin material, and Vs250 denotes the simulation used Soil Type II for the basin material.

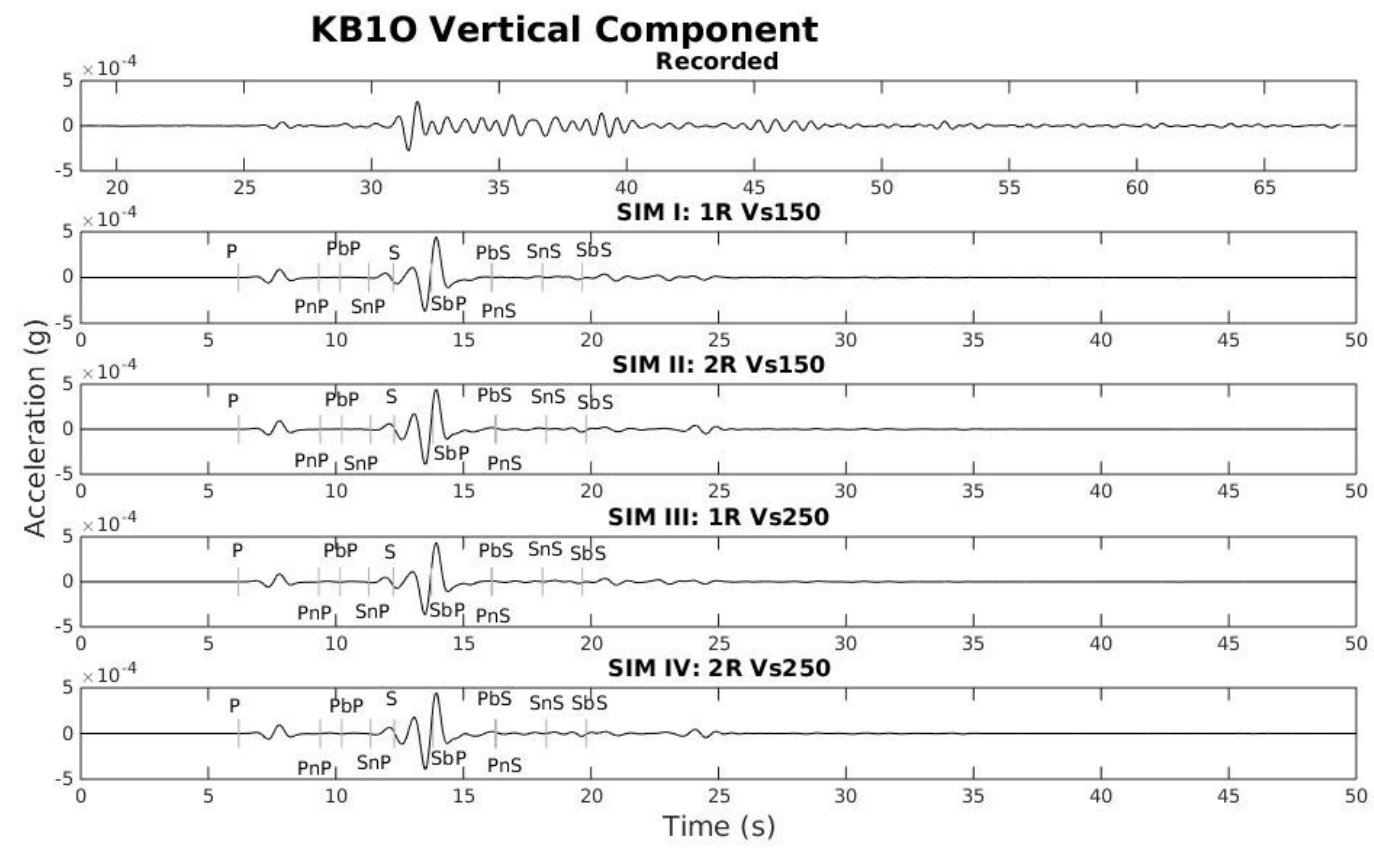

Figure 6.23: Vertical acceleration time series at station $\mathrm{KB} 10$ for the simulations with the global centroid moment tensor solution source. $1 R$ indicates the simulation used one rock type, $2 R$ is for a simulation which uses two rock types, Vs 150 denotes the simulation used Soil Type I for the basin material, and Vs250 denotes the simulation used Soil Type II for the basin material. 


\begin{tabular}{|c|c|c|c|c|c|c|}
\hline \multirow{2}{*}{ Model } & \multicolumn{2}{|c|}{ Peak at KB1O (g) } & \multicolumn{2}{c|}{ Peak at KB2O (g) } & \multicolumn{2}{c|}{ KB2O/KB1O } \\
\cline { 2 - 7 } & Horizontal & Vertical & Horizontal & Vertical & Horizontal & Vertical \\
\hline Recording & 0.00028 & 0.00028 & 0.00280 & 0.00120 & 10.12 & 4.33 \\
\hline $\begin{array}{c}\text { SIM I: } \\
\text { IRVs150 }\end{array}$ & 0.00024 & 0.00044 & 0.00046 & 0.00046 & 1.92 & 1.04 \\
\hline $\begin{array}{c}\text { SIM II: } \\
\text { 2RVs150 }\end{array}$ & 0.00023 & 0.00044 & 0.00056 & 0.00046 & 2.43 & 1.04 \\
\hline $\begin{array}{c}\text { SIM III: } \\
\text { 1RVs250 }\end{array}$ & 0.00024 & 0.00044 & 0.00047 & 0.00047 & 1.96 & 1.07 \\
\hline $\begin{array}{c}\text { SIM IV: } \\
\text { 2RVs250 }\end{array}$ & 0.00023 & 0.00045 & 0.00077 & 0.00048 & 3.35 & 1.07 \\
\hline
\end{tabular}

Table 6.4: Maximum acceleration values of the simulations for the global centroid moment tensor solution source at KB1O and KB2O. 1R indicates the simulation used one rock type, $2 \mathrm{R}$ is for a simulation which uses two rock types, Vs 150 denotes the simulation used Soil Type I for the basin material, Vs250 denotes the simulation used Soil Type II for the basin material, and Vs275 denotes the simulation used Soil Type III properties for the basin material.
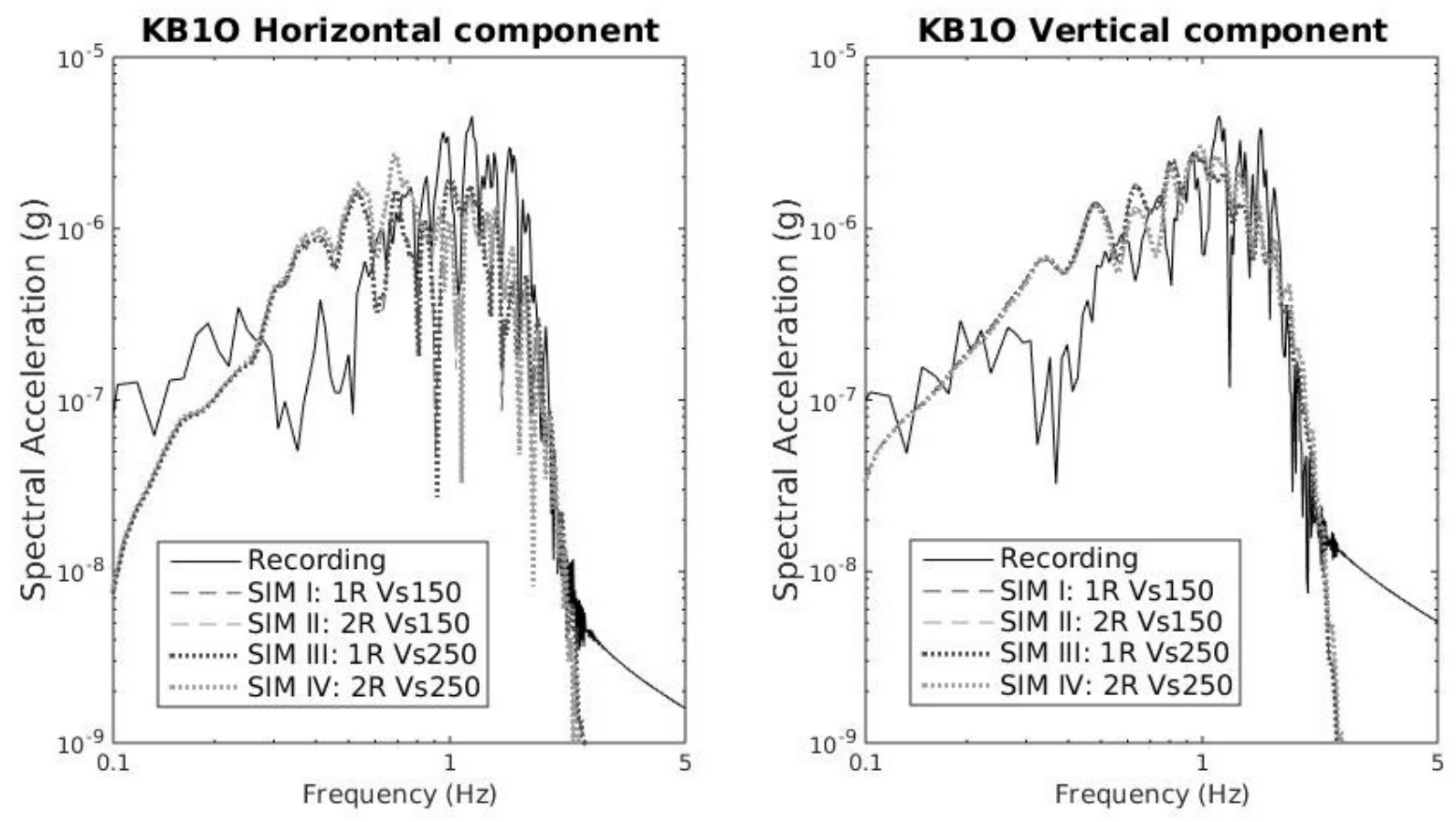

Figure 6.24: Fourier acceleration spectrum for the horizontal (left) and vertical (right) components of motion at station KB1O. 1R indicates the simulation used one rock type, $2 \mathrm{R}$ is for a simulation which uses two rock types, Vs150 denotes the simulation used Soil Type I for the basin material, and Vs250 denotes the simulation used Soil Type II for the basin material.

Figure 6.25 and Figure 6.26 show the acceleration time series at the soil site KB2O for the horizontal and vertical components of motion, respectively, for each simulation and the 
recordings. In both these components of motion, the simulated P-wave amplitude is comparable to the recorded amplitude, however the simulated amplitude of the S-wave is smaller than the recorded amplitude for the earthquake. From Table 6.4, the peak values within the simulations are at least 2.5 times smaller than the recordings. However, in the acceleration time series of the recordings, the peak arrivals are much later than the S-wave arrival. The recorded peak values at the S-wave arrival are $0.00130 \mathrm{~g}$ in the horizontal component and $0.00066 \mathrm{~g}$ in the vertical component. These peak values are more closely comparable to the peak values in the simulations. SIM IV, the simulation with two rock types and the Soil Type II properties, has the Swave amplitude which is the most comparable to the recorded amplitude.

There are also several later arriving events in the recorded motions which are not present in the simulations. These events were present at KB1O as well, indicating they are a result of either a complicated crustal structure between the earthquake and receiver, or there are several finite fault effects not being taken into account. Although there are later arrivals at KB2O in the simulations, they are much weaker than the ones seen in the recordings. This is likely due to the simulations containing only energy from resonances due to the main wave packets, whereas the recorded motions keep getting energy added within the basin from the later arrivals seen at KB1O. 


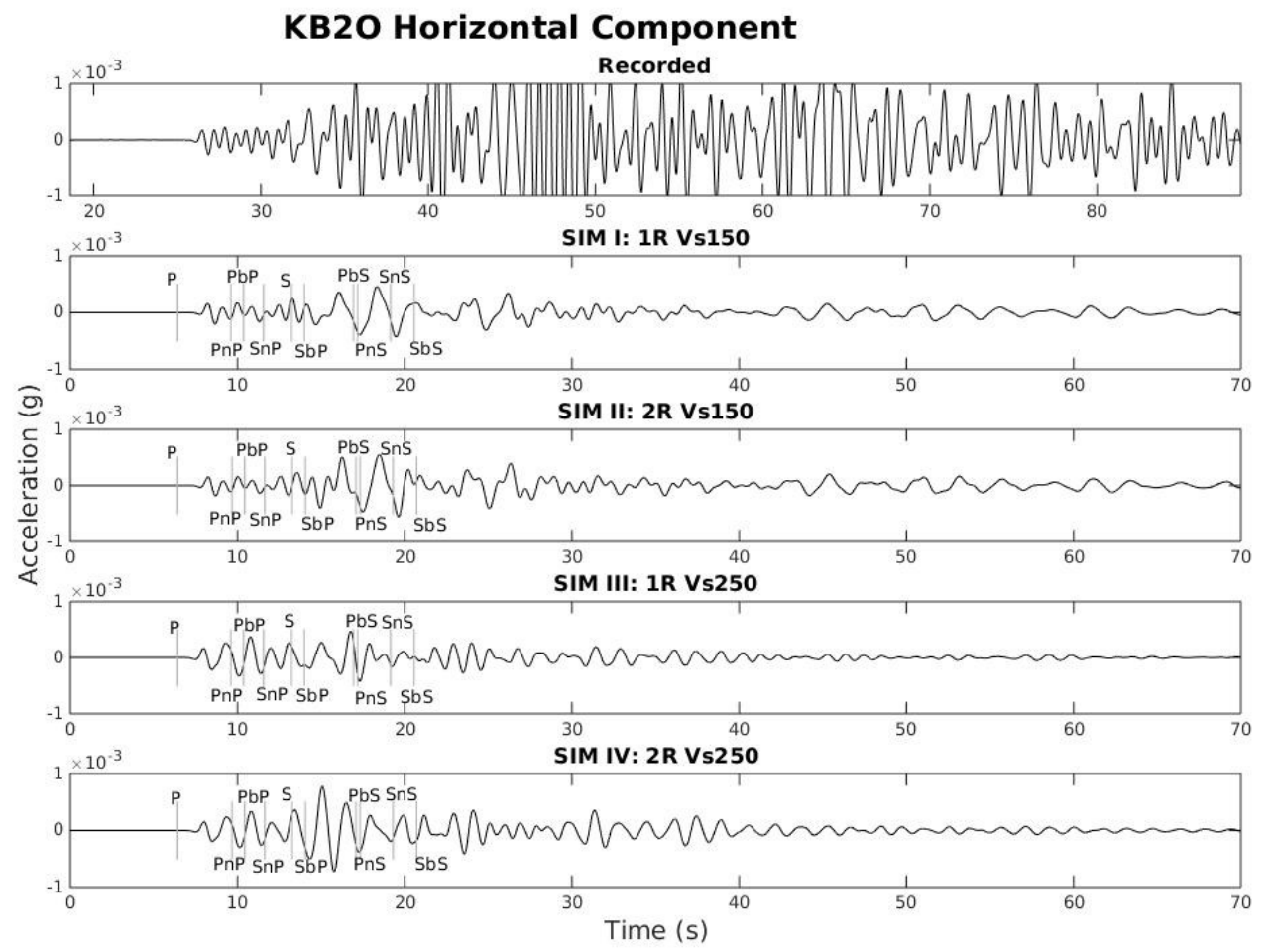

Figure 6.25: Horizontal acceleration time series at station $\mathrm{KB} 2 \mathrm{O}$ for the simulations with the centroid moment tensor solution source. $1 \mathrm{R}$ indicates the simulation used one rock type, $2 \mathrm{R}$ is for a simulation which uses two rock types, Vs150 denotes the simulation used Soil Type I for the basin material, and Vs250 denotes the simulation used Soil Type II for the basin material. 

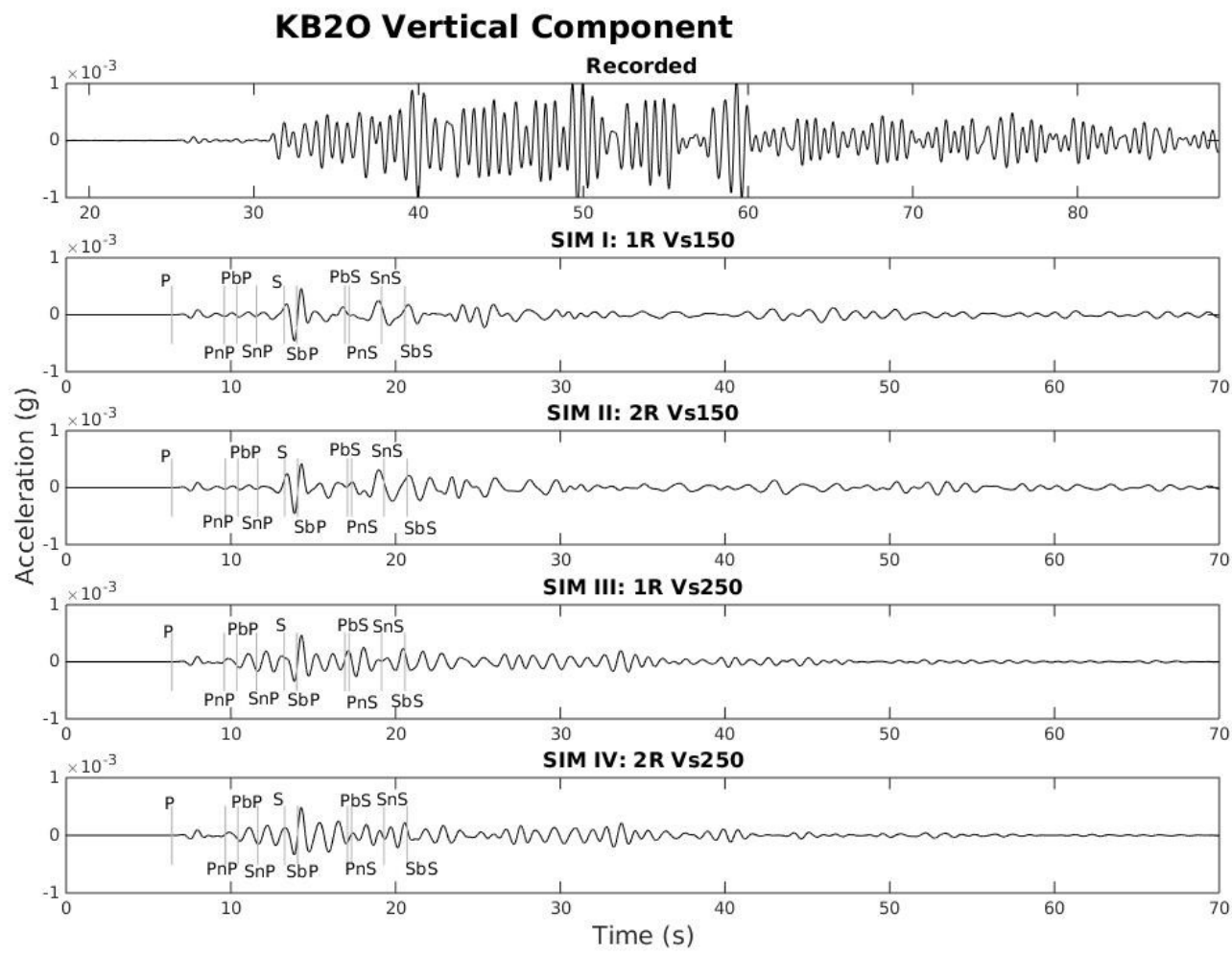

Figure 6.26: Vertical acceleration time series at station KB2O for the simulations with the centroid moment tensor solution source. $1 \mathrm{R}$ indicates the simulation used one rock type, $2 \mathrm{R}$ is for a simulation which uses two rock types, Vs150 denotes the simulation used Soil Type I for the basin material, and Vs250 denotes the simulation used Soil Type II for the basin material.

The simulations do show a strong resonating effect similar to the recording, and the dominant frequency is different between the models with Soil Type I properties and Soil Type II properties. The simulations with the Soil Type I properties, SIM I and SIM III, seem to have a lower dominant frequency than the recorded values, and the simulations with the Soil Type II properties, SIM II and SIM IV have almost the same dominant frequency, as seen in Figure 6.27 the spectral acceleration at KB2O.

The difference in dominant frequencies, shown in Figure 6.27 which is the acceleration spectrum for the horizontal and vertical motions at KB2O. The dominant frequency, displayed by the first peak in the spectral acceleration, is lower in frequency but comparable in amplitude to the recording for the simulations which use the Soil Type I properties, SIM I and SIM III. The 
dominant frequency of the simulations which used the Soil Type II properties is almost identical from the simulations to the recorded values. All the simulations have accelerations which attenuate more strongly at frequencies above $1.5 \mathrm{~Hz}$. The overlap of the two spectral accelerations with different rock types in the model shows that the material surrounding the basin had little effect on the motions within the basin compared to the material properties used within the basin.

The above could be explained by the estimation method of the fundamental period of this site as given by Dobry et al. (1976). The simulations were assigned a constant velocity within the basin material, however the soft sediment layer has been measured to have an increasing velocity with depth, as discussed in Section 2.1. According to Dobry et al. (1976) the dominant period with an increasing velocity according to a power, $p$, of depth is given as:

$$
T_{0}=\frac{4 \pi H^{(2-p / 2)}}{(2-p) V_{0} q_{1}}
$$

Here $0 \leq p<2, V_{0}$ is the velocity at the surface, and $q_{1}$ is the first root of $J_{n}\left(q_{1}\right)$, where $J_{n}()$ is the Bessel function of order $n=(p-1) /(2-p)$. This can be transformed in the equivalent of Eq. 1.6 as:

$$
T_{0}=\frac{4 H}{V_{e q}}
$$

where $V_{e q}$ is the velocity at the equivalent depth $z_{e q}$ :

$$
z_{e q}=\left(\frac{2-p}{\pi} q_{1}\right)^{2 / p}
$$


Using the soil properties for the city of Ottawa, as determined in Section 2.1, the equivalent depth, determined from Figure 5 in Dobry et al. (1976), is 58m instead of the layer thickness of $100 \mathrm{~m}$ as assigned. The resulting velocity at the equivalent depth is $175 \mathrm{~m} / \mathrm{s}$, however this can be further complicated since there is a thin overconsolidated layer of material (Dobry et al., 1976), which is also present on surface of the Kinburn basin. The combination of the increasing velocity as a power of depth, along with the high Vs velocity overconsolidated layer of material could be responsible for the shift from the expected fundamental frequency at this location.

The spectral ratio from $\mathrm{KB} 2 \mathrm{O}$ to $\mathrm{KB} 10$ for the recorded motions and the simulations is shown in Figure 6.28. These curves are approximately the transfer functions from the rock to soil within the basin. For both the horizontal and vertical motions, the spectral ratio of the recorded motions is much higher between $1-2 \mathrm{~Hz}$, and also below $0.2 \mathrm{~Hz}$. Between $0.2-1 \mathrm{~Hz}$ the peak values are similar between the recorded motions and the simulations, but the frequency of these peaks are different. This showed that the energy in the higher and lower frequencies was not transmitting into the basin as much as expected from the recording, but the large amplification ratio between the soil and rock was preserved within the simulations. 

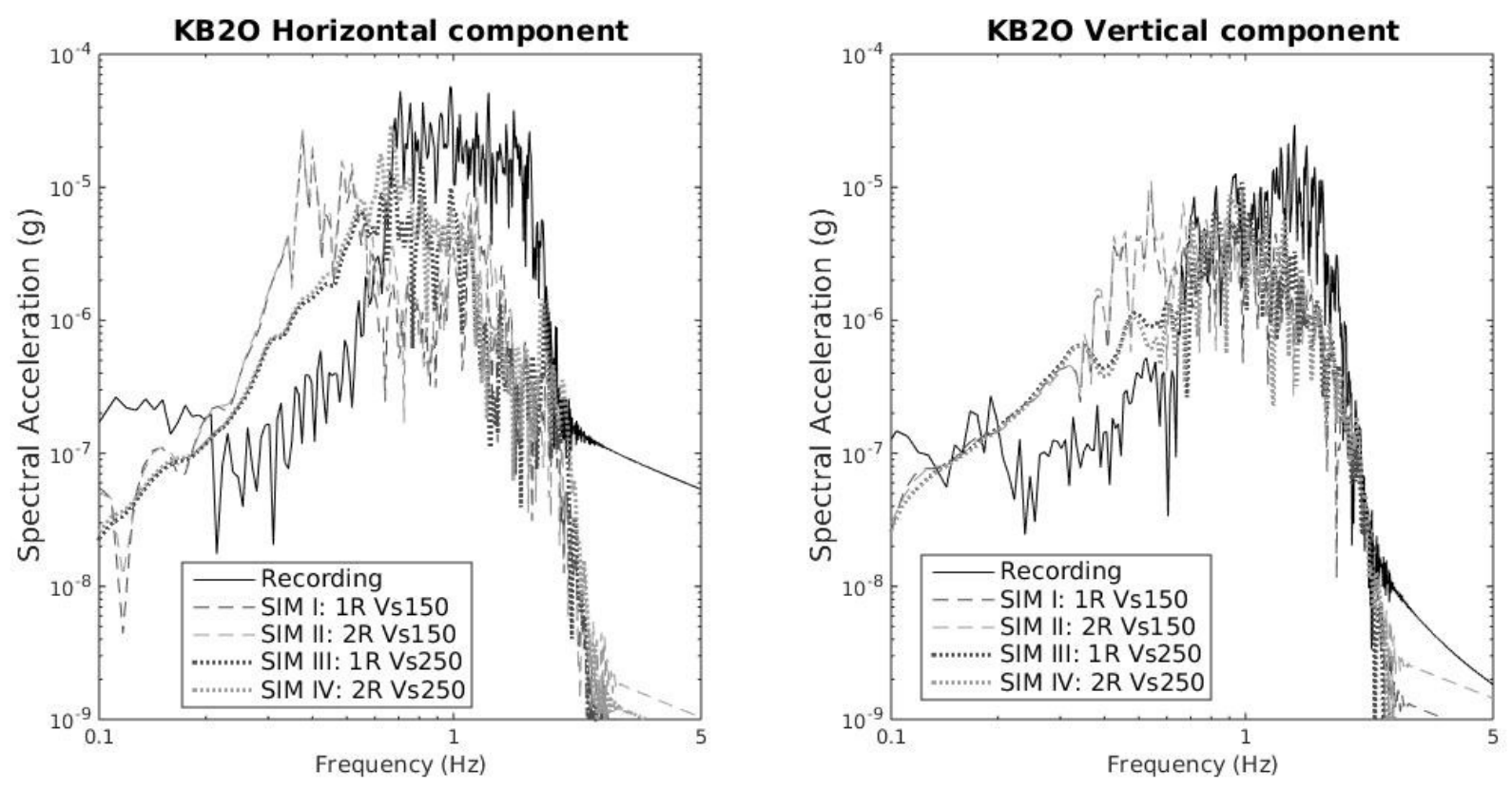

Figure 6.27: The Fourier acceleration spectrum for the horizontal (left) and vertical (right) components of motion at station KB2O. $1 R$ indicates the simulation used one rock type, $2 R$ is for a simulation which uses two rock types, Vs 150 denotes the simulation used Soil Type I for the basin material, and Vs250 denotes the simulation used Soil Type II for the basin material.
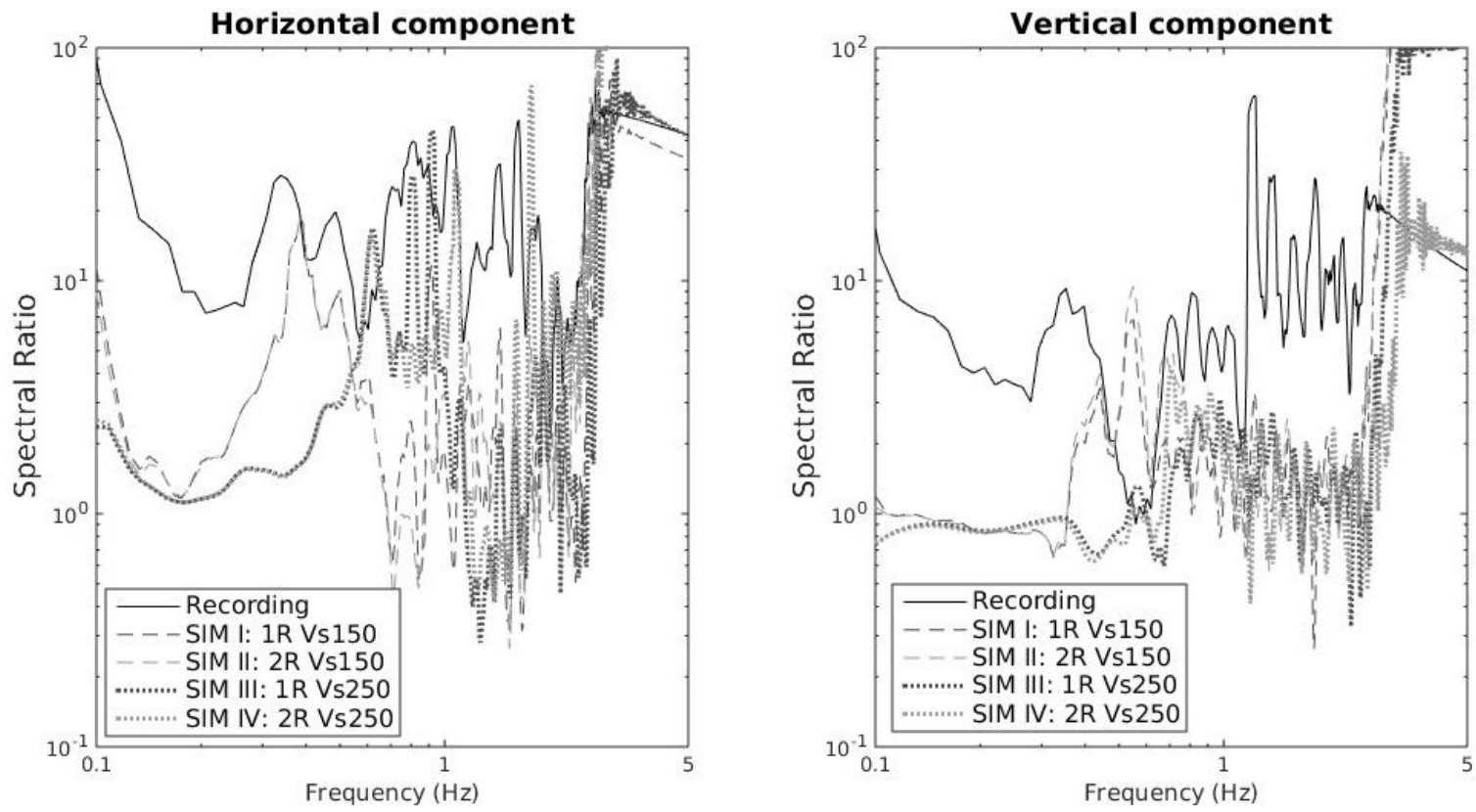

Figure 6.28: The spectral acceleration ratios for the horizontal (left) and vertical (right) components of motion for station KB2O/KB1O. 1R indicates the simulation used one rock type, 2R is for a simulation which uses two rock types, Vs150 denotes the simulation used Soil Type I for the basin material, and Vs250 denotes the simulation used Soil Type II for the basin material. 


\subsubsection{P-wave and S-wave Comparison}

The acceleration time series for the recorded motions and simulations SIM I, SIM II, SIM III, and

SIM VI were are windowed over five seconds before the onset of the P-wave, to five seconds after the arrival of the S-wave. This was done to reduce the effects of any later arriving wave packets in the recorded motion, producing a better comparison of the amplification effects of the main P-wave and S-wave packet. The horizontal motions at KB1O are shown in Figure 6.29, and the vertical motion at this station is shown in Figure 6.30. Although there is still some later motion in the recorded motion, the signal is primarily the P-wave and S-wave.
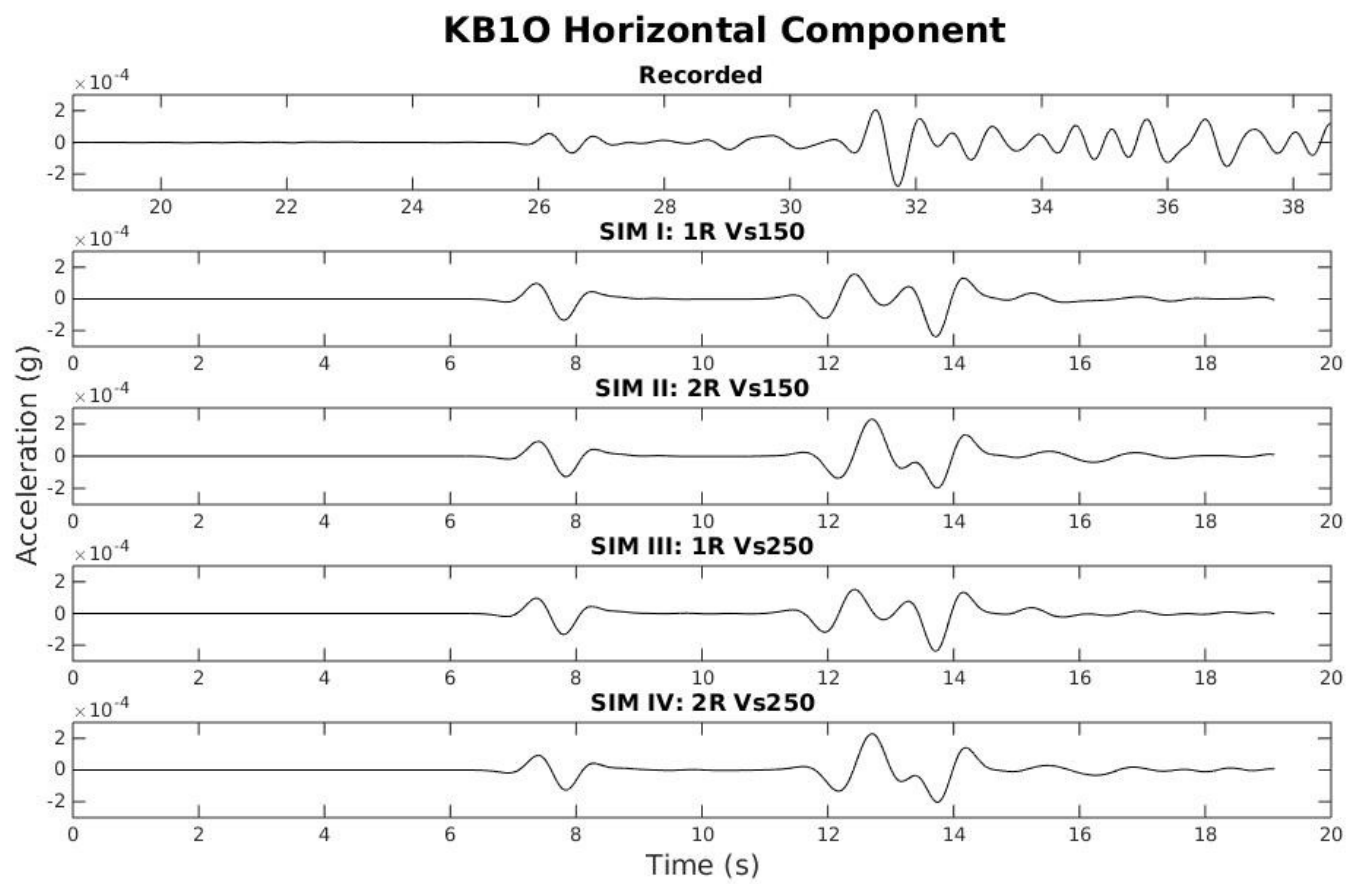

Figure 6.29: The windowed horizontal acceleration time series at station KB1O for the simulations with the global centroid moment tensor solution source. $1 R$ indicates the simulation used one rock type, $2 \mathrm{R}$ is for a simulation which uses two rock types, Vs150 denotes the simulation used Soil Type I for the basin material, and Vs250 denotes the simulation used Soil Type II for the basin material. 


\section{KB10 Vertical Component}
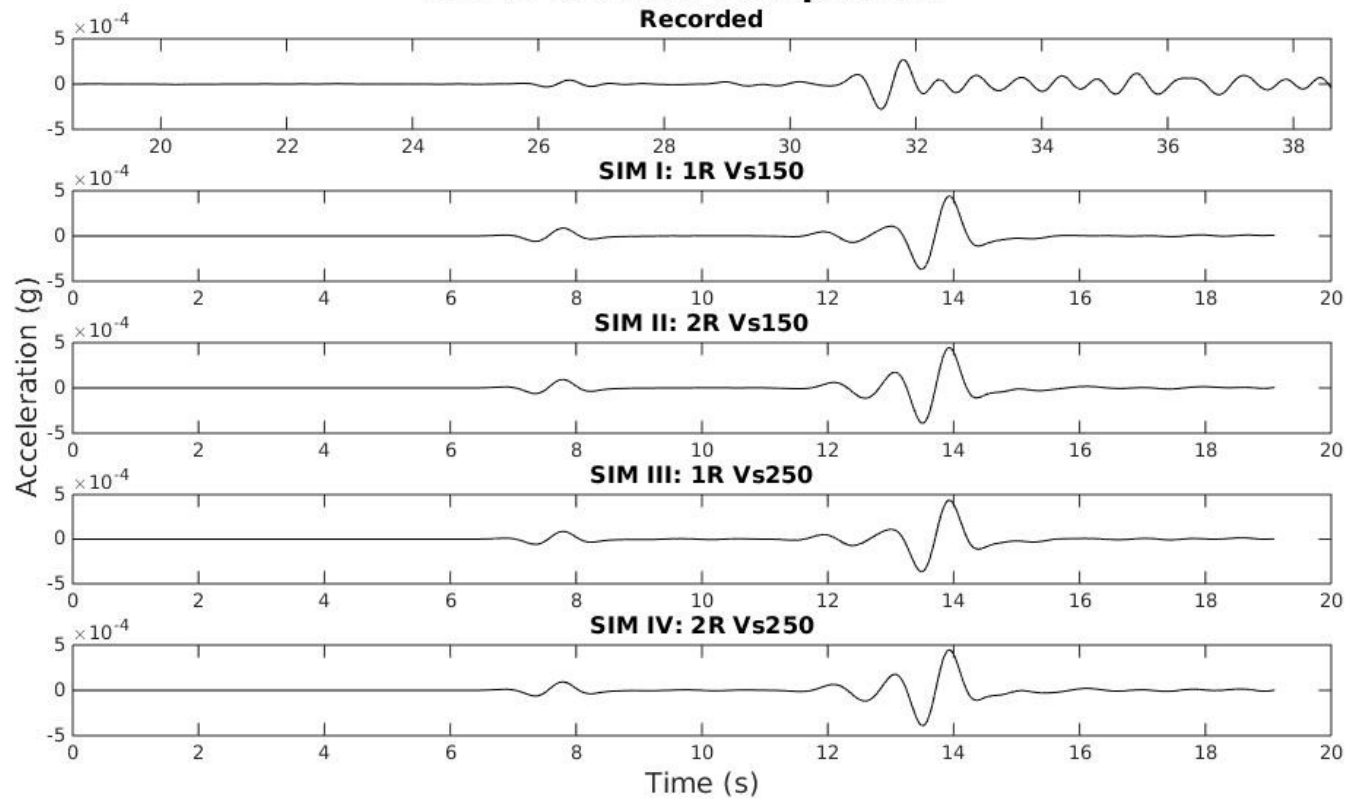

Figure 6.30: The windowed vertical acceleration time series at station KB1O for the simulations with the global centroid moment tensor solution source. $1 \mathrm{R}$ indicates the simulation used one rock type, $2 \mathrm{R}$ is for a simulation which uses two rock types, Vs150 denotes the simulation used Soil Type I for the basin material, and Vs250 denotes the simulation used Soil Type II for the basin material.

The acceleration time series of the recorded and simulated motions for station KB2O is shown in Figure 6.31 for the horizontal motion and Figure 6.32 for the vertical motion. The simulations show a later arriving S-wave; however, it has a larger amplitude in both the horizontal and vertical components. Table 6.5 lists the peak values of the recorded motions and the simulations for the windowed time series. The peaks are actually larger in the simulations, but the soil/rock ratio is still lower than the recorded motions for most of the frequency spectrum. This is shown in the displays of the spectral acceleration of KB2O/KB1O in Figure 6.33 for the simulations and recording using only 20 seconds of the time series. The simulated spectral ratios are very similar to the recorded motions and are closer than using the entire time series, as seen previously in Figure 6.28. 


\section{KB20 Horizontal Component}
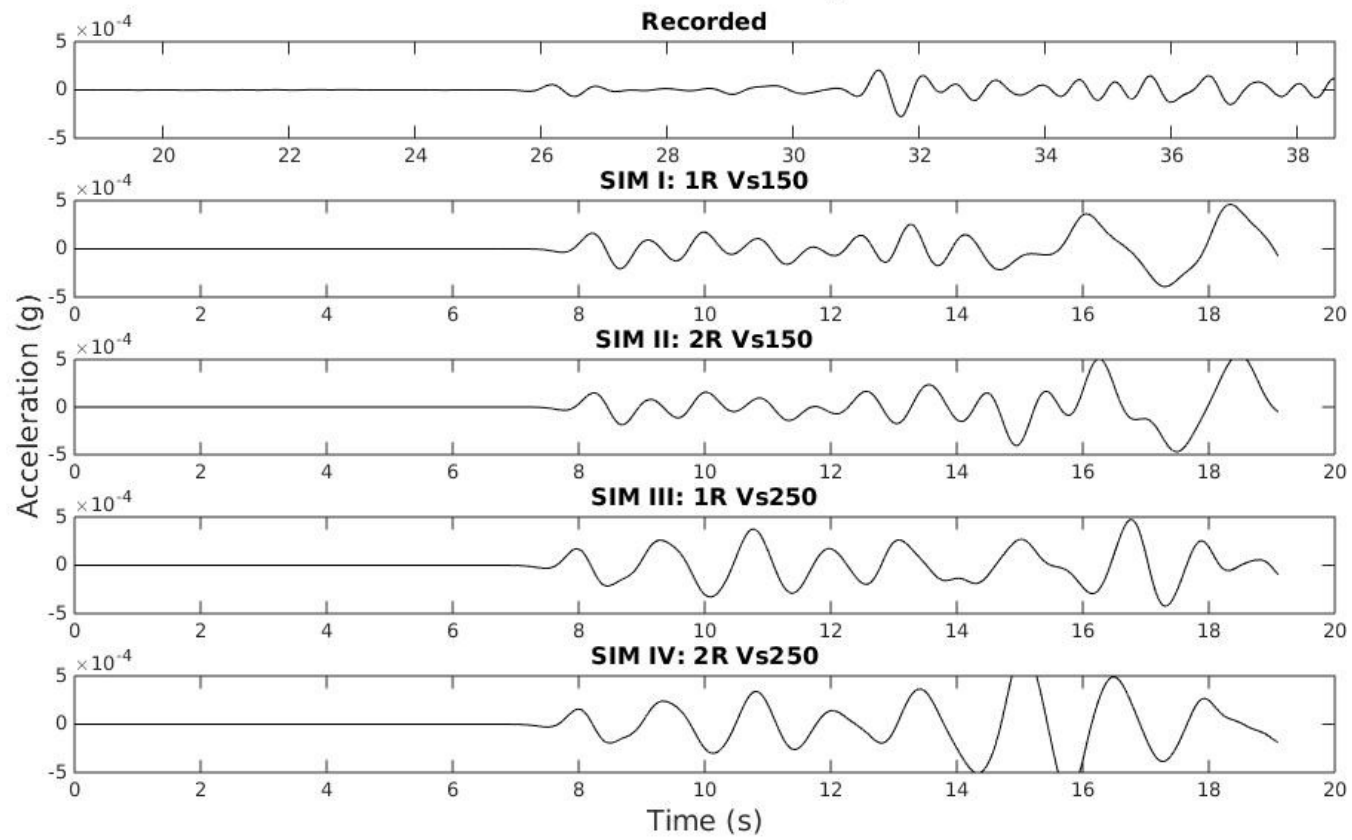

Figure 6.31: The windowed horizontal acceleration time series at station KB2O for the simulations with the global centroid moment tensor solution source. $1 \mathrm{R}$ indicates the simulation used one rock type, $2 \mathrm{R}$ is for a simulation which uses two rock types, Vs150 denotes the simulation used Soil Type I for the basin material, and Vs250 denotes the simulation used Soil Type II for the basin material. 

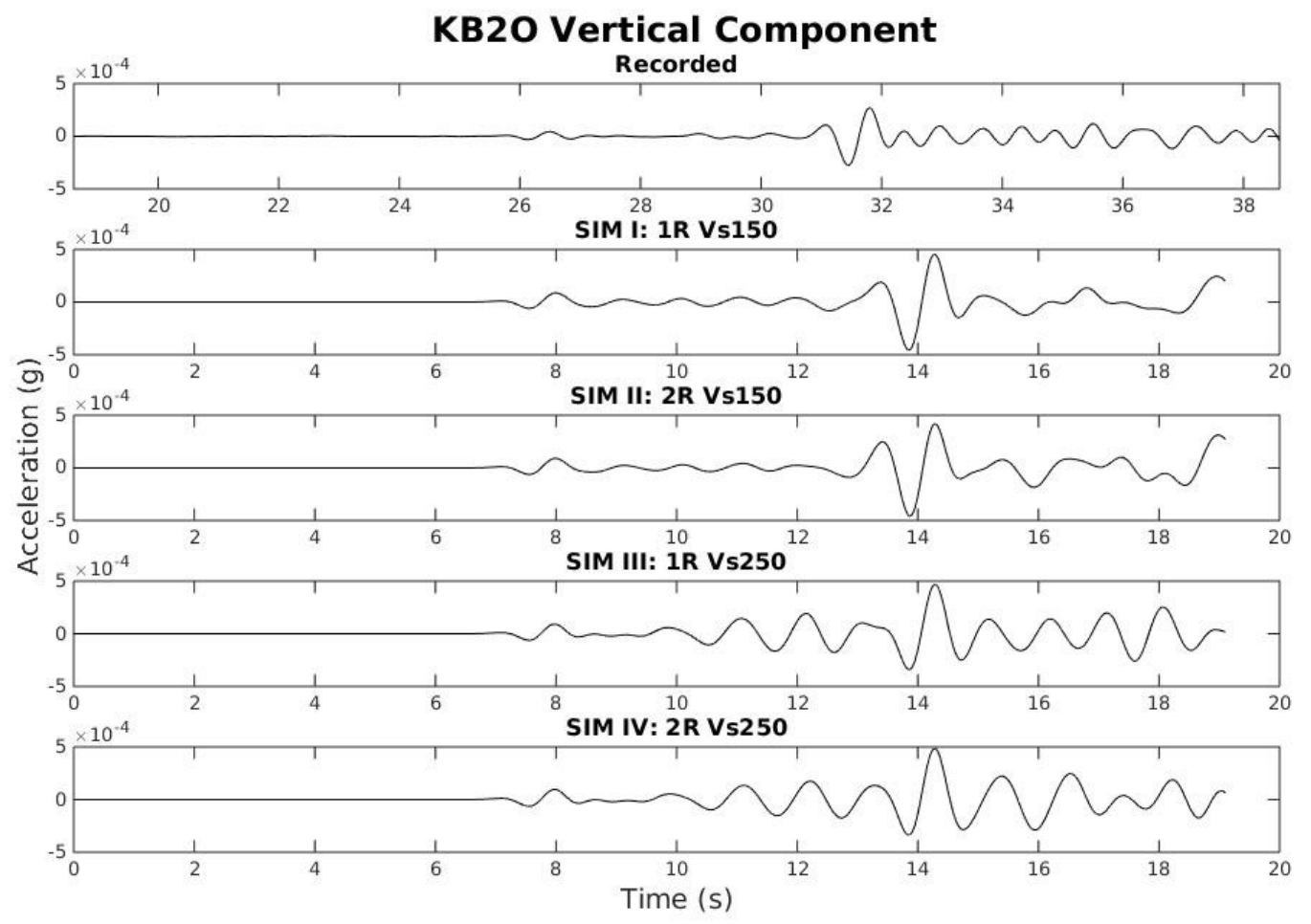

Figure 6.32: The windowed horizontal acceleration time series at station KB1O for the simulations with the global centroid moment tensor solution source. $1 R$ indicates the simulation used one rock type, $2 R$ is for a simulation which uses two rock types, Vs150 denotes the simulation used Soil Type I for the basin material, and Vs250 denotes the simulation used Soil Type II for the basin material.

\begin{tabular}{|c|c|c|c|c|c|c|}
\hline \multirow{2}{*}{ Model } & \multicolumn{2}{|c|}{ Peak at KB1O (g) } & \multicolumn{2}{c|}{ Peak at KB2O (g) } & \multicolumn{2}{c|}{ KB2O/KB1O } \\
\cline { 2 - 7 } & Horizontal & Vertical & Horizontal & Vertical & Horizontal & Vertical \\
\hline Recording & 0.00028 & 0.00028 & 0.00130 & 0.00066 & 4.64 & 2.36 \\
\hline $\begin{array}{c}\text { SIM I: } \\
\text { 1RVs150 }\end{array}$ & 0.00024 & 0.00044 & 0.00046 & 0.00046 & 1.92 & 1.04 \\
\hline $\begin{array}{c}\text { SIM II: } \\
\text { 2RVs150 }\end{array}$ & 0.00023 & 0.00044 & 0.00056 & 0.00046 & 2.43 & 1.04 \\
\hline $\begin{array}{c}\text { SIM III: } \\
\text { 1RVs250 }\end{array}$ & 0.00024 & 0.00044 & 0.00047 & 0.00047 & 1.96 & 1.07 \\
\hline $\begin{array}{c}\text { SIM IV: } \\
\text { 2RVs250 }\end{array}$ & 0.00023 & 0.00045 & 0.00077 & 0.00048 & 3.35 & 1.07 \\
\hline
\end{tabular}

Table 6.5: Maximum acceleration values of the simulations for the global centroid moment tensor solution source at KB10 and KB2O for the P-wave and S-wave arrivals. $1 \mathrm{R}$ indicates the simulation used one rock type, $2 \mathrm{R}$ is for a simulation which uses two rock types, Vs150 denotes the simulation used Soil Type I for the basin material, Vs 250 denotes the simulation used Soil Type II for the basin material, and Vs275 denotes the simulation used Soil Type III properties for the basin material. 

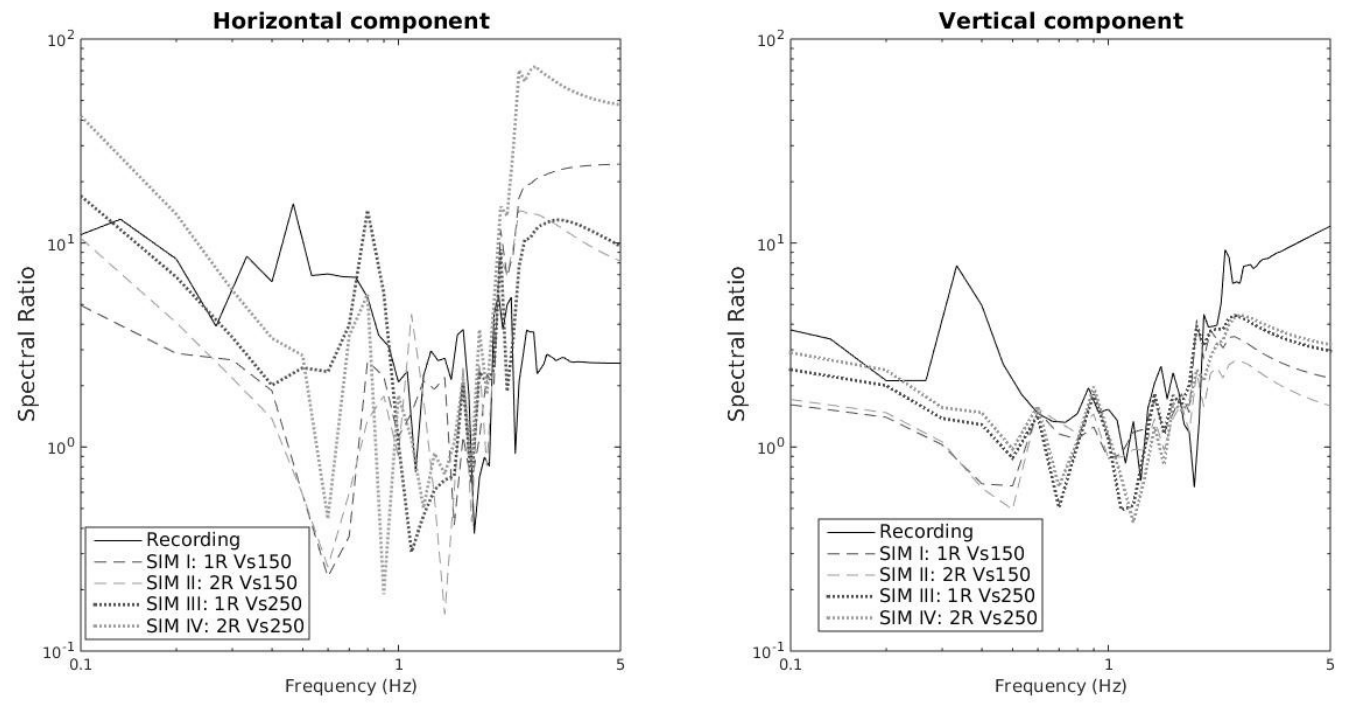

Figure 6.33: The spectral acceleration ratios for the horizontal (left) and vertical (right) components of motion for station KB2O/KB1O using the windowed time series. $1 \mathrm{R}$ indicates the simulation used one rock type, $2 \mathrm{R}$ is for a simulation which uses two rock types, Vs150 denotes the simulation used Soil Type I for the basin material, and Vs250 denotes the simulation used Soil Type II for the basin material.

\subsubsection{Spectral Acceleration Matching}

The spectral acceleration matching simulations use of the moment tensor solution from Bent et al. (2015) as it produced the most accurate spectral accelerations, specifically at frequencies between 1-2Hz, when simulating motions at KB1O (Chapter 4.3.1). There were five simulations completed using this source. The first simulation, SIM V, used one rock type and the Vs150, Soil Type I, properties. The second simulation, SIM VI, used two rock types and the Vs150, Soil Type I, properties. The third simulation, SIM VII, used one rock type and the Vs250, Soil Type II, properties. The fourth simulation, SIM VIII, used two rock types and the Vs250, Soil Type II properties. The last simulation, SIM IX, used two rock types and the Vs275, Soil Type III, properties. All of the properties used for the different surfaces in the simulations are listed in Table 6.3. 
The acceleration time series for the simulations and the recorded values were filtered with an 8-pole Butterworth bandpass filter between 0.1 and $3.2 \mathrm{~Hz}$. The recorded motions were then filtered with a 12-pole Butterworth low-pass filter with a cutoff frequency of $3 \mathrm{~Hz}$ to remove the higher frequencies as discussed in Appendix III.

The acceleration time series at KB1O for each simulation and the recorded values is shown in Figure 6.34 for the horizontal motions and in Figure 6.35 for the vertical motions. The acceleration time series at KB2O is shown for each simulation and the recorded values in Figure 6.36 for the horizontal motions and in Figure 6.37 for the vertical motions. Although the amplitudes of the motions are very different between the recorded values and simulated values, the timing of arrivals for the rock and soil station are similar between the recording and simulations.

Table 6.6 shows the peak values for the horizontal and vertical components at both the rock and soil location for the recording and each simulation, along with the ratio between the peak soil and peak rock amplitude. The ratios in the vertical component are less than half, with the model with two rock types and the Vs150 soil properties, SIM VIII, having the largest amplification. The models with only one rock type show a significant decrease in relative amplitudes which are roughly an order of magnitude smaller for the horizontal component at station KB2O. The models with two rock types show a closer relation between the peak horizontal ratio, but are still smaller in value. The difference is caused by the location of the peak values at KB2O. The peak acceleration is occurring much later in time than the P-wave or S-wave arrival, 
so the comparison of peak values in the recording is not comparing the strength of similar

arrivals.

KB10 Horizontal Component
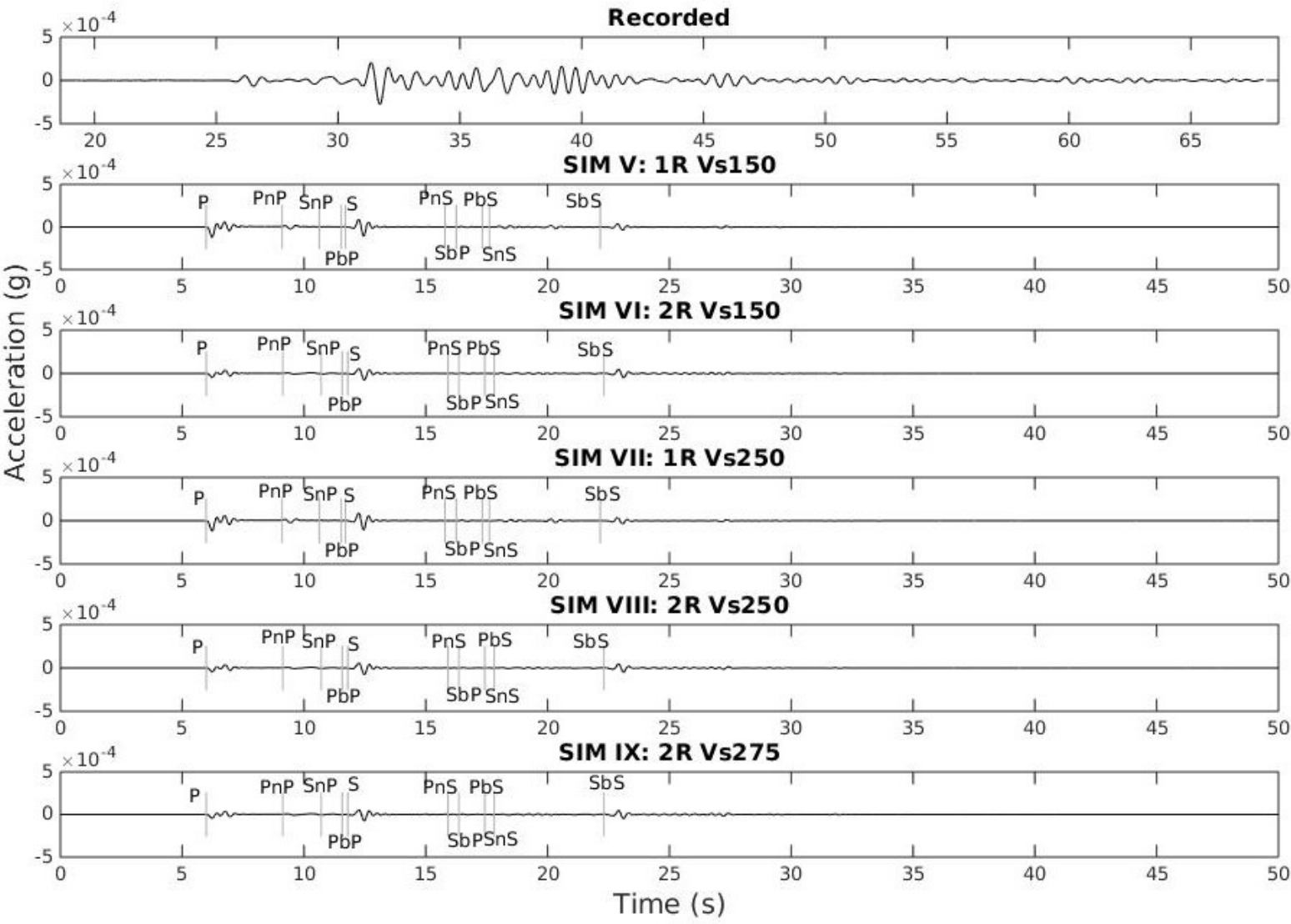

Figure 6.34: Horizontal acceleration time series at station KB1O for the simulations with the moment tensor solution source from Bent et al. (2015). 1R indicates the simulation used one rock type, $2 \mathrm{R}$ is for a simulation which uses two rock types, Vs150 denotes the simulation used Soil Type I for the basin material, Vs250 denotes the simulation used Soil Type II for the basin material, and Vs275 denotes the simulation used Soil Type III properties for the basin material. 
KB10 Vertical Component
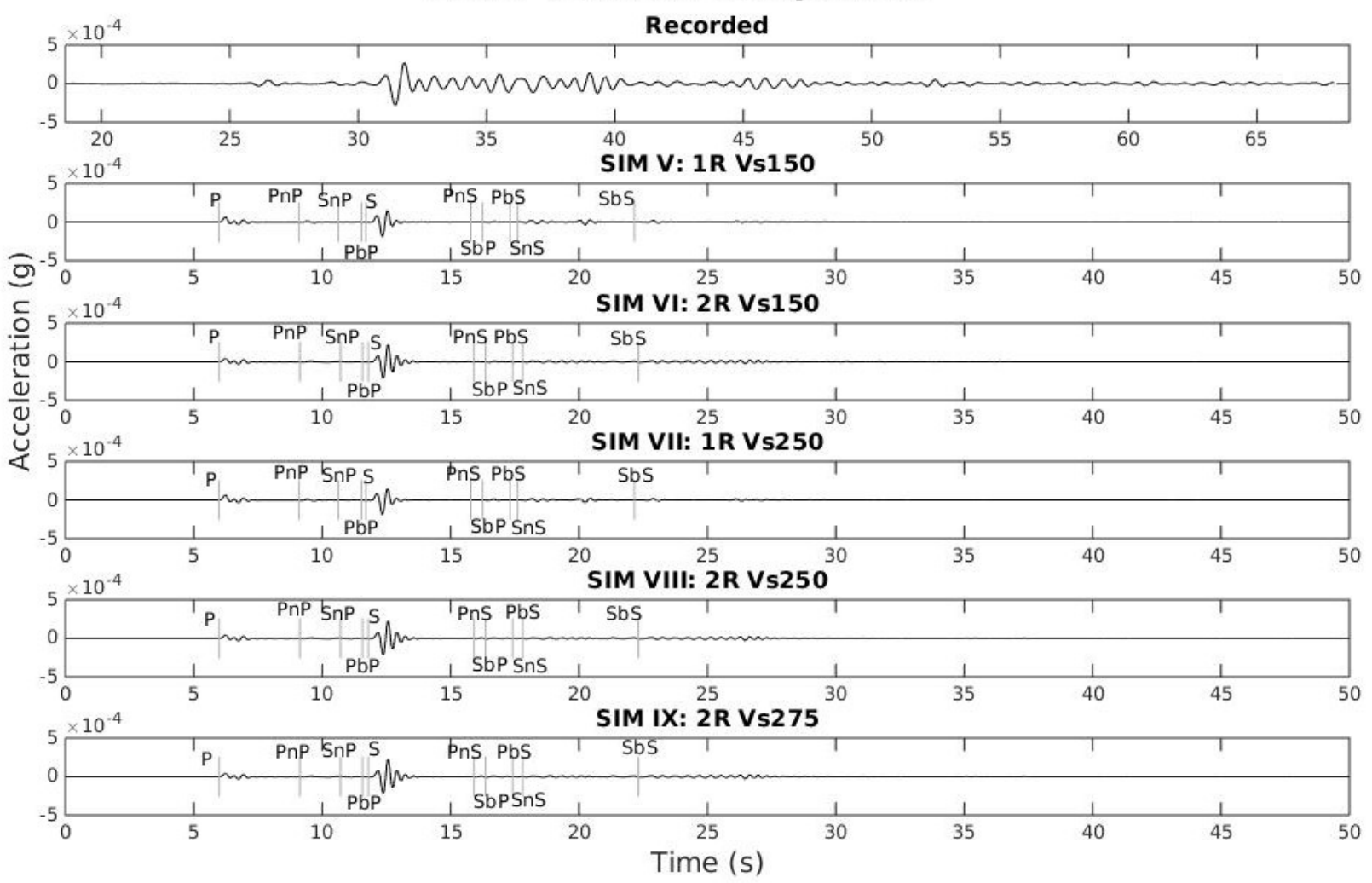

Figure 6.35: Vertical acceleration time series at station KB1O for the simulations with the moment tensor solution source from Bent et al. (2015). 1R indicates the simulation used one rock type, $2 R$ is for a simulation which uses two rock types, Vs 150 denotes the simulation used Soil Type I for the basin material, Vs250 denotes the simulation used Soil Type II for the basin material, and Vs275 denotes the simulation used Soil Type III properties for the basin material. 


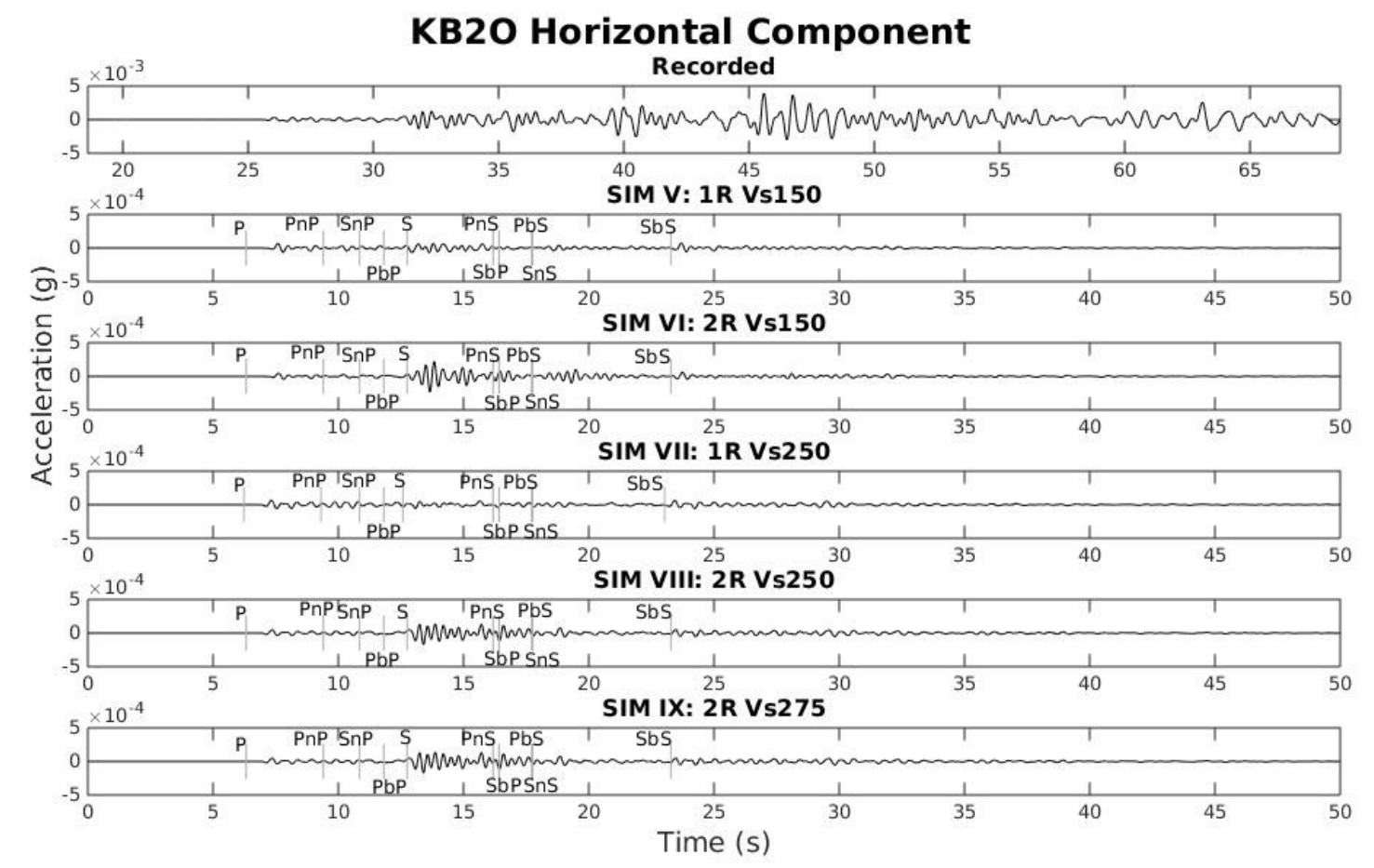

Figure 6.36: Horizontal acceleration time series at station KB2O for the simulations with the moment tensor solution source from Bent et al. (2015). 1R indicates the simulation used one rock type, $2 R$ is for a simulation which uses two rock types, Vs 150 denotes the simulation used Soil Type I for the basin material, Vs250 denotes the simulation used Soil Type II for the basin material, and Vs275 denotes the simulation used Soil Type III properties for the basin material. 


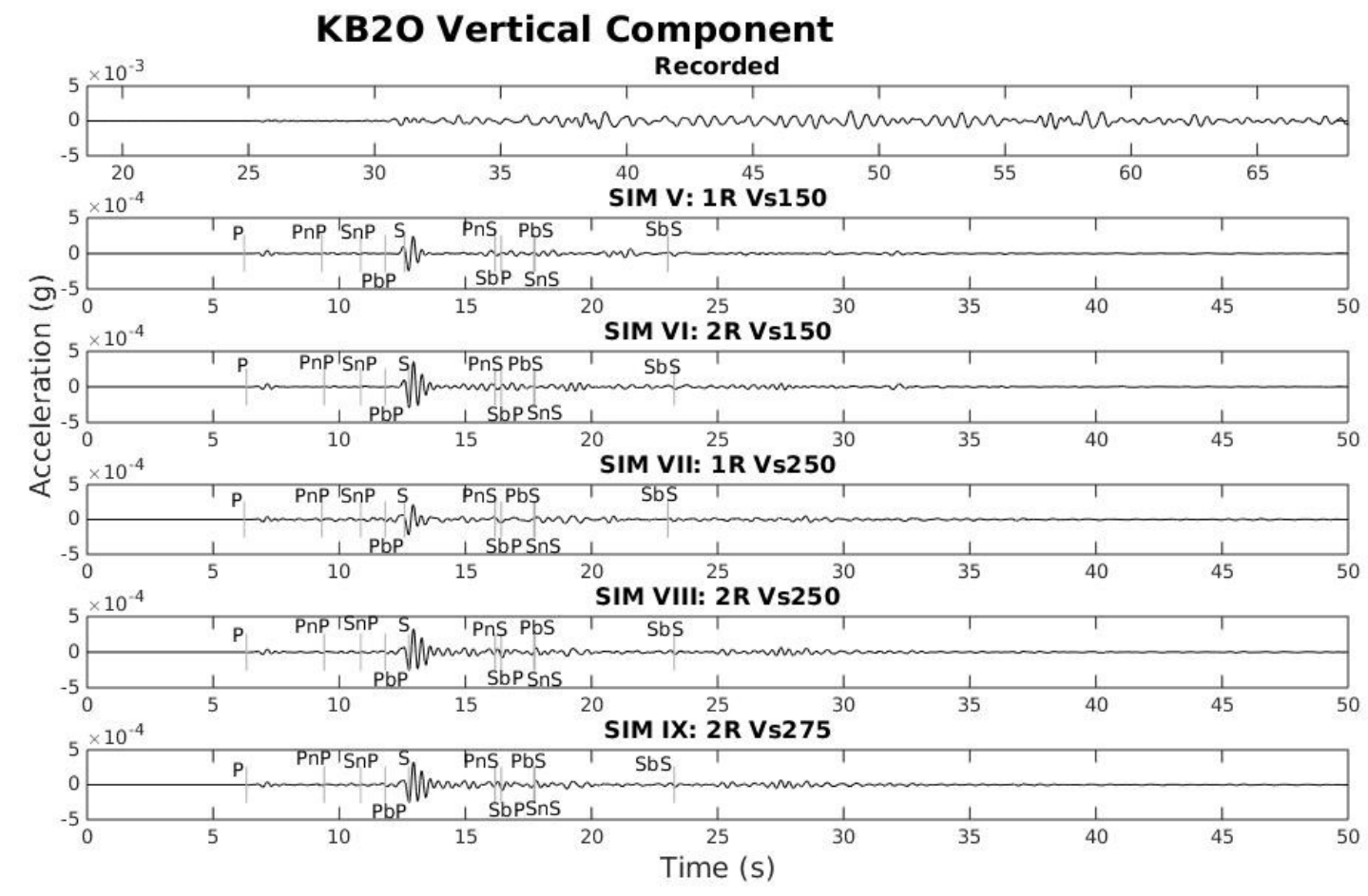

Figure 6.37: Vertical acceleration time series at station KB2O for the simulations with the moment tensor solution source from Bent et al. (2015). 1R indicates the simulation used one rock type, 2R is for a simulation which uses two rock types, Vs150 denotes the simulation used Soil Type I for the basin material, Vs250 denotes the simulation used Soil Type II for the basin material, and Vs275 denotes the simulation used Soil Type III properties for the basin material.

\begin{tabular}{|c|c|c|c|c|c|c|}
\hline \multirow{2}{*}{ Model } & \multicolumn{2}{|c|}{ Peak at KB1O (g) } & \multicolumn{2}{c|}{ Peak at KB2O (g) } & \multicolumn{2}{c|}{ KB2O/KB1O } \\
\cline { 2 - 7 } & Horizontal & Vertical & Horizontal & Vertical & Horizontal & Vertical \\
\hline Recording & 0.00025 & 0.00033 & 0.00270 & 0.00120 & 10.8 & 3.63 \\
\hline $\begin{array}{c}\text { SIM V: } \\
\text { 1RVs150 }\end{array}$ & 0.00011 & 0.00018 & 0.00007 & 0.00025 & 0.63 & 1.37 \\
\hline $\begin{array}{c}\text { SIM VI: } \\
\text { 2RVs150 }\end{array}$ & 0.00008 & 0.00023 & 0.00023 & 0.00036 & 2.88 & 1.57 \\
\hline $\begin{array}{c}\text { SIM VII: } \\
\text { 1RVs250 }\end{array}$ & 0.00011 & 0.00018 & 0.00006 & 0.00022 & 0.57 & 1.20 \\
\hline $\begin{array}{c}\text { SIM VIII: } \\
\text { 2RVs250 }\end{array}$ & 0.00008 & 0.00023 & 0.00017 & 0.00033 & 2.16 & 1.43 \\
\hline $\begin{array}{c}\text { SIM IX: } \\
\text { 2RVs275 }\end{array}$ & 0.00008 & 0.00023 & 0.00018 & 0.00031 & 2.25 & 1.38 \\
\hline
\end{tabular}

Table 6.6: Maximum acceleration values of the simulations with the moment tensor source from Bent et al. (2015) at KB10 and KB2O. 1R indicates the simulation used one rock type, $2 \mathrm{R}$ is for a simulation which uses two rock types, Vs 150 denotes the simulation used Soil Type I for the basin material, Vs250 denotes the simulation used Soil Type II for the basin material, and Vs275 denotes the simulation used Soil Type III properties for the basin material.

The Fourier acceleration spectrum for KB1O is shown in Figure 6.38 for the horizontal and the vertical component for the recorded and simulated values. The simulated models were very 
similar to each other but are about 10 times less than the recorded values at this location between $0.5-2 \mathrm{~Hz}$. These values were also larger than the simulations in Chapter 4.3.1. It is unclear if the difference here was caused by the presence of a soil basin, or the reduced mesh sizing in the model compared to the simulation in Section 4.3.1.
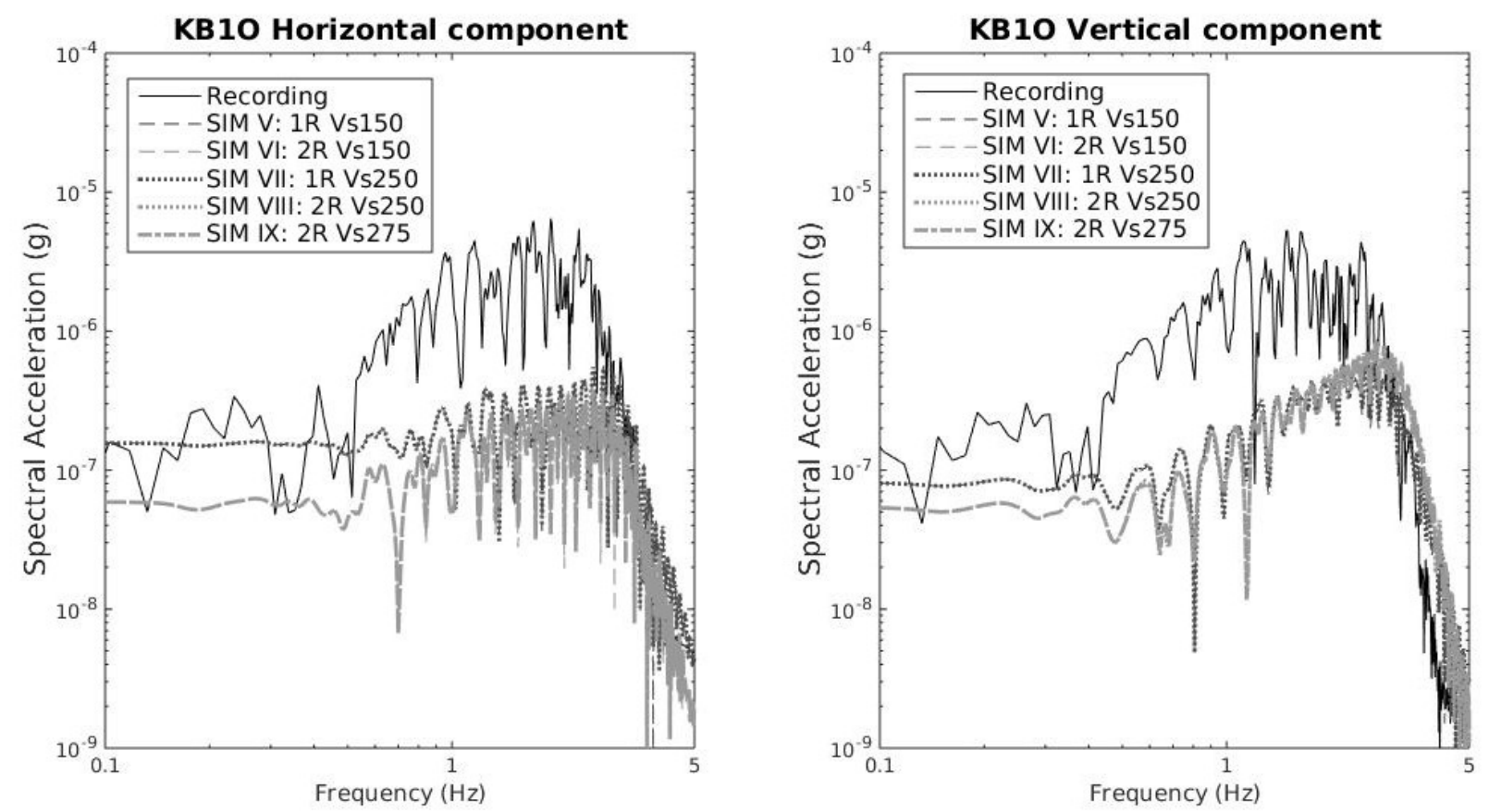

Figure 6.38: The Fourier acceleration spectrum at KB1O for the horizontal (left) and vertical (right) component for the simulations with the moment tensor solution from Bent et al. (2015). $1 \mathrm{R}$ indicates the simulation used one rock type, $2 \mathrm{R}$ is for a simulation which uses two rock types, Vs150 denotes the simulation used Soil Type I for the basin material, Vs250 denotes the simulation used Soil Type II for the basin material, and Vs275 denotes the simulation used Soil Type III properties for the basin material.

The horizontal and vertical components of the Fourier acceleration spectrum recorded at soil station, KB2O, is shown in Figure 6.39. The models with the Vs150, or Soil Type I, properties have a lower dominant frequency than expected from the recordings for both the horizontal and vertical components. The models with the Vs250, Soil Type II, and Vs275, Soil Type III, properties match very closely to the shape of the recorded response of the receiver in the basin, but are also about 10 times less. The ability to match the shape of the spectral frequencies for a wide 
pulse width around the peak frequency is an indication that the $2 \mathrm{D}$ simulation was producing some of the basin effects which had been recorded. One dimensional modelling of this soil profile beneath the receiver only produced a narrow band of large amplifications about the peak frequency (Hayek 2016).
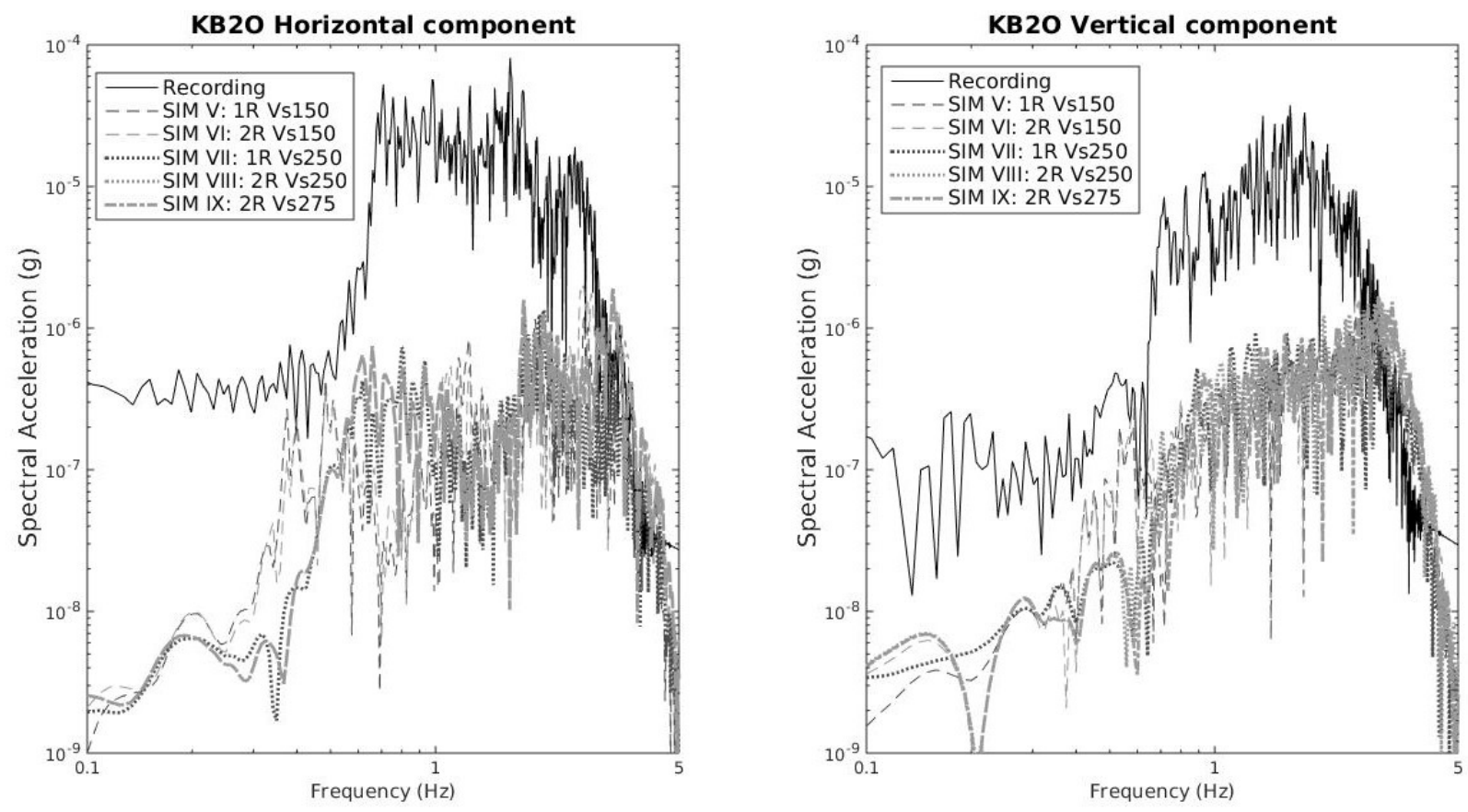

Figure 6.39: The Fourier acceleration spectrum at KB2O for the horizontal (left) and vertical (right) component for the simulations with the moment tensor solution from Bent et al. (2015). $1 R$ indicates the simulation used one rock type, $2 R$ is for a simulation which uses two rock types, Vs150 denotes the simulation used Soil Type I for the basin material, Vs250 denotes the simulation used Soil Type II for the basin material, and Vs275 denotes the simulation used Soil Type III properties for the basin material.

The spectral ratios from KB2O to KB1O are shown in Figure 6.40 for the simulations and recorded motions. In these simulations the transfer functions from the rock to soil are lower than the recorded motions, even though we observed some amplification at higher frequencies. There are only a few peaks from the simulations which match the recorded amplification. This indicates a lack of energy that was transmitted into the basin from the surrounding material within the simulations. 

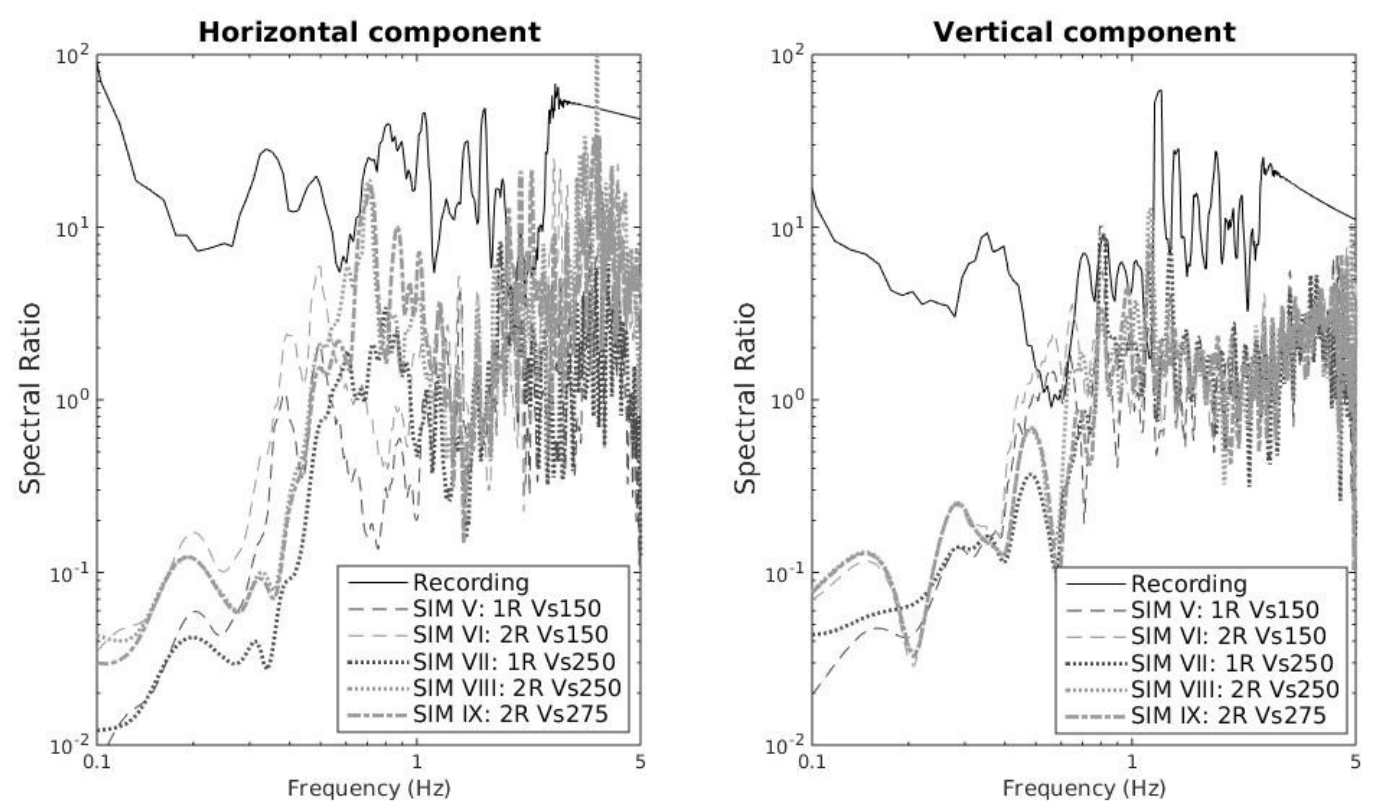

Figure 6.40: The spectral ratios for the horizontal (left) and vertical (right) components of motion at station KB2O divided by station KB1O. $1 \mathrm{R}$ indicates the simulation used one rock type, $2 \mathrm{R}$ is for a simulation which uses two rock types, Vs150 denotes the simulation used Soil Type I for the basin material, , Vs250 denotes the simulation used Soil Type II for the basin material, and Vs275 denotes the simulation used Soil Type III properties for the basin material.

\subsubsection{P-wave and $S$-wave Comparison}

The acceleration time series for the recorded motions and the simulations SIM V, SIM VI, SIM VII, SIM VIII, and SIM IX were shortened to correspond with about 5 seconds before the P-wave and 5 seconds after the S-wave arrival. This eliminated the extra arrivals at both recording stations, $\mathrm{KB} 1 \mathrm{O}$ and $\mathrm{KB} 2 \mathrm{O}$, and allowed for a better comparison of the energy transfer between the rock and basin for the main pulses. The horizontal acceleration time series is shown in Figure 6.41, and the vertical acceleration time series is shown in Figure 6.42 for KB1O. The windowed acceleration time series at $\mathrm{KB} 2 \mathrm{O}$ is shown in Figure 6.43 for the horizontal component and Figure 6.44 for the vertical component. Although the relative amplitudes within the time series are unchanged, the peak motions, listed in Table 6.7, in the recordings are much closer to the simulated values. 
KB10 Horizontal Component
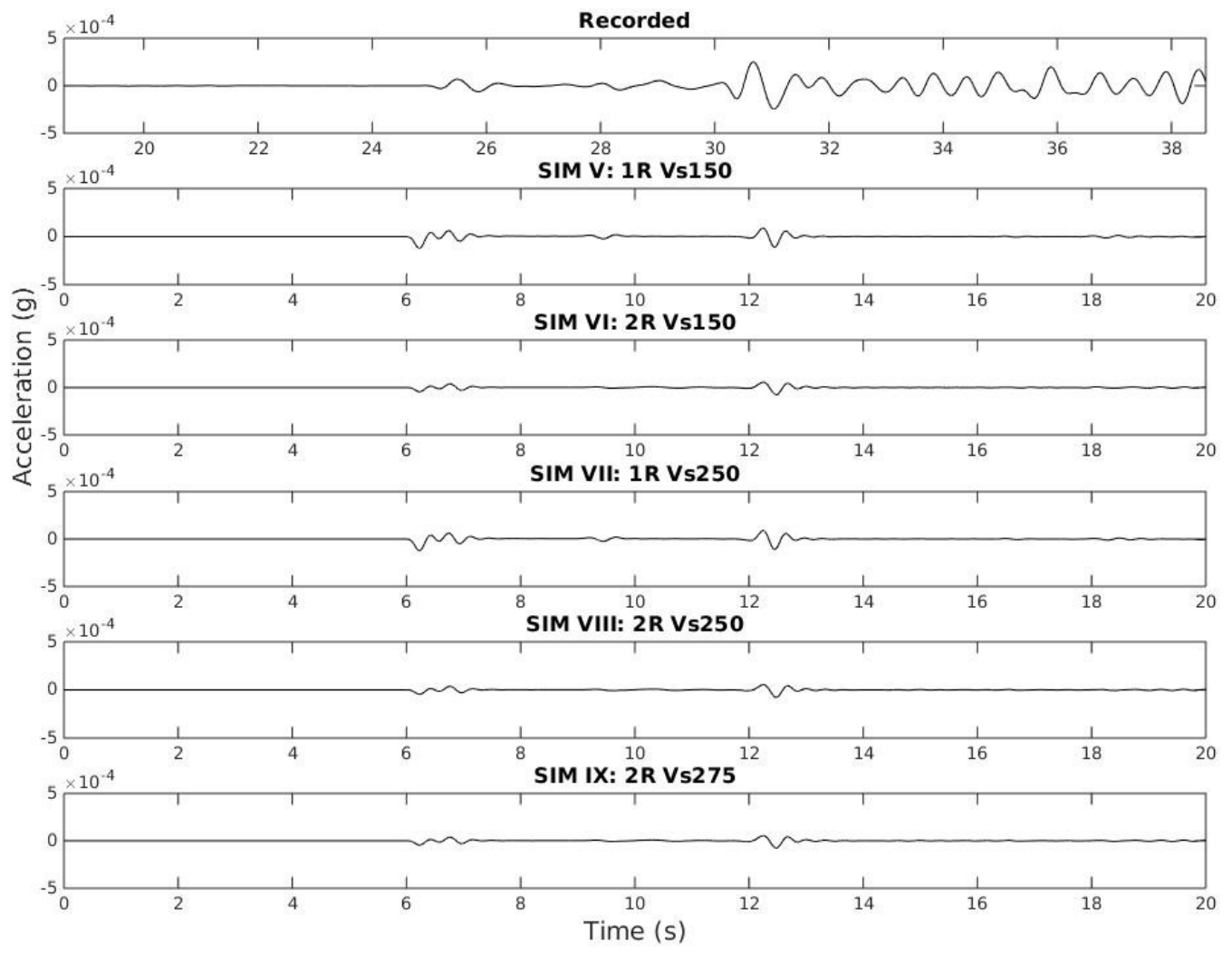

Figure 6.41: The windowed horizontal acceleration time series at station KB1O for the simulations with the moment tensor solution source from Bent et al. (2015). $1 \mathrm{R}$ indicates the simulation used one rock type, $2 \mathrm{R}$ is for a simulation which uses two rock types, Vs150 denotes the simulation used Soil Type I for the basin material, Vs250 denotes the simulation used Soil Type II for the basin material, and Vs275 denotes the simulation used Soil Type III properties for the basin material. 


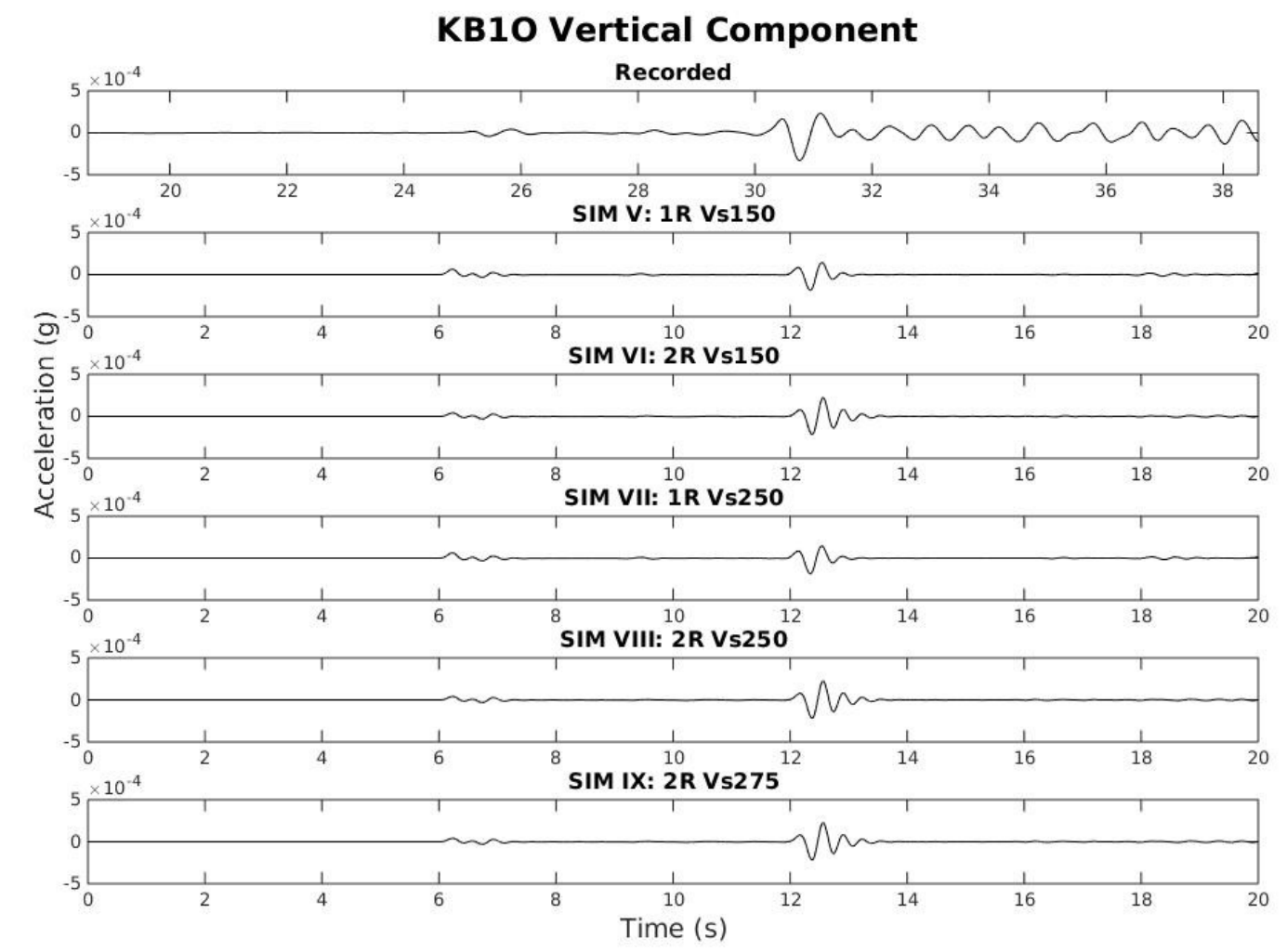

Figure 6.42: The windowed vertical acceleration time series at station KB1O for the simulations with the moment tensor solution source from Bent et al. (2015). 1R indicates the simulation used one rock type, $2 \mathrm{R}$ is for a simulation which uses two rock types, Vs150 denotes the simulation used Soil Type I for the basin material, Vs250 denotes the simulation used Soil Type II for the basin material, and Vs275 denotes the simulation used Soil Type III properties for the basin material. 
KB20 Horizontal Component
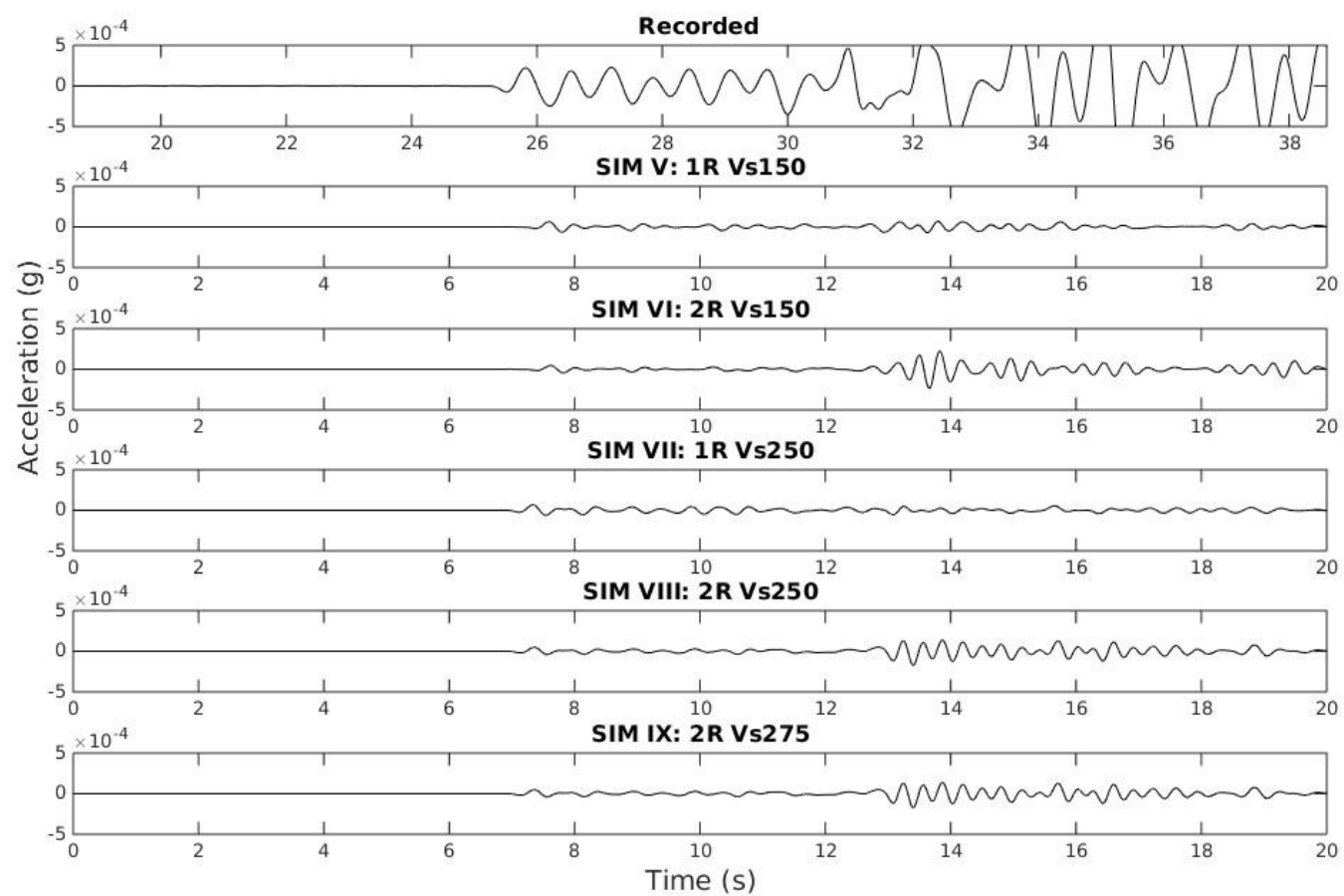

Figure 6.43: The windowed horizontal acceleration time series at station $\mathrm{KB} 2 \mathrm{O}$ for the simulations with the moment tensor solution source from Bent et al. (2015). 1R indicates the simulation used one rock type, 2R is for a simulation which uses two rock types, Vs150 denotes the simulation used Soil Type I for the basin material, Vs250 denotes the simulation used Soil Type II for the basin material, and Vs275 denotes the simulation used Soil Type III properties for the basin material. 
KB2O Vertical Component
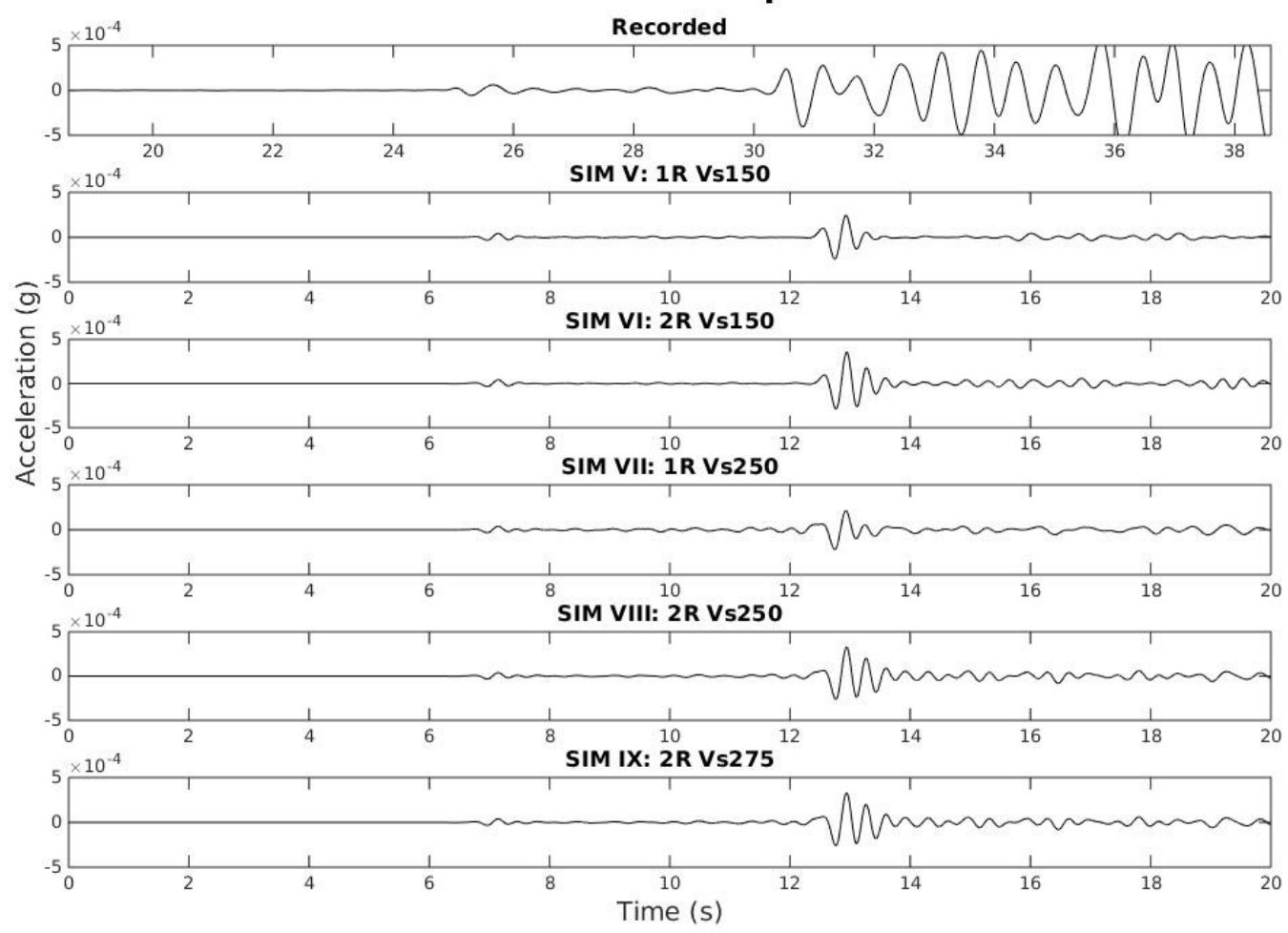

Figure 6.44: The windowed horizontal acceleration time series at station KB1O for the simulations with the moment tensor solution source from Bent et al. (2015). $1 R$ indicates the simulation used one rock type, $2 R$ is for a simulation which uses two rock types, Vs150 denotes the simulation used Soil Type I for the basin material, Vs250 denotes the simulation used Soil Type II for the basin material, and Vs275 denotes the simulation used Soil Type III properties for the basin material.

\begin{tabular}{|c|c|c|c|c|c|c|}
\hline \multirow{2}{*}{ Model } & \multicolumn{2}{|c|}{ Peak at KB1O (g) } & \multicolumn{2}{c|}{ Peak at KB2O (g) } & \multicolumn{2}{c|}{ KB2O/KB1O } \\
\cline { 2 - 7 } & Horizontal & Vertical & Horizontal & Vertical & Horizontal & Vertical \\
\hline Recording & 0.00048 & 0.00062 & 0.00190 & 0.00091 & 3.96 & 1.46 \\
\hline $\begin{array}{c}\text { SIM V: } \\
\text { 1RVs150 }\end{array}$ & 0.00011 & 0.00018 & 0.00007 & 0.00025 & 0.63 & 1.37 \\
\hline $\begin{array}{c}\text { SIM VI: } \\
\text { 2RVs150 }\end{array}$ & 0.00008 & 0.00023 & 0.00023 & 0.00036 & 2.88 & 1.57 \\
\hline $\begin{array}{c}\text { SIM VII: } \\
\text { 1RVs250 }\end{array}$ & 0.00011 & 0.00018 & 0.00006 & 0.00022 & 0.57 & 1.20 \\
\hline $\begin{array}{c}\text { SIM VIII: } \\
\text { 2RVs250 }\end{array}$ & 0.00008 & 0.00023 & 0.00017 & 0.00033 & 2.16 & 1.43 \\
\hline $\begin{array}{c}\text { SIM IX: } \\
\text { 2RVs275 }\end{array}$ & 0.00008 & 0.00023 & 0.00018 & 0.00031 & 2.25 & 1.38 \\
\hline
\end{tabular}

Table 6.7: Maximum acceleration values of the simulations with the moment tensor source from Bent et al. (2015) at KB10 and KB2O for only the P- and S-wave arrivals. $1 \mathrm{R}$ indicates the simulation used one rock type, $2 \mathrm{R}$ is for a simulation which uses two rock types, Vs150 denotes the simulation used Soil Type I for the basin material, Vs250 denotes the simulation used Soil Type II for the basin material, and Vs275 denotes the simulation used Soil Type III properties for the basin material. 
The spectral ratios for this time length for the simulations as well as the recorded motions are shown in Figure 6.45. Over this short time span, the spectral acceleration ratios from the rock to soil receivers were closer in value. This is an indication that the energy being transferred into the basin at the pulse of each wave was comparable between the simulations and recorded motions. The absence of energy within the basin for the longer simulations may have been caused by the lack of strong wave arrivals as observed in the recorded acceleration time series for KB1O, and were seemingly amplified at KB2O.
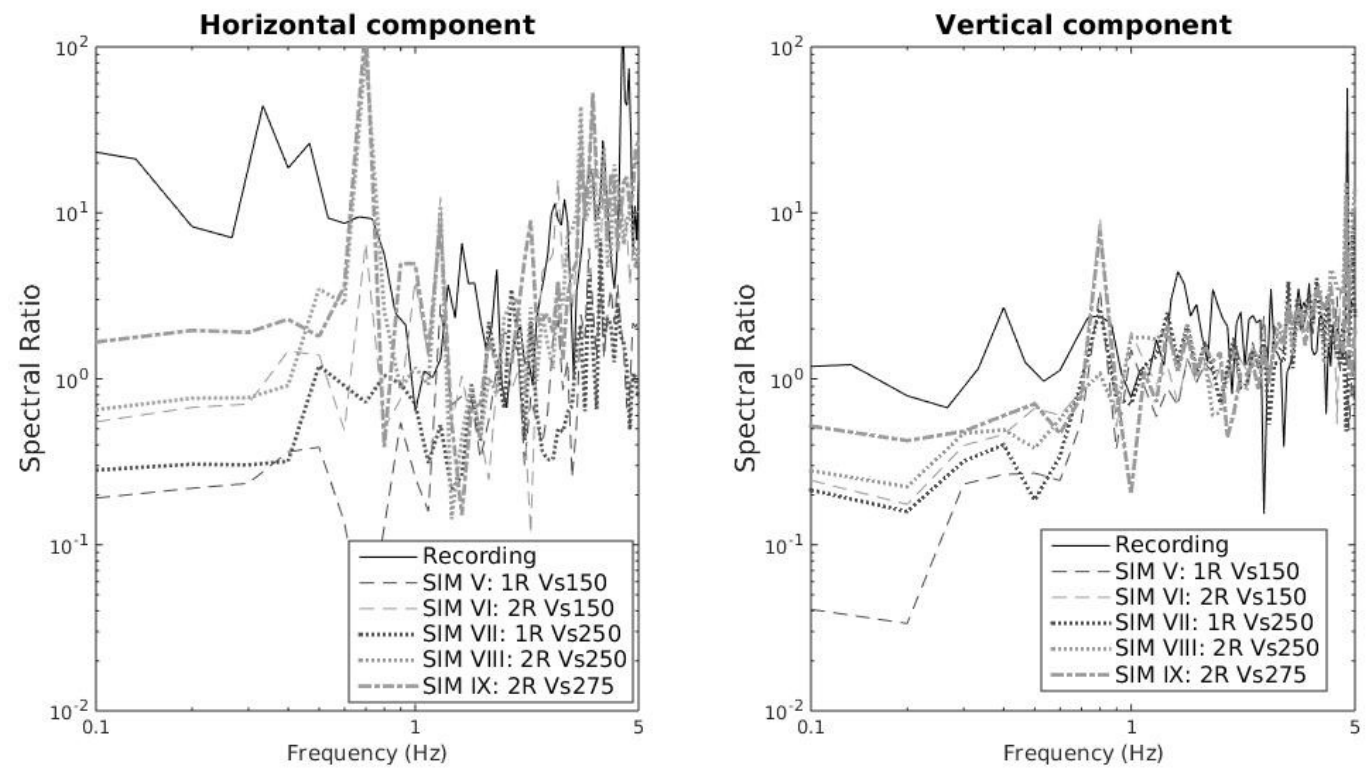

Figure 6.45: The spectral acceleration ratios for the horizontal (left) and vertical (right) components of motion for station KB2O/KB1O using the windowed time series. $1 \mathrm{R}$ indicates the simulation used one rock type, $2 \mathrm{R}$ is for a simulation which uses two rock types, Vs150 denotes the simulation used Soil Type I for the basin material, Vs250 denotes the simulation used Soil Type II for the basin material, and Vs275 denotes the simulation used Soil Type III properties for the basin material.

\subsubsection{Simulated Basin Effects}

A row of 50 receivers were placed across a total distance of 10 kilometres in the model, and extended roughly 400 metres from either edge of the soil filled basin for each of the simulations corresponding to the 2013 Ladysmith earthquake. Figure 6.46 shows the horizontal and vertical 
components of motion for the first 50 seconds of these receivers in SIM VI, the simulation with two rock types and the basin filled with the Vs150, Soil Type I material properties. The figure also displays a vertically exaggerated NWSE profile basin geometry and the location of the Kinburn Seismic Array receivers within this profile. The first two and last three receivers along this array are located on rock material, and the other receivers are placed on varying depths of soil. The shape of the soil basin is evident in the horizontal component of motion, based on the delayed arrivals of the dominant wave phases and stronger resonances in the deeper areas of the basin. The vertical component of motion doesn't show the same delay of arrivals but does show some resonances and other travelling waves.

Figure 6.47 shows the horizontal and vertical components of motion for the first 50 seconds in the simulation with two rock types and the Vs275 soil properties in the basin, SIM IX. The vertical component of motion behaved very similarly to the vertical component as shown in Figure 6.46 which had different soil parameters. The horizontal component of motion shows less chaotic motions and shows evidence of several wave fronts travelling in the opposite direction of the source beginning at about 3.5 kilometres from the end of the line of receivers. These waves start at the same location and are caused by each wave phase arrival. Several waves travelling in the opposite direction from the source were present in Figure 6.46. However, it is difficult to determine if these waves were caused by the basin edge or the model boundary, as these two interfaces are close in position; hence the waves cannot be traced back to their source clearly enough to determine their exact origin position. 

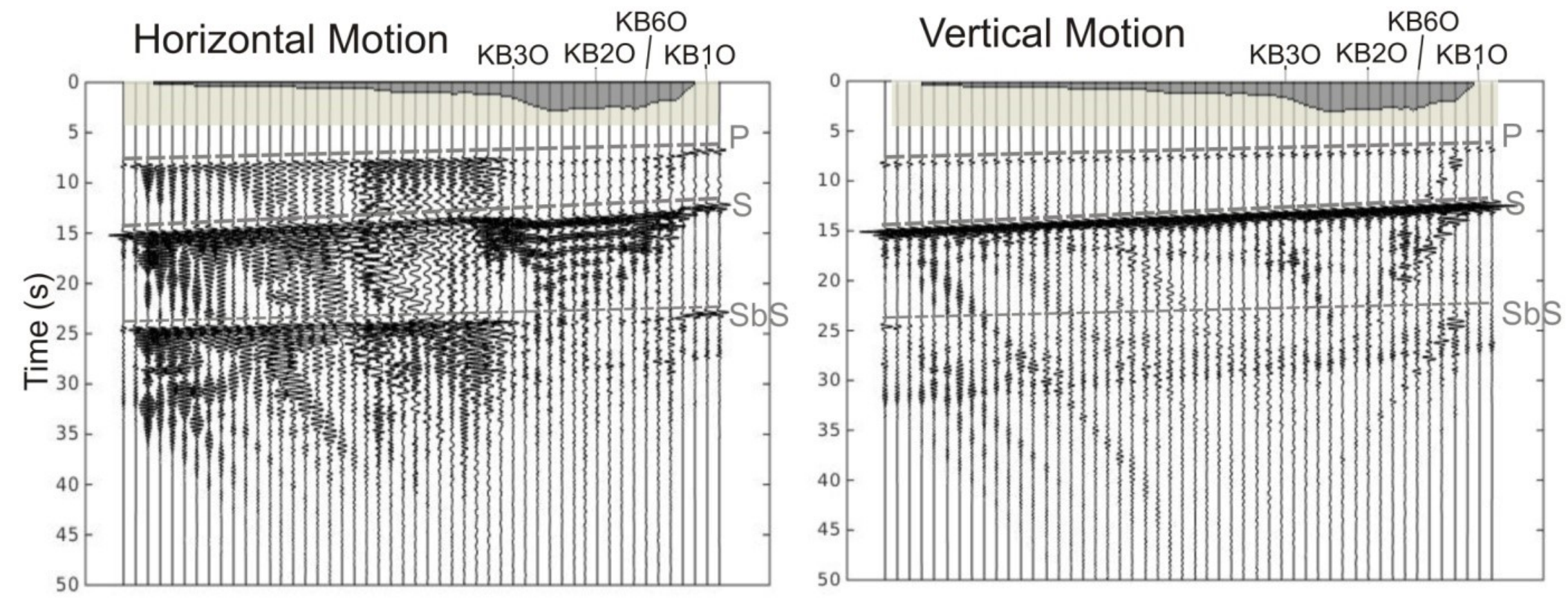

Figure 6.46: Horizontal (left) and vertical (right) components of motion across the basin for the model with two rock types and Soil Type I properties, SIM VI. The dashed lines display the theoretical arrival times of the P-wave (P), S-wave (S) and an S-wave reflected off the bottom of the model (SbS). 

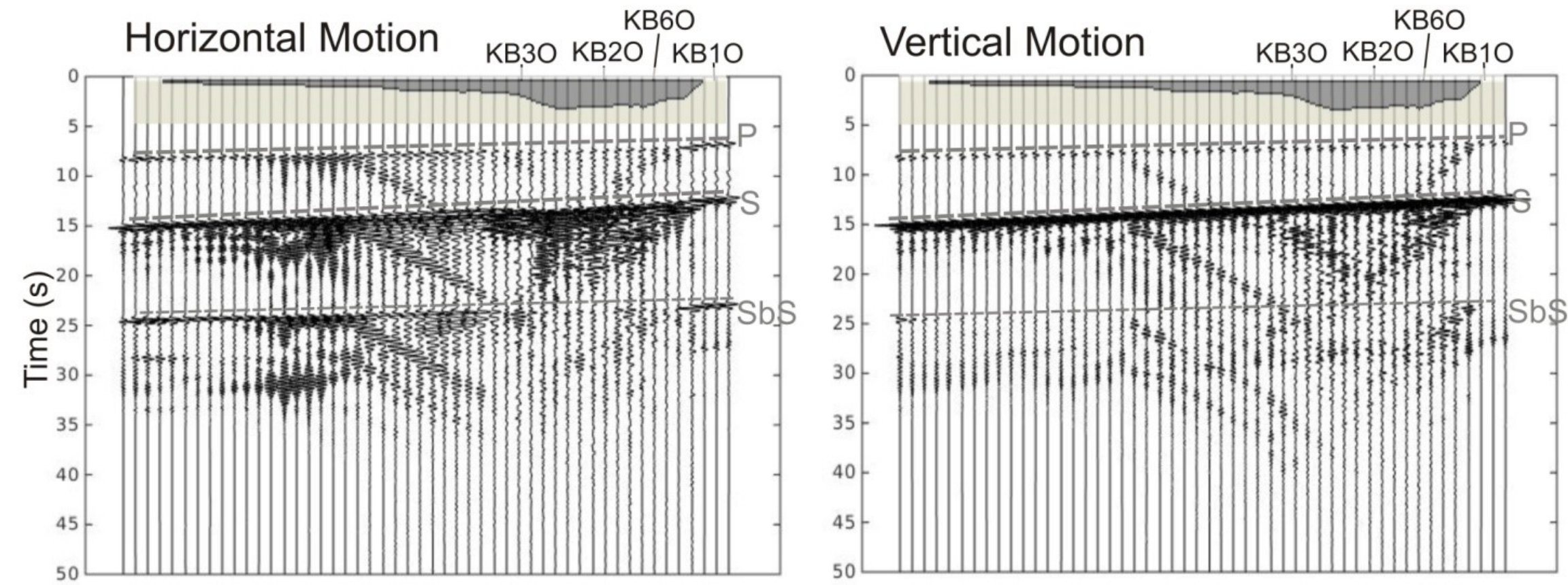

Figure 6.47: Horizontal (left) and vertical (right) components of motion across the basin for the model with two rock types and Soil Type III properties, SIM IX. The dashed lines display the theoretical arrival times of the P-wave (P), S-wave (S) and an S-wave reflected off the bottom of the model (SbS). 


\subsubsection{Simulated Motions at the Kinburn Seismic Array}

Figure 6.48 shows the horizontal and vertical component of the Fourier acceleration spectrum at the stations which best correlate with the positioning of the seismic stations KB1O, $\mathrm{KB} 2 \mathrm{O}, \mathrm{KB} 30$ and $\mathrm{KB} 60$. The simulation was completed with two rock types and the Vs250 soil properties, SIM VIII. The vertical spectral acceleration was very similar for all stations above $0.6 \mathrm{~Hz}$, but decrease at different rates for the lower frequencies. The response of KB2O and KB6O are very similar for the horizontal component, as their soil depths at these locations are similar, and KB3O displays a higher dominant frequency as expected with the smaller soil depth.

Figure 6.49 shows the spectral ratio from each station to KB1O for both the horizontal and vertical components, along with the recorded ratio KB2O/KB1O. As expected the behaviour was similar with $\mathrm{KB} 2 \mathrm{O}$ and $\mathrm{KB} 60$ showing the largest amplifications from KB1O at roughly the same dominant frequency. KB3O has a higher dominant frequency and a smaller peak amplification compared to the other two stations. All the simulated spectral ratios were significantly lower than the recorded spectral ratios indicating that the energy transfers at these frequencies were lower than expected in the simulation. 

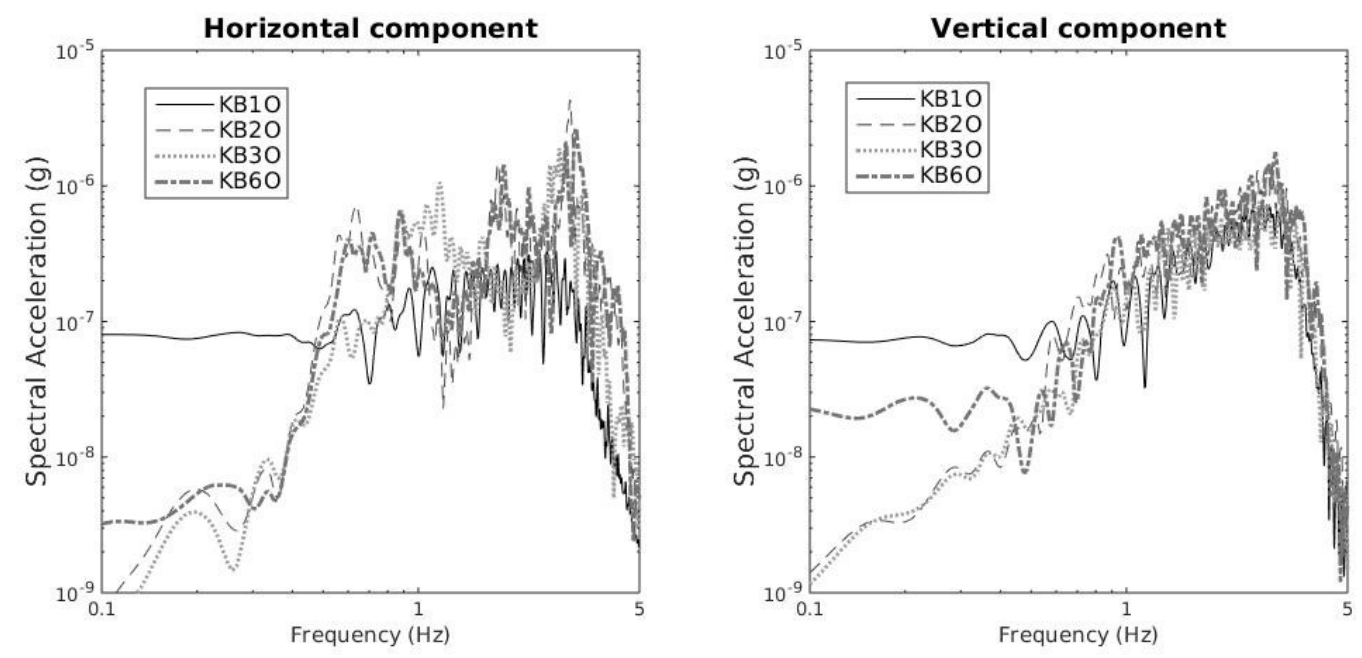

Figure 6.48: The smoothed horizontal (left) and vertical (right) components of the simulated Fourier acceleration spectrum at stations KB1O, KB2O, KB3O, and KB6O from SIM VIII.
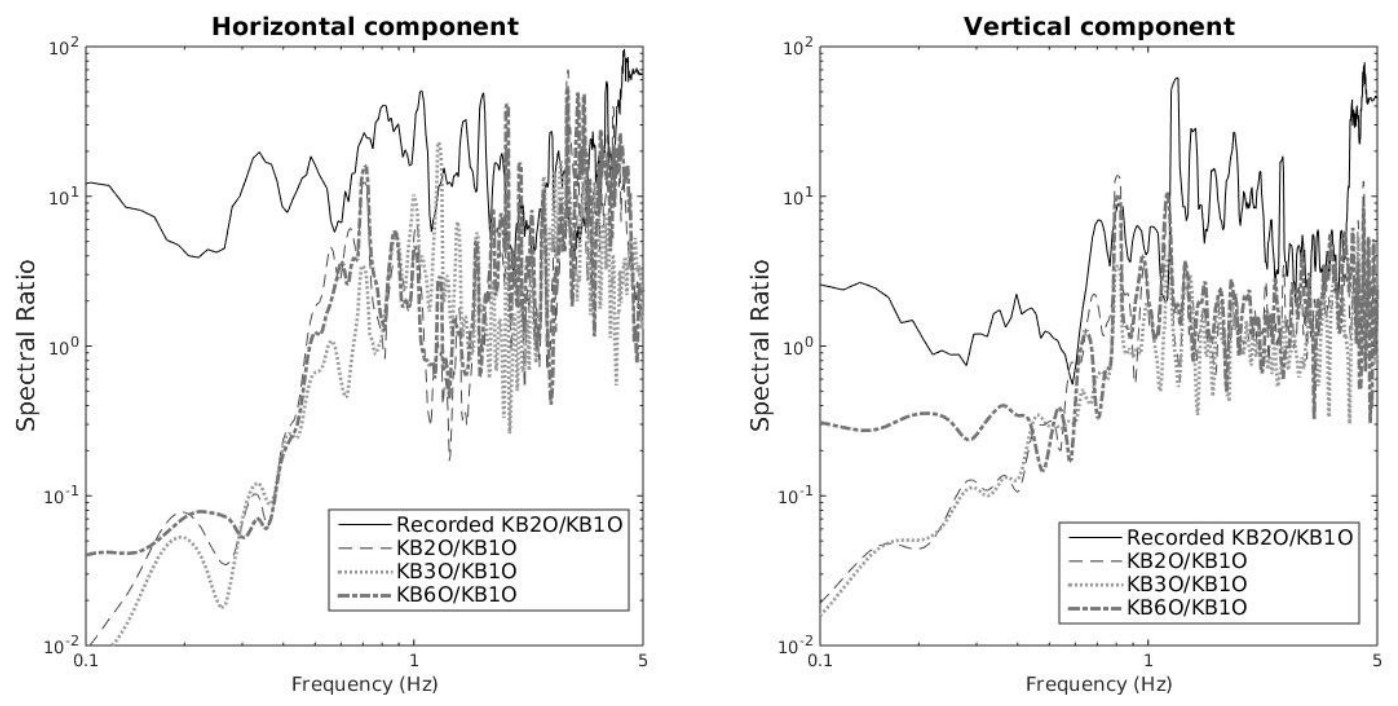

Figure 6.49: The smoothed spectral ratios of the Fourier acceleration spectrum at stations KB2O, KB3O, and KB6O compared to KB1O from SIM VIII.

The first 50 seconds of the acceleration time series of the stations KB1O, KB2O, KB30 and $\mathrm{KB} 60$ are shown in Figure 6.50. As seen in the simulation at KB10, the dominant wave arrivals are the P-wave, S-wave and the S-wave reflected from the bottom of the model (SbS). Although this reflection should correspond to the reflection off the Moho boundary, it appears the timing is different from the recorded motions. This can be seen from the previous comparisons, Figure 
6.34 and Figure 6.35 , where there was more pulses of strong motions after the S-wave arrival in the recorded motions compared to the simulations. This may be an indication of either a model depth which is different from the crustal thickness in this area, or an inaccurate velocity structure in the model.

In the simulation, station $\mathrm{KB} 2 \mathrm{O}$ had the longest strong motion duration after the arrival of the S-wave phase, followed by KB6O with a slightly shorter stong motion duration, and then KB3O which has about half the strong motion duration as at KB2O. In each of the soil stations, $\mathrm{KB} 2 \mathrm{O}, \mathrm{KB} 3 \mathrm{O}$, and $\mathrm{KB} 6 \mathrm{O}$, there appears to be another wave corresponding to the SbS wave phase seen in KB1O. However, the amplification of this wave appears to be smaller compared to the amplification of the other waves. From the simulated acceleration time series and spectral accelerations, it is possible to determine that the different positions of the Kinburn Seismic Array elements have an effect on the expected motions at these locations. The spectral ratios in Figure 6.49 are the simulated rock-to-soil transfer functions for the Kinburn Seismic Array. 

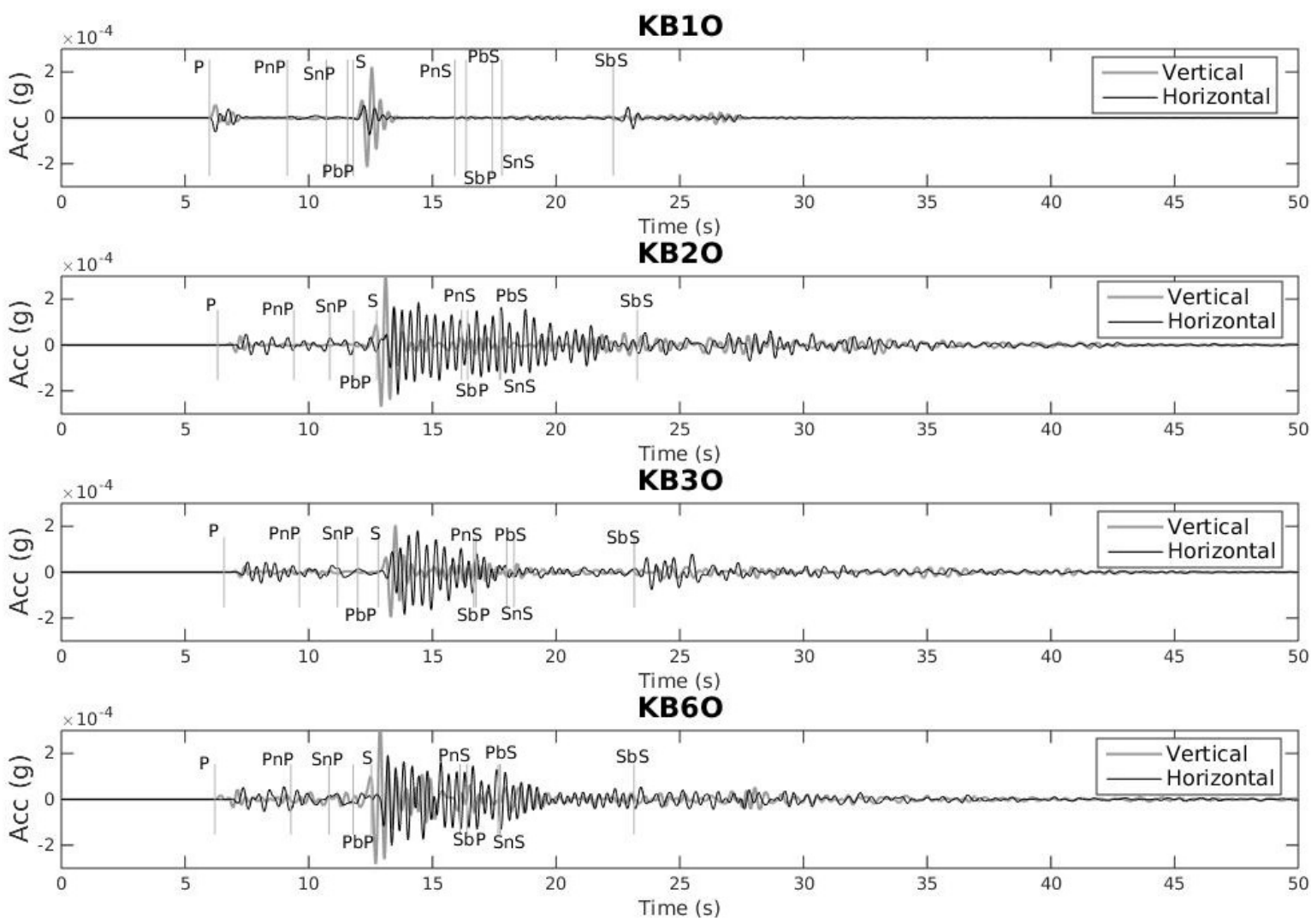

Figure 6.50: Simulated acceleration time series at stations KB1O, KB2O, KB3O and KB6O from SIM VIII.

\subsubsection{Particle Motions}

Figure 6.51, Figure 6.52, Figure 6.53, and Figure 6.54 shows the particle motion plots at different time slices and the displacement time series for KB1O, KB2O, KB3O, and KB6O, respectively. Each particle motion plot was normalized to the maximum motion during the time slice to give a better indication of movement. The particle motion was completed in an attempt to discriminate the wave type at the given time slices. The P-wave first arrival tends to be the simplest motion as it does not overlap with the other arrivals. The other waves tend to be a combination of several waves arriving close together. 
KB1O has the clearest particle motions as this receiver is on the rock within the model. The P-wave arriving has a clear orientation of motion at roughly $20^{\circ}$, which it the expected angle of P-wave emergence. The other time slices for KB1O appear to have overlapping wave types as there are varying motions inconsistent with any expected motion from a given wave type. The $12-14 \mathrm{~s}$ and $14-16 \mathrm{~s}$ seem to have possible retrograde elliptical motions, however it is oriented away from the source. This is possibly an indication of surface waves created at the basin edge but reflected away from the basin into the rock.

All the other stations, $\mathrm{KB} 2 \mathrm{O}, \mathrm{KB} 30$, and $\mathrm{KB} 6 \mathrm{O}$, seem to have the first wave arrival oriented horizontally. Neither of these stations have a discernible direction of motion for the P-wave or the S-wave arrival. This is consistent with the recordings within the basin, as there are many high frequency events that particle motions are often difficult to determine (Hayek, 2016).

Later time slices within the basin seem to indicate waves that are surface waves, based on their motions. These surface waves within the basin have a much larger horizontal to vertical ratio compared to other surface waves at the rock station KB1O, which may have emerged from the basin. This large horizontal motion is consistent with the result of Konno and Omachi (1998), where a large impedance contrast were shown to cause the horizontal motions to be larger than the vertical for Rayleigh wave motion. Tracing these waves back in the surface receiver plots (Figure 6.47), it appears that these waves originate at the basin boundary, and are basin-edge generated surface waves. 


\section{KB10 Particle Motion}
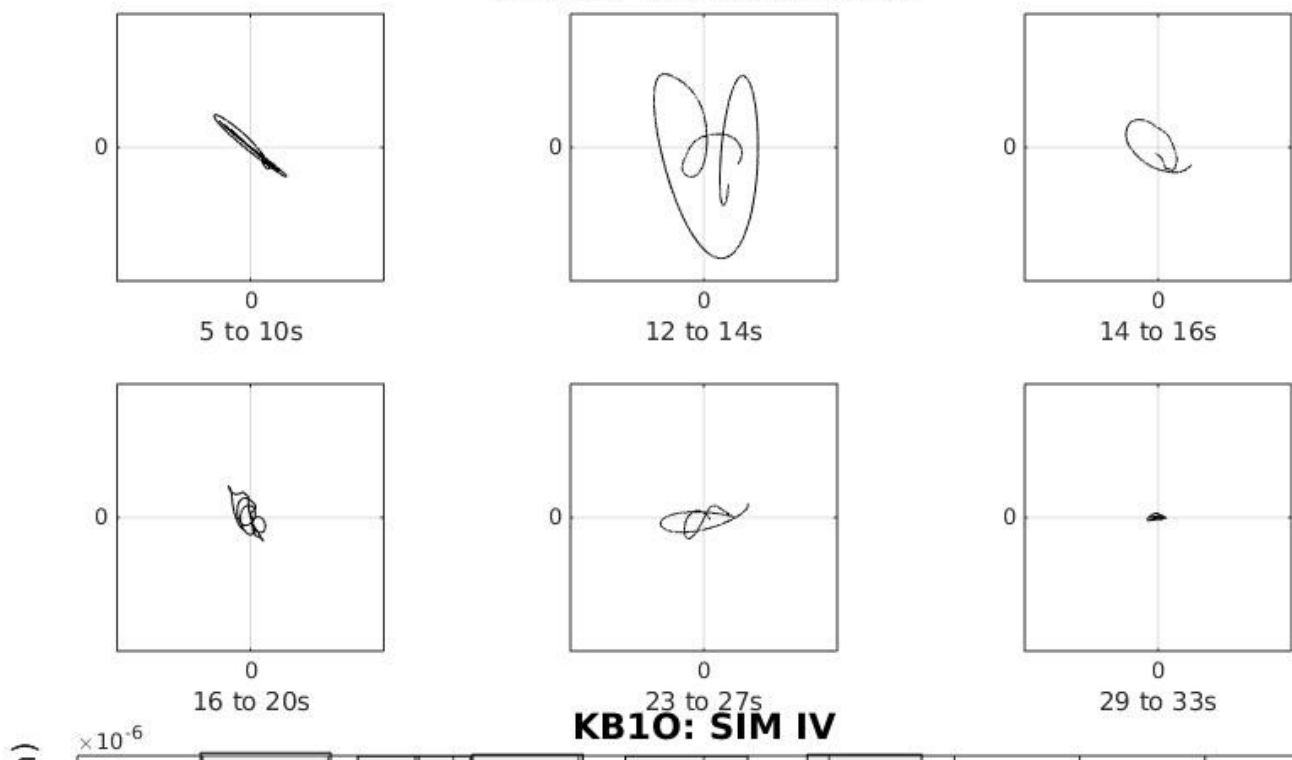

KB1O: SIMM IV
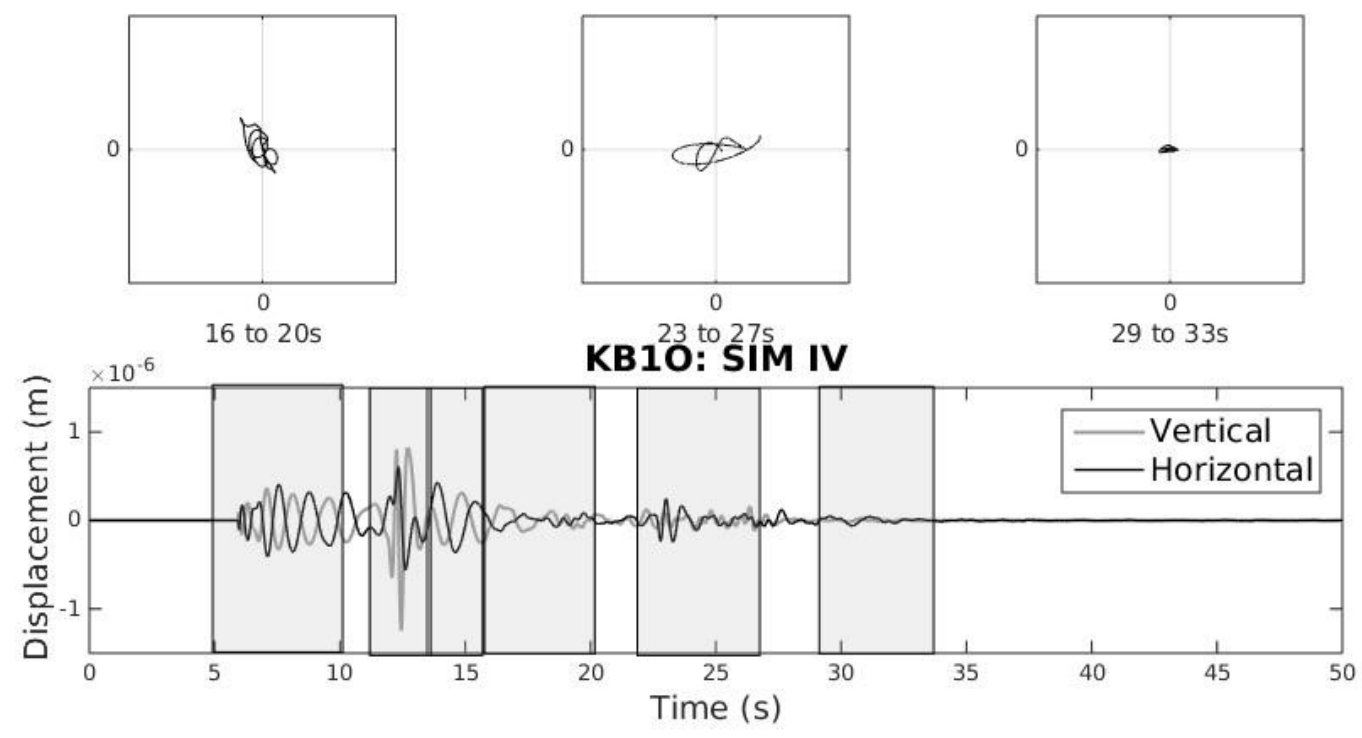

Figure 6.51: Particle motion plots (top) at KB1O with the time slices outlined in the displacement time series (below). 


\section{KB2O Particle Motion}
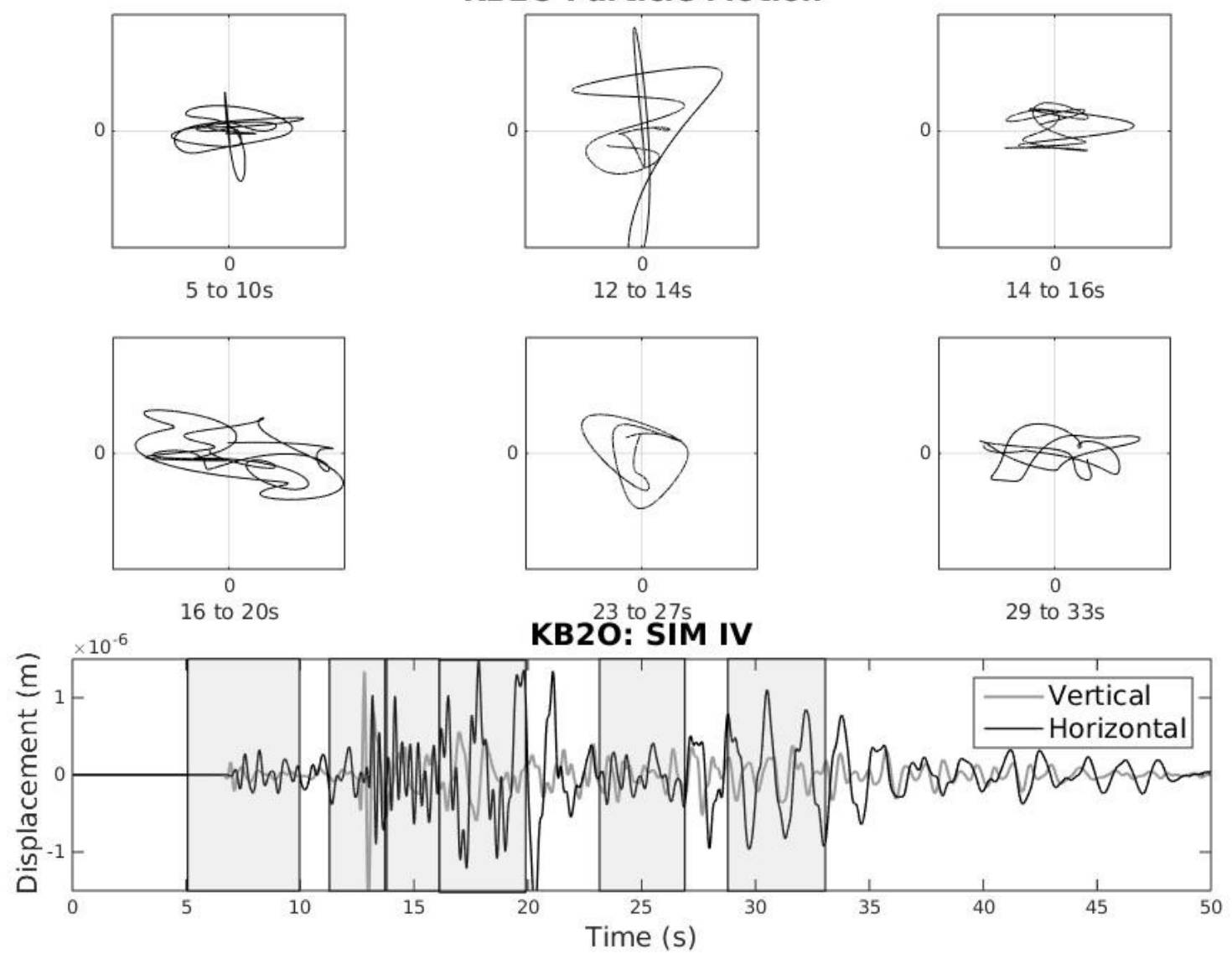

Figure 6.52: Particle motion plots (top) at KB2O with the time slices outlined in the displacement time series (below). 


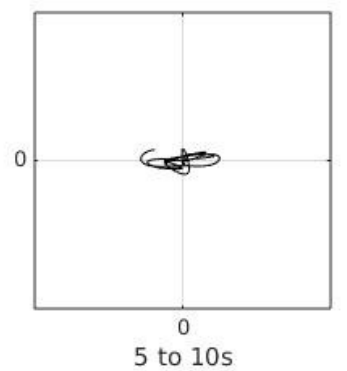

\section{KB30 Particle Motion}
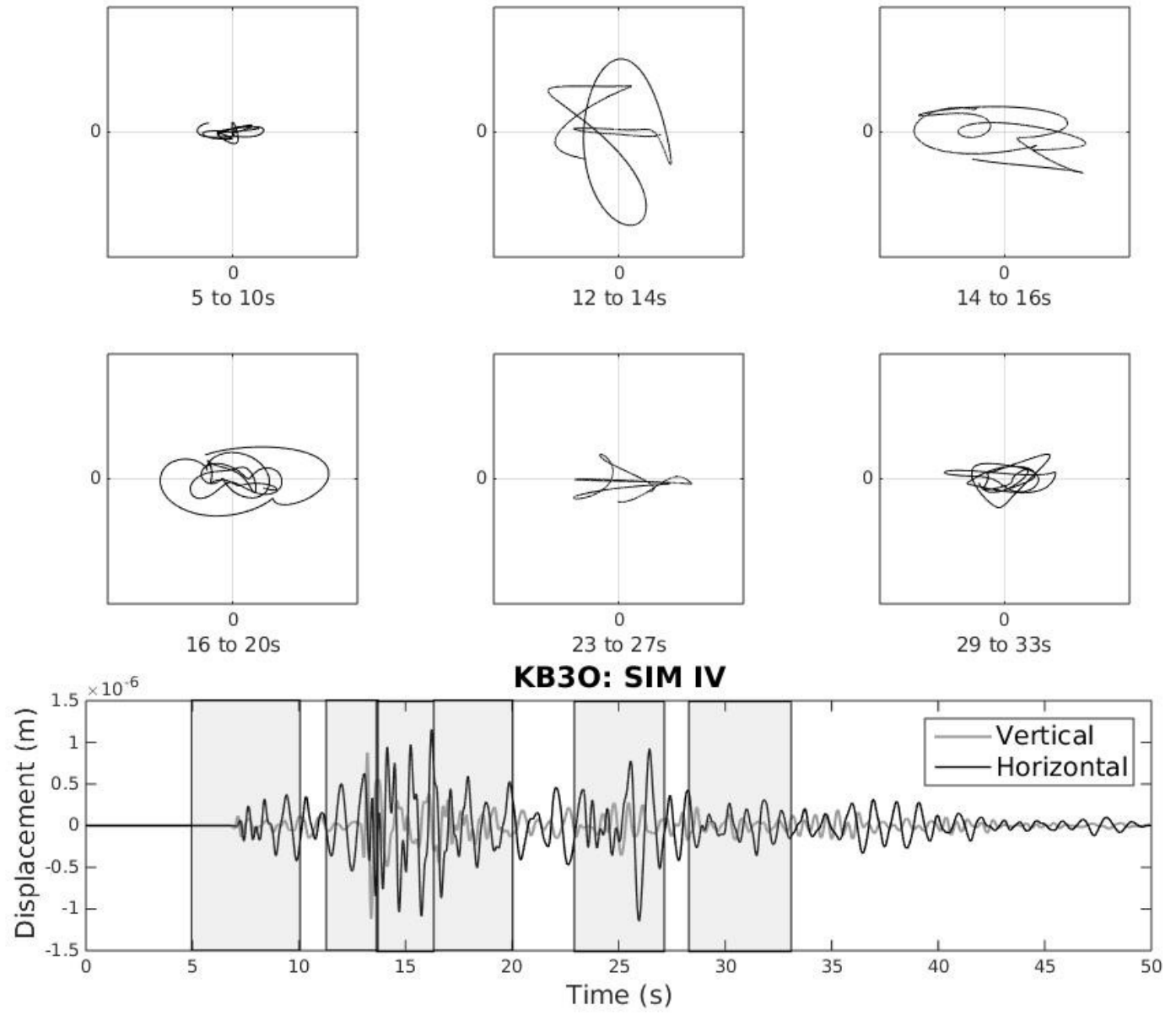

Figure 6.53: Particle motion plots (top) at KB3O with the time slices outlined in the displacement time series (below). 


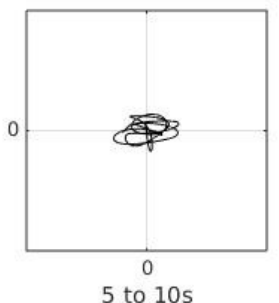

\section{KB60 Particle Motion}
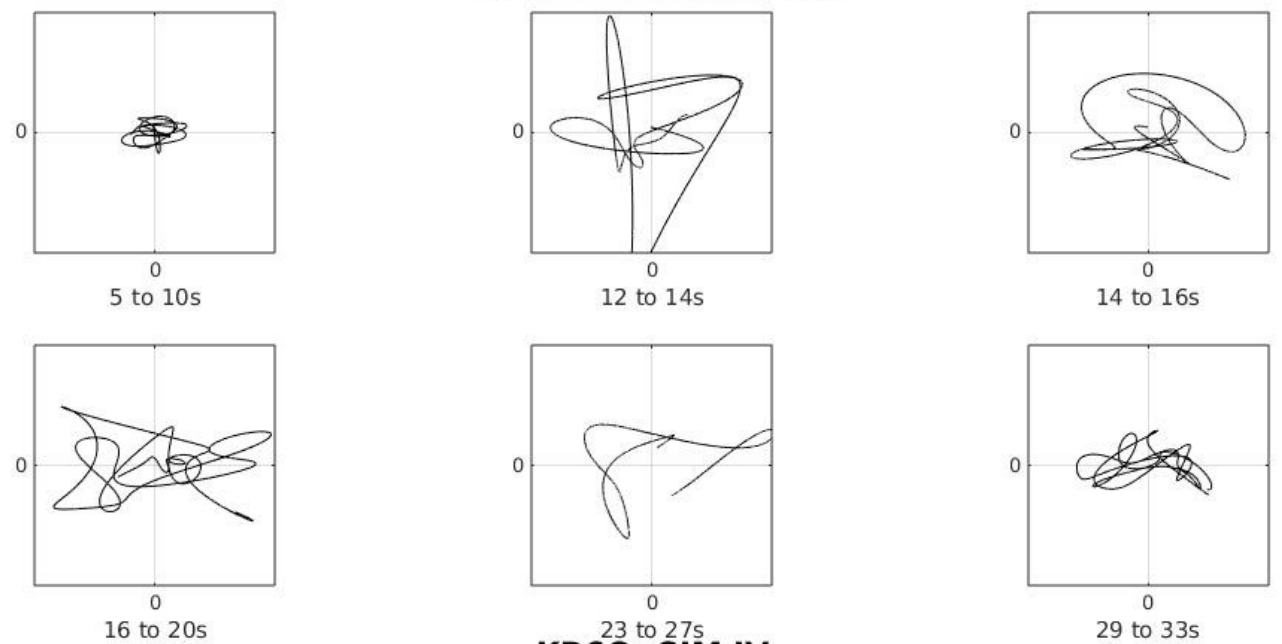

KB6O: 23 to 27 IM IV
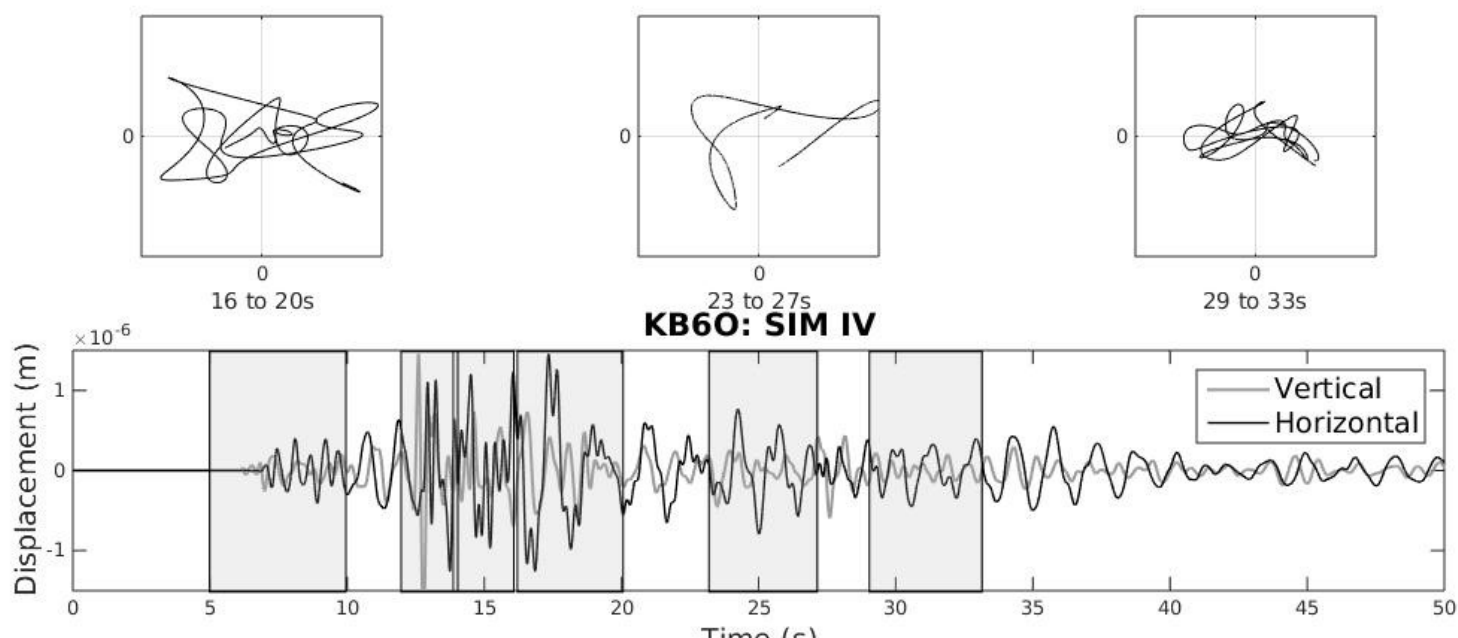

Figure 6.54: Particle motion plots (top) at KB6O with the time slices outlined in the displacement time series (below). 


\subsection{References}

Bent, A.L., M. Lamontagne, V. Peci, S. Halchuk, G.R. Brooks, D. Motazedian, J.A. Hunter, J. Adams, C. Woodgold, J. Drysdale, S. Hayek, W. N. Edwards (2015). The 17 May 2013 M 4.6 Ladysmith, Quebec, Earthquake, Seismol. Res. Lett., 86, 460-476. doi: 10.1785/0220140138

Dobry, R., I. Oweis, and A. Urzua (1976). Simplified procedures for estimating the fundamental period of a soil profile. Bull. Seism. Soc. Am., 66, 1293-1321.

Dziewonski, A. M., T.-A. Chou and J. H. Woodhouse (1981). Determination of earthquake source parameters from waveform data for studies of global and regional seismicity, J. Geophys. Res., 86, 2825-2852. doi:10.1029/JB086iB04p02825

Ekström, G., M. Nettles, and A. M. Dziewonski (2012) The global CMT project 2004-2010: Centroid-moment tensors for 13,017 earthquakes, Phys. Earth Planet. Inter., 200-201, 1-9. doi:10.1016/j.pepi.2012.04.002

Hayek, S. (2016). Seismic basin effects over soft-sediment filled basins in Ottawa, Canada. M.Sc. Thesis, Department of Earth Sciences, Carleton University.

Hunter, J.A., H.L. Crow, G.R. Brooks, M. Pyne, D. Motazedian, M. Lamontagne, A.J.-M. Pugin, S.E. Pullan, T. Cartwright, M. Douma, R.A. Burns, R. L. Good, K. Kaheshi-Banab, R. Caron, M. Kolaj, I. Folahan, L. Dixon, K. Dion, A. Duxbury, A. Landriault, V. Ter-Emmanuil, A. Jones, G. Plastow, D. Muir (2010). Seismic site classification and site period mapping in the Ottawa area using geophysical methods. Geological Survey of Canada. Open File 6273.

Konno, K., and T. Ohmachi (1998). Ground motion characteristics estimated from spectral ratio between horizontal and vertical components of microtremor. Bull. Seism. Soc. Am., 88, 228241.

Motazedian, D., J.A. Hunter, H. Torabi, S. Crane, S. Hayek, and H.L. Crow (2015). Investigation of soil amplification factors for the Ottawa area. Part I - Fa, in proceedings, 11 Canadian Conference on Earthquake Engineering, Victoria, BC, July 2015. 


\section{Discussion}

A wide variety of different research projects have attempted to measure the effect of soft soil basins on ground motions from earthquakes. These studies range from: a wide selection of numerical simulations that isolate the effect of basin features; numerical simulations of known basins to correlate with recorded ground motions; and, comparisons between recorded ground motions within a basin to ground motions recorded near the basin. Although the results of these studies do vary, and there is some disagreement between responses from 1-D effects versus basin effects, there are some dominant consistencies throughout this area of research. Mostly it is observed that there is often a resonating frequency at the receiver and a spectral shift of the strong frequencies.

Many studies use different numerical methods to try and isolate the effects of different parameters on basin ground motion: Bard and Bouchon (1980a, 1980b, 1985) used several numerical 2D techniques to isolate the incident wave type on the basin, and provided a framework for a resonance in basins. Kawase and Aki (1989) used two types of soft basin models for incident waves to reference the motions seen in Mexico City in 1985. Narayan $(2010,2012)$ used a staggered-grid finite difference method to determine the effects of impedance contrast and soil thickness on waves within a basin. Kamal and Narayan (2014) extended this work to include the effects of basin-shape ratio.

The above abundant research provides a clear example of the probable results with the application of numerical techniques to simulate basin effects. The work completed in this thesis does correlate well with the results given by these previous authors. When examining the 
response of the basin incident waves we see similar resonances to those found in Bard and Bouchon (1980a, 1980b, 1985) and the extended duration found by Kawase and Aki (1989). Although outside the limits used in the studies by Narayan $(2010,2012)$ and Kamal and Narayan (2014), the impedance contrast and basin shape have similar effects on the ground motion. Specifically, there is an increased amplitude around a dominant frequency, however there were also a few basin generated surface waves that were traced along the surface receivers of the basin at later times.

Studies which have used numerical techniques to simulate ground motions within basins are extensive and have had varying degrees of success: Numerical simulations in California were able to reproduce the amplification and extended durations seen in long period surface waves from the Los Angeles Basin (Olsen 2000), the Santa Clara Valley (Frankel and Vidale 1992) and the San Bernardino Valley (Frankel 1993). Similar effects have been simulated in other areas of North America including the Salt Lake basin in Utah (Olsen et al., 1995), the Seattle basin in Washington (Frankel et al., 2009), and the Georgia basin in British Columbia (Molnar et al., 2014a; Molnar et al., 2014b).

Other basins with comparable characteristics to those in this present study have been modeled and numerical simulations have produced accurate results, such as those by Chaljub et al., (2010) for the Grenoble valley in France and Maufroy et al. (2015) for the Mygdonian basin in Greece. These particular studies were accurate to higher frequencies compared to others and also model softer infill basin material. The Mygdonian basin study (Maufroy et al., 2015) included an extensive test for numerical simulations up to $4 \mathrm{~Hz}$ with contributions by several research 
teams. The basin was modelled to contain three layers of different thickness of sediment, with the shear wave velocities ranging from 200 to $650 \mathrm{~m} / \mathrm{s}$. The simulations from the different numerical methods were compared to one another using several baseline earthquake recordings

to determine their effectiveness. Two of the teams have used an identical numerical method as that which was utilized in this present research project resulting in similar results, which compared well with the other methods used in the Mygdonian basin study (Maufroy et al., 2015).

\subsection{Limitations}

A full scale 3D simulation of the Ladysmith earthquake source and Kinburn basin would have required a great deal of computing resources. In this study the limiting factor was the ability to create a realistic model which could produce a mesh suitable for the numerical simulations. As the spectral element method uses an unstructured hexahedral mesh, the mesh between the rock and soil interface must be consistent with one another. This dictated the maximum velocity to minimum mesh point distance to be very large, requiring a very small time step for a numerical simulation. This in turn required an extremely large amount of computing resources in order to complete each individual simulation.

The models used in the simulations by necessity had to be simplified from their known true form. The sediment within the basin was taken as a single substance with the same properties from the surface down to the basin interface, even though there were measured velocity profiles in the basin indicating some soil variance. This was also the case for most of the rock within the model, as it was taken to have an average value for each parameter and was taken to be consistent across the model. Despite these simplifications the results produced from 
the simulations corresponded well within the frequency range of interest. The rock station provided realistic ground motions and the basin produced motions which were consistent with previous studies.

\subsection{Conclusion}

This research project was successfully completed in an attempt to quantify some of the basin effects recorded in the Kinburn Basin through the use of 2D and 3D numerical simulations. From the recordings at seismic stations KB1O (the rock outcrop station) and KB2O (the thick soil stations) along with extensive modelling of the structure below the soil station, it is clear that there was a large portion of amplified ground motions which cannot be explained only using onedimensional rock to soil techniques (Hayek, 2016). The 2D and 3D simulations of this thesis were able to reproduce some of the ground motions recorded at KB2O that were not present at KB1O, even with a simplified model of the Kinburn Basin. Longer duration of strong motions was successfully simulated in the different models, and the dominant frequency of the station KB2O was best matched using a shear wave velocity of $250 \mathrm{~m} / \mathrm{s}$ for the soil within the basin. Although the measured average shear wave velocity of the Leda Clay is slightly lower than this value, using this value the dominant frequency is similar to the dominant frequency estimated using the process from Dobry et al. (1976). The spectral accelerations observed from earthquakes measured within the basin were very close matches to these simulations within the range of approximately $0.3 \mathrm{~Hz}$ to 1.5 or $3 \mathrm{~Hz}$ (depending on the simulation). This frequency range contained the several frequency peaks observed within the soil station recordings. Using the acceleration time series, we observed that the amplitude of the seismic waves were well matched to the first arrivals; however, there are several stronger phases that arrived later in the recordings which 
were not present in the simulations. These arrivals were observed at both the rock station and soil station earthquake recordings, suggesting that they are likely caused by processes outside the scope of this study.

The simulations of ground motions within the Kinburn Basin were completed using two different earthquake source locations in order to see if there were any effects within the soil basin dependent on the incoming waves. The first source, the global centroid moment tensor solution (CMT), had a lower dominant frequency but a larger seismic moment. The second source, a moment tensor from Bent et al. (2015), had a higher dominant frequency but a lower seismic moment. Both sources showed similar peak frequencies and amplifications within the basin material, proving these effects are not dependant on the original source or frequency content of the arriving wave packets.

Using a line of receivers within the simulations it was possible to trace some of the waves across the basin. In particular it was possible to determine the effect of the shape of the basin across the surface, and also to locate the position where reflected basin waves were produced. These traces were able to provide strong evidence of the different basin effects which occur within the Kinburn basin. Although a full scale 3D simulation was not possible for a complete detailed model of the Kinburn basin extending to the source of the Ladysmith earthquake, the 2D models provided a good description of the in-line array both for the source horizontal component and the vertical component of motion. 


\subsubsection{Significant Results}

The research completed during the course of this project was extensive and covered a wide variety of results. The significant contributions are summarized as: an accurate geometrical model of the subsurface topography of the Kinburn Basin; the installation of the Kinburn Seismic array; validity of 2D modelling of ground motions within a soft-sediment basin; comparisons of simulated ground motions from a basin and from different types of layering; matching of spectral acceleration amplifications from a rock to soil recording using 2D simulations; matching of filtered time series amplitudes from rock and soil recordings using 2D simulations; providing evidence of basin generated surface waves within the Kinburn Basin; and providing evidence of subsurface structure dependency on surface ground motions.

\subsubsection{Implications on Seismic Hazard}

The results of this research project indicate that the National Building Code of Canada (NBCC) may not be suitable for providing the proper design seismic hazard for sites located within a soft sediment basin. The NBCC uses the spectral acceleration values at the periods $0.2,0.5,1.0,2.0$, 5.0, and 10.0 seconds at different localities for a reference site class of $C$. These values are then adjusted based on the site class at a specific location based on the $\mathrm{Vs}_{30}$ value of the site.

For soft soil basins in eastern Canada, the deeper portions of the basin would be classified as a site class $\mathrm{E}$ (or $\mathrm{F}$ depending on the sediment properties) based on a low value of $\mathrm{Vs}_{30}$ which would measure only a small portion of the top layer of sediment. The design spectral acceleration values would then be adjusted according to factors, $F(T)$, which are dependent on the period and amplitude of the spectral acceleration and correlating to the site class E conditions. 
The fundamental site period is dependent on the depth to bedrock and the velocity of the top layers of material, which may not be near one of the spectral periods used for the NBCC. The amplification at and around this frequency is not confined to a narrow frequency band as seen in the recorded motions within the Kinburn Basin. The amplifications of the spectral accelerations and the extended duration of strong ground motions is not considered by the NBCC for a basin subsurface structure, even though it has been shown these are significant effects within one. Although this research focused on one basin, these effects have been measured in other basins (Hayek, 2016). Therefore more research should be completed on these basins, and the effects they generate on ground motions. It may become necessary to adapt the design seismic hazard within a basin to account for these effects, provided further research can provide a suitable method of quantifying the spectral amplifications and a reasonable method of estimating seismic hazard within a basin can be completed without the need for extensive research on each basin.

\subsection{References}

Bard, P.-Y. and M. Bouchon, (1980a). The seismic response of sediment-filled valleys. Part I. The case of incident SH waves, Bull Seismol Soc Am, 70, 1263-1286.

Bard, P.-Y. and M. Bouchon, (1980b). The seismic response of sediment-filled valleys. Part II. The case of incident $P$ and SV waves, Bull Seismol Soc Am, 70, 1921-1941.

Bard, P.-Y. and M. Bouchon, (1985). The two-dimensional resonance of sediment-filled valleys, Bull Seismol Soc Am, 75, 519-541.

Bent, A.L., M. Lamontagne, V. Peci, S. Halchuk, G.R. Brooks, D. Motazedian, J.A. Hunter, J. Adams, C. Woodgold, J. Drysdale, S. Hayek, W. N. Edwards (2015). The 17 May 2013 M 4.6 Ladysmith, Quebec, Earthquake, Seismol. Res. Lett., 86, 460-476. doi: 10.1785/0220140138

Chaljub, E., P. Moczo, S. Tsuno, P.-Y. Bard, J. Kristek, M. Käser, M. Stupazzini, and M. Kristeková (2010). Quantitative comparison of four numerical predictions of 3D ground motion in the Grenoble valley, France. Bull. Seismol. Soc. Am., 100, 1427-1455. 
Dobry, R., I. Oweis, and A. Urzua (1976). Simplified procedures for estimating the fundamental period of a soil profile. Bull. Seism. Soc. Am., 66, 1293-1321.

Frankel, A. (1993). Three-dimensional simulations of ground motions in the San Bernardino valley, California, for hypothetical earthquakes on the San Andreas fault, Bull. Seis. Soc. Am., 83, 1020-1041.

Frankel, A., and J. Vidale (1992). A three-dimensional simulation of seismic waves in the Santa Clara valley, California, from a Loma Prieta aftershock, Bull. Seis. Soc. Am., 82, 2045-2074.

Frankel, A., W.J. Stephenson, and D. Carver, (2009). Sedimentary basin effects in Seattle, Washington: Ground-motion observations and 3D simulations, Bull. Seis. Soc. Am., 99, 15791611.

Hayek, S. (2016). Seismic basin effects over soft-sediment filled basins in Ottawa, Canada. M.Sc. Thesis, Department of Earth Sciences, Carleton University.

Kamal, and J.P. Narayan (2015). 3D basin-shape ratio effects on frequency content and spectral amplitudes of basin-generated surface waves and associated spatial ground motion amplification and differential ground motion. J. Seismol, 19, 293-316.

Kawase, H., and K. Aki (1989). A study on the response of a soft basin for incident S, P, and Rayleigh waves with special reference to the long duration observed in Mexico City, Bull. Seis. Soc. Am., 79, 1361-1382.

Maufroy, E., E. Chaljub, F. Hollender, J. Kristek, P. Moczo, P. Klin, E. Priolo, A. Iwaki, T. Iwata, V. Etienne, F. De Martin, N.P. Theodoulidis, M. Manakou, C. Guyonnet-Benaize, K. Pitilakis, and P.-Y. Bard (2015). Earthquake ground motion in the Mygdonian basin, Greece: The E2VP verification and validation of 3D numerical simulation up to $4 \mathrm{~Hz}$. Bull. Seis. Soc. Am., 105, 1398-1418.

Molnar, S., J.F. Cassidy, K.B. Olsen, S.E. Dosso, and J. He (2014a). Earthquake ground motion and 3D Georgia Basin amplification in Southwestern British Colombia: Deep Juan De Fuca Plate scenario earthquakes, Bull. Seis. Soc. Am., 104, 301-320.

Molnar, S., J.F. Cassidy, K.B. Olsen, S.E. Dosso, and J. He (2014b). Earthquake ground motion and 3D Georgia Basin amplification in Southwestern British Colombia: Shallow Blind-thrust scenario earthquakes, Bull. Seis. Soc. Am., 104, 321-335.

Narayan, J.P. (2010). Effects of impedance contrast and soil thickness on basin-transduced Rayleigh waves and associated differential ground motion. Pure Appl. Geophys., 167, 14851510. 
Narayan, J.P. (2011). Effects of P-wave and S-wave impedance contrast on the characteristics of basin transduced Rayleigh waves. Pure Appl. Geophys., 169, 693-709.

Olsen, K.B., (2000). Site amplification in the Los Angeles basin from three dimensional modelling of ground motion, Bull. Seis. Soc. Am., 90, S77-S94.

Olsen, K.B., J.C. Pechmann, and G.T. Schuster, (1995). Simulation of 3D elastic wave propagation in the Salt Lake basin, Bull. Seis. Soc. Am., 85, 1688-1710. 


\section{Appendix I. Spectral Element Method}

\section{I.A. Constructing the Weak form of the momentum equation}

The strong form, or derivative form of the equations of motion is governed by the momentum equation given by:

$$
\rho \ddot{\boldsymbol{s}}=\boldsymbol{\nabla} \cdot \mathbf{T}+\mathbf{f}
$$

where $\rho$ is the density distribution, $\boldsymbol{S}$ is the displacement vector with a dot denoting differentiation with respect to time, $\mathbf{T}$ is the stress tensor, which is linearly related to the displacement vector in an elastic, anisotropic medium by:

$$
\mathbf{T}=\boldsymbol{c}: \boldsymbol{\nabla} \boldsymbol{s}
$$

where $\mathbf{c}$ is the fourth-order elastic tensor. It is related to the entire strain history in an attenuating medium:

$$
\mathbf{T}(t)=\int_{-\infty}^{\infty} \dot{\boldsymbol{c}}\left(t-t^{\prime}\right): \nabla \boldsymbol{s}^{\prime} d t^{\prime}
$$

The earthquake source is produced as a point force $f$, written in terms of a moment tensor $\mathbf{M}$ and point location $\mathbf{x}$ :

$$
\mathbf{f}=-\mathbf{M} \cdot \boldsymbol{\nabla} \delta\left(\mathbf{x}-\mathbf{x}_{\mathbf{s}}\right) S(t)
$$

where $\delta$ is the Dirca delta distribution and $S(t)$ is the source time function. The boundary condition at the Earth's surface must satisfy a stress-free momentum equation.

$$
\mathbf{T} \cdot \hat{\mathbf{n}}=0
$$

where $\hat{\mathbf{n}}$ is the unit outward normal vector associated with the surface boundary. At every internal boundary the traction $\mathrm{T} \cdot \hat{\mathbf{n}}$ and displacement vector $s$ both need to be continuous. At 
the model boundary, denoted as $\Gamma$, the waves travelling out of the volume, $\Omega$, must be absorbed in order to reduce reflections off the model boundaries. Although this is not easy to implement efficiently (Clayton \& Engquist 1977; Quarteroni et al., 1998; Komatitsch et al., 1999) the approximate boundary condition used is:

$$
\mathbf{T} \cdot \hat{\mathbf{n}}=\rho\left[v_{n}(\hat{\boldsymbol{n}} \cdot \dot{\mathbf{s}}) \widehat{\mathbf{n}}+v_{1}\left(\hat{\mathbf{t}}_{\mathbf{1}} \cdot \dot{\mathbf{s}}\right) \hat{\mathbf{t}}_{\mathbf{1}}+v_{2}\left(\hat{\mathbf{t}}_{\mathbf{2}} \cdot \dot{\mathbf{s}}\right) \hat{\mathbf{t}}_{\mathbf{2}}\right]
$$

Eq. 7.6

where $\hat{\mathbf{t}}_{\mathbf{1}}$ and $\hat{\mathbf{t}}_{\mathbf{2}}$ are the orthogonal unit vectors tangential to the boundary $\Gamma, v_{n}$ is the P-wave velocity of waves travelling in the $\widehat{\mathbf{n}}$ direction, $v_{1}$ is the S-wave velocity of waves oriented in the $\hat{\mathbf{t}}_{\mathbf{1}}$ direction, and $V_{2}$ is the S-wave velocity of the waves oriented in the $\hat{\mathbf{t}}_{\mathbf{2}}$ direction.

The weak form the equations of motion is an integrated form of the momentum equation and approximated boundary conditions. This is achieved by dotting the momentum equation with a test vector $\boldsymbol{w}$ and integrating by parts over the volume $\Omega$. Imposing the stress-free boundary condition and the absorbing boundary conditions based upon traction, the resulting equation, which is called weak form the equations of motion, is:

$$
\begin{aligned}
\int_{\Omega} \rho \boldsymbol{w} \cdot \ddot{\boldsymbol{s}} d^{3} \boldsymbol{x} & =-\int_{\Omega} \boldsymbol{\nabla} \boldsymbol{w}: \mathbf{T} d^{3} \boldsymbol{x}+\boldsymbol{M}: \boldsymbol{\nabla} \boldsymbol{w}\left(\boldsymbol{x}_{\boldsymbol{s}}\right) S(t) \\
& +\int_{\Gamma} \rho\left[v_{n}(\widehat{\boldsymbol{n}} \cdot \dot{\mathbf{s}}) \widehat{\mathbf{n}}+v_{1}\left(\hat{\mathbf{t}}_{\mathbf{1}} \cdot \dot{\mathbf{s}}\right) \hat{\mathbf{t}}_{\mathbf{1}}+v_{2}\left(\hat{\mathbf{t}}_{\mathbf{2}} \cdot \dot{\mathbf{s}}\right) \hat{\mathbf{t}}_{\mathbf{2}}\right] \cdot \mathbf{w} \mathrm{d}^{2} \mathbf{x}
\end{aligned}
$$

\section{I.B. Mesh Construction and Elemental Representation}

The model is subdivided into a mesh of hexahedral elements such that the entire volume is encompassed by the sum of the volume of each mesh element. Similarly, the entire boundary of the volume is composed of the sum of individual boundary mesh elements. A three-dimensional volume element is mapped into a local reference cube with the reference vector $\xi(\xi, \eta, \zeta)$ where 
$-1 \leq \xi \leq 1,-1 \leq \eta \leq 1$, and $-1 \leq \zeta \leq 1$. Each volume element is defined in terms of eight anchors $\mathbf{x}_{a}=$ $\mathbf{x}\left(\xi_{a}, \eta_{a}, \zeta_{a}\right), a=1, \ldots, 8$ and eight shape function $\mathrm{N}_{\mathrm{a}}(\xi)$. The mapping between the hexahedral mesh element and the reference cube is:

$$
\boldsymbol{x}(\xi)=\sum_{a=1}^{8} N_{a}(\xi) \boldsymbol{x}_{a}
$$

The shape functions are triple products of degree 1 Lagrange polynomials.

Any volume $d x d y d z$ within a given volume element can be related to a volume $d \xi d \eta d \zeta$ in the reference cube by:

$$
d x d y d z=J_{e} d \xi d \eta d \zeta
$$

where $J_{e}$ is the volumetric Jacobian given by:

$$
J_{e}=\left|\frac{\partial(x, y, z)}{\partial(\xi, \eta, \zeta)}\right|
$$

The hexahedral mesh elements need to be constructed in such a way that the Jacobian never vanishes, providing a strong constraint on the construction of the mesh. This is to ensure that mapping from the reference cube to the element is unique and invertible.

In order to solve the weak form of the equation of motion, the entire volume and boundaries are subdivided into elements, which are then solved with an integration over the element. The shape functions have been defined as low-degree Lagrange polynomials, however the functions on the elements are expressed as a higher-order Lagrange polynomial of order $n$. The control points $\xi_{\alpha}$, where $\alpha=1, \ldots, n_{l}$ for the Lagrange polynomial of order $n_{l}$, is taken as the $n_{l}$ +1 Gauss-Lobatto-Legendre points. These are the roots of: 


$$
\left(1-\xi^{2}\right) P_{n l}^{\prime}(\xi)=0
$$

where $P_{n l}^{\prime}$ is the derivative of the Legendre polynomial of degree $n_{l}$. These points are computed by numerical resolution of the above equation (Canuto et al. 1988, pg. 61). A function $f$, for each element volume is interpolated with triple products of Lagrange polynomials of degree $n_{l}$ as:

$$
f(\boldsymbol{x}(\xi, \eta, \zeta)) \approx \sum_{\alpha, \beta, \gamma=0}^{n_{l}} f^{\alpha \beta \gamma} l_{\alpha}(\xi) l_{\beta}(\eta) l_{\gamma}(\zeta)
$$

where

$$
f^{\alpha \beta \gamma}=f\left(\boldsymbol{x}\left(\xi_{\alpha}, \eta_{\beta}, \zeta_{\gamma}\right)\right.
$$

Integration of this function over an elemental volume can be approximated using the GaussLobatto-Legendre integration rule:

$$
\begin{aligned}
\int_{\Omega_{e}} f(\boldsymbol{x}) d^{3} \boldsymbol{x} & =\int_{-1}^{1} \int_{-1}^{1} \int_{-1}^{1} f(\boldsymbol{x}(\xi, \eta, \zeta)) J_{e}(\xi, \eta, \zeta) d \xi d \eta d \zeta \\
& \approx \sum_{\alpha, \beta, \gamma=0}^{n_{l}} \omega_{\alpha} \omega_{\beta} \omega_{\gamma} f^{\alpha \beta \gamma} J_{e}^{\alpha \beta \gamma}
\end{aligned}
$$

where $J_{e}^{\alpha \beta \gamma}=J_{e}\left(\xi_{\alpha}, \eta_{\beta}, \zeta_{\gamma}\right)$, and $\omega_{\alpha}, \alpha=1, \ldots, n_{I}$ is the Gauss-Lobatto-Legendre weights of integration computed numerically (Canuto et al. 1988, pg. 61).

\section{I.C. The Global System}

The global system incorporates the entire volume and is composed of the complete mesh of hexahedral elements. The second order differential equation governing the time dependence of the global system is defined as: 


$$
M \ddot{U}+C \dot{U}+K U=F
$$

where $U$ is the displacement vector of the global system, $M$ is the global mass matrix, $C$ is the global absorbing boundary matrix, $K$ is the global stiffness matrix, and $F$ is the source term. The displacement vector of the global system contains all the displacement vectors at each grid point of the entire mesh and is referred to as the global degrees of freedom of the system (Komatitsch and Tromp 1999).

The global stiffness matrix is formed through the first term of the weak form of the equation of motion and at the elemental level it is given as:

$$
\int_{\Omega_{\mathrm{e}}} \rho \boldsymbol{w} \cdot \ddot{\boldsymbol{s}} d^{3} \boldsymbol{x}=\int_{-1}^{1} \int_{-1}^{1} \int_{-1}^{1} \rho(x(\xi)) w(x(\xi)) \cdot \ddot{\mathbf{s}}(x, t) J(\xi) d^{3} \xi \quad \text { Eq. } 7.16
$$

The displacement vector $\boldsymbol{s}$ is expanded on an element as:

$$
\boldsymbol{s}(\boldsymbol{x}(\xi, \eta, \zeta)) \approx \sum_{j=1}^{3} \widehat{\boldsymbol{x}}_{j} \sum_{\sigma, \tau, v}^{n l} s_{j}^{\sigma \tau v} l_{\sigma}(\xi) l_{\tau}(\eta) l_{v}(\zeta)
$$

and similarly a test vector $\mathrm{w}$ is expressed as:

$$
\boldsymbol{w}(\boldsymbol{x}(\xi, \eta, \zeta))=\sum_{i=1}^{3} \widehat{\boldsymbol{x}}_{i} \sum_{\alpha, \beta, \gamma}^{n l} w_{i}^{\alpha \beta \gamma} l_{\alpha}(\xi) l_{\beta}(\eta) l_{\gamma}(\zeta)
$$

Using the displacement and test vector, and with the Gauss-Lobatto-Legendre integration rule we get the elemental mass matrix: 


$$
\begin{aligned}
& \int_{\Omega_{\mathrm{e}}} \rho \boldsymbol{w} \cdot \ddot{\boldsymbol{s}} d^{3} \boldsymbol{x} \\
& \approx \sum_{\alpha^{\prime}, \beta^{\prime}, \gamma^{\prime}} \omega_{\alpha^{\prime}} \omega_{\beta^{\prime}} \omega_{\gamma^{\prime}} J_{e}^{\alpha^{\prime} \beta^{\prime} \gamma^{\prime}} \rho^{\alpha^{\prime} \beta^{\prime} \gamma^{\prime}} \sum_{i, j=1}^{3} \widehat{\boldsymbol{x}}_{i} \cdot \widehat{\boldsymbol{x}}_{j} \\
& \times \sum_{\alpha, \beta, \gamma} w_{i}^{\alpha \beta \gamma} l_{\alpha}\left(\xi_{\alpha^{\prime}}\right) l_{\beta}\left(\eta_{\beta^{\prime}}\right) l_{\gamma}\left(\zeta_{\gamma^{\prime}}\right) \sum_{\sigma, \tau, v} s_{j}^{\sigma \tau v} l_{\sigma}\left(\xi_{\alpha^{\prime}}\right) l_{\tau}\left(\eta_{\beta^{\prime}}\right) l_{v}\left(\zeta_{\gamma^{\prime}}\right) \\
& =\sum_{\alpha, \beta, \gamma} \omega_{\alpha} \omega_{\beta} \omega_{\gamma} J_{e}^{\alpha \beta \gamma} \rho^{\alpha \beta \gamma} \sum_{i=1}^{3} w_{i}^{\alpha \beta \gamma} \ddot{s}_{i}^{\alpha \beta \gamma}(t)
\end{aligned}
$$

where $\rho^{\alpha \beta \gamma}=\rho\left(x\left(\xi_{\alpha}, \eta_{\beta}, \zeta_{\gamma}\right)\right)$. The weak form of the equation of motion holds for any test vector $\boldsymbol{w}$, so the global system is built by independently setting the factors $w_{1}^{\alpha \beta \gamma}, w_{2}^{\alpha \beta \gamma}$, and $w_{3}^{\alpha \beta \gamma}$ to zero, creating a diagonal mass matrix.

The elemental stiffness matrix is determined from the integral:

$$
\int_{\Omega_{e}} \nabla w: \mathbf{T} d^{3} x
$$

where the stress tensor on an element is:

$$
\mathbf{T}\left(\boldsymbol{x}\left(\xi_{\alpha}, \eta_{\beta}, \zeta_{\gamma}\right), t\right)=\boldsymbol{c}\left(\boldsymbol{x}\left(\xi_{\alpha}, \eta_{\beta}, \zeta_{\gamma}\right)\right): \boldsymbol{\nabla} \boldsymbol{s}\left(\boldsymbol{x}\left(\xi_{\alpha}, \eta_{\beta}, \zeta_{\gamma}\right), t\right)
$$

The integrand is written as:

$$
\boldsymbol{\nabla} \boldsymbol{w}: \mathbf{T}=\sum_{i, j=1}^{3} T_{i j} \partial_{j} \omega_{i}=\sum_{i, k=1}^{3}\left(\sum_{j=1}^{3} T_{i j} \partial_{j} \xi_{k}\right) \frac{\partial w_{i}}{\partial \xi_{k}}
$$

and the integral becomes: 


$$
\sum_{i, k=1}^{3} \int_{\Omega_{e}} F_{i k} \frac{\partial w_{i}}{\partial \xi_{k}} d^{3} \boldsymbol{x}=\sum_{i, k=1}^{3} \int_{-1}^{1} \int_{-1}^{1} \int_{-1}^{1} F_{i k} \frac{\partial w_{i}}{\partial \xi_{k}} J_{e} \partial \xi \partial \eta \partial \zeta
$$

where:

$$
F_{i k}=\sum_{j=1}^{3} T_{i j} \partial_{j} \xi_{k}
$$

The nine matrix elements of $F_{i k}$ are evaluated on the Guass-Lobatto-Legendre integration points: $F_{i k}^{\sigma \tau v}=F_{i k}\left(\boldsymbol{x}\left(\xi_{\sigma}, \eta_{\tau}, \zeta_{v}\right)\right)$. Substituting the test vector from before and using the Gauss-LobattoLegendre integration rule gives the elemental stiffness matrix:

$$
\begin{aligned}
\int_{\Omega_{e}} \boldsymbol{\nabla} \boldsymbol{w}: \mathbf{T} d^{3} \boldsymbol{x} & \approx \sum_{i=1}^{3} w_{i}^{\alpha \beta \gamma}\left[\omega_{\beta} \omega_{\gamma} \sum_{\alpha^{\prime}=0}^{n l} \omega_{\alpha^{\prime}} J_{e}^{\alpha^{\prime} \beta \gamma} F_{i 1}^{\alpha^{\prime} \beta \gamma} l_{\alpha}^{\prime}\left(\xi_{\alpha^{\prime}}\right)\right. \\
& +\omega_{\alpha} \omega_{\gamma} \sum_{\beta^{\prime}=0}^{n l} \omega_{\beta^{\prime}} J_{e}^{\alpha \beta^{\prime} \gamma} F_{i 2}^{\alpha \beta^{\prime} \gamma} l_{\beta}^{\prime}\left(\eta_{\beta^{\prime}}\right) \\
& \left.+\omega_{\alpha} \omega_{\beta} \sum_{\gamma^{\prime}=0}^{n l} \omega_{\gamma^{\prime}} J_{e}^{\alpha \beta \gamma^{\prime}} F_{i 3}^{\alpha \beta \gamma^{\prime}} l_{\gamma}^{\prime}\left(\zeta_{\gamma^{\prime}}\right)\right]
\end{aligned}
$$

The source term $\mathbf{M}: \boldsymbol{\nabla} \boldsymbol{w}$ is written as:

$$
\mathbf{M}: \boldsymbol{\nabla} \boldsymbol{w}=\sum_{i, j=1}^{3} M_{i j} \partial_{j} \omega_{i}=\sum_{i, k=1}^{3}\left(\sum_{j=1}^{3} M_{i j} \partial_{j} \xi_{k}\right) \frac{\partial w_{i}}{\partial \xi_{k}}
$$

Defining:

$$
G_{i k}=\sum_{j=1}^{3} M_{i j} \partial_{j} \xi_{k}
$$

and $G_{i k}^{\sigma \tau v}=G_{i k}\left(\boldsymbol{x}\left(\xi_{\sigma}, \eta_{\tau}, \zeta_{v}\right)\right)$, and using the test vector from above, give the source term: 


$$
\begin{aligned}
\mathbf{M}: \boldsymbol{\nabla} \boldsymbol{w}\left(\boldsymbol{x}_{\boldsymbol{s}}\right) \approx & \sum_{i=1}^{3} w_{i}^{\alpha \beta \gamma}\left\{\sum_{\sigma, \tau, v=0}^{n l} l_{\sigma}\left(\xi_{\alpha_{s}}\right) l_{\tau}\left(\eta_{\beta_{s}}\right) l_{v}\left(\zeta_{\gamma_{s}}\right)\right. \\
& \times\left[G_{i 1}^{\sigma \tau v} l_{\alpha}^{\prime}\left(\xi_{\alpha_{s}}\right) l_{\beta}\left(\eta_{\beta_{s}}\right) l_{\gamma}\left(\zeta_{\gamma_{s}}\right)\right. \\
& +G_{i 2}^{\sigma \tau v} l_{\alpha}\left(\xi_{\alpha_{s}}\right) l^{\prime}{ }_{\beta}\left(\eta_{\beta_{s}}\right) l_{\gamma}\left(\zeta_{\gamma_{s}}\right) \\
& \left.\left.+G_{i 3}^{\sigma \tau v} l_{\alpha}\left(\xi_{\alpha_{s}}\right) l_{\beta}\left(\eta_{\beta_{s}}\right) l^{\prime}{ }_{\gamma}\left(\zeta_{\gamma_{s}}\right)\right]\right\}
\end{aligned}
$$

where $\boldsymbol{x}_{\boldsymbol{s}}=\boldsymbol{x}\left(\xi_{\alpha_{s}}, \eta_{\beta_{s}}, \zeta_{\gamma_{s}}\right)$. If this source location coincides with a Gauss-Lobatto-Legendre point, then the above equation reduces to:

$$
\begin{aligned}
\mathbf{M}: \boldsymbol{\nabla} \boldsymbol{w}\left(\boldsymbol{x}_{\boldsymbol{s}}\right) \approx & \sum_{i=1}^{3} w_{i}^{\alpha \beta \gamma}\left[G_{i 1}^{\alpha_{s} \beta_{s} \gamma_{s}} l_{\alpha}^{\prime}\left(\xi_{\alpha_{s}}\right) \delta_{\beta \beta_{s}} \delta_{\gamma \gamma_{s}}\right. \\
& \left.+G_{i 2}^{\alpha_{s} \beta_{s} \gamma_{s}} \delta_{\alpha \alpha_{s}} l_{\beta}^{\prime}\left(\eta_{\beta_{s}}\right) \delta_{\gamma \gamma_{s}}+G_{i 3}^{\alpha_{s} \beta_{s} \gamma_{s}} \delta_{\alpha \alpha_{s}} \delta_{\beta \beta_{s}} l^{\prime}{ }_{\gamma}\left(\zeta_{\gamma_{s}}\right)\right]
\end{aligned}
$$

Lastly the global absorbing boundary matrix is found from the absorbing boundary integral approximated as:

$$
\begin{aligned}
\int_{\Gamma} \rho\left[v_{n}(\hat{\boldsymbol{n}} \cdot \dot{\mathbf{s}}) \widehat{\mathbf{n}}\right. & \left.+v_{1}\left(\hat{\mathbf{t}}_{\mathbf{1}} \cdot \dot{\mathbf{s}}\right) \hat{\mathbf{t}}_{\mathbf{1}}+v_{2}\left(\hat{\mathbf{t}}_{\mathbf{2}} \cdot \dot{\mathbf{s}}\right) \hat{\mathbf{t}}_{\mathbf{2}}\right] \cdot \boldsymbol{w} d^{2} \boldsymbol{x} \\
& \approx \sum_{i=1}^{3} w_{i}^{\alpha \beta \gamma}\left[\omega _ { \alpha } \omega _ { \beta } \rho ^ { \alpha \beta \gamma } \left(v_{n}^{\alpha \beta \gamma} \hat{n}_{i} \sum_{j=1}^{3} \hat{n}_{j} \dot{s}_{j}^{\alpha \beta \gamma}\right.\right. \\
& \left.\left.+v_{1}^{\alpha \beta \gamma} \hat{t}_{1 i} \sum_{j=1}^{3} \hat{t}_{1 j} \dot{s}_{j}^{\alpha \beta \gamma}+v_{2}^{\alpha \beta \gamma} \hat{t}_{2 i} \sum_{j=1}^{3} \hat{t}_{2 j} \dot{s}_{j}^{\alpha \beta \gamma}\right) J_{b}^{\alpha \beta}\right]
\end{aligned}
$$

Here $\xi_{\alpha}$ and $\eta_{\beta}$ are grid points within the absorbing boundary and $\zeta_{\gamma}$ are grid points perpendicular to the boundary, and $J_{b}^{\alpha \beta}=J_{b}\left(\xi_{\alpha}, \eta_{\beta}\right)$ is the two dimensional Jacobian evaluated at the Gauss-Lobatto-Legendre points for the surface elements. 
The different elemental level equations can each be evaluated over the volume to create the different global matrices needed for the global second order differential equation. When the global system is created, it can then be solved numerically. The time steps are marched forward using a classical explicit second order Finite Difference scheme. This time stepping is conditionally stable, and the Courant stability condition is dependent upon the value of the minimum distance between grid cells and the maximum P-wave velocity. Depending on the mesh of the model and the number of time steps needed, this system is usually solved on a computing cluster.

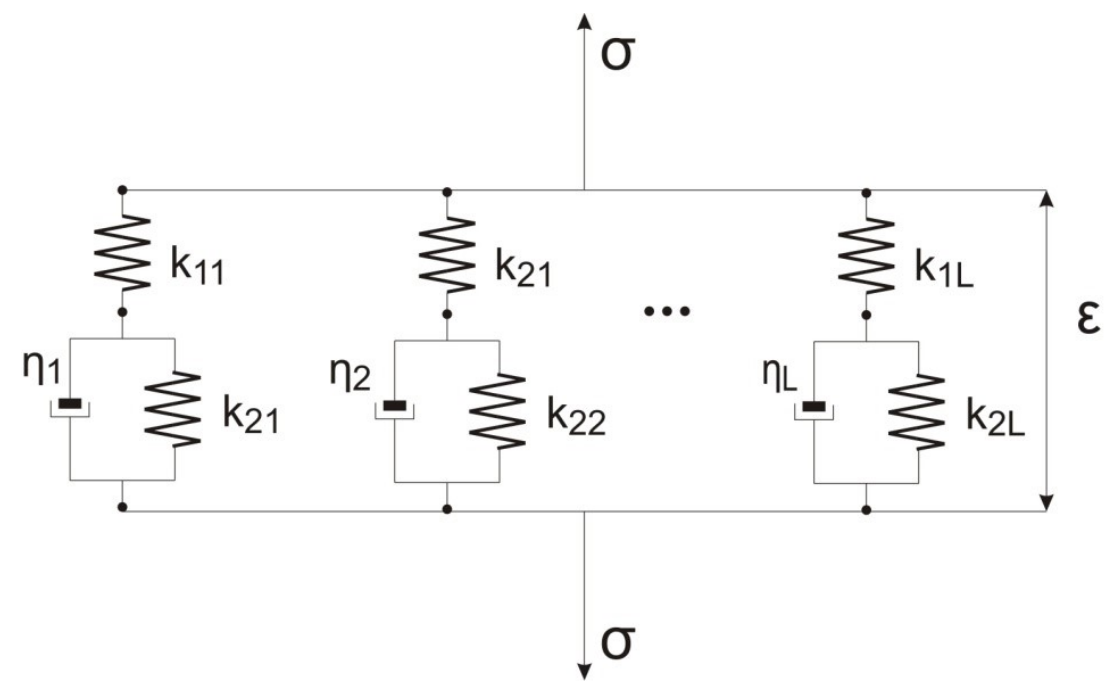

Figure I.1: The mechanical model of a generalized Zener Body

\section{I.D. Attenuating Medium}

As discussed in Eq. 7.3 an attenuating medium takes into account the entire strain history. This is implemented into SEM using a generalized Zener model, or several standard linear solids, to obtain a nearly constant quality factor over the range of seismic frequencies (Liu et al., 1976; Carcione, 2007). The mechanical model for this is shown in Figure l.1 with a system of Zener models connected in parallel. The stress-strain relation for an element is (Carcione, 2007): 


$$
\sigma_{i}+\tau_{\sigma i} \partial_{t} \sigma_{i}=M_{R i}\left(\epsilon+\tau_{\sigma i} \partial_{t} \epsilon\right), i=1, \ldots, N
$$

The relaxed modulus for each element is:

$$
M_{R i}=\frac{k_{1 i} k_{2 i}}{k_{1 i}-k_{2 i}}
$$

and the relaxation times are:

$$
\tau_{\sigma i}=\frac{\eta_{i}}{k_{1 i}+k_{2 i}}, \tau_{\epsilon i}=\frac{\eta_{i}}{k_{2 i}}
$$

Taking the Fourier transform for an element the complex modulus becomes:

$$
M_{i}(\omega)=M_{R i}\left(\frac{1+i \omega \tau_{\epsilon i}}{1+i \omega \tau_{\sigma i}}\right)
$$

The total stress acting on this system is $\sigma=\sum_{i=1}^{N} \sigma_{i}$, so the stress-strain relation in the frequency domain is

$$
\sigma=\sum_{i=1}^{N} M_{i} \epsilon
$$

Choosing $M_{R i}=M_{R} / N$ then the complex modulus is expressed as:

$$
M(\omega)=\sum_{i=1}^{N} M_{i}(\omega), \quad M_{i}(\omega)=\frac{M_{R}}{L}\left(\frac{1+i \omega \tau_{\epsilon i}}{1+i \omega \tau_{\sigma i}}\right)
$$

The relaxation function is obtained from the time-domain constitutive equation

$$
\sigma=\sum_{i=1}^{N} \sigma_{i}=\sum_{i=1}^{N} \psi_{i} * \partial_{t} \epsilon \equiv \psi * \partial_{t} \epsilon
$$

where $\psi_{i}$ has the form:

$$
\psi_{i}(t)=M_{R i}\left[1-\left(1-\frac{\tau_{\epsilon i}}{\tau_{\sigma i}}\right) \exp \left(-t / \tau_{\sigma i}\right)\right] H(t)
$$


and

$$
\psi(t)=M_{R i}\left[1-\frac{1}{N} \sum_{i=1}^{N}\left(1-\frac{\tau_{\epsilon i}}{\tau_{\sigma i}}\right) \exp \left(-t / \tau_{\sigma i}\right)\right] H(t) .
$$

At time $\mathrm{t}=0$ the unrelaxed modulus is:

$$
M_{U}=\frac{M_{R}}{N} \sum_{i=1}^{N} \frac{\tau_{\epsilon i}}{\tau_{\sigma i}}
$$

\section{I.D.1. Nearly Constant Q}

Using a constant-Q model is an efficient and convenient method to model attenuation in seismology. Several studies show a linear frequency dependence of attenuation over the frequencies commonly used in seismology (Carcione et al., 1988), justifying the use of constant$Q$ models. The technique used to obtain a constant $Q$ value over a range of frequencies was to consider equally spaced relaxation mechanisms in a $\log (\omega)$ scale (Liu et al., 1976).

Following Carcione (2007) each element of a generalized Zener model can have a more physical parameterization with a center frequency $\omega_{0}=\tau_{0}^{-1}$, and the value of the quality frequency at this center frequency:

$$
Q_{0}=\frac{2 \tau_{0}}{\tau_{\epsilon}-\tau_{\sigma}}
$$

The frequency dependent quality factor is then:

$$
Q(\omega)=Q_{0}\left(\frac{1+\omega^{2} \tau_{0}^{2}}{2 \omega \tau_{0}}\right)
$$


Solving for the relaxation times given the relaxation peak at the central frequency being $\tau_{0}=$ $\sqrt{\tau_{\sigma} \tau_{\epsilon}}$

and the quality factor at the center frequency:

$$
\tau_{\epsilon}=\frac{\tau_{0}}{Q_{0}}\left(\sqrt{Q_{0}^{2}+1}+1\right), \text { and } \tau_{\sigma}=\frac{\tau_{0}}{Q_{0}}\left(\sqrt{Q_{0}^{2}+1}-1\right) .
$$

To obtain a constant quality factor in a given frequency band centered around $\omega_{0 m}=$ $1 / \tau_{0 m}$, a set of relaxation times $\tau_{\epsilon i}$ and $\tau_{\sigma i}$ must be found. Here $m$ is the mechanism in the middle of the generalized Zener body being used. The quality factor of the entire system is:

$$
Q(\omega)=\frac{\operatorname{Re}(M)}{\operatorname{Im}(M)}=\frac{\operatorname{Re}\left(\sum_{i=1}^{N} M_{i}\right)}{\operatorname{Im}\left(\sum_{i=1}^{N} M_{i}\right)}
$$

where $M_{i}$, is shown in Eq. 7.36. Using the quality factor of each element as $Q_{i}=$ $\operatorname{Re}\left(M_{i}\right) / \operatorname{Im}\left(M_{i}\right)$, the above equation becomes:

$$
Q(\omega)=\frac{\sum_{i=1}^{N} Q_{i} \operatorname{Im}\left(M_{i}\right)}{\sum_{i=1}^{N} \operatorname{Im}\left(M_{i}\right)}
$$

where

$$
Q_{i}(\omega)=Q_{0 i}\left(\frac{1+\omega^{2} \tau_{0 i}^{2}}{2 \omega \tau_{0 i}}\right)
$$

Using equation Eq. 7.41, and the low-loss approximation $\left(\tau_{\sigma i} \approx \tau_{0 i}\right)$ then:

$$
\operatorname{Im}\left(M_{i}\right)=\frac{M_{R}}{N}\left[\frac{\omega\left(\tau_{\epsilon i}-\tau_{\sigma i}\right)}{1+\omega^{2} \tau_{\sigma i}^{2}}\right] \approx \frac{M_{R}}{N}\left[\frac{2 \omega \tau_{0 i}}{Q_{0 i}\left(1+\omega^{2} \tau_{0 i}^{2}\right)}\right]=\frac{M_{R}}{N Q_{i}}
$$

Substituting the above equation into Eq. 7.45 and choosing $Q_{0 i}=Q_{0}$, we obtain: 


$$
Q(\omega)=N Q_{0}\left(\sum_{i=1}^{N} \frac{2 \omega \tau_{0 i}}{1+\omega^{2} \tau_{0 i}^{2}}\right)^{-1}
$$

Setting $Q\left(\omega_{0 m}\right)=\bar{Q}$, the desired value of the quality factor, and $\tau_{0 i}$ to be distributed along the $\log (\omega)$ axis, then:

$$
Q_{0}=\frac{\bar{Q}}{N} \sum_{i=1}^{N} \frac{2 \omega_{0 m} \tau_{0 i}}{1+\omega_{0 m}^{2} \tau_{o i}^{2}}
$$

gives a constant $\mathrm{Q}$ which is equal to the desired $\mathrm{Q}$. In this application with a narrow frequency band $N=2$ or 3 give a constant Q over the desired frequencies (Carcione et al., 1988).

\section{I.E. References:}

Canuto, C., M.Y. Hussaini, A. Quarteroni, and T.A. Zang, (1988). Spectral Methods in Fluid Dynamics, Springer-Verlag, New York.

Carcione, J.M. (1993). Seismic modelling in viscoelastic media, Geophysics, 58, 110-120.

Carcione, J.M. (2007). Wave fields in real media: Theory and numerical simulation of wave propagation in anisotropic, anelastic, porous and electromagnetic media. Elsevier Science, Amsterdam, The Netherlands, second edition.

Carcione, J.M., Kosloi, D. \& Kosloi, R., (1988). Wave propagation simulation in a linear viscoelastic medium, Geophys. J. Int., 95, 597-611.

Clayton, R. \& B. Engquist, (1977). Absorbing boundary conditions for acoustic and elastic wave equations, Bull. Seism. Soc. Am., 67, 1529-1540.

Komatitsch, D., and J. Tromp, (1999). Introduction to the spectral-element method for 3-D seismic wave propagation, Geophys. J. Int., 139, 806-822. 
Komatitsch, D., J.P. Vilotte, R. Vai, J.M. Castillo-Covarrubias, and F.J. Sanchez-Sesma, (1999). The spectral element method for elastic wave equations: application to 2D and 3D seismic problems, Int. J. Num. Meth. Eng., 45, 1139-1164.

Liu, H.P., Anderson, D.L. \& Kanamori, H., (1976). Velocity dispersion due to anelasticity: implications for seismology and mantle com-position, Geophys. J. R. astr. Soc., 47, 41-58.

Quarteroni, A., A. Tagliani, and E. Zampieri, (1998). Generalized Galerkin approximations of elastic waves with absorbing boundary conditions, Comp. Meth. appl. Mech. Eng., 163, 323341. 


\section{Appendix II. Simulation Properties by Chapter}

\section{II.A. Chapter 4:}

\section{II.A.1. 2D Homogeneous Volume: Section 4.2 and 4.3}

Description: A two dimensional homogenous volume $60 \mathrm{~km}$ x $40 \mathrm{~km}$ with a grid spacing of $200 \mathrm{~m}$.

This model has 62 receivers.

\begin{tabular}{|c|c|}
\hline \multicolumn{2}{|c|}{ Volume Properties } \\
\hline Parameter & Surface 1 \\
\hline Vp (m/s) & $6500 \mathrm{~m} / \mathrm{s}$ \\
Vs (m/s) & $3500 \mathrm{~m} / \mathrm{s}$ \\
Density & $2800 \mathrm{~kg} / \mathrm{m}^{3}$ \\
$\left(\mathrm{~kg} / \mathrm{m}^{3}\right)$ & 1000 \\
$\mathrm{Q}$ & \\
\hline
\end{tabular}

This model was used for 5 different simulations. The first two simulations used a unit force one in the X-direction, one in the Z-direction. The other simulations used different moment tensor sources rotated in the direction of KB1O from the epicenter for the 2013 Ladysmith earthquake. All simulations have the same properties.

\begin{tabular}{|c|c|}
\hline \multicolumn{2}{|c|}{ Simulation Properties } \\
\hline Parameter & Value \\
\hline Min grid size & $200 \mathrm{~m}$ \\
Time step & $0.001 \mathrm{~s}$ \\
Total number of time steps & 100000 \\
Total time simulated & $100 \mathrm{~s}$ \\
Courant Number & 0.1882 \\
\hline \multicolumn{2}{|c|}{ Computer Resources } \\
\hline System used & PC \\
Number of Processors & 8 \\
Run Time (sec) & 11138.41
\end{tabular}




\section{II.A.2. $\quad$ 3D Homogeneous Volume: Section 4.2 and 4.3}

Description: A three dimensional homogenous volume $60 \mathrm{~km} \times 20 \mathrm{~km} \times 40 \mathrm{~km}$ with a grid spacing of $200 \mathrm{~m}$.

\begin{tabular}{|c|c|}
\hline \multicolumn{2}{|c|}{ Volume Properties } \\
\hline Parameter & Volume 1 \\
\hline Vp $(\mathrm{m} / \mathrm{s})$ & $6500 \mathrm{~m} / \mathrm{s}$ \\
Vs $(\mathrm{m} / \mathrm{s})$ & $3500 \mathrm{~m} / \mathrm{s}$ \\
Density & \\
$\left(\mathrm{kg} / \mathrm{m}^{3}\right)$ & $2800 \mathrm{~kg} / \mathrm{m}^{3}$ \\
$\mathrm{Q}$ & 1000 \\
\hline
\end{tabular}

This model was used for 5 different simulations. The first two simulations used a unit force one in the X-direction, one in the Z-direction. The other simulations used different moment tensor sources for the 2013 Ladysmith Earthquake. All simulations have the same properties.

\begin{tabular}{|c|c|}
\hline \multicolumn{2}{|c|}{ Simulation Properties } \\
\hline Parameter & Value \\
\hline Min grid size & $200 \mathrm{~m}$ \\
Time step & $0.001 \mathrm{~s}$ \\
Total number of time steps & 100000 \\
Total time simulated & $100 \mathrm{~s}$ \\
Courant Number & 0.1882 \\
\hline Computer Resources \\
\hline System used & Mp2 \\
Number of Processors & 720 \\
Run Time (sec) & 107960.56 \\
Total Core*hours & $21592.11 /$ simulation \\
\hline
\end{tabular}




\section{II.B. $\quad$ Chapter 5:}

\section{II.B.1. 2D Reference Model}

Description: The reference model has 3 surfaces. One surface is the Northeast to Southwest profile of the Kinburn basin. The second surface is a square surface which surrounds this basin and extend to a depth of $400 \mathrm{~m}$, with about $150 \mathrm{~m}$ on either side of the basin. The last surface is a square surface $60 \mathrm{~km} \times 40 \mathrm{~km}$ with the smaller square surface cut out of it. This model was used for eight simulations, each with the same source.

\section{Simulation 1: Sections 5.2.1 and 5.3}

\begin{tabular}{|c|c|c|c|}
\hline \multicolumn{4}{|c|}{ Volume Properties } \\
\hline Parameter & Surface 1 & Surface 2 & Surface 3 \\
\hline$V p(m / s)$ & 1600 & 6500 & 6500 \\
\hline Vs $(\mathrm{m} / \mathrm{s})$ & 150 & 3500 & 3500 \\
\hline $\begin{array}{l}\text { Density } \\
\left(\mathrm{kg} / \mathrm{m}^{3}\right)\end{array}$ & 1600 & 2800 & 2800 \\
\hline $\mathrm{Q}$ & 100 & 1000 & 1000 \\
\hline \multicolumn{3}{|c|}{ Simulation Properties } & \\
\hline \multicolumn{2}{|c|}{ Parameter } & \multicolumn{2}{|l|}{ Value } \\
\hline \multicolumn{2}{|c|}{ Min grid size } & \multicolumn{2}{|l|}{$4.79 \mathrm{~m}$} \\
\hline \multicolumn{2}{|c|}{ Time step } & \multicolumn{2}{|l|}{$0.0001 \mathrm{~s}$} \\
\hline \multicolumn{2}{|c|}{ Total number of time steps } & \multicolumn{2}{|l|}{600000} \\
\hline \multicolumn{2}{|c|}{ Total time simulated } & \multicolumn{2}{|l|}{$60 s$} \\
\hline \multicolumn{2}{|c|}{ Courant Number } & 0.5588 & \\
\hline \multicolumn{3}{|c|}{ Computer Resources } & \\
\hline \multirow{4}{*}{\multicolumn{2}{|c|}{$\begin{array}{c}\text { System used } \\
\text { Number of Processors } \\
\text { Run Time (sec) } \\
\text { Total Core*hours }\end{array}$}} & \multicolumn{2}{|l|}{ PC } \\
\hline & & \multicolumn{2}{|l|}{6} \\
\hline & & \multicolumn{2}{|l|}{34336.0} \\
\hline & & \multicolumn{2}{|l|}{57.22} \\
\hline
\end{tabular}

\section{Simulation 2: Section $\mathbf{5 . 2 . 2}$}




\begin{tabular}{|c|c|c|c|}
\hline \multicolumn{4}{|c|}{ Volume Properties } \\
\hline Parameter & Surface 1 & Surface 2 & Surface 3 \\
\hline$V p(m / s)$ & 1600 & 6000 & 6500 \\
\hline Vs $(\mathrm{m} / \mathrm{s})$ & 150 & 2600 & 3500 \\
\hline $\begin{array}{l}\text { Density } \\
\left(\mathrm{kg} / \mathrm{m}^{3}\right)\end{array}$ & 1600 & 2700 & 2800 \\
\hline Q & 100 & 1000 & 1000 \\
\hline \multicolumn{3}{|c|}{ Simulation Properties } & \\
\hline \multicolumn{2}{|c|}{ Parameter } & Value & \\
\hline \multicolumn{2}{|c|}{ Min grid size } & $4.79 m$ & \\
\hline \multicolumn{2}{|c|}{ Time step } & \multicolumn{2}{|l|}{$0.0001 \mathrm{~s}$} \\
\hline \multicolumn{2}{|c|}{ Total number of time steps } & \multicolumn{2}{|l|}{600000} \\
\hline \multicolumn{2}{|c|}{ Total time simulated } & \multicolumn{2}{|l|}{$60 \mathrm{~s}$} \\
\hline \multicolumn{2}{|c|}{ Courant Number } & 0.5159 & \\
\hline \multicolumn{3}{|c|}{ Computer Resources } & \\
\hline \multicolumn{2}{|c|}{ System used } & \multicolumn{2}{|l|}{$\mathrm{PC}$} \\
\hline \multicolumn{2}{|c|}{ Number of Processors } & \multicolumn{2}{|l|}{8} \\
\hline \multicolumn{2}{|c|}{ Run Time (sec) } & \multicolumn{2}{|l|}{26446.55} \\
\hline \multicolumn{2}{|c|}{ Total Core*hours } & \multicolumn{2}{|l|}{58.77} \\
\hline
\end{tabular}

Simulation 3: Section 5.3.1

\begin{tabular}{|c|c|c|c|}
\hline \multicolumn{4}{|c|}{ Volume Properties } \\
\hline Parameter & Surface 1 & Surface 2 & Surface 3 \\
\hline Vp (m/s) & 1600 & 6500 & 6500 \\
Vs (m/s) & 150 & 3500 & 3500 \\
Density & 1600 & 2800 & 2800 \\
$\left(\mathrm{~kg} / \mathrm{m}^{3}\right)$ & 100 & 750 & 750 \\
$\mathrm{Q}$ & Simulation Properties \\
\hline \multicolumn{3}{|c|}{ Parameter } & Value \\
\hline \multicolumn{3}{|c|}{ Min grid size } & $0.79 \mathrm{~m}$ \\
Time step & $60001 \mathrm{~s}$ & \\
Total number of time steps & $60 \mathrm{~s}$ \\
Total time simulated & 0.5588 \\
Courant Number & PC \\
\multicolumn{3}{|c|}{ Computer Resources } \\
System used \\
Number of Processors \\
Run Time (sec) \\
Total Core*hours
\end{tabular}


Simulation 4: Section 5.3.1

\begin{tabular}{|c|c|c|c|}
\hline \multicolumn{4}{|c|}{ Volume Properties } \\
\hline Parameter & Surface 1 & Surface 2 & Surface 3 \\
\hline$V p(m / s)$ & 1600 & 6500 & 6500 \\
\hline Vs (m/s) & 150 & 3500 & 3500 \\
\hline $\begin{array}{l}\text { Density } \\
\left(\mathrm{kg} / \mathrm{m}^{3}\right)\end{array}$ & 1600 & 2800 & 2800 \\
\hline $\mathrm{Q}$ & 100 & 500 & 500 \\
\hline \multicolumn{3}{|c|}{ Simulation Properties } & \\
\hline \multicolumn{2}{|c|}{ Parameter } & Value & \\
\hline \multicolumn{2}{|c|}{ Min grid size } & $4.79 \mathrm{~m}$ & \\
\hline \multicolumn{2}{|c|}{ Time step } & $0.0001 \mathrm{~s}$ & \\
\hline \multicolumn{2}{|c|}{ Total number of time steps } & 600000 & \\
\hline \multicolumn{2}{|c|}{ Total time simulated } & $60 s$ & \\
\hline \multicolumn{2}{|c|}{ Courant Number } & 0.5588 & \\
\hline \multicolumn{3}{|c|}{ Computer Resources } & \\
\hline \multicolumn{2}{|c|}{ System used } & PC & \\
\hline \multicolumn{2}{|c|}{ Number of Processors } & 8 & \\
\hline \multicolumn{2}{|c|}{ Run Time (sec) } & 26286.25 & \\
\hline \multicolumn{2}{|c|}{ Total Core*hours } & 58.41 & \\
\hline
\end{tabular}

Simulation 5: Section 5.3.2

\begin{tabular}{|c|c|c|c|}
\hline \multicolumn{4}{|c|}{ Volume Properties } \\
\hline Parameter & Surface 1 & Surface 2 & Surface 3 \\
\hline $\mathrm{Vp}(\mathrm{m} / \mathrm{s})$ & 1600 & 6500 & 6500 \\
\hline Vs (m/s) & 150 & 3500 & 3500 \\
\hline $\begin{array}{l}\text { Density } \\
\left(\mathrm{kg} / \mathrm{m}^{3}\right)\end{array}$ & 1600 & 2800 & 2800 \\
\hline$Q$ & 50 & 1000 & 1000 \\
\hline \multicolumn{3}{|c|}{ Simulation Properties } & \\
\hline \multicolumn{2}{|c|}{ Parameter } & \multicolumn{2}{|l|}{ Value } \\
\hline \multicolumn{2}{|c|}{ Min grid size } & \multicolumn{2}{|l|}{$4.79 m$} \\
\hline \multicolumn{2}{|c|}{ Time step } & \multicolumn{2}{|l|}{$0.0001 \mathrm{~s}$} \\
\hline \multicolumn{2}{|c|}{ Total number of time steps } & \multicolumn{2}{|l|}{600000} \\
\hline
\end{tabular}




\begin{tabular}{|c|c|}
\hline $\begin{array}{c}\text { Total time simulated } \\
\text { Courant Number }\end{array}$ & $60 \mathrm{~s}$ \\
\hline \multicolumn{2}{|c|}{ Computer Resources } \\
\hline System used & PC \\
Number of Processors & 8 \\
Run Time (sec) & 26534.17 \\
Total Core*hours & 58.96 \\
\hline
\end{tabular}

Simulation 6: Section 5.3.2

\begin{tabular}{|c|c|c|c|}
\hline \multicolumn{4}{|c|}{ Volume Properties } \\
\hline Parameter & Surface 1 & Surface 2 & Surface 3 \\
\hline $\mathrm{Vp}(\mathrm{m} / \mathrm{s})$ & 1600 & 6500 & 6500 \\
\hline Vs (m/s) & 150 & 3500 & 3500 \\
\hline $\begin{array}{l}\text { Density } \\
\left(\mathrm{kg} / \mathrm{m}^{3}\right)\end{array}$ & 1600 & 2800 & 2800 \\
\hline$Q$ & 20 & 1000 & 1000 \\
\hline \multicolumn{3}{|c|}{ Simulation Properties } & \\
\hline \multicolumn{2}{|c|}{ Parameter } & Value & \\
\hline \multicolumn{2}{|c|}{ Min grid size } & $4.79 m$ & \\
\hline \multicolumn{2}{|c|}{ Time step } & $0.0001 \mathrm{~s}$ & \\
\hline \multicolumn{2}{|c|}{ Total number of time steps } & 600000 & \\
\hline \multicolumn{2}{|c|}{ Total time simulated } & $60 \mathrm{~s}$ & \\
\hline \multicolumn{2}{|c|}{ Courant Number } & 0.5588 & \\
\hline \multicolumn{3}{|c|}{ Computer Resources } & \\
\hline \multicolumn{2}{|c|}{ System used } & \multicolumn{2}{|l|}{ PC } \\
\hline \multicolumn{2}{|c|}{ Number of Processors } & \multicolumn{2}{|l|}{8} \\
\hline \multicolumn{2}{|c|}{ Run Time (sec) } & \multicolumn{2}{|l|}{26590.72} \\
\hline \multicolumn{2}{|c|}{ Total Core*hours } & \multicolumn{2}{|l|}{59.09} \\
\hline
\end{tabular}

Simulation 7: Section 5.3.3

\begin{tabular}{|c|c|c|c|}
\hline \multicolumn{4}{|c|}{ Volume Properties } \\
\hline Parameter & Surface 1 & Surface 2 & Surface 3 \\
\hline Vp (m/s) & 1700 & 6500 & 6500 \\
Vs (m/s) & 250 & 3500 & 3500
\end{tabular}




\begin{tabular}{|c|c|c|c|}
\hline $\begin{array}{c}\text { Density } \\
\left(\mathrm{kg} / \mathrm{m}^{3}\right) \\
\mathrm{Q}\end{array}$ & $\begin{array}{c}1600 \\
100 \\
\end{array}$ & $\begin{array}{l}2800 \\
1000\end{array}$ & $\begin{array}{r}2800 \\
1000 \\
\end{array}$ \\
\hline \multicolumn{3}{|c|}{ Simulation Properties } & \\
\hline \multicolumn{2}{|c|}{ Parameter } & Value & \\
\hline \multicolumn{2}{|c|}{ Min grid size } & $4.79 \mathrm{~m}$ & \\
\hline \multicolumn{2}{|c|}{ Time step } & $0.0001 \mathrm{~s}$ & \\
\hline \multicolumn{2}{|c|}{ Total number of time steps } & 600000 & \\
\hline \multicolumn{2}{|c|}{ Total time simulated } & $60 \mathrm{~s}$ & \\
\hline \multicolumn{2}{|c|}{ Courant Number } & 0.5588 & \\
\hline \multicolumn{3}{|c|}{ Computer Resources } & \\
\hline \multirow{4}{*}{\multicolumn{2}{|c|}{$\begin{array}{c}\text { System used } \\
\text { Number of Processors } \\
\text { Run Time (sec) } \\
\text { Total Core*hours }\end{array}$}} & \multirow{4}{*}{$\begin{array}{c}P C \\
8 \\
26426.86 \\
58.73\end{array}$} & \\
\hline & & & \\
\hline & & & \\
\hline & & & \\
\hline
\end{tabular}

Simulation 8: Section 5.3.3

\begin{tabular}{|c|c|c|c|}
\hline \multicolumn{4}{|c|}{ Volume Properties } \\
\hline Parameter & Surface 1 & Surface 2 & Surface 3 \\
\hline$V p(m / s)$ & 1800 & 6500 & 6500 \\
\hline $\mathrm{Vs}(\mathrm{m} / \mathrm{s})$ & 350 & 3500 & 3500 \\
\hline $\begin{array}{l}\text { Density } \\
\left(\mathrm{kg} / \mathrm{m}^{3}\right)\end{array}$ & 1800 & 2800 & 2800 \\
\hline Q & 100 & 1000 & 1000 \\
\hline \multicolumn{3}{|c|}{ Simulation Properties } & \\
\hline \multicolumn{2}{|c|}{ Parameter } & Value & \\
\hline \multicolumn{2}{|c|}{ Min grid size } & $4.79 \mathrm{~m}$ & \\
\hline \multicolumn{2}{|c|}{ Time step } & $0.0001 \mathrm{~s}$ & \\
\hline \multicolumn{2}{|c|}{ Total number of time steps } & 600000 & \\
\hline \multicolumn{2}{|c|}{ Total time simulated } & $60 s$ & \\
\hline \multicolumn{2}{|c|}{ Courant Number } & 0.5588 & \\
\hline \multicolumn{3}{|c|}{ Computer Resources } & \\
\hline \multicolumn{2}{|c|}{ System used } & \multicolumn{2}{|l|}{$P C$} \\
\hline \multicolumn{2}{|c|}{ Number of Processors } & \multicolumn{2}{|l|}{6} \\
\hline \multicolumn{2}{|c|}{ Run Time (sec) } & \multicolumn{2}{|l|}{34377.30} \\
\hline \multicolumn{2}{|c|}{ Total Core*hours } & 57.30 & \\
\hline
\end{tabular}




\section{II.B.2. 2D Large Box Model}

Description: The large box model has 4 surfaces. One surface is the Northeast to Southwest profile of the Kinburn basin. The second surface is a square surface which surrounds this basin and extend to a depth of $400 \mathrm{~m}$, with about $150 \mathrm{~m}$ on either side of the basin. The third surface is another square which extends to $4 \mathrm{~km}$ and has the surface 2 cut out of it. The last surface is a square surface $60 \mathrm{~km} \times 40 \mathrm{~km}$ with the surface 3 cut out of it. This model was used for three simulations, each with the same source.

Simulation 1: Section 5.2.1 and 5.2.3

\begin{tabular}{|c|c|c|c|c|}
\hline \multicolumn{5}{|c|}{ Volume Properties } \\
\hline Parameter & Surface 1 & Surface 2 & Surface 3 & Surface 4 \\
\hline $\mathrm{Vp}(\mathrm{m} / \mathrm{s})$ & 1600 & 6500 & 6500 & 6500 \\
\hline Vs $(\mathrm{m} / \mathrm{s})$ & 150 & 3500 & 3500 & 3500 \\
\hline $\begin{array}{l}\text { Density } \\
\left(\mathrm{kg} / \mathrm{m}^{3}\right)\end{array}$ & 1600 & 2800 & 2800 & 2800 \\
\hline Q & 100 & 1000 & 1000 & 1000 \\
\hline \multicolumn{3}{|c|}{ Simulation Properties } & & \\
\hline \multicolumn{2}{|c|}{ Parameter } & Value & & \\
\hline \multicolumn{2}{|c|}{ Min grid size } & $4.50 \mathrm{~m}$ & & \\
\hline \multicolumn{2}{|c|}{ Time step } & $0.0001 \mathrm{~s}$ & & \\
\hline \multicolumn{2}{|c|}{ Total number of time steps } & 600000 & & \\
\hline \multicolumn{2}{|c|}{ Total time simulated } & $60 \mathrm{~s}$ & & \\
\hline \multicolumn{2}{|c|}{ Courant Number } & 0.5588 & & \\
\hline \multicolumn{3}{|c|}{ Computer Resources } & & \\
\hline \multicolumn{2}{|c|}{ System used } & PC & & \\
\hline \multicolumn{2}{|c|}{ Number of Processors } & 8 & & \\
\hline \multicolumn{2}{|c|}{ Run Time (sec) } & 26274.98 & & \\
\hline \multicolumn{2}{|c|}{ Total Core*hours } & 58.39 & & \\
\hline
\end{tabular}

Simulation 2: Section 5.2.2 and 5.2.3

\begin{tabular}{|c|c|c|c|c|}
\hline \multicolumn{5}{|c|}{ Volume Properties } \\
\hline Parameter & Surface 1 & Surface 2 & Surface 3 & Surface 4 \\
\hline
\end{tabular}




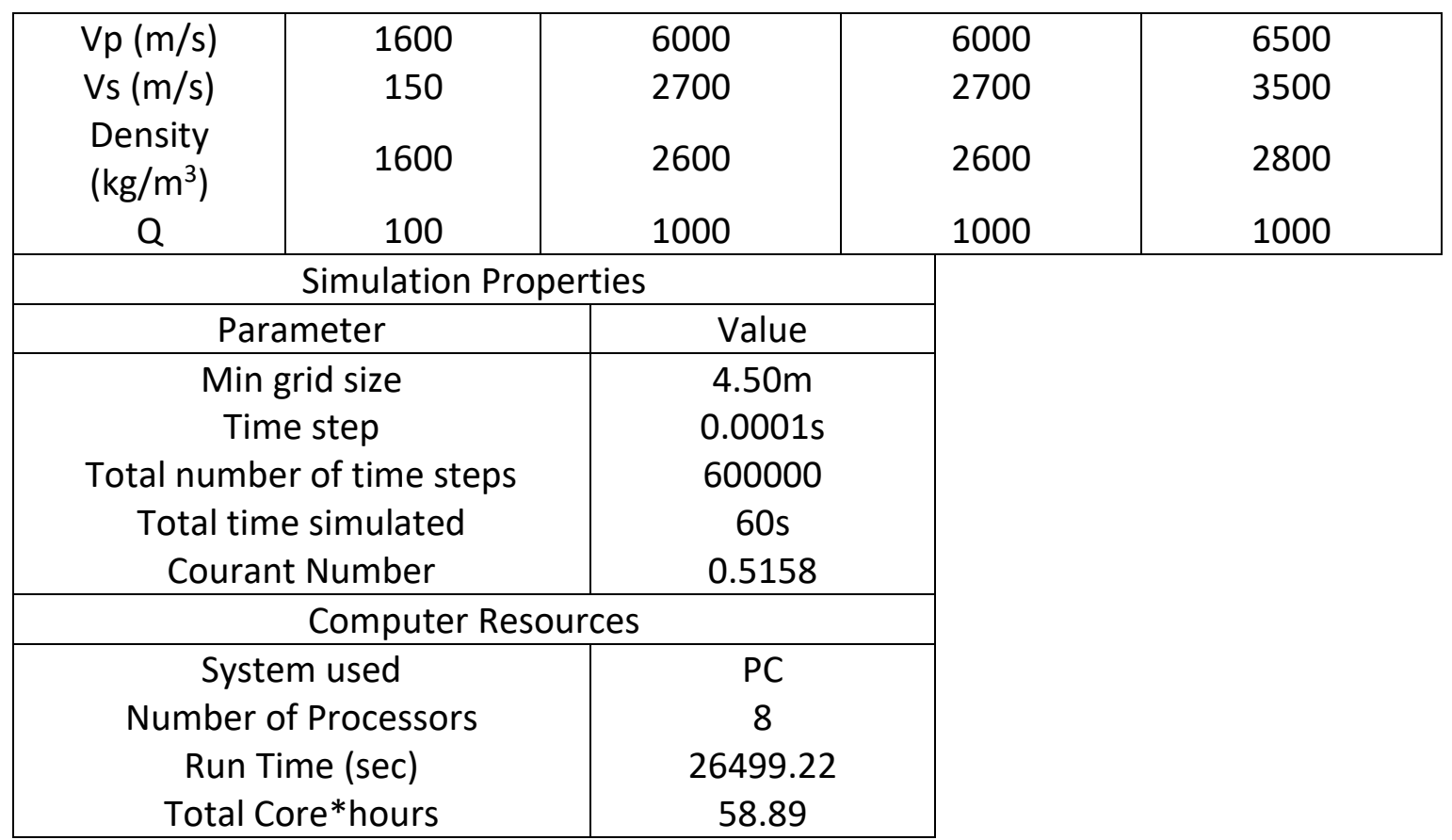

Simulation 3: Section 5.2.3

\begin{tabular}{|c|c|c|c|c|}
\hline \multicolumn{5}{|c|}{ Volume Properties } \\
\hline Parameter & Surface 1 & Surface 2 & Surface 3 & Surface 4 \\
\hline $\mathrm{Vp}(\mathrm{m} / \mathrm{s})$ & 1600 & 6000 & 6250 & 6500 \\
\hline $\mathrm{Vs}(\mathrm{m} / \mathrm{s})$ & 150 & 2700 & 3100 & 3500 \\
\hline $\begin{array}{l}\text { Density } \\
\left(\mathrm{kg} / \mathrm{m}^{3}\right)\end{array}$ & 1600 & 2600 & 2700 & 2800 \\
\hline $\mathrm{Q}$ & 100 & 1000 & 1000 & 1000 \\
\hline \multicolumn{3}{|c|}{ Simulation Properties } & & \\
\hline \multicolumn{2}{|c|}{ Parameter } & Value & & \\
\hline \multicolumn{2}{|c|}{ Min grid size } & $4.50 \mathrm{~m}$ & & \\
\hline \multirow{2}{*}{\multicolumn{2}{|c|}{$\begin{array}{l}\text { Time step } \\
\text { Total number of time steps }\end{array}$}} & $0.0001 \mathrm{~s}$ & & \\
\hline & & 600000 & & \\
\hline \multicolumn{2}{|c|}{ Total time simulated } & $60 \mathrm{~s}$ & & \\
\hline \multicolumn{2}{|c|}{ Courant Number } & 0.5158 & & \\
\hline \multicolumn{3}{|c|}{ Computer Resources } & & \\
\hline \multirow{4}{*}{\multicolumn{2}{|c|}{$\begin{array}{c}\text { System used } \\
\text { Number of Processors } \\
\text { Run Time (sec) } \\
\text { Total Core*hours }\end{array}$}} & \multirow{2}{*}{$\begin{array}{l}\mathrm{PC} \\
8\end{array}$} & & \\
\hline & & & & \\
\hline & & 26623.98 & & \\
\hline & & 59.16 & & \\
\hline
\end{tabular}




\section{II.B.3. 2D Layered Model}

Description: The Layered model has 9 surfaces. The first surface is a Northeast to Southwest profile of the Kinburn basin. The second surface surrounds the basin and extends down to $400 \mathrm{~m}$ depth from the free surface. The other surfaces are horizontally layered surfaces with boundaries at $1.2 \mathrm{~km}, 2.0 \mathrm{~km}, 4.0 \mathrm{~km}, 10 \mathrm{~km}, 20 \mathrm{~km}$, and $30 \mathrm{~km}$. This model was used for 4 simulations, each with the same source.

Simulation 1: Section 5.2.1and 5.2.4

\begin{tabular}{|c|c|c|c|c|c|c|c|c|c|}
\hline \multicolumn{10}{|c|}{ Volume Properties } \\
\hline Parameter & $\begin{array}{c}\text { Surface } \\
1\end{array}$ & $\begin{array}{c}\text { Surface } \\
2\end{array}$ & $\begin{array}{c}\text { Surface } \\
3\end{array}$ & $\begin{array}{c}\text { Surface } \\
4\end{array}$ & $\begin{array}{c}\text { Surface } \\
5\end{array}$ & $\begin{array}{l}\text { Surface } \\
6\end{array}$ & $\begin{array}{c}\text { Surface } \\
7\end{array}$ & $\begin{array}{l}\text { Surface } \\
8\end{array}$ & $\begin{array}{l}\text { Surface } \\
9\end{array}$ \\
\hline $\mathrm{Vp}(\mathrm{m} / \mathrm{s})$ & 1600 & 6500 & 6500 & 6500 & 6500 & 6500 & 6500 & 6500 & 6500 \\
\hline $\mathrm{Vs}(\mathrm{m} / \mathrm{s})$ & 150 & 3500 & 3500 & 3500 & 3500 & 3500 & 3500 & 3500 & 3500 \\
\hline $\begin{array}{l}\text { Density } \\
\left(\mathrm{kg} / \mathrm{m}^{3}\right)\end{array}$ & 1600 & 2800 & 2800 & 2800 & 2800 & 2800 & 2800 & 2800 & 2800 \\
\hline Q & 100 & 1000 & 1000 & 1000 & 1000 & 1000 & 1000 & 1000 & 1000 \\
\hline \multicolumn{10}{|c|}{ Simulation Properties } \\
\hline \multicolumn{3}{|c|}{ Parameter } & \multicolumn{3}{|c|}{ Value } & & & & \\
\hline \multicolumn{3}{|c|}{ Min grid size } & \multicolumn{2}{|r|}{$4.79 m$} & & & & & \\
\hline \multicolumn{3}{|c|}{ Time step } & \multicolumn{2}{|r|}{$0.0001 \mathrm{~s}$} & & & & & \\
\hline \multicolumn{3}{|c|}{ Total number of time steps } & \multicolumn{2}{|r|}{600000} & & & & & \\
\hline \multicolumn{3}{|c|}{ Total time simulated } & \multicolumn{2}{|r|}{$60 \mathrm{~s}$} & & & & & \\
\hline & urant Nur & ber & & 0.5073 & & & & & \\
\hline \multicolumn{6}{|c|}{ Computer Resources } & & & & \\
\hline \multicolumn{3}{|c|}{ System used } & \multicolumn{2}{|r|}{$P C$} & & & & & \\
\hline \multirow{2}{*}{\multicolumn{3}{|c|}{$\begin{array}{c}\text { Number of Processors } \\
\text { Run Time (sec) }\end{array}$}} & \multicolumn{2}{|r|}{8} & & & & & \\
\hline & & & \multirow{2}{*}{\multicolumn{2}{|c|}{$\begin{array}{c}30398.00 \\
67.55\end{array}$}} & & & & & \\
\hline \multicolumn{3}{|c|}{ Total Core*hours } & & & & & & & \\
\hline
\end{tabular}

Simulation 2: Section 5.2.2 and 5.2.4

\begin{tabular}{|c|c|c|c|c|c|c|c|c|c|}
\hline \multicolumn{10}{|c|}{ Volume Properties } \\
\hline \multirow{2}{*}{ Parameter } & Surface & Surface & Surface & Surface & Surface & Surface & Surface & Surface & Surface \\
& 1 & 2 & 3 & 4 & 5 & 6 & 7 & 8 & 9 \\
\hline
\end{tabular}




\begin{tabular}{|c|c|c|c|c|c|c|c|c|c|}
\hline$V p(m / s)$ & 1600 & 6000 & 6500 & 6500 & 6500 & 6500 & 6500 & 6500 & 6500 \\
\hline $\mathrm{Vs}(\mathrm{m} / \mathrm{s})$ & 150 & 2700 & 3500 & 3500 & 3500 & 3500 & 3500 & 3500 & 3500 \\
\hline $\begin{array}{l}\text { Density } \\
\left(\mathrm{kg} / \mathrm{m}^{3}\right)\end{array}$ & 1600 & 2600 & 2800 & 2800 & 2800 & 2800 & 2800 & 2800 & 2800 \\
\hline Q & 100 & 1000 & 1000 & 1000 & 1000 & 1000 & 1000 & 1000 & 1000 \\
\hline \multicolumn{6}{|c|}{ Simulation Properties } & & & & \\
\hline \multicolumn{3}{|c|}{ Parameter } & \multicolumn{3}{|c|}{ Value } & & & & \\
\hline \multicolumn{3}{|c|}{ Min grid size } & \multicolumn{2}{|r|}{$4.79 m$} & & & & & \\
\hline \multicolumn{3}{|c|}{ Time step } & \multicolumn{2}{|r|}{$0.0001 \mathrm{~s}$} & & & & & \\
\hline \multicolumn{3}{|c|}{ Total number of time steps } & \multicolumn{2}{|r|}{600000} & & & & & \\
\hline \multicolumn{3}{|c|}{ Total time simulated } & \multirow{2}{*}{\multicolumn{2}{|c|}{$\begin{array}{c}60 \mathrm{~s} \\
0.4683\end{array}$}} & & & & & \\
\hline & Irant Nu & & & & & & & & \\
\hline \multicolumn{6}{|c|}{ Computer Resources } & & & & \\
\hline \multirow{4}{*}{\multicolumn{3}{|c|}{$\begin{array}{c}\text { System used } \\
\text { Number of Processors } \\
\text { Run Time (sec) } \\
\text { Total Core*hours }\end{array}$}} & \multirow{4}{*}{\multicolumn{2}{|c|}{$\begin{array}{c}P C \\
8 \\
29748.61 \\
66.11\end{array}$}} & & & & & \\
\hline & & & & & & & & & \\
\hline & & & & & & & & & \\
\hline & & & & & & & & & \\
\hline
\end{tabular}

Simulation 3: Section 5.2.4

\begin{tabular}{|c|c|c|c|c|c|c|c|c|c|}
\hline \multicolumn{10}{|c|}{ Volume Properties } \\
\hline Parameter & $\begin{array}{c}\text { Surface } \\
1\end{array}$ & $\begin{array}{c}\text { Surface } \\
2\end{array}$ & $\begin{array}{c}\text { Surface } \\
3\end{array}$ & $\begin{array}{c}\text { Surface } \\
4\end{array}$ & $\begin{array}{c}\text { Surface } \\
5\end{array}$ & $\begin{array}{c}\text { Surface } \\
6\end{array}$ & $\begin{array}{c}\text { Surface } \\
7\end{array}$ & $\begin{array}{c}\text { Surface } \\
8\end{array}$ & $\begin{array}{c}\text { Surface } \\
9\end{array}$ \\
\hline $\mathrm{Vp}(\mathrm{m} / \mathrm{s})$ & 1600 & 6000 & 6050 & 6080 & 6130 & 6250 & 6380 & 6500 & 6500 \\
\hline Vs (m/s) & 150 & 2700 & 2730 & 2790 & 2830 & 2990 & 3250 & 3500 & 3500 \\
\hline $\begin{array}{l}\text { Density } \\
\left(\mathrm{kg} / \mathrm{m}^{3}\right)\end{array}$ & 1600 & 2600 & 2650 & 2680 & 2700 & 2750 & 2780 & 2800 & 2800 \\
\hline Q & 100 & 1000 & 1000 & 1000 & 1000 & 1000 & 1000 & 1000 & 1000 \\
\hline \multicolumn{10}{|c|}{ Simulation Properties } \\
\hline \multicolumn{3}{|c|}{ Parameter } & \multicolumn{3}{|c|}{ Value } & & & & \\
\hline $\begin{array}{r}\text { Total } n \\
\text { Tota } \\
\mathrm{Cc}\end{array}$ & $\begin{array}{l}\text { Min grid s } \\
\text { Time ste } \\
\text { mber of } \\
\text { time sim } \\
\text { urant Nur }\end{array}$ & $\begin{array}{l}\text { me steps } \\
\text { lated } \\
\text { lber }\end{array}$ & & $\begin{array}{c}4.79 \mathrm{~m} \\
0.0001 \mathrm{~s} \\
600000 \\
60 \mathrm{~s} \\
0.4683\end{array}$ & & & & & \\
\hline \multicolumn{6}{|c|}{ Computer Resources } & & & & \\
\hline \multicolumn{3}{|c|}{$\begin{array}{c}\text { System used } \\
\text { Number of Processors } \\
\text { Run Time }(\mathrm{sec}) \\
\text { Total Core*hours }\end{array}$} & \multicolumn{2}{|r|}{$\begin{array}{c}P C \\
8 \\
30059.61 \\
66.80\end{array}$} & & & & & \\
\hline
\end{tabular}


Simulation 4: Section 5.2.4

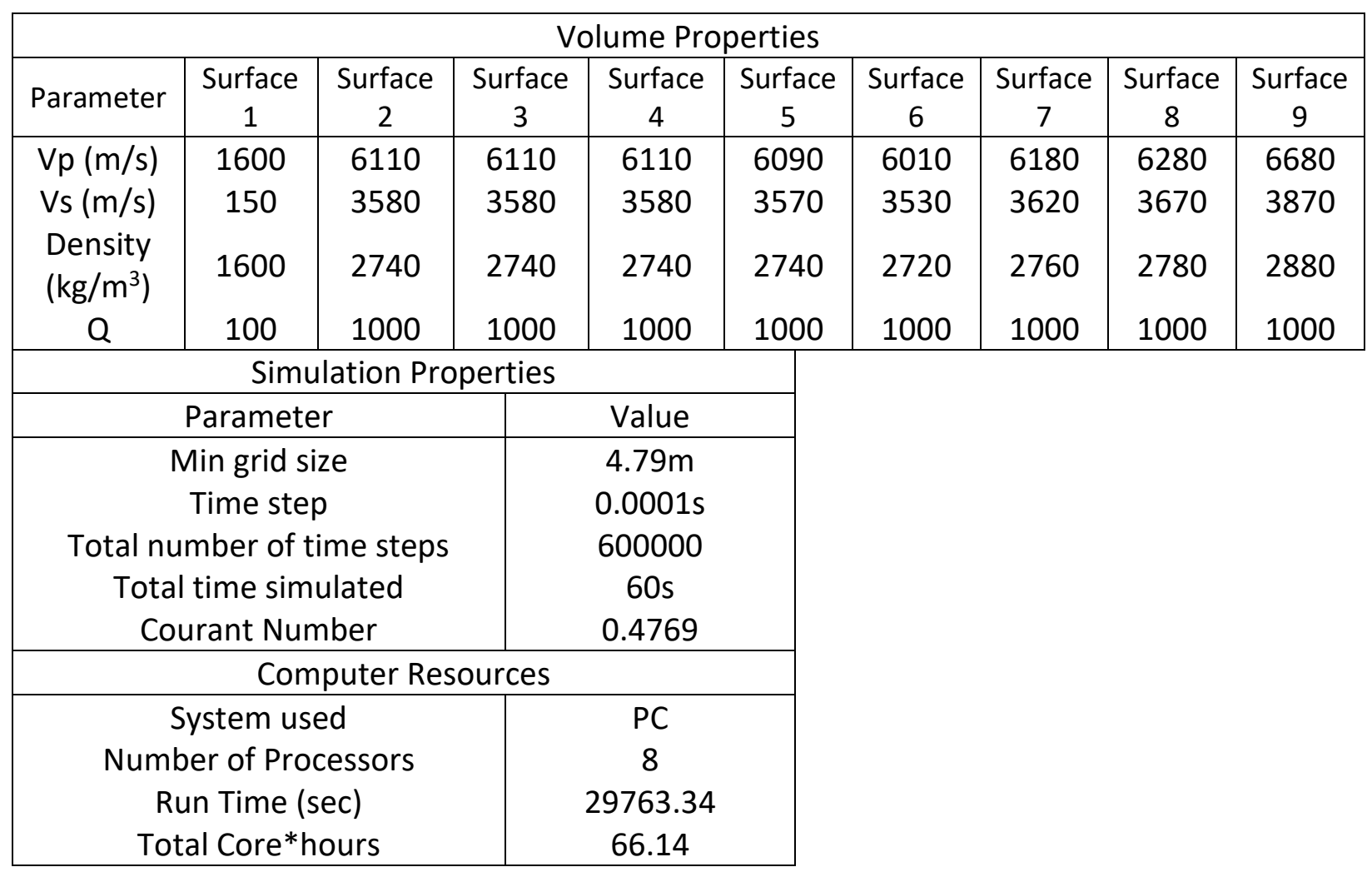

\section{II.C. Chapter 6}

\section{II.C.1. 2D Reference Model}

Description: The reference model has 3 surfaces. One surface is the Northeast to Southwest profile of the Kinburn basin. The second surface is a square surface which surrounds this basin and extend to a depth of $400 \mathrm{~m}$, with about $150 \mathrm{~m}$ on either side of the basin. The last surface is a square surface $60 \mathrm{~km} \times 40 \mathrm{~km}$ with the smaller square surface cut out of it. This model was used for 2 simulations with identical sources for both.

\section{Simulation 1: Section 6.1.1}




\begin{tabular}{|c|c|c|c|}
\hline \multicolumn{4}{|c|}{ Volume Properties } \\
\hline Parameter & Surface 1 & Surface 2 & Surface 3 \\
\hline Vp (m/s) & 6500 & 6500 & 6500 \\
Vs (m/s) & 3500 & 3500 & 3500 \\
Density & 2800 & 2800 & 2800 \\
$\left(\mathrm{~kg} / \mathrm{m}^{3}\right)$ & 1000 & 1000 & 1000 \\
$\mathrm{Q}$ & Simulation Properties \\
\hline \multicolumn{3}{|c|}{ Parameter } & Value \\
\hline \multicolumn{3}{|c|}{ Min grid size } & $4.79 \mathrm{~m}$ \\
Time step & $0.00005 \mathrm{~s}$ & \\
Total number of time steps & 1200000 \\
Total time simulated & $60 \mathrm{~s}$ \\
Courant Number & 0.3928 \\
\multicolumn{3}{|c|}{ Computer Resources } \\
\multicolumn{3}{|c|}{ System used } \\
Number of Processors \\
Run Time (sec) \\
Total Core*hours
\end{tabular}

Simulation 2: Section 6.1.1

\begin{tabular}{|c|c|c|c|}
\hline \multicolumn{4}{|c|}{ Volume Properties } \\
\hline Parameter & Surface 1 & Surface 2 & Surface 3 \\
\hline$V p(m / s)$ & 1600 & 6500 & 6500 \\
\hline $\mathrm{Vs}(\mathrm{m} / \mathrm{s})$ & 150 & 3500 & 3500 \\
\hline $\begin{array}{l}\text { Density } \\
\left(\mathrm{kg} / \mathrm{m}^{3}\right)\end{array}$ & 1600 & 2800 & 2800 \\
\hline $\mathrm{Q}$ & 100 & 1000 & 1000 \\
\hline \multicolumn{3}{|c|}{ Simulation Properties } & \\
\hline \multicolumn{2}{|c|}{ Parameter } & \multicolumn{2}{|l|}{ Value } \\
\hline \multicolumn{2}{|c|}{ Min grid size } & \multicolumn{2}{|l|}{$4.79 m$} \\
\hline \multicolumn{2}{|c|}{ Time step } & \multicolumn{2}{|l|}{$0.0001 \mathrm{~s}$} \\
\hline \multicolumn{2}{|c|}{ Total number of time steps } & \multicolumn{2}{|l|}{600000} \\
\hline \multicolumn{2}{|c|}{ Total time simulated } & \multicolumn{2}{|l|}{$60 s$} \\
\hline \multicolumn{2}{|c|}{ Courant Number } & \multicolumn{2}{|l|}{0.5588} \\
\hline \multicolumn{3}{|c|}{ Computer Resources } & \\
\hline \multicolumn{2}{|c|}{ System used } & \multicolumn{2}{|l|}{$P C$} \\
\hline \multicolumn{2}{|c|}{ Number of Processors } & \multicolumn{2}{|l|}{6} \\
\hline \multicolumn{2}{|c|}{ Run Time (sec) } & \multicolumn{2}{|l|}{34336.0} \\
\hline \multicolumn{2}{|c|}{ Total Core*hours } & \multicolumn{2}{|l|}{57.22} \\
\hline
\end{tabular}




\section{II.C.2. 2D Layered Model}

Description: The Layered model has 9 surfaces. The first surface is a Northeast to Southwest profile of the Kinburn basin. The second surface surrounds the basin and extends down to 400m depth from the free surface. The other surfaces are horizontally layered surfaces with boundaries at $1.2 \mathrm{~km}, 2.0 \mathrm{~km}, 4.0 \mathrm{~km}, 10 \mathrm{~km}, 20 \mathrm{~km}$, and $30 \mathrm{~km}$. This model was used for ten simulations, using several sources.

Simulation 1: Section 6.2.1

\begin{tabular}{|c|c|c|c|c|c|c|c|c|c|}
\hline \multicolumn{10}{|c|}{ Volume Properties } \\
\hline Parameter & $\begin{array}{c}\text { Surface } \\
1\end{array}$ & $\begin{array}{c}\text { Surface } \\
2\end{array}$ & $\begin{array}{c}\text { Surface } \\
3\end{array}$ & $\begin{array}{c}\text { Surface } \\
4\end{array}$ & $\begin{array}{l}\text { Surface } \\
5\end{array}$ & $\begin{array}{l}\text { Surface } \\
6\end{array}$ & $\begin{array}{c}\text { Surface } \\
7\end{array}$ & $\begin{array}{l}\text { Surface } \\
8\end{array}$ & $\begin{array}{l}\text { Surface } \\
9\end{array}$ \\
\hline $\mathrm{Vp}(\mathrm{m} / \mathrm{s})$ & 1600 & 6500 & 6500 & 6500 & 6500 & 6500 & 6500 & 6500 & 6500 \\
\hline $\mathrm{Vs}(\mathrm{m} / \mathrm{s})$ & 150 & 3500 & 3500 & 3500 & 3500 & 3500 & 3500 & 3500 & 3500 \\
\hline $\begin{array}{l}\text { Density } \\
\left(\mathrm{kg} / \mathrm{m}^{3}\right)\end{array}$ & 1600 & 2800 & 2800 & 2800 & 2800 & 2800 & 2800 & 2800 & 2800 \\
\hline Q & 100 & 1000 & 1000 & 1000 & 1000 & 1000 & 1000 & 1000 & 1000 \\
\hline \multicolumn{10}{|c|}{ Simulation Properties } \\
\hline \multicolumn{3}{|c|}{ Parameter } & \multicolumn{3}{|c|}{ Value } & & & & \\
\hline \multicolumn{3}{|c|}{ Min grid size } & \multicolumn{2}{|r|}{$4.79 \mathrm{~m}$} & & & & & \\
\hline \multicolumn{3}{|c|}{ Time step } & \multicolumn{2}{|r|}{$0.0001 \mathrm{~s}$} & & & & & \\
\hline \multicolumn{3}{|c|}{ Total number of time steps } & \multicolumn{2}{|r|}{600000} & & & & & \\
\hline \multicolumn{3}{|c|}{ Total time simulated } & \multicolumn{2}{|r|}{$60 s$} & & & & & \\
\hline \multicolumn{3}{|c|}{ Courant Number } & & 0.5073 & & & & & \\
\hline \multicolumn{6}{|c|}{ Computer Resources } & & & & \\
\hline \multicolumn{3}{|c|}{ System used } & \multicolumn{2}{|r|}{$P C$} & & & & & \\
\hline \multirow{2}{*}{\multicolumn{3}{|c|}{$\begin{array}{c}\text { Number of Processors } \\
\text { Run Time (sec) }\end{array}$}} & \multicolumn{2}{|r|}{8} & & & & & \\
\hline & & & \multirow{2}{*}{\multicolumn{2}{|c|}{$\begin{array}{c}30398.00 \\
67.55\end{array}$}} & & & & & \\
\hline \multicolumn{3}{|c|}{ Total Core*hours } & & & & & & & \\
\hline
\end{tabular}

Simulation 2: Section 6.2.1 


\begin{tabular}{|c|c|c|c|c|c|c|c|c|c|}
\hline \multicolumn{10}{|c|}{ Volume Properties } \\
\hline Parameter & $\begin{array}{c}\text { Surface } \\
1\end{array}$ & $\begin{array}{c}\text { Surface } \\
2\end{array}$ & $\begin{array}{c}\text { Surface } \\
3\end{array}$ & $\begin{array}{c}\text { Surface } \\
4\end{array}$ & $\begin{array}{c}\text { Surface } \\
5\end{array}$ & $\begin{array}{c}\text { Surface } \\
6\end{array}$ & $\begin{array}{c}\text { Surface } \\
7\end{array}$ & $\begin{array}{c}\text { Surface } \\
8\end{array}$ & $\begin{array}{c}\text { Surface } \\
9\end{array}$ \\
\hline $\mathrm{Vp}(\mathrm{m} / \mathrm{s})$ & 1600 & 6500 & 6500 & 6500 & 6500 & 6500 & 6500 & 6500 & 6500 \\
\hline $\mathrm{Vs}(\mathrm{m} / \mathrm{s})$ & 150 & 3500 & 3500 & 3500 & 3500 & 3500 & 3500 & 3500 & 3500 \\
\hline $\begin{array}{l}\text { Density } \\
\left(\mathrm{kg} / \mathrm{m}^{3}\right)\end{array}$ & 1600 & 2800 & 2800 & 2800 & 2800 & 2800 & 2800 & 2800 & 2800 \\
\hline Q & 100 & 1000 & 1000 & 1000 & 1000 & 1000 & 1000 & 1000 & 1000 \\
\hline \multicolumn{10}{|c|}{ Simulation Properties } \\
\hline \multicolumn{3}{|c|}{ Parameter } & \multicolumn{3}{|c|}{ Value } & & & & \\
\hline $\begin{array}{r}\text { Total } n \\
\text { Tot } \\
\text { C }\end{array}$ & $\begin{array}{l}\text { Min grid s } \\
\text { Time ste } \\
\text { mber of } t \\
\text { time sim } \\
\text { urant Nur }\end{array}$ & $\begin{array}{l}\text { me steps } \\
\text { lated } \\
\text { lber }\end{array}$ & & $\begin{array}{c}4.79 \mathrm{~m} \\
0.0001 \mathrm{~s} \\
1200000 \\
120 \mathrm{~s} \\
0.5073\end{array}$ & & & & & \\
\hline \multicolumn{6}{|c|}{ Computer Resources } & & & & \\
\hline Num & $\begin{array}{l}\text { ystem us } \\
\text { er of Pro } \\
\text { al Time ( } \\
\text { al Core*r }\end{array}$ & $\begin{array}{l}\text { d } \\
\text { essors } \\
\text { ec) } \\
\text { ours } \\
\end{array}$ & \multicolumn{2}{|r|}{$\begin{array}{c}8 \\
63522.91\end{array}$} & & & & & \\
\hline
\end{tabular}

Simulation 3: Section 6.2.1

\begin{tabular}{|c|c|c|c|c|c|c|c|c|c|}
\hline \multicolumn{10}{|c|}{ Volume Properties } \\
\hline Parameter & $\begin{array}{c}\text { Surface } \\
1\end{array}$ & $\begin{array}{c}\text { Surface } \\
2\end{array}$ & $\begin{array}{c}\text { Surface } \\
3\end{array}$ & $\begin{array}{c}\text { Surface } \\
4\end{array}$ & $\begin{array}{c}\text { Surface } \\
5\end{array}$ & $\begin{array}{c}\text { Surface } \\
6\end{array}$ & $\begin{array}{c}\text { Surface } \\
7\end{array}$ & $\begin{array}{c}\text { Surface } \\
8\end{array}$ & $\begin{array}{c}\text { Surface } \\
\quad 9\end{array}$ \\
\hline$V p(m / s)$ & 1600 & 6000 & 6500 & 6500 & 6500 & 6500 & 6500 & 6500 & 6500 \\
\hline Vs $(\mathrm{m} / \mathrm{s})$ & 150 & 2700 & 3500 & 3500 & 3500 & 3500 & 3500 & 3500 & 3500 \\
\hline $\begin{array}{l}\text { Density } \\
\left(\mathrm{kg} / \mathrm{m}^{3}\right)\end{array}$ & 1600 & 2700 & 2800 & 2800 & 2800 & 2800 & 2800 & 2800 & 2800 \\
\hline Q & 100 & 1000 & 1000 & 1000 & 1000 & 1000 & 1000 & 1000 & 1000 \\
\hline \multicolumn{10}{|c|}{ Simulation Properties } \\
\hline \multicolumn{3}{|c|}{ Parameter } & \multicolumn{3}{|c|}{ Value } & & & & \\
\hline \multicolumn{3}{|c|}{ Min grid size } & \multicolumn{2}{|r|}{$4.79 \mathrm{~m}$} & & & & & \\
\hline \multirow{2}{*}{\multicolumn{3}{|c|}{ Total number of time steps }} & \multicolumn{2}{|r|}{$0.0001 \mathrm{~s}$} & & & & & \\
\hline & & & \multicolumn{2}{|r|}{1200000} & & & & & \\
\hline \multicolumn{3}{|c|}{ Total time simulated } & \multicolumn{2}{|r|}{$120 s$} & & & & & \\
\hline \multicolumn{3}{|c|}{ Courant Number } & \multirow{2}{*}{\multicolumn{2}{|c|}{0.4683}} & & & & & \\
\hline \multicolumn{4}{|c|}{ Computer Resources } & & & & & & \\
\hline \multicolumn{3}{|c|}{$\begin{array}{c}\text { System used } \\
\text { Number of Processors }\end{array}$} & & $\begin{array}{c}P C \\
8\end{array}$ & & & & & \\
\hline
\end{tabular}




\begin{tabular}{c|c|} 
Run Time $(\mathrm{sec})$ & 63486.61 \\
Total Core*hours & 141.08 \\
\hline
\end{tabular}

Simulation 4: Section 6.2.1

\begin{tabular}{|c|c|c|c|c|c|c|c|c|c|}
\hline \multicolumn{10}{|c|}{ Volume Properties } \\
\hline Parameter & $\begin{array}{c}\text { Surface } \\
1\end{array}$ & $\begin{array}{c}\text { Surface } \\
2\end{array}$ & $\begin{array}{c}\text { Surface } \\
3\end{array}$ & $\begin{array}{c}\text { Surface } \\
4\end{array}$ & $\begin{array}{c}\text { Surface } \\
5\end{array}$ & $\begin{array}{c}\text { Surface } \\
6\end{array}$ & $\begin{array}{c}\text { Surface } \\
7\end{array}$ & $\begin{array}{c}\text { Surface } \\
8\end{array}$ & $\begin{array}{c}\text { Surface } \\
9\end{array}$ \\
\hline $\mathrm{Vp}(\mathrm{m} / \mathrm{s})$ & 1650 & 6500 & 6500 & 6500 & 6500 & 6500 & 6500 & 6500 & 6500 \\
\hline Vs $(\mathrm{m} / \mathrm{s})$ & 250 & 3500 & 3500 & 3500 & 3500 & 3500 & 3500 & 3500 & 3500 \\
\hline $\begin{array}{l}\text { Density } \\
\left(\mathrm{kg} / \mathrm{m}^{3}\right)\end{array}$ & 1600 & 2800 & 2800 & 2800 & 2800 & 2800 & 2800 & 2800 & 2800 \\
\hline Q & 100 & 1000 & 1000 & 1000 & 1000 & 1000 & 1000 & 1000 & 1000 \\
\hline \multicolumn{10}{|c|}{ Simulation Properties } \\
\hline \multicolumn{3}{|c|}{ Parameter } & \multicolumn{3}{|c|}{ Value } & & & & \\
\hline \multicolumn{3}{|c|}{ Min grid size } & \multicolumn{2}{|r|}{$4.79 m$} & & & & & \\
\hline \multicolumn{3}{|c|}{ Time step } & \multicolumn{2}{|r|}{$0.0001 \mathrm{~s}$} & & & & & \\
\hline \multicolumn{3}{|c|}{ Total number of time steps } & \multicolumn{2}{|r|}{1200000} & & & & & \\
\hline \multicolumn{3}{|c|}{ Total time simulated } & \multicolumn{2}{|r|}{$120 s$} & & & & & \\
\hline & urant Nur & nber & & 0.5073 & & & & & \\
\hline \multicolumn{6}{|c|}{ Computer Resources } & & & & \\
\hline \multicolumn{3}{|c|}{ System used } & \multicolumn{2}{|r|}{ PC } & & & & & \\
\hline \multicolumn{3}{|c|}{ Number of Processors } & \multicolumn{2}{|r|}{8} & & & & & \\
\hline \multicolumn{3}{|c|}{ Run Time (sec) } & \multicolumn{2}{|r|}{60542.24} & & & & & \\
\hline \multicolumn{3}{|c|}{ Total Core*hours } & \multicolumn{2}{|r|}{134.54} & & & & & \\
\hline
\end{tabular}

Simulation 5: Section 6.2.1

\begin{tabular}{|c|c|c|c|c|c|c|c|c|c|}
\hline \multicolumn{10}{|c|}{ Volume Properties } \\
\hline Parameter & \begin{tabular}{|c|} 
Surface \\
1 \\
\end{tabular} & $\begin{array}{c}\text { Surface } \\
2\end{array}$ & $\begin{array}{c}\text { Surface } \\
3\end{array}$ & $\begin{array}{c}\text { Surface } \\
4\end{array}$ & $\begin{array}{c}\text { Surface } \\
5\end{array}$ & $\begin{array}{c}\text { Surface } \\
6\end{array}$ & $\begin{array}{c}\text { Surface } \\
7\end{array}$ & $\begin{array}{c}\text { Surface } \\
8\end{array}$ & $\begin{array}{c}\text { Surface } \\
9\end{array}$ \\
\hline $\mathrm{Vp}(\mathrm{m} / \mathrm{s})$ & 1650 & 6000 & 6500 & 6500 & 6500 & 6500 & 6500 & 6500 & 6500 \\
\hline Vs (m/s) & 250 & 2700 & 3500 & 3500 & 3500 & 3500 & 3500 & 3500 & 3500 \\
\hline $\begin{array}{l}\text { Density } \\
\left(\mathrm{kg} / \mathrm{m}^{3}\right)\end{array}$ & 1600 & 2700 & 2800 & 2800 & 2800 & 2800 & 2800 & 2800 & 2800 \\
\hline $\mathrm{Q}$ & 100 & 1000 & 1000 & 1000 & 1000 & 1000 & 1000 & 1000 & 1000 \\
\hline \multicolumn{10}{|c|}{ Simulation Properties } \\
\hline \multicolumn{3}{|c|}{ Parameter } & \multicolumn{3}{|c|}{ Value } & & & & \\
\hline \multicolumn{3}{|c|}{ Min grid size } & \multicolumn{2}{|r|}{$4.79 m$} & & & & & \\
\hline
\end{tabular}




\begin{tabular}{|c|c|} 
Time step & $0.0001 \mathrm{~s}$ \\
Total number of time steps & 1200000 \\
Total time simulated & $120 \mathrm{~s}$ \\
Courant Number & 0.4683 \\
\hline \multicolumn{2}{|c|}{ Computer Resources } \\
\hline System used & PC \\
Number of Processors & 8 \\
Run Time (sec) & 60043.87 \\
Total Core*hours & 133.43 \\
\hline
\end{tabular}

Simulation 6: Section 6.2.2

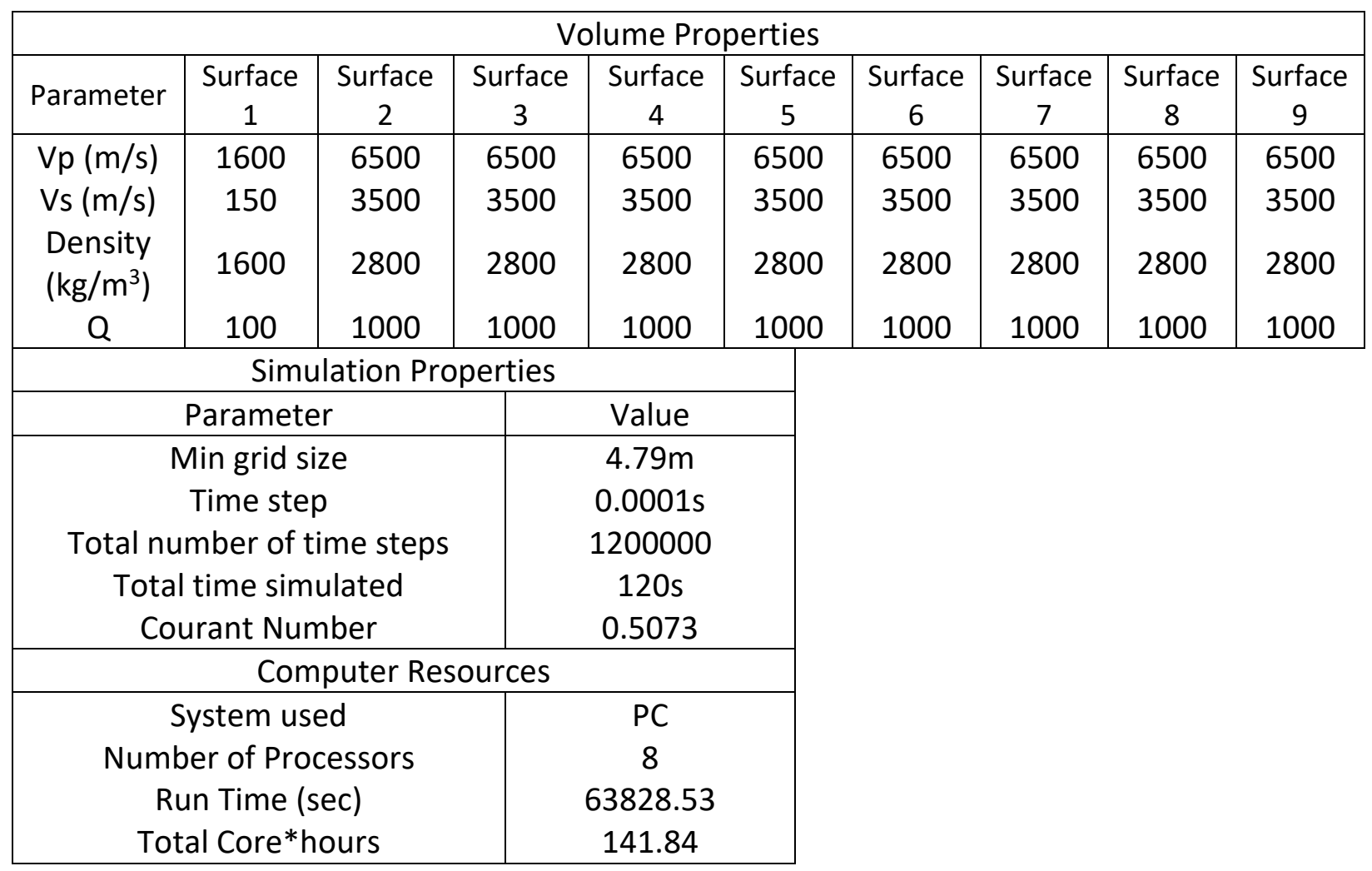

Simulation 7: Section 6.2.2

\begin{tabular}{|c|c|c|c|c|c|c|c|c|c|}
\hline \multicolumn{10}{|c|}{ Volume Properties } \\
\hline Parameter & Surface & Surface & Surface & Surface & Surface & Surface & Surface & Surface \\
8 & Surface \\
& 1 & 2 & 3 & 4 & 5 & 6 & 7 & 8 \\
\hline $\mathrm{Vp}(\mathrm{m} / \mathrm{s})$ & 1600 & 6000 & 6500 & 6500 & 6500 & 6500 & 6500 & 6500 & 6500
\end{tabular}




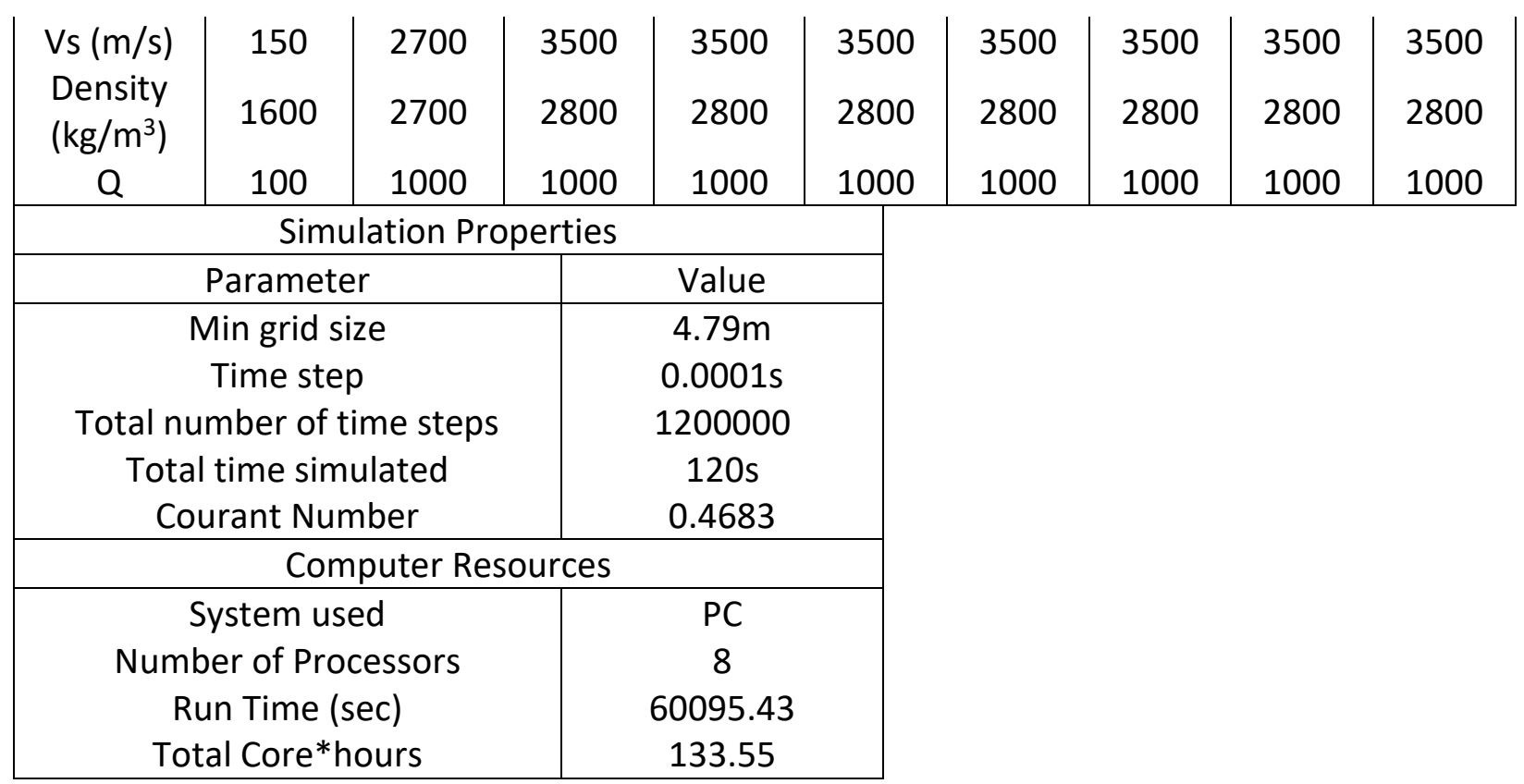

Simulation 8: Section 6.2.2

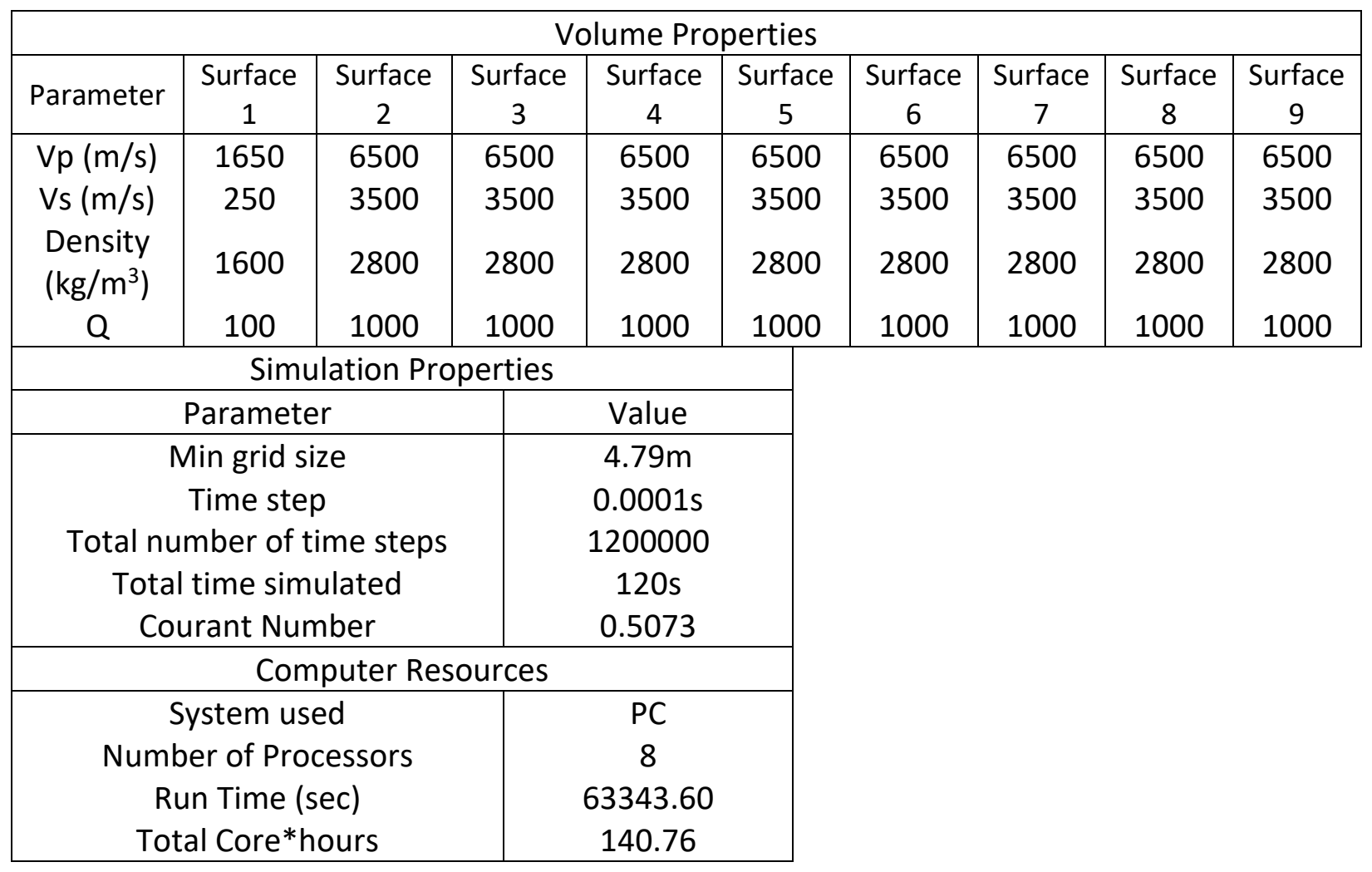


Simulation 9: Section 6.2.2

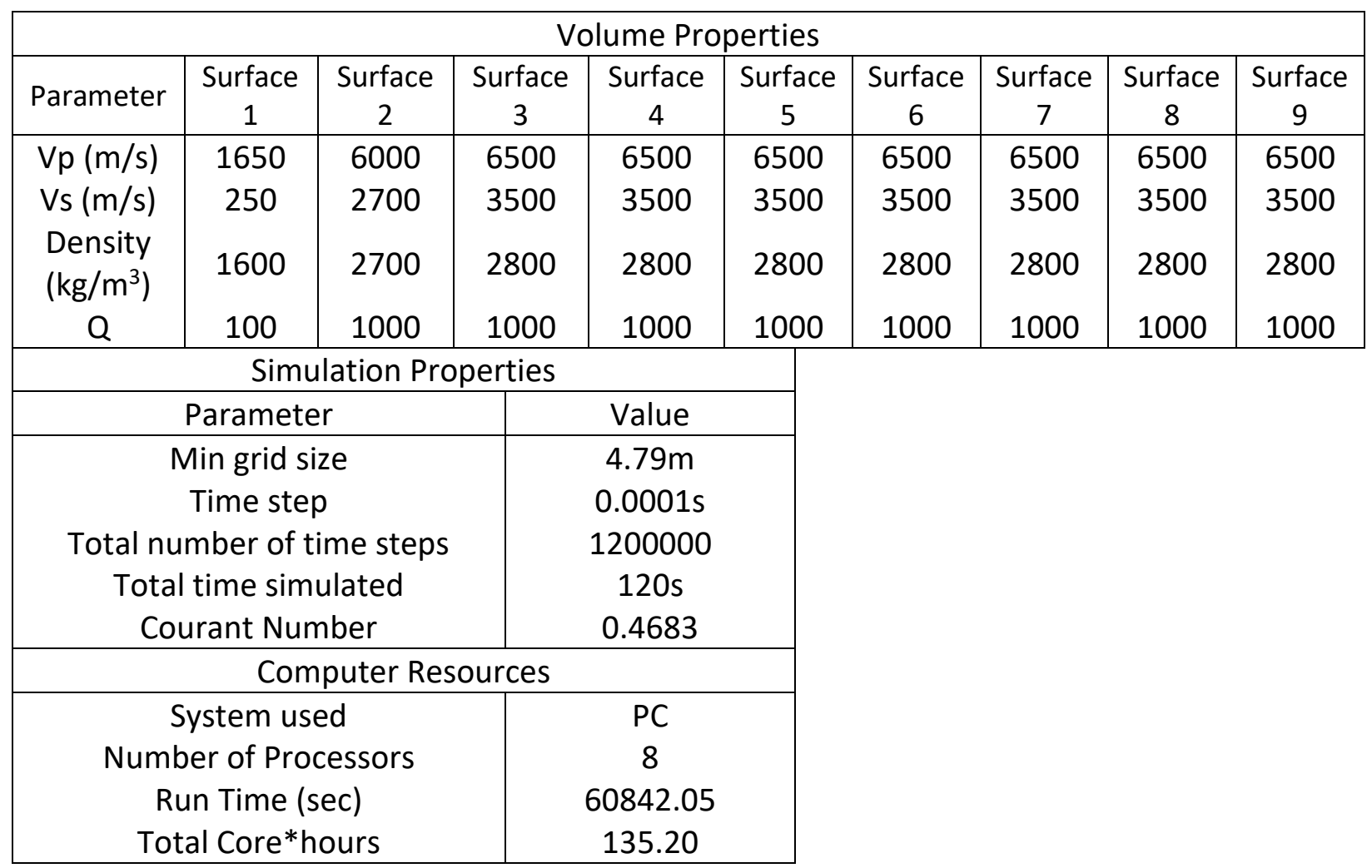

Simulation 10: Section 6.2.2

\begin{tabular}{|c|c|c|c|c|c|c|c|c|c|}
\hline \multicolumn{10}{|c|}{ Volume Properties } \\
\hline Parameter & \begin{tabular}{|c} 
Surface \\
1
\end{tabular} & $\begin{array}{c}\text { Surface } \\
2\end{array}$ & $\begin{array}{c}\text { Surface } \\
3\end{array}$ & $\begin{array}{c}\text { Surface } \\
4\end{array}$ & $\begin{array}{c}\text { Surface } \\
5\end{array}$ & $\begin{array}{c}\text { Surface } \\
6\end{array}$ & $\begin{array}{c}\text { Surface } \\
7\end{array}$ & $\begin{array}{c}\text { Surface } \\
8\end{array}$ & $\begin{array}{c}\text { Surface } \\
9\end{array}$ \\
\hline $\mathrm{Vp}(\mathrm{m} / \mathrm{s})$ & 1650 & 6000 & 6500 & 6500 & 6500 & 6500 & 6500 & 6500 & 6500 \\
\hline $\mathrm{Vs}(\mathrm{m} / \mathrm{s})$ & 275 & 2700 & 3500 & 3500 & 3500 & 3500 & 3500 & 3500 & 3500 \\
\hline $\begin{array}{l}\text { Density } \\
\left(\mathrm{kg} / \mathrm{m}^{3}\right)\end{array}$ & 1600 & 2700 & 2800 & 2800 & 2800 & 2800 & 2800 & 2800 & 2800 \\
\hline Q & 100 & 1000 & 1000 & 1000 & 1000 & 1000 & 1000 & 1000 & 1000 \\
\hline \multicolumn{10}{|c|}{ Simulation Properties } \\
\hline \multicolumn{3}{|c|}{ Parameter } & \multicolumn{3}{|c|}{ Value } & & & & \\
\hline \multicolumn{3}{|c|}{$\begin{array}{c}\text { Min grid size } \\
\text { Time step } \\
\text { Total number of time steps }\end{array}$} & \multicolumn{2}{|r|}{$\begin{array}{c}4.79 \mathrm{~m} \\
0.0001 \mathrm{~s} \\
1200000\end{array}$} & & & & & \\
\hline
\end{tabular}




\begin{tabular}{|c|c|}
\hline $\begin{array}{l}\text { Total time simulated } \\
\text { Courant Number }\end{array}$ & $\begin{array}{c}120 \mathrm{~s} \\
0.4683\end{array}$ \\
\hline \multicolumn{2}{|c|}{ Computer Resources } \\
\hline System used & PC \\
\hline Number of Processors & 8 \\
\hline Run Time (sec) & 60610.89 \\
\hline Total Core*hours & 134.69 \\
\hline
\end{tabular}

\section{II.C.3. 2D Plane Layered Model}

Description: The Plane Layered model has 8 surfaces. The surfaces are horizontally layered surfaces with boundaries at $100 \mathrm{~m}, 400 \mathrm{~m}, 1.2 \mathrm{~km}, 2.0 \mathrm{~km}, 4.0 \mathrm{~km}, 10 \mathrm{~km}, 20 \mathrm{~km}$, and $30 \mathrm{~km}$. This model was used for two simulations.

\section{Simulation 1: Section 6.1.2}

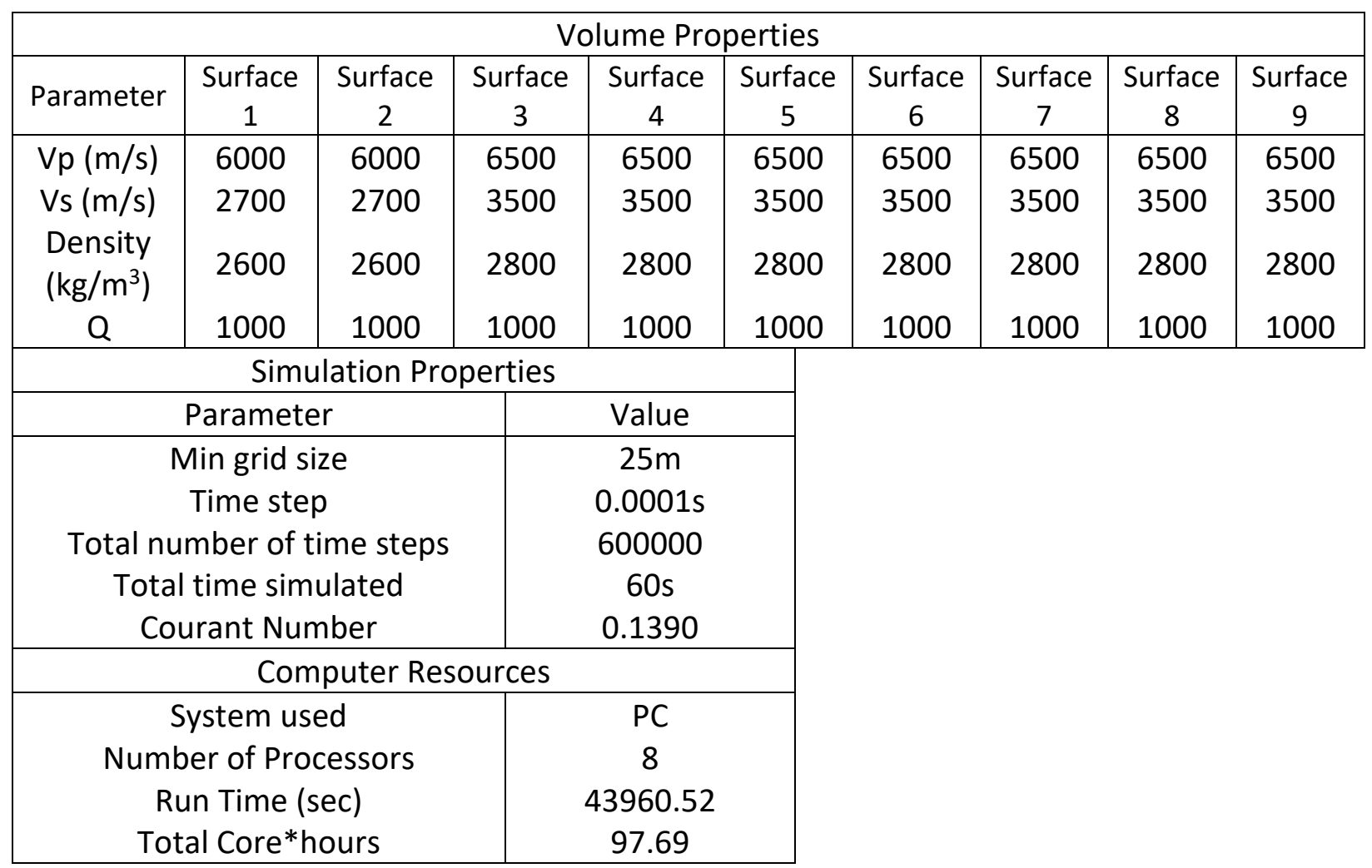


Simulation 2: Section 6.1.2

\begin{tabular}{|c|c|c|c|c|c|c|c|c|c|}
\hline \multicolumn{10}{|c|}{ Volume Properties } \\
\hline Parameter & \begin{tabular}{|c|} 
Surface \\
1
\end{tabular} & $\begin{array}{c}\text { Surface } \\
2\end{array}$ & $\begin{array}{c}\text { Surface } \\
3\end{array}$ & $\begin{array}{c}\text { Surface } \\
4\end{array}$ & $\begin{array}{c}\text { Surface } \\
5\end{array}$ & $\begin{array}{c}\text { Surface } \\
6\end{array}$ & $\begin{array}{c}\text { Surface } \\
7\end{array}$ & $\begin{array}{c}\text { Surface } \\
8\end{array}$ & $\begin{array}{c}\text { Surface } \\
9\end{array}$ \\
\hline $\mathrm{Vp}(\mathrm{m} / \mathrm{s})$ & 1600 & 6000 & 6500 & 6500 & 6500 & 6500 & 6500 & 6500 & 6500 \\
\hline $\mathrm{Vs}(\mathrm{m} / \mathrm{s})$ & 150 & 2700 & 3500 & 3500 & 3500 & 3500 & 3500 & 3500 & 3500 \\
\hline $\begin{array}{l}\text { Density } \\
\left(\mathrm{kg} / \mathrm{m}^{3}\right)\end{array}$ & 1600 & 2600 & 2800 & 2800 & 2800 & 2800 & 2800 & 2800 & 2800 \\
\hline Q & 100 & 1000 & 1000 & 1000 & 1000 & 1000 & 1000 & 1000 & 1000 \\
\hline \multicolumn{6}{|c|}{ Simulation Properties } & & & & \\
\hline \multicolumn{3}{|c|}{ Parameter } & \multicolumn{3}{|c|}{ Value } & & & & \\
\hline $\begin{array}{r}\text { Total } n \\
\text { Tot } \\
\mathrm{C}\end{array}$ & $\begin{array}{l}\text { Min grid } \mathrm{s} \\
\text { Time ste } \\
\text { mber of } t \\
\text { time sim } \\
\text { urant Nur }\end{array}$ & $\begin{array}{l}\text { me steps } \\
\text { lated } \\
\text { lber }\end{array}$ & & $\begin{array}{c}25 \mathrm{~m} \\
0.0001 \mathrm{~s} \\
600000 \\
60 \mathrm{~s} \\
0.1390\end{array}$ & & & & & \\
\hline \multicolumn{6}{|c|}{ Computer Resources } & & & & \\
\hline $\begin{array}{r}\text { Num } \\
\mathrm{TC} \\
\mathrm{T}\end{array}$ & $\begin{array}{l}\text { ystem us } \\
\text { er of Pro } \\
\text { un Time ( } \\
\text { al Core*r }\end{array}$ & $\begin{array}{l}\text { d } \\
\text { essors } \\
\text { ec) } \\
\text { ours }\end{array}$ & & $\begin{array}{c}P C \\
8 \\
43907.26 \\
97.69 \\
\end{array}$ & & & & & \\
\hline
\end{tabular}

\section{II.C.4. $\quad$ 2D Kinburn NESW Profile Model: Section 6.1.3}

Description: The Kinburn NWSE model has 2 surfaces. One surface is the shape of a Northeastto-Southwest profile of the Kinburn basin as derived from geophysical measurements. The second surface extends $10 \mathrm{~km}$ wide with a depth of $1 \mathrm{~km}$ surrounding the Kinburn Basin surface. This model was used for one simulation.

\begin{tabular}{|c|c|c|}
\hline Parameter & Surface 1 & Surface 2 \\
\hline Vp (m/s) & 1600 & 6000 \\
Vs (m/s) & 150 & 2700 \\
Density & 1600 & 2700 \\
$\left(\mathrm{~kg} / \mathrm{m}^{3}\right)$ & 70 & 1000 \\
$\mathrm{Q}$ & Simulation Properties \\
\hline \multicolumn{2}{|c|}{ Parameter } \\
\hline \multicolumn{2}{|c|}{} \\
\hline
\end{tabular}




\begin{tabular}{|c|c|}
\hline $\begin{array}{c}\text { Min grid size } \\
\text { Time step } \\
\text { Total number of time steps } \\
\text { Total time simulated } \\
\text { Courant Number }\end{array}$ & $\begin{array}{c}5.16 \mathrm{~m} \\
0.000025 \mathrm{~s} \\
950000 \\
23.75 \mathrm{~s} \\
0.1684 \\
\end{array}$ \\
\hline \multicolumn{2}{|c|}{ Computer Resources } \\
\hline $\begin{array}{c}\text { System used } \\
\text { Number of Processors } \\
\text { Run Time (sec) } \\
\text { Total Core*hours }\end{array}$ & $\begin{array}{c}P C \\
8 \\
35697.73 \\
79.32\end{array}$ \\
\hline
\end{tabular}

\section{II.C.5. 2D Kinburn NWSE Profile Model: Section 6.1.3}

Description: The Kinburn NWSE model has 2 surfaces. One surface is the shape of a Northwestto-Southeast profile of the Kinburn basin as derived from geophysical measurements. The second surface extends $10 \mathrm{~km}$ wide with a depth of $1 \mathrm{~km}$ surrounding the Kinburn Basin surface. This model was used for one simulation.

\begin{tabular}{|c|c|c|}
\hline Parameter & Surface 1 & Surface 2 \\
\hline Vp (m/s) & 1600 & 6000 \\
Vs (m/s) & 150 & 2700 \\
Density & 1600 & 2700 \\
$\left(\mathrm{~kg} / \mathrm{m}^{3}\right)$ & 70 & 1000 \\
$\mathrm{Q}$ & Simulation Properties \\
\hline \multicolumn{2}{|c|}{ Varameter } & $3.63 \mathrm{~m}$ \\
\hline \multicolumn{2}{|c|}{ Pin grid size } & $0.000025 \mathrm{~s}$ \\
Time step & 950000 \\
Total number of time steps & $23.75 \mathrm{~s}$ \\
Total time simulated & 0.1737 \\
Courant Number & PC \\
\multicolumn{2}{|c|}{ Computer Resources } \\
\hline \multicolumn{2}{|c|}{ System used } & 8 \\
Number of Processors & 99507.83 \\
Run Time (sec) & 221.12 \\
Total Core*hours
\end{tabular}




\section{II.C.6. $\quad 3 D$ Kinburn Basin Model: Section 6.1.3}

Description: The 3D Kinburn basin model has 2 volumes. One volume is a $6 \mathrm{~km} \times 7.02 \mathrm{~km}$ square with a depth of at least $10 \mathrm{~m}$, and the bottom surface the shape of the Kinburn basin as derived from geophysical measurements. The second surface extends to a depth of $1 \mathrm{~km}$ below the Kinburn Basin volume. This model was used for one simulation.

\begin{tabular}{|c|c|c|}
\hline Parameter & Volume 1 & Volume 2 \\
\hline Vp (m/s) & 1600 & 6000 \\
Vs (m/s) & 150 & 2700 \\
Density & 1600 & 2700 \\
$\left(\mathrm{~kg} / \mathrm{m}^{3}\right)$ & 70 & 1000 \\
$\mathrm{Q}$ & Simulation Properties \\
\hline \multicolumn{2}{|c|}{ Varameter } & $0.16 \mathrm{~m}$ \\
\hline \multicolumn{2}{|c|}{ Min grid size } & $0.000025 \mathrm{~s}$ \\
Time step & 950000 \\
Total number of time steps & $23.75 \mathrm{~s}$ \\
Total time simulated & 0.2500 \\
Courant Number & Mp2 \\
\multicolumn{2}{|c|}{ Computer Resources } \\
\hline \multicolumn{2}{|c|}{ System used } & 480 \\
Number of Processors & 25896.03 \\
Run Time (sec) & \\
Total Core*hours &
\end{tabular}




\section{Appendix III. Signal Processing}

The signal processing completed in this study varied for different purposes. It included determining the instrument response to calculate recorded motions, applying filters to different data sets, and converting acceleration data to displacement. A brief description of each process is described in this appendix, along with the implementation. All signal processing was completed using MATLAB (Simulink).

\section{III.A. Instrument Response}

The instrument response of a system can be given as the Fourier transform of the impulse response of a system. If we have a linear time-invariant system with input $x(t)$, and output $y(t)$, which is the convolution of the input with an impulse response, $h(t)$, given as:

$$
y(t)=\int_{-\infty}^{\infty} x(t) h(t-\tau) d \tau
$$

Then taking a Fourier transform of the system we obtain:

$$
Y(\omega)=H(\omega) X(\omega)
$$

The convolution in the time domain becomes a multiplication in the frequency domain. The instrument response is the transfer function of the system $H(\omega)$, which can be written as:

$$
H(\omega)=\frac{\prod_{j=1}^{m}\left(i \omega-z_{j}\right)}{\prod_{i=1}^{n}\left(i \omega-p_{i}\right)}
$$


where $=\sqrt{-1}, z_{j}$ are the zeros of the system, $p_{i}$ are the poles of the system, $m$ is the number of zeros, and $n$ is the number of poles. Both the zeros and poles of the system are complex numbers.

The poles and zeros for the Guralp CMG 3ESP series seismometers installed in the Kinburn basin are listed in Table III.1.

\begin{tabular}{|c|c|c|c|}
\hline \multicolumn{2}{|c|}{ Zeros } & \multicolumn{2}{c|}{ Poles } \\
\hline$z_{1}$ & 0 & $p_{1}$ & $(0.147+0.147 i)$ \\
\hline$z_{2}$ & 0 & $p_{2}$ & $(-0.147-0.147 i)$ \\
\hline
\end{tabular}

Table III.1: The zeros and poles of a CMG 3ESP by Guralp.

\section{III.B. Filters}

Several filters were applied to different data sets throughout this study. These filters were completed using the Signal Processing Toolbox for MATLAB (Simulink). The filters were created using the zeros, poles and gain method for digital filters, where the transfer function is given as (Simulink):

$$
H(z)=k \frac{\left(1-z(1) z^{-1}\right)\left(1-z(2) z^{-1}\right) \cdots\left(1-z(n) z^{-1}\right)}{\left(1-p(1) z^{-1}\right)\left(1-p(2) z^{-1}\right) \cdots\left(1-p(n) z^{-1}\right)}
$$

Here $z(n)$ are the zeros of the system, $p(n)$ are the poles of the system, and $k$ is the gain. The filter designs were Butterworth filters of different orders, usually order 4 if a weak filter was needed and order 8 or 12 if a strong filter was needed. 
An example script to apply a $4^{\text {th }}$ order Butterworth bandpass filter with cut-off frequencies of 0.1 and $5 \mathrm{~Hz}$ is shown below.

AccelX(1:n) = Acceleration time series in the $X$-direction

Time $(1: n)=$ the time steps for the acceleration data.

fs = sampling frequency

$\mathrm{fn}=\mathrm{fs} / 2$ \#Nyquist frequency

$[z, p, k]=$ butter $\left(4,\left[0.1 / \mathrm{fs}^{*} 2 * \mathrm{pi}, 5 / \mathrm{fs} * 2 * \mathrm{pi}\right],{ }^{\prime}\right.$ bandpass'); \#find the zeros, poles and gain for the filter BF4 = zp2sos $(z, p, k) ; \#$ create a filter object (requires Signal Processing Toolbox)

FiltAccelX = filter(BF4,AccelX); \#filtered acceleration time series

Plot(Time,AccelX,Time,FiltAccelX); \#create a plot showing both the filtered and unfiltered acceleration time series (Figure III.2)

loglog (Time,AccelX,Time,FiltAccelX); \#create a log-log plot showing both the filtered and unfiltered Fourier spectral acceleration (Figure III.3)
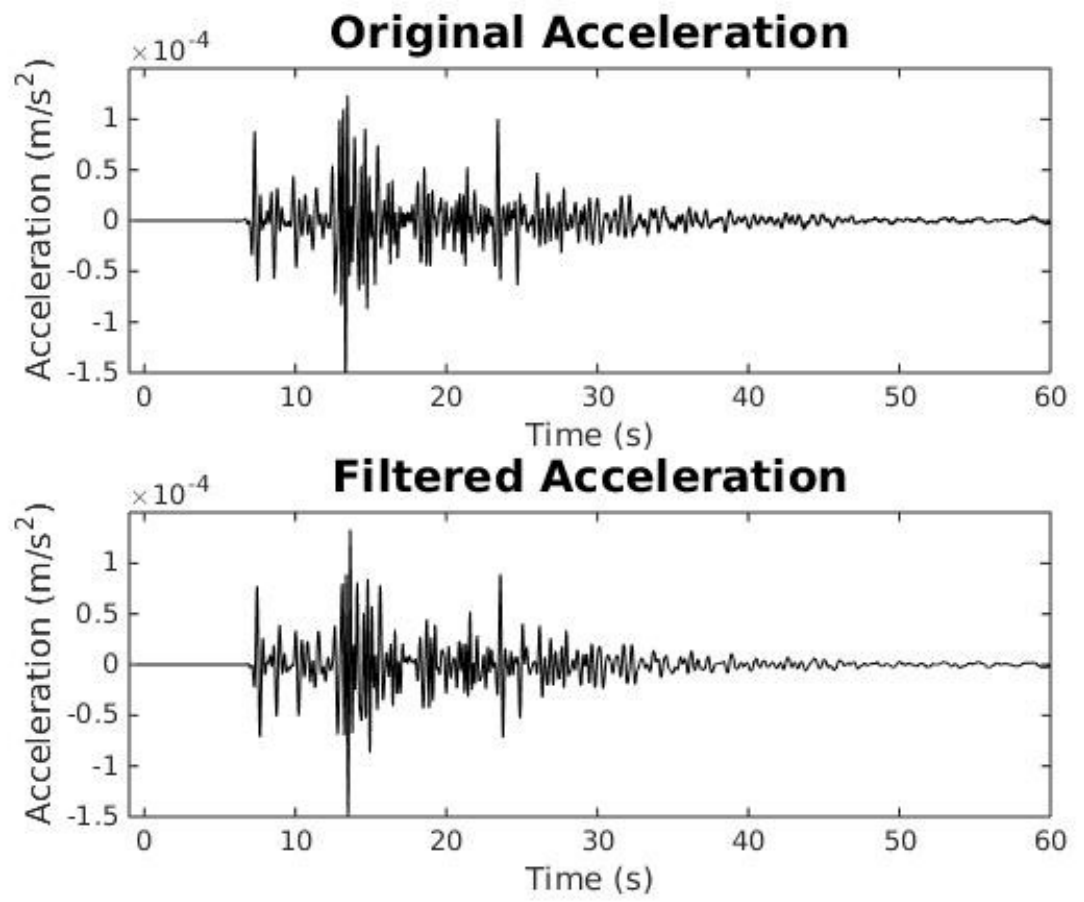

Figure III.2: Acceleration time series without a filter applied (top) and with a 4th order Butterworth bandpass filter with cutoff frequencies 0.1 and $5 \mathrm{~Hz}$ (bottom). 


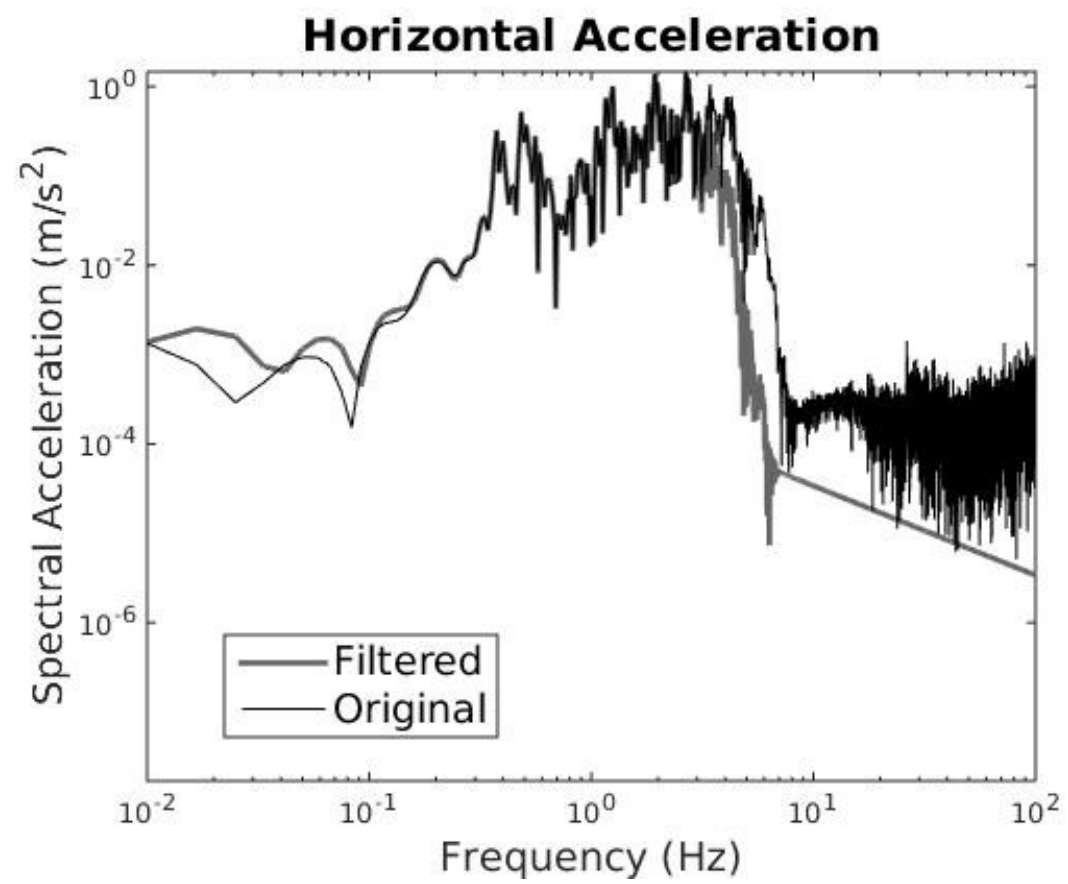

Figure III.3: Spectral acceleration without a filter applied and with a 4th order Butterworth bandpass filter with cutoff frequencies 0.1 and $5 \mathrm{~Hz}$.

\section{III.C. High Frequency Reduction}

As seen in Sections 4.3 and 6.2, the recorded accelerations have a much higher frequency content than the simulated acceleration values. This is due to the low frequency simulation method being used, and the seismicity in eastern North America having high frequency characteristics. In order to compare the simulated values to the recordings, the high frequency content of the recorded motions is reduced as much as possible. This is completed using a strong low-pass filter on the recorded motions. A comparison of using a low-pass filter and using the same filter for both the simulated and recorded motions is discussed below.

An example of the horizontal acceleration for the recorded and simulated motions is shown in Figure III.4 for the acceleration time series and Figure III.5 for the Fourier spectral acceleration. The simulated motions start to drop off in the spectrum at about $2-3 \mathrm{~Hz}$, however 
the recorded motions continue to have a strong presence above these frequencies. The amplitude in the time series is much greater for the recorded motions.
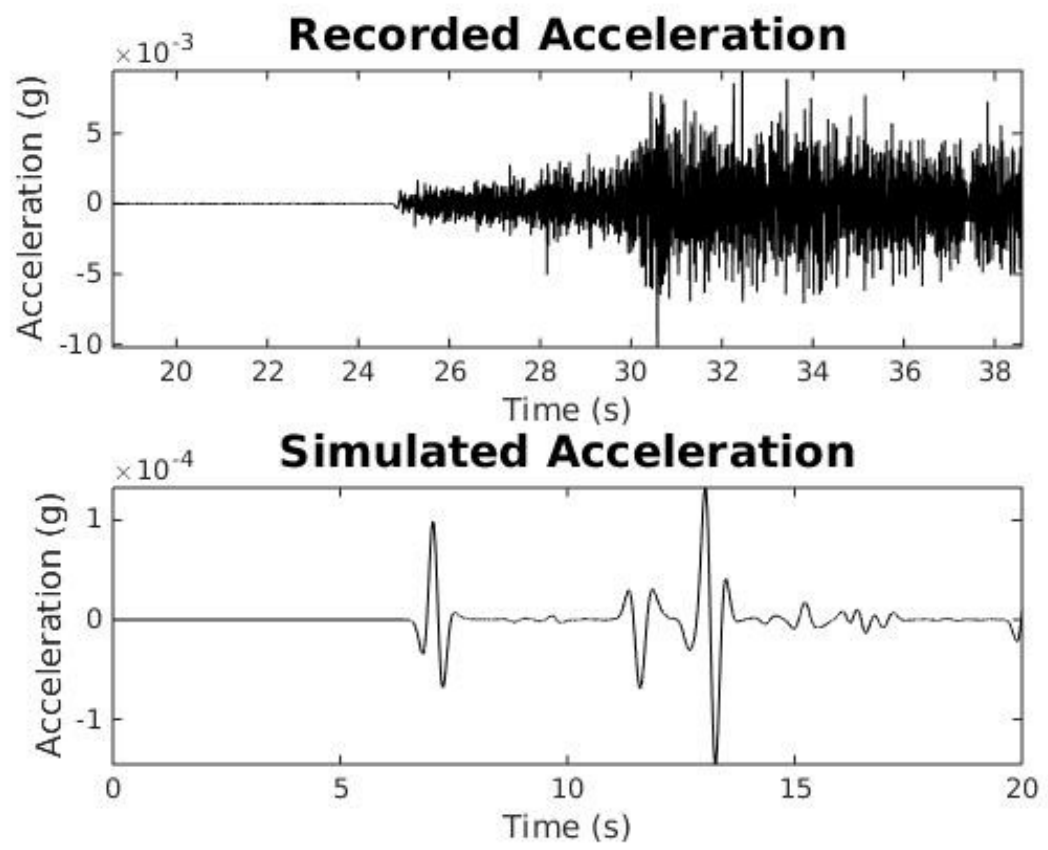

Figure III.4: Recorded acceleration (top) and simulated acceleration (bottom) without any filters applied.

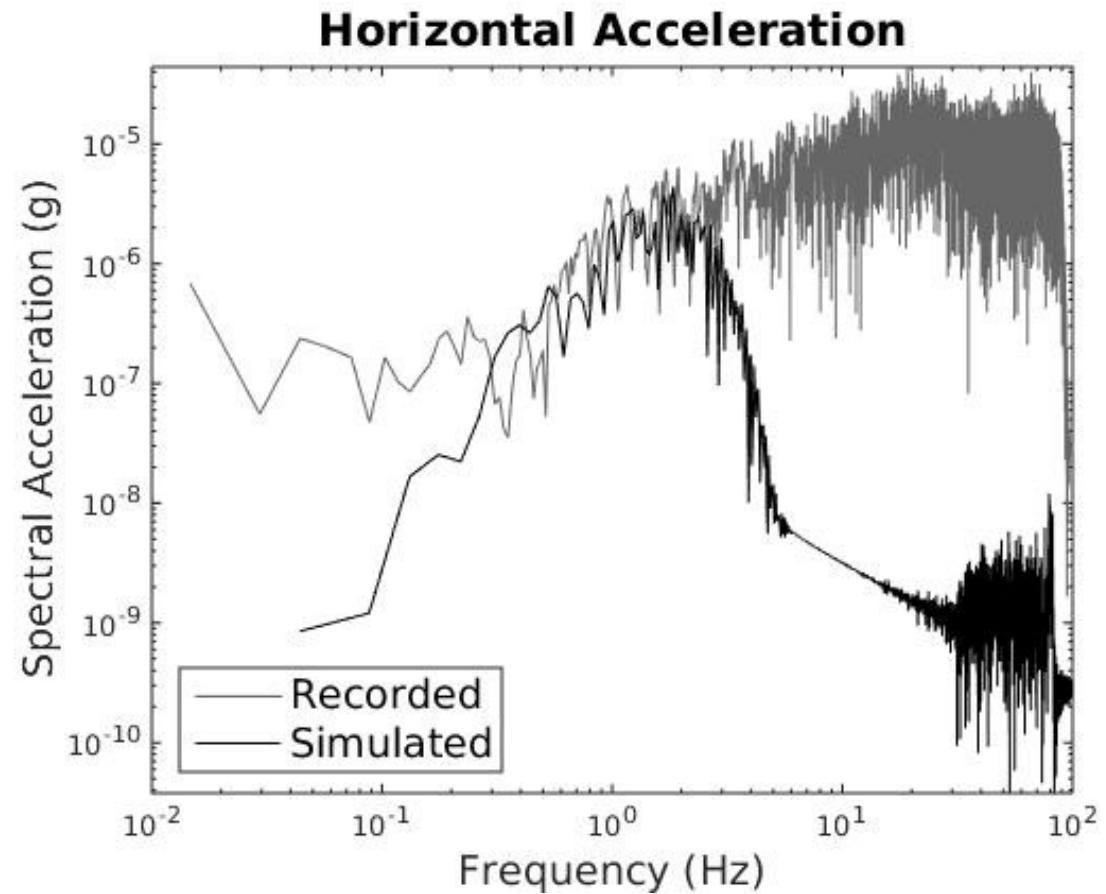

Figure III.5: Recorded spectral acceleration and simulated spectral acceleration without any filters applied. 
Applying a Butterworth bandpass filter of $8^{\text {th }}$ order with cutoff frequencies of 0.3 and $2.5 \mathrm{~Hz}$, the resulting Fourier spectra of motions is shown in Figure III.6. Both the recorded and simulated motions have a decreasing frequency content above $2.5 \mathrm{~Hz}$, however the simulated motions drop off much faster than the recorded motions. As seen in the acceleration time series for the filtered data in Figure III.7, the amplitude of the recorded motion is still greater than the amplitude of the simulated motions.

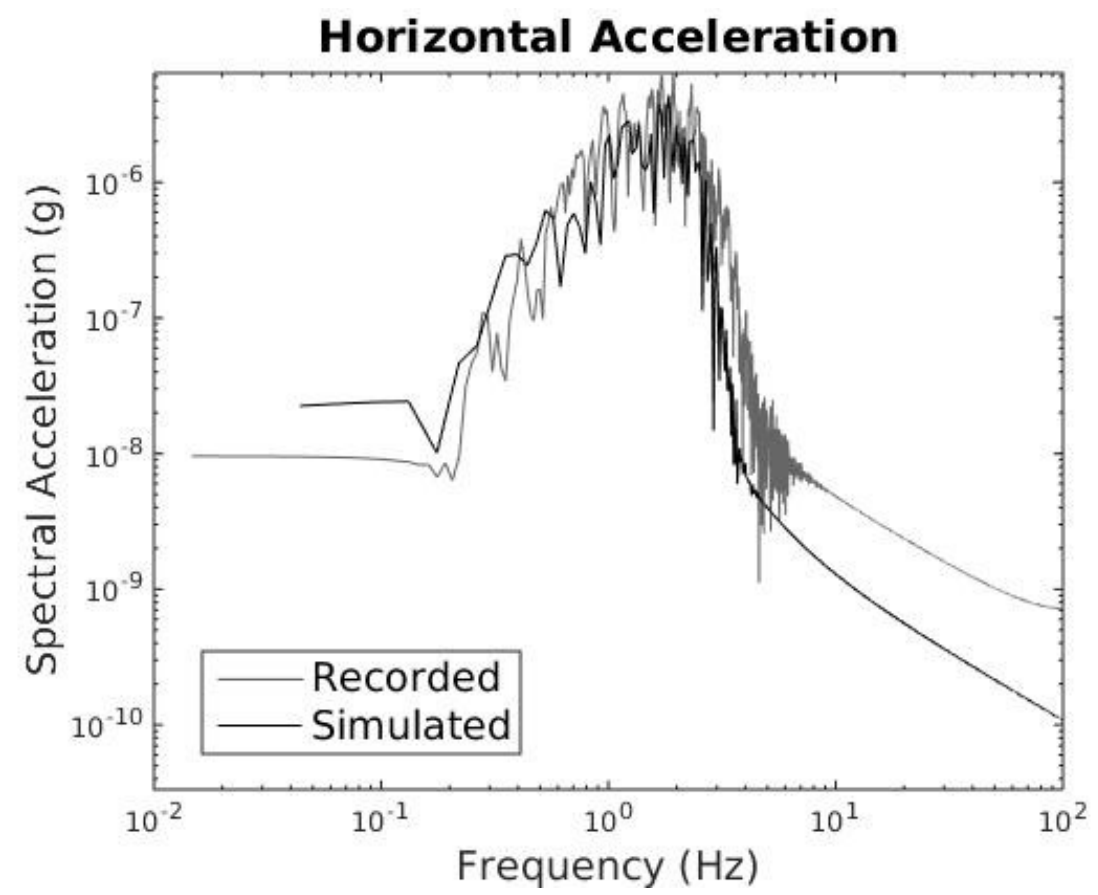

Figure III.6: Recorded spectral acceleration and simulated spectral acceleration with a Butterworth bandpass filter of $8^{\text {th }}$ order with cutoff frequencies of 0.3 and $2.5 \mathrm{~Hz}$ applied. 

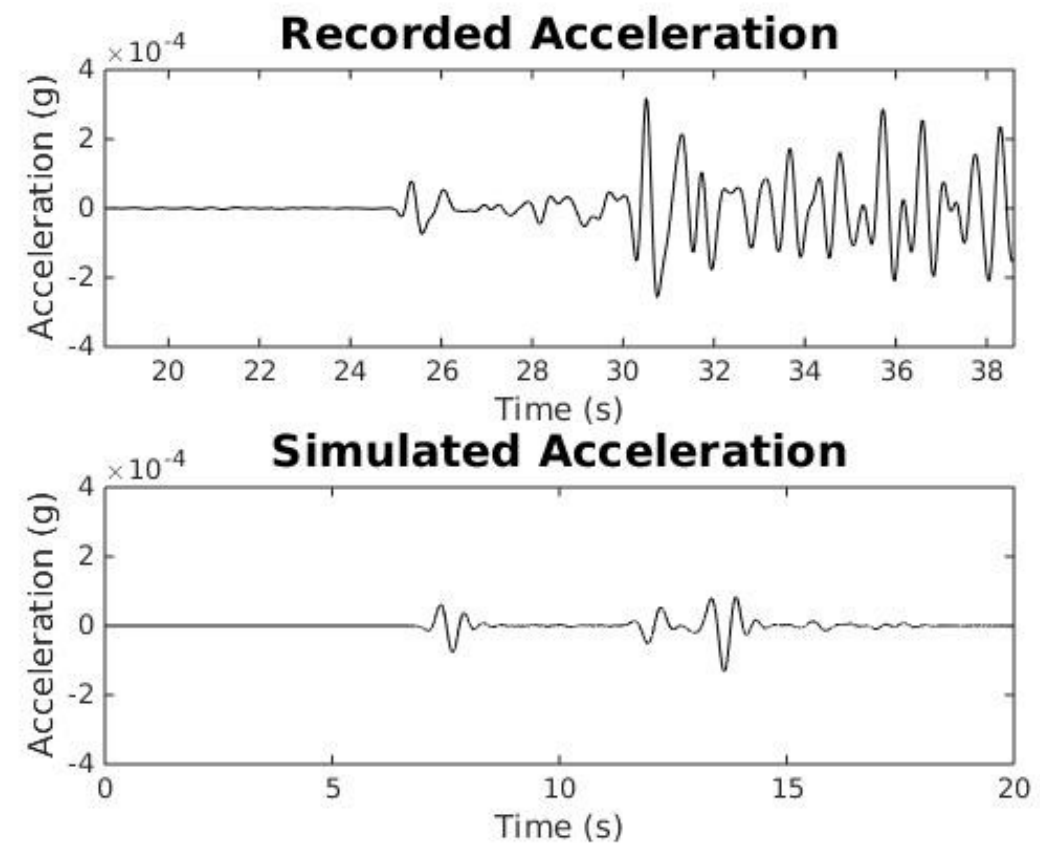

Figure III.7: Recorded acceleration (top) and simulated acceleration (bottom) Butterworth bandpass filter of 8th order with cutoff frequencies of 0.3 and $2.5 \mathrm{~Hz}$

A low-pass Butterworth filter of order 12 and cutoff frequency of $2.5 \mathrm{~Hz}$ is applied only to the recorded acceleration. Figure III.8 shows the Fourier spectra of both the simulated motion with the bandpass filter applied, and the recorded motion with a bandpass and low-pass filter applied. Figure III.9 displays the acceleration time series for the motions with the above filters. Here the recorded spectral acceleration drops off at a more comparable rate to the simulated motions. 


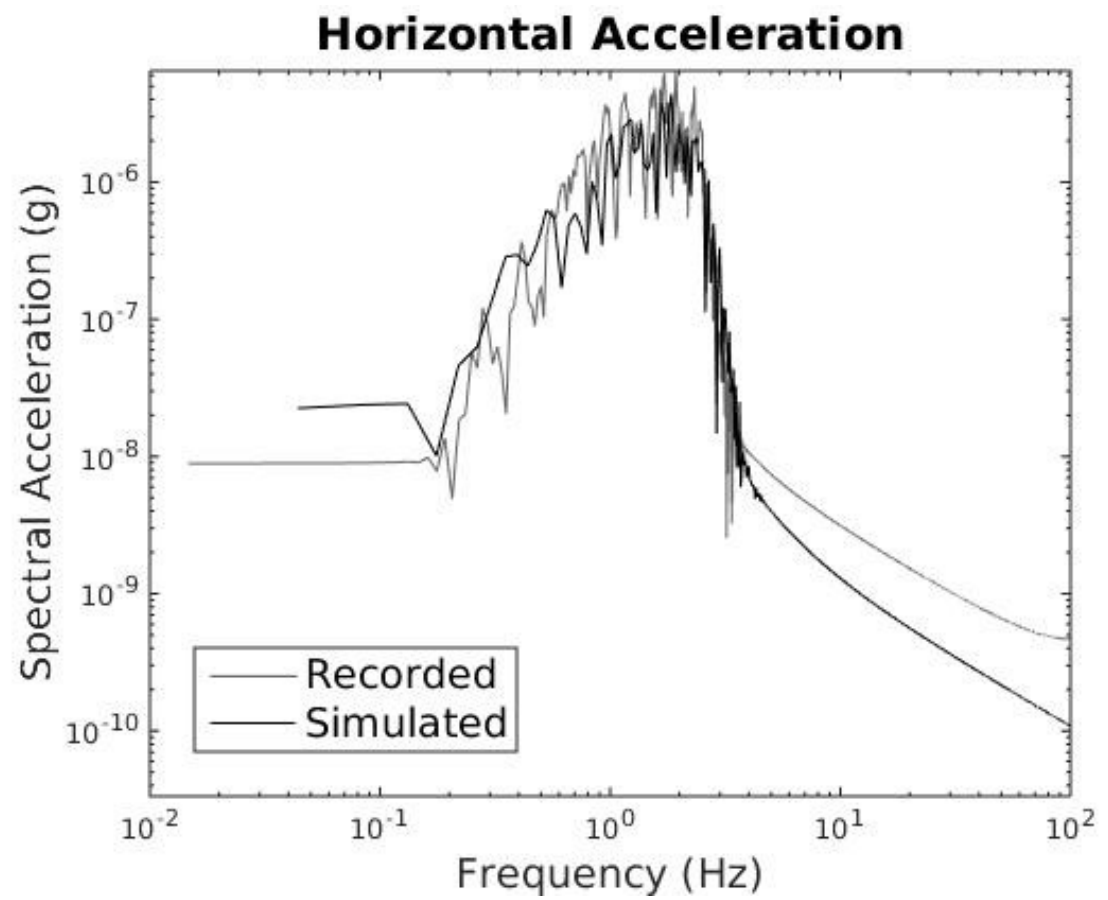

Figure III.8: Recorded spectral acceleration and simulated spectral acceleration with a Butterworth bandpass filter of 8th order with cutoff frequencies of 0.3 and $2.5 \mathrm{~Hz}$ applied, and a 12th order low-pass Butterworth filter with cutoff frequency of $2.5 \mathrm{~Hz}$ applied to the recorded acceleration.
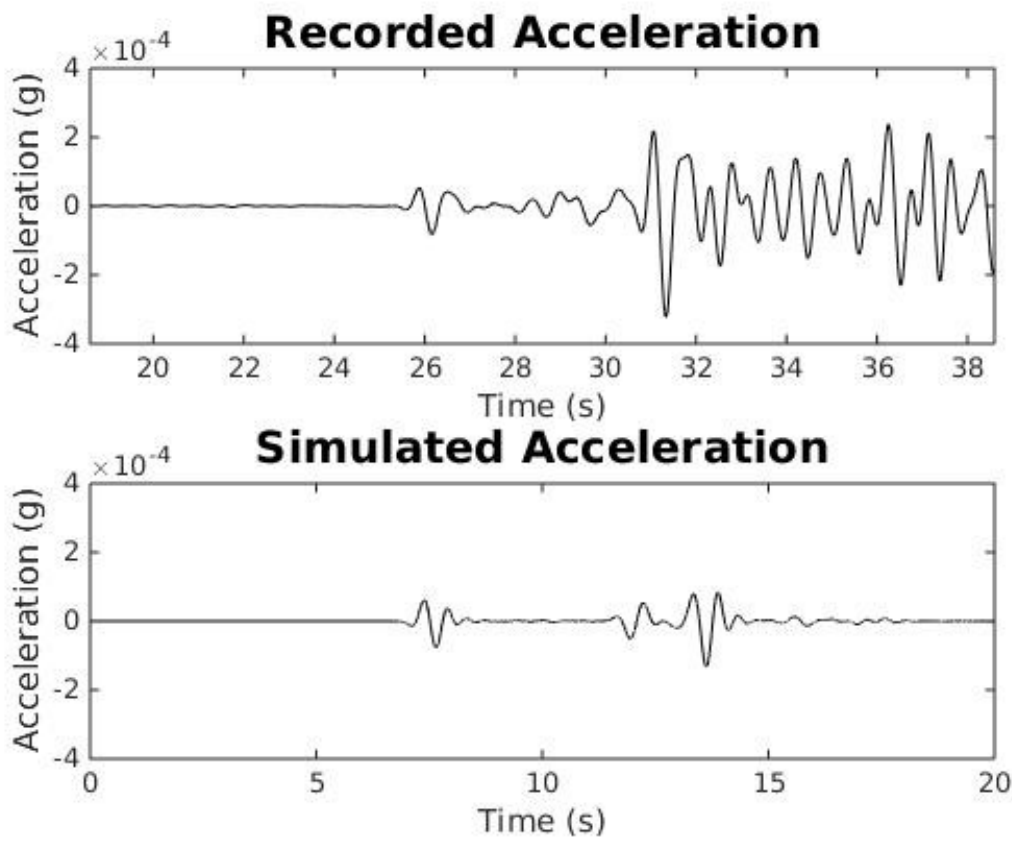

Figure III.9: Recorded acceleration (top) and simulated acceleration (bottom) Butterworth bandpass filter of 8th order with cutoff frequencies of 0.3 and $2.5 \mathrm{~Hz}$, and a 12th order low-pass Butterworth filter with cutoff frequency of $2.5 \mathrm{~Hz}$ applied to the recorded acceleration. 


\section{III.D. Acceleration to Displacement}

In order to obtain the displacement time series when the values were given as an acceleration time series, there were a few steps which needed to be followed. All steps were completed in MATLAB (Simulink), a computer software for mathematical solutions. The first step was to use the cumulative trapezoidal rule to integrate the acceleration values given the time steps. The trapezoidal rule in the general form is given as:

$$
\int_{a}^{b} f(t) \approx(b-a)\left[\frac{f(a)+f(b)}{2}\right]
$$

where $f$ is a function of $t$, evaluated at points a and $\mathrm{b}$. Applying the cumulative relationship for the acceleration the velocity time series is calculated. When present, the instrument drift response is removed from the velocity time series using a highpass filter to remove low frequencies (see above).

After the velocity time series is corrected, the cumulative trapezoidal rule is applied to this function, resulting in the displacement time series. Again, when present, the instrument drift is removed from this data using a highpass filter. Figure III.10 shows the steps in order to calculate the displacement time series from an acceleration time series. 

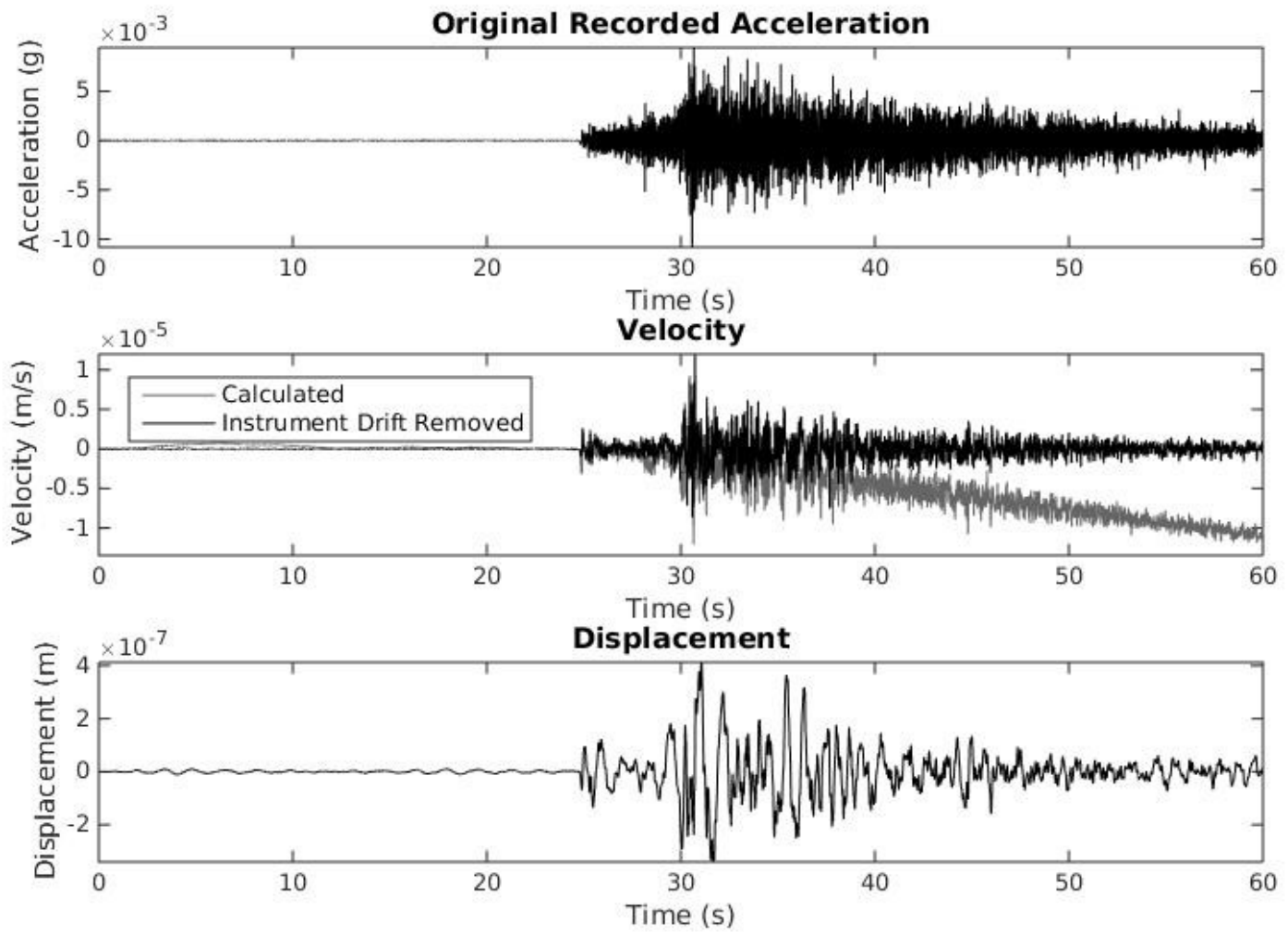

Figure III.10: The steps to calculating a displacement time series from acceleration data. The top plot is the original acceleration. The middle plot shows the calculated velocity, and the velocity time series with the instrument drift removed. The bottom plot is the resulating displacement time series. 